PETER SCHMIDT

\title{
DIE WAHL DES \\ RENTENALTERS
}




\section{PETER SCHMIDT}

\section{DIE WAHL DES RENTENALTERS}

Die Alterung der westlichen Industrienationen führt $\mathrm{zu}$ einer erheblichen Belastung der Sozialversicherungssysteme. Der Autor zeigt dies am Beispiel der bundesdeutschen Rentenversicherung auf. Die ökonomische Analyse ergibt, daß die deutsche Gesetzliche Rentenversicherung (GRV) deutliche Anreize zur Frühverrentung setzt, deren Abbau das Sozialversicherungssystem merklich entlasten würde. Das Rentenzugangsverhalten in Deutschland wird in einem Verweildauermodell mikroökonometrisch untersucht. Der Autor schlägt eine neuerliche Rentenreform in Form einer geänderten Rentenberechnung vor und zeigt anhand von Simulationsrechnungen deren Entlastungswirkungen für die GRV-Finanzen auf. Die Arbeit behandelt ein aktuelles Feld der sozialpolitischen Diskussion und leistet einen wissenschaftlichen Beitrag zur Versachlichung der öffentlichen Diskussion.

Peter Schmidt studierte Wirtschafts- und Sozialwissenschaften an der Universität Dortmund. Nach dem Abschluß als Diplom-Volkswirt arbeitete er von 1989 bis 1994 am Lehrstuhl für Makroökonomik und Wirtschaftspolitik an der Universität Mannheim im Gebiet Sozialpolitik. Derzeit arbeitet der Autor am Zentrum für Europäische Wirtschaftsforschung ZEW in Mannheim im Forschungsbereich Industrieökonomik mit dem Schwerpunkt Regionalökonomie. 
Die Wahl des Rentenalters

Theoretische und empirische Analyse des Rentenzugangsverhaltens

in West- und Ostdeutschland 


\section{ALLOKATION IM MARKTWIRTSCHAFTLICHEN SYSTEM}

\section{Herausgegeben von}

Heinz König, Hans-Heinrich Nachtkamp,

Ulrich Schlieper, Eberhard Wille

Band 36

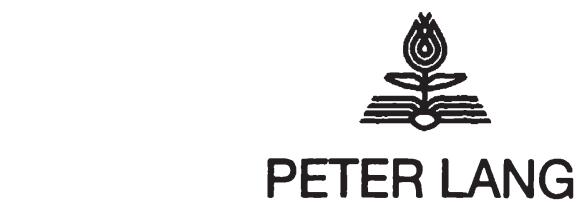

Frankfurt am Main · Berlin · Bern · New York · Paris · Wien

Peter Schmidt - 978-3-631-75568-6

Downloaded from PubFactory at 01/11/2019 03:28:55AM

via free access 


\section{PETER SCHMIDT}

\section{DIE WAHL \\ DES RENTENALTERS \\ Theoretische und empirische Analyse des Rentenzugangsverhaltens in West- und Ostdeutschland}

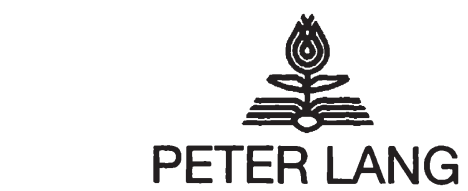

Europäischer Verlag der Wissenschaften 
Die Deutsche Bibliothek - CIP-Einheitsaufnahme

Schmidt, Peter:

Die Wahl des Rentenalters : theoretische und empirische Analyse des Rentenzugangsverhaltens in West- und Ostdeutschland / Peter Schmidt. - Frankfurt am Main ; Berlin ; Bern ; New York ; Paris ; Wien : Lang, 1995

(Allokation im marktwirtschaftlichen System ; Bd. 36)

Zugl.: Mannheim, Univ., Diss., 1994

ISBN 3-631-48903-X

Open Access: The online version of this publication is published on www.peterlang.com and www.econstor.eu under the international Creative Commons License CC-BY 4.0. Learn more on how you can use and share this work: http://creativecommons. org/licenses/by/4.0.

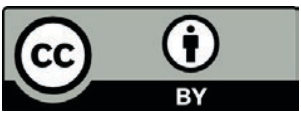

This book is available Open Access thanks to the kind support of ZBW - Leibniz-Informationszentrum Wirtschaft.

NE: GT

\author{
D 180 \\ ISSN 0939-7728 \\ ISBN 3-631-48903-X \\ ISBN 978-3-631-75568-6 (eBook) \\ (C) Peter Lang GmbH \\ Europäischer Verlag der Wissenschaften \\ Frankfurt am Main 1995 \\ Alle Rechte vorbehalten.
}

Das Werk einschließlich aller seiner Teile ist urheberrechtlich geschützt. Jede Verwertung außerhalb der engen Grenzen des Urheberrechtsgesetzes ist ohne Zustimmung des Verlages unzulässig und strafbar. Das gilt insbesondere für

Vervielfältigungen, Übersetzungen, Mikroverfilmungen und die Einspeicherung und Verarbeitung in elektronischen Systemen. 
für Jutta,

Maria, Korbinian und Dorothea 
Peter Schmidt - 978-3-631-75568-6

Downloaded from PubFactory at 01/11/2019 03:28:55AM

via free access 


\section{Vorwort}

Die vorliegende Arbeit ist die überarbeitete Fassung meiner Dissertation, die im November 1994 von der Fakultät für Volkswirtschaftslehre und Statistik der Universität Mannheim angenommen wurde. Das Thema entstammt inhaltlich dem Bereich der Sozialpolitik, die mit empirischen Methoden der Ökonometrie untersucht wird. Ich wünsche mir, daß die Arbeit damit ein Stückchen weit eine Brücke zwischen diesen Bereichen schlagen hilft. Nur mit ökonomischen Handlungsempfehlungen kann kaum konsensfähige Sozialpolitik gemacht werden, aber solche Empfehlungen können dazu beitragen, Diskussionen zu versachlichen.

Nicht nur als Doktor-, sondern auch als geistigem Vater dieser Arbeit, der mich für das Themengebiet begeisterte, möchte ich einen ganz herzlichen Dank an Prof. Axel Börsch-Supan, Ph.D. aussprechen. Er hat mich als akademischer Lehrer durch die Höhen und Täler der empirischen Sozialforschung begleitet, seine „Handschrift" findet sich an unterschiedlichsten Stellen der Arbeit wieder. Ich danke auch Prof. Dr. Wille, der das Koreferat übernommen hat und mir interessante Hinweise für diese Veröffentlichung gab. Über die Bereitschaft Prof. Dr. Eichhorn, als dritter Prüfer am Rigorosum teilzunehmen, habe ich mich sehr gefreut.

Mein besonderer Dank gilt Dr. Vera Lessat, die in der gegebenen Kürze der Zeit das gesamte Manuskript durchgesehen und mir mit hilfreichen Hinweisen den Weg zu einer lesbaren abschließenden Fassung erleichtert hat. Diskussionen mit Kollegen der Universität Mannheim in und außerhalb von Doktorandenseminaren gehen an unterschiedlichen Stellen in die Arbeit ein. Meine Kollegin Regina Riphahn, Ph.D. trug in konstruktiven Diskussionen zum Werden vor allem des vierten Kapitels bei.

Einen großen Teil der Lasten dieser Arbeit hatten meine Frau Jutta, die große Teile der Arbeit geduldig mit inhaltlicher und stilistischer Brille redigiert hat, und unsere Kinder zu tragen. Sie mußten nicht nur das Auf und Ab des Werdens, sondern vor allem auch mein Ausbleiben an Abenden, Wochenenden und Ferien ertragen und ausgleichen. Ihnen ist dieses Buch gewidmet, das ohne ihre Geduld und Unterstützung nicht hätte geschrieben werden können.

Ketsch im Mai 1995

Peter Schmidt 
Peter Schmidt - 978-3-631-75568-6

Downloaded from PubFactory at 01/11/2019 03:28:55AM

via free access 


\section{KURZÜBERSICHT}

1 Einleitung 1

2 Implikationen der Altersstrukturverschiebung - eine Übersicht 5

2.1 Altersökonomik als wissenschaftliches Forschungsgebiet .........................................9

2.2 Altersstrukturverschiebung und langfristige Finanzierung der GRV .........................21

3 Institutionelle Rahmenbedingungen der Ruhestandsentscheidung 28

3.1 Grundlegende Aspekte von Alterssicherungssystemen .............................................29

3.2 Das Alterssicherungssystem der Bundesrepublik Deutschland ................................... 33

3.3 Geschichte der GRV, Rentenreformen und wesentliche Regeländerungen..................55

3.4 Die deutsche Wiedervereinigung und Überführung des DDR-Rentenrechtes...............62

3.5 Anreizstrukturen des Rentenversicherungssystems ..................................................69

3.6 Zusammenfassung der GRV-Änderungen und Arbeitshypothesen ..............................74

4 Entwicklung der westdeutschen Rentenzugänge 1970 bis 1991

4.1 Arbeitsmarkt und Verrentungsentscheidung............................................................... 78

4.2 Die Entwicklung der Rentenzugänge in Deutschland seit 1968 .................................. 81

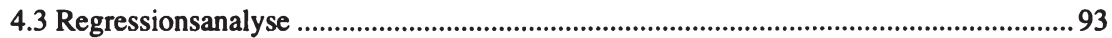

4.4 Zusammenfassung und Bewertung der makroökonomischen Untersuchung ............... 106

5 Empirische Analysen der individuellen Ruhestandsentscheidung 109

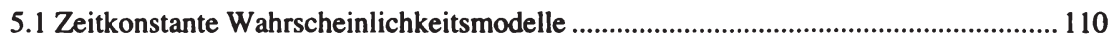

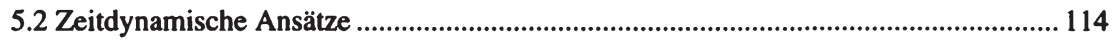

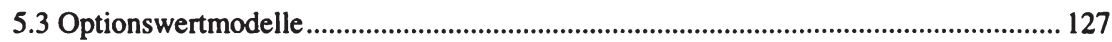

5.4 Zusammenfassung und Aufstellung von Arbeitshypothesen ..................................... 137

6 Ein Hazardratenmodell der Wahl des Rentenalters 144

6.1 Zeitraten- oder Verweildaueranalyse ..................................................................... 144

6.2 Spezifikation eines parametrischen Proportional Hazard Modelles ............................ 154

7 Empirische Analyse der individuellen Verrentungsentscheidung 157

7.1 Datengrundlage: Das Sozioökonomische Panel (SOEP) ............................................ 157

7.2 Deskriptive Betrachtung der Austritte aus dem ......................................................... 168

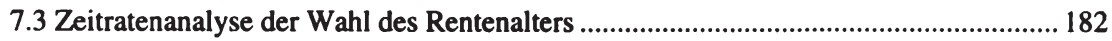

7.4 Zusammenfassung der Schätzergebnisse für Ost- und Westdeutschland ...................... 192

8 Volkswirtschaftliche und politische Implikationen 196

9 Abschließende Betrachtung und Ausblick 207

$\begin{array}{ll}\text { Anhang } & 213\end{array}$

$\begin{array}{ll}\text { Literaturverzeichnis } & 225\end{array}$

Schlagwortindex 234 


\section{INHALTSVERZEICHNIS}

Verzeichnisse ix

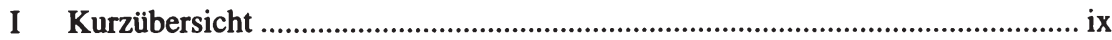

II Inhaltsverzeichnis ................................................................................................

III Abbildungsverzeichnis .................................................................................. xiii

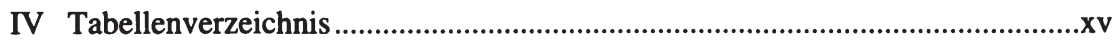

V Verzeichnis der Abkürzungen ...................................................................... xvii

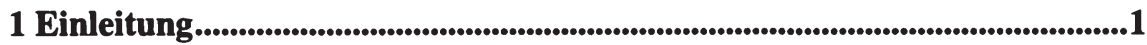

2 Implikationen der Altersstrukturverschiebung - eine Übersicht .................5

2.1 Altersökonomik als wissenschaftliches Forschungsgebiet......................................9

2.2 Altersstrukturverschiebung und langfristige Finanzierung der GRV ..................21

3 Institutionelle Rahmenbedingungen der Ruhestandsentscheidung ...........28

3.1 Grundlegende Aspekte von Alterssicherungssystemen.........................................29

3.2 Das Alterssicherungssystem der Bundesrepublik Deutschland.............................33

3.2.1 Die Gesetzliche Rentenversicherung (GRV) ................................................35

3.2.2 Betriebliche und private Altersversorgung ......................................................45

3.2.3 Angrenzende Gebiete der sozialen Sicherung .............................................52

3.3 Geschichte der GRV, Rentenreformen und wesentliche Regeländerungen.........55

3.3.1 Entstehung und Entwicklung der GRV bis 1991 ........................................56

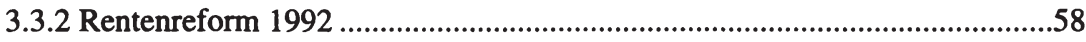

3.4 Die deutsche Wiedervereinigung und Überführung des DDR-Rentenrechtes ....62

3.4.1 Struktur und Rentenformeln der Pflicht- und der Freiwilligen Zusatzversicherung der DDR .......................................................................62

3.4.2 Anpassung der laufenden DDR-Renten an das westdeutsche System...........66

3.5 Anreizstrukturen des Rentenversicherungssystems: Altersanpassung und versicherungsmathematische Fairne $B$.

3.6 Zusammenfassung der GRV-Änderungen und Entwicklung von Arbeitshypothesen für die empirische Untersuchung. 
4 Entwicklung der westdeutschen Rentenzugänge 1970 bis 1991 .76

4.1 Arbeitsmarkt und Verrentungsentscheidung ..........................................................78

4.2 Die Entwicklung der Rentenzugänge in Deutschland seit 1968 ..........................81

4.2.1 Absolute Zugangszahlen zur GRV .................................................................81

4.2.2 Messung der Erwerbsbevölkerung...................................................................87

4.2.3 Bereinigte Rentenzugänge - Verrentungsraten ..............................................91

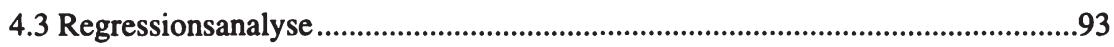

4.3.1 Schätzmethode ........................................................................................93

4.3.2 Regressionsanalyse der Rentenzugänge 1970 bis 1991 .................................96

4.3.3 Arbeitsmarktsituation und Rentenneigung .............................................103

4.4 Zusammenfassung und Bewertung der makroökonomischen Untersuchung ...106

5 Empirische Analysen der individuellen Ruhestandsentscheidung...........109

5.1 Zeitkonstante Wahrscheinlichkeitsmodelle .....................................................110

5.2 Zeitdynamische Ansätze ...................................................................................114

5.2.1 Modelle der dynamischen Programmierung ................................................115

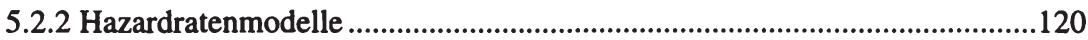

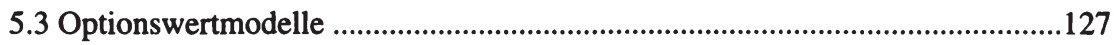

5.3.1 Grundlegendes Modell von LUMBSDAINE, STOCK UND WISE.....................129

5.3.2 Verbindung eines Discrete-Choice Modelles mit dem

Optionswertgedanken durch BÖRSCH-SUPAN

5.4 Zusammenfassung der wesentlichen Ergebnisse und Aufstellung von Arbeitshypothesen

6 Ein Hazardratenmodell der Wahl des Rentenalters......................................144

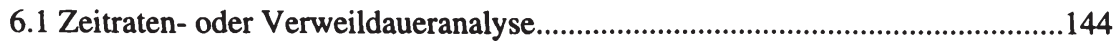

6.1.1 Wahrscheinlichkeitstheoretische Grundlagen..........................................145

6.1.2 Die Hazardrate als zentrales Konzept ....................................................147

6.1.3 Gebräuchliche Verweildauermodelle......................................................150

6.2 Spezifikation eines parametrischen Proportional Hazard Modelles unter

Berücksichtigung des Optionswertes 
7 Empirische Analyse der individuellen Verrentungsentscheidung in West- und Ostdeutschland......................................................................................157

7.1 Datengrundlage: Das Sozioökonomische Panel (SOEP) ………........................157

7.2 Deskriptive Betrachtung der Austritte aus dem Arbeitsmarkt in West- und Ostdeutschland 168

7.3 Zeitratenanalyse der Wahl des Rentenalters..............................................182

7.3.1 Verweildaueranalyse für Westdeutschland ...................................................182

7.3.2 Verweildaueranalyse für die neuen Bundesländer.........................................188

7.4 Zusammenfassung der Schätzergebnisse für Ost- und Westdeutschland. 192

8 Volkswirtschaftliche und politische Implikationen der dargestellten Ergebnisse.

9 Abschließende Betrachtung und Ausblick 207

Anhang

A.1 Regressionsanalysen der Rentenzugänge 1970-1991 ....................................214

A.2 Literatur zur makroökonomischen Entwicklung der GRV...............................220

A.3 OLS-Schätzung des normalisierten Einkommens aus dem SOEP für Westdeutschland 1988-1990.

A.4 Regressionsergebnisse der Übergangsratenmodelle für West- und

Ostdeutschland - Weibull-Spezifikationen.

Literaturverzeichnis 


\section{ABBILDUNGSVERZEICHNIS}

Abbildung 2-1: Geburten- und Heiratsziffern ................................................................

Abbildung 2-2: Einfache Lebenszyklushypothese....................................................11

Abbildung 2-3: Verrentungsprofil in der Bundesrepublik Deutschland.........................15

Abbildung 2-4: Verrentungsprofil in den USA ............................................................16

Abbildung 2-5: Wohnungskonsum und Mobilität im Alter.............................................18

Abbildung 2-6: Entwicklung des durchschnittlichen Rentenalters...............................25

Abbildung 2-7: Anteile der einzelnen Rentenzugangsalter männlicher Arbeiter ausgewählte Jahre ....................................................................................26

Abbildung 3-1: Die drei Säulen der Rentenversicherung ..............................................34

Abbildung 3-2: Rentenarten und Zugangsalter in der GRV ............................................41

Abbildung 3-3: Verbreitung betrieblicher Altersversicherung .........................................46

Abbildung 3-4: Höhe der Betriebsrenten in Deutschland...........................................47

Abbildung 3-5: Anreizwirkungen der betrieblichen Altersversorgung eines deutschen Großunternehmens ..........................................................50

Abbildung 3-6: Kumulierte Anreize aus betrieblicher und gesetzlicher Altersversorgung ..................................................................................50

Abbildung 3-7: Neuabgeschlossene Lebensversicherungen in Deutschland..................51

Abbildung 3-8: DDR-Rentenhöhe in Abhängigkeit von den Beitragsjahren ................64

Abbildung 3-9: Vergleich der Rentenhöhen nach Versicherungsjahren DDR/BRD ....66

Abbildung 4-1: Arbeitslosigkeit und Rentenalter Westdeutschlands in der langfristigen Entwicklung

Abbildung 4-2: Anteil einzelner Renteneintrittsalter an den Gesamtzugängen eines Jahres

Abbildung 4-3: Anteil einzelner Renteneintrittsalter 1966 - 1991 (Angestellte) ...........83

Abbildung 4-4: Anteil einzelner Renteneintrittsalter und der unter 60 jährigen an den Rentenzugängen.

Abbildung 4-5: Anteile der Leistungsarten an den Rentenzugängen 1966 bis 1990.....85

Abbildung 4-6: Bevölkerungsaufbau 1978 ................................................................8

Abbildung 4-7: Männliche Bevölkerung 1966-1990. 
Abbildung 4-8: Männliche Erwerbspersonen nach Mikrozensus...................................90

Abbildung 4-9: Erwerbsquoten im Jahresvergleich (1970 - 1991)...................................91

Abbildung 4-10: Verrentungsraten Angestellte 1970-1991..........................................92

Abbildung 4-11: Arbeitslosenquoten unterschiedlicher Altersgruppen .......................105

Abbildung 5-1: Empirische Dichte und Hazardfunktion aus der Stichprobe von Sueyoshi..................................................................................................121

Abbildung 5-2: Grundidee des Optionswertes......................................................128

Abbildung 6-1: Wahrscheinlichkeitsdichtefunktion der Normalverteilung ..................146

Abbildung 6-2: Verteilungsfunktion der Normalverteilung .......................................147

Abbildung 6-3: Überlebensfunktion der Normalverteilung............................................147

Abbildung 6-4: Baseline-Hazard des Gompertz Modelles .............................................151

Abbildung 6-5: Die Hazard-Rate des Weibull Modelles.............................................152

Abbildung 7-1: Mögliche Spellbeobachtungen in der Datenbasis ...............................162

Abbildung 7-2: Ermittlung des Optionswertes durch FORTRAN-Programm .................166

Abbildung 7-3: Verteilung der VDR-Rentenzugänge 1988 für Männer und Frauen auf Einzelalter.........................................................................................169

Abbildung 7-4: VDR-Rentenzugänge Frauen nach Einzelalter, ausgewählte Kalenderjahre

Abbildung 7-5: Alter bei Beendigung der Erwerbstätigkeit, West-SOEP 1984 1990

Abbildung 7-6: Alter bei Beendigung der Erwerbstätigkeit, Ost-SOEP, 1990 1992

Abbildung 7-7: Prozentanteil nicht erwerbstätiger Befragter - Westdeutschland ........174

Abbildung 7-8: Prozentanteil nicht erwerbstätiger Befragter - Ostdeutschland...........174

Abbildung 8-1: Ablaufschema der Simulation des Rentenalters mittels unterschiedlicher GRV-Szenarien

Abbildung 8-2: Kumulierte Zugangswahrscheinlichkeiten in die GRV unter verschiedenen GRV-Szenarien. 


\section{TABELLENVERZEICHNIS}

Tabelle 2-1: Entwicklung und Prognose der Wohnbevölkerung und der Belastungsquotienten für Gesamtdeutschland

Tabelle 2-2: Alterslastquotient in ausgewählten OECD-Staaten (in Prozent)..................9

Tabelle 2-3: Kennzahlen zur GRV Entwicklung ............................................................24

Tabelle 3-1: Die Gesetzliche Rentenversicherung im Kurzüberblick .............................44

Tabelle 3-2: Nettorentenniveau in der Bundesrepublik und den Vereinigten

Staaten

Tabelle 3-3: Die Rentenformel vor und nach der Rentenreform 1992

Tabelle 3-4: Rentenversicherungssysteme der Bundesrepublik und der ehemaligen DDR.

Tabelle 3-5: Fest- und Mindestbeträge in der Pflichtversicherung der DDR ................65

Tabelle 3-6: Anpassungen der DDR-Renten an die GRV ..........................................68

Tabelle 3-7: Nicht-verzerrende Anpassung der Rentenhöhe nach Zugangsalter............71

Tabelle 3-8: Rentenberechnung und Altersanpassung in der GRV ................................72

Tabelle 3-9: Relevante Regeländerungen der letzten 25 Jahre ......................................75

Tabelle 4-1: OLS-Schätzung der Verrentungsraten......................................................99

Tabelle 4-2: OLS-Schätzung der Verrentungsraten, polynomiale Spezifikationen.....103

Tabelle 5-1: Regressionsergebnisse der Studie von BERKOVEC UND STERN...............118

Tabelle 5-2: Regressionsergebnisse bei SUEYOSHI ......................................................122

Tabelle 5-3: Determinanten der Verrentungswahrscheinlichkeit im einfachen

Logit-Modell von BÖRSCH-SUPAN

Tabelle 5-4: Determinanten der Verrentungswahrscheinlichkeit im erweiterten

Logit-Modell von BÖRSCH-SUPAN.

Tabelle 5-5: In der Literatur untersuchte Determinanten der Verrentung 139

Tabelle 5-6: Arbeitshypothesen für die Verweildauerschätzung.

Tabelle 7-1: Untersuchte Personen und Austritte aus der Erwerbstätigkeit im SOEP

Tabelle 7-2: Das rapide Absinken der Erwerbsbeteiligung in Ostdeutschland 175

Tabelle 7-3: Durchschnittliches Alter bei Beendigung der Erwerbstätigkeit in der SOEP Stichprobe der über 50-jährigen 
Tabelle 7-4: Durchschnittliches Rentenzugangsalter 1980-91 - VDR Statistik für Westdeutschland

Tabelle 7-5: Varianzanalyse des durchschnittlichen Alters bei Beendigung der Erwerbstätigkeit in der SOEP Stichprobe der über 50-jährigen - West .....177

Tabelle 7-6: Varianzanalyse des durchschnittlichen Alters bei Beendigung der Erwerbstätigkeit in der SOEP Stichprobe der über 50-jährigen - Ost ....179

Tabelle 7-7: Variable der Verweildauerschätzung: Mittelwerte für Ost- und Westdeutschland

Tabelle 7-8: Verweildaueranalyse für West-Deutschland - Spezifikationen des Gompertz-Modelles .....

Tabelle 7-9: Verweildaueranalyse für Ost-Deutschland - Spezifikationen des Gompertz-Modelles

Tabelle 7-10: Signifikante Einflußfaktoren und Wirkungsrichtungen in der Verweildauerschätzung - Vergleich der alten und neuen Bundesländer

Tabelle 8-1: Kumulierte Wahrscheinlichkeiten eines Ruhestandes in unterschiedlichen GRV-Szenarien für ausgewählte Alter

Tabelle 8-2: Veränderung des durchschnittlichen Verrentungsalters in unterschiedlichen GRV-Szenarien.

Tabelle 8-3: Implikationen der modellierten GRV-Systeme auf den Alterskoeffizienten und den GRV-Beitragssatz .203

Tabelle 8-4: Entlastung der GRV durch die Einfuhrung einer unverzerrenden Rentenformel .203

\section{Tabellenanhang:}

Tabelle A-1: Übersicht über die verwendeten Variablennamen und -kürzel .214

Tabelle A-2: OLS-Schätzung der Zugangsraten zur GRV, Spezifikationen 1 bis 3 ...215 Tabelle A-3: OLS-Schätzung der Zugangsraten zur GRV, Spezifikationen 4 bis 5 ...216 Tabelle A-4: OLS-Schätzung der Zugangsraten zur GRV, Spezifikationen 6 bis 8 ...218 Tabelle A-5: Tabellarische Literaturübersicht .220

Tabelle A-6: OLS-Schätzung des Normalisierten Einkommens aus dem SOEP für Westdeutschland 1988-1990.

Tabelle A-7: Verweildaueranalyse für West-Deutschland - Spezifikationen des Weibull-Modelles

Tabelle A-8: Verweildaueranalyse für Ost-Deutschland - Spezifikationen des Weibull-Modelles 


\section{VERZEICHINIS DER ABKÜRZUNGEN}

$\begin{array}{ll}\text { Abb. } & \text { Abbildung } \\ \text { AnV } & \text { Rentenversicherung der Angestellten } \\ \text { ArV } & \text { Arbeiterrentenversicherung } \\ \text { AVG } & \text { Angestelltenversicherungsgesetz } \\ \text { BetrAVG } & \begin{array}{l}\text { Gesetz zur Verbesserung der betrieblichen Altersversorgung } \\ \text { (Betriebsrentengesetz) }\end{array} \\ \text { BA } & \text { Bundesanstalt für Arbeit (Nürnberg) } \\ \text { BAV } & \text { Betriebliche Altersversorgung } \\ \text { BfA } & \text { Bundesversicherungsanstalt für Angestellte (Berlin) } \\ \text { BMA } & \text { Bundesminister für Arbeit und Sozialordnung } \\ \text { BSP } & \text { Bruttosozialprodukt } \\ \text { bzw. } & \text { beziehungsweise } \\ \text { BU } & \text { Berufsunfähigkeit } \\ \text { EStG } & \text { Einkommensteuergesetz } \\ \text { EU } & \text { Erwerbsunfähigkeit } \\ \text { FZR } & \text { Freiwillige Zusatzrentenversicherung (ehemalige DDR) } \\ \text { GRV } & \text { Gesetzliche Rentenversicherung } \\ \text { GKV } & \text { Gesetzliche Krankenversicherung } \\ \text { KVdR } & \text { Krankenversicherung der Rentner } \\ \text { LVA } & \text { Landesversicherungsanstalt } \\ \text { ML } & \text { Maximum Likelihood } \\ \text { OECD } & \text { Organisation for Economic Cooperation and Development } \\ \text { o.g. } & \text { oben genannt } \\ \text { OV } & \text { Optionswert (Optionvalue) } \\ \text { PSID } & \text { Panel Study of Income Dynamics (Haushaltspanel USA) } \\ \text { Prob } & \text { Wahrscheinlichkeit (Probability) } \\ \text { RV } & \text { Rentenversicherung } \\ \text { RVO } & \text { Reichsversicherungsordnung } \\ \text { SERPS } & \text { State Earnings Related Pension Scheme (England) } \\ \text { SGB } & \text { Sozialgesetzbuch } \\ \text { SOEP } & \text { Sozioökonomisches Panel } \\ \text { SSI } & \text { Supplementary Security Income (USA: Zusatzrente) } \\ \text { u.a. } & \text { unter anderem / und andere } \\ \text { VR } & \text { Verrentungsrate (Definition im 4. Kapitel) } \\ \text { VBL } & \text { Versorgungsanstalt des Bundes und der Länder } \\ \text { VDR } & \text { Verband Deutscher Rentenversicherungsträger } \\ \text { vgl. } & \text { vergleiche } \\ \text { Z.B. } & \text { zum Beispiel } \\ & \end{array}$


Peter Schmidt - 978-3-631-75568-6

Downloaded from PubFactory at 01/11/2019 03:28:55AM

via free access 
Mit Eifer hab ich mich der Studien beflissen; zwar weiß ich viel, doch möcht ich alles wissen. ${ }^{1}$

\section{Einleitung}

Die westlichen Industrienationen stehen am Anfang eines dramatischen demographischen Umstrukturierungsprozesses, der gerade erst begonnen hat und dessen Dimensionen und Auswirkungen noch nicht überschaubar sind. Durch den doppelten Alterungsproze $\beta$ der Gesellschaften, in denen die Menschen immer älter werden und gleichzeitig die Geburtenhäufigkeit abnimmt, verschieben sich die zahlenmäßigen Verhältnisse zwischen den Generationen. Der Anteil der Menschen über 65 Jahre wird sich von 15,5\% im Jahre 1990 auf prognostizierte 27,6 \% im Jahre 2040 fast verdoppeln, der Alterslastquotient steigt im gleichen Zeitraum von 23,4 auf 48,8 Prozent ${ }^{2}$. Dieser Altersquotient wird zusätzlich zum demographischen Wandel durch ein deutliches Absinken des Rentenalters erhöht, welches das Verhältnis von Pensionären zu Erwerbstätigen weiter verschärft. Wie in keinem anderen Gebiet laufen dabei die offenen Fragen, wie diese Verschiebungen das Sparverhalten, das Arbeitsangebot, den Ruhestand und die soziale Sicherung tangieren, beim Thema Rentenversicherung zusammen.

In der politischen Diskussion taucht entsprechend die Frage nach der Sicherheit und Stabilität des bundesdeutschen Rentenversicherungssystems in den letzten Jahrzehnten immer wieder auf. Die Diskussion hat vor allem zwei Dimensionen, eine finanztechnische und eine sozialpolitische. Die in der Öffentlichkeit geführte Auseinandersetzung konzentriert sich vornehmlich auf den zweiten Aspekt, wobei die Argumente nicht immer sachbezogen sind, sondern auch aus (partei-) politischen Gründen vorgebracht werden. Dies gilt auch nach der jüngsten Rentenreform 92, die zwar als Gesamtkonsens aller großen Fraktionen des Bundestages zustande kam, bei der aber einige ursprünglich geplante Maßnahmen nicht verwirklicht wurden, obwohl sie ökonomisch sinnvoll waren.

\footnotetext{
1 Dieses und alle folgenden Eingangszitate entstammen GOETHES „FAUST“ (erster Teil).

2 OECD (1988); Der Alterslastquotient (Dependency-Ratio) ist definiert als der Quotient: „Anzahl der Personen über 65 Jahre / potentiell Erwerbstätige“ und stellt somit eine Maßzahl für den Alterungsprozeß einer Gesellschaft und die Belastung der erwerbstätigen Bevölkerung durch die Altersstruktur dar.
} 
Auf diesem Hintergrund leistet die vorliegende Arbeit einen wissenschaftlichen Beitrag zur Messung des staatlichen Einflusses auf die Rentenalterentscheidung älterer Arbeitnehmerinnen und Arbeitnehmer. ${ }^{3}$

In jüngerer Zeit wurden mehrere Simulationsrechnungen zur Entwicklung und langfristigen Finanzierbarkeit des Rentenversicherungssystems veröffentlicht. Diese Studien, ${ }^{4}$ die unterschiedliche potentielle Bevölkerungsentwicklungen modellieren, kommen zu dem Ergebnis, daß das Sozialversicherungssystem in seiner heutigen Ausgestaltung spätestens in 20 bis 40 Jahren nicht mehr tragbar sein wird. Der Beitrag der Erwerbstätigen zur Rentenversicherung wird sich danach von derzeit unter 20 Prozent des Bruttoeinkommens auf mehr als 33 Prozent erhöhen. Ähnliche Entwicklungen sind - in abgeschwächter Form - für die Kranken- und die Pflegeversicherung zu erwarten, auch die steuerliche Belastung kann durch den Alterungsprozeß steigen. Zusammengenommen ergibt sich eine Abgabenbelastung für den einzelnen Arbeitnehmer von deutlich über 50\% des Bruttoeinkommens. Diese Höhe ist mit den ökonomischen Gesichtspunkten der Anreizkompatibiliät und Leistungsentlohnung schwer zu vereinbaren.

Auf dieser Basis ist es eine Kernfrage der Sozialpolitik, ob und wie der Gesetzgeber die kommenden Probleme durch eine Veränderung der Rentenversicherungsgesetzgebung verringern kann. Zentral für die Frage, wie das Sozialversicherungssystem geändert werden sollte bzw. muß, ist eine fundierte Aussage darüber, ob die gesetzlichen Rahmenbestimmungen tatsächlich in der Lage sind, das Verhalten älterer Arbeitnehmer bei der Wahl ihres Rentenalters so maßgeblich zu verändern, daß eine Entlastung des Gesamtsystems spürbar wird. Dieses Thema ist in der Literatur umstritten, weshalb in der vorliegenden Arbeit empirische Analysen zur Quantifizierung von Anreizwirkungen der Gesetzlichen Rentenversicherung (GRV) durchgeführt werden.

Als Kernergebnis der vorliegenden Analyse wird in beiden durchgeführten empirischen Untersuchungen deutliche Evidenz für eine spürbare Wirkung institutioneller Veränderungen des Sozialversicherungssystems gefunden. Darauf aufbauend wird eine anreizneutrale Rentenberechung als Reformvorschlag formuliert und dessen Wirkung in Simulationsrechungen quantifiziert. Diese zeigen eine merkliche Entlastung der

${ }^{3}$ Wenn im folgenden für die Bezeichnung einzelner Gruppen der Bevölkerung nur die männliche Form verwendet wird, so dient dies lediglich der besseren Lesbarkeit und schlieBt die Frauen selbstverständlich mit ein.

4 SCHMÄHL (1988), FELDERER (1992), DINKEL und LEBOK (1993), DUDEY (1993), BÖRSCH-SUPAN, MEIER UND REIL-HELD (1994). 
Sozialversicherung durch die modellierte Umgestaltung der GRV. Diese Resultate sollten als Ermutigung und Aufforderung an die sozialpolitischen Entscheidungsträger verstanden werden, die existierenden Probleme der Sozialversicherung aktiv anzugehen und dies rechtzeitig zu tun. Eine zu lange Verzögerung der notwendigen und teilweise einschneidenden Schritte verschärft nicht nur die Situation, sondern durch die sich ändernde Altersstruktur und damit der Mehrheitsverhältnisse innerhalb der Bevölkerung werden sie langfristig politisch nicht mehr durchsetzbar sein.

\section{Aufbau des Buches}

Die Analyse unterteilt sich in drei wesentliche Schritte. Vorbereitend wird nach einer einführenden Betrachtung der Problematik gesellschaftlicher Altersstrukturverschiebung und ihrer ökonomischen Konsequenzen (Kapitel 2) der institutionelle Rahmen der deutschen GRV dargestellt (Kapitel 3). Daraufhin wird als erster Schritt im 4. Kapitel gezeigt, da $B$ die Rentenversicherungsgesetzgebung die historische Entwicklung der Rentenzugangsalter geprägt hat. Zur Quantifizierung des institutionellen Einflusses wird als zweiter Schritt auf Basis einer Literaturübersicht (Kapitel 5) im sechsten Kapitel ein Modell der individuellen Ruhestandsentscheidung entworfen und im 7. Kapitel für beide Teile des wiedervereinigten Deutschland getrennt ökonometrisch geschätzt. Die Schätzergebnisse unterstreichen die Relevanz ökonomischer Einflußfaktoren für die persönliche Entscheidung. Auf Basis der quantitativen Ergebnisse werden als dritter Schritt Simulationsrechnungen für die Entwicklung der gesetzlichen Rentenversicherung bis zur Mitte des nächsten Jahrhunderts durchgeführt (Kapitel 8). Der Vergleich einer Status-Quo Prognose mit einem versicherungsmathematisch fairen System zeigt, daß der Gesetzgeber nennenswerte Gestaltungsspielräume zur Finanzierung des Rentenversicherungssystems hat, die in naher Zukunft genutzt werden sollten.

Das folgende Kapitel 2 gibt eine einführende Übersicht über die wichtigsten Implikationen der Altersstrukturverschiebung, die insbesondere in Deutschland eine große Herausforderung an die Politik darstellt. Es wird hier deutlich, daß eine neuerliche Reform des Rentenversicherungssystems unausweichlich ist. Ebenso zeigt die Betrachtung der historischen Entwicklung der Rentenzugänge und des durchschnittlichen Rentenalters, daß es notwendig ist, den gesetzlichen Rahmen der GRV gründlich aufzuarbeiten und auch deren Entwicklungsgeschichte zu betrachten. Diese institutionelle und historische Aufbereitung ist Thema des Kapitels 3, in dem weiterhin eine Darstellung der Situation in Ostdeutschland erfolgt. An dieser Stelle ist es erforderlich, die aus der notwendigen Umstellung des DDR-Rentenversicherungssystems resultierenden 
Übergangseffekte und ihre Wirkungen zu erfassen. Das dritte Kapitel schließt mit der Bewertung des bestehenden GRV-Systems aus versicherungsmathematischer Sicht und der Entwicklung eines nicht verzerrenden Rentenversicherungssystems als Grundlage der in den folgenden Kapiteln diskutierten Reformvorschläge.

Im vierten Kapitel werden als erster Hauptschritt des Buches die Rentenzugänge der Jahre 1970 bis 1991 und deren Wechselwirkung mit den Änderungen des Rentensystems auf gesamtwirtschaftlicher Ebene untersucht. Basisgröße dieser Analyse sind altersspezifische Verrentungsraten, die als Rentenzugangswahrscheinlichkeiten durch kohortenbereinigte Zugangszahlen in die Gesetzliche Rentenversicherung, bezogen auf die Anzahl der noch arbeitenden Personen pro Altersgruppe, ermittelt werden. Die Analyse zeigt, daß die GRV deutliche Auswirkungen auf das Rentenzugangsverhalten der Arbeitnehmer hat. Im dort verwendeten makroökonomischen Regressionsmodell ist jedoch eine Quantifizierung der Einzeleinflüsse und damit eine Simulation zukünftigen Verhaltens aus methodischen Gründen nicht möglich.

Im zweiten Schritt wird daher ein mikroökonometrischer Ansatz gewählt. Nach der Diskussion ausgewählter Analysen aus der Literatur im fünften Kapitel wird ein Modell der individuellen Verrentungsentscheidung entwickelt (Kapitel 6), das im siebten Abschnitt ökonometrisch geschätzt wird. Kernvariable der Untersuchung ist der Optionswert einer Verlängerung der Lebensarbeitszeit, welcher eine Operationalisierung ökonomischer Anreizeffekte der GRV darstellt und als Erklärungsvariable in das verwendete Verweildauermodell eingeht. Die Analyse bestätigt die Relevanz ökonomischer Determinanten auf eindrucksvolle Weise. Die Schätzungen beruhen auf mikroökonomischen Längsschnittdaten des Sozioökonomischen Panels für West- und Ostdeutschland, so daß ein Vergleich der beiden Landesteile nach der Wiedervereinigung durchgeführt werden kann.

Aus den ermittelten Schätzkoeffizienten werden im Kapitel 8 als dritter Schritt der Analyse Simulationen unterschiedlicher Politikstrategien durchgeführt. Diese Analyse zeigt, daß durch eine geänderte, mehr anreizkompatible Ausgestaltung der GRV die Probleme der Altersstrukturverschiebung entschärft werden können. Die gleichzeitige Berücksichtigung unterschiedlicher Entwicklungen der Bevölkerungsstruktur v.a. bezüglich Migration und Fertilität zeigt einen Weg für eine durchführbare und für alle Gruppen tragbare Rentenpolitik in der ersten Hälfte des 21. Jahrhunderts. Politische Schritte in diese Richtung müssen jedoch in den nächsten Jahren eingeleitet werden, da die Veränderung der Alterszusammensetzung ihre politische Durchsetzung in der $\mathrm{Zu}$ kunft erheblich erschweren wird. 
Was Du ererbt von Deinen Vätern, Erwirb es, um es zu benützen; Was man nicht nützt, ist eine schwere Last, Nur was der Augenblick erschafft, das kann er nützen.

\section{IMPLIKATIONEN DER ALTERSSTRUKTURVERSCHIEBUNG - EINE ÜBERSICHT}

Die Altersstruktur der meisten Industriegesellschaften wird sich in den kommenden Dekaden auf dramatische Weise verändern. Eine steigende Lebenserwartung wird begleitet durch sinkende Heiratsziffern und Geburtenzahlen, so daß sich das Verhältnis von jungen und alten Menschen bereits heute wandelt und im nächsten Jahrhundert weiter erheblich wandeln wird. Dieser aus den genannten beiden Gründen sinkender Fertilität und steigender Lebenserwartung resultierende Prozeß wird als „Double Aging" bezeichnet5.

Tabelle 2-1: Entwicklung und Prognose der Wohnbevölkerung und der Belastungsquotienten für Gesamtdeutschland

\begin{tabular}{|c|c|c|c|c|c|c|c|}
\hline \multirow[b]{2}{*}{\begin{tabular}{|c} 
1. Januar \\
des \\
jeweiligen \\
Jahres
\end{tabular}} & \multicolumn{4}{|c|}{ Bevölkerung (in 1.000) } & \multicolumn{3}{|c|}{ Belastungsquotienten } \\
\hline & Gesamt & $\begin{array}{l}\text { Jugend } \\
\text { unter } \\
20 \text { Jahren }\end{array}$ & $\begin{array}{c}\text { potentielle } \\
\text { Erwerbs- } \\
\text { personen } \\
20-59 \\
\text { Jahre }\end{array}$ & $\begin{array}{l}\text { Ältere } \\
60 \text { Jahre } \\
\text { und älter }\end{array}$ & $\begin{array}{l}\text { Jugend(last)- } \\
\text { quotient } \\
\text { (Spalte 2 I } \\
\text { Spalte 3) }\end{array}$ & $\begin{array}{c}\text { Alters(last) } \\
\text { quotient } \\
\text { (Spalte 4/ } \\
\text { Spalte 3) }\end{array}$ & $\begin{array}{c}\text { Gesamtlast- } \\
\text { quotient } \\
\text { (Spalte } 5+ \\
\text { Spalte 6) }\end{array}$ \\
\hline Spalte: & 1 & 2 & 3 & 4 & 5 & 6 & 7 \\
\hline 1950 & 69.346 & 22.104 & 37.119 & 10.123 & $59,5 \%$ & $27,3 \%$ & $86,8 \%$ \\
\hline 1955 & 71.350 & 22.474 & 37.558 & 11.318 & $59,8 \%$ & $30,1 \%$ & $90,0 \%$ \\
\hline 1960 & 74.247 & 22.111 & 39.404 & 12.732 & $56,1 \%$ & $32,3 \%$ & $88,4 \%$ \\
\hline 1965 & 76.336 & 23.002 & 38.941 & 14.394 & $59,1 \%$ & $37,0 \%$ & $96,0 \%$ \\
\hline 1970 & 78.069 & 24.490 & 38.012 & 15.568 & $64,4 \%$ & $41,0 \%$ & $105,4 \%$ \\
\hline 1975 & 78.465 & 23.689 & 38.769 & 16.007 & $61,1 \%$ & $41,3 \%$ & $102,4 \%$ \\
\hline 1980 & 78.398 & 22.258 & 40.966 & 15.174 & $54,3 \%$ & $37,0 \%$ & $91,4 \%$ \\
\hline 1985 & 77.661 & 19.648 & 42.487 & 15.525 & $46,2 \%$ & $36,5 \%$ & $82,8 \%$ \\
\hline 1990 & 79.753 & 18.410 & 45.080 & 16.263 & $40,8 \%$ & $36,1 \%$ & $76,9 \%$ \\
\hline 1992 & 80.975 & 17.403 & 47.073 & 16.499 & $37,0 \%$ & $35,0 \%$ & $72,0 \%$ \\
\hline 2000 & 83.741 & 17.757 & 46.662 & 19.322 & $38,1 \%$ & $41,4 \%$ & $79,5 \%$ \\
\hline 2010 & 83.433 & 15.505 & 47.129 & 20.799 & $32,9 \%$ & $44,1 \%$ & $77,0 \%$ \\
\hline 2020 & 81.184 & 13.912 & 44.357 & 22.915 & $31,4 \%$ & $51,7 \%$ & $83,0 \%$ \\
\hline 2030 & 77.414 & 13.020 & 38.370 & 26.023 & $33,9 \%$ & $67,8 \%$ & $101,8 \%$ \\
\hline 2040 & 72.413 & 11.650 & 36.217 & 24.546 & $32,2 \%$ & $67,8 \%$ & $99,9 \%$ \\
\hline
\end{tabular}

Quelle: Werte für 1950 bis 1990: Bundesministerium für Familie und Senioren (1994), Seite 211 f. Werte für 1992 bis 2040: 8. koodinierte Bevölkerungsvorausberechnung des Statistischen Bundesamtes, Variante II (mittlere Variante); in: Sommer (1994), Seite 501.

${ }^{5} \mathrm{Vgl} \mathrm{z.B.} \mathrm{BORSCH-SUPAN} \mathrm{(1990} \mathrm{a).}$ 
Tabelle 2-1 zeigt die Entwicklung der Wohnbevölkerung sowohl in absoluten Zahlen der Altersgruppen (,Jugend“ „Erwerbspersonen“ und „Ältere“) 6 als auch die relative Verteilung dieser Generationen und ihrer Stellung im Lebenszyklus in Form der Lastquotienten. Beide Aspekte der doppelten Alterung können aus dem zukünftig fallenden Jugend- (Spalte 5) und dem steigenden Altersquotienten (Spalte 6) abgelesen werden.

Die Motivation der Ermittlung von Lastquotienten ist, daß die „Erwerbspersonen“ durch ihre produktive Tätigkeit das Sozialprodukt für alle Bevölkerungsgruppen erwirtschaften müssen und somit durch die beiden anderen Generationen „belastet" werden. Ein wichtiger Aspekt ist die Umverteilung zwischen den Generationen. Fand diese Umverteilung an die eigenen Kinder und Eltern bis zum Anfang des Jahrhunderts noch im Kreise der Familie, also auf privater Ebene statt und tut es im Falle der Kinder nach wie vor, so hat sich die Versorgung alter Menschen heute weitgehend auf die gesellschaftliche bzw. staatliche Ebene verlagert. Dies geschieht durch die unterschiedlichen Formen der sozialen Sicherung, sowohl der Versicherungssysteme im Renten-, Krankheits- und Pflegebereich als auch der staatlichen Sozialhilfe. Das rapide Ansteigen des Alterslastquotienten, der den Gesamtlastquotienten 2030 auf über 100\% steigen lassen wird, zeigt die Dimension der innergesellschaftlichen Umverteilung. Diese Größenordnung bedeutet, daß in diesem Jahr die Erwerbstätigen einen größeren Teil ihres Einkommens an andere Gruppen der Gesellschaft weitergeben müssen, als zum eigenen Konsum zur Verfügung steht. ${ }^{7}$ Ein intergenerationaler Konflikt ist somit vorgezeichnet. Es ist Aufgabe der Sozialpolitik, die finanziellen Aspekte zu quantifizieren und die zukünftige Umverteilung auf einem möglichst breiten gesellschaftlichen Konsens zu basieren. Die konkreten Auswirkungen der beschriebenen Phänomene auf die Rentenversicherung werden im Verlauf dieses Kapitels diskutiert.

Die Gründe für den beschriebenen gesellschaftlichen Wandel sind vielfältig und können an dieser Stelle nicht erschöpfend diskutiert werden. BIRG begründet die „Vermeidung langfristiger biographischer Festlegung“8 durch die Industriegesellschaft

${ }^{6}$ Es ist zu beachten, daß diese Meßgrößen nicht die tatsächlichen Anzahlen der Erwerbspersonen darstellen, sondern nur eine Annäherung über das Alter. Insofern wird der intertemporale Vergleich dadurch erschwert, daB die relevanten Alter sich im Betrachtungszeitraum ändern. Dies kann sowohl durch eine Verlängerung der Ausbildungszeit, als auch eine Verlagerung des Rentenalters (siehe folgende Kapitel) geschehen.

7 Dies gilt unter der Annahme eines gleichen Konsumniveaus beider Gruppen.

8 BIRG (1989), Seite 40. 
selbst. Durch die Anreize, aber auch Ansprüche, die eine arbeitsteilige Gesellschaft mit sich bringe, ergebe sich ein Zielkonflikt zu familienorientierten Lebenszielen. Ökonomisch läuft dieses Begründung auf ein Opportunitätskostenargument hinaus, die Menschen widmen sich lieber der Arbeit (als Einkommensquelle) bzw. dem eigenen Konsum, als sich auf die Gründung einer Familie zu konzentrieren, ${ }^{9}$ weil mit den steigenden Konsummöglichkeiten auch die Opportunitätskosten der Erziehung gestiegen sind.

\section{Abbildung 2-1: Geburten- und Heiratsziffern}

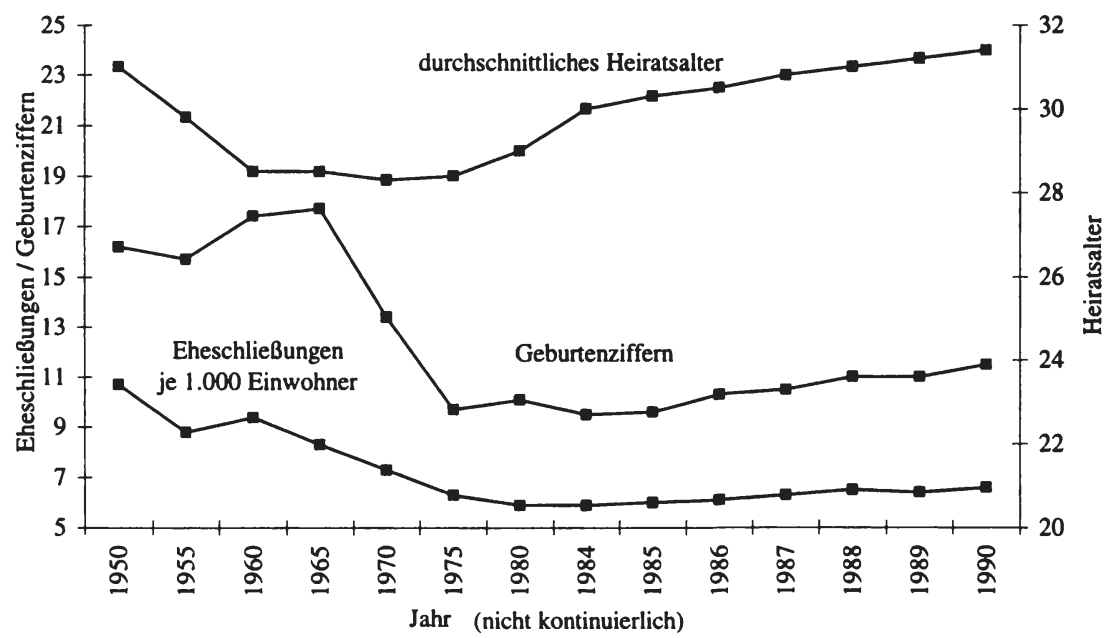

Quelle: Statistisches Jahrbuch 1992, Geburts- und Heiratsziffer je 1.000 Einwohner (Westdeutschland), eigene Darstellung.

Abbildung 2-1 zeigt einige Aspekte der beschriebenen Fakten. Die relativ hohen Geburtenziffern (Geburten je 1.000 Einwohner) der Nachkriegszeit fallen nach den geburtenstarken Jahrgängen der 60er Jahre bis zur Mitte der 70er Jahre stark ab. Der seither zu beobachtende Anstieg ist flach und bei näherer Betrachtung resultiert er daraus, daß in den 80er Jahren die geburtenstarken Jahrgänge selbst Familien gründen. Gemessen

\footnotetext{
${ }^{9}$ Auf Basis des oben diskutierten zeigt sich diese Individualsichtweise der Menschen als kurzsichtig und falsch. Durch die geringen Geburtenzahlen sinkt die Anzahl der zukünfitgen Erwerbspersonen und damit erhöht sich die individuelle Belastung durch Steuern und Sozialabgaben weiterhin. Dieser fiskalische Aspekt muß allerdings getrennt betrachtet werden von der persönlichen Entscheidung gegen eigene Kinder, die aus individuellen Nutzenerwägungen heraus erfolgt und nicht primär finanziell motiviert sein dürfte.
} 
an dieser Tatsache erscheint der leichte Anstieg als ein Artefakt, der sich bei einer kohortenbereinigten Betrachtung umkehrt ${ }^{10}$. Gleiches gilt für die ebenfalls seit Beginn der 80er Jahre leicht ansteigenden Heiratsziffern (Eheschließungen je 1.000 Einwohnern). Zeitgleich steigt das durchschnittliche Heiratsalter der Bundesbürger seit 1970 kontinuierlich an, wodurch der Anstieg der Heiratsziffer gegenläufig zur Altersstruktur wieder gedämpft wird".

Auch eine internationale Betrachtung stützt die Opportunitätskosten-Hypothese. Der Alterslastquotient in den Ländern Afrikas und Asiens ist in der Regel gering, was auf eine gemischte Altersverteilung hindeutet, eine Ausnahme bildet der Industriestaat Ja$\operatorname{pan}^{12}$. Dies bedeutet, daß in den Ländern mit niedrigem Einkommen mehr Kinder geboren werden als in reichen Staaten. Der Alterslastquotient (Dependency-Ratio) ist definiert als der Quotient: „Anzahl Personen über 65 Jahre / potentiell Erwerbstätige“ und stellt somit eine Maßzahl für den Alterungsprozeß einer Gesellschaft dar. Gleichzeitig mißt er die finanzielle Belastung der arbeitenden Generationen durch die Menschen im Ruhestand ${ }^{13}$, die durch das erwirtschaftete Sozialprodukt mit versorgt werden müssen.

Tabelle 2-2 enthält die derzeitigen und prognostizierten Alterslastquotienten einiger OECD-Staaten im Vergleich. Dabei wurden bewußt Staaten mit unterschiedlicher Wirtschaftsstruktur und unterschiedlichem Sozialprodukt ausgewählt. Die Tabelle zeigt, daß die Überalterung der bundesrepublikanischen Gesellschaft wesentlich weiter fortgeschritten ist, als die anderer westlicher Industrienationen. Lediglich die Schweiz weist eine noch krassere Altersstruktur auf. In der Bundesrepublik Deutschland stellt

10 Die „Geburtenziffern" des Statistischen Bundesamtes erweisen sich an dieser Stelle als nicht dazu geeignet, dem beschriebenen Wandel der Bevölkerungsstruktur Rechnung zu tragen. Eine Messung der Geburten pro 1.000 Einwohner läßt die Altersstruktur, insbesondere die Frage, wieviele Menschen im „reproduktionsfähigen“ Alter sind, außer Acht. Ein etwas besseres MaB sind die „NettoReproduktionsziffern", bei denen der artifizielle Anstieg nicht mehr auftritt (siehe: OECD Health Systems (1993) The socio economic environment; Volume II).

"Interessante Aspekte zum Thema Familienbildung, Sozialstruktur und (Planung der) Kinderzahl werden in der weitgehend deskriptiven aber sehr detaillierten Veröffentlichung „Familien heute Strukturen, Verläufe und Einstellungen“" (STATISTISCHES BUNDESAMT, 1990) dargestellt.

12 OECD (1988 a, 1988 b).

13 Auch hier werden nur zwei Potentialgrößen miteinander verglichen. Es werden nicht exakt die Arbeitenden und die Rentner ins Verhältnis gesetzt, sondern zwei Altersgruppen. Damit schwankt die Relevanz dieser Größe damit, wieweit das angenommene Renteneintrittsalter, hier also 65 Jahre, im betreffenden Land wirklich relevant ist. Im weiteren Verlauf der Analyse wird sowohl deutlich werden, da $B$ in Deutschland ein erheblich geringeres durchschnittliches Rentenalter herrscht, als auch, daß die Veränderung ebendieses Rentenalters ein wichtiges Mittel der Politik bei der Stabilisierung des Rentenversicherungssystems ist. 
die Altersstrukturverschiebung somit wie in kaum einem anderen Land eine Herausforderung an die Politik dar. Hingegen liegt die Belastung in den Vereinigten Staaten von Amerika unter dem OECD-Durchschnitt. Auf dieser Basis überrascht es, daß in den USA die Diskussion des Altersstrukturwandels erheblich weiter fortgeschritten ist als in den meisten europäischen Staaten. Auch das Themengebiet der Altersökonomik wurde vornehmlich in den USA entwickelt. Da aus diesem Grunde einige ausführliche Analysen aus Nordamerika vorliegen, die es für Deutschland noch nicht gibt, werden diese an unterschiedlichen Stellen der Arbeit als Vergleichsbasis für die vorliegenden bundesdeutschen Daten zitiert.

Tabelle 2-2: Alterslastquotient in ausgewählten OECD-Staaten (in Prozent)

\begin{tabular}{|l|cccccccc|}
\hline & 1980 & 1990 & 2000 & 2010 & 2020 & 2030 & 2040 & 2050 \\
\hline Schweiz & 21,2 & 25,0 & 31,7 & 40,0 & 48,1 & 50,1 & 46,0 & 46,0 \\
Deutschland & 23,4 & 22,5 & 25,1 & 30,3 & 33,2 & 43,4 & 48,8 & 42,3 \\
Italien & 13,5 & 13,8 & 15,3 & 17,3 & 19,4 & 21,9 & 24,2 & 22,6 \\
Frankreich & 14,0 & 13,8 & 15,3 & 16,3 & 19,5 & 21,8 & 22,7 & 22,3 \\
Türkei & 7,0 & 8,1 & 8,6 & 10,1 & 13,6 & 16,4 & 17,6 & 17,6 \\
USA & 17,1 & 18,7 & 18,3 & 18,5 & 25,0 & 31,7 & 32,4 & 31,8 \\
Australien & 14,8 & 16,6 & 17,4 & 18,6 & 23,7 & 29,2 & 32,4 & 32,0 \\
Kanada & 9,5 & 11,4 & 12,8 & 14,6 & 18,6 & 22,4 & 22,5 & 21,3 \\
Japan & 13,4 & 16,6 & 22,4 & 27,5 & 33,7 & 31,8 & 37,5 & 37,6 \\
\hline OECD Durchschnitt & 19,0 & 19,8 & 21,2 & 23,5 & 28,8 & 34,4 & 36,9 & 35,4 \\
\hline G7 & 19,1 & 19,9 & 21,9 & 24,2 & 29,4 & 35,2 & 38,6 & 36,3 \\
\hline
\end{tabular}

Quelle: OECD (1988 a), Seite 22

Die Überalterung der Gesellschaft findet ihren Niederschlag in vielen Bereichen des öffentlichen Lebens. In diesem Kapitel werden die wichtigsten ökonomischen Aspekte der Thematik angesprochen, indem im ersten Teil (Kapitel 2.1) die Geschichte und Gebiete der altersökonomischen Forschung angesprochen werden. Es wird dabei deutlich, daß die Wahl eines geeigneten Rentenalters aus theoretischer Sicht nicht eindeutig determiniert ist, sondern aus verschiedenen ökonomischen Gesichtspunkten betrachtet werden kann und muß. Im zweiten Teil (Kapitel 2.2) wird das Kernthema dieser Arbeit - die individuelle Wahl des Rentenalters - am konkreten Fall der Bundesrepublik Deutschland einleitend behandelt.

\subsection{Altersökonomik als wissenschaftliches Forschungsgebiet}

Neben der Gerontologie, die als älteres und umfassenderes Feld der Altersforschung betrachtet werden kann, ${ }^{14}$ beginnt sich bereits in den $60 \mathrm{er}$ Jah-

14 Vgl. z.B. LEHR (1987). 
ren das Feld der Altersökonomik als Teil der VWL zu etablieren. ANDO UND MODIGLIANI (1963) beschreiben die „Life-Cycle-Hypothesis of Saving“ die im folgenden Abschnitt bei der Diskussion des Sparverhaltens diskutiert wird. Allgemeine Einführungen in die ökonomischen Aspekte des Alterns finden sich bei R. MURRAY (1968): „Economic aspects of pensions: A summary report“ oder ORBACH UND TIBBITS (1963): „Aging and the Economy“. Ein umfassender Übersichtsartikel zum Thema „Economics of Aging: A Survey“ von ClARK, KREBS, UND SPENGLER erscheint 1978 im Journal of Economic Literature (JEL), 1990 veröffentlicht MICHAEL HURD an gleicher Stelle die Zusammenschau „Issues and Results from Research on the Elderly“. BÖRSCH-SUPAN (1991) verbindet in seinem Artikel „Aging Population“ drei Aspekte: einen Überblick über die demographischen Phänomene, volkswirtschaftliche Theorie der betroffenen Wirtschaftssegmente (vgl. unten) und empirische Ergebnisse einer kritischen Überprüfung dieser Theorien. Im folgenden werden wichtige Bereiche der Altersökonomik angesprochen und im Zusammenhang mit der Rentenversicherung diskutiert.

\section{Spar- und Konsumverhalten}

Für eine ökonomische Analyse des Lebenszyklus von Menschen bzw. Generationen bietet sich die Betrachtung des jeweiligen Stromes von Einkommen einerseits und Konsumausgaben andererseit an. Der Lebenszyklus eines Menschen unterteilt sich aus dieser Sicht in drei wesentliche Phasen: die Kinder- und Jugendzeit vor der Erzielung eigenen Erwerbseinkommens, die Erwerbsphase und die Ruhestandsphase, in der keine Erwerbseinkünfte mehr erzielt werden. Der Zeitpunkt des Renteneintritts trennt die zweite von der dritten Phase, und prägt somit dieses Profil.

Die oben erwähnte Arbeit "The Life-Cycle-Hypothesis of Saving" von ANDO UND MODIGLIANI (1963), ist eine der bekanntesten Arbeiten in diesem Bereich ${ }^{15}$. Aus der Frage nach Alters-Einkommensprofilen ergibt sich auch die Frage nach der Verwendung dieses Einkommens, also der Aufteilung in Konsum und Ersparnis. Das laufende Einkommen schwankt über den Lebenszyklus stark: niedriges Einkommen in der Kindheit - überproportionale Steigerung in der frühen Erwerbsphase - Stagnation des Niveaus um das fünfzigste Lebensjahr - deutlicher Abfall nach dem Ende der Erwerbstätigkeit. Dagegen erweist sich das Konsumverhalten im Lebenszyklus als relativ stabil. Die ökonomische Interpretation für dieses Phänomen ist, daß Individuen ihren

15 Einen ausführlichen Überblick über die unterschiedlichen verwendeten Modelle und Ergebnisse bietet HURD (1990, Kapitel V - Seite 606 ff ). 
Konsum nicht am laufenden, sondern am permanenten Einkommen ausrichten. Diese Überlegung führt zu einem wohldefinierten Lebenszyklus des Sparens. Das einfache Lebenszyklusmodell besagt, daß - auf Basis perfekter Information über Einkommenshöhe, Verrentungszeitpunkt und Lebenserwartung - ein Mensch während seines Erwerbslebens spart und ab dem Tag der Verrentung beginnt, von diesem Ersparten zu leben. Es wird genau so viel gespart, daß er für die gesamte Dauer des Ruhestandes das gewohnte Konsumniveau beibehalten kann. Dieser Verlauf wird in Abbildung 2-2 verdeutlicht.

Abbildung 2-2: Einfache Lebenszyklushypothese.

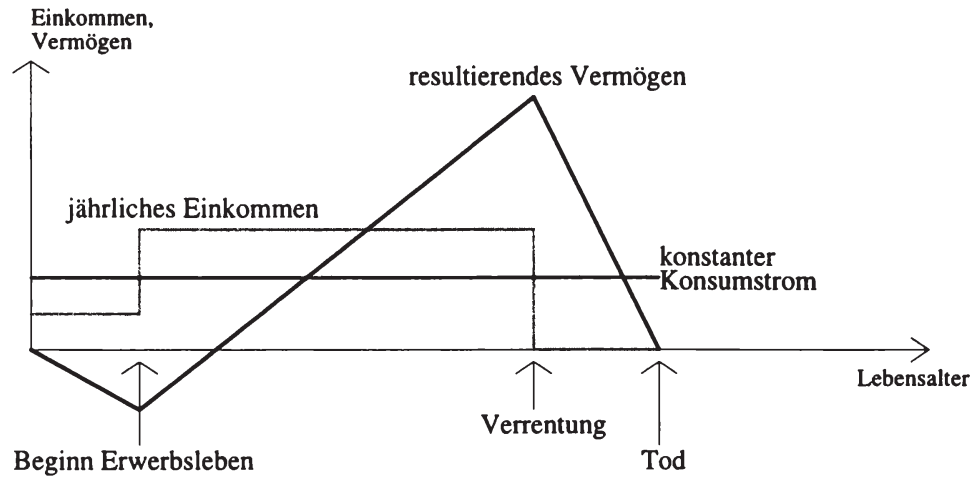

Diese einfache Darstellung des Lebenszyklusmodelles läßt viele Fragen offen und diskutiert relevante Aspekte nicht, die jedoch das Thema dieser Arbeit übersteigen würden. So muß das Modell um die Existenz einer Rentenversicherung, aber auch um intertemporale Elemente, vor allem der Vererbung, erweitert werden. Eine sehr interessante Darstellung möglicher Erweiterungen findet sich in HOMBURG (1988). ${ }^{16}$

Einer empirischen Überprüfung hält die Lebenszyklushypothese allerdings nicht stand. BÖRSCH-SUPAN und STAHL (1991) 17 untersuchen das Alters-Sparprofil der Bundesrepublik Deutschland und finden dabei Sparprofile, die unmittelbar nach dem Zeitpunkt der Verrentung signifikant ansteigen, statt abzufallen. Erst mit steigendem Alter sinken die Konsumausgaben wieder ab. Die Renteneinkommen hingegen sind in jedem Lebensalter gleich hoch, so daß alte Menschen in der ersten Rentenphase das verfügbare

\footnotetext{
${ }^{16}$ Kapitel 4 „Altersvorsorge und individuelle Ersparnis“.

17 ,Life cycle Savings and Consumption Constrains: Theory, empirical evidence, and Fiscal Implications".
} 
Einkommen ausgeben oder sogar Ersparnisse auflösen, wogegen sie in höherem Alter nicht mehr die ganze Rente ausgeben und somit neue Ersparnisse bilden.

Die Autoren formulieren auf Basis dieser Beobachtung eine alternative theoretische Spezifikation. Ihr Hauptargument ist, daß wenn alte Rentner mehr Rente bekommen, als sie für Konsum ausgeben, diese zwangsläufig sparen müssen ${ }^{18}$. Der Effekt dieser „Zwangssparhypothese“ wird dadurch verstärkt, daß die Konsummöglichkeiten alter Menschen im hohen Alter zurückgehen. Insbesondere die Ausgaben für Reisen, aber auch die für Kleidung und Essen sinken bei sehr alten Menschen. Die Argumentation wird weitergeführt in BÖRSCH-SUPAN (1992 a-c) ${ }^{19}$, in dem er das Phänomen des Alterssparens auch nach einer Kohortenbereinigung bestätigt findet. Insbesondere in Bezug auf die Sparraten findet der Autor Altersprofile, die zwar vom Alter 50 bis etwa 65 Jahre abfallen, dann aber bis ins Alter 80 fast wieder auf den alten Stand ansteigen ${ }^{20}$.

Im Bezug auf die Rentenversicherung stellt sich die Frage, wieweit das Sparverhalten älterer Menschen durch deren Existenz und Ausgestaltung beeinflußt wird. Diese Diskussion, die „FELDSTEIN - BARRO Kontroverse“, ist als „fast schon historischer Streit" ${ }^{21}$ seit 20 Jahren in der Literatur aktuell. Eine umfassende Darstellung der Inhalte und des Argumentationsverlaufes findet sich bei SCHOLZ (1993). FELDSTEIN geht von einem deutlichen Einfluß der Sozialversicherung auf das Sparverhalten der Individuen aus, BARRO hingegen negiert diesen Zusammenhang22. Beide belegen ihre Argumente und widerlegen die des anderen mehrfach durch empirische Untersuchungen.

18 Die Autoren bezeichnen diesen Effekt als „Über-Annuisierung“ („over annutized“). Das zunächst zu erwartende Verhalten, in der frühen Rentenphase das spätere Einkommen auf dem Kapitalmarkt zu beleihen, zeigt sich als nicht durchführbar. Dieser Effekt resultiert daraus, daß die Renten als Annuitäteneinkommen oder Transfereinkommen den Rentnern für die Dauer ihres Lebens zustehen, dieses Einkommen aber im Sinne einer Barwertbetrachtung keine ex ante kalkulierbare Größe ist, weil sie von der individuellen Lebensdauer abhängen. Die monatliche Rente ist festgelegt, der zeitpunktbezogene Wert dieses Einkommensstromes (etwa zum Zeitpunkt der Ruhestandsentscheidung) nicht. Deshalb sind diese Einkünfte auf dem Kaptialmarkt nicht beleihbar. Aus diesem Grunde müssen Rentner für die oben beschriebene erste Phase hoher Konsumausgaben Ersparnisse bilden, auch wenn in der späteren Phase Einkommen ,auflaufen“, die sie nicht mehr ausgeben können.

19 „Saving and consumption Patterns of the elderly - The German Case“ sowie 2 Teile des Papiers: "Household Savings in Germany“.

20 Weitere empirische Arbeiten in diesem Bereich sind etwa HIRVONEN (1993): „Alterssicherung und Alterseinkommensverteilung“, SCHNIEWIND (1989): „Gesetzliche Rentenversicherung und Konsum“ oder KIM (1992) „Sozialversicherungskapital und das Sparen der privaten Haushalte in der Bundesrepublik Deutschland 1961-1988“ mit weiteren kommentierten Literaturhinweisen (Seite $22 \mathrm{ff}$ ).

${ }^{21}$ SCHOLZ (1993).

22 Vergleiche hierzu bespielhaft: FELDSTEIN $(1974,1976,1982)$, BARRO $(1974,1978)$. 
Die theoretische Basis der Kontroverse ist der Modellkontext überlappender Generationen. Deren Grundidee ist eine intertemporale Betrachtung des Lebenszyklus von Personengruppen, die zu „Generationen“ zusammengefaßt werden ${ }^{23}$. Die Generationen bilden unterschiedliche Akteure verschiedenen Alters, deren Verhalten in Abhängigkeit von der Position im Lebenszyklus variiert. Auf diese Weise ist ein Spar- bzw. Entsparverhalten vor und nach dem Ruhestand theoretisch modellierbar ${ }^{24}$. Die Kontroverse zeigt jedoch, daß Modelle in diesem Kontext hochsensibel auf die strukturellen Annahmen reagieren.

Schon diese kursorische Betrachtung des Themenbereiches "Ersparnisbildung" zeigt, $\mathrm{da} B$ diesem für eine Modellierung des Verrentungsalters eine wichtige Rolle zukommt. Die oben angeschnittenen Modelle gehen jeweils von der Prämisse aus, daß Individuen bei gegebenem Rentenzeitpunkt zu Beginn ihres (Erwerbs-) Lebens eine Entscheidung über einen Komsum- und Sparpfad treffen. Tatsächlich ist der Verrentungszeitpunkt aber keine exogene Größe, sondern kann von den Individuen selbst bestimmt werden. Die alternative, dem Ansatz dieser Arbeit näher liegende Spezifikation ist somit ein Kontext, indem ältere Menschen auf Basis eines Einkommensstromes und ihrer Lebenserwartung eine optimale Wahl des Zeitpunktes treffen, in dem sie aus dem Erwerbsleben ausscheiden und ab dem sie Rente beziehen. ${ }^{25}$ Im Sinne der oben geführten Überlegungen hängt dieser Zeitpunkt davon ab, ob bereits genug Kapital akkumuliert wurde, um für die Dauer der Ruhestandsphase die Lücke zwischen Renteneinkommen und Konsumwünschen füllen zu können. Die aus dieser Theorie abgeleitete Hypothese für die empirische Untersuchung ist damit, daß Menschen mit großen Ersparnissen ceteris paribus früher aus dem Arbeitsmarkt ausscheiden, als andere ${ }^{26}$. Die konkrete

${ }^{23}$ Die Grundlagen dieser Modellklasse sind die Artikel von SAMUELSON (1958) und DiAmOND (1965).

24 Ein weiteres anschauliches Modell dynamischer Lebenszyklusentscheidungen entwirft etwa ZIMMERMANN (1988).

25 Dieser Zeitpunkt muß aus theoretischer Sicht nicht der gleiche sein, sondern es ist denkbar, daß Arbeitnehmer ab einem bestimmten Alter Renten beziehen, aber noch einige Jahre zusätzlich arbeiten. In den USA ist dies rechtlich möglich und führt zu einer dynamischen Neuberechnung der Rente, wenn weitere Beiträge gezahlt werden. Durch die Teilrente der Rentenreform 1992 wird ein ähnliches Modell auch in Deutschland eingeführt.

${ }^{26}$ Wie bereits oben erwähnt, liegt dieser Erwartung die Annahme zugrunde, daß das Renteneinkommen kleiner ist als das zur Befriedigung der Konsumwünsche benötigte. Dies widerspricht teilweise den Beobachtungen von BÖRSCH-SUPAN UND STAHL (1991) und BÖRSCH-SUPAN (1992 a), allerdings heben diese hervor, da $\beta$ nicht nur das (gesetzliche) Renteneinkommen die Lebensgrundlage für Rentner bildet, sondern auch andere Annuitätseinkommen wie Betriebs- und Zusatzrenten oder annuisierte Lebensversicherungen und da $B$ die Summe dieser Annuitätseinkommen die bewerteten Peter Schmidt - 978-3-631-75568-6 
empirische Berücksichtigung von Vermögenswerten auf die individuelle Verrentungsentscheidung wird in Kapitel 6 diskutiert.

\section{Arbeitsmarkt und Wahl des Rentenalters}

Ein Themengebiet, in dem sich das ältere ökonomische Thema des Arbeitsmarktes mit dem neueren der Altersökonomik überlappt, ist die Ruhestandsentscheidung im Sinne der Wahl eines geeigneten Rentenalters. James H. SCHULZ überschreibt dieses Thema treffend mit der Frage „To Work or not to Work“27. Neben diesem Aspekt, der „ArbeitFreizeit Abwägung"28 als Angebotseffekt auf dem Arbeitsmarkt,29 sind als weitere Dimensionen der Wahl des Rentenalters zu beachten:

- direkte Nachfrageeffekte des Arbeitsmarktes: fehlende Arbeitsplätze für ältere Arbeitnehmer, Entlassungen, ('unfreiwillige') Arbeitslosigkeit;

- indirekte Nachfrageeffekte durch (finanzielle) Anreize zur Frühverrentung, Betriebsrentenprofile, Sozialpläne;

- Gesundheitsaspekte: körperliche Fähigkeiten, Behinderungen, Berufs- oder Erwerbsunfähigkeiten;

- institutionelle Rahmenbedingungen in Form von Mindest- / Höchstrentenaltern, altersabhängige Rentenhöhen.

Bereits aus dieser kursorischen Betrachtung möglicher Motive und Anreize für die Wahl des Verrentungszeitpunktes wird deutlich, da $B$ diese Aspekte genauer Einzeldiskussion unterzogen und ihre Relevanz empirisch getestet werden sollte, was in den folgenden Kapiteln sowohl theoretisch (Kapitel 5), als auch für die beiden Teile des wiedervereinigten Deutschland empirisch (Kapitel 4 und 6) geschieht.

Für eine Bewertung der bundesdeutschen Rentenversicherung im internationalen Kontext bietet sich eine Gegenüberstellung von Rentenprofilen an, aus denen abgelesen werden kann, in welchem Alter die Arbeitnehmer eines Landes in Rente gehen bzw. aus dem Arbeitsmarkt ausscheiden. Die Abbildungen 2-3 und 2-4 zeigen solche

Konsumwünsche übersteigt. Tabelle 2-3 zeigt für die Gesetzliche Rentenversicherung GRV, daß für diese die Ersatzquote in Deutschland zwischen 60 und $70 \%$ des letzten Nettoeinkommens liegt.

Damit ist an dieser Stelle die theoretische Annahme eines Entsparens mit reiner Sicht auf die gesetzliche Rentenversicherung korrekt, für die empirische Analyse muß jedoch dem o.a. Effekt Rechung getragen werden.

${ }^{27}$ SCHulz (1988), Chapter 3, Seite $64 \mathrm{ff}$.

${ }^{28}$ Vgl. ebenda, Seite 64: „The Work-Leisure Trade-off“.

29 FrANZ (1991) modelliert diese Entscheidung im Zusammenhang eines Lebenszyklusmodelles, womit eine Verbindung zur Sparentscheidung hergestellt ist (Kapitel 2.3, Seite $56 \mathrm{ff}$ ). Auf Basis einer intertemporalen Nutzenmaximierung über Konsum und Freizeit wird ein optimaler Pensionierungszeitpunkt ermittelt, der zusätzlich die institutionellen Rahmenbedingungen des gesetzlichen Rentenversicherungssystems berücksichtig. 
Profile als Vergleich der Bundesrepublik mit den Vereinigten Staaten von Amerika ${ }^{30}$.

\section{Abbildung 2-3: Verrentungsprofil in der Bundesrepublik Deutschland}

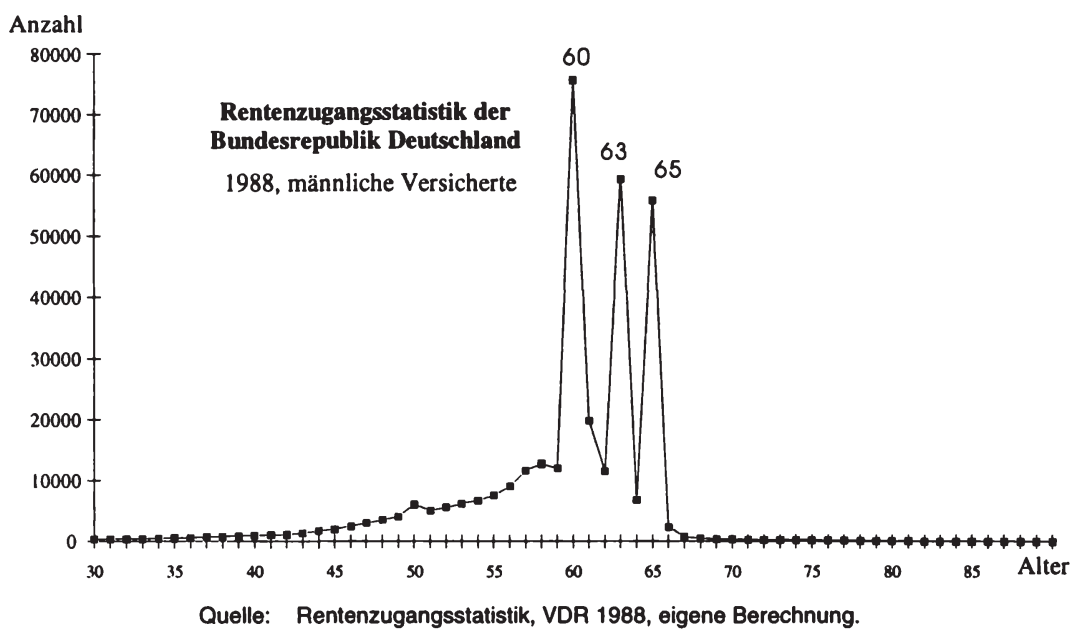

Bei dieser Betrachtung der beiden Länder zeigen sich erhebliche Unterschiede im Verhalten. Während das Verrentungsprofil der amerikanischen Arbeitnehmer -bis auf eine Abweichung beim Alter 63- einer regelmäßigen Verteilung folgt, wirkt das der bundesdeutschen Ruheständler sehr verzerrt. Es treten hier 3 deutliche „VerrentungsSprünge" bei den Lebensaltern 60, 63 und 65 auf, ein erheblicher Teil der Rentenzugänge verteilt sich auf diese drei Einzelalter. Zur Erklärung eines solchen Profils bietet sich von den oben aufgezählten Punkten am ehesten die institutionellen Vorgaben des Rentenversicherungssystems an, da weder eine so krasse Präferenzverteilung, sei es von Anbietern oder Nachfragern auf dem Arbeitsmarkt, noch ein schlagartiges Nachlassen der Gesundheit zu festen Lebensaltern glaubwürdig erscheint.

Diese Hypothese wird im Kapitel 4 empirisch getestet und deutlich unterstützt. Tatsächlich handelt es sich bei den drei hier hervortretenden Zugangsaltern um die Eckpunkte des deutschen GRV-Systems: Um das Regelrentenalter von 65 Jahren herum gibt es das „Fenster“ des flexiblen Ruhestandes zwischen 63 und 67, womit auch die Bedeutung des Alters 63 erklärt wird. Das Alter 60 schließlich ist die erste Möglichkeit

\footnotetext{
${ }^{30}$ Die beiden Vergleichsbasen sind unterschiedlich, da für die Bundesrepublik die gesamten Zugänge in die Gesetzliche Rentenversicherung gezählt werden (ca. 330.000 Männer), wogegen das Bild für die USA auf Basis einer Mikrostichprobe aus der Panel Study of Income Dynamics (PSID) erstellt wurde.
} 
einer vorgezogenen Verrentung für Langzeitarbeitslose und Personen, deren Berufsbzw. Erwerbsfähigkeit eingeschränkt ist ${ }^{3 !}$. Aber auch das regelmäßiger verteilte Bild für die Vereinigten Staaten weist eine erkennbare Struktur auf, indem -nach dem Anstieg ab 58 Jahren- die Alter von 62 bis 65 die größten Anteile an den Rentenzugängen aufweisen. Diese Alter stellen das entsprechende „Verrentungsfenster" für die USA $\mathrm{dar}^{32}$.

\section{Abbildung 2-4: Verrentungsprofil in den USA}

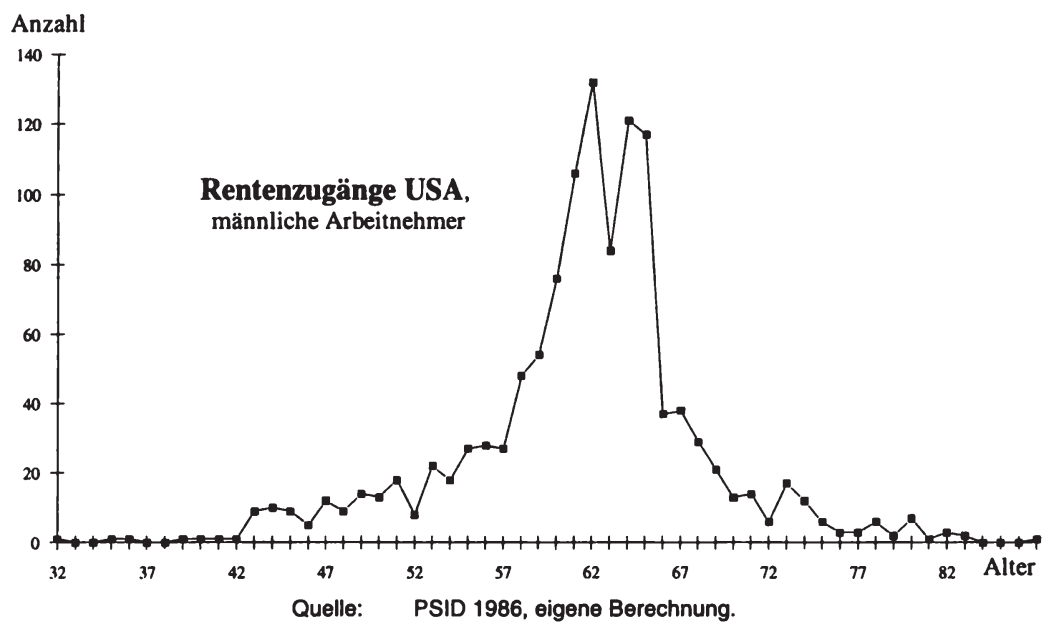

\section{Wohnungsmarkt}

Auch der Wohnungsmarkt als ökonomisches Themengebiet nimmt in der Altersökonomik einen breiten Raum ein. In der Nachfrageanalyse ist die Tatsache, daß alte Menschen überproportional viel Wohnraum konsumieren, ein bekanntes empirisches Phänomen ${ }^{33}$. Dieses wird in BÖRSCH-SUPAN (1992 d) genauer untersucht, indem der Autor in einer Längsschnittstudie die auftretenden Kohorteneffekte analysiert. Er zeigt, daß deren Berücksichtigung zwar wichtig ist, das angesprochene Phänomen aber bestehen

31 Vergleiche hierzu im Einzelnen die Darstellungen der Kapitel 3 und 4.

32 Für eine ausführliche Darstellung des US-amerikanischen Rentenversicherungssystems siehe CASMIR (1989), Seite 395 ff. BÖRSCH-SUPAN (1991) beschreibt analoge Ergebnisse.

${ }^{33} \mathrm{Vgl}$. z.B. STAHL (1989), Seite $98 \mathrm{ff}$, v.a.Tabellen 3.5 A, $3.5 \mathrm{~B}$ und 3.7. Es ist allerdings wichtig an dieser Stelle zwischen dem Haushalts- und dem Pro-Kopf-Konsum zu unterscheiden. Ein theoretisch basiertes und ökonometrisch getestetes Modell für die USA präsentieren VENTI und WISE (1990). 
bleibt. Ein wesentlicher Grund für den steigenden Pro-Kopf Konsum von Wohnraum ist, da $\beta$ alte Menschen auch dann in den relativ großen Wohnungen bleiben, wenn der Haushalt personenmäßig kleiner wird, sei dies durch Auszug von Kindern oder durch Tod des Partners. Hier zeigt sich die Interdependenz zu den bereits diskutierten Themengebieten der Altersökonomik: Die Wohnungswahl ist ein Teil der Konsumentscheidung, welche das Sparverhalten determiniert. Auf der anderen Seite kann die Ersparnis direkt in Wohnungs-, sprich Immobilienvermögen erfolgen. Wenn dieses Immobilienvermögen im Lebenszyklus eine Höhe erreicht hat, die einen gleichmäßigen Wohnungskonsum in der Ruhephase sicherstellt, ergibt sich möglicherweise die oben (,Spar- und Konsumverhalten“) skizzierte Rückwirkung auf eine frühere Pensionierungsentscheidung.

BÖRSCH-SUPAN (1991) zeigt in einer weiteren Untersuchung auf, daß ein erheblicher Anteil des Volksvermögens in Immobilien vorliegt ${ }^{34}$ und da $B$ alte Menschen, gemessen an einem unverzerrten Wohnungsmarkt, in zu großen Wohnungen leben. Als Verzerrungen nennt der Autor dabei sowohl die Wohnungsmarktpolitik, die generell die Mobilität drossele ${ }^{35}$, als auch „Lock-In"-Effekte, dergestalt, daß Senioren quasi in ihrem Hausvermögen „eingesperrt“ bleiben können, weil der Kapitalmarkt nicht die Möglichkeit bietet, das Immobilienvermögen in laufendes Einkommen umzuwandeln. ${ }^{36}$

Die oben aufgestellte Hypothese, daß hohes Immobilienkapital eine frühere Verrentung begünstigt, gilt somit nur, wenn dieses auch tatsächlich zu Konsumzwecken zur Verfügung steht bzw. von den Entscheidungsträgern als zur Verfügung stehend bewertet wird. Dazu müßten Pensionäre tatsächlich eigengenutztes Wohneigentum verkaufen und in kleinere (Miet-) Wohnungen ziehen. Tatsächlich ist aber die Mobilität älterer

\footnotetext{
${ }^{34}$ BÖRSCH-SUPAN (1991), Seite 126 f (Tabelle 16). Der Anteil des Immobilienvermögens sinkt sowohl in Deutschland als auch den USA -die eine höhere Eigentümerquote aufweisen- nur für die sehr alten Menschen (über 80 Jahre) unter $50 \%$, für die mittleren Alter um 50 liegt er in Deutschland bei fast $3 / 4$ des Gesamtvermögens.

${ }^{35}$ Dies gilt insbesondere auf dem Mietwohnungsmarkt, wo sich durch die Kündigungsschutzgesetzgebung die Verweildauern von Mietern erhöht werden und sich die Mieten hauptsächlich durch Mieterwechseln anpassen können, so daß die Mieten in bestehenden Mietverhältnissen regelmäßig unter der durchschnittlichen Marktmiete liegen. Dieser Abstand erhöht sich mit steigender Mietdauer.

${ }^{36} \mathrm{Obwohl}$ eine solche Annuisierung von Immobilienvermögen aus Sicht von Kreditinstituten und Versicherungen rentabel wäre. Die Institute könnten gegen die Überschreibung des Vermögens eine lebenslange Rente garantieren und diese Verträge auf Basis der gegebenen Lebenserwartungen risikoneutral formulieren. In den Vereinigten Staaten existieren solche Modelle bereits, etwa die "reverse annuity mortgages" und die „home equity loans“.
} 
Menschen nach der Verrentung eher gering und sinkt mit zunehmendem Alter, vergleiche Abbildung 2-537. Interessanterweise sinkt aber gleichzeitig die Eigentümerquote und auch der Anteil des in Hauseigentum gehaltenen Vermögens am Gesamtvermögen fällt von 73,3 Prozent im Alter von 50 auf unter 50 Prozent ab 75 Jahren ${ }^{38}$.

\section{Abbildung 2-5: Wohnungskonsum und Mobilităt im Alter}

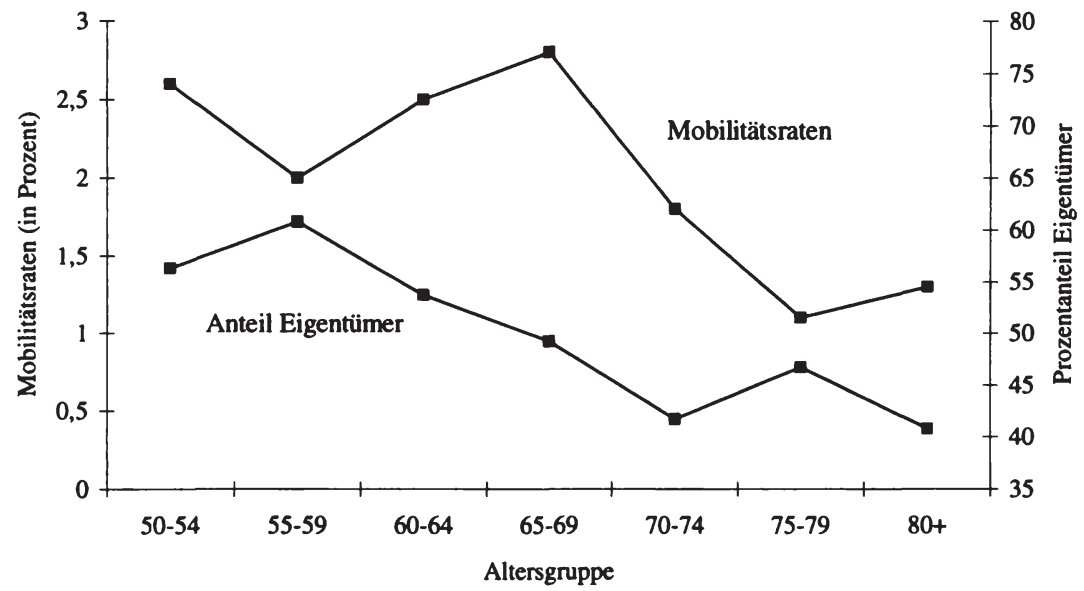

Quelle: BORSch-SuPAN (1991), Tabellen 16 und 17, eigene Darstellung. Die Mobilitătsrate ist der Anteil der umziehenden Haushalte an der Gesamtzahl der Haushalte.

Ob und wie stark das vorhandene Hausvermögen eine Rolle bei der Wahl des Rentenalters spielt, bleibt damit als offene Frage für die empirische Untersuchung des siebten Kapitels.

\section{Gesamtwirtschaftliche Implikationen der Altersstrukturverschiebung}

Die Frage nach gesamtwirtschaftlichen Implikationen der Altersstrukturverschiebung ist Gegenstand unterschiedlicher ökonomischer Modellierungen. In vielen Ansätzen dominiert der Fokus auf das Alterssicherungssystem. Gemeint ist hiermit die Frage der Finanzierung, also eines Kapitaldeckungs- versus Umlageverfahrens. Letzteres wird in der Mehrzahl der Staaten praktiziert, auch in der Bundesrepublik. Diese theoretische Diskussion wird zu Beginn des Kapitels 3 dargestellt.

\footnotetext{
${ }^{37}$ Mit Ausnahme der ganz hohen Alter über 80 Jahren; in diesem Alter treten oft gesundheitsbedingte Umzüge, etwa in Alters- oder Pflegeheime ein.
}

38 BÖRSCH-SUPAN (1991), Tabelle 16, Seite 126. 
Die Monographien von HOMBURG (1988): „Theorie der Alterssicherung“, BREYER (1990): „Ökonomische Theorie der Alterssicherung“ und VON WEIZsÄCKER (1993): „Bevölkerungsentwicklung, Rentenfinanzierung und Einkommensverteilung“ sind drei Beispiele theoretischer Modelle der Alterssicherung. In diesen Arbeiten wird teilweise Bezug auf die Altersstrukturverschiebung genommen, allerdings eher aus Gründen der Motivation und nicht in einer konkreten Auseinandersetzung mit den empirischen Fakten. VON WEIZSÄCKER nimmt zwar explizit Bezug auf die Situation in Deutschland und errechnet mit Hilfe seines Modelles für die gesetzlichen Regelungen vor und nach der Rentenreform 1992 stilisierte Kenngrößen der GRV wie Ungleichheitsmaße und „Elastizitäten der intergenerationalen Budgetinzidenz und Lastenverteilung“399, bezieht sich jedoch dabei nicht auf die tatsächliche Bevölkerungsstruktur und deren Entwicklung.

Solche ausschließlich theoretischen Studien können zwar Orientierungen für eine idealtypische Ausgestaltung von GRV-Systemen geben, tragen aber zu Politikempfehlungen bei konkreten Fragestellungen und zur Entwicklung politisch durchsetzbarer Reformschritte nicht so viel bei, wie dies landesspezifische empirische Analysen tun.

Dagegen beinhalten empirische Ansätze wie JAHNKE (1990) „Wirkungen der Bevölkerungsentwicklung bis zum Jahre 2000“, FELDERER (1992) „Can Immigration Policy Help to Stabilize Social Security Systems?“ oder BÖRSCH-SUPAN, MEIER UND REILHELD (1994) „Macroeconomic Implications of Population Aging“ konkrete Analysen der Situation in Deutschland mit der Möglichkeit von Simulationsrechnungen für alternative Politikstrategien. Die letzten beiden Arbeiten zeigen zudem auf, daß außer der Veränderung der Alterszusammensetzung ein weiteres Phänomen, das der Einwanderungen, eine zunehmende Relevanz sowohl für die gesamtwirtschaftliche Situation im Allgemeinen als auch die der Rentenversicherung im Besonderen gewinnt.

JAHNKE (1990) benutzt das makroökonometrische Modell der deutschen Bundesbank für eine Untersuchung der Auswirkungen demographischen Wandels auf die gesamtwirtschaftliche Entwicklung der Bundesrepublik für den Zeitraum 1985-2000. Sein Fokus ist nicht die Sozialversicherung, sondern die „Wirkung der Bevölkerungsentwicklung auf die Einkommensverteilung und die öffentlichen Haushalte"40. Bei letzteren erwähnt er zwar die steigenden „Transferzahlungen an Rentner“, rechnet diese aber

\footnotetext{
39 VON WEIZSÄCKER (1993), Kapitel VI.

40 JAHNKE (1990), Seite 222 f, für eine Übersicht vgl. Tabelle Seite 223.
} 
mit den sinkenden Aufwendungen für Arbeitslose sowie Transferleistungen an Familien gegen und ermittelt auf diese Weise, daß die „Belastungen, die in Zukunft von der Bevölkerungsentwicklung auf die öffentlichen Haushalte zukommen, zumindest bis zum Jahre 2000, verhältnismäßig begrenzt bleiben" ${ }^{41}$ werden. Diese Ergebnis überrascht nicht aufgrund der oben genannten Tatsache, daß die Änderungen der Bevölkerungsstruktur erst nach $2010 \mathrm{zu}$ den beschriebenen drastischen Konsequenzen führen. Dagegen beschäftigt sich FELDERER (1992) quantitativ mit der Immigrationssituation in der Bundesrepublik Deutschland bis zur Mitte des 21. Jahrhunderts. In Simulationsrechnungen ermittelt er die notwendigen Einwandererzahlen, bei denen die Beitragssätze zur GRV konstant blieben. Aus den unrealistisch hohen Werten von bis zu 4,2 Millionen notwendigen Immigranten pro Jahr schließt der Autor, daß das Sozialversicherungssystem der Bundesrepublik nicht dauerhaft überlebensfähig sein kann. FELDERER führt die Probleme vornehmlich auf die Finanzierung der GRV als Umlageverfahren und schlägt aus diesem Grunde einen Wechsel zu einem kapitalgedeckten System vor. ${ }^{42}$ Die Diskussion unterschiedlicher Finanzierungsverfahren einer Rentenversicherung wird in Kapitel 3.1 angesprochen, dieser theoretische Disput kann jedoch in der vorliegenden Arbeit nicht vertieft werden.

Eine weitere empirische Analyse, die sowohl demographische als auch makroökonomische Aspekte berücksichtigt, ist die Studie von BöRSCH-SUPAN, MEIER UND REILHELD (1994). Die Autoren errechnen im ersten Schritt Status-Quo Prognosen der Auswirkungen des demographischen Wandels. Die ermittelten Beitragssätze zur Rentenversicherung mit einem Maximum von 34,6 Prozent im Jahre 2036 erreichen zusammen mit den weiteren Abgaben (Einkommensteuer, Beiträge zur Kranken-, Arbeitslosen- und Pflegeversicherung) ein nicht mehr tragbares Belastungsniveau. Die Autoren widersprechen allerdings der Schlußfolgerung von FELDERER, daß das deutsche Rentenversicherungsystem nicht überlebensfähig sei bzw. durch Einwanderungen nicht stabilisiert werden könne. Sie verweisen darauf, da $B$ Immigrationspolitik nur eines der möglichen Mittel zur Stabilisierung des Systemes ist und nicht notwendig dazu benutzt werden muß, die Beitragssätze auf dem derzeitigen Stand zu halten, sondern daß Einwanderung zu einer spürbaren Abschwächung des Trends führen kann. So hielte beispielsweise eine konstante Zuwanderung von 300.000 Personen pro Jahr den Beitragssatz unter 25\%, was eine ganz erhebliche Entlastung des Systems darstellt ${ }^{43}$.

\footnotetext{
${ }^{41}$ Ebenda, Seite 225.

42 FELDERER (1992), Seite 8 und Seite 17 als SchluBfolgerung.

43 BÖRSCH-SUPAN, MEIER UND REIL-HELD (1994), Seite 23.
} 
Im weiteren Verlauf der Studie wird ein makroökonomisches Modell einer offenen Volkswirtschaft benutzt, in welchem die Wechselwirkungen unterschiedlicher demographischer Szenarien und politischer Vorgaben betrachtet werden. Kernergebnis ist, $\mathrm{da} ß$ eine internationale Zusammenarbeit von Ländern mit unterschiedlicher Altersstrukur (etwa Ost- und West-Europas) einen Großteil der Probleme abfangen kann. Auch BÖRSCH-SUPAN, MEIER UND REIL-HELD kommen zu dem Ergebnis, daß die (teilweise) Umstellung auf ein kapitalgedecktes Finanzierungssystem notwendig ist. Die folgenden beiden Beispiele zeigen die konkrete quantitative Ausrichtung der Studie und der ablesbaren Politikempfehlungen:

Möglichkeiten, den Alterslastquotienten im Jahre 2030 um 1 Prozentpunkt zu senken ${ }^{44}$ :

- Erhöhung der Arbeitszeit um 1 Stunde / Woche (2,65\% Erhöhung), oder

- Steigerung der weiblichen Enwerbsbeteiligung um 5,95\% (von 34,7 auf 36,8 ), oder

- Erhöhung des Rentenalters um 0,8 Jahre $(1,4 \%$ von 59,9 auf 60,7$)$, oder

- Netto-Zuwanderung von 112.500 Personen pro Jahr.

Möglichkeiten, den Beitragssatz der GRV um einen Prozentpunkt zu senken:

- Erhöhung der Arbeitszeit um 50 MinutenWoche (2,71\% Erhöhung), oder

- Steigerung der weiblichen Erwerbsbeteiligung um 4,2\% (von 34,7 auf 36,2 ), oder

- Erhöhung des Rentenalters um $1 / 2$ Jahr $(0,86 \%$ von 59,9 auf 60,4$)$, oder

- Netto-Zuwanderung von 68.000 Personen pro Jahr.

Die hier angesprochenen gesamtwirtschaftlichen Implikationen der Altersstrukturverschiebung zeigen den ökonomischen Zusammenhang, in dem die Rentenversicherung steht. In diesem Sinne behandelt die vorliegende Arbeit einen Teileffekt der Gesamtproblematik, der allerdings sowohl aus arbeitsmarkt- wie sozialpolitischer Sicht als zentral zu bezeichnen ist. Im folgenden Teilkapitel 2.2 wird daher konkreter auf die Rentenversicherung der Bundesrepublik eingegangen und die Implikationen der demographischen Verschiebung auf die Finanzierung der Gesetzlichen Rentenversicherung (GRV) diskutiert.

\subsection{Altersstrukturverschiebung und langfristige Finanzierung der GRV}

Bereits bei der Betrachtung von Alterslastquotienten im internationalen Vergleich in Tabelle 2-2 war deutlich abzulesen, da $\mathrm{B}$ insbesondere das deutsche Rentenversicherungssystem vor wachsenden Aufgaben steht. In diesem Abschnitt wird kurz diskutiert, welche Probleme in der GRV durch eine Verschiebung der Altersstruktur entstehen und welche grundlegenden Möglichkeiten einer staatlichen Regulierung existieren.

44 Ebenda, Exhibits 4-16 und 4-17. 
Für eine nähere Betrachtung der GRV sei eine einfache Budgetgleichung des Gesamtsystems betrachtet ${ }^{45}$ :

$$
\text { Einnahmen }=\text { Ausgaben }{ }^{46}
$$

was sich konkret darstellt als die Beziehung:

$$
\mathrm{N} \cdot \overline{\mathrm{W}} \cdot \mathbf{b}=\mathbf{R} \cdot \mathrm{RH}
$$

mit: $\quad \frac{N}{W} \quad$ Anzahl Beschäftigte

$\overline{\mathrm{W}}$ Durchschnittslohn

b Beitragssatz zur Gesetzlichen Rentenversicherung

R Anzahl Rentner

RH durchschnittliche Rentenhöhe

so daB: $\quad \mathrm{N} \cdot \overline{\mathrm{W}}$ die Lohnsumme und

R.RH die Rentensumme des Gesamtsystems darstellen.

Wird auf der linken Seite dieser Gleichung vereinfachend auf einen durchschnittlichen Lohn $\overline{\mathrm{W}}$ von 1 normiert, so kann statt der Rentenhöhe das Rentenniveau angegeben werden (Gleichung 2-2).

$$
\mathbf{N} \cdot \mathbf{b}=\mathbf{R} \cdot \mathbf{R N}
$$

$\begin{array}{lll}\text { mit: } & \text { N } & \text { Anzahl Beschäftigte } \\ \text { b } & \text { Beitragssatz zur Gesetzlichen Rentenversicherung } \\ \text { R } & \text { Anzahl Rentner } \\ \text { RN } & \text { Rentenniveau, } \\ & \text { definiert als: } \frac{\text { Durchschnittsrente }}{\text { Durchschnittslohn }} .\end{array}$

Das Rentenniveau als gesamtwirtschaftliches $\mathrm{Maß}$ mißt also den relativen Wohlstand der Ruheständler im Vergleich zur erwerbstätigen Bevölkerung. Diese Maßzahl ist geeignet, die durchschnittliche Situation der Rentenempfänger im Vergleich zur arbeitenden Bevölkerung anzugeben. Der VDR berichtet diese Größe als ,(Netto-) Rentenniveau", dessen Entwicklung in Tabelle 2-3 dargestellt ist.

45 Diese Budgetgleichung basiert auf dem Umlageverfahren, nach dem die deutsche GRV organisiert ist. Dies bedeutet, daB die Einnahmen jeweils zeitgleich identisch mit den Ausgaben sein müssen. Gesetzlich ist eine Schwankungsreserve von mindestens einer Monatsausgabe vorgeschrieben, darüber hinaus existiert kein Kapitalstock der GRV.

46 Diese idealtypische Budgetidentität der (parafiskalischen) Versicherungsanstalten im Umlageverfahren unterscheidet sich damit grundlegend vom Budget fiskalischer Haushalte, die einer solchen Identität durch die Möglichkeit von Defiziten und intertemporalen Übertragungen typischerweise nicht streng unterliegen. 
Eine nah verwandte Maßzahl, die teilweise ebenfalls als Rentenniveau bezeichnet wird, ist die Ersatzquote. Diese mißt auf individueller Ebene den Rentenbetrag eines Neu-Rentners in Prozent des vorherigen Einkommens $\frac{\text { Rentenbetrag (bei Rentenbeginn) }}{\text { Erwerbseinkommen (vor Rentenbeginn) }}$. Diese Ersatzquote stellt aus Sicht des einzelnen die relevante Planungsgröße dar, mit der er oder sie den Lebensstandard im Ruhestand mit dem vorhergehenden vergleichen kann.

Der Unterschied zwischen den Maßzahlen „Rentenniveau“ und der „Ersatzquote“ besteht somit einerseits in der Bezugsgröße (alle Rentner versus Einzelbetrachtung), aber auch im Zeitbezug (Zeitpunktbetrachtung versus Vergleich zweier Perioden).

Schon diese einfachen Gleichungen zeigen die grundlegenden Möglichkeiten zur Ausgestaltung bzw. Regulierung einer staatlichen Rentenversicherung. Die Identität der Budgetgleichung kann durch die Variation eines oder mehrerer der drei folgenden $\mathrm{Pa}$ rameter beibehalten bzw. wiederhergestellt werden:

- Beitragssatz b der Erwerbstätigen,

- Rentenniveau RN der Ruheständler (Durchschnittsrente in Prozent des letzten durchschnittlichen Lohnes),

- Rentenalter zur Änderung der relativen Anteile von Erwerbspersonen $\mathrm{N}$ und Rentnern $\mathrm{R}$.

Damit sind die drei wesentlichen Mittel der Politik in der Rentenversicherungsdebatte genannt. Ein steigender Anteil älterer Menschen kann durch erhöhte Beitragssätze, ein niedrigeres Rentenniveau oder ein steigendes Rentenalter, bzw. einer Mischung aus diesen Maßzahlen kompensiert werden.

Der Blick auf die Entwicklung der Gesetzlichen Rentenversicherung in Tabelle 2-3 demonstriert, daß sowohl das sinkende Verrentungsalter als auch das zunehmende Rentenniveau der letzten 25 Jahre aus dieser Sicht eine Entwicklung in die "falsche“ Richtung darstellen. Steigende Beitragssätze stellten in der Vergangenheit den Preis dieser Leistungssteigerung dar. Es sei betont, daß der Begriff falsch hier lediglich technisch gebraucht ist, in dem Sinne, da $\beta$ er einem Budgetausgleich bei steigendem Anteil älterer Menschen entgegenwirkt. Ein steigendes Rentenniveau und damit eine bessere Absicherung im Ruhestand können ebenso einen Nutzengewinn für die Gesellschaft darstellen, wie die Möglichkeit, diesen Ruhestand durch eine frühere Verrentung zu verlängern. 
Tabelle 2-3: Kennzahlen zur GRV Entwicklung

\begin{tabular}{|c|ccc|ccc|c|c|}
\hline & $\begin{array}{c}\text { Durchschnittliches } \\
\text { Verrentungsalter } \\
\text { (Männer) }\end{array}$ & \multicolumn{2}{|c|}{$\begin{array}{c}\text { Entwicklung des Bun- } \\
\text { deszuschusses in \% }\end{array}$} & \multirow{2}{*}{$\begin{array}{c}\text { Beitrags- } \\
\text { sätze }\end{array}$} & $\begin{array}{c}\text { Netto } \\
\text { Renten- } \\
\text { niveau }\end{array}$ \\
\cline { 2 - 6 } 1965 & Gesamt & AnV & ArV & Gesamt & ArV & AnV & 7,0 & 59,3 \\
1970 & $\mathbf{6 1 , 7 2}$ & 63,13 & 61,25 & 26,1 & 33,3 & 13,3 & 7,0 & 63,9 \\
1975 & $\mathbf{6 1 , 5 8}$ & 62,84 & 61,10 & 18,6 & 25,9 & 6,0 & 8,5 & 66,1 \\
1980 & $\mathbf{6 1 , 2 5}$ & 62,51 & 60,63 & 18,3 & 23,9 & 9,0 & 9,0 & 70,3 \\
1985 & $\mathbf{5 8 , 7 9}$ & 60,49 & 57,94 & 19,3 & 25,4 & 10,1 & 9,0 & 71,8 \\
1990 & $\mathbf{5 8 , 9 2}$ & 60,61 & 58,01 & 17,8 & 25,3 & 7,7 & 9,6 & 67,6 \\
\hline
\end{tabular}

In Tabelle 2-3 zeigt sich weiterhin, daß das durchschnittliche Alter bei Rentenbeginn im betrachteten Zeitraum deutlichen Schwankungen unterworfen war. Eine genauere Darstellung des durchschnittlichen Renteneintrittsalters männlicher Arbeiter und Angestellten für die gesamte Zeitperiode (1966 bis 1991) bietet Abbildung 2-6.

Es zeigt sich hier eine erstaunlich gradlinige Entwicklung um die beiden Eckpunkte der Jahre 1973 und 1981 herum. Die Verläufe sind jedoch aus den bisher angestellten Überlegungen heraus nicht zu erklären. Die deutlichen Trendwenden sind so frappierend, daß es naheliegt, institutionelle Änderungen als ihre Ursachen zu vermuten. Mögliche Kohorteneffekte können zwar potentiell für Verschiebungen eines Durchschnittsalters verantwortlich sein, nicht aber so deutliche Einschnitte erzeugen.

Die Überprüfung der Hypothese, daß gesetzliche Reformen die Ursache für die beobachteten Verhaltensänderungen sind, wird Thema der folgenden Kapitel sein.

Die bereits in der Tabelle gesehene Entwicklung eines im Zeitablauf sinkenden Durchschnittsrentenalters wird in Abbildung 2-6 zwar auch deutlich, aber doch modifiziert, da in den Jahren 1981 - 1990 eine moderate Steigerung erkennbar ist. Insgesamt ist das gemeinsame Durchschnittsalter vom Höchststand des Jahres $1965(61,7)$ über das 'Zwischentief' von 58,4 im Jahre 1981 auf den 1991er Wert von 59,7 deutlich abgesunken.

47 Quotient aus der Jahresrente eines „Standardrentners“ mit 45 Beitragsjahren und dem durchschnittlichem Jahresarbeitsentgelt aller Arbeitnehmer. Eine Standardrentner hat über sein gesamtes Erwerbsleben immer genau das Durchschnittseinkommen bezogen.

48 Jeweils von Arbeitgeber und Arbeitnehmer zu zahlen, so daß der der Rentenversicherung zugehende Prozentsatz das Doppelte des hier angegebenen beträgt. 


\section{Abbildung 2-6: Entwicklung des durchschnittlichen Rentenalters}

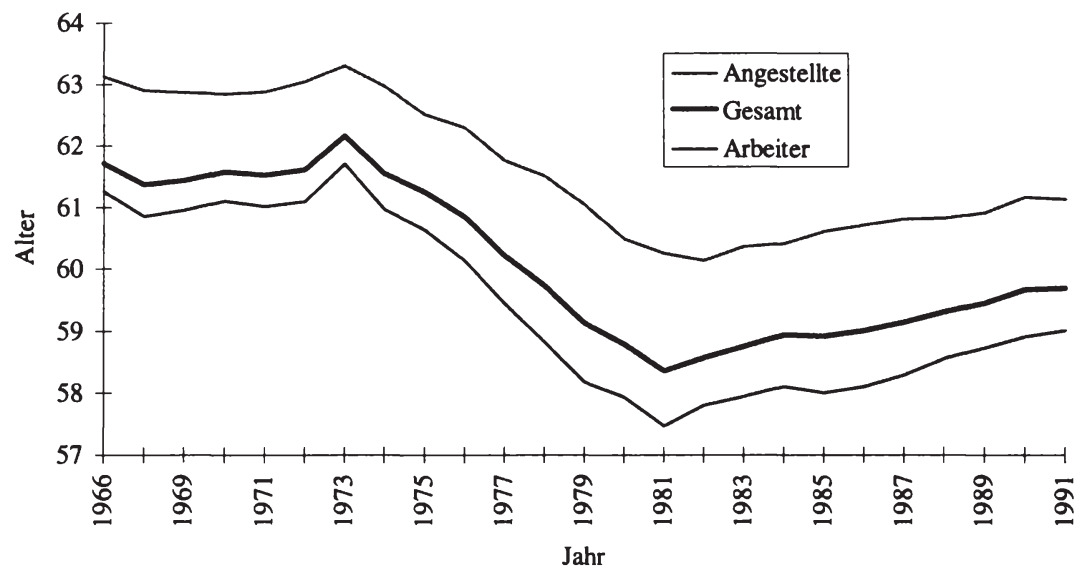

Quelle: VDR Rentenzugangsstatistik, Gesetzliche Rentenversicherung der Mānner, div. Jahrgănge, eigene Berechnung 49

Weiterhin verdeutlicht diese Abbildung den erheblichen Niveauunterschied im Rentenzugangsverhalten zwischen Arbeitern und Angestellten. Die strukturelle Entwicklung beider Gruppen ist allerdings ähnlich. Beide Phänomene präsentieren sich im Zeitablauf als erstaunlich stabil. Lediglich vor 1973 und in den Jahren 1981 bis 85 weichen die Kurvenverläufe voneinander ab. Arbeiter und Angestellte werden im empirischen Teil der Untersuchung getrennt behandelt.

Für eine nähere Betrachtung der Gründe für die beobachtete Entwicklung sei in einem weiteren Schritt auf die einzelnen Rentenalter geschaut, die Alter also, mit denen Arbeitnehmer aus dem Erwerbsleben ausscheiden und sich zur Ruhe setzen. Es ist die Frage, wie die Rentenzugänge auf Einzelalter verteilt sind, ob einzelne Zugangsalter dominieren und ob sich diese Verteilung im Betrachtungszeitraum geändert hat. Abbildung 2-7 gibt hierauf eine klare Antwort, indem sie zeigt, daß sich die Verhältnisse der Zugangsalter massiv verändert haben.

49 „Gesamt“ bezeichnet hier den gewichteten Durchschnitt aus Arbeitern und Angestellten. Die Versicherten der Knappschaft und anderer kleiner Kassen sind nicht berücksichtigt, so daß sich geringfügige Abweichungen zu anderen Zeitreihen des VDR ergeben können. 
Abbildung 2-7: Antelle der einzelnen Rentenzugangsalter männlicher Arbeiter - ausgewählte Jahre

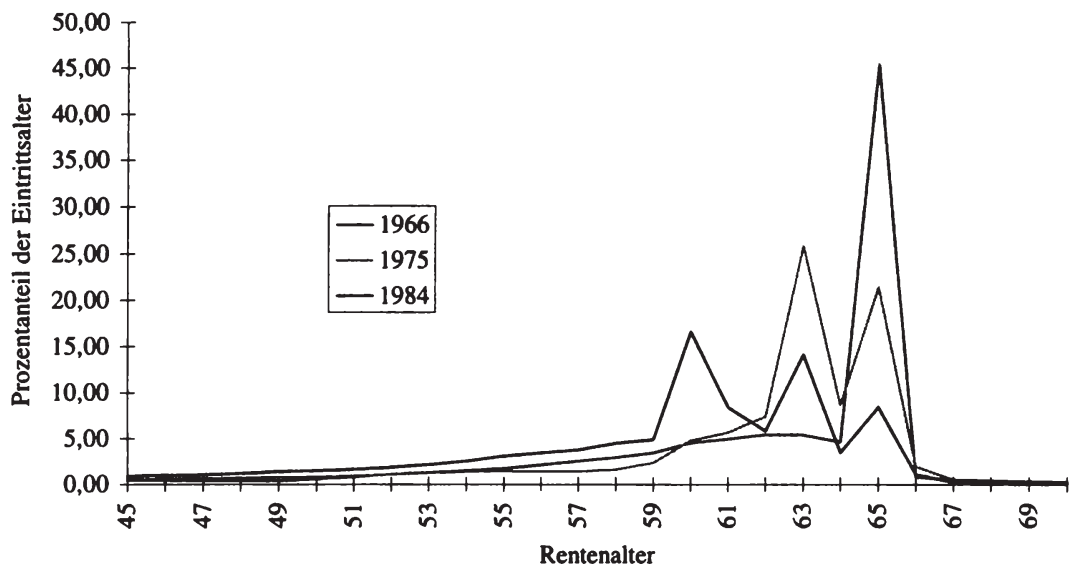

Quelle: VDR: Rentenzugangsstatistik, diverse Jahrgånge, eigene Berechnung und Darstellung. ${ }^{50}$

In der Abbildung wird deutlich, daß sich die drei Alter: 60, 63 und 65 als die heute mit Abstand wichtigsten Zugangsalter herausstellen, in denen sich gemeinsam jeweils ca. $50 \%$ der Neurentner zur Ruhe setzen. Die relativen Anteile dieser drei genannten Alter haben sich aber im Zeitverlauf augenscheinlich erheblich verändert. Traten 1966 noch nahezu $50 \%$ der Arbeiter mit 65 in den Ruhestand, so überwogen bereits 1975 die 63jährigen das Regelrentenalter von 65. Das Bild für 1984 ist noch einmal krasser, indem der stärkste Anteil der Neurentner nur 60 Jahre alt war, erst dahinter folgen die 63- und 65- jährigen. Weiterhin ist interessant zu beobachten, daß bereits ab dem Alter von etwa 50 Jahren nennenswerte Prozentsätze der Rentenzugänger zu verzeichnen sind. Dies gilt in allen drei gezeigten Kalenderjahren und stellt den Grund für das deutliche Auseinanderfallen des jeweiligen Modus und dem (in Abbildung 2-6 dargestellten) Mittelwert dar.

50 In dieser und einigen folgenden Graphiken ist in der Regel nur eine Gruppe Arbeiter oder Angestellte dargestellt. Diese Unterscheidung ist notwendig, da die beiden Gruppen einen deutlichen Niveauunterschied aufweisen (Unterschiede im Rentenalter von 2 Jahren und mehr, vgl. Abbildung 26), die die strukturelle Aussagen der Graphen verwaschen würden. Bis auf diese Niveaudifferenz ist jedoch das Verhaltensbild der beiden Gruppen so ähnlich, daß eine jeweilig doppelte Darstellung nur den Umfang erhöhen, nicht aber weitere Aussagen mit sich bringen würde. 
Diese Strukturveränderung läßt, wie oben bereits gesagt, das Vorliegen systematischer, d.h. durch die Rentengesetzgebung vorgegebener Gründe der Anteilsverschiebung vermuten. Als nächster Schritt der Analyse ist es daher erforderlich, die in Deutschland geltenden gesetzlichen Regelungen für den Rentenzugang, insbesondere das $\mathrm{Zu}$ gangsalter näher zu betrachten. Hierbei ist vor allem wichtig, die Veränderungen dieser Regeln im Zeitablauf zu verfolgen, da diese Aufschluß über die Gründe für das beobachtete Phänomen geben können. Dies geschieht im folgenden dritten Kapitel. 
Es erben sich Gesetz und Rechte,

Wie eine ewge Krankheit fort;

Sie schleppen vom Geschlecht sich zum Geschlechte.

\section{Institutionelle Rahmenbedingungen der Ruhestandsentscheidung}

In diesem Kapitel werden die gesetzlichen Rahmenbedingungen der persönlichen Wahl des Rentenalters aus verschiedenen Blickpunkten dargestellt. Die hier diskutierten alternativen Wege in den frühen Ruhestand bilden eine wesentliche Grundlage für die empirischen Untersuchungen der späteren Kapitel. Es wird sich dort zeigen, da $ß$ die hier geschilderten formalen Regelungen in der Praxis teilweise in anderem Sinne genutzt werden als der Gesetzgeber dies direkt intendierte. Aus der Sicht des einzelnen Arbeitnehmers ökonomisch sinnvolle Substitutionseffekte zwischen verschiedenen Formen der Verrentung (Rentenarten) bzw. Mitnahmeeffekte stellen aus Sicht des Gesetzgebers, der die Finanzierbarkeit des Versicherungssystems GRV erhalten muß, ein Problem dar. So wurde beispielsweise der 1972 eingeführte flexible Ruhestand, das sogenannte Verrentungsfenster, aber auch in späteren Jahren eingeführte Erleichterungen der frühen Verrentung, von den Arbeitnehmern in großem Umfang genutzt (vergleiche Kapitel 4), was zu Zeiten wirtschaftlichen Wachstums und einem ,jungen“ Erwerbspersonenpotential als sozialpolitischer Erfolg angesehen werden konnte. Es wird in diesem Kapitel gezeigt, daß hierbei die ökonomischen Konsequenzen nicht (ausreichend) bedacht wurden, so daß diese Regelungen zu unerwünschten Verzerrungen individueller Entscheidungen führen, die angesichts des Altersstrukturwandels erhebliche finanzielle Probleme für die Rentenversicherung mit sich bringen.

Der Abschnitt 3.5 beinhaltet eine ökonomische Bewertung der diskutierten Rentenversicherungssysteme aus versicherungsmathematischer Sicht und bildet damit die Basis für die vergleichende Analyse und die Rentenreformvorschläge des 8. Kapitels. Das Kapitel schließt mit der Aufstellung von Arbeitshypothesen für das folgende Kapitel 4.

Das Kapitel beginnt mit einer Diskussion unterschiedlicher Rentenversicherungssysteme und deren ökonomischen Eigenschaften (Abschnitt 3.1) mit einer „Typologie der Alterssicherungsverfahren".' Die bereits im zweiten Kapitel angesprochene Frage, ob ein solches umlagefinanziert sein sollte, also auf einem Generationenvertrag beru-

${ }^{1}$ Homburg (1988), Kapitel 2. 
hen, oder auf einem Kapitaldeckungsverfahren basieren sollte, zeigt sich auch hier als Grundsatzentscheidung jedes Alterssicherungssystems.

In den danach folgenden Teilkapiteln wird das Alterssicherungssystem der Bundesrepublik Deutschland zunächst in seinen Kernpunkten vorgestellt. Dabei ist eine Unterteilung in die Gesetzliche Rentenversicherung (GRV), weitere Komponenten der Sicherung im Alter (betriebliche und private Renten), sowie Steuern, Kranken- und andere Sozialversicherungen sinnvoll. Diese Punkte sind Thema des Abschnittes 3.2. Im Anschluß an diese „status-quo“-Betrachtung stellt das Unterkapitel 3.3 die Geschichte der GRV dar und nimmt dabei besonderen Bezug auf die Änderungen der Rentenzugangsregelungen im historischen Vergleich, deren Relevanz bereits im zweiten Kapitel angesprochen wurde und die Thema des vierten Kapitels sein werden. Eine geschichtliche Besonderheit des deutschen Rentenrechtes wurde durch die deutsche Wiedervereinigung ausgelöst mit der notwendigen Überführung des DDR-Rentenrechtes in das bundesdeutsche Recht unter Beachtung bestehender Anwartschaften. Diese Regelungen sind für die vorliegende Studie relevant, da in der Verweildaueranalyse (Kapitel 7) für die neuen Bundesländer Daten der Jahre 1990 - 1992 verwendet werden. Die rechtlichen Grundlagen sowie die dabei notwendigen Übergangsregelungen werden Abschnitt 3.4 beschrieben. Den Abschluß der institutionellen Darstellung der deutschen Rentenversicherung bildet eine ökonomische Operationalisierung und Bewertung der Anreizwirkungen, die das beschriebene System impliziert, bzw. im Zeitablauf impliziert hat in Abschnitt 3.5 sowie Arbeitshypothesen für die empirische Untersuchung (Unterkapitel 3.6).

\subsection{Grundlegende Aspekte von Alterssicherungssystemen}

In der Literatur wird an vielen Stellen die Finanzierungsfrage (Umlage- oder Kapitaldeckungsverfahren) als das einzige Kriterium zur Bewertung eines Rentenversicherungssystems und für Reformvorschläge diskutiert ${ }^{2}$. HOMBURG (1988) hingegen unterscheidet in einer „Typologie der Alterssicherungssysteme“ fünf Aspekte zur Differenzierung unterschiedlicher Systeme ${ }^{3}$ :

- Umlage- versus Kapitaldeckungsverfahren

Das in den meisten Staaten praktizierte Finanzierungsverfahren der staatlichen Rentenversicherung ist das Umlageverfahren. Es beruht auf dem „Generationen-

2 Siehe etwa die Beiträge in FELDERER (1987), FELDERER (1992), RAFFELHÜSCHEN UND KITTERER (1990) oder HOMBURG UND RICHTER (1990).

${ }^{3}$ HOMBURG (1988) „Theorie der Alterssicherung“, Kapitel 2, Seite $5 \mathrm{ff}$. 
vertrag“, einer (ungeschriebenen) Abmachung der in Kapitel 2 genannten Altersgruppen einer Gesellschaft, daß die jeweiligen Erwerbspersonen in ein Versicherungssystem Beiträge einzahlen, aus dem zeitgleich die Renten an die Generation im Ruhestand bezahlt werden. Die Erwerbspersonen als zukünftige Rentner wiederum werden im Alter von der dann arbeitenden Generation ihrer Kinder finanziert.

Aufgrund der zeitgleichen Finanzierung der Ausgaben durch die laufenden Einnahmen existiert in der reinen Form keinerlei Kapitalstock, ${ }^{4}$ und es gilt somit die in Kapitel 2.2 dargestellte Budgetbeschränkung (Gleichungen 2-1 und 2-2). Mit dieser istdas System den Schwankungen der relativen Generationenanteile (Alterslastquotient) voll ausgesetzt und muß diese wie dort beschrieben kompensieren.

Beim Kapitaldeckungsverfahren hingegen werden die Renten der Pensionäre ausschließlich aus dem Kapital heraus finanziert, das sie selbst im Laufe ihres Erwerbslebens angespart haben. Jede Person spart bis zum Verrentungszeitpunkt Kapital an, aus dem nach diesem Zeitpunkt das Ruhegehalt finanziert wird. Der Verlauf des angesparten Kapitalvermögens entspricht dem der einfachen Lebenszyklushypothese, die graphisch in Abbildung 2-2 dargestellt ist.

In diesem System besteht also zu jedem Zeitpunkt ein positiver Kapitalstock. Eine Budgetbeschränkung wie in den Gleichungen 2-1 und 2-2 dargestellt, gilt hier nicht.

- Staatliche versus Private Alterssicherung

Die älteste Form der Alterssicherung ist die der (Groß-) Familie: die drei Generationen leben zusammen und haben eine Aufgabenteilung derart, daB die mittlere Generation das notwendige Einkommen erwirtschaftet. Dieses System ist rein privatwirtschaftlich organisiert. Weitere Beispiele privater Altersvorsorge sind private Ersparnisse oder Lebensversicherungen, aber auch Rentenzusagen durch Arbeitgeber an ihre Mitarbeiter.

Ein staatliches Rentenversicherungssystem ist dagegen durch eine ,übergeordnete“ Organisation charakterisiert, wobei es unerheblich ist, ob es sich um einen rein staatlichen oder parafiskalischen Träger handelt.

- Zwangsversicherung versus freiwillige Sicherung

Im Gegensatz zur freiwilligen Altersabsicherung, bei der idealtypisch jede Person individuell entscheidet, ob und in welchem Umfang sie eine Vorsorge für den Ru-

\footnotetext{
${ }^{4}$ In dieser Hinsicht ist die deutsche Gesetzliche Rentenversicherung keine reine Form eines Umlageverfahrens, da gesetzlich eine Monatsausgabe als Schwankungsreserve vorgeschrieben ist. Tatsächlich lag dieser Wert in der Vergangenheit erheblich höher und schwankte vom Ende der 60er bis Mitte der 70er Jahre zwischen 7 und 9 Monatsausgaben, sank dann bis zum Tiefstand von 1984 $(0,9)$ stetig ab und hat sich derzeit um einen Wert von 2 Monatsreservern eingependelt. Von einem Kapitalstock konnte jedoch zu keinem Zeitpunkt die Rede sein, da ein solcher mehrere Jahresausgaben betragen müBte; vgl. MÜLLER UND ROPPEL (1990).
} 
hestand trifft, geht ein Zwangssicherungssystem mit einer staatlichen Organisation einher, die die Einhaltung der Regeln überwacht.

- Verfahren mit versus ohne Risikoausgleich

Den Begriff Risiko benutzt HoMBURG als Synonym für die individuell unsichere Lebenserwartung. Auch wenn die durchschnittliche Lebenserwartung aller Personen einer Gesellschaft zu jedem Zeitpunkt fixiert ist, muß jeder Einzelne mit dem „Risiko“ rechnen, wesentlich älter als der Durchschnitt zu werden. Daraus wird deutlich, daß bei einer individuellen Vorsorge ohne Risikoausgleich jeder Einzelne ein zu hohes Kapital ansparen muß, um bis ins potentiell hohe Alter gesichert zu sein.

- Verfahren mit und ohne Umverteilung

Auch der Begriff Umverteilung ist hier nur in einem speziellen Sinne gemeint, dem der Umverteilung zwischen Generationen, wie sie bereits in Kapitel 2 beschrieben wurde. Die Frage ist in diesem Zusammenhang, ob und wieweit Änderungen der Produktivität oder der Altersstruktur berücksichtigt werden. In einem System ohne Umverteilung sind bei positivem Wirtschaftswachstum die Rentner schlechter gestellt, da sie an dieser Entwicklung nicht teilhaben.

Nicht alle der Kombinationen dieser Charakteristiken sind kompatibel, sondern mehrere Aspekte schließen sich gegenseitig aus. HOMBURG stellt allerdings heraus, daß in der Literatur häufig nur exakt 2 der möglichen 32 Fälle miteinander verglichen werden,

- als „Umlageverfahren“ ein staatliches Rentenversicherungssystem mit Zwangsmitgliedschaft, mit Risikoausgleich und mit Umverteilung und

- als „Kapitaldeckungsverfahren“ ein privates Versicherungssystem ohne Zwang, ohne Risikoausgleich und ohne Umverteilungs

und $\mathrm{da} B$ dieses Vorgehen eine grobe Vereinfachung der Problemstellung ist.

Tatsächlich ist das bestehende bundesdeutsche System exakt vom ersten Typ. Die Frage ist aber, ob als Alternative lediglich das zweitgenannte idealtypische Kapitaldekkungsverfahren zur Verfügung steht, oder Mischformen denkbar sind.

Zur Frage, welches System das ökonomisch bessere ist, gibt es eine breite Literatur, die sowohl theoretische wie auch empirische Herangehensweisen umfaßt ${ }^{6}$. Die theore-

\footnotetext{
5 HOMBURG (1988), Seite 8.

6 Vergleiche hierzu die Diskussion des Kapitels 2.2 im letzten Abschnitt „gesamtwirtschaftliche Implikationen der Altersstrukturverschiebung“. Zu nennen sind als theoretische Arbeiten etwa die Beiträge in FELDERER (1987): „Kapitaldeckungsverfahren versus Umlageverfahren“" HOMBURG 
tischen Studien stellen heraus, daß es aus Effizienzgesichtspunkten sinnvoll sei, ein Kapitaldeckungsverfahren zu etablieren, da in diesem eine direkte Äquivalenz der eingezahlten Beiträge zur auszuzahlenden Rente besteht, die durch die vorliegende Altersstrukturverschiebung der Tendenz nach nicht so stark verzerrt wird, wie dies beim Umlageverfahren der Fall ist ${ }^{7}$. Auch die empirischen Studien von FELDERER (1992) und BÖRSCH-SUPAN, MEIER UND REIL-HELD (1994) kommen zu diesem Ergebnis. Dagegen hält z.B. ScHOLZ (1993) im Resümee einer theoretischen Analyse „ein Plädoyer dafür, im Rahmen einer zukünftigen Reform ein staatliches Alterssicherungssystem zu installieren, das aus einer umlagefinanzierten, weiterhin beitrags- und einkommensbezogen bleibt und durch eine Zwangsmitgliedschaft gekennzeichnet ist"

Das grundlegende Problem ist allerdings der potentielle Systemwechsel selbst. Die Änderung eines bestehenden Umlageverfahrens in ein kapitalgedecktes halten viele Autoren für faktisch nicht durchführbar, da dadurch die betroffene (Übergangs-) Generation der Erwerbspersonen doppelt belastet würde. Sie müßte zum einen die Rente der heutigen Pensionäre finanzieren, da für diese kein Kapitalstock besteht und gleichzeitig einen Kapitalstock für die eigene Ruhestandsphase schaffen. Eine staatliche Finanzierung des Anfangskapitalstocks sei ebenfalls nicht möglich, da sie entweder steuerfinanziert sein müßte, was theoretisch äquivalent zu erhöhten Rentenversicherungsbeiträgen wäre, ${ }^{9}$ oder auf dem Kapitalmarkt finanziert werden müßte, was bei den in Frage stehenden Dimensionen finanziell nicht leistbar sei. MÜLLER UND ROPPEL (1990) nehmen „Eine Abschätzung des Kapitalbedarfes bei einer vollständigen Kapitaldekkung der Gesetzlichen Rentenversicherung" vor und kommen zu dem Ergebnis, daß der für eine Systemumstellung nötige Kapitalstock den Gegenwert mehrerer Jahres-

(1988): „Theorie der Alterssicherung“ oder BREYER (1990): „Ökonomische Theorie der Alterssicherung " mit weiterführenden Literaturhinweisen.

${ }^{7}$ Eine - wenngleich kleinere - Verzerrung gibt es allerdings auch, da angespartes Kapital nur dadurch produktiv sein kann, daß mit ihm produziert wird. Durch einen steigenden Altersquotienten erhöht sich das Kapitalangebot bei gleichzeitig knapper werdenen Arbeitskräften. Daher verschieben sich die relativen Preise von Arbeit und Kapital, namentlich wird der Zinssatz sinken, so daß die Pensionärsgeneration tatsächlich relativ weniger Kapitalrückflüsse erhält, als unter strenger Einzahlungen / Auszahlungen - Äquivalenz notwendig wäre. Vgl. auch HOMBURG (1988), Kapitel 3, Seite 15ff.

${ }^{8}$ SCHOLZ (1993) „Ausgestaltung und Reform der gesetzlichen Alterssicherung“, Seite 257.

9 Tatsächlich werden die Rentenversicherungsbeiträge nur von den sozialversicherungspflichtig Beschäftigten getragen, wogegen ein Großteil der Selbständigen und Beamte hierdurch nicht belastet werden. Steuern hingegen werden von allen Bevölkerungsgruppen getragen, teilweise auch durch die Rentner selbst, wenn diese hohe sonstige Einkommen haben. 
Sozialprodukte hätte ${ }^{10}$, was nicht über den Kapitalmarkt zu leisten ist. In der Bundesrepublik wird somit ein Wechsel vom derzeitigen Umlageverfahren auf ein Kapitaldeckungssystem in absehbarer Zeit aufgrund der Finanzrestriktionen nicht möglich sein. Dies gilt unabhängig von der ökonomischen Bewertung der beiden Systeme.

\subsection{Das Alterssicherungssystem der Bundesrepublik Deutschland}

Die Begründung für die Existenz einer staatlich organisierten Rentenversicherung mit Zwangsmitgliedschaft ist in der staatlichen Fürsorge- bzw. Versorgungspflicht zu sehen. Wenn eine alle Bürger umfassende Versorgung gewünscht wird, kann dies nur auf Basis einer sowohl intergenerational wie auch interpersonal umverteilenden Versicherung mit Mitgliedschaftszwang und Risikoausgleich erfolgen."

Die Frage, ob der Staat diese Altersversorgung vollständig übernehmen oder ob der privaten Initiative der einzelnen Arbeitnehmer Raum gegeben werden sollte, wurde in der sozialpolitischen Diskussion viel besprochen. ${ }^{12}$ Als ein verbreiteter Ansatz hat sich das 3-Säulen Modell'3 etabliert, das in Abbildung 3-1 skizziert ist. Dieser Ansatz besagt, daß die Sicherung der Versorgung im Alter auf drei Standbeinen (Säulen) beruht. In Deutschland ist die Gesetzliche Rentenversicherung die mit Abstand wichtigste Säule, die für die meisten Menschen den Großteil des Alterseinkommens abdeckt. Die relative Bedeutung der einzelnen Säulen schwankt international erheblich. So ist in den USA die betriebliche Altersversorgung von wesentlich größerer Relevanz als in der Bundesrepublik. Neben der GRV ist in der Bundesrepublik Deutschland auch die betriebliche Altersversorgung gesetzlich geregelt (vgl. Kapitel 3.2.2). Dagegen liegt die private Absicherung in der Hand des Einzelnen, wird allerdings ebenfalls durch steuerliche Anreize und Versicherungsaufsicht staatlich kontrolliert ${ }^{14}$.

${ }^{10}$ Für eine theoretische Analyse vergleiche auch RAFFELHÜSCHEN UND KITTERER (1990), „Übergangsprobleme eines Systemwechsels in der sozialen Alterssicherung. Eine dynamische Systemanalyse".

1 Für eine umfassende Diskussion der Legitimation staatlicher Sozialpolitk vergleiche etwa FRERICH (1987), Seiten 30-35 sowie CASMIR (1989), Seite $65 \mathrm{ff}$.

12 Vgl z.B. Heinelt (1991), der den „Public/Private-Mix der Alterssicherung“ beschreibt (Seite 42 ff) mit weiteren Literaturhinweisen.

13 Vgl. etwa DürKoP (1992), Seite $12 \mathrm{ff}$ oder SCHMÄHL (1991 b), Seite $37 \mathrm{ff}$.

14 Die Frage, inweit eine Zwangsversicherung private Sparaktivität verdrängt wurde in Kapitel 2.2 diskutiert. 
Die relativen Anteile der Säulen hängen auch vom Selbstverständnis oder Sicherungsziel der staatlichen Altersversorgung ab. Wird diese - wie in der Bundesrepublik - als Lebensstandardsicherung mit Lohnersatzcharakter ${ }^{15}$ verstanden, so folgt die Dominanz dieser GRV über die anderen Säulen. Dagegen ist die US-Amerikanische staatliche Rentenversicherung ${ }^{16}$ als Mindestsicherung in Form eines Zuschusses zum allgemeinen Lebensbedarfs konzipiert ${ }^{17}$, so daß das dortige System stärker auf die Selbstverantwortung der Versicherten setzt und die individuelle Vorsorge betont. Das deutsche System der sozialen Sicherung ${ }^{18}$ ist nach dem Subsidiaritätsprinzip organisiert, diese Idee des „sozialen Netzes“ besagt, daß die Menschen, deren Absicherung (hier Ver-Sicherung) nicht ausreicht, durch staatliche Unterstützung (Sozialhilfe) versorgt werden. Thema dieses Abschnittes sind die gesetzlichen Regelungen der Alterssicherung, die sich wie erwähnt auf alle 3 Säulen auswirken, wenn auch in unterschiedlicher Stärke.

Abbildung 3-1: Die drel Säulen der Rentenversicherung

\section{A 1 t e rs sicherung}

\begin{tabular}{|c|c|c|}
\hline $\begin{array}{c}\text { I V } \\
\text { Individuelle } \\
\text { Vorsorge } \\
\begin{array}{c}\text { (private } \\
\text { Lebensver- } \\
\text { sicherung/ } \\
\text { Vermögen) }\end{array}\end{array}$ & B A V \\
\hline
\end{tabular}

Sinn dieses Abschnittes ist allerdings nicht die umfassende Darstellung des bundesdeutschen Rentenversicherungssystems, sondern lediglich die Aufarbeitung seiner für die empirische Analyse notwendigen Kernpunkte. Ein Schlagwortindex am Ende dieses Buches erleichtert das Auffinden der einzelnen Regelungen und Fachbegriffe. Für Details sei einerseits auf Lehrwerke der Sozialpolitk verwiesen, z.B. LAMPERT (1985),

\footnotetext{
15 BUNDESMINISTER FÜR ARBEIT UND SOZIALORDNUNG -BMA (Hg.), Kapitel 5, Seite 145 ff. 16 OASDHI: Old-Age, Survivors, Disability and Health Insurance.

17 Vgl. CASMIR (1989), Seiten 383-386; eine Umverteilung findet allerdings auch im Rahmen der OASDHI statt.
}

$18 \mathrm{Zu}$ diesem Sozialversicherungssystem sind neben der Rentenversicherung weiterhin die Arbeitslosen-, Kranken- und Unfallversicherung sowie zukünftig die Pflegeversicherung zu zählen. 
„Lehrbuch der Sozialpolitik“ oder FrERICH (1987) „Sozialpolitik“, der u.a. eine anschauliche geschichtliche Übersicht über das gesamte Sozialsystem der Bundesrepublik und dessen Entwicklung gibt. Daneben bieten auch die international vergleichenden Studien von CASMIR (1989) „Staatliche Rentenversicherungssysteme im internationalen Vergleich“ und DÜRKOP (1992) „Alterssicherung in der EG“ übersichtliche Darstellungen der Rentenversicherungssysteme. CASMIR bearbeitet über die reine Darstellung der Alterssicherungssysteme inhaltliche Fragestellungen ${ }^{19}$ und nimmt in diesem Rahmen teilweise (vergleichende) Bewertungen und Beispielrechnungen vor, die die Funktionsweise der Einzelregelungen veranschaulichen ${ }^{20}$.

Für die folgende Darstellung des bundesdeutschen Rentenrechtes ist es wichtig, zwischen den derzeit geltenden Gesetzen, die durch die Rentenreform 1992 in Kraft traten bzw. modifiziert wurden und den Regelungen, die vor 1992 in Kraft waren, zu unterscheiden. Letztere, also die „alten“ Regelungen (bis 1991) sind für die vorliegende Arbeit relevant, da sich beide empirischen Untersuchungen auf Daten der Jahre vor 1992 stützen. Aus diesem Grunde bezieht sich der Abschnitt 3.2.1 nur auf den Stand der Rentenversicherung bis 1991.

Der grundlegende Struktur der Rentengesetzgebung hat sich durch die Reform von 1992 nicht verändert. Allerdings wurde die Rentenformel neu strukturiert und bezieht neue Elemente ein, sowie diverse „Termini technici“ umbenannt und verändert. Die Rentenreform 1992 wird im Rahmen des Kapitels 3.3 vorgestellt, in dem die historische Entwicklung der GRV thematisiert wird. Dort findet sich auch ein Vergleich der alten und neuen Regelungen, insbesondere der Rentenformeln, der durch die Diskussion der ökonomischen Anreizwirkungen in Kapitel 3.5 abgerundet wird.

\subsubsection{Die Gesetzliche Rentenversicherung (GRV)}

Träger der GRV sind unterschiedliche Versicherungsanstalten, deren gleichzeitige Existenz historische Ursachen hat. Die Träger sind rechtsfähige Körperschaften des öf-

19 Diese Themen sind die „Vermeidung von Armut“, die „Stetigkeit des Einkommens“ sowie die „soziale Sicherung der Frauen“.

20 Für Detailfragen bieten sich juristisch orientierte Lehrbücher wie Ruland und Maydell (1989), "Sozialrechtshandbuch" oder Maydell (1986), "Lexikon des Rechts" an. Die wichtigste Primärquelle ist die Reichsversicherungsordnung (RVO), das Angestelltenversicherungsgesetz (AVG) und das Sozialgesetzbuch (SGB - allgemeiner Teil) mit entsprechenden Kommentaren. Diese Darstellungen gehen allerdings in ihrer Tiefe in der Regel weit über das in einer empirischen Arbeit relevante hinaus. 
fentlichen Rechts mit eigenem Haushalt, die einer begrenzten staatlichen Aufsicht unterliegen. Die Auszahlungen werden aus dem Bundeshaushalt durch den Bundeszuschuß mitfinanziert. Der Bundeszuschuß und andere Kenngrößen der deutschen GRV sind in Tabelle 2-3 wiedergegeben. Gemessen in von Hundert der gesamten Bundesausgaben liegt der Bundeszuschuß seit den 50er Jahren kontinuierlich bei etwa $5 \%$ und stellt somit einen nicht unerheblichen Ausgabenfaktor dar.

Die wichtigsten Einzelträger der GRV sind die Bundesversicherungsanstalt für Angestellte (BfA) in Berlin, die für alle Angestellten im Bundesgebiet zuständig ist, und die inzwischen 23 Landesversicherungsanstalten (LVA) für Arbeiter. ${ }^{21}$ Dazu kommen einige kleinere Anstalten, in denen Arbeiter aus eng definierten Bereichen versichert sind, wie die Bundesknappschaft, die Bundesbahnversicherungsanstalt, die Seekasse und weitere Einzeleinrichtungen.

Im öffentlichen Sektor ist zum einen die Beamtenversorgung zu nennen, die allerdings nicht in Form einer Versicherung organisiert ist, da deutsche Beamte nach der Pensionierung ein Ruhegehalt aus dem laufenden Staatshaushalt beziehen. Dieses stellt ökonomisch ein reines Umlageverfahren dar. Ebenfalls zu diesem Bereich gehört die $\mathrm{Zu}$ satzversicherung für Angestellte und Arbeiter im öffentlichen Dienst durch die Versorgungsanstalt des Bundes und der Länder (VBL), die ebenfalls als Umlageverfahren (mit Zwangsmitgliedschaft) organisiert ist.

Die Rentenversicherungsträger sind zusammengeschlossen in einer Dachorganisation, dem Verband Deutscher Rentenversicherungsträger (VDR), der administrative und statistische Aufgaben zentral wahrnimmt. Der VDR ist ein privatrechtlicher Zusammenschluß der Träger in Form eines eingetragenen Vereines und unterliegt damit keiner direkten staatlichen Aufsicht. 22

\section{Erfaßter Personenkreis}

Als Pflichtversicherung, erfaßt die GRV alle Arbeitnehmer (Arbeiter, Angestellte, Auszubildende) automatisch im Rahmen der Sozialversicherungspflicht. Die Versicherungspflicht ist gesetzlich verankert, die Mitgliedschaft kann nicht abgelehnt werden. Unter die Versicherungspflicht fallen:23

21 Die LVA's sind nicht nach Bundesländern, sondern Versicherungsbezirken organisiert, in den neuen Bundesländern enstanden allerdings 5 Bezirke, die den Bundesländern entsprechen.

22 Vgl. auch FrERICH (1987), Kapitel 7

${ }^{23} \mathrm{Zu}$ den jeweils zutreffenden Beitragsregeln und -höhen vergleiche etwa CASMIR (1989) Seite 468 ff. 
- Pflichtversicherte sind alle abhängig Beschäftigten d.h. nichtselbständige Personen, ab einem Einkommen von 500,- DM ${ }^{24}$ pro Monat einschließlich Auszubildender. Einkünfte unter dieser Grenze gelten als 'geringfügige Beschäftigungen', mehrere solcher Einkünfte werden allerdings addiert. Die Versicherungspflicht ist unabhängig von Alter, Geschlecht und Nationalität. Im Ausland lebende Deutsche können an der Pflichtversicherung teilnehmen,

- Männer, die Wehr- oder Zivildienst im Rahmen der Wehrpflicht leisten. Ihnen wird für diese Zeit ein Einkommen von 70\% eines Durchschnittseinkommens zugerechnet,

- Pflichtversicherte Selbständige sind besonders definierte Gruppen, die trotz Selbständigkeit der Versicherungspflicht unterliegen: Selbständige Lehrer und Erzieher, Künstler und Publizisten sowie freiberuflich tätige Hebammen, Entbindungshelfer und Handwerker,

- Behinderte Personen in anerkannten Werkstätten sowie Auszubildenden in diesen Einrichtungen.

- Mitglieder geistlicher Genossenschaften und ähnliche Beschäftigte.

Versicherungsfrei sind hingegen:

- Selbständige,

- Arbeitslose, also Personen, die von der Bundesanstalt für Arbeit Arbeitslosengeld, Arbeitslosenhilfe oder Unterhaltsgeld beziehen, unterliegen seit dem 1.1.1983 nicht mehr der Pflichtversicherung,

- Rehabilitanten und Bezieherinnen von Mutterschaftsgeld seit dem 1.1.84 ebenfalls nicht mehr,

- Vollrentner, auch wenn sie Erwerbseinkommen erzielen,

- Studenten;

- sowie auf Antrag:

- Personen, die einem besonderen Versorgungssystem angehören, z.B. berufsständischer Versorgungseinrichtungen: Ärzte, Rechtsanwälte, Notare und Architekten.

Hingegen können sich die meisten dieser Personen in der freiwilligen Versicherung versichern ${ }^{25}$. Freiwillig Versicherte haben die gleichen Rechte und Pflichten wie Pflichtversicherte.

\section{Beiträge}

Die RV-Beiträge werden bei abhängig Beschäftigten im Rahmen der Sozialabgaben als Pflichbeiträge in Höhe von z.Zt. ca. 19\% des Brutto-Einkommens (vgl. Tabelle 23) direkt durch den Arbeitgeber vom Gehalt einbehalten und an den RV-Träger abgeführt. Der Beitrag wird jeweils zur Hälfte von Arbeitgeber und Arbeitnehmer getragen, so daß nur der Arbeitnehmerbeitrag zu dessen steuerpflichtigem Einkommen gehört.

Bei geringfügigen Beschäftigungen (unterhalb eines Freibetrages) wird der gesamte Beitrag durch den Arbeitgeber getragen.

\footnotetext{
${ }^{24}$ Wert für 1992, 1989 betrug er 410,- DM. Er errechnet sich als 1/7 der monatlichen Bezugsgröße nach § 18 SGB IV ('durchschnittliches Arbeitsentgelt aller Versicherten der Rentenversicherung der Arbeiter und Angestellten ohne Auszubildende im vorhergehenden Kalenderjahr), vgl. SGB $\S 8$ i.V.m. §18, sowie CASMIR (1989), Seite 466).

${ }^{25}$ Dies steht allen Personen offen, die nicht einer Pflichtversicherung unterliegen. Nicht allerdings Vollrentnern, Studierenden und Beamten.
} 


\section{Rentenarten}

Unter dem Begriff „Rentenarten“ oder „Leistungsarten“ wird in der GRV die Unterteilung in definierte Gruppen von Rentenleistungen verstanden. Die erste Unterscheidung ist die der Versichertenrenten und der Hinterbliebenenrenten. Die letzteren bilden eine Sondergruppe, da den an sie zu zahlenden Leistungen keine eigenen Beiträge gegenüberstehen. Personen mit bestehendem Anspruch auf eine Alters- oder Hinterbliebenenrente, die nicht (aktiv) versichert sind, werden als latent Versicherte bezeichnet. Dies trifft auf Arbeitnehmer (oft Frauen) zu, die in früherer Erwerbstätigkeit einen Anspruch erworben haben, aber nicht aktiv am Erwerbsleben teilnehmen. Für die in dieser Arbeit betrachtete individuelle Ruhestandsentscheidung spielt diese Gruppe keine Rolle, da sie naturgemäß keine Entscheidung zum Austritt aus dem Erwerbsleben trifft.

Die Versichertenrenten wiederum unterteilen sich in Invaliditätsrenten und Altersrenten und gliedern sich nach dem Rentenzugangsalter wie folgt: ${ }^{26}$

- Normales Altersruhegeld:

kann bezogen werden nach dem Erreichen des Regelrentenalters und der Erfüllung besonderer Versicherungszeiten (Wartezeiten ${ }^{27}$ ). Das Regelrentenalter liegt für alle Versicherten bei 65 Jahren, die Wartezeit beträgt (seit 1980) 60 Monate;

- Hinausgeschobenes Altersruhegeld: (seit 1972) wird bezogen, wenn Anspruch auf normales Altersruhegeld besteht, aber ein bis zu zwei Jahren hinausgeschobener Rentenbeginn gewählt (d.h. 66 bzw. 67 Jahre) wurde. Für diese Verschiebung von bis zu 2 Jahren gibt es pro Jahr eine Erhöhung der Rente um 7\%.

- Vorgezogenes (flexibles) Altersruhegeld: (seit 1972)

alle Versicherten mit dem Erreichen des 63. Lebensjahres und einer Wartezeit von 35 Versicherungsjahren;

Im Zusammenhang dieser freien Wahl des flexiblen Ruhestandes zwischen dem 63. und dem 67. Lebensjahr, wird in der Praxis oft vom Verrentungsfenster gesprochen

- Vorzeitiges Altersruhegeld für Frauen:

kann nach Erreichen des 60. Lebensjahres bezogen werden, wenn die Versicherte in den letzten 20 Jahren für mehr als der Hälfte der Monate Pflichbeiträge gezahlt hat;

- Vorzeitiges Altersruhegeld wegen Arbeitslosigkeit: (seit 1957)

Versicherte, die mindestens 52 Wochen aus den letzten anderhalb Jahren arbeitslos gemeldet waren, können mit Erreichen des 60. Lebensjahres vorzeitiges Altersruhegeld beziehen. Diese

26 Vgl. VDR (1992): „Zeitreihen“ - Glossar.

27 Wartezeiten sind die zur Entstehung eines Leistungsanspruches erforderlichen Mindestversicherungszeiten. Zu den Wartezeiten zählen die Beitrags-, sowie die Kindererziehungs-, Ersatz- und Ausfallzeiten.

Ersatzzeiten sind gegeben, wenn der Versicherte aus Gründen, die er nicht zu vertreten hat, keine Beiträge zahlen konnte. Dies trifft sowohl auf Wehr- und Zivildienst, also auch auf Vertreibung, Verfolgung, Um- und Aussiedlung;

Ausfallzeiten liegen vor, wenn der Versicherte aus persönlichen Gründen an einer Beitragsleistung gehindert war. Dies trifft vor allem zu bei Krankheiten, einer Schul-, Fach- oder Hochschulausbildung und bei Arbeitslosigkeit (vgl. VDR (1992): ,Zeitreihen“ - Glossar) 
Möglichkeit ist vor allem für Männer interessant, da Frauen das o.a. vorzeitige Ruhegeld zusteht, wenn die Wartezeit erfüllt ist.

- Flexibles Altersruhegeld für Schwerbehinderte, Berufs- und Erwerbsunfähige: (erleichtert seit 1980)

Die genannten Versichertengruppen können das vorgezogenen Altersruhegeld bereits mit dem 60. Lebensjahr in Anspruch nehmen. Die Wartezeit beträgt auch hier grundsätzlich 35 Versicherungsjahre, wobei allerdings Zurechnungszeiten ${ }^{28}$ berücksichtigt werden.

\section{Arten der Invalidtätsrenten:}

Das deutsche Rentenrecht unterscheidet zwischen Schwerbehinderung, Berufsunfähigkeit (BU) und Erwerbsunfähigkeit (EU) ${ }^{29}$ :

- Allgemeine Regelung -Invalidität:

Für Renten bei Invalidität gibt es keine Altersbegrenzung, sie beginnen mit Ablauf des Monats, in dem die Behinderung eingetreten ist.

Für die Berechnung der Renten werden die Zurechnungszeiten (s.o. bzw. Fußnote) in die Berechnung einbezogen. Es besteht allerdings erst dann ein Anspruch auf Zahlung einer Rente, wenn Halbbelegung (s.o.) vorliegt.

- Erwerbsunfähigkeit (EU):

Die Erwerbsunfähigkeit eines Arbeitnehmers ist gegeben, wenn er infolge einer Krankheit oder Behinderung auf nicht absehbare Zeit keine Erwerbstätigkeit oder nur eine mit geringfügigem Einkommen ausüben kann. Eine Versicherungszeit von 60 Monaten muß erfüllt sein.

- Berufsunfähigkeit $(\boldsymbol{B U})^{30}$ :

Eine Berufsunfähigkeit liegt vor, wenn die Erwerbsfähigkeit einer Person in ihrem ausgeübten Beruf nur noch weniger als die Hälfte der eines gesunden Menschen beträgt. Sie kann nach einer Versichenungszeit von 60 Monaten gewährt werden. Die BU-Rente ist ein Drittel niedriger (Steigerungssatz St $=1,0$ ) als eine Alters- oder EU-Rente. Dies ist in der Annahme begründet, $\mathrm{da} \beta$ berufsunfähige Personen trotzdem noch einer geringfügigen Erwerbstätigkeit nachgehen können. Entsprechend haben sie auch eine Hinzuverdienstmöglichkeit von bis zu 50\% ihres ehemaligen Einkommens, ohne Anrechnung auf die Rentenhöhe.

\section{Formen der Frühverrentung - Alternative Wege in den frühen Ruhestand:}

Im zweiten Kapitel wurde gezeigt, daß eine erhebliche Anzahl von Arbeitnehmern schon vor dem flexiblen Ruhestand von 63 Jahren und auch vor 60, dem Zeitpunkt der

${ }^{28}$ Die Zurechnungszeit ist die Zeit zwischen dem Eintreten eines Versicherungsfalles (Invalidität) und dem 55. Lebensjahr. Diese Jahre werden dem Invaliden also zusätzlich zu seinen Beitragsjahren als Versicherungsjahre angerechnet, allerdings nur, wenn die Bedingung der Halbbelegung erfüllt ist.

Halbbelegung liegt vor, wenn zwischen dem Eintritt des Versicherten in die Versicherung und dem Versicherungsfall (Invalidität) für mindestens die Hälfte, mindestens jedoch 60, der Monate Pflichbeiträge gezahlt worden sind. (vgl. VDR (1992): „Zeitreihen“- Glossar).

29 Vgl. CASMIR (1989) Seite 476 und genauer: Seite $482 \mathrm{ff}$.

${ }^{30}$ Zur Behandlung der BU-Renten in der Rechtsprechung vgl. den folgenden Abschnitt zur Frühverrentung. 
vorzeitigen Verrentung, in den Ruhestand treten. Die Konsequenz dieser Entwicklung ist, daß das durchschnittliche Renteneintrittsalter unter 60 Jahren liegt. Es gilt an dieser Stelle aufzuzeigen, welche Wege in den Ruhestand die Frührentnern offenstehen.

Die Frage nach Formen und Ursachen der Frühverrentung werden in vielen sozialpolitischen Arbeiten diskutiert. Eine grundlegende und sehr sorgfältige Analyse ist die von JACOBS, KOHLI UND REIN (1991) „Pathways to Retirement“. Die potentiellen Strategien der verschiedenen Akteure, „Verrentungswillige“ und Firmen, werden dargestellt (vgl deren Kapitel 6.3, Seite $198 \mathrm{ff}$ ) und als wichtigste 3 Wege festgehalten: Arbeitslosigkeit (59er Regel), Vorruhestand und Gesundheitsgründe in ihren vielfachen Ausprägungen.

Die beiden Rentenarten $\boldsymbol{B} \boldsymbol{U}$ und $\boldsymbol{E} \boldsymbol{U}$ sind zusammen mit der Arbeitslosigkeitsregel zentral für diese Frage, da sie - insbesondere für Männer - die gesetzlich vorgegebenen Möglichkeiten darstellen, mit 60 Jahren in den Ruhestand zu treten.

Die Regelung zur Arbeitslosigkeit wurde in den Jahren 1984-1988 durch die „Vorruhestandsregelung31" erweitert, einer primär arbeitsmarktpolitischen Maßnahme, bei der Arbeitnehmer ab einem Alter von 58 Jahren in den Ruhestand gehen konnten. Die Firma zahlte ein Vorruhestandsgeld von mindestens $65 \%$ (Praxis: 75-80 \%) des bisherigen Bruttogehaltes und erhielt dafür bei Einstellung eines jüngeren Arbeitnehmers einen Zuschuß der Bundesanstalt für Arbeit (BA). Die Arbeitnehmer waren weiterhin sozialversichert und konnten mit dem Erreichen des 60. Lebensjahres in die reguläre Altersrente eintreten.

Eine ähnliche Form des vorgezogenen Ruhestandes ist die ,59er Regel 32 “, mit der Unternehmen ältere Arbeitnehmer ,freisetzen“, indem diese offiziell entlassen und daher arbeitslos werden. Das Unternehmen zahlt auch hier einen Zuschlag auf das Arbeitslosengeld, dieser wird teilweise mit Betriebsräten bzw. Gewerkschaften im Rahmen von Sozialplänen ausgehandelt. Obwohl diese Praxis legal im Sinne der Einhaltung der Gesetze ist, läuft sie doch der Intention, der sozialen Absicherung älterer Arbeitsloser, entgegen. Faktisch werden hier betriebliche Kosten von Unternehmen, die ihre Belegschaft verjüngen wollen, auf die Solidargemeinschaft der Versicherten in der Arbeitslosen- und Rentenversicherung abgewälzt. Durch diesen „Mißbrauch“ der Arbeitslo-

31 Vergleiche die Darstellung auf Seite 53 im Rahmen des Kapitels 3.2.3.

321986 wurde der Zeitraum, für den Arbeitslosengeld bezogen werden konnte von 12 auf 32 Monate erhöht, so daß die hier beschriebene Praxis schon mit 57 Jahren und 4 Monaten begonnen werden konnte und damit zur „57er Regel““ wurde. 
sigkeitsregel können die Arbeitnehmer mit 60 Jahren in den Ruhestand treten, diese Praxis entlastet Arbeitgeber und Arbeitnehmer, belastet allerdings die Bundesanstalt für Arbeit und die Rentenversicherung.

Ebenfalls zentral für die Frage der Frühverrentung sind die erwähnten $\boldsymbol{E} \boldsymbol{U} / \boldsymbol{B} \boldsymbol{U}$ Renten. Der oft geäußerte Verdacht des Mißbrauches ist eine Frage der Kausalität: Werden Arbeitnehmer zunehmend häufiger krank und der Arzt empfiehlt ihnen deshalb die vorgezogene Verrentung oder gehen 'pensionierungswillige' Beschäftigte zum Mediziner, um sich eine Erwerbsunfähigkeit bescheinigen zu lassen? Der oben beschriebene Unterschied zwischen EU und BU Rente, durch den letztere nur 2/3 der EU-Rente beträgt, erwies sich in der Praxis als weitgehend undurchführbar. Er wurde durch Sozialgerichtsentscheidungen dahingehend konkret interpretiert (im Gegensatz zur abstrakten Auslegung), daß Personen, die nach einem Jahr BU-Rente noch keine Teilzeitarbeit finden konnten, eine Umwandlung zur EU-Rente beantragen können.

Wie oben dargestellt, müssen die EU/BU - Renten unterteilt werden in die ,flexiblen Altersruhegelder für Schwerbehinderte, Berufs- und Erwerbsunfähige" ab dem 60. Lebensjahr (seit 1980) und die allgemeinen Invaliditätsrenten, die sich wiederum in Berufs- und Erwerbsunfähigkeitsrenten unterteilen, die aber keiner Altersbeschränkung unterliegen. Tatsächlich können nur die letztgenannten, altersunabhängigen Renten für ein durchschnittliches Rentenalter unter 60 Jahren verantwortlich sein.

Eine Veranschaulichung der beschriebenen unterschiedlichen Rentenarten und der zugehörigen Zugangsalter findet sich bei RIPHAHN, ${ }^{33}$ siehe Abbildung 3-2.

Abbildung 3-2: Rentenarten und Zugangsalter in der GRV

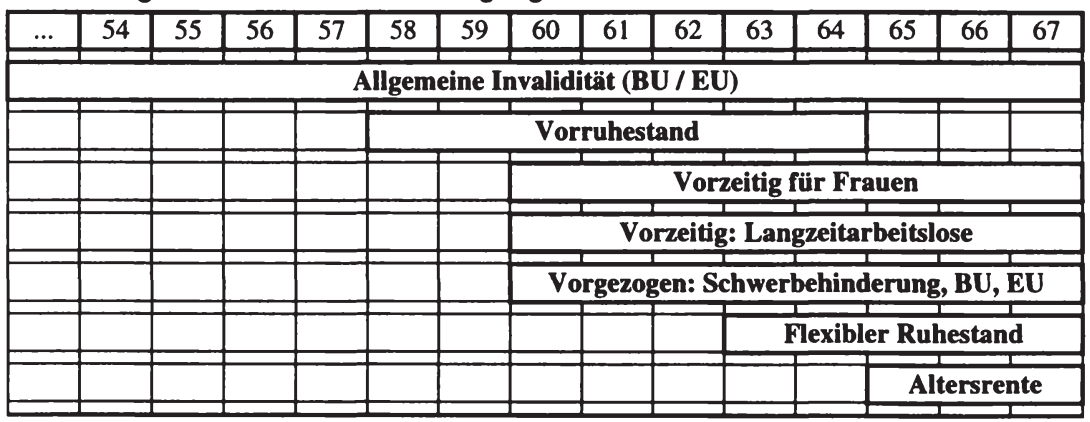

33 RIPHAHN (1995), Seite 3. 


\section{Leistungen und Rentenhöhe}

Die Rentenhöhe von Altersrenten berechnet sich nach der Rentenformel (3-1). Diese Formel hat sich durch die Rentenreform 1992 erheblich geändert. Sie ist in ihrer Struktur verändert worden, wodurch die hier aufgezählten Elemente in der nun gültigen Form teilweise umbenannt wurden, teilweise nicht mehr enthalten sind. Ein Vergleich der hier dargestellten mit der neuen Rentenformel findet sich in Kapitel 3.3.

Rentenformel bis 1991:

$$
\begin{aligned}
& \text { Jahresrente }=\mathrm{Vj} \cdot \mathrm{St} \cdot \mathrm{BG} \cdot \mathrm{Vhs} \\
& \mathrm{Vj} \quad \text { anrechnungsfähige Versicherungsj } \\
& \mathrm{St} \quad \text { Steigerungssatz } \\
& \mathrm{BG} \quad \text { allgemeine Bemessungsgrundlage } \\
& \mathrm{Vhs} \quad \text { persönlicher Vom-Hundert-Satz }
\end{aligned}
$$

mit: $\quad$ Vj anrechnungsfähige Versicherungsjahre

Dabei haben die Elemente der Rentenberechnung die folgenden Bedeutungen:

\section{Anrechnungsfähige Versicherungsjahre (Vj)}

Die Anzahl der Versicherungsjahre ergibt sich aus den unterschiedlichen Beitragszeiten (s.o.): Beitragszeiten, Ersatzzeiten, Ausfallzeiten, Kindererziehungszeiten und Zurechnungszeiten. ${ }^{34}$

Steigerungssatz (St)

Der Steigerungssatz legt einen von der Rentenart abhängigen Faktor fest, der die Rente korrigiert. Der normale St ist 1,5; für eine Berufsunfähigkeitsrente jedoch beträgt er nur 1,0, reduziert die Rente also um ein Drittel, da bei Berufsunfähigkeitsrenten eine zusätzliche Erwerbstätigkeit des Leistungsempfängers erwünscht ist. Ebenfalls über den St reduziert werden die Hinterbliebenenrenten.

\section{Allgemeine Bemessungsgrundlage (BG)}

Dieses Element der Rentenformel ist für die seit der Reform 1972 eingeführten Dynamisierung notwendig, d.h. der Koppelung der Rentenzahlungen an die Entwicklung der Bruttoarbeitsentgelte ${ }^{35}$. Es miBt die Veränderung der Durchschnittslöhne unter sich im Zeitverlauf ändernden Rechenregeln.

Persönlicher Vom-Hundert-Satz (Vhs)

Neben den Vj ist der Vhs der Bestandteil der Rentenberechnung, der die individuelle Anpassung der Rente an die persönlichen Anwartschaften sicherstellt. Verkürzt ausgedrückt mißt er die relative Einkommensposition des Versicherten im Vergleich zum Durchschnittsverdiener. Der Vhs wird für jedes Beitragsjahr gesondert ermittelt und abschließend über alle Versicherungsjahre gemittelt. Für die ersten fünf Versicherungsjahre sowie für unterschiedliche Beitragszeiten gelten besondere Berechnungskonventionen. ${ }^{36}$

$34 \mathrm{Zu}$ den einzelnen Definitionen vergleiche die Fußnote 27 im vorhergehenden Abschnitt oder CASMIR (1989) Seite 471.

35 Diese Anpassung der Renten an die Entwicklung der Bruttolöhne unter Vernachlässigung der steigenden relativen Steuerlast der Arbeitnehmer führte dazu, daB die Renten netto stärker stiegen als die Löhne, was naturgemäß Finanzierungsprobleme mit sich brachte. Mit der Rentenreform 1992 wird die Dynamisierung daher an die Netto-Lohnentwicklung gekoppelt

36 Vgl. CASMIR (1989), Seite $473 \mathrm{ff}$. 
In dieser Berechnung lassen sich drei Elemente der Rentenermittlung unterscheiden:

1. Allgemeine Komponenten (Steigerungssatz und allgemeine Bemessungsgrundlage). Diese unterliegen nicht dem Einfluß des Versicherten.

2. Messung der relativen Einkommensposition durch den persönlichen Vom-HundertSatz.

Diese ist durch den Versicherten bedingt beeinflußbar. Eine langfristige Entscheidung findet durch die Wahl von Ausbildung und Beruf statt. Kurz- und mittelfristig kann das Einkommen über das persönliche Arbeitsangebot, v.a. die Arbeitszeit variiert werden. Beide Einflußmöglichkeiten sind allerdings deutlich durch die Nachfragesituation determiniert.

3. Anpassung über die Anzahl der Beitragsjahre (Versicherungsjahre), d.h. die Lebensarbeitszeit.

Diese Größe steht im Mittelpunkt der vorliegenden Untersuchung. Durch die Wahl des Rentenzeitpunktes kann der Versicherte seine Lebensarbeitszeit direkt beeinflussen. Er unterliegt dabei allerdings den oben beschriebenen Mindestanforderungen, v.a. Versicherungszeiten, die zur Gewährung der unterschiedlichen Rentenarten notwendig sind.

Aus letzterem Punkt wird deutlich, daß das Vorziehen des Ruhestandes um ein Jahr nur einen sehr geringen Einfluß auf die Rentenhöhe hat. Das Rentenrecht sah (bis 1992, vgl. unten) keine explizite Regelung zur Adjustierung der Rente bei früherem Rentenzugang über die beschriebenen Wege vor. Es ergab sich lediglich eine Verminderung des Altersruhegeldes dadurch, daß weniger Beiträge gezahlt wurden, was sich auf die anrechnungsfähigen Versicherungsjahre $\mathrm{Vj}$ auswirkte. In Kapitel 3.5 wird gezeigt, da $B$ diese Anpassung aus versicherungsmathematischer Sicht nicht adäquat ist, sondern einen ökonomischen Anreiz zu einer früheren Verrentung bildet.

Tabelle 3-1 gibt eine Zusammenfassung der in diesem Abschnitt 3.2.1 aufgezeigten Kernpunkte der bundesdeutschen Gesetzlichen Rentenversicherung. 
Tabelle 3-1: Die Gesetzliche Rentenversicherung im Kurzüberblick

\begin{tabular}{|c|c|}
\hline \multicolumn{2}{|r|}{ Organisation } \\
\hline Zielsetzung & $\begin{array}{l}\text { GRV definiert sich als Regelsicherung für einen Großteil der } \\
\text { Bevölkerung. Zielsetzung ist die lebensstandardsichernde, } \\
\text { leistungsbezogene Rente. }\end{array}$ \\
\hline Konzeption & $\begin{array}{l}\text { Pflichtversicherung mit der Möglichkeit freiwilligen } \\
\text { Beitrittes. } \\
\text { Umlageverfahren }\end{array}$ \\
\hline Organisation & $\begin{array}{l}\text { Parafiskalische Körperschaften des öffentlichen Rechtes, für } \\
\text { Angestellte auf Bundes-, für Arbeiter auf Landesebene ( } 23 \\
\text { LVA's) organisiert, zusätzlich kleinere Einzelträger. Zusam- } \\
\text { mengeschlossen im Verband Deutscher Rentenversiche- } \\
\text { rungsträger (VDR). }\end{array}$ \\
\hline Finanzierung & $\begin{array}{l}\text { Pflichbeiträge von z.Zt. ca. 19\% des Brutto-Einkommens; je } \\
\text { zur Hälfte von Versichertem und Arbeitgeber zu tragen. } \\
\text { Staatlicher Zuschuß z.Zt. knapp } 20 \% \text { des Rentenvolumens. }\end{array}$ \\
\hline Erfaßter Personenkreis & $\begin{array}{l}\text { Pflichtversicherte: } \\
\text { - Arbeiter } \\
\text { - Angestellte } \\
\text { - Auszubildende } \\
\text { - Wehr- und Zivildienstleistende } \\
\text { - einzelne Selbständigengruppen } \\
\text { sowie freiwillig Versicherte. Dazu latent Versicherte sowie } \\
\text { Hinterbliebene. }\end{array}$ \\
\hline \multicolumn{2}{|r|}{ Rentenarten und Leistungen } \\
\hline Grundunterscheidung & $\begin{array}{l}\text { Versichertenrenten als Invaliditäts- oder Altersrenten und Hinter- } \\
\text { bliebenenrenten }\end{array}$ \\
\hline $\begin{array}{l}\text { Verrentung zum Re- } \\
\text { gelalter }\end{array}$ & $\begin{array}{l}\text { Normales Altersruhegeld mit } 65 \text { nach einer Wartezeit von } 35 \\
\text { Versicherungsjahren }\end{array}$ \\
\hline Frühere Verrentung & $\begin{array}{l}\text { - flexibler Ruhestand } \\
\text { - alle Versicherten ab } 63 \\
\text { - vorzeitiges Altersruhegeld für: } \\
\text { - Frauen ab } 60 \\
\text { - Arbeitslose ab } 60 \\
\text { - Schwerbehinderung, EU- / BU-Renten } \\
\text { - Allgemeine Invaliditätsrenten ohne Altersbegrenzung }\end{array}$ \\
\hline Spätere Verrentung & hinausgeschobenes Altersruhegeld \\
\hline
\end{tabular}




\subsubsection{Betriebliche und private Altersversorgung}

\section{Betriebliche Altersversorgung}

Die zweite Säule des oben genannten Drei-Säulen-Modelles ist die betriebliche Altersversorgung (BAV). Unter dieser ist die Zusage eines Unternehmens an seine Mitarbeiter zu verstehen, nach dem Austritt aus dem Erwerbsleben eine zusätzliche Rente zu zahlen. Tatsächlich sind die Betriebsrenten älter als die gesetzliche Rentenversicherung, denn bereits 1832 ist das erste Alterssicherungssystem eines deutschen Unternehmens aktenkundig und damit ein gutes halbes Jahrhundert vor der Etablierung einer staatlichen Altersvorsorge ${ }^{37}$. Die Motivationen der Unternehmer für die Einrichtung eines solchen Systems haben sich seither gewandelt. Dürften die erwähnten ersten BAV noch aus dem Fürsorgemotiv der Unternehmer heraus entstanden sein, entwickelte sich diese Einrichtung nach dem 2. Weltkrieg zu einem Instrument der betrieblichen Personalpolitk. So konnte eine zugesagte Betriebsrente als Mittel dazu benutzt werden, Arbeitnehmer im Unternehmen zu halten, da sie den Rentenanspruch im Falle eines Arbeitsplatzwechsels verloren. Dieser und die weiteren Kritikpunkte:

- fehlende oder unzureichende Anpassung der Rentenhöhen an die Preisentwicklung;

- Verlust des Rentenanspruches bei Insolvenz des Unternehmens;

- willkürliche Gestaltung und Veränderung der Zahlungsbedingungen und Rentenhöhen (Anrechnung steigender GRV-Renten u.a.);

führten zur Verabschiedung des Betriebsrentengesetzes ${ }^{38}$ im Jahre 1974. Dessen Kernpunkte sind 39 :

- 'Unverfallbarkeit' betrieblicher Versorgungsanwartschaften dem Grunde und der Höhe nach;

- Verbot der Anrechnung sonstiger Versorgungsanwartschaften, die aus Beiträgen des Versorgungsempfängers entspringen;

- Verpflichtung der Unternehmen zur Absicherung der Rentenzusagen gegen Insolvenz;

- einzelne Regelungen zur Anpassung (Dynamisierung) der Rentenzusagen, die jedoch nicht bindend sind.

Mit dem ersten Punkt haben die bundesdeutschen Betriebsrenten eine wesentliche Funktion der Personalpolitik verloren, der in anderen Staaten wesentlich stärker sind.

${ }^{37}$ Es handelt sich um die Gutehoffnungs-Hütte, Krupp und Henkel folgten 1858, Siemens 1872 , BASF 1879 und Hoechst 1882; vergleiche FRERICH (1987), Seite 104. Zur Gründung der GRV im Jahre 1889 vergleiche Kapitel 3.3.

38 BetrAVG; Gesetz zur Verbesserung der betrieblichen Altersversorgung.

39 Vgl. etwa FrERICH (1987), Seite 197. 
Die unten folgende Diskussion der Anreizwirkungen von Firmenpensionen in den USA zeigt, das dieses Instrument dort teilweise die Versorgung durch die gesetzliche Rentenversicherung der USA (OASDHI) ${ }^{40}$ übertrifft. Allerdings sind betriebliche Rentenzusagen weiterhin aus steuerlichen Gründen interessant für Unternehmen. Bei der Organisationsform ${ }^{41}$ der Direktzusage können die dafür notwendigen Beträge bilanziell in Form von Rückstellungen ausgewiesen werden und beinhalten damit steuerliche Vorteile. Aber auch die anderen Formen der BAV beinhalten steuerliche Vorteile auf Arbeitgeber und/oder Arbeitnehmerseite, so daß Rentenzusagen in diesem Bereich sich als günstige Lohnnebenleistungen für beide Seiten herausstellen können. Abbildung 3-3 zeigt die Verbreitung betrieblicher Altersversicherungen in der Bundesrepublik im Jahre 1982. Es zeigt sich, daß mehr als $60 \%$ der Industrieunternehmen und über $70 \%$ der dortigen Beschäftigten über eine Versicherung verfügen, im Handel liegen die Zahlen mit 20 bzw. $30 \%$ erheblich niedriger.

\section{Abbildung 3-3: Verbreitung betrieblicher Altersversicherung}
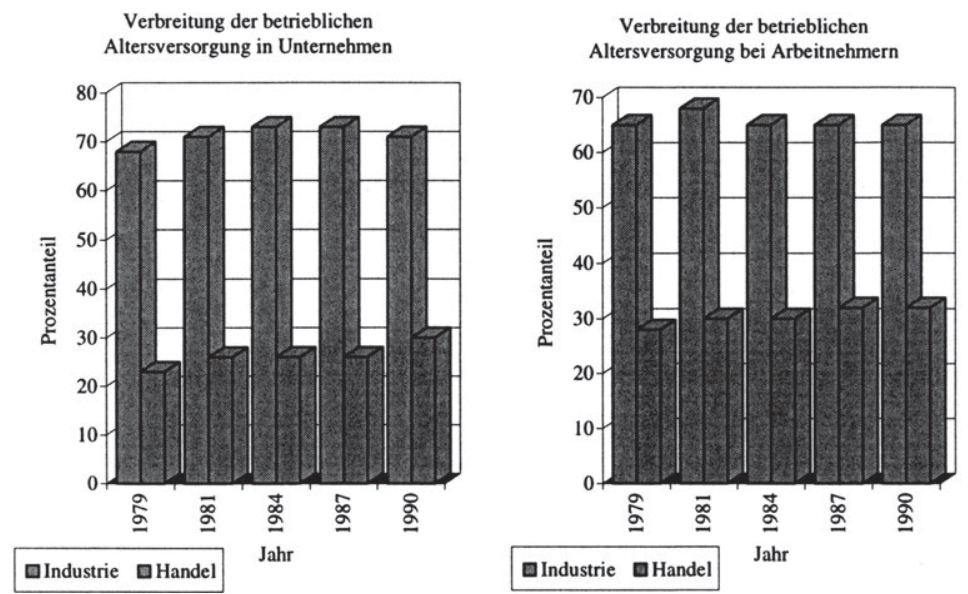

Quelle: DũRKoP (1993), Seite 111.

40 „Old-Age, Survivors, Disability and Health Insurance“.

41 Die wichtigsten Organisationsformen der BAV in Deutschland sind.

- Direktzusage (unmittelbare Versorgungszusage);

- Direktversicherung (im Rahmen einer Lebensversicherung);

- Pensionskasse (über Arbeitgeberbeiträge finanziertes eigenständiges Versicherungsunternehmen);

- Unterstützungskasse (unternehmenseigene Versorgungseinrichtung).

vergleiche hierzu z.B. HEMMER (1984), Seiten 7-10. 


\section{Abbildung 3-4: Höhe der Betriebsrenten in Deutschland}

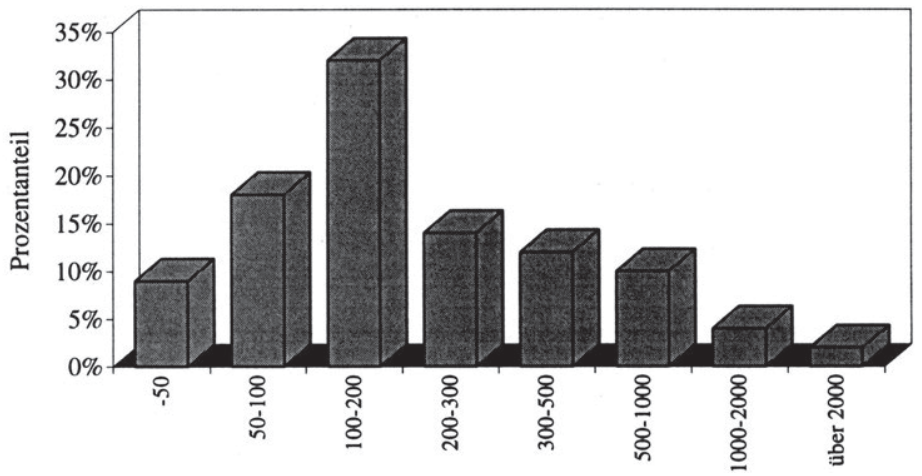

Monatliche Auszahlungsbeträge von Betriebesrenten im Jahre 1982 in DM; Infratest-Erhebung. Quelle: Dürkop (1993), Seite 116.

Allerdings sagen diese relativ hohen Anteile noch nichts über die tatsächliche Bedeutung der BAV für die heutige Rentnergeneration aus. Abbildung 3-4 zeigt für 1982, daß mehr als $60 \%$ derjenigen, die eine Zusatzversorgung bekommen, eine Summe unter 200,- DM pro Monat erhalten, so daß der Beitrag der BAV zur Lebensstandardsicherung gering ist. Im Sozio-ökonomischen Panel (SOEP, vgl. Kapitel 7.1) machen Betriebsrenten weniger als 5\% des Einkommens der befragten Personen aus. Allerdings sind diese Zahlen mit Vorsicht zu interpretieren, denn diese Auszahlungssummen beziehen sich auf die heute im Ruhestand befindliche Generation, während die derzeitige Erwerbsbevölkerung möglicherweise nicht nur über eine höhere Versorgung mit BAV-Zusagen verfügt, sondern mit diesen auch höhere Rentenzahlungen in der Zukunft verbunden sein können. ${ }^{42}$

\section{Ökonomische Anreizwirkungen der Betrieblichen Altersversorgung}

Für die Wahl des Rentenalters ist die BAV vor allem im Zusammenhang mit der Frage relevant, ob und in welcher Richtung der Verrentungszeitpunkt durch die betriebliche Altersversorgung beeinflußt wird. In den Vereinigten Staaten spielen die „Firm Pensions" eine wesentlich wichtigere Rolle, was vor allem aus zwei Gründen der Fall ist: Die amerikanische Sozialversicherung versteht sich nicht wie die deutsche GRV als lebensstandarderhaltend, sondern lediglich als Grundsicherung. Die staatlichen Renten sind entsprechend in den USA niedriger als in Deutschland. Tabelle 3-2 zeigt das 
Rentenniveau in beiden Ländern im Vergleich. ${ }^{43}$ Zweitens gibt es in den USA keine effektive Gesetzgebung, die verhindern würde, da $B$ die Unternehmen die Firmenpensionen als machtvolles Druckmittel gegen die Mitarbeiter einsetzen können und dies in Realität auch tun. ${ }^{44}$

\section{Tabelle 3-2: Nettorentennlveau in der Bundesrepublik und den Vereinigten Staaten}

\begin{tabular}{|l|cc|}
\hline Einkommensklasse & Netto-Ersatzquote (Rentenniveau) \\
\hline & BRD & USA \\
\hline 1/8 Durchschnittseinkommen & $188 \%$ & $191 \%$ \\
1/4 Durchschnittseinkommen & $130 \%$ & $99 \%$ \\
1/2 Durchschnittseinkommen & $82 \%$ & $61 \%$ \\
3/4 Durchschnittseinkommen & $66 \%$ & $55 \%$ \\
1 Durchschnittseinkommen & $71 \%$ & $53 \%$ \\
1,5 Durchschnittseinkommen & $77 \%$ & $45 \%$ \\
2 Durchschnittseinkommen & $75 \%$ & $41 \%$ \\
3 Durchschnittseinkommen & $53 \%$ & $30 \%$ \\
\hline
\end{tabular}

Quelle: CASMIR (1989), Seiten 508 und 512. Angaben für Versicherten mit 40 Beitragsjahren In Prozent.

Angestellte werden in den USA mit Hilfe der Firmenpläne im Unternehmen gehalten, da ihnen sonst der gesamte Anspruch auf die Betriebsrente verloren geht. Auf diese Weise schützen sich Unternehmen gegen das Abwandern von Mitarbeitern. So sind die meisten Firmenpläne auf ein genaues Rentenalter ausgelegt, das oft zwischen 55 und 60 Jahren liegt, obwohl in dieser Zeit die Arbeitnehmer noch gar keine gesetzliche Altersrente beziehen können ${ }^{45}$. Auf diese Weise bleibt den Arbeitnehmern oft nichts anderes übrig, als nach der „Verrentung“" aus ihrer Haupt-Berufstätigkeit („career Job“) bei einem anderen Arbeitgeber weiter $\mathrm{zu}$ arbeiten, bis sie in die gesetzliche Rentenversicherung eintreten können ${ }^{46}$.

Eine intensive Untersuchung der Daten einer großen Firma nehmen für die USA KOTLIKOFF und WISE vor. In diversen Artikeln präsentieren sie unterschiedliche

43 CASMIR errechnet die Rentenhöhen für Durchschnittsverdiener nach der Definition der OECD (Average Production Worker APW) und für Personen, die einen Teil oder ein Mehrfaches dieses Durchschnittsverdienstes bezogen haben. In beiden Ländern greifen Regelungen zur Unterstützung von Personen mit sehr geringen Einkommen. In Deutschland ist dies die „Rente nach Mindesteinkommen", die für die ersten drei Zeilen der Tabelle relevant ist.

44 BURKHAUSER \& QUINN (1989).

45 Jacobs (1990), Seite 156.

46 BURKHAUSER \& QUINN (1989), Seite 109 ff. JACOBS (1990) beschreibt, daB dies für viele USFirmen als günstige Gelegenheit genutzt wird, billige und erfahrene Arbeitskräfte zu bekommen (Seite 156). 
Aspekte des von ihnen benutzen Datensatzes ${ }^{47}$. Die Autoren zeigen auf, in welchen Altersjahren das Rentenprofil der Firma den Arbeitnehmern die größten Anreize bietet, in die Betriebsrente zu gehen. Ihre meistgewählte Darstellung ist die der ,accrual profiles“, von Alters-Barwert-Profilen, welche den finanziellen Effekt unterschiedlicher Ruhestandsalter modellieren. KOTLIKOFF und WISE stellen dabei nicht auf den Barwert selbst ab, sondern auf die Veränderung dieses Barwertes von einem potentiellen Renteneintrittsjahr zum nächsten. Ihre Analyse zeigt, daß die Barwert-Profile tatsächlich Rückschlüsse auf die zu beobachtenden Rententermine erlauben. ${ }^{48}$ Eine ökonometrische Analyse dieser Fragestellung mit dem genannten Datensatz von James STOCK, David WISE und Robin LUMBSDAINE ist das Optionswertmodell, das im fünften Kapitel ausführlich dargestellt wird.

JÄHNKE (1993) führt eine analoge Untersuchung für die Bundesrepublik Deutschland durch, indem sie die BAV-Regeln von fünf Firmen zunächst theoretisch analysiert und im zweiten Schritt entsprechende Barwert-Profile errechnet. Bei diesen Profilen ist zu unterscheiden zwischen den reinen Anreizwirkungen der BAV und den kumulierten Anreizen, die sich aus der Addition der Rentenanwartschaften von BAV und GRV ergeben. Abbildung 3-5 zeigt die graphische Darstellung eines solchen accrual-Profiles für die Betriebliche Altersversorgung eines deutschen Chemieunternehmens, aus dem deutlich zu erkennen ist, daß die Firma den Anreiz setzt, mit 55 Jahren auszuscheiden. Die Abbildung zeigt, daß die BAV-Ansprüche kontinuierlich mit der Betriebszugehörigkeit steigen und ein besonderer Anreiz bei einer Pensionierung mit 55 Jahren hinzukommt. Da sich die Anwartschaft nach dem 60. Lebensjahr nicht mehr erhöht, wird für die Barwertberechnung die sich verkürzende Lebenserwartung relevant, so daß die Barwerte sinken und die Zuwächse negativ werden.49 Das zweite Schaubild (Abbildung 3-6) verdeutlicht die Interaktion zwischen dem firmenspezifischen Profil und der GRV. Es wird hierbei deutlich, daß der Barwert durch beide Rentenarten steigen kann. Neben der bisher bereits erkennbaren Steigerung im Alter von 55 Jahren, ergibt sich eine solche beim vorgezogenen Ruhestandsalter von 60 Jahren. Die 7 prozentigen Aufschläge, die die GRV bei einer Verrentung mit 66 oder 67 Jahren bietet, drosseln zwar das altersbedingte Absinken des Barwertes, reichen jedoch nicht aus,

\footnotetext{
${ }^{47}$ KOTLIKOFF und WISE (1985) „Labor compensation and the structure of private pension plans: Evidence for contractual versus Spot labor markets“, dieselben (1988) „Employee Retirement and a Firms Pension Plan“ oder dieselben (1989) „The Wage Carrot and the Pension Stick“.

${ }^{48}$ KOTLIKOFF und WISE (1988): „Employee Retirement and a Firms Pension Plan“.

49 JÄHNKE (1994), Seite 6f.
} 
den Zuwachs positiv werden zu lassen. Dies drückt die fehlende versicherungsmathematische Anpassung der deutschen GRV aus, die in Kapitel 3.5.1 nachgewiesen wird.

\section{Abbildung 3-5: Anreizwirkungen der betrieblichen Altersversorgung eines deutschen Großunternehmens}

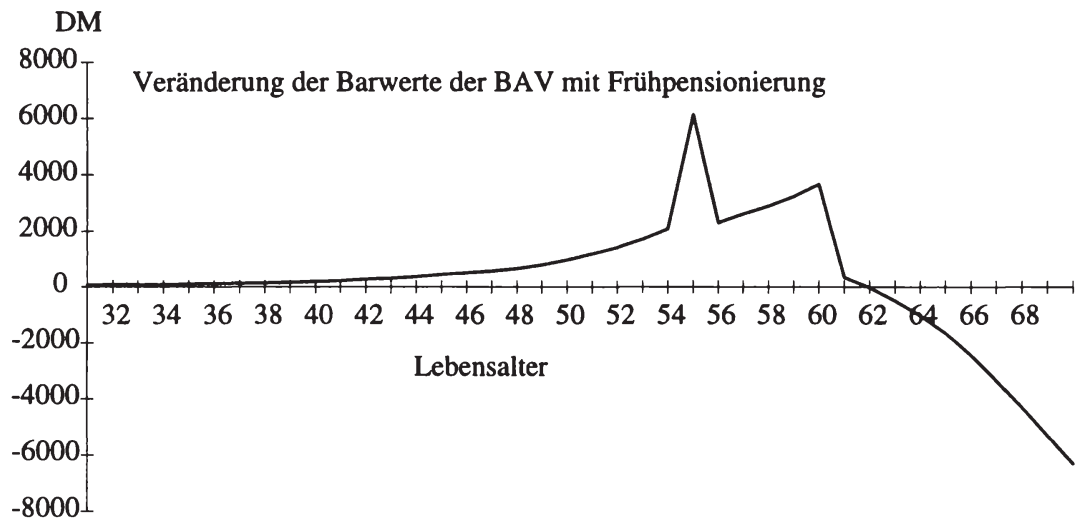

Quelle: JÄHNKE (1994), Seite 6.

Abbildung 3-6: Kumulierte Anreize aus betrieblicher und gesetzlicher Altersversorgung

DM

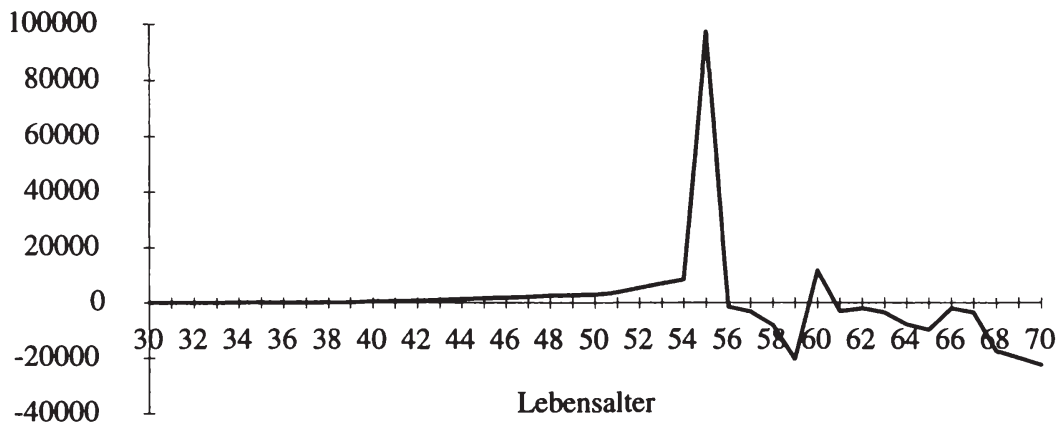

Quelle: JĀHNKE (1994), Seite 7.

Das hier beispielhaft vorgestellte Unternehmen ist ein spezieller Fall möglicher Ausgestaltungen betrieblicher Rentenversicherungssysteme. Für andere Unternehmen zeigt die Studie von JÄHNKE $(1993,1994)$ zwar andere Altersprofile, es wird jedoch deutlich, daß die Unternehmen durchaus in der Lage sind, deutliche ökonomische Anreize für gewünschte Rentenalter zu setzen und dies in der Praxis auch tun. Als Ergebnis ist 
festzuhalten, daß die betriebliche Altersversorgung auch in der Bundesrepublik Deutschland die individuelle Wahl des Rentenalters beträchtlich beeinflussen kann. Dieses Phänomen ist für die Vereinigten Staaten, wie oben dargestellt, seit langem bekannt, für den Fall der Bundesrepublik Deutschland ist es jedoch ein Feld, in dem empirische Untersuchungen weitgehend fehlen.

\section{Private Alterssicherung}

Als dritte Säule einer Altersversorgung ist die private Vorsorge zu nennen. Unter dieser können so unterschiedliche Dinge verstanden werden wie Familienhilfe, herkömmliches Sparen, Haus- und Grunderwerb, aber auch gezielte Sparformen wie Sparpläne und Lebensversicherungen. In Kapitel 2.1 wurde das Sparverhalten im Lebenszyklus, sowohl vor als auch nach der Verrentung, aus theoretischer Sicht angesprochen. In der Praxis sind in Deutschland, neben der Familienhilfe, Lebensversicherungen die mit Abstand häufigste Form privater Vorsorge.

\section{Abbildung 3-7: Neuabgeschlossene Lebensversicherungen in Deutschland}

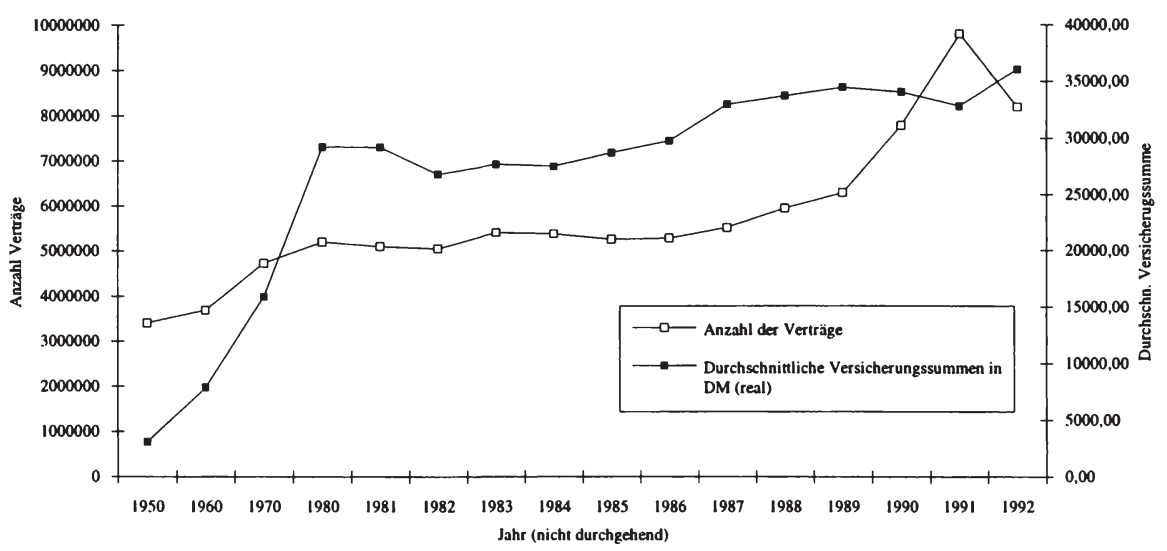

Quelle: Verband der Lebensversicherungsunternehmen e.V. (Zahlen direkt übermittelt), eigene Darstellung.

Abbildung 3-7 zeigt die Anzahl der jährlichen Neuabschlüsse von Lebensversicherungsverträgen und verdeutlicht, daß dieses Instrument der Ersparnisbildung nach wie vor an Wichtigkeit zunimmt, auch wenn die sprunghaften Zuwächse der 50er - 70er Jahre nicht mehr erreichbar sind. Ende 1990 bestanden knapp 63 Millionen Lebensversicherungsverträge über eine Vertragshöhe von 1.582 Milliarden DM, was bezogen 
auf die Zahl der Privathaushalte einen Durchschnittsbestand von 56.000 DM pro Haushalt in Lebensversicherungen ausmacht. ${ }^{50}$ Allerdings sind hier nicht nur private Verträge enthalten, sondern auch im Rahmen von Direktversicherungen, also betrieblicher Altersversorgung, abgeschlossene. ${ }^{51}$

Die Diskussionen dieses Abschnittes zeigen, daß es wichtig ist, neben der GRV zusätzlich die beiden anderen Säulen der Rentenversicherung zu betrachten und Anreizwirkungen auf bestimmte Ruhestandsalter auch von diesen beiden Sicherungssparten aus zu untersuchen. Diese Aufgabe ist allerdings schwierig, da in beiden Fällen die notwendigen Informationen in Form von Mikrodaten, die für die vorliegende empirische Arbeit verwendbar sind, sehr schwer zu bekommen sind.

\subsubsection{Angrenzende Gebiete der sozialen Sicherung}

Für die Bewertung eines Alterssicherungssystems sind nicht nur die institutionellen Regelungen zur Rentenversicherung selbst von Bedeutung, sondern auch die verwandter Bereiche, vor allem der Kranken-, und Arbeitslosenversicherung sowie der Sozialhilfe. Ein weiterer relevanter Punkt ist die steuerliche Behandlung der Beiträge und Ruhegelder.

\section{Krankenversicherung}

Durch das Gesetz über die Krankenversicherung der Rentner vom 12.6.1956 (KVdR) sind Rentenempfänger vollwertige Mitglieder der Krankenversicherung. Sie sind in der Krankenversicherung, in der sie vor der Verrentung versichert waren, versicherungspflichtig. Bis Juni 1989 zahlten sie dort einen Beitrag von 11,8\% ihres monatlichen Renteneinkommens, wobei sie einen Zuschuß von 7,3\% erhielten, so daß die faktische Eigenbeteiligung 4,5\% betrug. Seit dem 1.7.89 sind die Beiträge zur KVdR den allgemeinen Krankenversicherungsbeiträgen angepaßt, Leistungsempfänger und Rentenversicherung zahlen diesen Prozentsatz jeweils zur Hälfte. Auch ein eventueller Hinzuverdienst (s.u.) unterliegt der Krankenversicherungspflicht im Rahmen der gelten Beitragssätze 52 .

\footnotetext{
50 Quelle: Statistisches Jahrbuch 1992, Seiten 70 und 386, Angaben für das frühere Bundesgebiet.

51 Vgl DURKOP (1993), Seite $106 \mathrm{f}$.

52 Vgl. z.B. Bundesminister für Arbeit und Sozialordnung BMA (1991), Seiten 68f und 195. 


\section{Arbeitslosenversicherung und -unterstützung}

Die Relevanz der Arbeitslosenversicherung im Rahmen der Frühverrentung wurden im Kapitel 3.2.1.2 diskutiert. Bei der sogenannten 59er-Regel (siehe dort) wird die Arbeitslosenversicherung dazu benutzt, einen Ruhestandsbeginn vor dem 60. Lebensjahr zu finanzieren. Nach dem Eintritt in den Ruhestand ist die Arbeitslosenversicherung nicht mehr relevant, da selbst Personen, die nach der offiziellen Verrentung einer zusätzlichen Erwerbstätigkeit nachgehen, nicht mehr der ArbeitslosigkeitsPflichtversicherung angehören, im Gegensatz zur oben genannten Krankenversicherung. Dies ist sinnvoll, da die Absicherung eines Rentners durch die Rente unterstellt wird, so daß ein weiterer Schutz vor Arbeitsplatzverlust nicht notwendig erscheint.

\section{Exkurs: Die Vorruhestandsregelung - Ein arbeitsmarktorientiertes Modell}

Bereits oben erwähnt (Kapitel 3.2.1.2) wurde die Vorruhestandsregelung, die als arbeitsmarktpolitische Maßnahme am 1. Mai 1984 eingeführt wurde und zunächst auf fünf Jahre befristet war. Wegen des geringen Zuspruches wurde die Maßnahme nicht verlängert, so daß sie 1989 auslief.

Arbeitnehmer konnten im Alter von 58 Jahren in den Vorruhestand gehen. Der Arbeitgeber zahlte aufgrund eines Tarifvertrages oder einer Einzelvereinbarung das sogenannte Vorruhestandsgeld an freiwillig ausscheidende Arbeitnehmer. Kam es zu einer Wiederbesetzung der so freigemachten Stelle, dann gewährt die Bundesanstalt für Arbeit (BA) dem Arbeitgeber einen Zuschuß zur Vorruhestandsleistung53. Das arbeitsmarktpolitische Ziel war also deutlich die Einstellung jüngerer Arbeitsloser. Die Höhe des Vorruhestandsgeldes wurde über Tarifvertrag oder Einzelvereinbarung geregelt. Das Gesetz schrieb eine Mindesthöhe von $65 \%$ des bisherigen Bruttogehaltes vor (sonst zahlt die BA keinen Zuschuß). Diese war bis zu einem Betrag von 36.000,- DM p.a. steuerfrei. Die Praxis zeigte eine Höhe von 75 - 80\% des bisherigen Lohnes. Während des Vorruhestandes war der ausgeschiedene Arbeitnehmer sowohl in der Kranken- wie auch in der Rentenversicherung versichert, wobei sich Arbeitgeber und Arbeitnehmer die Beiträge wieder teilten ${ }^{54}$.

Im Gegensatz zur 59er-Regel (s.o.) fand das Vorruhestandsmodell bei den Unternehmen wenig Anklang, weil seine Durchführung wesentlich aufwendiger war als die erstgenannte. Die Neueinstellung eines vorher Arbeitslosen mußte nachgewiesen wer-

53 35\% der Leistung - vgl. FreRICH, Seite $521 \mathrm{f}$.

$54 \mathrm{Vgl} \mathrm{etwa} \mathrm{FrERICH} \mathrm{(1987),} \mathrm{Seiten} \mathrm{521-524.}$ 
den, diverse Formalitäten waren mit der Maßnahme verbunden. Dagegen stellt sich die Durchführung der 59er Regel aus unternehmerischer Sicht relativ unproblematisch dar.

\section{Sozialhilfe}

Die Sozialhilfe wird im Sinne des oben erwähnten Subsidiaritätsprinzip oft als „unterste Masche des sozialen Netzes“ bezeichnet. Es gilt der Grundsatz des „Nachranges“ der Sozialhilfe, in dem Sinne, daß diejenigen Unterstützung finden, die diese weder durch andere Träger der Sozialvorsorge noch durch eigene Kraft erreichen kann. Sie wird sowohl in Form finanzieller, aber auch sachlicher Unterstützung geleistet.

Für die Rentenversicherung ist sie insofern relevant, als Personen, die wenig Beitragsjahre und geringe Beiträge aufweisen, unter die „Armutsgrenze“ im Sinne des Sozialhilfesatzes fallen können. Zwar wurde 1972 das Prinzip der Renten nach Mindesteinkommen eingeführt, welches Personen mit mindestens 25 Beitragsjahren so stellt, als haben sie in allen Beitragsjahren $75 \%$ der durchschnittlichen Entgeltes verdient. Wer aber 25 Jahre Versicherungszeit nicht erfüllt, profitiert auch von dieser Regelung nicht. Das bekannteste Beispiel aus der öffentlichen Diskussion sind die sogenannten „Trümmerfrauen“, die wesentliche Aufbauarbeit geleistet haben, ohne dafür eine Altersabsicherung zu haben ${ }^{55}$. Tatsächlich sind es vor allem Frauen, die von Renten nach Mindesteinkommen profitieren. So wurde im Rentenzugangsjahr 1988 jede fünfte Rente an Frauen im Rahmen dieser Regelung angehoben, bei Männern dagegen nur jede 28. ${ }^{56}$

\section{Steuerliche Behandlung von Leistungen und Beiträgen}

Die Beiträge zur Gesetzlichen Rentenversicherung eines Arbeitnehmers sind in den Arbeitgeber- und den Arbeitnehmeranteil zu unterscheiden. Der Arbeitgeberanteil am Rentenversicherungsbeitrag ist für diesen Betriebsausgaben. Für den Versicherungspflichtigen zählen sie nicht zum steuerpflichtigen Einkommen. Die eigenen Beiträge des Versicherten sind im Rahmen des Vorwegabzuges und der Vorsorgeaufwendungen beschränkt steuerlich abzugsfähig. Faktisch können Geringverdiener ihre Beiträge von der Steuer absetzen, wogegen besser Verdienende den nicht abzugsfähigen Betrag aus dem versteuerten Einkommen zahlen müssen. Eine Sonderregelung gilt für Beamte, die nicht der Sozialversicherungpflicht unterliegen ${ }^{57}$.

\footnotetext{
$55 \mathrm{Vgl}$ dazu auch FRERICH (1987), Seite 160.

56 BMA (1991), Seite 179, Rz. 135.

57 Vgl. CASMIR (1989), Seite $494 \mathrm{f}$.
} 
Empfangene Rentenleistungen sind grundsätzlich als sonstige Einkünfte (§ $22 \mathrm{EStG}$ ) zu versteuern, allerdings nur ihr Ertragsanteil ${ }^{58}$. Unter Berücksichtigung der Freibeträge (insbesondere Altersfreibetrag) der Einkommensteuer bleibt bei Rentenbeginn mit 65 Jahren eine Rente bis 2830,- DM (Ehepaare 5.342,- DM) steuerfrei. Faktisch zahlt ein Großteil der Rentenempfänger in Deutschland keine Einkommensteuer.

\subsection{Geschichte der GRV, Rentenreformen und wesentliche Regelände- rungen}

Für die empirische Untersuchung der Rentenzugänge seit 1968 im Kapitel 4 ist es nötig, über die Beschreibung des institutionellen Rahmen hinaus die Veränderungen der einzelnen Regeln und Altersgrenzen im Zeitablauf zu betrachten. In diesem Abschnitt werden deshalb die wichtigsten Regeländerungen des Betrachtungszeitraumes beschrieben. ${ }^{59}$ Dazu werden die Rentenreformen der Jahre 1957 und 1972 sowie andere relevante Gesetze in ihren Grundzügen vorgestellt. Weiterhin wird die Rentenreform 1992 dargestellt, die bei der abschließenden Diskussion möglicher Reformalternativen (Kapitel 8 und 9) als Referenzsystem dienen wird.

Die jüngste Rentenreform in der Bundesrepublik Deutschland, die Rentenreform 1992 ist noch in der aktuellen Diskussion, ihre Wirkungen in der Politik umstritten. Ihre tatsächlichen Auswirkungen werden erst nach mehreren Jahrzehnten zu beobachten sein, da ihre Regelungen erst im Jahr 2013 komplett greifen. Sie wurde, wie einleitend erwähnt, nicht in die bisherige Darstellung des bundesdeutschen Rentenversicherungssystems einbezogen, da die im Kapitel 4 untersuchten Daten aus den Jahren 1968 bis 1991 und die des Kapitels 7 aus 1984-1990 stammen und daher Entscheidungen untersuchen, die auf Basis der vor der Reform gültigen gesetzlichen Regelungen getroffen wurden.

Eine ökonomische Bewertung der Rentenreform 92 ist Gegenstand der Simulationsstudien des achten Kapitels, in dem ihre Auswirkung auf die Ruhestandsentscheidung mit Hilfe der aus Vergangenheitsdaten gewonnenen Schätzkoeffizienten prognostiziert werden.

${ }^{58}$ Die Rente wird aufgesplittet in zurückfließende Tilgungsbeiträge (vom Sparkapital) und Zinserträge, welche zu versteuern sind. Der Ertragsanteil einer Rente wird über das Rentenzugangsalter approximiert. Der Ertragsanteil einer Rente, die mit dem 65 . Lebensjahr beginnt, beträgt etwa $24 \%$, die mit dem 60 . Lj beginnt etwa 29\%, die Prozentsätze sind einer Tabelle des Einkommensteuergesetzes zu entnehmen. (§22 EStG).

${ }^{59}$ Die Darstellung basiert teilweise auf der historischen Übersicht bei FRERICH (1987), Seite 80 ff, auch JACOBS, KOHLI UND REIN (1991) beschreiben teilweise die durchgeführten Reformschritte. 


\subsubsection{Entstehung und Entwicklung der GRV bis 1991}

Basis der deutschen Rentenversicherung ist das „Gesetz betreffend die Invaliditätsund Alterssicherung“, vom 22. Juni 1889, welches auf Initiative Otto von Bismarcks vom Kaiser Wilhelm I verkündet wurde und zum 1.1.1891 in Kraft trat. Drei wesentliche Rentenreformen gaben der GRV ihre heutige Gestalt.

\section{Rentenreform 1957}

- Neue Rentenformel:

- dynamische Rente gekoppelt an Lohnentwicklung,

- Rente mit Lohnersatzfunktion zur Lebensstandardsicherung;

- Anpassung der Arbeiterrentenversichenung an die der Angestellten;

- Einführung des vorgezogenen Ruhestandes für Frauen ab 60 Jahren;

- Einführung des vorgezogenen Ruhestandes für Arbeitslose ab 60 Jahren.

Als erste große Rentenreform stellte die Umgestaltung von 1957 eine grundlegende Neuordnung dar. Wesentliche Eigenschaften der deutschen Gesetzlichen Rentenversicherung bestehen seit Inkrafttreten dieser Reform. Kernstück war die Einführung der „dynamischen Rente“, d.h. die Koppelung der Rentenentwicklung an die der Löhne. Konkret wurde die Beitragsbemessungsgrenze, die der Lohnentwicklung angepaBt wird, als Maßstab für die Dynamisierung gewählt. Die Kopplung der Rentenhöhe an die Entwicklung des gesamtwirtschaftlichen Bruttoarbeitslohnes hat dazu geführt, da $B$ das reale Rentenniveau seit 1957 stärker anstieg als die Nettolöhne. Dieser (unerwünschte - weil langfristig nicht finanzierbare) Effekt wurde 1992 durch eine Koppelung der Renten an die Nettolöhne geändert. Die Dynamisierung war die Geburtsstunde des lohn- und gehaltsbezogenen Versicherungssystems. Damit verbunden war der Wandel von einem bloßen Zuschuß zum allgemeinen Lebensbedarf hin zur Lebensstandardsicherung vollzogen. Durch die dynamische Rente wird die Rentenhöhe (über die Rentenformel) an die relative Einkommensposition des Versicherten gebunden, sie erhält damit eine Lohnersatzfunktion.

Die Regelungen der Arbeiterrentenversicherung werden an die der Angestellten angepaßt. Durch die 57er Reform wurde die Möglichkeit für Frauen, sowie für männliche Arbeitslose eingeführt, mit 60 Jahren in den vorgezogenen Ruhestand zu treten. Diese Regelung ist bis heute in Kraft.

\section{Rentenreform 1972}

Die Auswirkungen der 72er Reform sind das Kernstück der Analyse des 4. Kapitels. Die wichtigsten Neuregelungen:

- die Einführung der flexiblen Altersgrenze (Verrentungsfenster zwischen 63 und 67 Jahren); 
- Einführung einer vorgezogenen Rente für Behinderte, Berufs- und Erwerbsunfähige ab dem Alter von 62 Jahren;

- Etablierung der Rente nach Mindesteinkommen;

- Öffnung der GRV für alle Bevölkerungsgruppen.

Mit dem Rentenreformgesetz 1972 wird erstmals das fixe Verrentungsalter von 65 Jahren gelockert. Vielmehr stehen seither langjährig Versicherte vor der Wahl, eines von 5 möglichen Rentenaltern aus dem 'Verrentungsfenster' zu wählen. Die Auswirkungen dieser Änderung auf das Rentenzugangsverhalten war dramatisch, wie das nächste Kapitel zeigen wird, mit einem sehr deutlichen Trend hin zu den möglichst frühen Rentenzeitpunkten.

Durch die Einführung einer „Versicherungspflicht auf Antrag“ (freiwillige Versicherung) für Selbständige, Hausfrauen u.a. und der Einräumung von Nachversicherungsmöglichkeiten wird die GRV weiten Bevölkerungsschichten geöffnet. Weitere strukturelle Leistungsverbesserungen sind Bestandteil der Reformpakets, wie etwa die Renten nach Mindesteinkommen, durch welche Personen mit mindestens 25 Beitragsjahren (Lohn-, Ersatz- und Zurechnungszeiten) mindestens so gestellt werden, als haben sie in allen Beitragsjahren mindestens $75 \%$ der durchschnittlichen Entgeltes verdient. Diese Regelung zielt auf die Verbesserung der Situation derer, die in der Vergangenheit sehr niedrig entlohnt wurden, v.a. gilt dies für Aufbauarbeiten nach dem zweiten Weltkrieg, haus- und landwirtschaftliche Arbeit und Entlohnung durch Sachbezüge, aber auch für Hausfrauenarbeit ${ }^{60}$.

\section{Einzelne Maßnahmen der 70er und 80er Jahre}

- 1979: vorgezogener Renteneintritt für Behinderte, Berufs- und Erwerbsunfähige ab dem Alter von 61 Jahren;

- 1980: vorgezogener Renteneintritt für Behinderte, Berufs- und Enwerbsunfähige ab dem Alter von 60 Jahren;

- 1984: Senkung der notwendigen Wartezeiten für Regelverrentung mit 65 Jahren;

- 1984: Verschärfung der Anspruchsvoraussetzungen für EU/BU-Renten.

Die Möglichkeit für Schwerbehinderte (Behinderte, Berufs- und Erwerbsunfähige), mit 62 Jahren in den vorgezogenen Ruhestand zu gehen, wird zum 1.1.1979 auf 61, ab 1.1.1980 auf 60 Jahre abgesenkt.

Mit dem Haushaltsbegleitgesetz 1983 werden einige kostensenkende Maßnahmen beschlossen, wie die Erhöhung des Beitragssatzes, Kürzung des Bundeszuschusses und der pauschalen Zahlungen an die Krankenversicherungen. Der den Rentnern gewährte Zuschuß zur Krankenversicherung der Rentner wird ,ab Mitte 83 schrittweise abge-

60 Vgl. FrERICH (1987), Seite 84 und 160, sowie Anmerkungen in Kapitel 3.2.3. 
schmolzen"61, auch für Rehabilitationsmaßnahmen wird eine Selbstbeteiligung eingeführt.

Durch das Haushaltsbegleitgesetz 1984 werden auf der einen Seite die für eine Regelverrentung mit 65 Jahren notwendigen Versicherungsjahre von 15 auf 5 gesenkt, als weitere Maßnahme werden die Bedingungen für EU/BU-Renten verschärft.

\subsubsection{Rentenreform 1992}

Diese jüngste Rentenreform, auf die sich 1989 alle großen Fraktionen des Bundestages einigten, ist, wie oben ausgeführt, für die empirischen Teile dieser Arbeit nicht relevant, sehr wohl aber für die Simulationsrechnungen des 8. Kapitels. Die Maßnahmen der Regeländerungen 1992 zielen teilweise auf eine mehr aus Anreizgesichtspunkten gestaltete Rentenberechnung ab, eine ökonomische Bewertung dieses Ansatzes erfolgt in Kapitel 3.5. Nach der Vorstellungen der wichtigsten Reformpunkte wird die neue Rentenformel im Vergleich zur vorher geltenden dargestellt.

Erklärtes Ziel der Neuregelung war eine 'ausgewogene Verteilung der zusätzlichen Belastungen' unter 'Festhalten an der lohn- und beitragsbezogenen lebensstandardsichernden Rente'. Die Reform trägt damit dem oben beschriebenen demographischen Strukturwandel Rechnung. Es geht letztlich darum, Anreize für eine spätere Verrentung zu geben bzw. solche für möglichst frühen Ruhestand abzubauen. Ihre Kernpunkte sind 62 :

- Einbeziehung einer Abweichung vom Regelrentenalter (s.u.) in die Rentenberechnung. Die neue Rentenformel stellt einen kleinen Schritt in Richtung auf eine versicherungsmathematisch faire Rentenanpassung dar ${ }^{63}$. Ein bis zu $3 \mathrm{Jahre}$ früherer Rentenbezug ist weiterhin möglich, allerdings mit 3.6\% Abschlag pro Jahr.

Das Fenster des flexiblen Ruhestandes beginnt somit zukünftig bereits mit $62 \mathrm{Jah}$ ren.

- Anhebung der Altersgrenzen: Diese werden ab 2001 um jährlich 3 Monate bis 2004, dann um jährlich 6 Monate erhöht. Hierdurch liegt dann die Altersgrenze der Männer 2006 und die der Frauen 2012 bei 65 Jahren.

Für Frauen bedeutet dies eine erhebliche Verschiebung des Regelalters. Für Männer wird lediglich das bisher geltende Regelrentenalter von 65 Jahren erneut fest-

61 Vgl. FrERICH (1987), Seite 85.

62 Bundesministerium für Arbeit und Sozialordnung BMA (1990 und 1993).

${ }^{63} \mathrm{Vgl}$. Kapitel 3.5. 
geschrieben, in Verbindung mit o.a. Punkt stellt diese Festschreibung eine Verringerung der Rente im Falle einer Frühverrentung dar.

Ein vorgezogener Rentenzugang aufgrund von Langzeitarbeitslosigkeit, Erwerbsoder Berufsunfähigkeit wird auch nach 1992 möglich sein.

- Neue Möglichkeit der Teilverrentung mit 1/3,1/2 oder 2/3 Rentenbezug. Der o.a. Abschlag wird dann nur auf diesen Teil berechnet, hinzuerworbene Ansprüche werden bei der Vollverrentung berücksichtigt.

Dieses neue Modell stell ein Angebot nicht nur an die Arbeitnehmer, sondern auch an die Unternehmen zur Flexibilisierung der Arbeitszeit dar. Für letztere kann es durchaus interessant sein, auf das Humankapital eines Mitarbeiters weiterhin zurückgreifen zu können, ohne diesen dafür vollzeit beschäftigen zu müssen. Dadurch können neue Stellen für jüngere Mitarbeiter geschaffen werden. Aus Arbeitnehmersicht stellt die Teilverrentung die Möglichkeit eines schrittweisen Überganges in den Ruhestand dar.

- Wechsel von der Brutto- zu einer Nettoanpassung der Rentenhöhe.

Die Dynamisierung der Rentenhöhe durch die Bruttolohnentwicklung führte v.a. in den 70er Jahren dazu, daß die Nettorenten stärker stiegen als die Nettolöhne, die durch Steuerprogression und erhöhte sonstige Abgaben gedämpft wurden. Es werden also bei der Ermittlung des aktuellen Rentenwertes (s.u.) sowohl die Entwicklung der Bruttolöhne als auch die der Abgabenbelastungen berücksichtigt.

- Die bisherigen „Ausfallzeiten“ werden zu „Anrechnungszeiten“ umgewandelt. Die Ausbildungszeit wird von 13 auf 7 Jahre verkürzt, die Kindererziehungszeit allerdings von 1 auf 3 Jahre erhöht. Das Institut der "Berücksichtigungszeiten " wird neu aufgenommen, dies können etwa bis zu 10 Jahren Kindererziehung und auch Zeiten der Pflegetätigkeit (unbegrenzt) sein.

- Der Bundeszuschuß wird erhöht und anschließend durch eine Kopplung an die Bruttolohnentwicklung und den Beitragssatz dynamisiert.

- Neue Berechnung der Rente (Rentenformel) zur Berücksichtigung der obigen Aspekte $\rightarrow$ vgl. unten.

- Kontinuität / zukünftige Entwicklung:

Beitragssatz, Bundeszuschuß und Rentenanpassung sind in Zukunft einem Selbstregulierungsproze $ß$ unterworfen und müssen damit nicht mehr über kurzfristig orientierte politische Entscheidungen gesetzt werden.

Die folgende Tabelle führt zum einen die Rentenformel zur neuen Berechnung der Monatsrente (bisher Berechnung einer Jahresrente) ab 1992 ein und stellt sie weiterhin im Vergleich zur bisherigen Formel dar, so daß Gemeinsamkeiten und Unterschiede sichtbar werden: 
Tabelle 3-3: Die Rentenformel vor und nach der Rentenreform 1992

\begin{tabular}{|c|c|}
\hline Rentenberechung ab 1957/72 & Rentenformel ab 1992 \\
\hline 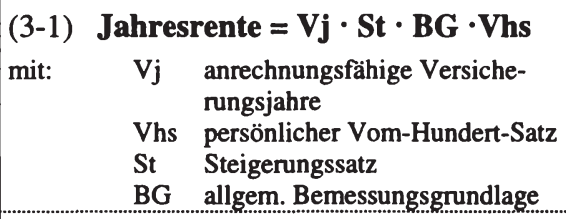 & $\begin{array}{lll}\text { (3-2) } & \text { Monatsrente }=\text { Ep } \cdot \text { Raf } \cdot \mathbf{a R} \cdot \mathbf{Z f} \\
\text { mit: } & \text { Ep } & \text { Summe der Entgeltpunkte } \\
& \text { Raf } & \text { Rentenartfaktor } \\
& \text { aR } & \text { aktueller Rentenwert } \\
& \text { Zf } & \text { Zugangsfaktor }\end{array}$ \\
\hline \multicolumn{2}{|c|}{ Dabei haben die Elemente der Rentenberechnungen die folgenden Bedeutungen: } \\
\hline $\begin{array}{l}\text { Vj Anrechnungsfähige Versiche- } \\
\text { rungsjahre } \\
\text { Die Anzahl der Versicherungsjahre ergibt } \\
\text { sich aus den unterschiedlichen Beitragszeiten } \\
\text { (vgl. Kapitel 3.2.1.2): Beitragszeiten, Ersatz- } \\
\text { zeiten, Ausfallzeiten, Kindererziehungszeiten } \\
\text { und Zurechnungszeiten. }\end{array}$ & $\begin{array}{l}\text { Ep } \rightarrow \text { Entgeltpunkte } \\
\text { Die neu eingeführten Entgeltpunkte er- } \\
\text { setzen die beiden bisherigen Elemente } \\
\text { der Vj und des VHS. }\end{array}$ \\
\hline $\begin{array}{l}\text { Vhs } \rightarrow \text { Persönlicher Vom-Hundert-Satz } \\
\text { Neben den Vj ist der Vhs das Bestandteil der } \\
\text { Rentenberechnung, das für die individuelle } \\
\text { Anpassung der Rente an den Versicherten } \\
\text { sorgt. Er mißt die relative Einkommenspositi- } \\
\text { on des Versicherten im Vergleich zum } \\
\text { Durchschnittsverdiener. Der Vhs wird für je- } \\
\text { des Beitragsjahr gesondert ermittelt und ab- } \\
\text { schließend über alle Versicherungsjahre } \\
\text { gemittelt. Für die ersten fünf Versicherungs- } \\
\text { jahre sowie für unterschiedliche Beitragszei- } \\
\text { ten gelten besondere Berechnungskonventio- } \\
\text { nen. }\end{array}$ & $\begin{array}{l}\text { Für jedes Versicherungsjahr wird das } \\
\text { Durchschnittsentgelt aller Arbeitnehmer } \\
\text { des betreffendes Jahres ermittelt (vgl. den } \\
\text { bisherigen Vhs). Eine Beitragsleistung, } \\
\text { die genau dem Jahresdurchschnittsbeitrag } \\
\text { entspricht, bringt dem Versicherten einen } \\
\text { Entgeltpunkt } \\
\text { Die Summe aller einzelnen Entgeltpunkte } \\
\text { stellt den Faktor EP in der Rentenformel } \\
\text { dar. } \\
\text { Eine Versicherter, der immer genau ein } \\
\text { Durchschnittseinkommen bezogen hat, } \\
\text { hat somit nach } 40 \text { Beitragsjahren } 40 \text { EP. } \\
\text { Jedes weitere Arbeitsjahr erhöht die An- } \\
\text { zahl der EP. }\end{array}$ \\
\hline $\begin{array}{l}\text { St } \rightarrow \text { Steigerungssatz } \\
\text { Der Steigerungssatz legt einen von der Ren- } \\
\text { tenart abhängigen Faktor fest, der die Rente } \\
\text { korrigiert. Der normale St ist 1,5; für eine Be- } \\
\text { rufsunfähigkeitsrente jedoch beträgt er nur } \\
\text { 1,0, reduziert die Rente also um ein Drittel, } \\
\text { da bei Berufsunfähigkeitsrenten eine zusätzli- } \\
\text { che Erwerbstätigkeit des Leistungsempfän- } \\
\text { gers erwünscht ist. Ebenfalls über den St re- } \\
\text { duziert werden die Hinterbliebenenrenten. }\end{array}$ & $\begin{array}{l}\text { Raf } \rightarrow \text { Rentenartfaktor } \\
\text { Differenzierung zwischen normalen Ren- } \\
\text { ten (Altersrenten, Schwerbeschädigtenren- } \\
\text { ten, Erwerbsunfähigkeitsrenten (EU) ...) } \\
\text { mit einem Raf von } 1.0 \text { und den Berufsun- } \\
\text { fähigkeitsrenten (BU) mit einem Raf von } \\
0.667 \text {, also } 2 / 3 \text { der bei einer anderen Zu- } \\
\text { gangsart auszuzahlenden Rente (verglei- } \\
\text { che Kapitel 3.2.1). }\end{array}$ \\
\hline
\end{tabular}




\begin{tabular}{|c|c|}
\hline Rentenberechung ab 1957/72 & Rentenformel ab 1992 \\
\hline $\begin{array}{l}\text { BG } \rightarrow \text { Allgemeine Bemessungsgrundlage } \\
\text { Dieses Element der Rentenformel ist für die } \\
\text { seit der Reform } 1972 \text { eingeführten Dy- } \\
\text { namisierung, d.h. die Koppelung der Renten- } \\
\text { zahlungen an die Entwicklung der Brutto- } \\
\text { arbeitsentgelte } 64 \text { notwendig. Es mißt die } \\
\text { Veränderung der Durchschnittslöhne unter } \\
\text { sich im Zeitverlauf ändernden Rechenregeln. }\end{array}$ & $\begin{array}{l}a \boldsymbol{R} \rightarrow \text { aktueller Rentenwert: } \\
\text { die frühere allg. Bemessungsgrundlage } \\
\text { multipliziert mit dem Steigerungssatz } 1,5 \\
\text { und geteilt durch } 12 \text {, da ab } 1992 \text { direkt } \\
\text { Monatsrenten berechnet werden }\end{array}$ \\
\hline & $\begin{array}{l}\text { Zf } \rightarrow \text { Zugangsfaktor } \\
\text { Neues Element der Rentenberechnung, } \\
\text { das dem Eintrittsalter in die GRV } \\
\text { Rechnung trägt. } \\
\text { - Abschlag bei Vorziehen der Rente (bis } \\
\text { zum } 62 \text {. Lebensjahr möglich): } 0,3 \% \text { pro } \\
\text { Monat entsprechend 3,6\% pro Jahr; } \\
\text { - Zuschlag bei Verschieben der Rente:0,5\% } \\
\text { pro Monat entsprechend 6\% pro Jahr. } \\
\text { (\$77 SGB) }\end{array}$ \\
\hline
\end{tabular}

Die Darstellung der Rentenreform 1992 kann an dieser Stelle nur auf die wichtigsten Punkte beschränkt bleiben, die wichtigen Wirkungen liegen teilweise im Detail. Aus ökonomischer Sicht zeigen sich zwei wesentliche Elemente: Die explizite Einbeziehung des Rentenalters in die Ermittlung der Rentenhöhe und der Wechsel von der Brutto- zur Nettoanpassung, die zukünftig verhindern wird, daß die Renten stärker steigen als die Arbeitseinkommen. Beide Punkte sind aus ökonomischer Sicht zu begrüßen, da sie Verzerrung des bestehenden Systems verringern. Es wird sich in Bezug auf die Berücksichtigung des Renteneintrittsalters jedoch zeigen (Kapitel 3.5), daß die $\mathrm{Zu}$ - und Abschläge aus versicherungsmathematischer Sicht nicht groß genug sind und das System weiterhin die Entscheidung der Arbeitnehmer in Richtung auf eine möglichst frühe Verrentung verzerrt. Neben der neu eingeführten Teilverrentung, die neue Flexibilität in die Ruhestandsentscheidung bringt, ist als wichtiges Element die Kürzung der Ausbildungszeiten zu nennen, die die Kassen der Versicherungsträger spürbar entlastet, da hiervon viele Rentenempfänger betroffen sind.

${ }^{64}$ Die Anpassung der Renten an die Entwiclung der Bruttolöhne unter Vernachlässigung der steigenden relativen Steuerlast der Arbeitnehmer führte dazu, daß die Renten netto stärker stiegen als die Löhne, was naturgemäß Finanzierungsprobleme mit sich brachte. Mit der Rentenreform 1992 wird die Dynamisierung daher an die Netto-Lohnentwicklung gekoppelt 


\subsection{Die deutsche Wiedervereinigung und Überführung des DDR-Renten- rechtes}

Die deutsche Wiedervereinigung wurde mit dem Fall der Mauer am 9. November 1989 an exakt dem selben Tag eingeleitet, an dem der Bundestag das Rentenreformgesetz 1992 verabschiedete. Damit kam am gleichen Tag, an dem der Gesetzgeber das Thema Rentenversicherung zunächst abschließend zu behandeln geplant hatte, auch auf die Rentenversicherung eine große Aufgabe zu: Die Integration der Versicherten der ehemaligen DDR, die Berechnung und Umbewertung der dort gesammelten Rentenanwartschaften. In diesem Kapitel wird zunächst die Struktur der DDR- Rentenversicherung dargestellt und daraus die Erfordernisse für ihre Überleitung in das bundesrepublikanische Recht abgeleitet. Anschließend wird die tatsächlich durchgeführte Überführung beschrieben. Hierzu ist teilweise ein Vergleich der beiden Systeme notwendig.

\subsubsection{Struktur und Rentenformeln der Pflicht- und der Freiwilligen Zusatzver- sicherung der DDR}

Die Rentenversicherung der DDR unterteilt sich in eine Pflichtversicherung und eine im Jahre 1971 eingeführte „Freiwillige Zusatzversicherung“ FZR. Die Besonderheit der Pflichtversicherung besteht darin, daß die in ihr enthaltene Beitragsbemessungsgrenze seit Einführung des Systems im Jahre 1947 konstant auf 600,- DM festgehalten wurde. Damit bekommt das DDR-System den Charakter eines statischen Rentenversicherungssystems ohne Anpassung der Beiträge an die Einkommens- oder Preisentwicklung. Die wesentlichen Unterschiede der Systeme sind in Tabelle 3-4 dargestellt:

Tabelle 3-4: Rentenversicherungssysteme der Bundesrepublik und der ehemaligen DDR

\begin{tabular}{|c|c|c|}
\hline & BRD & DDR \\
\hline $\begin{array}{l}\text { Sicherungs- } \\
\text { ziel }\end{array}$ & $\begin{array}{l}\text { leistungsbezogene, lebensstandardsi- } \\
\text { chernde Rente }\end{array}$ & Einheitliches Ruhestandseinkommen \\
\hline Organisation & $\begin{array}{l}\text { 3-Säulen-Modell: } \\
\text { - Pflichtversicherung: GRV } \\
\text { - betriebliche Zusatzversicherungen } \\
\text { (freiwillig) } \\
\text { - private Zusatzversicherungen } \\
\text { (freiwillig) }\end{array}$ & $\begin{array}{l}\text { ausschließlich staatlich organisiert: } \\
\text { - Pflichtversicherung } \\
\text { - FZR seit } 1971 \text { (freiwillig / staatlich) } \\
\text { - Zusatzregelungen für (mehr als 60) } \\
\text { einzelne Gruppen }{ }^{65}\end{array}$ \\
\hline
\end{tabular}

65 Vergleiche SCHMÄHL (1992), Seite $34 \mathrm{f}$. 


\begin{tabular}{|c|c|c|}
\hline & BRD & DDR \\
\hline $\begin{array}{l}\text { Höhe und } \\
\text { Struktur der } \\
\text { Renten }\end{array}$ & $\begin{array}{l}\text { einkommensbezogen, dynamisch } \\
\text { d.h. an Lohnentwicklung gekoppelt. }\end{array}$ & $\begin{array}{l}\text { statisch } \\
\text { durch niedrige Beitragsbemessungs- } \\
\text { grenzen }\end{array}$ \\
\hline Finanzierung & $\begin{array}{l}\text { Versicherungssytem; } \\
\text { Getrennte Träger für Gesundheits-, Al- } \\
\text { ters-, Unfall- und Arbeitslosenversiche- } \\
\text { rung; } \\
\text { Bundeshaushalt gibt Zuschuß (z.Zt. } \\
\text { knapp 20\%) }\end{array}$ & $\begin{array}{l}\text { Versicherungssystem; } \\
\text { ein Träger für alle Bereiche der Sozia- } \\
\text { len Sicherung. } \\
\text { Mehr als } 50 \text { prozentige Finanzierung } \\
\text { durch Staatshaushalt }\end{array}$ \\
\hline $\begin{array}{l}\text { Umverteilung } \\
\text { swirkungen }\end{array}$ & $\begin{array}{l}\text { lineare Formel } \\
\text { teilweise Umverteilung durch Beitrags- } \\
\text { bemessungsgrenze und Mindestrenten }\end{array}$ & $\begin{array}{l}\text { nichtlinear durch Mindestbeträge und } \\
\text { diverse Sprungstellen. } \\
\text { Deutliche Umverteilung auf niedrigem } \\
\text { Level }\end{array}$ \\
\hline Rentenalter & $\begin{array}{l}\text { Flexibler Ruhestand zwischen } 63 \text { und } 67 \\
\text { Frauen ab } 60 \text { Jahren, Sonderregelungen } \\
\text { ab } 60\end{array}$ & $\begin{array}{l}\text { Festes Rentenalter; } \\
\text { Frauen: } 60 \text { Jahre, Männer: } 65 \text { Jahre }\end{array}$ \\
\hline
\end{tabular}

Rentenformeln:

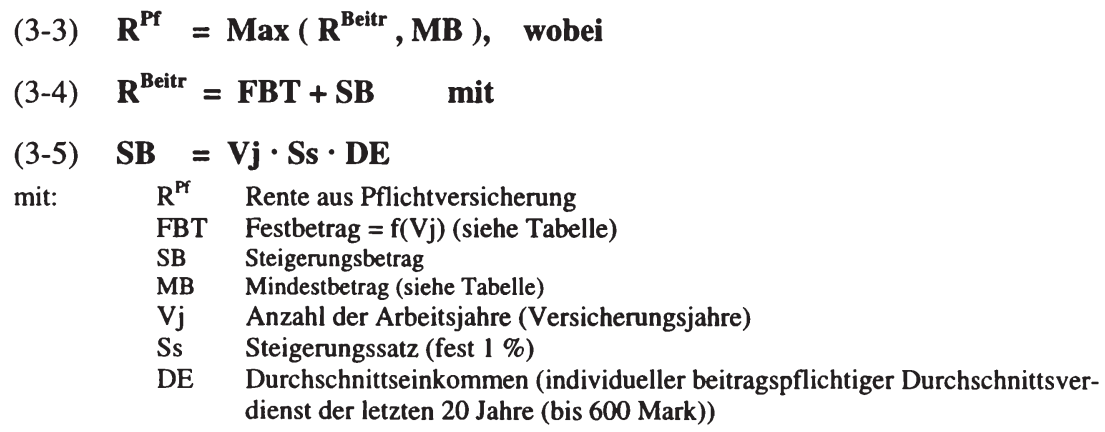

Durch die Zweiteilung der effektiven Rente in einen beitragsabhängigen Teil und einen garantierten Mindestbetrag und durch die gleichzeitige rigide Deckelung der Beiträge auf 600,- Mark pro Monat ist die erhaltene Rente aus der Pflichtversicherung $R^{\mathrm{Pf}}$ eine Stufenfunktion der Versicherungsjahre. Sie hängt kaum vom Durchschnittseinkommen der Versicherten ab, da über $90 \%$ der Beitragszahler die Beitragsbemessungsgrenze 
überschritten ${ }^{66}$. Diese Funktion wird in Abbildung 3-8 graphisch veranschaulicht. Die fett gezeichnete Linie zeigt den Verlauf der tatsächlich erhaltenen Monatsrente aus der Pflichtversicherung $\mathbf{R}^{\mathrm{Pf}}$. Es wird deutlich, daß $\mathbf{R}^{\mathrm{Pf}}$ bis etwa 34 Versicherungsjahren dem Mindestbetrag entspricht und danach geringfügig über diesem liegt. Die Höhe dieser Rente im Vergleich zum Lohneinkommen ist relativ gering. SCHWITZER (1990) errechnet ein Rentenniveau, das von $33 \% 1980$ bis 1989 zwar auf $45 \%$ gestiegen ist, damit aber für einen Erhalt des Lebensstandards nicht ausreichend ist.

\section{Abbildung 3-8: DDR-Rentenhöhe in Abhängigkeit von den Beitragsjahren}

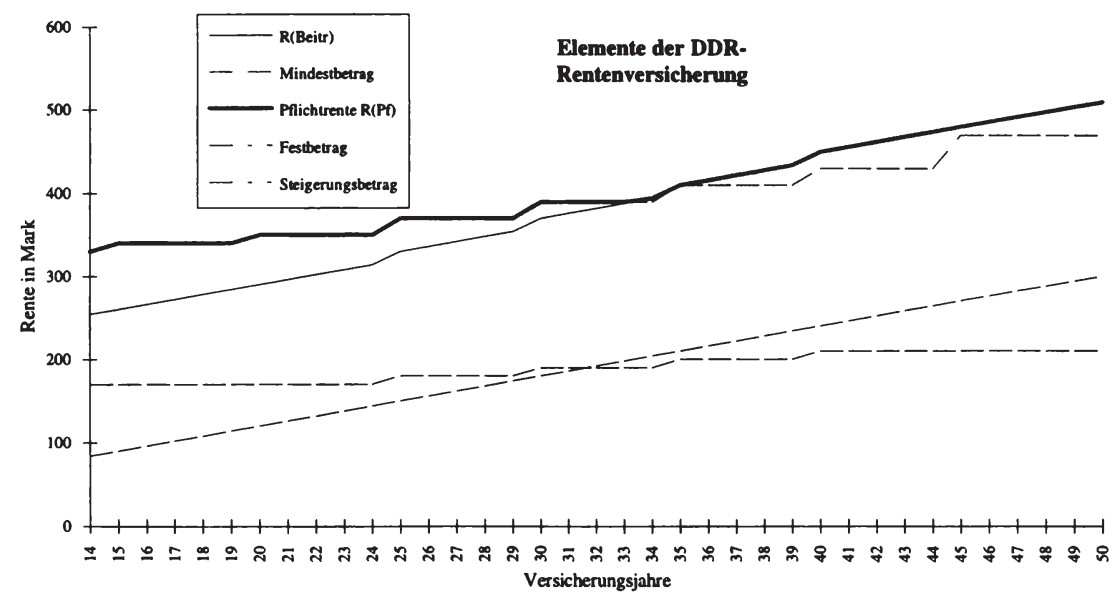

Quelle: Berechnung aus der Rentenformel für ein Durchschnittseinkommen der letzten 20 Jahre von mindestens 600,- Mark (Beitragsbemessungsgrenze); Darstellung in Anlehnung an SCHMAHL (1991 c) Seite 61.

Tabelle 3-5: Fest- und Mindestbeträge in der Pflichtversicherung der DDR

\begin{tabular}{|l|cc|}
\hline Anzahl der Arbeitsjahre & Festbetrag & Mindestbetrag \\
\hline unter 15 & 170 & 330 \\
15 bis 19 & 170 & 340 \\
20 bis 24 & 170 & 350 \\
25 bis 29 & 180 & 370 \\
30 bis 34 & 190 & 390 \\
35 bis 39 & 200 & 410 \\
40 bis 44 & 210 & 430 \\
45 und mehr & 210 & 470 \\
\hline
\end{tabular}

${ }^{66}$ LÖTSCH (1992), Seite 20. 
Diese geringe Ersatzquote liegt in der statischen Beitragsbemessungsgrenze von 600,Mark pro Monat begründet, die 1947 von der sowjetischen Besatzungsmacht festgesetzt wurde und durch die SED-Regierung nie erhöht wurde. Stattdessen führte die DDR im März 1971 ein zusätzliches System ein, die „Freiwillige Zusatzrentenversicherung" FZR. In dieser war das Einkommen oberhalb von 600,- Mark versicherbar, bis 1976 war auch hier der Beitrag auf weitere 600,- Mark festgelegt, danach wurde er bis zur vollen Höhe der Bezüge freigegeben ${ }^{67}$. Die Rentenformel der FZR war linear gestaltet:

$$
\mathbf{R}^{\mathrm{FZR}}=\mathbf{V j} \cdot \mathbf{S f} \cdot \mathbf{E g}
$$

mit: $\quad R^{\mathrm{FZR}} \quad$ Rente aus Freiwilliger Zusatzrentenversicherung

$\mathrm{Vj} \quad$ Anzahl der Arbeitsjahre (Versicherungsjahre)

Sf Steigerungssatz $(2,5 \%)$

Eg Versichertes Entgelt (oberhalb von 600,- Mark)

Die Gesamtrente ergibt sich schließlich als Summe aus Pflicht- und freiwilliger Versicherung:

$$
\mathbf{R}^{\text {Gesamt }}=\mathbf{R}^{\mathrm{pf}}+\mathbf{R}^{\mathrm{FZR}}
$$

Durch die Ausgestaltung der FZR wurde somit ein beitragsbezogenes Element in das Rentenversicherungssystem der DDR eingeführt. Allerdings sind die Auswirkungen der FZR bzw. der aus ihr resultierenden Anwartschaften hypothetisch, denn beim Zusammenbruch der DDR war sie nicht einmal 20 Jahre in Kraft, so daß es noch keine Rentner gab, die eine vollständig auf beiden Versicherungskomponenten beruhende Rente erhielten. So berechnet wiederum SCHWITZER (1990) ein Rentenniveau unter Berücksichtigung der FZR, das von $56 \% 1980$ bis 55\% 1989 nicht angestiegen ist. Aus der Einberechnung der FZR-Beiträge ergibt sich die kuriose Tatsache, daß Rentner, die vor 1971 in Rente gingen, keine zusätzliche Rente bezogen, Rentner 1972 bezogen einen geringen Anteil aufgrund des einen geleisteten Versicherungsjahres ${ }^{68}$, wogegen mit steigendem Abstand zum Jahr 1971 auch der Anteil der FZR-Rente an der Gesamtrente stieg und sich die Rente um den FZR-Anteil erhöhte.

\footnotetext{
${ }^{67}$ SCHMÄHL (1991 c) Seite $61 \mathrm{ff}$.

68 Gegeben, daß diese Person sich in der FZR versichert hat. SCHMÄHL (1991 c) nennt die Zahl, daß ca. $80 \%$ derjenigen, die FZR-berechtigt waren, diese auch abschlossen. Allerdings lag dieser Anteil bei den Frauen wesentlich geringer als bei Männern.
} 


\subsubsection{Anpassung der laufenden DDR-Renten an das westdeutsche System}

Nach der deutschen Wiedervereinigung war es notwendig, die laufenden „Ostrenten“ so neu zu berechnen, daß sie in die bundesdeutsche GRV überführt werden konnten. Aus der Tatsache, daß die FZR-Ansprüche mit dem Jahr des Rentenzuganges (nach 1971) ansteigen, folgt eine soziale Ungleichheit, die nicht übernommen werden konnte. Abbildung 3-9 zeigt die gesamte DDR-Rente aus Pflichtversicherung und FZR für einen „Eckrentner“ des Jahres $1990^{69}$.

\section{Abbildung 3-9: Vergleich der Rentenhöhen nach Versicherungsjahren DDR/BRD}

Rentenböhe nach Versicherungsjaluren - DDR versus BRD - Berechnung

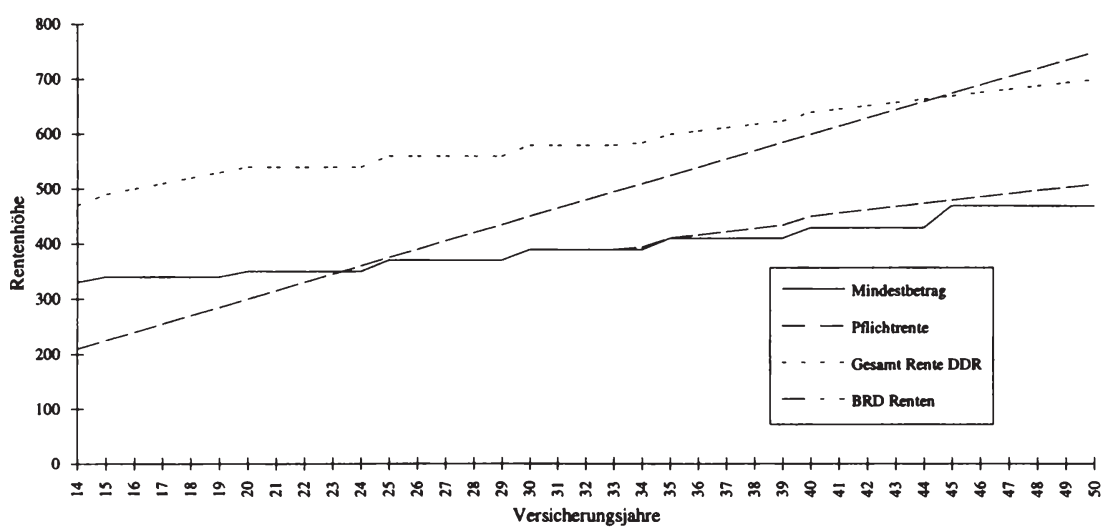

Quelle: Berechnung aus der Rentenformel unter Annahme eines Durchschnittseinkommens der letzten 20 Jahre von 1.000,- (D) Mark - Darstellung in Anlehnung an SCHMAHL (1991 c) Seite 61.

Die FZR steigt nur für die ersten 19 Versicherungsjahre (1971 bis 1990) an und bleibt danach konstant, da vorher die FZR nicht bestand. Die bundesdeutsche GRV hat dagegen einen linearen Verlauf in der Zahl der Beitragsjahre (siehe Rentenformel 3-1). Es wird deutlich, daß die Differenz zwischen West und Ostrente für jede Anzahl von Versicherungsjahren unterschiedlich ist.

Daraus ergab sich die Notwendigkeit einer vollkommenen Neubewertung der DDRRenten. Ein weiterer Grund hierfür war, daß die DDR-Renten keine reinen Versicherungsrenten im Sinne der GRV waren, sondern zusätzlich Sozialleistungsfunktionen

${ }^{69}$ Gemeint ist ein Arbeitnehmer mit einem konstanten Durchschnittseinkommen von 1.000 Mark, der also immer genau die Obergrenze von 600,- Mark in die Pflichtversicherung und für die Jahre ihres Bestehens weitere 400,- Mark in die FZR eingezahlt hat. 
beinhalteten, die in der Bundesrepublik durch die Sozialhilfe abgedeckt werden. Aus diesem Grunde liegt die DDR-Rente fast durchgängig über der hypothetischen WestRente. Insofern war eine Umrechnung der bisherigen DDR-Pensionen auf die lohnbezogenen Renten der GRV vorzunehmen. ${ }^{70}$ Der Unterschied der beiden Berechnungsarten wird ebenfalls in Abbildung 3-9 durch die bereits betrachtete Differenz der beiden Berechnungsarten deutlich, die für eine geringe Anzahl von Versicherungsjahren, d.h. niedrige Beitragszahlungen am größten ist. Dies verdeutlicht die starke Umverteilung des DDR-Systems in Richtung der Bezieher niedriger Einkommen. Hingegen findet in diesem Bereich keine Umverteilung durch die GRV statt, sondern die Bezieher niedriger Renten haben einen Anspruch auf Sozialhilfe.71

Die Umrechnung wurde im Rahmen der Wirtschafts- und Sozialunion durch das „Rentenangleichungsgesetz" vom 28.6.1990 geregelt. Grundlegende Maxime der Umstellung sowohl der laufenden Renten wie auch der Anwartschaften war, daß die Rente der neuen Bundesbürger $70 \%$ von deren Nettoarbeitsentgelt betragen sollte. ${ }^{72}$ Es ergab sich eine notwendige Höherbewertung der DDR-Anwartschaften in Abhängigkeit der Versicherungsjahre und des Renteneintrittsjahres ${ }^{73}$. Bei Inkrafttreten der Wirtschaftsund Sozialunion am 1.7.1990 differierten in der DDR die laufende Renten eines „Eckrentners“ zwischen 470,- und 602,- Mark pro Monat in Abhängigkeit vom Eintritt in den Ruhestand. Tabelle 3-6 zeigt diese grundsätzlichen Möglichkeiten für beispielhafte 5-Jahres Intervalle, wobei Spalte (7) die notwendige Höherbewertung der Rentenzahlung in Prozent angibt. Diese Anpassungen wurden zum 1.7.90 getätigt.

${ }^{70}$ Bundesministerium für Arbeit und Sozialordnung (1993), Seite $46 \mathrm{ff}$.

${ }^{71}$ Die für die West-GRV unrealistisch niedrigen Beiträge führen zu einer sehr geringen Rente, die im Westen ggf. durch die Sozialhilfe ergänzt worden wäre. Die Abbildung vergleicht in diesem Sinne nicht die absoluten Höhen der Renteneinkommen, da die Beitragsszahlungen nicht vergleichbar sind. In der DDR waren die angenommenen 1.000,- Mark Einkommen die Höchstversicherung, in Westdeutschland stellen sie ein niedriges Einkommen dar.

72 Die exakten Berechnungen können hier nicht wiedergegeben werden, für eine genaue Darstellung vergleiche SCHMÄHL (1991 c), Seite 72 ff.

${ }^{73}$ Renten, die höher waren, als die lohnbezogene Rente, wurden nicht abgewertet, sondern die Differenz wird als ,Auffiillbetrag “ weitergezahlt und auch die Rentenerhöhungen bis 1996 beziehen sich auf die Gesamtsumme. Ab 1997 werden sie jedoch im Rahmen der Rentenanpassungen schrittweise abgeschmolzen und somit langsam auf das lohnbezogene Niveau angepaßt. 
Tabelle 3-6: Anpassungen der DDR-Renten an die GRV

\begin{tabular}{|c|c|c|c|c|c|c|}
\hline 1 & 2 & 3 & 4 & 5 & 6 & 7 \\
\hline $\begin{array}{l}\text { Jahr des } \\
\text { Renten- } \\
\text { eintritts }\end{array}$ & $\begin{array}{l}\text { Pflichtver- } \\
\text { sicherung }\end{array}$ & FZR & $\begin{array}{c}\text { Summe } \\
\text { DDR-Rente }\end{array}$ & $\begin{array}{c}\text { DDR } \\
\text { Rentenniveau }\end{array}$ & $\begin{array}{c}\text { Differenz zu 70\% } \\
\text { Vorgabe für } \\
\text { Umstellung }\end{array}$ & $\begin{array}{c}\text { notwendige } \\
\text { Höherbewertung } \\
\text { am } 1.7 .90 \\
\text { (6) in \% von (5) }\end{array}$ \\
\hline bis 1970 & 470 & & 470 & 49,0 & 21,0 & 42,98 \\
\hline 1975 & 470 & 13 & 483 & 50,3 & 19,7 & 39,13 \\
\hline 1980 & 470 & 36 & 506 & 52,7 & 17,3 & 32,81 \\
\hline 1985 & 478 & 70 & 548 & 57,1 & 12,9 & 22,63 \\
\hline 1990 & 480 & 122 & 602 & 62,7 & 7,3 & 11,63 \\
\hline
\end{tabular}

Quelle: Berechnung der Renten aus Rentenformel; Annahme: 45 Versicherungsjahre und Einkommen von 960 Mark 74

Nach der vollzogenen Anhebung der DDR-Renten auf eine Ersatzquote von $70 \%$ blieb der Niveauunterschied zu westdeutschen Renten, der durch schrittweise Rentenerhöhungen abgebaut wird. Erhöhungen bis Mitte 93:

$\begin{array}{ll}\text { - } 1.1 .91 & \text { 15 Prozent } \\ \text { - } 1.7 .91 & 15 \text { Prozent } \\ \text { - } 1.1 .92 & 11,65 \text { Prozent } \\ \text { - } 1.1 .93 & \text { 12,79 Prozent } \\ \text { - } 1.7 .93 & \text { 14,1 Prozent }\end{array}$

Bis 1996 gilt ein Übergangsrecht im Gebiet der ehemaligen DDR, wodurch jeder neue Rentner, der nach DDR-Recht besser gestellt wäre, nach diesem bewertet wird. Ebenfalls bis zu diesem Zeitpunkt werden die ,Sozialzuschläge“ als Rentenbestandteile bezahlt, die ab 1.1.97 ggf. durch Wohngeld, Sozialhilfe o.a. Elemente der bundesdeutschen sozialen Sicherung ersetzt werden müssen. ${ }^{75}$ Die Rentenreform 1992 gilt mit ihrem Inkrafttreten ebenso in Ostdeutschland. Für die Ermittlung neuer Renten werden die Beitragszahlungen teilweise umbewertet (Ost-Entgeltpunkte). ${ }^{76}$

Aus ökonomischer Sicht ist es wichtig zu bedenken, daß ein Großteil der durchgeführten Maßnahmen nicht aus wirtschaftlichen, sondern aus politischen Motiven durchgeführt wurden und - aufgrund der sehr schnell vollzogenen Wiedervereinigung - durchgeführt werden mußten, um das Einkommensniveau in Ost- und Westdeutschland anzugleichen und weitere innerdeutsche Migration im verträglichen Rahmen zu halten.

\footnotetext{
74 Beispiel in Analogie zu SCHMÄHL (1992 b) Tabelle 1; Seite 15 gewählt. Für eine genaue Tabelle der prozentualen Anhebungen siehe Bundesministerium für Arbeit und Sozialordnung BMA (1990), Seiten 12 und 13.

75 Bundesministerium für Arbeit und Sozialordnung (1993), Seite 49.

76 Bundesministerium für Arbeit und Sozialordnung (1993), Seite $144 \mathrm{ff}$.
} 
SCHMÄHL schätzt die effektive Erhöhung der Ostrenten im ersten Jahr seit der Einführung der DM auf über 60\%. ${ }^{77}$ Die beschriebenen Aufwertungen konnten nicht aus dem Versicherungssystem der GRV finanziert werden, sondern etwa $40 \%$ der Zahlungen werden aus dem laufenden Staatshaushalt der Bundesrepublik getragen. ${ }^{78}$

Eine weitaus größere Belastung resultiert aus dem ostdeutschen Arbeitsmarkt. Massenhafte Unternehmensschließungen und Entlassungen ließen die Arbeitslosenzahlen in die Höhe schnellen, ohne daß es ein kurzfristiges Mittel zu Wiedereingliederung der Arbeitskräfte gäbe. Ein politisches Mittel in diesem Zusammenhang war und ist, älteren Arbeitnehmern die Möglichkeit einer Frühverrentung einzuräumen und damit die in der Statistik auszuweisende Arbeitslosenquote zu senken. Aus diesem Grunde wurde in Ostdeutschland ein neues Modell des Vorruhestandes gebildet, welches - ähnlich dem oben beschriebenen Vorruhestand in der Bundesrepublik 1984-88 - von der Bundesanstalt für Arbeit finanziert wird und durch das arbeitslose Erwerbspersonen bereits im Alter von 55 Jahren in den Ruhestand treten können und bei dem die Arbeitnehmer $70 \%$ ihres vorherigen Nettoeinkommens erhalten. Schmähl schätzt, daß diese Einrichtung die Arbeitslosenquote Ostdeutschlands 1991 um 37\% verringert hat. ${ }^{79}$

\subsection{Anreizstrukturen des Rentenversicherungssystems: Altersanpassung und versicherungsmathematische Fairneß}

Aus der Darstellung der demographischen Situation in Verbindung mit der historischen Entwicklung der Rentenzugänge und des Rentenzugangsalters in Kapitel 2 wird deutlich, daß die Finanzierung der GRV als Versicherungssystem durch das sinkende Rentenalter zunehmend problematisch wird. Dies folgt aus der dargestellten Budgetgleichung der Rentenversicherung, die durch die Verschiebung der relativen Anteile von Erwerbstätigen und Rentnern belastet wird (Formel 2-1 und 2-2). Um die Budgetidentität auch bei sinkendem Rentenzugangsalter zu garantieren, müssen die beiden wichtigsten Effekte eines früheren Renteneintritts:

- kürzere Beitragszahlung und

- längerer Rentenbezug80

bei der Berechnung der individuellen Rente berücksichtigt werden.

77 SCHMÄHL (1992 b), Seite 16.

${ }^{78}$ Ebenda, Seite 21.

${ }^{79}$ Ebenda, Seite 28.

${ }^{80}$ Unter zusätzlicher Berücksichtigung der jeweiligen, sich ebenfalls im Zeitverlauf ändernden, Lebenserwartung. 
$\mathrm{Zu}$ diesem Zweck wird in diesem Abschnitt ein versicherungsmathematisch faires Rentenversicherungssystems vorgestellt, in dem für einen Arbeitnehmer kein monetärer Anreiz besteht, mit einem bestimmten Alter in den Ruhestand zu treten, das System also die Wahl des Rentenalters nicht verzerrt. ${ }^{81}$

Es sei angenommen, daß ein Arbeitnehmer für die Wahl des Rentenalters die Barwerte aller zukünftigen Rentenzahlungen abzüglich der für Arbeitseinkommen zu zahlenden Beiträge für alle potentiellen Rentenalter vergleicht. Bezugsgröße ist dabei ein Renteneintritt mit 65 Jahren. Für jedes andere Zugangsalter wird die Adjustierung der Rente durch einen multiplikativen Adjustierungsfaktor $\mathrm{AF}(\mathrm{R})$ dargestellt:

$$
B W_{A}(R)=\sum_{t=R}^{\infty} A F(R) \cdot Y_{t}^{R}(65) \cdot a_{t} \cdot \delta^{i-A}-\sum_{t=A}^{R-1} Y_{t}^{L} \cdot B S \cdot a_{t} \cdot \delta^{t-A}
$$

$\begin{array}{lll}\text { mit: } & \mathrm{BW}_{\mathrm{A}}(\mathrm{R}) & \text { Barwert aller zukünftigen Zahlungsströme bezogen auf das aktuelle Alter } \mathrm{A} \\ \mathrm{A} & \text { Entscheidungsalter (aktuelles Alter eines Arbeitnehmers) } \\ \mathrm{R} & \text { Verrentungsalter } \\ \mathrm{AF}(\mathrm{R}) & \text { Anpassungsfaktor } \\ \mathrm{Y}^{\mathrm{R}}(65) & \text { Renteneinkommen für } \mathrm{R}=65 \\ \mathrm{Y} & \text { Arbeitseinkommen } \\ \mathrm{BS} & \text { Beitragssatz zur Rentenversicherung } \\ \mathrm{a}_{\mathrm{t}} & \text { Überlebenswahrscheinlichkeit im Alter t } \\ \delta & \text { Diskont Faktor. }\end{array}$

In einem verzerrungsfreien Rentenversicherungssystem ist dieser Barwert für alle potentiellen Rentenalter gleich. ${ }^{82}$ Es bietet sich an, als Referenzgröße jenen Barwert für einen Eckrentner zu betrachten, der zum Regelrentenalter von 65 Jahren in das System eintritt:

$$
B W_{A}(R)=B W_{A}(65) \quad \forall R
$$

Diese Identität kann durch die entsprechende Wahl der Anpassungsfaktoren erreicht werden. Die Auflösung von (3-8) unter der Annahme (3-9) nach AF ergibt [für AF(65) = 1] (3-10) als nicht-verzerrenden Faktor $\mathbf{A F}$.

\footnotetext{
81 Vgl. BÖRSCH-SUPAN (1992).

82 BÖRSCH-SUPAN (1992 e) weist darauf hin, daß das Konzept der versicherungsmathematischen Fairneß einen Schritt weitergeht und eine Identität zwischen Ein- und Auszahlungen in das Versicherungssystem verlangt, so daß der Barwert für alle Rentenalter $=0$ sein müsse. (BÖRSCH-SUPAN (1992 c), Seite 541) Dies gilt allerdings nur, wenn noch keine Einzahlungen in die Versicherung geleistet wurden, ansonsten müßte der zweite Teil von (3-8) entsprechend erweitert werden und die Summierung der Beitragszahlungen ab dem Eintritt ins Erwerbsleben laufen und nicht erst ab dem Entscheidungsalter A.
} 


$$
A F^{*}(R)=\frac{\sum_{t=65}^{\infty} Y_{t}{ }^{R} \cdot a_{t} \cdot \delta^{t-A}-\sum_{t=A}^{64} Y_{t}{ }^{L} \cdot B S \cdot a_{t} \cdot \delta^{t-A}+\sum_{t=A}^{R-1} Y_{t}{ }^{L} \cdot B S \cdot a_{t} \cdot \delta^{t-A}}{\sum_{t=R}^{\infty} Y_{t}{ }^{R} \cdot a_{t} \cdot \delta^{t-A}},
$$

so daß $\mathrm{AF}^{*}$ für alle $\mathrm{R}$ errechnet werden kann.

Tabelle 3-7: Nicht-verzerrende Anpassung der Rentenhöhe nach Rentenzugangsalter.

\begin{tabular}{|c|ccc|}
\hline $\begin{array}{c}\text { Diskontfaktor: } \\
\text { Zugangsalter: }\end{array}$ & $13,8 \%$ & $6 \%$ & $3 \%$ \\
\hline 55 & $40,9 \%$ & $50,1 \%$ & $70,0 \%$ \\
56 & $44,0 \%$ & $52,7 \%$ & $71,3 \%$ \\
57 & $47,4 \%$ & $55,5 \%$ & $72,9 \%$ \\
58 & $51,3 \%$ & $58,7 \%$ & $74,6 \%$ \\
59 & $55,6 \%$ & $62,4 \%$ & $76,7 \%$ \\
60 & $60,5 \%$ & $66,5 \%$ & $79,1 \%$ \\
61 & $66,2 \%$ & $71,3 \%$ & $81,9 \%$ \\
62 & $72,7 \%$ & $76,8 \%$ & $85,3 \%$ \\
63 & $80,3 \%$ & $83,3 \%$ & $89,3 \%$ \\
64 & $89,3 \%$ & $90,9 \%$ & $94,1 \%$ \\
65 & $100 \%$ & $100 \%$ & $100 \%$ \\
66 & $113,0 \%$ & $111,1 \%$ & $107,3 \%$ \\
67 & $129,0 \%$ & $124,8 \%$ & $116,4 \%$ \\
68 & $149,2 \%$ & $142,0 \%$ & $128,0 \%$ \\
69 & $175,4 \%$ & $164,5 \%$ & $143,3 \%$ \\
70 & $210,7 \%$ & $194,6 \%$ & $164,0 \%$ \\
\hline
\end{tabular}

Anmerkung: Angabe in Prozent einer für das Eintrittalter 65 Jahren errechneten Altersrente. Die Berechnung erfolgte auf Basis der 0.a. Formeln unter der Annahme einer Ersatzquote von $70 \%$, eines Beitragssatzes von $19 \%$ sowie altersspezitischer Mortalitătraten.

Tabelle 3-7 zeigt die auf diese Weise ermittelten nicht-verzerrenden Anpassungsfaktoren, wobei deutlich wird, daß deren Werte stark vom gewählten Diskontfaktor $\delta$ abhängen. Es stellt sich hier die Frage nach der Bedeutung dieses Faktors, der aus Sicht des Individuums als persönliche Zeitpräferenzrate interpretiert werden kann. In einer Mikro-Schätzung ermittelt BÖRSCH-SUPAN den in der Tabelle ebenfalls angegebenen Wert von 13,8\%,83 der eine individuell hohe Gegenwartspräferenz der Arbeitnehmer widerspiegelt. Dieser Wert liegt im Vergleich zu anderern in der Literatur ermittelten Zeitpräferenzraten sehr hoch, üblich sind geringere Werte etwa die hier angegebenen 3 und 6 Prozent, auch räumt der Autor ein, daß die statistische Identifikation des Wertes schwach ist. Es ist jedoch zu bedenken, daß dieser Wert auf Basis der selben Daten-

${ }^{83}$ BÖRSCH-SUPAN (1992 c) Seite 551. 
grundlage, dem SOEP, ermittelt wurde, auf dem auch die empirische Untersuchung des 7. Kapitels beruht. Er wird aus diesem Grunde im folgenden alternativ zu den anderen genannten Eckpunkten von 3 bzw. 6 Prozent für die Berechungen verwendet.

Tabelle 3-8: Rentenberechnung und Altersanpassung in der GRV

\begin{tabular}{|c|c|c|c|c|c|}
\hline \multirow[b]{2}{*}{ Eintrittsalter: } & \multicolumn{2}{|c|}{ Formel bis 1991} & \multirow{2}{*}{$\begin{array}{c}\text { unverzerrter } \\
\mathbf{A F}^{*}\end{array}$} & \multicolumn{2}{|c|}{ 1992er Reform } \\
\hline & $35 \mathrm{Vj}$ & $45 \mathrm{Vj}$ & & $35 \mathrm{Vj}$ & $45 \mathrm{Vj}$ \\
\hline 55 & 74,8 & - & $40,9 \%$ & 51,9 & - \\
\hline 56 & 77,0 & - & $44,0 \%$ & 55,4 & - \\
\hline 57 & 79,3 & . & $47,4 \%$ & 59,1 & - \\
\hline 58 & 81,6 & - & $51,3 \%$ & 63,2 & - \\
\hline 59 & 84,0 & $-^{*}$ & $55,6 \%$ & 67,4 & $-{ }^{*}$ \\
\hline 60 & 86,5 & 89,4 & $60,5 \%$ & 72,0 & 74,4 \\
\hline 61 & 89,1 & 91,4 & $66,2 \%$ & 76,9 & 78,9 \\
\hline 62 & 91,7 & 93,5 & $72,7 \%$ & 82,1 & 83,7 \\
\hline 63 & 94,4 & 95,6 & $80,3 \%$ & 87,7 & 88,8 \\
\hline 64 & 97,1 & 97,8 & $89,3 \%$ & 93,6 & 94,3 \\
\hline 65 & 100 & 100 & $100 \%$ & 100 & 100 \\
\hline 66 & 110,1 & 109,4 & $113,0 \%$ & 109,0 & 108,4 \\
\hline 67 & 121,1 & 119,6 & $129,0 \%$ & 118,9 & 117,4 \\
\hline 68 & 124,6 & 122,3 & $149,2 \%$ & 129,6 & 127,2 \\
\hline 69 & 128,1 & 125,0 & $175,4 \%$ & 141,3 & 137,8 \\
\hline 70 & 131,8 & 127,8 & $210,7 \%$ & 154,1 & 149,4 \\
\hline
\end{tabular}

Anmerkung:Angaben in Prozent der Eckrente mit 65 Jahren. Die kursiv dargestellten Angaben deuten an, daß mit diesen Lebensaltem ein offizieller Rentenzugang noch nicht vorgesehen ist, wohl aber ein Zugang über Invalidităts, EU-, BU- und Arbeitslosigkeitsrenten mőglich ist. Die „Verrentungsfenster" sind umrahmt, das Alter 60 stellt vor wie nach der Reform das erstmögliche Alter für eine vorgezogenen Ruhestand dar.

*: Angabe nicht sinnvoll, da der Beginn der Einzahlungen vor dem 15. Lebenjahr lăge.

Für eine Bewertung der GRV aus Anreizgesichtspunkten stellt sich die Frage, wieweit die tatsächliche Altersanpassung der Rentenbeträge in Bezug auf das Eintrittsalter dem oben aufgestellten Kriterium der Unverzerrtheit entspricht. Tabelle 3-8 zeigt die Anpassungsfaktoren, die sich aus den Rentenformeln vor und nach $1992 \mathrm{im}$ Vergleich zu den versicherungsmathematisch fairen bei einem Diskontsatzes von 13,8 \% ergeben. Bei der Rentenberechnung bis 1991 sind keine Anpassungen an das Eintrittsalter vorgesehen, so daß sich die Abschläge lediglich aus der geringeren Zahl der Beitragsjahre ergeben. Erhält ein Eckrentner (Eintritt in die GRV im Alter von 65 Jahren und Beitragszeit von 45 Versicherungsjahren) eine Rente von $100 \%$, so verringert sich diese Zahlung bei einem Eintritt in die GRV mit 64 Jahren um 1/45 = 2,2 Prozent. Für einen Arbeitnehmer mit 35 Versicherungsjahren beträgt dieser Abschlag 2,9 Prozent. Eine Verlängerung der Erwerbsphase auf 66 bzw. 67 Jahre wurde durch einen Rentenaufschlag von $7 \%$ honoriert. Die Tabelle zeigt, daß die in Prozent gemessenen Ab- 
schläge auch von der bisherigen Erwerbshistorie in Form der Beitragsjahre, die im Alter von 65 erreicht wären, abhängt. Ein Frührentner, der mit 65 auf 45 Versicherungsjahre gekommen wäre, mußte mit 55 Jahren nur einen um 5 Prozentpunkte niedrigeren Abschlag hinnehmen als jemand der 10 Versicherungsjahren weniger aufwies.

Die Berechnung der Rente nach der Rentenreform 92 berücksichtigt hingegen zusätzlich zu den geringeren Beitragsjahren den neu eingeführten Zugangsfaktor Zf, der die Rente pro Jahr vorgezogenen Ruhestandes um 3,6 Prozent verringert (vgl. Tabelle 3-3, Seite 60).

Die Tabelle zeigt, daß das bundesdeutsche Rentenversicherungssystem auch nach der Rentenreform 1992 von einer nicht-verzerrenden Architektur ein gutes Stück entfernt ist. Vor der Reform bekam ein langjährig Versicherter (Spalte 3) bei einem Rentenzugang mit 55 Jahren fast die doppelte Rente ( 74,8 statt 40,9 entspricht $83 \%$,zu viel“) als es aus versicherungsmathematischer Sicht sinnvoll ist. Spalte 5 zeigt, daß die Rentenreform 1992 diese Anreize durch die Einführung des Zugangsfaktors zwar erheblich verringert hat, diese gleichwohl weiterhin bestehen. Bezüglich des häufigsten Rentenzugangsalters von 60 Jahren lag die Rente langjährig versicherter Arbeitnehmer vor 1992 um 48 Prozent zu hoch, seit der Rentenreform immer noch um 40 Prozent (29 bzw. 24 Prozentpunkte). Auch die gesetzlich fixierten Anreize für eine Verrentung nach dem Regelrentenalter zeigen sich in dieser Betrachtung als nicht ausreichend, um einen Arbeitnehmer dazu zu bringen, seine Erwerbsphase zu verlängern. Für einen Arbeitnehmer die Kosten des Weiterarbeitens in Gestalt von Beitragszahlung und Rentenverzicht höher als der Gewinn in Form einer höheren Rente.

Somit ist aus ökonomischer Sicht von einem rationalen Arbeitnehmer zu erwarten, daß er einen möglichst frühen Renteneintritt anstrebt ${ }^{84}$. „Möglichst früh“ heißt in diesem Zusammenhang, daß die erste Möglichkeit ergriffen werden sollte, die sich auf Basis des Rentenversicherungssystems und des individuellen Gesundheitszustandes ergibt.

${ }^{84}$ Diese Betrachtung vereinfacht das Entscheidungskalkül des Arbeitnehmers. Weder wird die Frage gestellt, ob die erreichbare Rente für eine Lebensstandardsicherung ausreicht, noch werden Nutzenüberlegungen einbezogen. Beide Aspekte verändern jedoch die Grundaussage nicht. Sobald ein Reservations-Rentenniveau bzw. ein Reservationsnutzen erreicht sind, wird der Arbeitnehmer danach streben, sich so schnell wie möglich zu verrenten. 
Als Fazit werden im folgenden Gliederungspunkt die Kernpunkte der GRV-Historie des Kapitels 3.3 zusammengefaßt und daraus Arbeitshypothesen für eine Untersuchung des Rentenzugangsverhaltens im Zeitverlauf abgeleitet.

\subsection{Zusammenfassung der GRV-Änderungen und Entwicklung von Ar- beitshypothesen für die empirische Untersuchung}

Aus den in Kapitel 3.3 beschriebenen Regeländerungen und der hier entwickelten Annahme rationalen Verhaltens, daß Arbeitnehmer ein möglichst frühes Ruhestandsalter anstreben, lassen sich Hypothesen über die Wirkungen der institutionellen Änderungen von Renteneintrittsmöglichkeiten auf das Zugangsverhalten einzelner Altersgruppen ableiten.

Dies erfolgt in Tabelle 3-9, in der die erwarteten Wirkungen aller Gesetzesänderungen von 1972 bis 1991 auf die Wahl des Rentenalters zusammengefaßt sind. Die dritte Spalte dieser Tabelle stellt die erwarteten Wirkungsrichtungen der beschriebenen Gesetzesmaßnahmen auf die einzelnen Zugangsalter dar.

Die Notation sei am Beispiel der Rentenreform von 1972 erläutert. Durch die Einführung des flexiblen Ruhestandes ab dem Alter von 63 Jahren ist ein erhöhtes Ausscheiden aus dem Erwerbsleben im Alter von 63 und 64 zu erwarten. Dies wird durch die Notation 63,64 (+) angezeigt. Gleichzeitig sollte der Rentenzugang mit 65 Jahren seltener stattfinden, was durch 65 (-) dargestellt wird. Ein indirekter Effekt dürfte sich auf die Zugangsalter unter 63 Jahren ergeben, da es Menschen mit (leicht) eingeschränkter Erwerbsfähigkeit, die vor der Reform eine Invaliditätsrente (EU / BU) angestrebt hätten, nun möglich ist, mit Hinblick auf die kürzere Phase bis zu einem offiziellen Rentenalter 63, bis zu diesem weiterzuarbeiten. Dieser Effekt tritt vor allem bei berufsunfähigen Personen auf, die bei einer BU-Rente mit einer $33 \%$ niedrigeren Pension als der auskommen müßten, die sie bei einem Rentenzugang mit 63 Jahren erreichen können. Diese Hypothese wird als $<63$ (-) notiert. Die folgenden Hypothesen sind analog dargestellt und ergeben sich direkt aus den genannten Regeländerungen.

Aus der Analyse des Kapitels 3.3 wurde deutlich, daß sich die Rentenzugangsmöglichkeiten im Zeitablauf verändert haben. Eine empirische Überprüfung der hier aufgestellten These vom „Rationalen Arbeitnehmer" kann somit dadurch erfolgen, daß die Rentenzugänge in die unterschiedlichen Leistungsarten im Zeitablauf daraufhin untersucht werden, ob die Zugangszahlen auf die Änderungen der institutionellen Rahmenbedin- 
gungen reagieren und zum jeweils frühestmöglichen Zeitpunkt in den Ruhestand treten. Diese Analyse ist Thema des folgenden vierten Kapitels.

Tabelle 3-9: Relevante Regeländerungen der letzten 25 Jahre

\begin{tabular}{|c|c|c|}
\hline Zeitraum: & Regeländerungen & relevante Zugangsalter \\
\hline seit 1973 & $\begin{array}{l}\text { - Einfürürung der flexiblen Altersgrenze ab } 63 \\
\text { - Gewährung von Altersrenten an Schwerbe- } \\
\text { hinderte ab dem } 62 \text { Lebensjahr } \\
\text { - Einführung der Rente nach Mindestein- } \\
\text { kommen }\end{array}$ & $\begin{array}{l}<63(-) ; 63,64(+) ; 65(-) \\
62(+) \\
\text { Tendenz: jüngere Verren- } \\
\text { tung }\end{array}$ \\
\hline 1979 & $\begin{array}{l}\text { Herabsetzung der flexiblen Altersgrenze für } \\
\text { Schwerbehinderte auf das } 61 \text {. Lebensjahr }\end{array}$ & $61(+)$ \\
\hline seit 1980 & $\begin{array}{l}\text { Herabsetzung der flexiblen Altersgrenze für } \\
\text { Schwerbehinderte auf das } 60 \text {. Lebensjahr }\end{array}$ & $60(+)$ \\
\hline seit 1984 & $\begin{array}{l}\text { (Haushaltsbegleitgesetz 1984) } \\
\text { - Herabsetzung der Mindestversicherungszeit } \\
\text { für Altersruhegelder ab } 65 \text { von } 15 \text { auf } 5 \text { Jah- } \\
\text { re } \\
\text { - Erschwerte Anspruchsvoraussetzungen für } \\
\text { EU/BU Renten }\end{array}$ & $\begin{array}{l}65(+) \\
\Rightarrow \text { negative Wirkung auf angren- } \\
\quad \text { zende Alter } \\
60(-)\end{array}$ \\
\hline $\begin{array}{l}1984-1988 \\
1986- \\
1990\end{array}$ & $\begin{array}{l}\text { Vorruhestandsregelung } \\
\text { (Arbeitsmarktorientiert) } \\
\text { Wirkung durch reguläre Verrentung }\end{array}$ & $60(+)$ \\
\hline
\end{tabular}


Gebraucht der Zeit,

Sie geht so schnell von hinnen.

\section{Entwicklung der Westdeutschen RentenzugÄnge 1970 BIS 1991}

In diesem Kapitel werden die Zugänge zur Gesetzlichen Rentenversicherung (GRV) der Jahre 1970 bis 1991 im Hinblick auf die offenen Fragen des zweiten und dritten Kapitels untersucht.

In Kapitel 2 wurde aus theoretischen Überlegungen heraus die Frage formuliert:

Sind die gesetzlichen Rahmenbestimmungen tatsächlich in der Lage, das Verhalten älterer Arbeitnehmer bei der Wahl ihres Rentenalters so maßgeblich zu verändern, daß eine Entlastung des Gesamtsystems spürbar wird?

Die institutionelle Betrachtung des dritten Kapitels führte zu der

"Hypothese der rationalen Arbeitnehmer",

die aus finanziellen Erwägungen der intertemporalen Einkommensmaximierung unter den jetzigen GRV-Regeln einen möglichst frühen Rentenzeitpunkt anstreben sollten.

Beide Hypothesen gilt es empirisch zu überprüfen.

Die altersspezifische Betrachtung zeigt auf, daß sich das Verhalten der Neurentner sowohl allgemein als auch bei Betrachtung von Einzelaltern erheblich gewandelt hat. In der Literatur finden sich hierfür zwei unterschiedliche Erklärungsmuster. Das eine argumentiert angebotsorientiert, daß die Arbeitnehmer größer werdende Spielräume der GRV zu früherer Verrentung genutzt haben, während die Gegenposition von reinen Nachfrageeffekten ausgeht, die aus hoher Arbeitslosigkeit und daraus resultierender Frühverrrentung bestehen. Diese Diskussion wird in Kapitel 4.1 geführt.

Durch die empirische Analyse wird gezeigt, daß institutionelle Vorgaben des Rentenversicherungssystems in der Tat einen deutlichen Einfluß auf das Verhalten der Arbeitnehmer haben, wogegen die Arbeitsmarktsituation keinen eindeutigen Erklärungsanteil hat. Auf Basis der in Kapitel drei dargestellten institutionellen Rahmenbedingungen der Ruhestandsentscheidung und deren Entwicklung im Zeitverlauf wird in diesem vierten Kapitel die Änderung der Bevölkerungsstruktur und der Rentenzugänge im Betrachtungszeitraum analysiert. Datengrundlage sind die Anzahl der als Neurent- 
ner in die GRV eintretenden Männer nach Einzelalter. Diese Rentenzugänge werden in einem weiteren Schritt um die altersspezifische Entwicklung der Erwerbsbevölkerung bereinigt und auf dieses Weise als zentrale Untersuchungsgröße altersspezifische Verrentungsraten. Diese empirischen Rentenzugangswahrscheinlichkeiten sind für jedes Alter $t$ definiert als Anteilswert „Anzahl Rentenzugänge in t / Anzahl Erwerbspersonen in t". Sie ist der Betrachtung absoluter Zugangszahlen in die GRV vorzuziehen, da die Kohortengrößen der einzelnen Altersgruppen in den betrachteten Jahren stark schwanken. Sie mißt den Anteil derer, die in einem bestimmten Alter in Rente gehen, bezogen auf die Gesamtstärke dieser Altersklasse. Die Analyse des vierten Kapitels bezieht sich ausschließlich Männer in den alten Bundesländern.

Im dritten Abschnitt (4.3) wird der Einfluß der gesetzlichen Anreize in einer Regressionsanalyse quantifiziert. Als Datenquelle für diese Untersuchung dienen vor allem die Veröffentlichungen des Verbandes Deutscher Rentenversicherungsträger VDR ${ }^{\perp}$. Dieser publiziert seit den 50er Jahren die Zugänge zur Gesetzlichen Rentenversicherung GRV, differenziert nach den ausgewählten Personengruppen (Rentenversicherungsträgern): Arbeiter (Landesversicherungsanstalten LVA), Angestellte (Bundesversicherungsanstalt BfA) sowie den in der knappschaftlichen Rentenversicherung organisierten Bergleuten. Bereits grobe Betrachtungen der Zeitreihen zeigen die Notwendigkeit einer Trennung der Arbeiter und der Angestellten. ${ }^{2}$

Die Veränderung der Rentenzugänge über die Zeit ist bereits in einigen Arbeiten untersucht worden, v.a. der VDR veröffentlicht regelmäßig differenzierte Analysen über die Struktur des Rentenbestandes und auch der Neuzugänge in die GRV.

Tabelle A-5 im Anhang zeigt für einige ausgewählte wissenschaftliche Arbeiten die untersuchten Personen- und Altersgruppen sowie Untersuchungsjahre. Allen Studien ist gemeinsam, daß jeweils nur eine Unterauswahl aus diesen drei Kriterien betrachtet wird, in keiner Arbeit wird eine lange Zeitreihe mit einer differenzierten Analyse unterschiedlicher Altersjahre und Geburtskohorten betrachtet. MÖRSCHEL und REHFELD (1981) untersuchen zwar eine Zeitreihe von 17 Jahren, REHFELD (1991) sowie JACOBS, KOHLI und REIN (1991) sogar von bis zu 30 Jahren, aber diese Betrachtungen haben nur Altersgruppen zum Gegenstand. Aus der näheren Betrachtung der gesetzlichen

1 Der VDR ist der privatrechtliche Zusammenschluß aller Rentenversicherungsträger und nimmt deren administrative und statistische Aufgaben zentral wahr.

2 Der Anteil der knappschaftlichen Rentenversicherung ist verschwindend, so daß diese nicht betrachtet werden. 
Rahmenbedingungen des vorigen Kapitels wurde jedoch klar, daß diese nicht ausreichen können, um die in der Regel auf Einzelalter wirkenden Gesetzesänderungen zu messen. Viele Studien betrachten nur wenige oder einzelne Jahre und können somit keine Aussage über Gesetzesänderungen und deren Wirkung treffen. Die ausführlichste Untersuchung ist die von REIMANN (1985), der eine Zeitreihe von 22 Jahren bearbeitet und dabei in einer deskriptiven Analyse einzelne Kohorten und Zugangsalter unterscheidet.

Die zitierte Literatur legt nahe, die Struktur der Rentenzugänge im Zeitablauf gründlich nach den beiden Kriterien institutionelle Rahmenbedingungen und Kohortenstruktur zu untersuchen. Nur durch die Verbindung dieser beiden Effekte können gesicherte Rückschlüsse auf die Wirkung von Gesetzesänderungen gezogen werden. Gleichzeitig sollte eine möglichst lange Zeitreihe betrachtet werden, welche die Zeitpunkte der relevanten Rentenreformen beinhaltet, so daß das Verhalten vor und nach gesetzlichen Änderungen verglichen werden kann.

\subsection{Arbeitsmarkt und Verrentungsentscheidung}

Die Entscheidung über eine Verrentung und deren Zeitpunkt ist aus Sicht des Arbeitsmarktes der Entschluß, aus dem Erwerbsleben auszutreten. Bereits im Kapitel 2.1 wurde diese „Arbeit-Freizeit-Abwägung“ beschrieben. Als mögliche Motivationen eines älteren Arbeitnehmers für diese Entscheidung wurden genannt:

- Rentenentscheidung als Arbeitsangebotsentscheidung des Arbeitnehmers;

- Gesundheitsaspekte: körperliche Fähigkeiten, Behinderungen, Berufs- oder Erwerbsunfähigkeiten;

- direkte Nachfrageeffekte des Arbeitsmarktes: fehlende Arbeitsplätze für ältere Arbeitnehmer, Entlassungen, ('unfreiwillige') Arbeitslosigkeit;

- indirekte Nachfrageeffekte durch (finanzielle) Anreize zur Frühverrentung, Betriebsrentenprofile, Sozialpläne;

- institutionelle Rahmenbedingungen in Form von Mindest- / Höchstrentenaltern, altersabhängige Rentenhöhen.

Zusammengefaßt können drei Elemente der Ruhestandsentscheidung unterschieden werden, die persönliche Entscheidung auf Basis einer Nutzenmaximierung unter den Restriktionen des intertemporalen Budgetausgleichs und den persönlichen Fähigkeiten auf der einen Seite, sowie die äußeren Bedingungen des Arbeitsmarktes und des Gesetzgebers auf der andern. 
Arbeitsmarkt
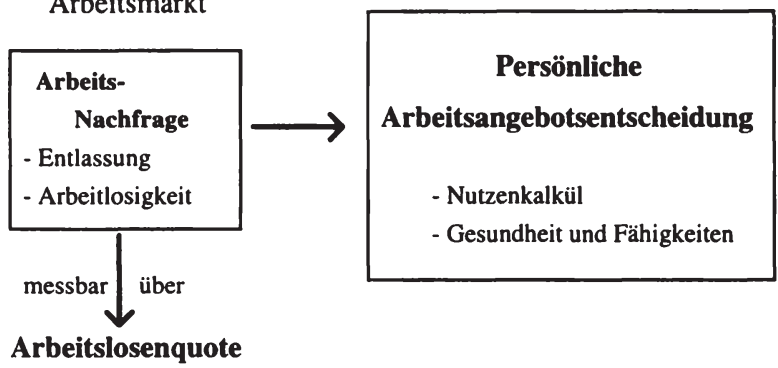

Gesetzliche

Regelungen

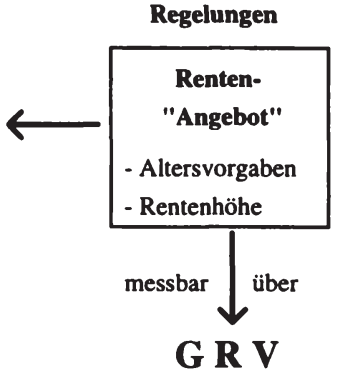

Die Diskussion, die in der Literatur über die Determinanten der Ruhestandsentscheidung geführt wird, ist letztlich die, in welchem Verhältnis diese Faktoren zueinander stehen, welches Element die anderen dominiert.

Einen Versuch der Operationalisierung dieser „Nachfrage-“" versus „Angebots“-Effekte nehmen etwa KOHLI und REIN (1991) vor, indem sie zwischen „Austritt aus dem Arbeitsmarkt" und „Frühverrentung“ unterscheiden. ${ }^{3}$ Die Autoren trennen damit sowohl die „Akteure“ in Arbeitnehmer, Unternehmer und Staat sowie die „Schauplätze“ in den Arbeitsmarkt und die Sozialpolitik. Diese Unterscheidung zeigt die wichtige Interaktion der Ökonomie mit der Politik auf, die bereits im zweiten Kapitel angeklungen ist. Keine Problemlösung kann nur auf einem der beiden Bereichen beruhen. Weder ist ein strikt ökonomischer Weg (zum Budgetausgleich) politisch durchsetzbar, noch ist eine ausschließlich umverteilungspolitisch orientierte Strategie finanzierbar. Daß sich diese Erkenntnis bereits bis in die konkrete Politik durchgesetzt hat, zeigt die Tatsache, daß die Rentenreform 92 als Gesamtkonsens aller großen Fraktionen des Bundestages beschlossen wurde.

In einer umfangreichen international vergleichenden Studie zeigen KOHLI et al. die „Wege in den Ruhestand“, der ihrer Auffassung nach nicht mit dem Austritt aus dem Arbeitsmarkt zeitgleich beginnen muß, für die verschiedenen Länder auf. ${ }^{4}$ Für die Bundesrepublik interpretieren sie die gesetzlich geschaffenen Möglichkeiten eines früheren „Austrittes aus dem Arbeitsmarkt“ als Reaktion auf die gespannte Situation auf

${ }^{3}$ KOHLI und REIN (1991), S. 5-7. Dies ist der Einleitungsartikel zu dem Buch „Time for Retirement“ von KOHLI, REIN, GuILlEMARD und VAN GUNSTEREN (Hrsg.), wobei die Terminologie sich durch alle Beiträge dieser international vergleichenden Arbeit zieht und letzlich deren Kernaussage ist .

${ }^{4}$ Mit den Autoren sind hier sowohl die Herausgeber KoHLI, REIN, GUILLEMARD UND VAN GUNSTEREN, als auch die Autoren der Einzelbeiträge gemeint. 
diesem Arbeitsmarkt, also als Politik gegen Arbeitslosigkeit. ${ }^{5}$ Damit wird eine weitere Dimension der genannten Unterscheidung zwischen „Austritt“ und „Verrentung“ eingeführt, das der Freiwilligkeit. Ein Austritt aus dem Beruf wird interpretiert als ein unfreiwilliger Akt, z.B. durch Arbeitslosigkeit, vornehmlich aber durch den Druck auf die Arbeitnehmer seitens der Firmen. Die These, ob die Gesetzesänderungen als Reaktion auf die Situation am Arbeitsmarkt geschaffen wurden, sei anhand der langfristigen Entwicklung von Arbeitslosigkeit und Rentenalter in Abbildung 4-1 betrachtet.

Abbildung 4-1: Arbeitslosigkeit und Rentenalter Westdeutschlands in der langtristigen Entwicklung

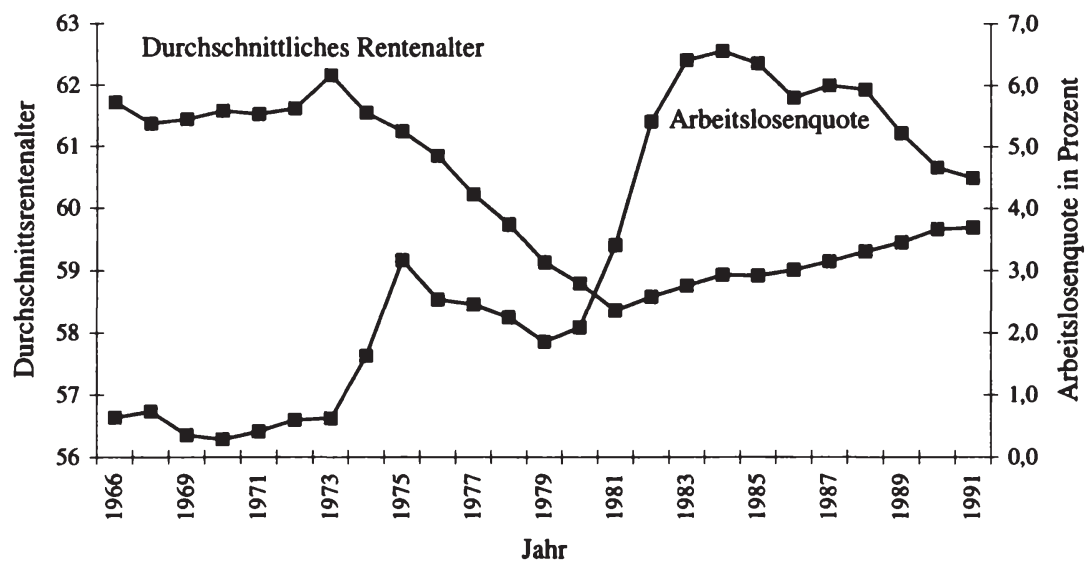

Quelle: Rentenzugangsstatistik des VDR, Arbeitslosenstatistik der Bundesanstalt für Arbeit, jeweils diverse Jahrgånge, eigene Berechnung.

Eine Unterstützung würde diese Annahme dadurch erfahren, daß in Zeiten hoher Arbeitslosigkeit das durchschnittliche Rentenalter sinkt, weil die betroffenen Arbeitnehmer zum Austritt aus dem Arbeitsmarkt gezwungen werden. Dies ist aus der Abbildung nicht zu erkennen. Die Rentenreform wurde zu Beginn der 70er Jahre entworfen und paraphiert, einer Phase in der eine Arbeitslosenquote unter einem Prozent lag. Das kurzzeitige Ansteigen des Rentenalters resultiert, wie später gezeigt wird, daraus, daß eine hohe Anzahl von Personen mit 63 und 64 Jahren in den Ruhestand tritt und damit die 1973 neu geschaffene Möglichkeit des flexiblen Ruhestandes ab 63 Jahren nutzt. Nach 1973 sinkt das Rentenalter kontinuierlich bis 1981. Die Jahre 1974 und 1975

5 JACOBS, KOHLI und REIN (1991) „Germany: The diversity of Pathways“. 
zeichnen sich tatsächlich durch einen sprunghaften Anstieg der Arbeitslosenquote aus (1. Ölpreisschock), allerdings sinkt diese nach 1975 wieder ab. Das gleichzeitige Sinken der Rentenalters widerspricht der aufgestellten These ebenso wie die Tatsache, da $B$ in der Zeit sehr hoher Arbeitslosigkeit ab 1982 das Rentenalter wieder steigt. Auch hier gilt, daß die erleichterten Renteneintrittsmöglichkeiten für 61- bzw. 60-jährige der Jahre 79 und 80 in einer Zeit des sich entspannenden Arbeitsmarktes beschlossen wurden.

Als Resümee dieses Abschnittes sei festgehalten, daß die „Nachfrage“-Wirkungen des Arbeitsmarktes auf den ersten Blick nicht ursächlich, im Sinne einer zeitlichen Predeterminiertheit, für das Verhalten der Rentenzugänger erscheinen. Allerdings sollte die Struktur der Rentenzugänge nicht ausschließlich am Durchschnittsrentenalter gemessen werden, sondern einer genaueren Betrachtung unterzogen werden.

\subsection{Die Entwicklung der Rentenzugänge in Deutschland seit 1968}

Auf Basis der oben dargestellten institutionellen Rahmenbedingungen wird in diesem Kapitel die Entwicklung der Rentenzugänge von 1968 bis 1991 genau betrachtet. Hierbei sind die absoluten Zugangszahlen kein adäquates $\mathrm{Ma} B$, da sie nicht nur das Rentenverhalten, sondern auch die Bevölkerungsstruktur widerspiegeln. Durch eine Bereinigung der absoluten Zahlen um diese Struktur werden in Abschnitt 4.2.3 Verrentungsraten gebildet, die für jede Altersgruppe eine kohortenbereinigte Maßzahl des Rentenzugangsverhaltens darstellen. Mit dieser Kenngröße ist eine genaue Beurteilung der zeitlichen Variation von Ruhestandsentscheidungen möglich. Zu diesem Zweck werden zunächst im Abschnitt 4.2.1 die absoluten Rentenzugänge zur GRV deskriptiv dargestellt und in Teil 4.2.2 die zur Kohortenbereinigung notwendigen Basiszahlen der Erwerbspersonen nach Einzelalter ermittelt. Datenbasis für dieses Kapitel ist die vom VDR herausgegebene Rentenzugangsstatistik, aus der die neu in die GRV eintretenden Männer, getrennt nach Arbeitern und Angestellten betrachtet werden.

\subsubsection{Absolute Zugangszahlen zur GRV}

Der bereits in Kapitel 2.2 dargestellte strukturelle Wandel der Rentenzugangsalter (vgl. auch Abbildungen 2-6 und 2-7) wird bei genauer Betrachtung der Prozentanteile relevanter Einzelalter im Zeitablauf noch deutlicher. Dies geschieht in Abbildung 4-2, in der die Zugangsalter 60, 63, 64 und 65 dargestellt sind. 


\section{Abbildung 4-2: Anteil einzelner Renteneintrittsalter an den Gesamtzugängen eines Jahres}

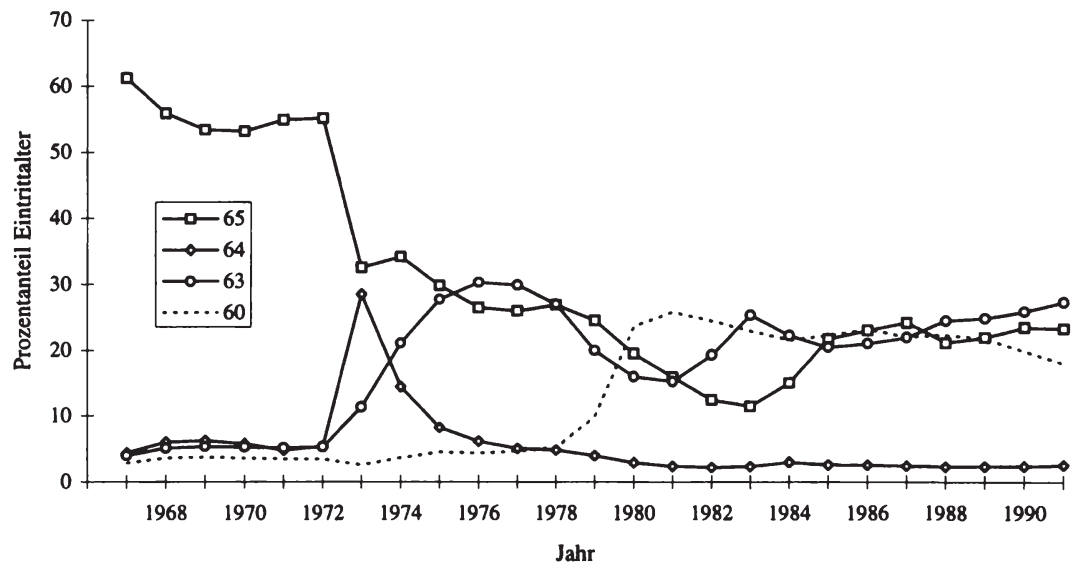

Quelle: VDR Rentenzugangsstatistik, eigene Berechnungen

Bis auf das Alter 64 sind dies die Eckzahlen, die sich aus der institutionellen Betrachtung des Kapitel 3 ergeben haben. Es zeigt sich in diesem Schaubild deutlich, daß die Änderungen des gesetzlichen Rahmens klare Auswirkungen auf das Verhalten der Neurentner hatten:

- Während bis 1972 der Anteil der im Regelrentenalter 65 in den Ruhestand tretenden über $50 \%$ lag, fällt er nach der 72er Reform drastisch ab und wird durch andere Alter in seiner Bedeutung ersetzt. Erst durch das Haushaltsbegleitgesetz von 1984 steigt die Bedeutung des Regelrentenalters wieder an, indem der Zugang in diesem Alter auch Personen mit wenigen Versicherungsjahren ermöglicht wurde;

- Auch das Rentenalter 63 zeigt genau den auf Basis der Gesetzesänderungen zu erwartenden Verlauf: Während es bis 1972 keine Bedeutung hatte, steigt sie nach diesem Termin sprunghaft an. Interessant sind hierbei zwei Phänomene: Das ansonsten unbedeutende Verrentungsalter 64 hat 1973 eine Anteil von fast 30\%, ein reiner Übergangseffekt, da Arbeitnehmer die 1972 noch nicht mit 63 gehen konnten, 1973 mit 64 in das 'Fenster' fallen; dieser Effekt dämpft den Anstieg des 63er Prozentanteiles leicht. Ein zweiter Übergangseffekt zeigt sich in den Jahren 1979/80 bis 1982. Es sind dies die Jahre, in denen der Rentenzugang im Alter 61 / 60 durch die EU / BU Renten erleichtert wurde und in denen also Personen, die unter diese Regelungen fallen, nicht mehr das Verrrentungsfenster in Anspruch nehmen müssen. Interessanterweise pendeln sich alle drei betrachteten Alter auf ähnlichem Niveau ein. 
- Der Verlauf des Zugangsalters 60 ist nun bereits mehrfach angesprochen worden. Der Einfluß dieses Alters ist bis 1978 unbedeutend und steigt dann sprunghaft auf einen Prozentsatz um 30.

Abbildung 4-3: Anteil einzelner Renteneintrittsalter 1966 - 1991 (Angestellte)

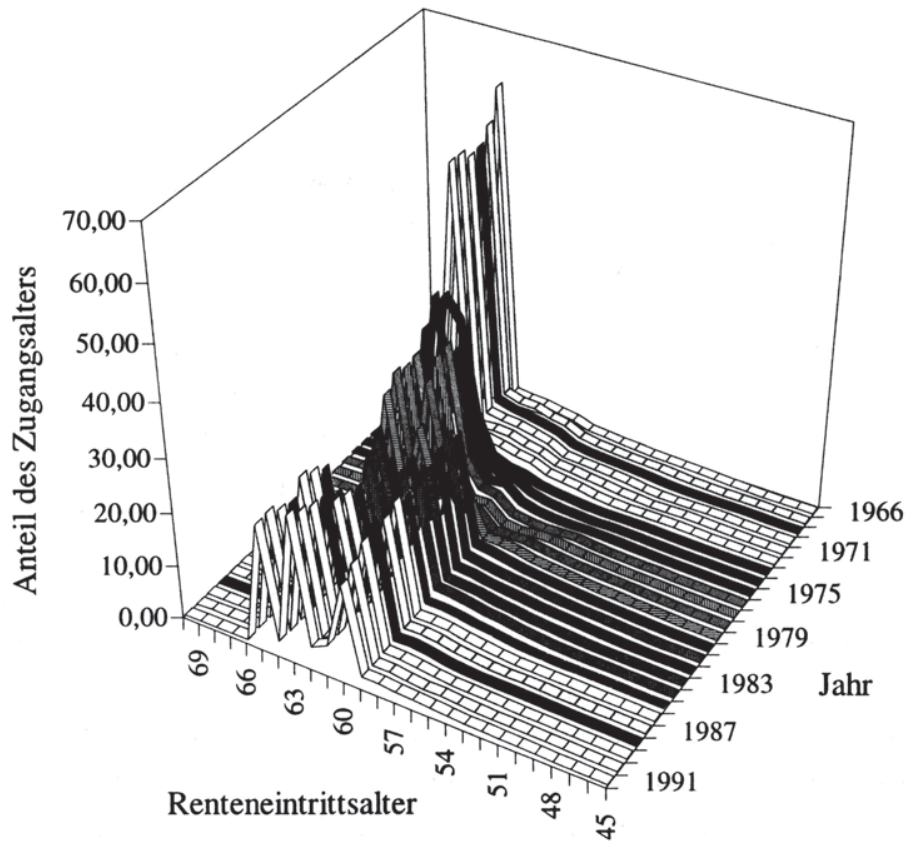

Quelle: VDR Rentenzugangsstatistik, eigene Berechnungen

Abbildung 4-3 zeigt diese eindrucksvolle Übereinstimmung mit dem institutionell zu erwartenden Verhalten in der intertemporalen Entwicklung aller Alter und Jahre. Es ist deutlich zu erkennen, daß aus dem einen Rentenalter 65 ein sich erweiterndes „Fenster“ möglicher Alter wird, das zuletzt von 60 bis 65 reicht.

Im steigenden Anteil des Zugangsalters 65 liegt eine mögliche Erklärung des in den 80er Jahren wieder steigenden Rentenalters (Abbildung 2-6 in Kapitel 2.2). Allerdings kann dies nur ein Teileffekt sein, da das Durchschnittsalter bereits seit 1981 steigt, wogegen der Anteil der 65-jährigen erst ab 1985 ein höheres Niveau erreicht. 
Abbildung 4-4: Anteil einzelner Renteneintrittsalter und der unter 60 jährigen an den Rentenzugăngen

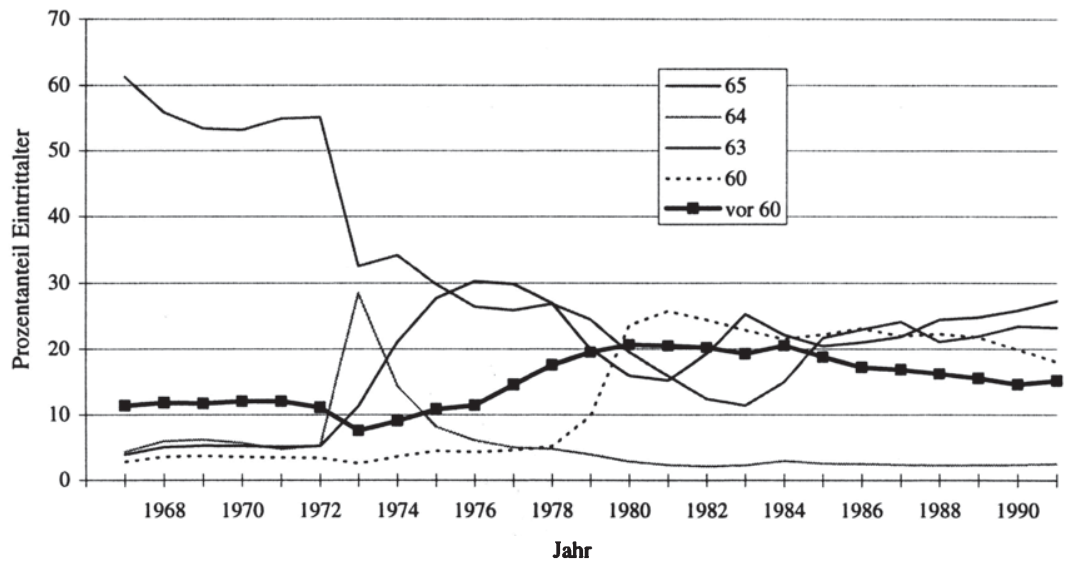

Quelle: VDR Rentenzugangsstatistik, eigene Berechnungen

Eine interessante Erweiterung der Abbildung 4-2 ist die in Abbildung 4-4 eingefügte Reihe der Personen, die vor dem 60. Lebensjahr in den Ruhestand treten, alle anderen Angaben entsprechen Abbildung 4-2. Aus dieser Darstellung ist zu erkennen, daß das vorher konstante Niveau durch die Rentenreform 72 gesenkt wird. Dies entspricht allerdings nicht einer zurückgehenden Zahl, sondern der gesunkene Anteil resultiert aus einer hohen, teilweise durch die Reform selbst induzierten Zahl der Personen, die mit 63 und 64 in den Ruhestand treten. Namentlich scheinen vor allem die 64-jährigen diesen Effekt hervorzurufen, die in den beiden Jahren 1973 und 74 einen künstlich hohen Anteil haben, da sie früher in Renten gehen, als es nach dem alten Recht möglich gewesen wäre.

Eine Erweiterung der einfachen Untersuchung von Rentenaltern bietet der Blick auf die Leistungsarten in Abbildung 4-5. Auch in dieser Darstellung wird die Ausrichtung der gewählten Leistungsart an den jeweilig gegebenen institutionellen Möglichkeiten deutlich ${ }^{6}$ :

- Die Regelaltersrente 65:

macht bis zur Rentenreform 1972 ca. $50 \%$ aller Rentenzugänge aus, ihr Anteil sinkt nach dieser Reform durch die Einführung des flexiblen Ruhestandes ab 63 Jahren bis 1983 ab. Ab 1984, mit der Erleichterung des Eintritts in die Regelaltersrente durch das Haushaltsbegleitgesetz 1984, steigt der Anteil wieder an.

6 Vergleiche Tabelle 3-9 mit einer Zusammenfassung der Rentenrechtsänderungen und Hypothesen über die relativen Anteile der Zugangsalter. 


\section{- Berufsunfähigkeitsrente $B U:^{7}$}

Spielt insgesamt eine untergeordnete Rolle. Schon vor der 72er Reform werden zunehmend EU statt BURenten beantragt (BU Renten sind 33\% niedriger).

- Allgemeine Erwerbsunfähigkeitsrente EU: ${ }^{7}$

Der Anteil der EU-Renten fällt durch die Rentenreform 1972 ab, steigt dann aber bis zum Beginn der 80er Jahre wieder an. Nach der Einführung des „Flexiblen Ruhegeldes für Schwerbehinderte, Berufs- und Erwerbsunfähige mit 60 Jahren“ im Jahre 1980 fällt die Bedeutung der EU-Rente.

- Vorzeitiges Ruhegeld für Langzeitarbeitslose:

Als einzige Leistungsart reagiert diese seit 1957 kontinuierlich bestehende Rente nicht auf die betrachteten Gesetzesänderungen.

Interessanterweise reagiert ihr Anteil ebensowenig auf die (in Abbildung 4-1 gezeigte) Arbeitslosenquote. Weder deren Anstieg 74/75, noch deren Abfall 76 bis 79 werden vom Anteil der Arbeitslosigkeitsrenten reflektiert, ebensowenig wie der starke Anstieg und anschließende Rückgang der Arbeitslosenzahlen in den 80er Jahren.

- Flexibles Ruhegeld für Schwerbehinderte, Berufs- und Erwerbsunfähige:

Diese Rente konnte 1972-1978 mit 62, 1979 mit 61 und seit 1980 mit 60 Jahren bezogen werden.

Der Anteil dieser Rentenart steigt zwar bereits nach der Einführung 1973 leicht an, springt jedoch durch die Senkungen der Zugangsalter 1979 und 1980 auf ein hohes Niveau.

- Flexibler Ruhestand ab 63 Jahren:

Nach dem „revolutionären Einschlagen“ des flexiblen Ruhestandes mit seiner Einführung 1973, sinkt dessen Anteil gegenläufig zur Steigerung der EU-Renten ab.

\section{Abbildung 4-5 Anteile der Leistungsarten an den Rentenzugängen 1966 bis 1990:}

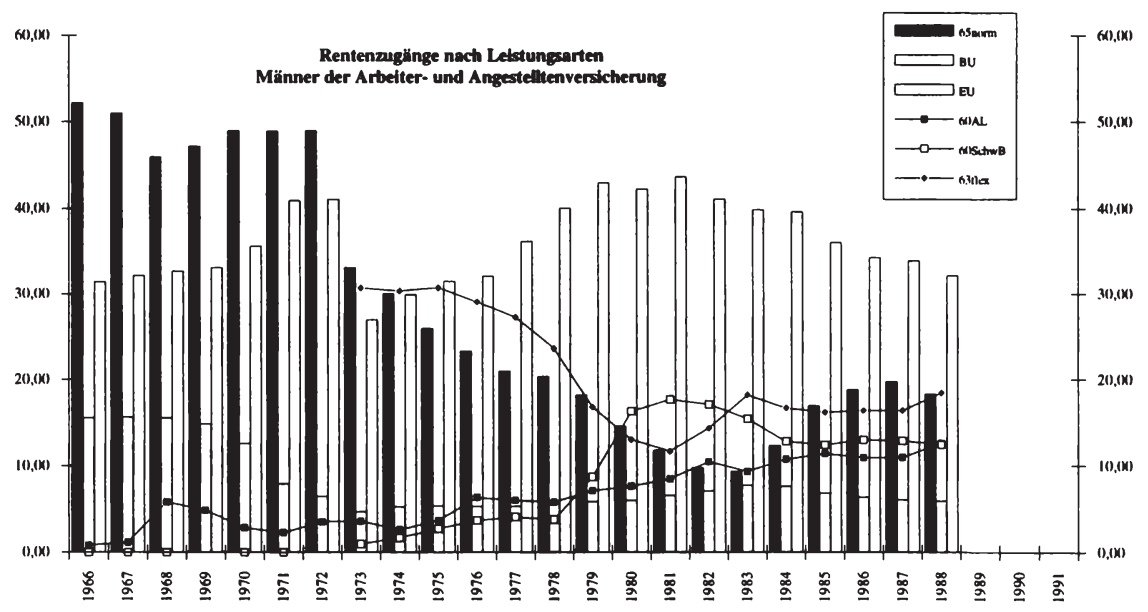

Quelle: Rentenzugangsstatistik des VDR, diverse Jahrgănge; eigene Berechnung.

${ }^{7}$ Diese EU- und BU-Renten im Rahmen der allgemeinen Invaliditätsrente unterliegen keinen Altersbeschränkungen und sind in sofern nicht zu verwechseln mit dem „flexiblen Altersruhegeld für Schwerbehinderte, Berufs- und Erwerbsunfähige“. 
Aus der Tatsache, daß das durchschnittliche Rentenalter in den 80er Jahren wieder ansteigt (Abbildung 2-6) wird allerdings deutlich, daß die Wirkungen einer einzelnen Gesetzesänderungen und die Anteilsverschiebung zwischen Leistungsarten auf das dort gezeigte durchschnittliche Rentenalter nicht immer klar zuzuordnen ist.

So kann die Liberalisierung von EU/BU-Renten im Alter von 60 Jahren auf den ersten Blick zu einer Senkung des durchschnittlichen Rentenalters führen, da diese Personen vor dem Verrentungsfenster in den Ruhestand treten können. Der gegenteilige Effekt ist aber auch denkbar, wenn durch dieselbe Maßnahme Arbeitnehmer, die vor 1979/80 schon unter 60 Jahren eine Invalditätsrente beantragt hätten, nun erst mit 60 gehen. Diese Versicherten hätten damit die Möglichkeit genutzt, statt einer Invaliditätsrenten eine (vorgezogene) Altersrente zu beziehen, die leichter zu beantragen ist.

Die Umsetzung solcher indirekten Effekte in empirisch überprüfbare Hypothesen ist in der hier durchgeführten makroökonomischen Analyse allerdings kaum möglich, da sie statistisch nicht identifiziert werden können. Hierzu ist eine mikroökonomische, d.h. auf einzelnen Individuen basierende Untersuchung notwendig.

\section{Datengrundlage}

Für die Bewertung der verwendeten Statistiken seien zwei Besonderheiten hervorgehoben:

\section{Definition von Verrentung}

Der VDR kann naturgemäß nur solche Personen als Neurentner registrieren, die als Zugänge zur Gesetzlichen Rentenversicherung ab einem bestimmten Stichtag eine Rentenzahlung erhalten. Damit werden diejenigen Personen nicht erfaßt, die zwar aus dem Erwerbsleben aus-, aber noch nicht in die Rentenversicherung eintreten. z.B. Arbeitnehmer im Vorruhestand, Arbeitslose, die nach der 59er-Regel ${ }^{8}$ 'verrenten' und andere Sozialplanregelungen. Diese Fälle sollten aus volkswirtschaftlicher Sicht als Austritte aus dem Arbeitsmarkt betrachtet werden, sind aber in den hier vorhandenen Daten nicht erfaßt. Konkret heißt dies, daß ein Arbeitnehmer, der mit 57 aufhört zu arbeiten und sich auch als Rentner fühlt, in den hier untersuchten Daten erst bei seinem offiziellen Eintritt in die Gesetzliche Rentenversicherung mit 60 Jahren als Neu-Rentner erfaßt wird. In diesem Sinne sind volkswirtschaftlich die hier gemessenen Zugangsraten in den Altern vor 60 unterschätzt.

${ }^{8}$ Bzw. 57er Regel - vgl. oben. 
Aus Sicht der Sozialversicherung ist diese Erfassung allerdings nicht nur die einzig technisch machbare, sondern auch die relevante, da es um die Einhaltung der Budgetrestriktion geht, so daß Personen, die keine Rente beziehen, auch nicht relevant sind.

Es sei festgehalten, daß hier nur die Zugänge zur Rentenversicherung und nicht die Austritte aus dem Arbeitsmarkt, im Sinne der Terminologie von KoHLI ET AL. betrachtet werden.

\section{Meßprobleme innerhalb der Rentenversicherung}

Herausgeber der Rentenzugangsstatistik ist der VDR, der seinerseits auf die Berichterstattung seiner Mitglieder, der Rentenversicherungsträger angewiesen ist. Auch diese führen die Rentenzugänge nicht selbst, sondern beziehen sich auf die geleisteten Zahlungen durch die Bundespost. Zwei mögliche Probleme der Datenbasis werden durch die VDR hervorgehoben:

- „Das Jahr des technischen Rentenzuganges muß nicht identisch mit dem Jahr des Versicherungsfalles sein ... besonders bei Frührenten “ (VDR Rentenzugangsstatistik 1988, Seite X).

- „Die Altersangaben werden grundsätzlich aus der Differenz zwischen dem Jahr des technischen Rentenbeginns und dem Geburtsjahr gewonnen. Das Alter bei Rentenbeginn ist daher nicht zwingend das Alter im Zeitpunkt des Versicherungsfalles“ (ebenda, Seite XII).

Ein relevanter Einwand bezüglich der Datenbasis ist, daß die oben betrachteten Prozentanteile basierend auf absoluten Zugangszahlen falsch gemessen sein können, wenn sie durch die jeweilige Kohortengröße verzerrt sind. So kann es Jahre geben, in denen die 65 jährigen in der Bevölkerung einen geringeren Anteil ausmachen, so daß sie natürlich in Folge dessen auch einen kleineren Anteil an den Rentenzugängen haben.

Um diese mögliche Fehlerquelle zu beseitigen, ist eine Bereinigung der jährlichen Rentenzugänge um die Basisgröße „Kohortenstärke“ notwendig, die im folgenden Abschnitt 4.2.2 dargestellt wird.

\subsubsection{Messung der Erwerbsbevölkerung}

Die deutsche Bevölkerungsstruktur ist deutlich durch demographische Einschnitte gekennzeichnet. Vor allem die beiden Weltkriege mit den starken Verlusten, insbesondere der männlichen Bevölkerung, und geringen Geburtenzahlen, aber auch die geburtenstarken Jahrgänge der 60er Jahre mit dem anschließenden „Pillenknick“ sind in 
Abbildung 4-6 zu erkennen. Diese Darstellungsart stellt die (männliche) Hälfte des als „Zwiebel“ bekannten Bevölkerungsaufbaues westlicher Gesellschaften dar. Es sind in ihr auch die Kohorten mit geringer Besetzung zu erkennen, in diesem Fall im Alter von 60-63 Jahren (Auswirkungen 1. Weltkrieg), von ca. 35 Jahren (Auswirkungen 2. Weltkrieg) und unter 10 Jahren (geburtenschwache Jahrgänge). Die erstgenannte Kohorte wird 1982 das Alter 65 erreicht haben und damit einen Einfluß auf die (relative) Anzahl der Rentenzugänge in unterschiedlichen Kalenderjahren haben.

\section{Abbildung 4-6: Bevölkerungsaufbau 1978}

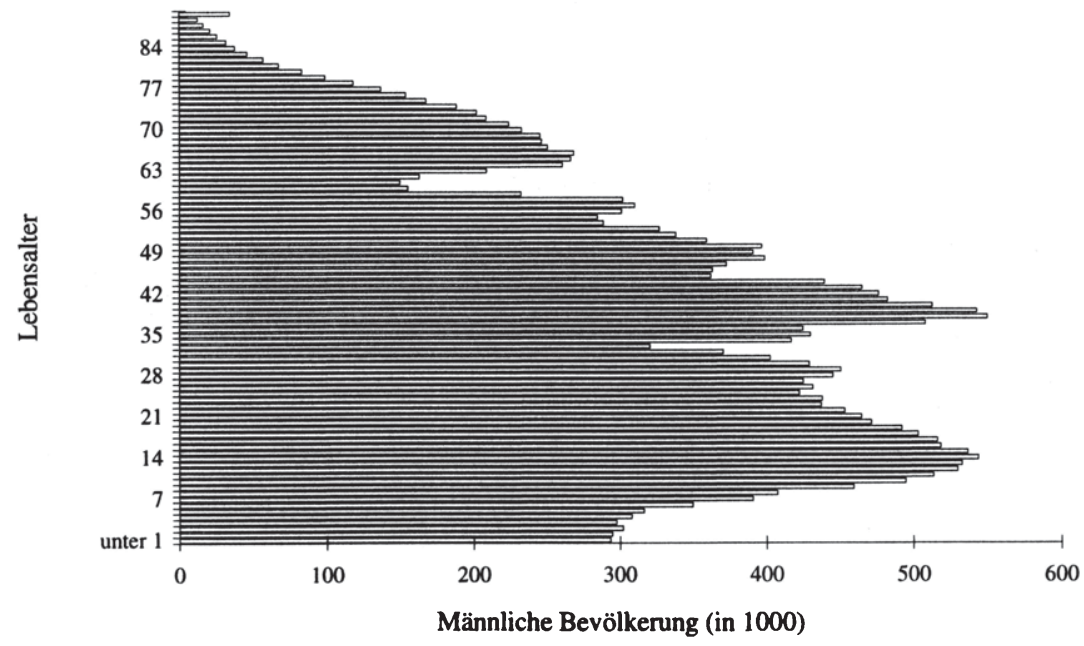

Quelle: Statistisches Jahrbuch 1979, eigene Darstellung

Diese zeitliche Struktur ist anschaulicher in der folgenden Abbildung 4-7 dargestellt, die die Wanderung der "Kohortentäler" über die Altersachse im Zeitablauf veranschaulicht.

Aus dieser Abbildung 4-7 ist auch das (wieder) steigende Rentenalter der 80er Jahre erklärbar (Vgl. Abbildung 4-1, Seite 80), da in diesem Zeitraum der Anteil der 60- und mehr jährigen deutlich ansteigt, während die Personen in der zweiten Hälfte des 5. Lebensjahrzehnts relativ weniger vertreten sind (durch die Verschiebung des „Kohortentales“ in diese Altersgruppe). Damit präsentiert sich das steigende Rentenalter in den 80er Jahren teilweise als demographischer Artefakt, welches aus der Verzerrung durch die Kohortenstruktur resultiert. Dies gilt in Verbindung mit Abbildung 4-2 (Seite 82) insbesondere für die Jahre nach 1985, in denen sich die Anteile der relevanten Zugangsalter nicht verändern, so daB die Steigerung des Durchschnittsalters nur 
aus der Kohortenstruktur resultieren kann. In den Jahren 1983 bis 85 ist hingegen eine Steigerung der Zugänge mit 65 Jahren auf das Niveau von etwa 30 Prozent beobachtbar, bedingt durch das Haushaltsstrukturgesetz von 1984. Dieser Anstieg entspricht jedoch - wie bei Abbildung 4-2 bereits verdeutlicht - nicht den Kalenderjahren des steigenden Rentenalters.

\section{Abbildung 4-7: Mãnnliche Bevölkerung 1966-1990}

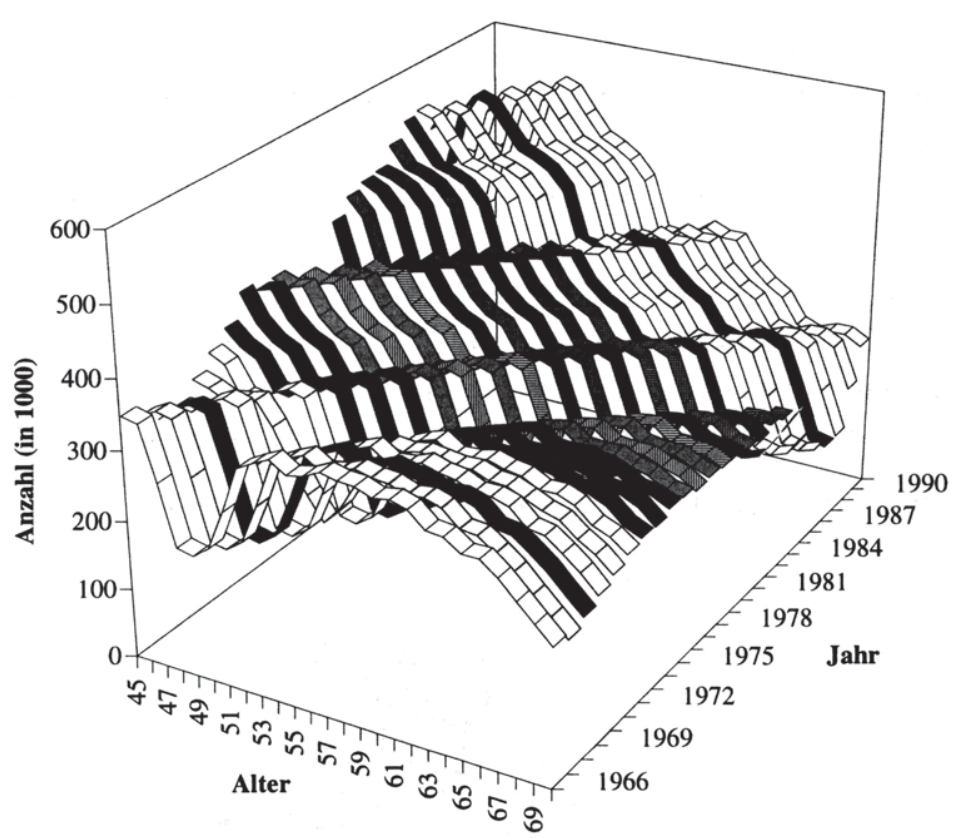

Quelle: Statistische Jahrücher, Wohnbevölkerung, Werte am Jahresende; eigene Darstellung

Allerdings ist es nicht die Wohn-, sondern die Erwerbsbevölkerung, die die Basis für einer Bereinigung der Rentenzugänge eines Jahrganges bildet. Dies gilt insbesondere, da in der vorliegenden Studie ausschließlich Männer betrachtet werden, bei denen latente Rentenanwartschaften selten vorkommen.

Für die empirische Messung dieser Bevölkerungsbasis bieten sich unterschiedliche Datenquellen an. Die korrekte Bezugsgröße wären die aktiv Versicherten in der GRV. Diese Maßzahl wird jedoch erst seit 1982 durch den VDR veröffentlicht und die Zahlen der davor liegenden Jahre liegen auch dem VDR nicht vor. Aus diesem Grunde wurden folgende mögliche Datenquellen betrachtet: 
Die Enwerbsquoten des Statistischen Bundesamtes

könnten auf die vorliegenden Bevölkerungszahlen angewendet werden und so die Anzahl der Erwerbspersonen errechnet werden. Die Quoten werden jedoch nur in 5jährigen Altersintervallen veröffentlicht, was eine ausreichend genaue Ermittlung der Erwerbspersonen in den relevanten Altersgruppen unmöglich macht.

- Die „Sozialversicherungspflichtig Beschäftigten“

werden von der Bundesanstalt für Arbeit erfaßt und bilden als Primärerhebung die zuverlässigste mögliche Datenquelle. Die Angaben liegen für Angestellte und Arbeiter getrennt nach Einzelalter bis zu 70 Jahren vor. Sie konnten in der vorliegenden Arbeit jedoch nicht verwendet werden, da diese Statistik erst seit 1976 existiert und damit die wichtige Rentenreform von 1972 aus dem Betrachtungszeitraum fallen würde.

Aus diesem Grunde basiert die Arbeit auf den Erwerbspersonen, die das Statistische Bundesamt auf Basis des jährlichen Mikrozensus veröffentlicht. Diese Datengrundlage beschränkt die beobachteten Altersjahre nach oben auf 65, eine Trennung von Arbeitern und Angestellten ist ebenfalls nicht möglich. Die Zeitreihe liegt erst ab 1970 vor.

\section{Abbildung 4-8: Männliche Enwerbspersonen nach Mikrozensus}

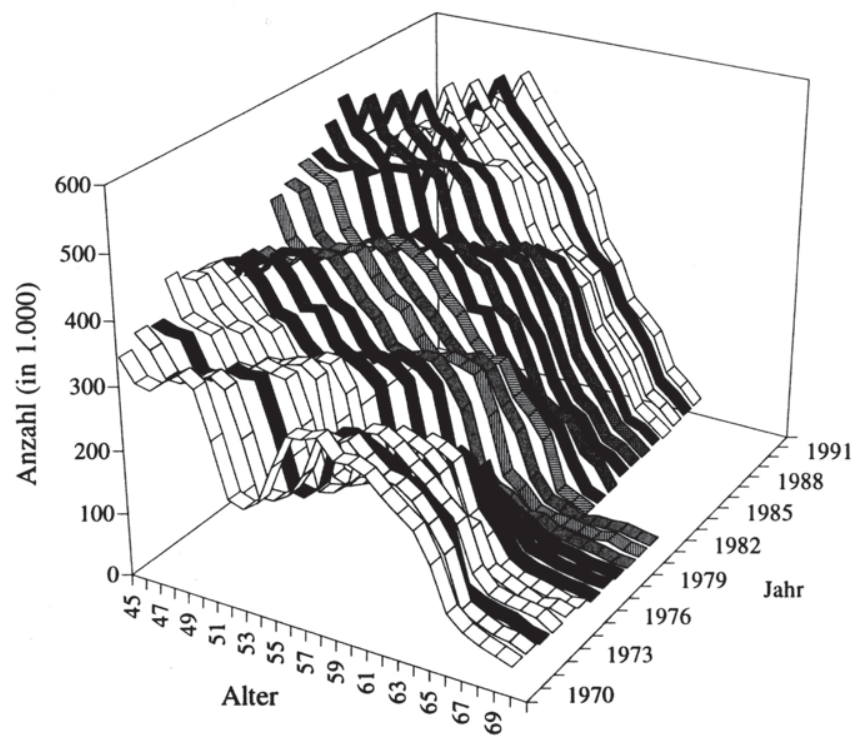

Quelle: Statistisches Bundesamt, Enwerbspersonen nach Mikrozensus; diverse Jahrgånge 
Die zeitliche Entwicklung dieser Datenbasis ist in Abbildung 4-8 dargestellt. Die Abbildung zeigt, daß sich die kohortenbedingten Einschnitte auch bei den Erwerbspersonen niederschlagen.

Abbildung 4-9 verdeutlicht dagegen, daß die altersspezifischen Erwerbsquoten (berechnet als Quotient aus Erwerbspersonen und Bevölkerung) nicht mehr die bisherige Kohortenstruktur aufweisen. Dies belegt, daß die unterschiedlichen Besetzungen der Erwerbspersonen in Altersklassen ein reiner Kohorteneffekt ist, für den es im empirischen Teil zu kontrollieren gilt. Allerdings ist in Abbildung 4-9 ebenfalls zu erkennen, daß die Erwerbsbeteiligung der 60- und mehr jährigen seit 1970 merklich zurückgegangen ist. Insbesondere die 65-jährigen wiesen 1970 und 71 noch eine Erwerbsbeteiligung von $52 \%$ auf, die $72 / 73$ auf unter $30 \%$ und 1991 auf $10 \%$ absinkt.

Abbildung 4-9: Enwerbsquoten im Jahresvergleich (1970 - 1991)

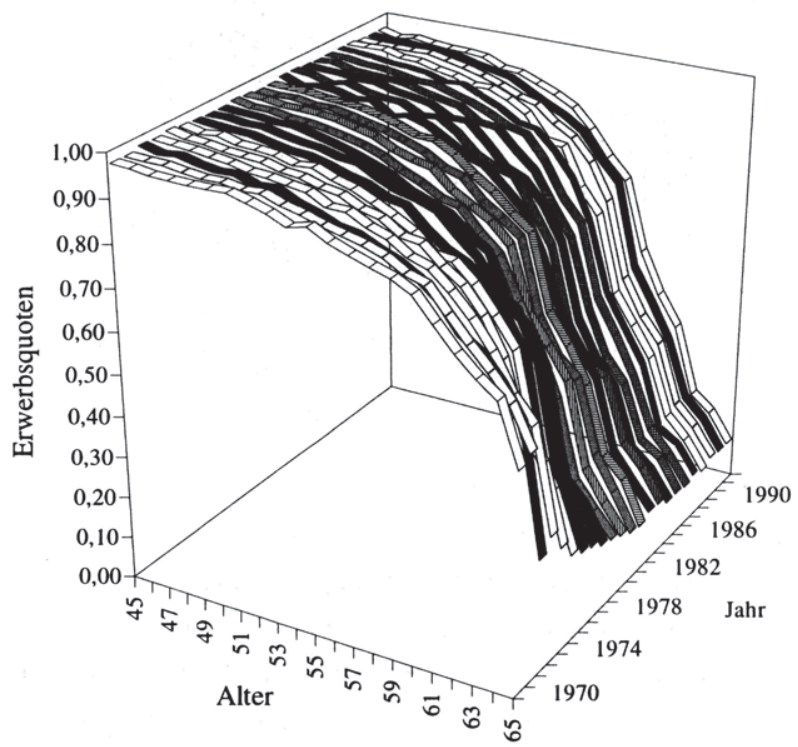

Quelle: Statistisches Bundesamt, Wohnbevölkerung und Erwerbspersonen nach Mikrozensus; diverse Jahrgänge; eigene Berechnungen

\subsubsection{Bereinigte Rentenzugänge - Verrentungsraten}

Auf Basis der absoluten Rentenzugänge und der Erwerbspersonen werden als zentrale Bezugsgröße dieser Untersuchung 


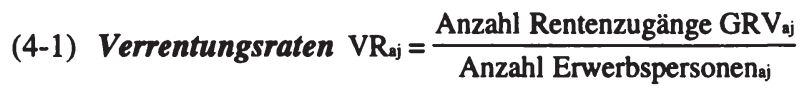
im Alter a zum Zeitpunkt j gebildet.

\section{Abbildung 4-10: Verrentungsraten Angestellte 1970-1991}

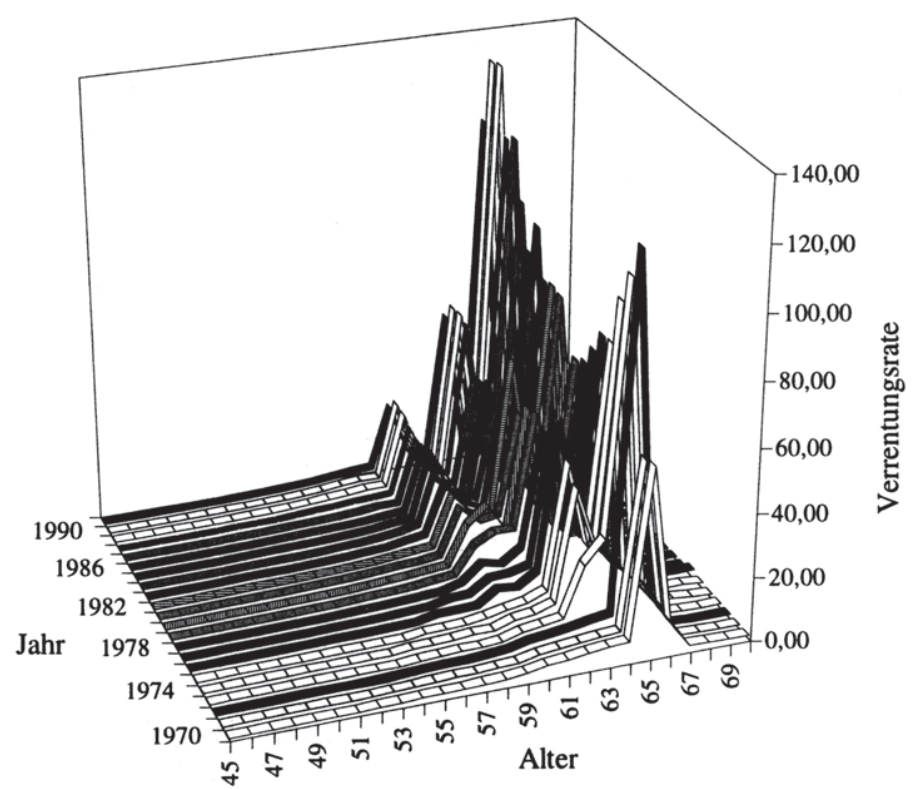

Quelle: VDR-Rentenzugănge, Enwerbspersonen nach Mikrozensus; eigene Berechnung

Theoretisch liegt dieser Quotient immer im Intervall $\{0,1\}$ und stellte eine empirische Hazardrate dar, indem die Rentenzugänge in Relation gestellt würden zu den noch ,at risk" " befindlichen Erwerbspersonen der jeweiligen Altersgruppe. Da die Erwerbspersonen als Bezugsgröße jedoch nicht aus der selben Grundgesamtheit stammen, sondern durch eine - teilweise fortgeschriebene - Stichprobenbefragung ermittelt wurden, ergeben sich hier z.T. Verrentungsraten über 1,0, d.h. es gehen mehr Personen eines Alters in Rente, als in dieser Gruppe Erwerbstätige gezählt wurden. Dieses prinzipiell unmögliche Phänomen ergibt sich aus den unterschiedlichen Datenquellen von Rentnern und Erwerbspersonen, vgl. Kapitel 4.2.2. Da diese Verzerrung als Niveaufaktor zeitkonstant ist, ändert sie nichts an der strukturellen Interpretation der Verrentungsraten.

\footnotetext{
${ }^{9}$ In der Terminologie der Verweildauermodelle, vgl. Kapitel 6.
} 
Abbildung 4-10 zeigt die resultierenden Verrentungsraten im Zeitvergleich. Es wird deutlich, daß die bekannten Eckpunkte 60, 63 und 65 auch bei den Verrentungsraten relevant bleiben, allerdings sind die Größenverhältnisse gegenüber Abbildung 4-3 verändert. Während dort seit Mitte der 80er Jahre das Alter 60 dominiert, sind die größten Verrentungsraten eindeutig beim Alter $65 \mathrm{zu}$ finden. Dies ist ein Effekt der jeweiligen Basis: Die Verrentungsrate ist eine Maßzahl, die auf dem altersspezifischen Bestand basiert. Je mehr Arbeitnehmer mit 60 bzw. 63 bereits verrentet sind, desto höher ist die Verrentungsrate der 65 jährigen, weil in diesem Alter nahezu alle noch aktiven Erwerbspersonen in den Ruhestand treten.

An dieser Stelle kann als wichtiges $Z$ wischenergebnis festgehalten werden, daß auch die deskriptive empirische Analyse die aufgestellte Hypothese unterstützt, daß Arbeitnehmer frühestmöglich in den Ruhestand treten, sobald sich Ihnen die gesetzliche Möglichkeit bietet. Die im Kapitel 4.2 dargestellten Veränderungen des Rentenzugangsverhaltens, die für diese Hypothese sprechen, sind kein Artefakt, der aus unterschiedlichen Kohortenstärken resultiert.

\subsection{Regressionsanalyse}

Im vorhergehenden Abschnitt wurden die eingangs gestellten Fragen deskriptiv anhand von Grafiken untersucht. Im folgenden wird diese Betrachtung durch eine multivariate Analyse abgesichert, die es ermöglicht, die Wirkung einzelner Einflußgrößen unter der ceteris paribus-Annahme zu messen. So können damit die Korrelationen zwischen den Verrentungsraten und der Höhe der Arbeitslosigkeit unter Konstanthaltung der gesetzlichen Rahmenbedinungen untersucht und damit die in Abschnitt 4.1 gestellten Fragen weitergehend analysiert werden. Zur Messung der Korrelationen wird eine Regressionsanalyse in Form einer multivariaten linearen Regression durchgeführt.

\subsubsection{Schätzmethode}

In der Regressionsanalyse werden die eingangs dieses Kapitels gestellten Fragen untersucht. Die Schätzungen werden dabei in zwei Schritten durchgeführt. Im ersten, technisch motivierten Schritt werden die Korrelationsstrukturen als Varianzanalyse betrachtet, indem die Verrentungsraten nur auf Indikatorvariablen für Alter, Jahr und Kohorte regressiert werden..$^{10}$ Im zweiten - inhaltlichen - Schritt dienen diese Indika-

${ }^{10}$ Es ist dies keine „Re-gression“ im Wortsinne (Rückführung), der eine kausale Interpretation zugrunde liegt, sondern an dieser Stelle wird eine OLS-Schätzung zur Betrachtung partieller Korrelationskoeffizienten benutzt. 
torvariablen als Kontrollgrößen für die Messung des Einflusses der gesetzlichen Rahmenbedingungen und der Wirtschaftsentwicklung.

Die Schätzungen erfolgen in einer gepoolten OLS-Regression, ${ }^{11}$ in der die exogenen Variablen Alter A, Beobachtungsjahr J und daraus resultierend Kohorten $\mathrm{K}$ verbunden werden. Es ergibt sich damit als Dimension für die abhängige Variable Verrentungsrate: 22 Jahre 21 Altersstufen 2 Gruppen (Arbeiter, Angestellte) $=924$ Datenpunkte. Die Regressionsgleichung lautet:

(4-2) $\mathrm{VR}_{\mathrm{A}}=\mathrm{a} \cdot \mathrm{A}+\mathrm{j} \cdot \mathrm{J}+\mathrm{k} \cdot \mathrm{K}+\mathrm{g} \cdot \mathrm{GRV}+\mathrm{m} \cdot$ Makro $+\varepsilon$

mit: $\mathrm{VR}_{\mathrm{A}} \quad$ Verrentungsrate im Alter a
A Altersindikatorvariable $[(0,1)$ codiert $]$
J Jahresindikatoren $[(0,1)$ codiert $]$
$\mathrm{K} \quad$ Kohortenindikatoren $[(0,1)$ codiert; $\mathrm{K}=\mathrm{A}-\mathrm{J}]$
GRV Indikatoren der Gesetzlichen Rentenversicherung
Makro Makroökonomische Indikatoren
a,j,k,g,m Regressionskoeffizienten
$\varepsilon \quad$ Residuum der OLS-Regression;

so daß die Verrentungsrate VR sich ergibt als Linearkombination der Schätzkoeffizienten und Indikatorvariablen für Alter, Jahr und Kohorte sowie weiterhin interaktiver GRV-Indikatoren und jahresspezifischer makroökonomischer Kenngrößen.

Eine Anschauung der gepoolten Regression bietet die Matrix-Schreibweise, hier für den einfachen Fall von 3 Jahren (1970-1972) und 3 Altersstufen (60, 63 und 65) $)^{12}$ :

\begin{tabular}{|c|c|c|c|c|c|c|c|c|c|c|c|c|c|c|c|c|c|c|c|c|c|c|c|}
\hline & \multirow{2}{*}{\multicolumn{4}{|c|}{$\begin{array}{c}\text { Jahr j } \\
707172\end{array}$}} & \multirow{2}{*}{\multicolumn{3}{|c|}{$\begin{array}{c}\text { Alter a } \\
606365\end{array}$}} & \multicolumn{10}{|c|}{ Kohorte k } & \multirow{2}{*}{\multicolumn{3}{|c|}{ GRV - Variablen }} & \multicolumn{3}{|c|}{ Makro Variablen } \\
\hline & & & & & & & & & 5 & 67 & 78 & 89 & 9 & 10 & 111 & & & & & & & & \\
\hline$\left(V_{R_{60}^{20}}^{70}\right)$ & $(1$ & 0 & 0 & & $(1$ & 0 & $0)$ & & $(0$ & 0 & 0 & 0 & 0 & 1 & 0 & & & & 0 & 0 & & $\left(\triangle B S P x_{0}\right.$ & \\
\hline $\mathrm{VR}_{\mathrm{ni}}^{20}$ & 1 & 0 & 0 & & 0 & 1 & 0 & & 0 & 0 & 1 & 0 & 0 & 0 & 0 & 0 & & & & 0 & & $\triangle \mathrm{BSP}_{7}$ & \\
\hline $\mathrm{VR}_{\mathrm{hs}}^{20}$ & 1 & 0 & 0 & & 0 & 0 & 1 & & 1 & 0 & 0 & 0 & 0 & 0 & 0 & 0 & & & & 0 & $\left(g_{1}\right)$ & $\triangle B_{S P} P_{0}$ & \\
\hline$V_{R}^{\prime \prime}$ & $\mathrm{c}$ & 1 & 0 & $\left(j_{10}\right)$ & 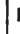 & 0 & 0 & $\left(a_{40}\right)$ & 0 & 0 & 0 & 0 & 0 & 0 & 1 & 0 & & & GI & 0 & $\mathrm{~g}_{2}$ & $\triangle \mathrm{BSP}_{n}$ & $\left(m_{1}\right)$ \\
\hline $\mathbf{V R}_{63}^{7 !}=$ & $=0$ & 1 & 0 & $\mathbf{j}_{n}+$ & & 1 & 0 & $a_{63}+$ & +0 & 0 & 0 & 1 & 0 & 0 & 0 & 0 & $x_{8}$ & $+\ldots+$ & & 0 & + & $+\triangle \mathrm{BSP}_{1} \ldots$ & .. $+\varepsilon$ \\
\hline $\mathbf{V R}_{\mathrm{s}}^{\prime \prime}$ & c & 1 & 0 & $\left(j_{n_{2}}\right)$ & o & 0 & 1 & $\left(a_{k s}\right)$ & 0 & 1 & 0 & 0 & 0 & 0 & 0 & 0 & & & & 0 & . & $\triangle B S P$, & \\
\hline$V_{R}^{72}$ & & 0 & 1 & & & 0 & 0 & & 0 & 0 & 0 & 0 & 0 & 0 & 0 & 1 & & & G1 & 0 & . $)$ & $\Delta \mathrm{BSP}_{n}$ & \\
\hline $\mathrm{VR}_{63}^{2}$ & C & 0 & 1 & & 0 & 1 & 0 & & 0 & 0 & 0 & 0 & 1 & 0 & 0 & 0 & & & 0 & G2 & & $\triangle \mathrm{BSP}_{n}$ & \\
\hline $\mathrm{VR}_{\mathrm{s}}^{\prime 2}$ & c & 0 & 1 & & & 0 & 1 & & 0 & 0 & 1 & 0 & 0 & 0 & 0 & 0) & & & 0 & G2 & & $\triangle \mathrm{BSP}_{n}$ & \\
\hline
\end{tabular}

11 In einer gepoolten Regression werden Beobachtungen aus unterschiedlichen Jahren und für verschiedene Gruppen von Befragungspersonen (hier Arbeiter, Angestellte) in einer Schätzung zusammengefaBt. Die Identifikation erfolgt hier über Indikator- oder Dummy-Variablen, die nur die Werte 0 und 1 annehmen können und den ceteris-paribus-Einfluß einer Ausprägung einer exogenen Variablen messen. Dieses Vorgehen wird in Formel 4-3 deutlich.

12 Die Schreibweise der kompletten Matrizen A, J und K erfolgt in dieser Abbildung zur besseren Anschauung. In der Schätzung kann nur eine dieser Matrizen komplett aufgenommen werden, die anderen müssen um eine Spalte verringert werden, damit keine lineare Abhängigkeit entsteht, da sich $\mathrm{K}=\mathrm{J}$ - A linear ergibt. 
Es ergeben sich in diesem Beispiel 8 verschiedene Kohorten (Geburtsjahrgänge) von 1905 bis 1912, wobei der Geburtsjahrgang 1907 zweimal, alle anderen einmal vorkommen. Am Beispiel der Altersindikatoren sei $a_{60}$, das erste Element des Vektors a betrachtet: Die erste Spalte der Matrix A nimmt nur für das Alter 60 den Wert 1 an und der Koeffizient $a_{60}$ quantifiziert damit die Interaktion der Altersausprägung $60 \mathrm{mit}$ der Verrentungsrate VR ceteris paribus, also unabhängig von den Ausprägungen der anderen Erklärungsvariablen J, K, G und $\mathbf{M}$.

Die GRV-Indikatorvariable G1 nimmt für alle Personengruppen im Alter 60 und für alle Jahre ab 1971 den Wert 1 an, so daß deren Koeffizient $g_{1}$ die Korrelation zwischen den VR und diesen Ausprägungen der Erklärungsvariablen quantifiziert. Diese (Beispiel-) Variable G1 könnte den Einfluß einer gesetzlichen Maßnahme messen, die eine Verrentung mit 60 Jahren erleichtert und ab 1971 in Kraft ist.

Diese Struktur erlaubt eine systematische Untersuchung der Bestimmungsfaktoren von Verrentungsraten, indem zunächst die Hauptfaktoren Alter, Jahr und Kohorte einzeln als ausschließliche Regressoren verwendet werden (4-4 a bis c) und danach Kombinationen dieser Größen (z.B. 4-4 d und e).

$(4-4)^{12}$
(a) $\mathrm{VR}=\mathrm{a} \cdot \mathrm{A}+\varepsilon_{\mathrm{a}}$
(b) $\mathrm{VR}=\mathrm{k} \cdot \mathrm{K}+\varepsilon_{\mathrm{b}}$
(c) $\mathrm{VR}=\mathrm{j} \cdot \mathrm{J}+\varepsilon_{\mathrm{c}}$
(d) $V R=a \cdot A+j \cdot J+k \cdot K+\varepsilon_{d}$
(e) $V R=a \cdot A+k \cdot K+\varepsilon_{c}$

Aus dieser technischen Betrachtung wird die Grundstruktur des Rentenzugangsverhaltens deutlich. Wenn sich Altersdummies als die einzig signifikanten Regressoren ergeben und Jahres- und Kohortenvariablen ohne Erklärungsgehalt bleiben, so könnte eine intertemporale Veränderung des Zugangsverhaltens ausgeschlossen werden. Ein solches Ergebnis ist jedoch auf Basis der kohortenbereinigten deskriptiven Analyse nicht zu erwarten. Bis zu diesem Schritt wird die Regressionsanalyse nicht als kausales Modell betrachtet, sondern es werden lediglich die Korrelationsstrukturen verglichen.

Wird (4-4 d) nun zunächst um die exogenen makroökonomischen Faktoren erweitert und anschließend mit der kompletten Regression (4-2) verglichen, kann der Erklärungszuwachs durch die GRV-Variablen quantifiziert werden. In diesem zweiten Schritt wird die oben beschriebene Korrelationsanalyse um die GRV-Kenngrößen als echte exogene Variablen erweitert, so daß eine kausale Interpretation des Erklärungszuwachses möglich wird. 


\section{Alternative Spezifikation auf Polynom-Basis}

Die bisher betrachteten Spezifikationen auf Basis von Dummyvariablensätzen haben den Vorteil, daß diese einen recht genauen Funktionsverlauf für die abhängige Variable abbilden können. Die Nachteile dieser Modellierung sind einerseits, daß sehr viele Freiheitsgrade für diese Anpassung gebraucht werden. Weiterhin kann immer nur ein Satz von Indikatorvariablen komplett geschätzt werden, da die Regression sonst aufgrund linearer Abhängigkeit der erklärenden Variablen nicht identifiziert ist. Das Auslassen einzelner Kohorten und Jahre ${ }^{13}$ bringt Referenzkategorien mit sich, deren Auswahl eine nicht vorherbestimmbare Wirkung auf die anderen Schätzkoeffizienten hat. Als alternative Spezifikation bietet sich daher eine Abbildung des zugrunde liegenden Funktionsverlaufes als Polynom von Alters-, Kohorten- und Jahreseffekten an. Einzelheiten zu dieser Modellierung finden sich in einem ähnlichen Ansatz zur Untersuchung der Lohnentwicklung von FITZENBERGER, HUJER, MACURDY UND SCHNABEL (1994). Die Modellgleichung:

(4-5) $\mathrm{VR}=\alpha+\beta \cdot A+\gamma \cdot J+\delta \cdot \mathrm{K}+\mathrm{g} \cdot \mathrm{GRV}+\mathrm{m} \cdot \mathrm{Makro}+\varepsilon$

wobei $A$ den Vektor $\left(A, A^{2}, A^{3}, A^{4}, \ldots, A^{n}\right)$ darstellt, $J$ und $K$ analog. Die GRV- und Makrovariablen werden nach wie vor als Indikatorvariablen modelliert.

wird für Polynome vierten und fünften Grades modelliert und reduziert daher die Dimension der Schätzung erheblich.

Das polynomiale Modell ist allgemeiner als das Modell (4-2), die ersten drei Terme A, $\mathrm{J}$ und $\mathrm{K}$ beschreiben eine Trendfunktion, auf deren Basis die Wirkungen der exogenen Größen G und M gemessen werden können. Auch für diese Spezifikation wird das Referenzmodell

(4-6) $\mathrm{VR}=\alpha+\beta \cdot A+\gamma \cdot J+\delta \cdot K+\varepsilon$

im Vergleich zur Gesamtspezifikation (4-5) getestet.

\subsubsection{Regressionsanalyse der Rentenzugänge 1970 bis 1991}

Die Regressionsanalyse ergibt, daß die GRV-Indikatoren die dominierenden Erklärungsgrößen für die Bestimmung der Verrentungsraten sind. Die wichtigsten besprochenen Regressionen sind im Text abgedruckt (Tabelle 4-1, Seite 99), die Schätzer-

13 Unter der Annahme, daß ein Komplettsatz Altersvariable spezifiziert wird, muß bei den Jahres- und Kohortenvariablen jeweils eine Referenzkategorie gebildet werden, die nicht zur Schätzung benutzt wird. 
gebnisse der weiteren Regressionsanalyse finden sich in den Tabellen des Anhanges A.1, die verwendeten Variablenbezeichnungen können der Anhangtabelle A-1 entnommen werden.

Tabelle 4-1 (Seite 99) zeigt die Schätzergebnisse der Regressionsanalyse der Verrentungsraten. Schätzung I stellt die Varianzanalyse der Gleichung (4-4 e) dar. Diese Regression hat keine kausale Interpretation, sondern bildet lediglich Korrelationen ab. Sie zeigt das erwartete Ergebnis, daß die zeitliche Streuung der altersspezifischen Verrentungsraten sowohl mit der Varianz der Zugangsalter A als auch der Kohortenindikatoren $\mathrm{K}$ korrespondiert, welche die intertemporale Strukturverschiebung der Verrentungsraten repräsentieren. ${ }^{14}$ Alle Schätzungen enthalten die Variable AB_Ind, eine Indikatorvariable für die Gruppe der Arbeiter. Da die Verrentungswahrscheinlichkeiten der Arbeiter, wie oben gesehen, durchweg über denen der Angestellten liegen, dient diese Variable der Niveauanpassung. Es ist ein positiver Koeffizient zu erwarten, der auch in allen betrachteten Spezifikationen vorliegt.

Im zweiten Schritt der Schätzung II dienen die Indikatorvariablen des Alters und der Kohorten als statistische Kontrollterme und ermöglichen somit eine strukturelle Interpretation der inhaltlichen Erklärungsgrößen. Die GRV-Variablen sind Indikatorvariablen, die als Interaktionsterme aus Alters- und Jahresvariablen die in Tabelle 3-9 (Kapitel 3.5.2) zusammengefaßten Regeländerungen abbilden. ${ }^{15}$ Durch die Hereinnahme dieser strukturellen Erklärungsgrößen verschwindet die Signifikanz der Altersund Kohortendummies gänzlich, lediglich das Alter 65 behält positive Signifikanz. Das liegt daran, daß diese Altersvariable A65 in diesem Kontext zu einer qualifizierten GRV-Variable wird. Wie im Kapitel 3 deutlich wurde, ist das Alter 65 über die gesamte Periode das relevante Regelrentenalter. Aus diesem Grunde (keine Veränderung

\footnotetext{
${ }^{14}$ Eine Regression aller drei Faktoren A, J und K erwies sich trotz der Verminderung von J und $\mathrm{K}$ um eine Dimension zur Beseitigung des Kolliniearitätsproblemes aus technischen Gründen als nicht durchführbar. Die Anhangtabelle A-2 beinhaltet die Regressionen (4-4 a) bis (4-4 c), von denen lediglich die Spezifikation 1 (Gleichung 4-4 a) einen nennenswerten Erklärungsgehalt aufweist, in dem Sinne daß die Variation der Regressoren $87 \%$ der Variation der abhängigen Variable „erklärt“, was aus dem Gütema $\beta R^{2}$ abzulesen ist. Dieses Ergebnis war zu erwarten, da die Verrentungsraten vor allem mit dem Alter schwanken. Die Ergebnisse der Spezifikation 3 bedeuten, da $B$ die Kalenderjahre für sich genommen, keinerlei Erklärungsgehalt haben. Dies widerspricht nicht der These einer intertemporalen Verschiebung der Verrentungsraten, sondern zeigt, daß die undifferenzierte Betrachtung einzelner Kalenderjahre nicht in der Lage ist, eine solche Strukturveränderung abzubilden. Es wird aus diesem Grund im weiteren mit einer Kombination aus Alters- und Kohortenvariablen gearbeitet.

15 Die Nomenklatur für die die GRV-Variablen ist in Anhangtabelle A-1 erläutert.
} 
über die Zeit) wird es nicht als GRV-Variable benannt, sollte aber als solche interpretiert werden.

Die GRV-Indikatoren erklären einen Großteil der Varianz der Verrentungsraten, in beiden Schätzungen liegt das korrigierte Bestimmtheitsmaß $\overline{R^{2}}$ über $94 \%$.

\section{Anreizwirkungen der GRV}

Rentenreform 1972

Die Variablen GJ73... repräsentieren die Einflüsse der großen Rentenreform von 1972, in der die flexible Altersgrenze (ab dem Alter 63) eingeführt wurde. Entsprechend wurde aus den theoretischen Vorüberlegungen (Tabelle 3-9, Kapitel 3.5.2) erwartet, daß die Alter 62 bis 64 positiv, die Alter 65 und alle vor 62 durch die Reformmaßnahmen negativ beeinflußt werden. Das wichtigste betroffene Alter sollte 63 sein (GJ73_63), bei dem die neu eingeführte flexible Altersgrenze beginnt. Die Koeffizienten zeigen durchweg das erwartete Vorzeichen, allerdings sind nur die Werte für das Alter 63 und für Personen unter 62 Jahren statistisch signifikant. Daß die Schwerbehindertenrenten mit 62 Jahren bis zur Absenkung dieser Altersgrenze im Jahre 1979 kaum ausgenutzt wurden, wird in der Literatur bestätigt (vgl. z.B. JACOBS, KOHLI UND REIN, 1991), augenscheinlich liegt dieses Alter zu nahe am flexiblen Ruhestandsalter 63.

Es wird also nicht nur die Erwartung der Wirkungsrichtungen, sondern auch die der relativen Wichtigkeit der Einzeleinflüsse der 72er Reform eindrucksvoll bestätigt.

\section{Änderungen des Zugangsalters für BU/EU}

Die nächste Gruppe der GRV-Variablen betreffen die Regeländerungen des Jahres 1979, durch die die flexible Altersgrenze für Schwerbehinderte auf das 61. Lebensjahr herabgesetzt wurde. Aus theoretischer Sicht ist hieraus zu erwarten, daß die Verrentungsraten für dieses Lebensalter 61 höher ist als in anderen Altersstufen. Diese Vermutung impliziert die empirische Tatsache, daß dieser Verrentungsgrund durch einen erheblichen Anteil der Neurentner genutzt wird (s.o.). Die Schätzergebnisse stützen auch diese Hypothese. Während die negativen Wirkungen auf andere Rentenzugangsalter im Jahr 79 nicht signifikant sind, wird die positive Wirkung des Eintrittsalters 61 mit einem t-Wert von 5,5 markant deutlich.

Die weitere Absenkung derselben flexiblen Altersgrenze für Schwerbehinderte im folgenden Jahr 1980 auf das 60. Lebensjahr läßt analog erhöhte Verrentungsraten für dieses Zugangsalter 60 und niedrigere für die angrenzenden Alter ab dem Jahr 1980 ver- 
Tabelle 4-1: OLS-Schätzung der Verrentungsraten

\begin{tabular}{|c|c|c|c|c|c|c|c|c|c|c|c|}
\hline 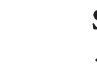 & $\begin{array}{l}\text { Schätzung } \\
\text { Alters- ur }\end{array}$ & $\begin{array}{l}\text { I } \\
d\end{array}$ & & & & $\begin{array}{l}\text { chätzu } \\
\text { Llter, } \mathrm{K}\end{array}$ & $\begin{array}{l}\text { ng II } \\
\text { Kohor }\end{array}$ & $\begin{array}{l}\text { (Gleichu } \\
\text { e, GRV, }\end{array}$ & $\begin{array}{l}\text { g 4-2) } \\
\text { Makro }\end{array}$ & & \\
\hline Koh & ortenvari & ablen & & & (Teil 1 & & & & (Teil 2) & & \\
\hline Variable & Koeffizent & $t$-Wert & Sign & Variable & Koeffizent & t-Wert & Sign. & Variable & Koeffizent & t-Wert & Sign. \\
\hline A45 & $-42,908$ & $-4,20$ & $*$ & A45 & $-30,0$ & $-1,48$ & & GJ73_u62 & $-1,1$ & $-1,88$ & 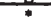 \\
\hline A46 & $-42,926$ & -4.20 & $*$ & A46 & $-30,0$ & $-1,48$ & & GJ73_62 & 4,7 & 0,80 & \\
\hline A47 & $-42,890$ & $-4,20$ & ** & A47 & $-29,8$ & $-1,47$ & & GJ73_63 & $\mathbf{3 8 , 0}$ & 4,10 & 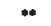 \\
\hline A48 & $-42,871$ & $-4,19$ & $*$ & A48 & $-29,7$ & $-1,46$ & & GJ73_64 & 7,1 & 0,64 & \\
\hline A49 & $-42,795$ & $-4,19$ & $*$ & A49 & $-29,4$ & $-1,45$ & & GJ73_65 & $-0,3$ & $-0,02$ & \\
\hline A50 & $-42,691$ & $-4,18$ & ** & A50 & $-29,2$ & $-1,44$ & & GJ79_u61 & 0,1 & 0.26 & \\
\hline A51 & $-42,697$ & $-4,18$ & $*$ & A5I & $-29,1$ & $-1,44$ & & GJ79_61 & 7,0 & 5,46 & $* *$ \\
\hline A52 & $-42,530$ & $-4,17$ & $*$ & A52 & $-28,9$ & $-1,43$ & & GJ79_62 & $-1,1$ & $-0,49$ & \\
\hline A53 & $-42,320$ & $-4,15$ & ** & A53 & $-28,6$ & $-1,41$ & & GJ79_63p & $-1,7$ & $-0,63$ & \\
\hline A54 & $-42,069$ & $-4,12$ & $*$ & A54 & $-28,4$ & $-1,40$ & & GJ80_u60 & $-0,8$ & $-1,37$ & \\
\hline A55 & $-41,612$ & $-4,08$ & ** & Ass & $-27,9$ & $-1,38$ & & GJ80_60 & 10,4 & 13,11 & ** \\
\hline A56 & $.41,156$ & $-4,03$ & ** & A56 & $-27,5$ & $-1,36$ & & GJ80_61 & $-2,7$ & $-1,56$ & \\
\hline A57 & -40.611 & $-3,98$ & $* *$ & A57 & $-27,0$ & $-1,33$ & & GJ80_62p & 1,4 & 0,56 & \\
\hline A.58 & -39.938 & $-3,92$ & $*$ & A58 & $-26,3$ & -1.30 & & GJ84_u60 & $-1,0$ & $.2,44$ & $*$ \\
\hline A59 & $-39,217$ & $-3,85$ & $*$ & A59 & $-25,7$ & $-1,27$ & & GJ84_60 & 2,4 & 1,99 & $*$ \\
\hline A60 & $.29,116$ & $-2,85$ & $* *$ & A60 & -22.2 & $-1,10$ & & GJ84_61 & $-5,5$ & $-3,65$ & $*$ \\
\hline A61 & $-33,938$ & $-3,33$ & $* *$ & A61 & $-22,4$ & $-1,12$ & & GJ84_62 & $-6,3$ & $-5,72$ & ** \\
\hline A62 & $-.33,410$ & $-3,27$ & ** & A62 & $-23,5$ & $-1,28$ & & GJ84_63 & 1.9 & 0,70 & \\
\hline A63 & $-3,286$ & $-0,32$ & & A63 & $-26,5$ & $-1,44$ & & GJ84_64 & $-9,7$ & $-5,01$ & * \\
\hline A64 & $.27,802$ & $-2,70$ & $*$ & A64 & $-21,0$ & $-1,2.3$ & & GJ84_65 & 28,3 & 6,51 & $*$ \\
\hline A65 & 42,110 & 4,35 & ** & A65 & 41.4 & 4,26 & ** & GJ73_64 & 8,8 & 1,28 & \\
\hline $\mathrm{K} 6$ & 14.961 & 1,11 & & $\mathrm{~K} 6$ & $i i, 3$ & 0.76 & & BSP & 0.1 & 0,68 & \\
\hline K7 & 26,346 & 1,88 & $*$ & K7 & 31,2 & 1,62 & & Anpass & 0.1 & 1,06 & \\
\hline K8 & 25,435 & 2,04 & ** & K8 & 26,4 & 1,49 & & Alq_Ges & 0,2 & 1,51 & \\
\hline K9 & 31.511 & 2,75 & $*$ & K9 & 26,6 & 1,39 & & & & & \\
\hline K10 & 30,898 & 2,94 & $*$ & K10 & 20,6 & 1,02 & & & & & \\
\hline KII & 34,080 & 3,31 & $*$ & KII & 22,6 & 1,10 & & & & & \\
\hline $\mathrm{K} 12$ & 34,976 & 3,39 & ** & $\mathrm{K} 12$ & 23,2 & 1,13 & & & & & \\
\hline K13 & 36,110 & 3,49 & ** & K13 & 24,2 & 1,18 & & & & & \\
\hline K14 & 36,414 & 3,52 & $*$ & $\mathrm{~K} 14$ & 24,5 & 1,20 & & & & & \\
\hline K15 & 36,382 & 3,52 & $*$ & K15 & 24,4 & 1,20 & & & & & \\
\hline K16 & 39,069 & $\mathbf{3 , 8 4}$ & $*$ & K16 & 27,0 & 1,33 & & & & & \\
\hline K17 & 39,174 & 3,85 & $*$ & K17 & 26,9 & 1,32 & & & & & \\
\hline K18 & 39,662 & 3,90 & $*$ & K18 & 26,7 & 1,31 & & & & & \\
\hline K19 & 42.914 & 4.22 & $*$ & K19 & 28,1 & 1,38 & & & & & \\
\hline K20 & 40,598 & 4.01 & $*$ & K20 & 25,9 & 1,27 & & & & & \\
\hline K21 & 40,779 & 4.02 & $*$ & K21 & 26,0 & 1,28 & & & & & \\
\hline $\mathrm{K} 22$ & 41,814 & 4.08 & ** & K22 & 27.5 & 1,35 & & & & & \\
\hline K23. & 41,340 & 4,05 & $*$ & $\mathrm{~K} 23$ & 27,4 & 1,35 & & & & & \\
\hline $\mathrm{K} 24$ & 42,274 & 4,08 & $* *$ & K24 & 28,3 & 1,39 & & & & & \\
\hline K25 & 42,741 & 4,13 & $*$ & K25 & 28,9 & 1,42 & & & & & \\
\hline K26 & 41,572 & 4,07 & $*$ & $\mathrm{~K} 26$ & 27,9 & 1,37 & & & & & \\
\hline K27 & 40,988 & 4,01 & $*$ & $\mathrm{~K} 27$ & 28,1 & 1,38 & & & & & \\
\hline K28 & 41,178 & 4,04 & $* *$ & $\mathrm{~K} 28$ & 28,0 & 1,37 & & & & & \\
\hline K29 & 40,899 & 4.01 & ** & K29 & 28,1 & 1,38 & & & & & \\
\hline $\mathrm{K} .30$ & 41,165 & 4,04 & $*$ & K.30 & 28,2 & 1,38 & & & & & \\
\hline K31 & 41,218 & 4,04 & ** & K.31 & 28,2 & 1,38 & & & & & \\
\hline K.32 & 41,084 & 4,03 & $* *$ & $\mathrm{~K} 32$ & 28,5 & 1,40 & & & & & \\
\hline K33 & 41,191 & 4,04 & $*$ & $\mathbf{K} 33$ & 28,7 & 1.41 & & & & & \\
\hline K34 & 41,228 & 4,04 & $*$ & $\mathrm{~K} 34$ & 28,8 & 1.41 & & & & & \\
\hline K.35 & 41,234 & 4,04 & *. & K.35 & 28,9 & 1.42 & & & & & \\
\hline K36 & 41,290 & 4,05 & ** & K.36 & 29,0 & 1.42 & & & & & \\
\hline K.37 & 41,326 & 4,05 & $* *$ & K.37 & 29,1 & 1.42 & & & & & \\
\hline K38 & 41,337 & 4,05 & $*$ & K.38 & 29.2 & 1,43 & & & & & \\
\hline K39 & 41,355 & 4,05 & $* *$ & K39 & 29,4 & 1,44 & & & & & \\
\hline K40 & 41,345 & 4,05 & ** & K40 & 29,5 & 1,44 & & & & & \\
\hline K41 & 41,369 & 4,05 & $*$ & K41 & 29,6 & 1.44 & & & & & \\
\hline K42 & 41,363 & 4,05 & ** & K42 & 29,6 & 1.45 & & & & & \\
\hline K43. & 41,372 & 4,05 & $*$ & $\mathbf{K} 43$ & 29,7 & 1,45 & & & & & \\
\hline K44 & 41,340 & 4,04 & $*$ & K44 & 29,8 & 1,45 & & & & & \\
\hline K45 & 41,345 & 4,04 & ** & K45 & 29.8 & 1.45 & & & & & \\
\hline K46 & 41,319 & 4,02 & ** & K46 & 29,9 & 1,45 & & & & & \\
\hline$A B_{\text {_Ind }}$ & 3,579 & 9,24 & $*$ & AB_Ind & 3,6 & 12,55 & $*$ & & & & \\
\hline Beob. & 924 & & & & & & & Beob. & 924,0 & & \\
\hline$R^{2} /$ korr $R^{2}$ & $90.4 \%$ & $89,7 \%$ & & & setzung ob & & & $\mathrm{R}^{2} /$ kor. $\mathrm{R}^{2}$ & $94.8 \%$ & $94,25 \%$ & \\
\hline DW & 2,197 & & & & & & & DW & 1.8 & & \\
\hline
\end{tabular}

Anmerkungen: A:Alter, K:Kohorte, G:GRV-Indikator; AB_Ind: Indikatorvariable Arbeiter; Beispiel: GJ73_u62: Indikatorvariable, die in allen Jahren ab 1973 für Personen unter 62 Jahren den Wert 1 annimmt. - Die genauen Variablenbezeichnungen können der Tabelle A-1 entnommen werden. *": signifikant auf dem $95 \%$-Niveau; ": signifikant auf dem $90 \%-N i v e a u$ 
muten. Die empirischen Ergebnisse bestätigen diese These, da der Altersdummy für das Alter 60, GJ80_60 mit positivem Vorzeichen einen sehr hohen t-Wert aufweist. Die angrenzenden Alter GJ80_u60 und GJ80_61 weisen erwartungsgemäß negative Koeffizienten auf, deren t-Werte nur knapp unter der 90\%-Signifikanz liegen.

Es ist zu beachten, daß die Regeländerungen des Jahres 1980 einen permanenten Effekt auf die Rentengesetzgebung $a b$ dem Jahr 1980 haben, wogegen die Variablen GJ79... lediglich die Auswirkungen einer einjährigen Regeländerung messen.

\section{Haushaltsbegleitgesetz 1984}

Der letzte Block der GRV-Variablen quantifiziert die Wirkungen des Haushaltsbegleitgesetzes von 1984, in welchem neben der Herabsetzung der Mindestversicherungszeit für Altersruhegelder ab 65 von 15 auf 5 Jahre auch erschwerte Anspruchsvoraussetzungen für EU/BU Renten eingeführt wurden. Die Kombination beider Maßnahmen läßt daher höhere Verrentungsraten für das Alter 65 erwarten in Verbindung mit geringeren Raten in den jüngeren Zugangsaltern, insbesondere dem Alter 60. Dies impliziert eine Substitution von EU/BU und Schwerbehindertenrenten durch eine normale Regelaltersrente (mit 65) für Personen, die geringe Versicherungszeiten aufweisen. Der Koeffizient für GJ84_65 bestätigt die erste Vermutung mit hoher Signifikanz und auch die entsprechenden Einflußgrößen GJ84_61, GJ84_62 und GJ84_64 wirken theoriekonform negativ. Die positive Wirkungsrichtung des Zugangsalters 60 (GJ84_60) stellt den einzigen Widerspruch der Regressionsergebnisse mit den geäuBerten Erwartungen dar. Der signifikant negative Koeffizient der Variable GJ84_u60, also für alle unter 60 jährigen ab 1984, resultiert aus dem bereits in Abbildung 4-4 (Seite 84) sichtbaren nach 1984 sinkenden Anteil der Personen, die vor dem Alter von 65 in die Rentenversicherung eintreten.

\section{Gesamtwirtschaftliche Indikatoren}

Als Variablen zur Messung der gesamtwirtschaftlichen Situation dienen in dieser Gleichung die Änderung des realen Bruttosozialproduktes, die jährliche Rentenanpassung der GRV'16 sowie die gesamtwirtschaftliche Arbeitslosenquote. Die ersten beiden Kenngrößen repräsentieren die Entwicklung der Produktion und des Einkommens, zei-

${ }^{16}$ Diese wird jährlich durch die Bundesregierung festgelegt und orientiert sich an der Lohnentwicklung der Arbeitnehmer. Die Anpassung erfolgte teilweise nicht regelmäßig (1972 2 Anpassungen), teilweise zum 1.1., seit 1983 zum 1.7. jeden Jahres (vgl BMA „Rentenversicherung in Zahlen“ (1992), Seite 25“). 
gen aber keinen signifikanten Einfluß auf das Rentenzugangsverhalten. Die Anpassung der gesamtwirtschaftlichen Arbeitslosenquote zeigt ebenfalls keine Signifikanz, wodurch die These der (Arbeitsmarkt-) nachfrageinduzierten Ruhestandsentscheidung nicht unterstützt werden kann. Diese Variable wird allerdings im folgenden Abschnitt 4.3.3 weitergehend diskutiert.

Die Schätzergebnisse zeigen eine sehr eindrucksvolle Bestätigung der theoretisch bzw. institutionell basierten Hypothesen des 3. Kapitels.

\section{Alternative Spezifikation auf Polynom-Basis}

Tabelle 4-2 beinhaltet drei Spezifikationen des Polynomialmodelles (4-5). Schätzung P1 ist das Analogon zur oben beschriebenen Schätzung I, allerdings können hier zusätzlich auch die Jahresangaben berücksichtigt werden. ${ }^{17}$ Wie in den vorangegangenen Schätzungen zeigt sich auch hier, daß die Jahresvariablen keinen Erklärungsgehalt für die Verrentungsraten haben ${ }^{18}$, dieser setzt sich augenscheinlich aus Alters- und Kohorteneffekten zusammen. Allerdings ist die statistische Anpassung, gemessen durch das Gütemaß $R^{2}$ in der Schätzung P1 mit 73,6\% erheblich geringer als die der Schätzung I. Dies erklärt sich statistisch aus der wesentlich geringeren Zahl der Freiheitsgrade, mit denen das Modell angepaßt wird in Verbindung mit dem aus den vorigen Kapiteln bekannten unstetigen Verlauf der Altersfigur. Diese wird abgebildet durch die Hereinnahme dreier Indikatorvariablen für die Kernalter 60, 63 und 65 in Schätzung P2 (,Spitzendummies“). Das Gütema $\overline{\mathrm{R}^{2}}$ zeigt, da $B$ diese Spezifikation trotz der geringeren Dimension die Anpassung von Schätzung I sogar leicht übertrifft. Die eingefügten 3 Altersindikatoren dominieren das Alterspolynom eindeutig, was ein weiterer Hinweis auf die absolute Wichtigkeit der GRV-Indikatoren ist, denn diese drei Kernalter sind die wichtigsten Rentenalter der GRV. Insofern können diese Variablen auch als Modellierung simpler GRV-Indikatoren interpretiert werden, die alle drei erwartungsgemäß hochsignifikant positiv wirken. Die fünf Variablen des Alterspolynomes sind weiterhin gemeinsam signifikant, da ein F-Test die partielle Null-Hypothese für diese

${ }^{17}$ Dies wird aufgrund der wesentlich geringeren Dimension der Polynome gegenüber den Indikatorvariablen möglich. Allerdings kann auch hier in der dritten Dimension „Jahr“" kein Term erster Ordnung steht, da sich das Jahr linear aus der Summe Alter + Kohorte errechnet und die Schätzgleichung damit kollinear wäre. Dies gilt für die höherwertigen Polynome nicht mehr, da kein linearer Zusammenhang besteht.

18 Dies bestätigt sich auch mit einem F-Test der Hypothese, daß alle Jahres-Variablen Null sind. Dieser F-Test liegt mit einem Wert von 0,88 weit unter der kritischen Grenze $\left(F_{3,1000}=3,80\right)$, so daß diese Null-Hypothese nicht verworfen werden kann. 
Variablen deutlich verwirft. ${ }^{19}$ Die Schätzung P2 dient als technische Referenzgröße im Rahmen der Varianzanalyse für die inhaltlich interpretierbare Schätzung P3:

Die Regressionsschätzung P3 stellt das Pendant zu Schätzung II dar, es beinhaltet sowohl die qualifizierten GRV-Indikatoren als auch die Makrovariablen. Das Schätzergebnis nach Hinzunahme der qualifizierten GRV-Indikatoren ist genauso eindrucksvoll wie in der oben beschrieben Schätzreihe auf Basis von Indikatorvariablensätzen. Die Gesetzliche Rentenversicherung dominiert deutlich das beobachtete Verrentungsverhalten. Die dem Modell zugrunde liegenden Polynome, die den Alters-, Kohorten- und Jahrestrend modellieren sind nicht mehr statistisch signifikant, auch der Altersdummy für das Alter 63 ist nicht mehr signifikant, wogegen die Altersvariable 60 einen signifikanten Erklärungsgehalt behält. Die Altersvariable 65 bleibt weiterhin signifikant, wie bereits beschrieben ist sie als qualifizierte GRV-Variable des sich im Beobachtungszeitraum nicht ändernden Regelrentenalters $65 \mathrm{zu}$ interpretieren.

Die wichtigen Variablen der Rentenreformen, GJ73_63 für den flexiblen Ruhestand ab 1973, die Indikatoren des 1979 und 80 gesenkten Einstiegsalters für Schwerbehinderte GJ79_61 und GJ80_60 erweisen sich als theoriekonform in dem Sinne, daß die Arbeitnehmer die sich bietende Möglichkeiten einer Frühverrentung genutzt haben. Die hier betrachtete polynomiale Spezifikation erweist sich im Fall der bisher insignifikanten Variable GJ73_n64, die nur im Jahr 1973 für den einmaligen Effekt der Einführung des flexiblen Ruhestandes kontrolliert, als sensibler in Bezug auf einzelne „Ausreißer“ aus der Trendfunktion. ${ }^{20}$

Die deutlichsten Auswirkungen auf das Rentenzugangsverhalten zeigen die Änderungen des Haushaltsbegleitgesetzes von 1984. Alle Variablen wirken in der oben beschriebenen Form (Schätzung II), allerdings gilt auch hier wieder als einzige Ausnahme einer nicht aus den GRV-Änderungen zu erwartenden Verhaltensänderung der positive Effekt im Sinne höherer Verrentungsraten im Alter 60 ab 1984. Dies kann nur dahingehend interpretiert werden, daß die Verschärfung der Anforderungen an BU/EU Renten nicht die erhoffte Wirkung einer verringerten Inanspruchnahme dieser Frührenten-Leistungsart bewirkt hat.

${ }^{19} \mathrm{~F}$-Wert $=22,11$ bei einem Schwellenwert ( $99 \%$ Signifikanzniveau) von $\mathrm{F}_{5,1000}=3,02$

20 Dieser artifizielle Effekt ist in Abbildung 4-2 (Steite 82) zu sehen und dort auch erläutert. Die Signifikanz dieses „Ausreißers“ in der polynomialen Spezifikation verdeutlicht noch einmal die grundlegende Modellstruktur, die im Polynomfall auf Trendfunktionen aufbaut. In diesen können einzelne Ausreißer nicht modelliert werden, wogegen dies im Fall einer großen Zahl einzelner Dummy-Variablen möglich ist. 
Tabelle 4-2: OLS-Schätzung der Verrentungsraten, polynomiale Spezifikationen

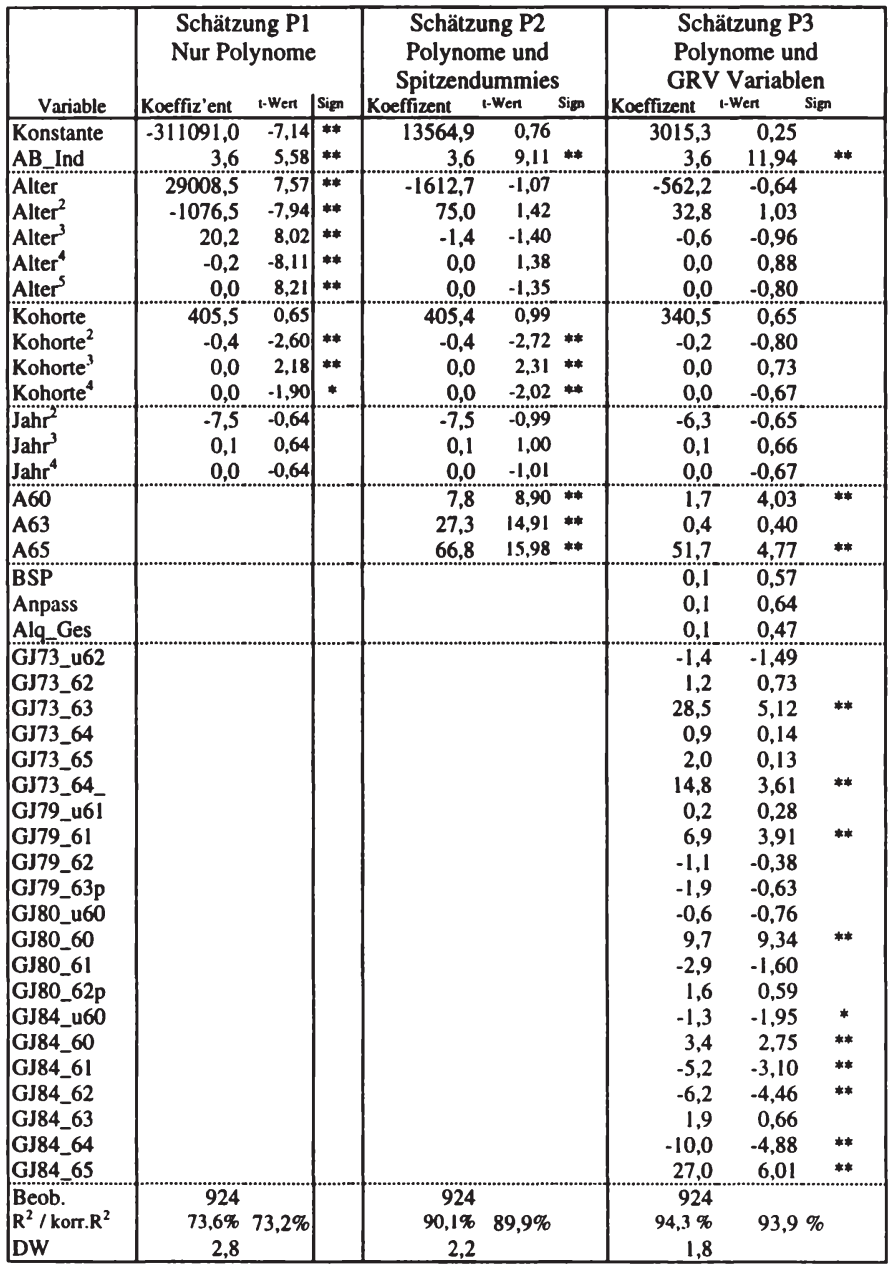

\subsubsection{Arbeitsmarktsituation und Rentenneigung}

In diesem Abschnitt wird die Diskussion des Kapitels 4.1 aufgegriffen, in dem aus theoretischer Sicht die Frage gestellt wurde, ob und wie stark die Nachfrage auf dem Arbeitsmarkt die persönliche Arbeitsangebotsentscheidung von Arbeitnehmern beeinflußt. 
In der oben betrachteten Schätzung II bleibt die Einflußgröße „Alq_Ges“, die gesamtwirtschaftliche Arbeitslosenquote ohne meßbaren Einfluß auf die Verrentungsentscheidung. Eine differenziertere Betrachtung der Arbeitsnachfrage unterscheidet zwei mögliche Einzeleffekte.

\section{Relevanz altersspezifischer Arbeitslosenquoten}

Wenn die Arbeitslosenquoten für die relevanten Altersgruppen von der gesamtwirtschaftlich beobachteten abweichen, liegt die Vermutung nahe, daß diese altersgruppenspezifische Arbeitslosenziffer die Entscheidung älterer Arbeitnehmer stärker beeinfluBt, als die für alle Altersgruppen gültige. In jedem Fall ist die aus der Theorie zu erwartende Wirkung von Arbeitslosenquoten die, daß bei hoher Erwerbslosigkeit eher auf die Rente „ausgewichen“ wird, die Verrentungsraten in diesen Jahren also höher sind. Dies entspricht einem positiven Vorzeichen des Regressionskoeffizienten.

Abbildung 4-11 zeigt die Arbeitslosenziffern unterschiedlicher Altersgruppen. Die als „Gesamt" angegebene Kurve bezieht sich auf alle Erwerbspersonen. ${ }^{21}$ Es wird deutlich, daß die Quote der 40-45 jährigen unter der gesamtwirtschaftlichen liegt. Dies leuchtet aus Sicht eines Erwerbs-Lebenszyklus unmittelbar ein. Die Schwankungen der Quote dieser Gruppe repräsentieren die gesamtwirtschaftliche Beschäftigungsentwicklung. Die beiden anderen betrachteten Altersgruppen älterer Arbeitnehmer weisen überdurchschnittliche Arbeitslosenzahlen auf. Im Falle der 50-55 jährigen ist auf Basis der bisherigen Ausführungen die Frage der Kausalität nicht eindeutig zu beantworten, da die hohe Arbeitslosigkeit auch aus Vorruhestandsregelungen, Sozialplänen und anderen Formen der 59er Regel resultieren können und somit eine Folge der Rentenpolitik sein könnten, statt diese kausal zu beeinflussen. Tabelle A-4 im Anhang zeigt neben der bereits diskutierten Schätzung II zwei alternative Spezifikationen der EinfluBgröße „Arbeitsmarkt“. In Schätzung III ist die Wirkung der altersspezifischen Arbeitslosenziffer der 60 bis 64 jährigen auf alle Arbeitnehmer modelliert und diese übt tatsächlich einen positiven Einfluß auf, der $\mathbf{9 0} \%$ signifikant ist. Diese Wirkung unterstuitzt die Hypothese arbeitsnachfrageinduzierter Ruhestandsentscheidungen. Allerdings ist die Arbeitslosenziffer der 60 bis 64 jährigen die einzig signifikante. Weder die gesamtwirtschaftliche (Schätzung II), noch die der Altersgruppen 45-50, 50-54, 55 $60^{22}$ weisen eine meßbaren Einfluß auf. Tatsächlich zeigt Abbildung 4-11, daß die

\footnotetext{
${ }^{21}$ Angegeben Altersgruppen von „unter 20“ bis „über 65“.

22 Es wurden weitere Regressionanalysen durchgeführt, die nicht einzeln tabelliert sind.
} 
Quote der Altersgruppe 60-64 einen anderen Verlauf aufweist, als die gesamtwirtschaftliche. Sie bleibt auch während der Konjunkturberuhigung der späten 70er Jahre relativ hoch und unterliegt in den 80er Jahren starken Schwankungen, welche die Gesamtquote kaum widerspiegelt. Es darf aber nicht unerwähnt bleiben, daß generell Arbeitslosenzahlen und -quoten in Altersstufen über 55 Jahre äußerst problematisch sind. Die Bundesanstalt für Arbeit weist für diese Alter keine Arbeitslosenquote aus, mit der Begründung, daß die Basis der Erwerbstätigen nicht ausreichend genau definiert sei. In der vorliegenden Arbeit wurden aus den veröffentlichten absoluten Zahlen der Bundesanstalt für Arbeit und den in Kapitel 4.2 diskutierten Erwerbspersonen des statistischen Bundesamtes altersspezifische Arbeitslosenquoten errechnet.

Abbildung 4-11: Arbeltslosenquoten unterschledlicher Altersgruppen

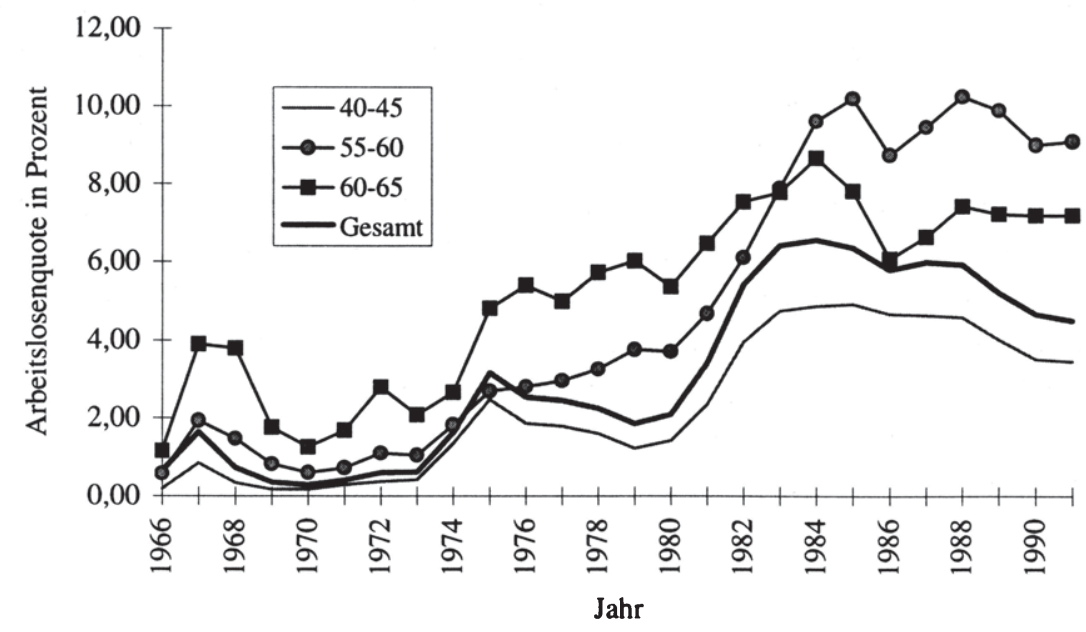

Quelle: Amtliche Nachrichten der Bundesanstalt für Arbeit, diverse Jahrgănge.

Auf dieser Basis sei ein positiver Koeffizient in mehreren alternativen Spezifikationen durchaus mit Vorsicht interpretiert. Alternativ wurde daher die Erwerbslosigkeit auf eine andere Weise modelliert:

\section{Unterschiedliche Reaktion verschiedener Altersgruppen auf die Arbeitslosigkeit}

Die im vorliegenden Modell betrachteten Arbeitnehmer im Alter von 45 bis 65 Jahren befinden sich an unterschiedlichen Stellen ihres Lebenszyklus. Während für die ersten das Ende des Erwerbslebens selbst bei früher Verrentung noch weit entfernt ist, arbeiten von den ältesten nur noch sehr wenige. In Schätzung III (Anhangtabelle A-4) erfolgt eine Untersuchung der Wirkung der gesamtwirtschaftlichen Arbeitslosenquote 
auf unterschiedliche Altersgruppen. Das Ergebnis zeigt unterschiedliche Vorzeichen: Während die 45 bis 49 und die über 60 jährigen ${ }^{23}$ auf höhere Arbeitslosigkeit mit erhöhten Verrentungsraten reagieren, ist die Reaktion der 55 bis 60 jährigen signifikant negativ und die der 50 bis 54 jährigen ist nicht meßbar. Die positiven Koeffizienten der Gruppen 45-49 und 60 plus unterstützen die in Kapitel 4.1 diskutierten Theorie von JACOBS, KOHLI UND REIN, wogegen der negative Koeffizient der Gruppe 55-59 dieser widerspricht. Ein möglicher Einwand gegen diesen Widerspruch ist die umgekehrte Kausalität: Weil die 55-59 jährigen verstärkt in Rente gehen, ${ }^{24}$ ist in dieser Gruppe die Arbeitslosigkeit geringer und die Regressionsanalyse ergibt fälschlicherweise ein negatives Vorzeichen. Dieser Einwand gilt für eine altersspezifische Arbeitslosenquote der gleichen Altersgruppe. Für die - hier betrachtete - Gesamtquote wäre er nur dann zutreffend, wenn diese durch das Verhalten der 50-59 jährigen maßgeblich beeinflußt würde. Dies ist aber, wie in Abbildung 4-11 zu sehen, nicht der Fall. In den Jahren 1975 bis 1980, 85 - 88 und nach 1990 ist der Funktionsverlauf deutlich unterschiedlich und eine Wirkung der älteren Arbeitnehmer auf die Gesamtquote nicht zu erkennen.

Als Ergebnis kann damit festgehalten werden, daß keine eindeutigen Korrelationen zwischen der Arbeitsnachfrage und den Rentenzugängen gefunden werden konnte. Die Hypothese, die Frühverrentung resultiere vornehmlich aus Arbeitsmarktbedingungen, wie sie etwa von JACOBS, KOHLI und REIN (1991) vertreten wird, kann durch diese Regressionsanalyse nicht gestützt werden. Die Gesamtbetrachtung der Schätzung I zeigt keinen Einfluß, differenzierte Einzeluntersuchungen erbringen widersprüchliche Ergebnisse.

\subsection{Zusammenfassung und Bewertung der makroökonomischen Unter- suchung}

Als Resümee kann festgehalten werden, daß die im dritten Kapitel auf Basis der einzelnen gesetzlichen Änderung entwickelten Erwartungen an das Verhalten der Rentenzugänger durch die empirische Analyse auf eindrucksvolle Weise bestätigt werden. Die Hypothese, daß das Verhalten der Arbeitnehmer mit den institutio-

${ }^{23}$ Mit statistischen Abstrichen. Der t-Wert von 1,63 für die über 60 jährigen liegt direkt unter der kritischen Schwelle von 1,64, ab der eine 90 \% - Signifikanz gegeben wäre.

${ }^{24}$ Aufgrund der unterschiedlichen, in Kapitel 3 und 4.1 diskutierten Wege (Vorruhestandregelungen, Sozialpläne und andere Formen der 59er Regel). 
nellen Veränderungen korreliert ist, wird durch die Regressionen deutlich unterstützt. Dies gilt für beide betrachteten Modellierungen gleichermaßen. Auch die „Hypothese der rationalen Arbeitnehmer" wird durch die diskutierten Schätzergebnisse gestuitzt. Die untersuchten Arbeitshypothesen sind so formuliert, daß sie auf dem Ziel eines frühestmöglichen Ruhestandes basieren. Durch ihre empirische Untermauerung wird auch dieses Ziel als sinnvolle Operationalisierung des Entscheidungskalüls bestätigt.

Die vorliegende Untersuchung hatte sich eine Analyse der Rentenzugänge der 22 Jahre von 1970 bis 1991 zum Ziel gesetzt. Die eingangs gestellte Kernfrage war dabei, ob die gesetzlichen Rahmenbestimmungen tatsächlich in der Lage sind, das Verhalten älterer Arbeitnehmer bei der Wahl ihres Rentenalters so maßgeblich zu verändern, daß eine Entlastung des Gesamtsystems spürbar wird. Für die Analyse wurden im ersten Schritt die absoluten Zugangszahlen zur GRV erhoben und diese dann um die Kohortenstärken bereinigt. Die deskriptive Auswertung der so ermittelten Verrentungsraten zeigt ebenso wie die Regressionsanalyse des Abschnittes 4.2 eine deutliche Änderung in der Struktur der Rentenzugangsalter, die mit den Reformen der gesetzlichen Rahmenbedingungen hoch korreliert sind. Die Untersuchung der These von JACOBS, KOHLI UND REIN, die nachfrageinduzierte Verhaltensänderung der Arbeitnehmer in Zeiten hoher Arbeitslosigkeit vermuten, ergibt keine klaren Aussagen. Die statistische Evidenz spricht eher gegen diese These.

Das klare Ergebnis der makroökonometrischen Studie ist, daß die Wahl des Rentenalters älterer Arbeitnehmer stark mit den Änderungen der staatlichen Anreize des Rentenversicherungssystems korrespondiert. Sie zeigen, daß das Verhalten der älteren Erwerbstätigen den gesetzlichen Regeländerungen unmittelbar zeitlich folgt.

Die Architektur der Regressionsmodelle als Mittel zu Korrelationsanalysen erlauben weder kausale Aussagen im Sinne einer Bestimmung des Ruhestandsverhaltens durch die gesetzlichen Regelungen, noch liefern sie quantitative Ergebnisse in dem Sinne, daß mit ihnen die Wirkungen zukünftiger Rentenreformen, etwa der Reform von 1992 prognostiziert werden könnten. Diese wäre jedoch für konkrete Politikempfehlungen notwendig. Mit ihnen könnten Gesetzesänderungen so entworfen werden, daß die 
„Rentennachfrage“ der Arbeitnehmer in erwünschter Weise reagiert, in Richtung auf eine Entlastung des umlagebasierten GRV-Systems.

Hierzu wäre eine Operationalisierung der GRV-Änderungen nötig, am sinnvollsten in der Form einer Quantifizierung der finanziellen Auswirkungen von Regeländerungen aus Sicht des einzelnen bzw. eines repräsentativen potentiellen Pensionärs. Eine solche Analyse macht allerdings auf der hier betrachteten aggregierten Ebene wenig Sinn, so $\mathrm{da} ß$ die Analyse mit einer mikroökonometrischen Untersuchung des individuellen Rentenzugangsverhaltens fortgesetzt wird. Eine solche Analyse wird nun vorgestellt, indem, aufbauend auf den im fünften Kapitel vorgestellten Literaturanalysen, im sechsten Abschnitt ein mikroökonometrisches Verrentungsmodell entwickelt wird, dessen Umsetzung auf die Datenbasis und Durchführung der Schätzung Thema des Kapitels 7 sind. Auf Basis der dort modellierten individuellen Wahl des Rentenalters wird eine Kausalanalyse des staatlichen Einflusses auf die Ruhestandsentscheidung möglich. 
Es ist ein groß Ergetzen, Sich in den Geist der Zeiten zu versetzen; $\mathrm{Zu}$ schaun, wie vor uns ein weiser Mann gedacht, Und wie wir's dann zuletzt so herrlich weit gebracht.

\section{EMPIRISCHE ANALYSEN DER INDIVIDUELLEN RUHESTANDSENTSCHEIDUNG}

Dieses Kapitel beinhaltet einen Überblick über einige in der Literatur veröffentlichte Modelle der Ruhestandsentscheidung auf Basis von Einzeldaten. Es untergliedert sich nach Art der benutzten Modelle und zeichnet auf diese Weise eine „Typologie der Ruhestandsmodelle".

Ziel dieses Abschnittes ist es einerseits, die Vorteile und Schwächen der dargestellten Modelltypen zu diskutieren und daraus eine sinnvolle Modellwahl für die in den nächsten Kapiteln folgende empirische Analyse zu treffen. Weiterhin werden die in den vorgestellten Modellen benutzten Erklärungsvariablen der Ruhestandsentscheidung im Hinblick auf die eigene Untersuchung ausgewertet und aus diesen Informationen Arbeitshypothesen für die eigene empirische Analyse gebildet.

Es ist eine naheliegende Interpretation, daß die Verrentung eines Arbeitnehmers eine Entscheidung über sein Arbeitsangebot ist. In der ökonomischen Theorie der Arbeitsmärkte wird die Angebotsentscheidung in der Regel nicht diskretionär, als Ja / Nein - Entscheidung modelliert, sondern der potentielle Arbeitnehmer wählt eine für ihn optimale Anzahl von Stunden, die er auf dem Arbeitsmarkt anbieten möchte. ${ }^{1}$ Somit ist die Entscheidung, in den Ruhestand zu treten, eine Entscheidung zu 0 Stunden Arbeit. ${ }^{2}$ Die hier angesprochene Optimierung eines Konsumenten über die Entscheidungsgrößen Konsum und Freizeit wurde bereits im zweiten Kapitel einleitend in

\footnotetext{
I Die institutionelle Beschränkung dieses Marktes, daß dort oft nur klar definierte Stundenkontingente nachgefragt werden, ist in diesem Kalkül eine Nebenbedingung.

2 Tatsächlich wird in einem großen Teil der angelsächsischen Studien der Status „Verrentung“ über die Anzahl der Arbeitsstunden gemessen. Dies resultiert aus dem im dritten Kapitel dargestellten Phänomen, daß amerikanische Arbeitnehmer aus Gründen der betrieblichen Rentenversicherung bereits vor einem offiziell möglichen Rentenzugangsalter zur staatlichen Alterssicherung ihre Firma verlassen und die Jahre bis zum Eintritt in die Social Security mit Teilzeitarbeit überbrücken. In der Bundesrepublik sind die „Zustände“ der Arbeitnehmer stärker institutionell motiviert, diese sind entweder in (GRV-) Rente oder berufstätig. Durch die im dritten Kapitel beschriebenen Sozialplanund 59er Regelungen werden allerdings auch in Deutschland die Grenzen undeutlicher.
} 
Bezug auf die Lebenszyklushypothese des Sparens angesprochen. Mit demselben Ansatz kann auch die intertemporale Arbeitsangebotsentscheidung modelliert werden. In diesem Fall weist die Budgetbeschränkung eine Unstetigkeit auf, indem ab einem gesetzlich vorgegebenen Alter ein externes Renteneinkommen bezogen werden kann, welches jedoch niedriger ist als das bisher bezogene Arbeitseinkommen. Aus der Arbeitsmarktsicht ist die Verrentung also eine Frage der Erwerbsbeteiligung (,labor force participation") älterer Menschen.

Neben diesen auf der Arbeitsmarktforschung basierenden Modellen haben sich in der Altersökonomik explizite Modelle der Ruhestandsentscheidung etabliert, die stärker auf den Status (erwerbstätig, arbeitslos, verrentet) der beobachteten Personen abstellen. Die wichtigsten Typen dieser Ruhestandsmodelle werden im vorliegenden Kapitel diskutiert.

\subsection{Zeitkonstante Wahrscheinlichkeitsmodelle}

Im Gegensatz zu vielen arbeitsmarktökonomischen Ansätzen, die eine stetige Wahl eines in Stunden gemessenen Arbeitsangebotes modellieren, beschreiben Ruhestandsmodelle die Wahl eines Status (erwerbstätig, Rentner u.a.) als Entscheidung zwischen einer diskreten Anzahl von Alternativen. ${ }^{3}$ Für deren ökonometrische Modellierung bieten sich Discrete Choice Modelle an, deren Konzept die Beschreibung von Wahrscheinlichkeiten für die betrachteten Zustände ist. ${ }^{4}$ Die individuellen Wahrscheinlichkeiten können für jede beobachtete Person auf Basis ihrer persönlichen Charakteristika ermittelt werden. Die Alternative mit der höchsten Wahrscheinlichkeit wird durch das Modell als Verhaltensprognose für die untersuchten Einzelpersonen vorhergesagt. Die bekanntesten Modelle dieser Klasse sind das LOGIT- und das PROBIT-Modell, deren Namen sich aus den zugrundeliegenden Verteilungsfunktionen (Wahrscheinlichkeitsdichtefunktionen) ableitet. ${ }^{5}$

AARTS UND DE JONG (1993) untersuchen die Frühverrentung holländischer Arbeiter mit besonderem Bezug auf die niederländische Erwerbsunfähigkeitsversicherung (Social Security Disability Insurance Program). Sie modellieren die Wahrscheinlich-

${ }^{3}$ Dies gilt auch, wenn nicht nur die beiden Möglichkeiten Arbeit und Rente, sondern auch weitere Formen (Arbeitslosigkeit, Teilzeitbeschäftigung) betrachtet werden.

${ }^{4}$ Vgl. etwa MADDALA (1983).

5 Vgl etwa MAIER UND WEISS (1990), inbes. Seiten $121 \mathrm{ff}$ oder RONNING (1991), Seite 29 ff. 
keit, daß eine beobachtete Person einen vorgezogenen Ruhestand gewählt hat ${ }^{6}$ als die Wahrscheinlichkeit:?

(5-1) Prob $(E U)=1$ wenn $\quad \operatorname{Prob}(\mathrm{U}(\mathrm{EU})-\mathrm{U}(\mathrm{A})>0)$

mit: Prob Wahrscheinlichkeit (Probability),

EU Erwerbsunfähigkeitsrente,

U Nutzen,

A Arbeit,

wobei die Nutzen für die Alternativen EU und A Funktionen von Einkommen und Freizeit sind und der gesamte Ausdruck der Nutzendifferenz modelliert wird in Abhängigkeit von den Erwartungswerten für Einkommen und Freizeit sowie einem Nutzenparameter:

(5-2) Prob $(\mathrm{EU})=f(\mathrm{GW}(\mathrm{Y}), \mathrm{GW}(\mathrm{L}), \beta)$.

mit GW Gegenwartswert (Diskontfaktor $\delta$ ),

$\mathrm{Y}$ Differenz der Erwartungseinkommen $\mathrm{EY}_{\mathrm{A}}-\mathrm{EY}_{\mathrm{EU}}$,

$\mathrm{EY}_{\mathrm{A}}$ erwartetes Arbeitseinkommen,

EY $_{\mathrm{EU}}$ erwartetes Renteneinkommen,

L (Nutzen aus) erwartetem Freizeitgewinn

tion).

$\beta \quad$ Nutzenparameter, mit $\beta=\mathrm{f}$ (Behinderungsgrad, Arbeitsvertrag und Arbeitsmarktsitua-

Durch diese Art der Modellierung über Gegenwartswerte künftiger Ein- und Auszahlungen wird das Alter der Personen zu einem wichtigen Einflußfaktor der Wahrscheinlichkeit einer Verrentung. Dies ist intuitiv einleuchtend. Die Erwartungswerte werden in Abhängigkeit entsprechender Kovariate modelliert.

Die Autoren schätzen eine reduzierte Form dieses Modelles mit Hilfe einer PROBITRegression, wobei sich die folgenden Einflußgrößen als wichtigste Determinanten ergeben:

1. Einkommensverlust (-)

2. Wiedereinstellungswahrscheinlichkeit auf dem Arbeitsmarkt (-) 8

3. geistige Behinderung (+)

3. Aufstiegschancen in der Firma (-)

5. körperliche Behinderung (+)

${ }^{6}$ Gemessen dadurch, daß er zum Zeitpunkt der Befragung eine Erwerbsunfähigkeitsrente bezieht.

7 AARTS UND DE JONG (1993), Seite 34.

${ }^{8}$ Diese Variable ist im Zusammenhang mit der Arbeitslosigkeitsvariable (Nummer 8) zu sehen, die erwartungsgemäß einen positiven Einfluß auf die EU-Wahrscheinlichkeit hat. Gegeben diese positive Wirkung ist die negative Richtung der persönlichen Wiedereinstellungswahrscheinlichkeit plausibel. 
6. Gesundheitsindikator (+) (Kumulierte Krankheitsjahre / Alter)

7. Freizeitgewinn (+)

8. Wahrscheinlichkeit für Arbeitslosigkeit (+)

8. Arbeitszufriedenheit $(+)^{9}$

Die Vorzeichen in Klammern stellen die Richtung des Einflusses auf die Wahrscheinlichkeit einer EU-Rente dar. Damit spielen die Gesundheitsvariablen geistige und körperliche Behinderung sowie der konstruierte Gesundheitsindikator (6.) ebenso wichtige Rollen wie die ökonomischen Indikatoren des Einkommens und der Aufstiegschancen, wogegen die Abwägung des Freizeitwertes und die Arbeitszufriedenheit keinen dominanten Einfluß ausüben.

Die wichtigste Erklärungsgröße „Barwert der Einkommensverluste bei Verrentung“ spiegelt dabei die Regelungen der gesetzlichen Rentenversicherung wider, so daß auch in dieser mikroökonometrischen Studie das Ergebnis des vierten Kapitels, die Dominanz der Anreizwirkungen durch das GRV-System, für die Niederlande unterstützt wird.

Die Autoren modellieren das Alter nicht als explizite Erklärungsvariable, ${ }^{10}$ gleichwohl übt es als Bestimmungsgröße erheblichen Einfluß aus, da es zur Definition vieler Variabler verwendet wird. Dies gilt außer den Barwerten unter andrem für die Einkommensvariable und den Gesundheitsindikator. AARTS UND DE JONG interpretieren die Insignifikanz der direkten Altersvariable als Gütekriterium für ihr Modell, da die Alterseffekte sämtlich durch strukturelle Variablen abgedeckt seien.

Im Gegensatz dazu modelliert BÖRSCH-SUPAN (1992 e) die Ruhestandswahrscheinlichkeit in einem LOGIT-Modell, in dem er das Alter der untersuchten Personen als Indikatorvariablen explizit in die Regression einbezieht." Die Altersvariablen sind auch in diesem Modell insignifikant, was Ausdruck der richtigen Spezifikation inhaltlicher Variablen ist. Dies ist vor allem die ökonomische Variable „Optionswert“, der

9 Vergleiche AARTS UND DE JONG (1993), Tabelle 2.5, Seite 40. Die Relevanz der Einflüsse wird durch Multiplikation der geschätzten Koeffizienten mit den Stichproben-Standardfehlern der entsprechenden Variablen gebildet. Auf diese Weise entsteht eine zwischen den exogenen Variablen vergleichbare Meßgröße, die in der Dimension „Änderung der Wahrscheinlichkeit bei Änderung der erklärenden Variablen um eine Standardabweichung“ einem Elastizitätskonzept nahe kommt.

${ }^{10}$ Mit der Begründung, es sei in Vorstudien insignifikant gewesen: AARTS UND DE JONG (1993), Seite 39, vergleiche aber auch die Interpretation Seite 43.

11 BÖRSCH-SUPAN (1992 c), Seite 548 ff. 
unten (Kapitel 5.3) eingehend besprochen wird, sowie Gesundheitsvariablen und das Geschlecht.

1. Optionswert (vergleiche unten, Kap. 5.3) (-)

2. Nächte im Krankenhaus in den letzten drei Monaten (-)

3. weibliches Geschlecht (+)

4. Anzahl Arztbesuche in den letzten drei Monaten ( + )

Trotz der Relevanz zweier (von 5) Gesundheitsindikatoren, ist eine inhaltlich sinnvolle Aussage in diesem Punkt nicht möglich. Die Anzahl der Arztbesuche erhöht die Wahrscheinlichkeit, in Rente zu sein, 12 wogegen die Anzahl der Nächte im Krankenhaus diese senken, ein kontraintuitives Ergebnis. Der Autor bezeichnet die verfügbaren Gesundheitsvariablen als unzureichende Approximationen des wirklichen Gesundheitszustandes.

Auch in dieser Untersuchung zeigt sich die ökonomische Erklärungsgröße (Optionswert) als wichtigster Einflußfaktor der (bereits getroffenen) Rentenentscheidung.

Eine weitere Forschungsarbeit zeitpunktbezogener Ruhestandswahrscheinlichkeiten führen KRUEGER UND PISCHKE (1991) für die Vereinigten Staaten durch, die allerdings die Zeitdimension durch eine Kohortenanalyse explizit modellieren. Die Autoren bilden auf Basis einer wiederholten Befragung ${ }^{13}$ ein Quasi-Panel, mit dem sie gepoolte Regressionen durchführen und die Einflüsse von Alter und Beobachtungsjahr quantifizieren. Kernanliegen der Arbeit ist die Untersuchung der „Notch-Generation“, damit sind die Kohorten gemeint, die durch die amerikanische Rentenrechtsänderung von 1977 betroffen waren. Diese zeitpunktbezogene Regeländerung sorgte dafür, daß Arbeitnehmer ab dem Geburtsjahrgang 1917 bei gleichen Beiträgen zur Rentenversicherung substantiell niedrigere Pensionen bekamen als Personen, die vor diesem Zeitpunkt geboren sind. Aus dieser Fragestellung ergibt sich der Ansatz, Alter und Rentenzugangsjahre getrennt zu betrachten, also Kohorten zu unterscheiden.

\footnotetext{
12 An dieser Stelle ist wiederum die Kausalität nicht eindeutig. Sind die häufigen Arztbesuche Indikator für einen schlechten Gesundheitszustand oder haben Rentner mehr Zeit, zum Arzt zu gehen ?

13 Datengrundlage ist der Current Population Survey (CPS) für die Jahre 1976 bis 1988. Die untersuchten Daten sind keine Mikrodatensätze, sondern Mittelwerte aus 117 Zellen einer Matrix der Dimensionen „Geburtsjahr / Kalenderjahr“ KRUEGER UND PISCHKE (1991), Seite 12.
} 
Entsprechend haben sowohl Alters- als auch Kohortenvariablen hohe Signifikanz in den Regressionsanalysen. ${ }^{14}$ Das Ergebnis der Studie zeigt allerdings nicht den erwartet starken Einfluß der Rentenanwartschaft, weder in absoluter Höhe noch in Wachstumsraten, sobald die Kohortenkontrolle in die Schätzung genommen wird. Daher lautet das Resümee, daß es in den Vereinigten Staaten nicht die Renten aus der gesetzlichen Sozialversicherung sind, die die Wahl des Rentenalters dominieren. Dies ist jedoch ein landesspezifisches Ergebnis, welches den wesentlich geringeren Anteil der „Säule“ gesetzliche Rentenversicherung in den USA widerspiegelt. Die Arbeiten von STOCK, WISE UND LUMBSDAINE, die unten vorgestellt werden, zeigen tatsächlich auf, daß in den Vereinigten Staaten die betriebliche Altersversorgung einen wesentlich stärkeren Einfluß auf den Zeitpunkt des Ausscheidens aus dem Arbeitsmarkt hat als die gesetzliche.

Die in diesem Abschnitt besprochene Modellklasse zeitkonstanter Wahrscheinlichkeitsmodelle hat den Vorteil, relativ einfach auf einen gegebenen Querschnittsdatensatz anwendbar zu sein, indem modelliert wird, ob sich die Befragungspersonen $z u$ diesem Zeitpunkt im Ruhestand befinden oder nicht. Damit sind grundlegende Aussagen über unterschiedliche Eigenschaften von Rentnern und Erwerbstätigen möglich, es ist jedoch nicht möglich, den Übergang in den Ruhestand zu modellieren. Dies erweist sich als Nachteil dieser Modelle, da es für politikrelevante Fragestellungen darauf ankommt, das zukünftige Verhalten der Erwerbstätigen und ihrer Ruhestandsentscheidung $\mathrm{zu}$ modellieren und prognostizieren. Ohne eine Information über diesen Entscheidungsproze $B$ selbst, ist eine solche Aussage jedoch nur schwer möglich. Es kommt deshalb darauf an, die zeitliche Dimension des Übergangs vom Erwerbsleben in den Ruhestand zu berücksichtigen und diese Dynamik im verwendeten Modell abzubilden.

\subsection{Zeitdynamische Ansätze}

Das „klassische“ Modell ökonomischer Modellierung individueller Entscheidungsprozesse im intertemporalen Kontext ist das der ,dynamischen Programmierung" oder „Optimal Control“.15 Da die Modellierung auf einem individuellen Kalkül einzelner Personen beruht beruht, ist es sehr gut für mikroökonometrische Modelle geeignet, es

\footnotetext{
14 KRUEGER UND PischKe (1991) Table 4, Seite 19.

15 Siehe etwa KAMIEN UND SCHWARTZ (1981) oder BELLMANN (1971).
} 
findet aber auch weite Anwendung in der makroökonomischen Wachstumstheorie. Grundidee dieser Ansätze ist die intertemporale Nutzenmaximierung. Allerdings erfordern Dynamic-Programming Ansätze einen sehr hohen programmiertechnischen Aufwand und benötigen sehr lange Rechenzeiten.

Die zweite hier beschriebene zeitdynamische Schätzmethode ist die der Verweildaueranalyse, die sich inzwischen in der Literatur zu Rentenalterentscheidungen weitgehend durchgesetzt hat. Verweildauermodelle sind nicht nur einfacher $\mathrm{zu}$ handhaben und schneller zu rechnen, sondern sie sind von der Modellarchitektur her für die betrachtete Fragestellung besonders gut geeignet. Abhängige Variable ist die Zeitdauer eines $\mathrm{Zu}$ standes, im vorliegenden Fall des Erwerbslebens. Aus diesem Grunde muß nicht wie in der dynamischen Programmierung der Gesamtzeitraum modelliert werden, sondern eine individuelle Nutzenabwägung, gegeben den erreichten Status (Anzahl der Arbeitsjahre, eingezahlte Beiträge) kann für jeden Zeitpunkt des Erwerbslebens getroffen werden.

Tatsächlich sind die beiden Modellklassen eng verwandt und lassen sich oft in das jeweils andere umformulieren. Von der Grundidee her ist das Verweildauermodell eine Vereinfachung der dynamischen Programmierungsansätze.16

\subsubsection{Modelle der dynamischen Programmierung}

Auf Basis einer longitudinalen Datenerhebung, des amerikanischen Retirement History Survey (RHS) modellieren GUSTMAN UND STEINMEIER (1991) ein Lebenszyklusmodell des intertemporalen Arbeitsangebotes. Die Basis ihrer Überlegungen bildet die von ihnen beobachtete Tatsache, daß Arbeitnehmer, die von einer Vollzeitbeschäftigung in eine Teilzeittätigkeit wechseln, dabei niedrigere Stundenlöhne in Kauf nehmen müssen, unabhängig davon, ob sie die Firma wechseln oder nicht. Vor diesem Hintergrund wird die Entscheidung zwischen

- Vollzeiterwerbstätigkeit,

- Teilzeitbeschäftigung und

- (Vollzeit-) Ruhestand

modelliert als intertemporale Nutzenmaximierung unter der Nebenbedingung einer nichtlinearen Budgetbeschränkung. ${ }^{17}$

16 Vgl. LUMSDAINE, STOCK UND WISE (1992).

17 GUSTMAN UND STEINMEIER (1991), Kapitel 2, Seite 556-560. 
Das ökonometrische Modell wird geschätzt auf den ersten 4 Wellen des RHS (1969 1975), so daß maximal drei Übergänge von einem Status zum andern beobachtet werden können. Die ökonometrisch behandelte Frage ist, bis zu welchem Zeitpunkt in einem Zustand verharrt werden sollte, bevor der Wechsel in einen andern effizient ist. Diese Fragestellung macht die Thematik zu einem „optimal stopping“ Problem. ${ }^{18}$ Die Lohngleichung beinhaltet drei Komponenten: Lohn (in Abhängigkeit von der Wochenstundenzahl), staatliche Rente und sonstige Rente (betrieblich und privat). Der von der Wochenstundenzahl abhängige Stundenlohn wird in einer ersten Schätzstufe per linearer Regression ermittelt und dient als erklärende Variable in den Modellen, die variable Stundenlöhne unterstellen. Die Autoren unterscheiden vier Szenarien:

- unterschiedliche Lohnsätze, flexible Wochenstunden bei Teilzeitarbeit,

- fester Lohnsatz, flexible Wochenstunden bei Teilzeitarbeit,

- unterschiedliche Lohnsätze, fixe Wochenstunden bei Teilzeitarbeit,

- fester Lohnsatz, fixe Wochenstunden bei Teilzeitarbeit, die in einzelnen Regressionsmodellen geschätzt werden.

Außer einer Indikatorvariable für einen langfristig schlechten Gesundheitszustand spezifizieren die Autoren in der Schätzung keine weiteren Kovariate, so daß lediglich das Alter, der Geburtsjahrgang und der imputierte Lohn als erklärende Größen eingehen. Darüber hinaus werden Parameter für die Freizeitpräferenz geschätzt, die sich aus der Struktur der intertemporalen Nutzenmaximierung ergeben. Als wichtigste Erklärungsgrößen ergeben sich die Freizeitpräferenz (relatives Gewicht der Freizeit gegenüber Konsum) und der Gesundheitsindikator. ${ }^{19}$

Die überaus komplizierte Modellstruktur erlaubt keine weitere Untersuchung von Kovariaten. ${ }^{20}$ Die Autoren errechnen aber aus den finanziellen Anreizen die altersspezifischen Rentenzugänge und können mit dem vollständigen Modell die Häufigkeitsverteilung der Rentenzugänge nach Alter ${ }^{21}$ reproduzieren. Diese korrekte Simulation der gewählten Rentenalter resultiert aus der Berücksichtigung der altersspezifischen Ren-

18 Diese Formulierung zeigt die Nähe zu Verweildauermodellen.

${ }^{19}$ Ebenda, Tabelle III, Seite 575.

${ }^{20}$ Dies gilt für die intertemporale Nutzenfunktion, die in der Datenbasis vorhandene individuellen Informationen werden in der Lohnermittlung (Regressionsergebnisse in Tabelle II, Seite 568) benutzt und gehen damit indirekt in die Nutzenermittlung ein.

21 Diese zeigen die häufigsten Rentenzugangsalter bei 62, 64 und 65 Jahren (GUSTMAN UND STEINMEIER (1991), Seite 579), die in der vorliegenden Arbeit bereits im 2. Kapitel, Abbildung 2-4 dargestellt wurden. 
tenzahlungen, ${ }^{22}$ so daß auch diese Studie die Hypothese unterstützt, daß sich das Rentenzugangsverhalten von Arbeitnehmern an den finanziellen Anreizen der Sozialversicherung orientiert.

Die bereits erwähnten Problem bei der empirischen Umsetzung dynamischer Optimierungsmodelle, der erhebliche Programmierungs- und Rechenaufwand, die auch die Möglichkeiten des GUSTMAN UND STEINMEIER Modelles einschränken, werden in einer Arbeit von BERKOVEC UND STERN (1991) durch die neue Schätzmethode der simulierten Momente verringert, ${ }^{23}$ so da $B$ die Autoren die Verrentungsentscheidung in einem breiteren Rahmen von Erklärungsvariablen beschreiben. Allerdings ist die strukturelle Problematik dieser Modellklasse damit nur verringert und nicht aufgehoben. So nennen die Autoren bereits in der Einleitung wünschenswerte Erweiterungen des Modelles, die aber „die EDV-technischen Anforderungen des Schätzverfahrens steigern würden". ${ }^{24}$

Das Modell erlaubt, wie das von GUSTMAN UND STEINMEIER, drei verschiedene Status der Beobachtungspersonen: Erwerbstätigkeit, Teilzeitarbeit und Ruhestand. Allerdings wird die Teilzeitarbeit hier nicht flexibel (in Stunden) gemessen, sondern ist ein fest definierter Zustand. Zielgröße der dynamischen Optimierung ist der erwartete Wert $\operatorname{EV}(Z, t, s)$ der drei Zustände $Z \in\{F, P, R\}$, mit

$\mathrm{t}=$ Aktuelles Alter,

$s=$ Eintrittstermin in der aktuellen Firma,

F = Vollzeiterwerbstätigkeit,

$P=$ Teilzeitarbeit und

$\mathbf{R}=$ Ruhestand.

Der Lohn setzt sich hier aus einem zufälligen und einem deterministischen Teil zusammen, der unter anderem von der Zugehörigkeit zur Firma (t-s) abhängt, so daß diese Zugehörigkeit als Zustandsgröße modelliert werden kann.

22 Ebenda, Seite 580.

${ }^{23}$ Das rechentechnische Problem besteht darin, daß zur Lösung der dynamischen Optimierung die Zielfunktion mehrdimensionale Integrale enthält. Da diese nicht explizit gelöst werden können, müssen deren erste Ableitungen während des Optimierungsprozesses numerisch ermittelt werden. Diese Ermittlung übersteigt bereits ab geringen Dimensionen des Problems die EDV-technischen Möglichkeiten.

Die „Method of simulated moments“ löst dieses Problem, indem die gesuchten Funktionswerte nicht errechnet, sondern auf Basis einer unterstellten Verteilung mit Pseudo-Zufallszahlen simuliert werden. Dies verringert den Rentenaufwand erheblich, ohne dabei wesentlich Verzerrungen zu ergeben. Vgl etwa McFadden (1989).

${ }^{24}$ BERKOVEC UND STERN (1991), Seite 190. 
Zur ökonometrischen Schätzung dieses Modelles benutzen die Autoren einen amerikanischen Panel-Datensatz, den National Longitudinal Survey of Mature Men (NLS), aus der ihnen knapp 2500 Erwerbshistorien von Männern aus den Jahren 1966 bis 1983 zur Verfügung stehen. Die Autoren beobachten neben den ,erwarteten“ Zustandsübergängen von der Erwerbstätigkeit in den Ruhestand auch eine erhebliche Zahl umgekehrter Wege, also Rentner, die trotz Bezuges einer Rente wieder eine (volloder teilzeit) Arbeit aufnehmen. ${ }^{25}$

Die Schätzung beinhaltet das errechnete Einkommen für eine Vollzeitbeschäftigung, die strukturellen Variablen sowie Indikatoren für Ausbildung, Hautfarbe, Alter und Gesundheit. Diese Einflußgrößen werden spezifisch für alle drei Status geschätzt. Darüber hinaus testen die Autoren, ob die Struktur des modellierten Prozesses dynamisch ist oder nicht. Sie fixieren zu diesem Zweck den internen Diskontsatz $\beta$ in einem Fall auf 0 (,statisches Modell“) und im anderen auf 0,95 (,dynamisches Modell“).

Tabelle 5-1: Regressionsergebnisse der Studie von BERKOVEC UND STERN

\begin{tabular}{|c|c|c|c|c|c|c|}
\hline Variable & $\begin{array}{r}\text { Rut } \\
\text { statisch }\end{array}$ & $\begin{array}{l}\text { estand } \\
\text { dynamisch }\end{array}$ & $\begin{array}{r}\text { Teil } \\
\text { statisch }\end{array}$ & $\begin{array}{l}\text { itarbeit } \\
\text { dynamisch }\end{array}$ & $\begin{array}{r}\text { Vollzeit E } \\
\text { statisch } \\
\end{array}$ & $\begin{array}{l}\text { bstätigkeit } \\
\text { dynamisch }\end{array}$ \\
\hline Bildung & - & - & & & + & ++ \\
\hline Hautfarbe & - & - & & & - & \\
\hline Alter & + & + & + & & - & + \\
\hline Alter $^{2}$ & - & & & & & - \\
\hline Gesundheitsprobleme & ++ & ++ & & + & - & - \\
\hline Firmenzugehörigkeit & .1. &. & & & ++ & \\
\hline Transferkosten & & Statisch: & ++ & dynamisch & +++ & \\
\hline
\end{tabular}

Quelle: Berkovec Und STERn (1991) Tabelle VI, eigene Berechnung und Darstellung. Die Vorzeichen stellen die Vorzeichen der Regressionskoeffizienten dar, die Anzahl der Zeichen verdeutlicht die relative Stärke der statistischen Signifikanz innerhalb des Status;.$/ .=$ in diesem Status nicht implementiert; kein Eintrag = nicht statistisch signifikant.

Das Ergebnis der Regressionsanalyse ist in Tabelle 5-1 zu einer Übersicht zusammengestellt. Die statische und die dynamische Analyse stellen jeweils eine Schätzung dar, in der für die drei Status sowohl ein Absolutglied als auch die exogenen Variablen getrennt geschätzt werden. Lediglich die aus der Lohngleichung ermittelte Erklärungsgröße „Transferkosten“ geht nur einmal in die Gleichung ein und quantifiziert damit den Einfluß der Kosten eines Wechsels von einem beliebigen Zustand in einen andern.

${ }^{25}$ BERKOVEC UND STERN (1991), Tabellen III, IV und V, Seiten $195 \mathrm{f}$. 
Die abhängige Variable ist die Wahrscheinlichkeit der Wahl einer der drei Alternativen und folgt einer multinomialen LOGIT-Spezifikation. ${ }^{26}$ Die Tabelle zeigt die Vorzeichen und die statistische Signifikanz der exogenen Erklärungsvariablen des Modelles, die Strukturvariablen des dynamischen Optimierungsmodells sind nicht aufgeführt.

Der Vergleich des statischen und dynamischen Ansatzes zeigt weitgehend gleiche Ergebnisse, lediglich im Teil der Vollerwerbstätigkeit zeigen sich deutliche Abweichungen, indem die Einflußgröße Alter vom statischen Modell als hochsignifikant negativ, vom dynamischen jedoch als positiv auf die Wahrscheinlichkeit eines Vollzeitberufes ermittelt wird. Die Dauer der Firmenzugehörigkeit wird nur im statischen Modell identifiziert und steigert dort die Wahrscheinlichkeit der vollen Erwerbstätigkeit. Die Autoren stellen heraus, daß es keine Möglichkeit der Wertung der beiden Modelle gegeneinander gibt, ${ }^{27}$ bewerten jedoch anhand möglicher Indikatoren und eines Vergleichs der erzeugten mit den tatsächlichen Hazard-Verläufen die Anpassung des dynamischen Modelles als besser.

Als wichtigste Einflußgröße werden die für alle Zustände implementierten Transferkosten ermittelt. Der Faktor Gesundheit erweist sich auch in diesem Ansatz als wichtige Einflußgröße, Krankheit erhöht erwartungsgemäß die Wahrscheinlichkeit der Verrentung und senkt sie für Vollerwerbstätigkeit. Der Tatsache, daß mit zunehmendem Alter die Wahrscheinlichkeit der Verrentung steigt, wird lediglich in der dynamischen Spezifikation dadurch widersprochen, daß auch die Wahrscheinlichkeit einer Vollzeitarbeit positiv mit dem Alter korreliert. Ein im Vergleich zu den bisher genannten Studien neuer Aspekt ist der der Ausbildung, die die Wahrscheinlichkeit einer längeren Erwerbstätigkeit erhöht und die einer vollständigen Verrentung senkt. Diese Variable kann als Indikator sowohl für Arbeitsbedingungen, Arbeitszufriedenheit, aber auch Motivation des Arbeitnehmers interpretiert werden. Die Bedeutung der Hautfarbe ist ein US-amerikanischer Effekt, der in vielen Studien ermittelt wird. Die geringere Wahrscheinlichkeit einer frühen Verrentung dürfte in diesem Modell daraus herrühren, daß die Hautfarbe nicht in der Lohngleichung spezifiziert ist, so daß unter der Annahme, daß nicht-Weiße ein geringeres Einkommen haben, dieser Nachteil durch eine längere Lebensarbeitszeit kompensiert werden muß.

\footnotetext{
${ }^{26}$ Ebenda, Gleichung (4.5), Seite 198. Die LOGIT-Form ergibt aus der unterstellen Extremwertverteilung.

${ }^{27}$ Der Versuch, den für die Dynamik verantwortlichen Diskontfaktor $\beta$ in der Schätzung zu identifizieren, mißlang.
} 


\subsubsection{Hazardratenmodelle}

Das zuletzt vorgestellte dynamische Programmierungs-Modell von BERKOVEC UND STERN beinhaltete bereits wesentliche Elemente der nun zu diskutierenden Verweildauermodelle:

- Unterscheidung unterschiedlicher Zustände (Status) der Beobachtungspersonen,

- Betrachtung von Übergängen und Übergangszeitpunkten zwischen diesen Zuständen,

- Bestimmung dieser beiden Kenngrößen auf Basis von Übergangswahrscheinlichkeiten.

Die formale Darstellung von Verweildauer- oder Hazardratenmodellen erfolgt im Kapitel 6, so daß der Fokus an dieser Stelle die inhaltliche Ausgestaltung und die Ergebnisse der Modelle vorgestellt werden.

In einer ausführlichen Analyse untersucht SUEYOSHI (1989) die Determinanten von Voll- und Teilverrentung in den USA für den Zeitraum von 1969 bis 1979. Als Datenbasis dient ihm der (bereits bei GUSTMAN UND STEINMEIER angesprochene) Retirement History Survey RHS der amerikanischen Rentenversicherung, aus dem ihm 5 Ziehungswellen mit Beobachtungsreihen von gut 1600 Personen zwischen 58 und 63 Jahren zur Verfügung stehen. ${ }^{28}$

Auch SUEYOSHI stellt heraus, daß nicht nur die beiden Status „erwerbstätig“ und „verrentet“, sondern auch die Möglichkeit eines „Teilruhestandes“ in einer Schätzung der Rentenzugänge berücksichtigt werden sollte. Für sein Übergangsmodell bedeutet dies, daß die Individuen im Anfangszustand „erwerbstätig“ zwei verschiedenen „Risiken29“" unterliegen: dem einer Teilverrentung oder dem eines vollständigen Ruhestandes. Im Falle unterschiedlicher Zielzustände werden diese als „konkurrierende Risiken“ („,competing risks“) bezeichnet. SUEYOSHI modelliert die Ruhestandsentscheidung durch einen breiten Kranz erklärender Variabler, die er in die Hauptpunkte Berufsvariablen, ökonomische Kenngrößen und Kontrollterme unterteilt (s.u.). Die Spezifikation des Schätzmodelles ergibt sich, wie in Kapitel 6 näher beschrieben wird, als die bedingte Wahrscheinlichkeit, in einem bestimmten Alter $t$ in einen der betrachteten Zustände zu wechseln, unter der Bedienung, daß bis zu diesem Alter gearbeitet wurde. Damit ergibt sich die Hazard-Rate als (5-3).

28 SUEYOSHI (1989), Seite $12 \mathrm{f}$.

29 Der Begriff des „Risiko“ entspringt der Terminologie der Verweildauermodelle, die in den ursprünglichen Anwendungen etwa Werkstücke beschrieben, die dem Risiko eines Defektes unterliegen. 


$$
\lambda_{j}\left(t, X_{j}, \beta_{j}, v_{j}\right)=v_{j} \cdot \lambda_{0,}(t) \cdot \Phi_{j}\left(X_{j}, \beta_{j}\right)
$$

mit: $\quad \lambda_{j} \quad$ Hazard-Rate für das Risiko $j$;

j „Risiko“: Teilruhestand, Vollruhestand;

t Entscheidungszeitpunkt $t$;

$\mathrm{X}_{\mathrm{j}} \quad$ Matrix der exogenen Variablen für Risiko $\mathrm{j}$ zum Zeitpunkt $\mathrm{t}$;

$\beta_{\mathrm{j}} \quad$ Koeffizientenvektor für erklärende Variable und Risiko j;

$v_{j} \quad$ nichtnegativer Fehlerterm, der unbeobachtete Eigenschaften repräsentiert;

$\Phi \quad$ stetig differenzierbare, nicht negative Aggregationsfunktion von $\mathfrak{R}^{\mathbf{k}} \rightarrow \mathfrak{R}^{+}$

$\lambda_{\text {oj }}(t)$ Baseline-Hazard (vgl. Kapitel 6).

Von den von ihm untersuchten 1633 Personen gingen im Verlaufe des zehnjährigen Beobachtungszeitraumes 1490 in Ruhestand, für 143 Personen konnte kein Ruhestandstermin beobachtet werden, d.h. sie arbeiteten über die Beobachtungsdauer hinaus, oder konnten aus anderen Gründen nicht mehr befragt werden. Die beobachteten Rentenzugänge sind in Abbildung 5-1 dargestellt. Die „empirische Dichte“ (linke Achse) zeigt den Prozentanteil der Einzelalter an allen Rentenzugängen, wogegen die „empirische Hazardfunktion“ (rechte Achse) zeigt, wieviel Prozent der in einem Lebensalter noch erwerbstätigen Personen in Rente gingen. ${ }^{30}$ Diese Maßzahl entspricht den „Verrentungsraten“ des vierten Kapitels.

\section{Abbildung 5-1: Empirische Dichte und Hazardfunktion aus der Stichprobe von Su-} eyoshi

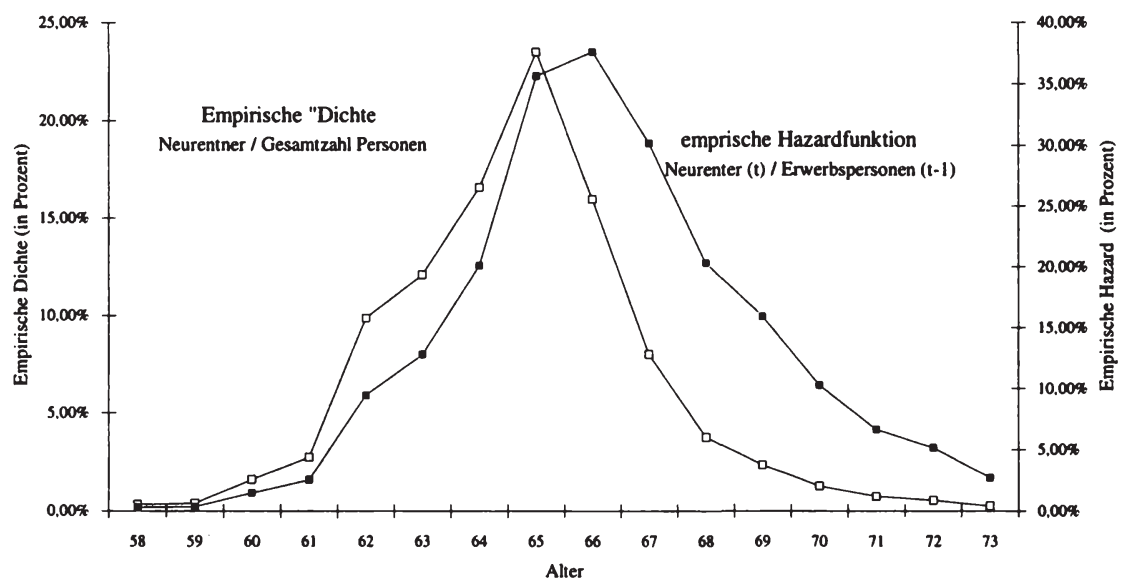

Quelle: SUEYOSH (1989), Tabelle 1, Seite 25, eigene Berechnung.

${ }^{30}$ Die Bezeichnung „empirisch“ wurde als Abgrenzung von den theoretischen Konzepten der Dichte und Hazard gewählt, die hier nicht betrachtet werden 
Tabelle 5-2: Regressionsergebnisse bel SUEYOSHI

\begin{tabular}{|c|c|c|c|c|c|c|}
\hline & \multicolumn{2}{|c|}{ Risiken unabhängig } & \multicolumn{3}{|c|}{ Risiken korreliert } & \multirow[b]{2}{*}{ Teilrente } \\
\hline & Vollrente & Teilrente & Vollrente & Teilrente & Vollrente & \\
\hline \multicolumn{7}{|c|}{ I. Kontrollvariablen (persönliche Charakteristika): } \\
\hline Ausbildung & $\begin{array}{l}-0,020^{*} \\
-1,667\end{array}$ & $\begin{array}{l}-0,028^{*} \\
-1,750\end{array}$ & $\begin{array}{l}-0,034 * * \\
-3,778\end{array}$ & $\begin{array}{l}-0,032 * * \\
-3,200\end{array}$ & $\begin{array}{l}-0,019 * \\
-1,900\end{array}$ & $\begin{array}{l}-0,021^{*} \\
-1,909\end{array}$ \\
\hline Gesundheit eingeschränkt & $0,474 * *$ & $-0,012$ & $0,368 * *$ & $0,268 * *$ & $0,384 * *$ & $0,220 * *$ \\
\hline t-Werte: & 6,493 & $-0,120$ & 5,750 & 3,080 & 5,908 & 2,245 \\
\hline Verheiratet & $-0,480^{* *}$ & 0,001 & $-0, \mathbf{4 4 2 * *}$ & $-0,337 * *$ & $-0, \mathbf{4 4 3} * *$ & $-0,300^{* *}$ \\
\hline 1.Werie: & $-3,582$ & 0,006 & $-4,420$ & $-2,982$ & $-4,430$ & $-2,564$ \\
\hline Hautfarbe nicht weiß & $-0,120$ & $-0,350 * *$ & $-0,089$ & $-0,169^{*}$ & $-0,087$ & $-0,177^{*}$ \\
\hline t-Werte: & $.0,976$ & $-2,134$ & $-0,908$ & $\cdot 1,707$ & $-0,853$ & $-1,788$ \\
\hline Haushaltsgröße & 0,017 & 0,010 & $0,044^{* *}$ & 0,037 & $0,041^{*}$ & 0,034 \\
\hline t-Werte: & 0,654 & 0,263 & 2,095 & 1,609 & 1,952 & 1,417 \\
\hline Pflichtverrentung & $0,466 * *$ & 0,081 & $0,305^{* *}$ & $0,218 * *$ & $0,293 * *$ & $0,164^{*}$ \\
\hline t-Werte: & 6,563 & 0,802 & 4,766 & 2,659 & 4,508 & 1,907 \\
\hline \multicolumn{7}{|l|}{ II. Berufsvariablen: } \\
\hline Bürotätigkeit & $\begin{array}{l}0,073 \\
0,388\end{array}$ & $\begin{array}{l}-0,029 \\
-0,124\end{array}$ & & & $\begin{array}{l}-0,220^{*} \\
-1,864\end{array}$ & $\begin{array}{l}-0,064 \\
-0,474\end{array}$ \\
\hline Handwerk & 0,164 & $0,465^{* *}$ & & & 0,081 & 0,176 \\
\hline t-Werte: & 0,868 & 2,039 & & & 0,653 & 1,275 \\
\hline Arbeiter & $0,407 * *$ & 0,115 & & & $0,221^{*}$ & 0,121 \\
\hline t-Werte: & 2,236 & 0,511 & & & 1,905 & 0,858 \\
\hline Manager & $-0,097$ & $-0,090$ & & & $-0,218$ & $-0,196$ \\
\hline t-Werie: & $-0,473$ & $-0,338$ & & & $-1,580$ & $-1,265$ \\
\hline Technische Berufe & $-0,208$ & 0,236 & & & $-0,209$ & $-0,038$ \\
\hline t-Werte: & $-0,889$ & 0,852 & & & $-1,314$ & $-0,218$ \\
\hline \multicolumn{7}{|c|}{ III. Ökonomische Kenngrößen: } \\
\hline Einkommen & 0,001 & $-0,028 * *$ & $-0,009$ & $-0,014 * *$ & $-0,003$ & $-0,012^{*}$ \\
\hline t-Werte: & 0,100 & $-2,000$ & $-1,286$ & $-2,000$ & $-0,375$ & $-1,714$ \\
\hline Vermögen & 0,009 & $0,036^{* *}$ & 0,011 & 0,016 & 0,015 & $0,022 *$ \\
\hline t-Werte: & 0,060 & 2,250 & 0,917 & 1,455 & 1,154 & 1,833 \\
\hline Betriebsrente Vollzusage & 0,111 & $-0,065$ & 0,077 & 0,037 & 0,090 & 0,032 \\
\hline t-Werte: & 1,144 & $-0,496$ & 0,987 & 0,451 & 1,125 & 0,386 \\
\hline Betriebsrente Teilzusage & $-0,057$ & 0,201 & 0,022 & 0,071 & 0,006 & 0,079 \\
\hline t-Werre: & $-0,594$ & 1,718 & 0,301 & 0,934 & 0,080 & 0,988 \\
\hline Gesetzliche Rente mit 62 & $0,587^{* *}$ & 0,084 & $0,411^{* *}$ & $0,310^{* *}$ & $0,411^{* *}$ & $0,257^{* *}$ \\
\hline t-Werte: & 7,623 & 0,840 & 6,323 & 3,974 & 6,134 & 3,024 \\
\hline Differenz zu 62er Rente & 0,020 & $-0,100^{* *}$ & $-0,039 * *$ & $-0,061^{* *}$ & $-0,035 * *$ & $-0,071^{* *}$ \\
\hline t-Werte: & 1,333 & $-4,762$ & $-3,250$ & $-3,813$ & $-2,692$ & $-4,176$ \\
\hline
\end{tabular}

Quelle: SueYosh (1989), Tabelle 4, Seite 29 f; eigene Darstellung. Die erste Zeile zeigt die Schätzkoeffizienten der verschiedenen Modelle, die zweite Zeile die aus den angegebenen Standardfehlem ermittelten tWerte.

*: $95 \%$ signifikant,

* $90 \%$ Signifikanz.

Die Zahlenangaben der empirischen Dichte stellen die Aufteilung aller im Gesamtzeitraum beobachteten Rentenzugänge auf Einzelalter dar, addieren sich also zu 1, was für die Hazard nicht der Fall ist. Die Hazardrate ist für jedes Lebensalter einzeln definiert 
und somit auf keine Obergrenze skaliert. Interessanterweise zeigt sich jedoch die Struktur der empirischen Hazard sehr ähnlich der Dichte. Dies muß nicht notwendig der Fall sein, so ergaben sich in Kapitel 4 in hohen Altersstufen überproportional hohe Verrentungsraten, die aus der geringen Zahl von Erwerbstätigen in diesen Zellen resultieren. Tatsächlich liegt die empirische Hazardrate einiges höher als die empirische Dichte, was durch die verschiedenen Skalen unterdrückt wird.

Die Schätzergebnisse von SUEYOSHI sind in Tabelle 5-2 abgedruckt. Die Schätzungen werden für die beiden Zustände $\mathrm{j}$ (Teil- oder Vollverrentung) sowohl als einzelne, voneinander unabhängige Entscheidungen spezifiziert als auch als korrelierte Risiken in einer verbundenen Schätzung.

Die Ergebnisse zeigen die Relevanz der in den bisher vorgestellten Analysen nicht oder nur knapp modellierten Einflußfaktoren. Dies gilt vor allem für die persönlichen Charakteristika, abgeschwächt auch für die beruflichen Indikatoren. Die Vorzeichen der Koeffizienten beziehen sich auf die Wahrscheinlichkeit einer (frühen) Verrentung.

Personen mit besserer Ausbildung tendieren daher ebenso zu späterer Verrentung wie verheiratete Personen und nichtweiße, wobei der letzte Effekt nur für eine Teilrente meßbar ist. Dies deutet darauf hin, daß -im Gegensatz zum BERKOVEC UND STERN Modell- die ökonomischen Nachteile von Farbigen in den entsprechenden Variablen richtig modelliert sind, wogegen die negativen Koeffizienten im Falle der Teilverrentung die Schwierigkeiten für Farbige abbilden, eine Teilzeitstelle zu bekommen. Auch ein größerer Haushalt wirkt signifikant auf eine frühe Verrentung hin.

Die Berufsindikatoren zeigen nur in einigen Fällen statistische Signifikanz, das Modell der korrelierten Risiken zeigt mit diesen Variablen eine bessere Anpassung, ${ }^{31}$ was dafür spricht, diese Terme als Kontrollgrößen in einer Rentenzugangsschätzung aufzunehmen.

Auch in dieser Studie sind es erneut die ökonomischen Variablen, die deutliche Signifikanz zeigen, abgesehen von den Betriebsrentenindikatoren. In allen Schätzungen wirkt ein hohes Einkommen negativ auf die Rentenzugangswahrscheinlichkeit, wogegen größeres Vermögen augenscheinlich die Entscheidung zu einer teilweisen Verrentung erleichtert. Die von SUEYOSHI errechneten Indikatoren der gesetzlichen Rente erweisen sich als relevant und zeigen erwartungsgemäße Vorzeichen. Eine hohe Rentenanwartschaft (im Alter von 65 Jahren) erleichtert die Entscheidung zu einer früh-

${ }^{31}$ Gemessen in einem geringeren Wert der Loglikelihoodfunktion, SUEYOSHI (1989), Seite 30. 
zeitigen Verrentung, wogegen die Differenz zwischen der heutigen Rente und dieser Eckrente zu längerem Weiterarbeiten motiviert. Dies kann ein Alterseffekt dergestalt sein, daß jüngere Arbeitnehmer, die noch relativ weit vom 65 . Lebensjahr entfernt sind, eine geringe Rentenanwartschaft aufweisen. Die Wirkungsrichtung entspricht jedoch auch der Idee des im nächsten Unterpunkt 5.3 diskutierten Optionswertes.

Eine Schwäche des SUEYOSHI-Modelles scheint die Modellierung der betrieblichen Altersversorgung zu sein, denn weder haben die benutzten Indikatoren signifikante Wirkungen auf das Verhalten der untersuchten Personen, noch kann der Autor in den auf den Schätzkoeffizienten basierenden Simulationsstudien die tatsächliche Entwicklung der 70er Jahre reproduzieren. ${ }^{32}$ Die Ergebnisse der bisher vorgestellten USamerikanischen Studien legen nahe, daß dies auf die fehlende Möglichkeit der adäquaten Abbildung der Anreize aus betrieblicher Altersversorgung resultiert.

In einer jüngeren Studie für Großbritannien benutzen MEGHIR UND WHITEHOUSE (1993) ebenfalls ein Verweildauermodell zur Beschreibung der Arbeitsmarktaustritte. Das britische System der gesetzlichen Rentenversicherung unterscheidet sich von den bisher beschriebenen vor allem dadurch, daß es keine Flexibilität des Ruhestandsalters gibt. Dies ist für Frauen auf 60 und Männer auf 65 festgelegt. Die Rente setzt sich aus diversen Teilen zusammen; die staatliche Basisrente (,basic state pension“) beträgt zur Zeit pauschal £45,- pro Woche und wird an einen Großteil der Rentenempfänger gezahlt. Der zweite Hauptteil ist das „State Earnings Related Pension Scheme“ (SERPS), das nach einer Rentenformel in Bezug auf das durchschnittliche Lebenseinkommen berechnet wird. ${ }^{33} \mathrm{Zu}$ diesen beiden Grundbestandteilen werden unterschiedliche Alterszusatzbeträge und Übergangsregelungen sowie Sonderleistungen gezahlt. ${ }^{34}$ Die starren Altersgrenzen zusammen mit hohen Mindestversicherungsjahren von 49 Jahren für Männer und 45 Jahren für Frauen führen dazu, daß ein vorgezogener Ruhestand fast immer eine Übergangszeit ohne den Bezug einer offiziellen Rente bedeutet. Aus diesem Grunde können die Autoren für die Untersuchung von Frühverrentung keinen direkten Übergang von Erwerbstätigkeit in einen Rentenstatus betrachten, son-

\footnotetext{
32 ebenda, Seite 24.

33 BRUGiavini, DiSNeY UND Whitehouse (1993), Seite 4 ff.

$34 \mathrm{Vgl}$. CASMIR (1989), Seite $270 \mathrm{ff}$.
} 
dern messen den Übergang von Erwerbstätigkeit in Arbeitslosigkeit.35 $\mathrm{Da}$ das Gesamtmodell somit verschiedene Wege in die unterschiedlichen Arten des Ruhestandes umfaßt (staatliche, betrieblich und private Rentenarten, die zu unterschiedlichen Zeitpunkten beginnen und die Übergänge zwischen diesen Zuständen), erfolgt die empirische Schätzung der Übergangsraten als reduzierte Form, bei der eine Kontrolle für unbeobachtete Heterogenität vorgenommen wird. ${ }^{36}$ Weiterhin werden Kohorteneffekte durch Dummyvariablen kontrolliert.

Die Regressionsergebnisse von MEGHIR UND WHITEHOUSE ergeben folgende Determinanten des Arbeitsmarktaustrittes: ${ }^{37}$

- Gesundheitsindikatoren ( $(+)$

2 Indikatorvariablen für "leichte“ und „erheblich Gesundheitsprobleme“ wirken beide signifikant positiv auf den Arbeitsmarktaustritt;

- Arbeitslosenrate (+) ;

- Indikatorvariable „Ehepartner arbeitet“ (-);

- Berufscharakteristiken von fünf spezifizierten Branchenindikatoren wirkt der für Bürotätigkeit signifikant positiv (+), so daß diese Branche durch frühere Austritte gekennzeichnet ist. In einigen Spezifikationen erweisen sich Manager als länger arbeitende Berufsgruppe. Die Bezugsgruppe ist die der ungelernten Arbeiter, für die also eine spätere Verrentung als für Büroangestellte ermittelt wird;

- Ausbildungsdauer (+) das Polynom 2ten Grades ist gemeinsam signifikant und zeigt eine degressiv steigende Figur in den Ausbildungsjahren nach dem 14. Lebensjahr;

- Kontrollvariablen: Alter, Beschäftigungsdauer. ${ }^{38}$

Es ergeben sich somit auch bei MEGHIR UND WHITEHOUSE für Großbritannien die Indikatoren der Gesundheit als wichtige Einflußgrößen der Entscheidung, die Lebensarbeitszeit zu beenden. Der positive Einfluß der Arbeitslosenrate war angesichts der

35 MEGHIR UND WhITEHOUSE (1993), Seite 3. Die Autoren beziehen sich auf eigene frühere Arbeiten und betonen, daß mit dieser Messung nicht alle Austritte aus dem Erwerbsleben erfaßt werden können, da auch eine Rückkehr aus der Arbeitslosigkeit möglich ist. In diesem Sinne ist der hier modellierte „Ruhestand“" kein absorbierender Endzustand.

${ }^{36}$ Die Kontrolle erfolgt nach der Methode von HECKMAN UND SINGER, es werden drei Subpopulationen modelliert (vgl. SCHNEIDER (1991), Seite $173 \mathrm{ff}$ ).

37 MEghiR UND WhITEHouse (1993), Tabelle 2, Seite 18. Abhängige Variable ist die Rate der Austritte aus dem Arbeitsmarkt, es werden unterschiedliche Spezifikationen bezüglich der Heterogenitätskorrektur sowie zweier Indikatoren der Erwerbstätigkeit des Ehepartners präsentiert. Die Ergebnisse sind in der Reihenfolge ihrer statistischen Signifikanz dargestellt.

38 Jeweils als Polynom dritten Grades spezifiziert. 
Modellstruktur zu erwarten, da ein Unterschied zwischen einer freiwilligen Verrentung und dem Verlust des Arbeitsplatzes hier empirisch nicht unterscheidbar sind.

Eine Wechselwirkung der Rentenentscheidung im Falle von Ehepartnern erscheint plausibel, das negative Vorzeichen impliziert, daß Arbeitnehmer, deren Partner noch berufstätig ist, einen späteren Rentenzeitpunkt wählen, ein gemeinsamer Eintritt in den Ruhestand ist hier zu vermuten. Dies Ergebnis stimmt auch mit Resultaten von ZWEIMÜLLER, WINTER-EBMER UND FALKINGER (1993) überein, die diesen Zusammenhang für Österreich untersuchen. ${ }^{39}$

Im Vergleich zu den anderen diskutierten Untersuchungen modellieren MEGHIR UND WHITEHOUSE keine expliziten ökonomischen Anreize. Eine Rentenberechnung dürfte auf Basis der unterschiedlichen Ruhestands, bzw. Nichterwerbstätigkeitsarten und dauern nicht möglich gewesen sein. Die Höhe des Einkommens dürfte sich in zwei betrachteten Erklärungsgrößen widerspiegeln, der Berufsgruppe und der Ausbildung. Im Fall der Branche ist das Ergebnis, daß Büroangestellte früher in Rente gehen. Es kann unterstellt werden, daß erstere ein höheres Einkommen erzielen und die Wirkung dieses Einkommens (Absicherung, Ersparnisbildung) die Wahl des Rentenalters beeinflußt. Da der Gesundheitszustand in einer eigenen Variable modelliert wird, ist dieser mittelbare Einfluß der Berufsgruppe hier nicht relevant. Das positive Vorzeichen der Ausbildungsjahre kann in diesem Zusammenhang ebenfalls als Indikator für höheres Einkommen und damit höhere Opportunitätskosten interpretiert werden. Damit wäre der Widerspruch zu den Ergebnissen von BERKOVEC UND STERN erklärt. Letztere hatten hochsignifikant positive Vorzeichen der Ausbildung ermittelt (Tabelle 5-1, Seite 118), die dort als Indikator für Arbeitszufriedenheit interpretiert wurden, gleiches gilt für die Ergebnisse von SUEYOSHI (siehe Tabelle 5-2, Seite 122).

Als Zusammenschau wird deutlich, daß zeitdynamische Modellierungen dem Phänomen der Rentenzugänge besser gerecht werden, als dies von zeitpunktbezogenen (Wahrscheinlichkeits-) Modellen geleistet werden kann. Innerhalb dieser Klasse sind Modelle der dynamischen Programmierung flexibler gestaltbar, haben aber einen wesentlich höheren programmierungs- und rechentechnischen Aufwand, der die theoretischen Möglichkeiten in der empirischen Praxis wieder beschneidet. Dagegen seien

39 ZWEIMÜLLER, WINTER-EBMER UND FALKINGER (1993) „Joint Retirement of spouses and Social Security Reform“. 
Verweildauermodelle durch MEGHIR UND WHITEHOUSE beschrieben: „The advantage of our approch is that it does not impose too much on the data and serves as a good descripitve framework ". ${ }^{40}$ Innerhalb der Verweildauermodelle wurden unterschiedliche Ansätze diskutiert, die deren Möglichkeiten, etwa der Modellierung konkurrierender Risiken oder auch der Kontrolle für heterogene Populationen zeigte.

Strukturell liegen die Verweildauermodelle in Bezug auf die Fragestellung näher, da die abhängige Variable, die bedingte Rentenzugangswahrscheinlichkeit im Alter $t$, gegeben, daß bis t-1 gearbeitet wurde, die Entscheidungssituation der Arbeitnehmer besser abbildet. Die abhängige Variable in Hazardratenmodellen ist die Verweildauer in einem Zustand, hier der Erwerbstätigkeit. Diese Dauer kann in den Meßgrößen „Jahre der Erwerbstätigkeit“, aber auch „Lebensalter“ gemessen werden. Letztere Möglichkeit berücksichtigt die institutionellen Vorgaben des Rentenversicherungssytems, die in Kapitel 3 diskutiert wurden.

\subsection{Optionswertmodelle}

In Kapitel 5.2 wurden 2 Arten von Modellen vorgestellt. Das „klassische“ Modell der dynamischen Optimierung wurde dabei auch mit seinen Nachteilen, der komplizierten Umsetzbarkeit und Handhabung für empirische Umsetzungen beschrieben. Diese ist sicher ein Grund dafür, daß sich in der Literatur die Klasse der Verweildauermodelle zunehmend durchsetzt. Das in diesem Kapitel beschriebene Optionswertmodell kann als weitere „Alternativstrategie“ zu dynamischen Optimierungsansätzen verstanden werden. Die technische Idee ist diesen Modellen durchaus verwandt. ${ }^{41}$

In diesem Kapitel 5.3 wird in einem ersten Schritt die Grundidee eines Optionswertmodelles diskutiert, wie es Ende der 80er Jahre von James STOCK, David WISE und später Robin LUMBSDAINE in verschiedenen Variationen vorgestellt wurde. Die genannten Autoren verstehen dieses Modell als Alternative zu dynamischen Programmierungsansätzen. Der zweite Teil dieses Kapitels 5.3 befaßt sich mit der Implementierung der Idee des Optionswertes in einfachere Wahrscheinlichkeitsmodelle. Der Optionswert wird hierbei verstanden als eine Möglichkeit der Operationalisierung ökono-

\footnotetext{
40 MEGHIR UND WHITEHOUSE (1993), Seite 1.

41 Für Vergleiche des Optionswertmodelles mit dynamischen Programmierungsspezifikationen vergleiche LUMBSDAINE, STOCK UND WISE (1992 und 1993).
} 
mischer Anreizwirkungen, die ein Arbeitnehmer bei seiner Wahl des Ruhestandsalters abwägen muß.

Der Name Optionswert steht für den „Wert der Option, die sich ein Arbeitnehmer dadurch offen läßt, daß er zu einem gegebenen Alter nicht in den Ruhestand tritt." Der Ruhestand ist hierbei als absorbierender Endzustand Endzustand, verstanden, als ein Status, der nicht mehr geändert werden kann. Ein Arbeitnehmer, der in Pension gegangen ist, kann später nicht mehr auf dem Arbeitsmarkt aktiv werden. Damit wird die Verrentung zu einer „Einbahnstraße“, die, einmal beschritten, den weiteren Pfad der Einkünfte fest vorgibt. Im Gegensatz dazu läßt die Entscheidung eines Arbeitnehmers, ein weiteres Jahr zu arbeiten, die Optionen offen, diese Entscheidung im nächsten Jahr neu zu treffen. Abbildung 5-2 verdeutlicht die Grundidee dieses Ansatzes aus Sicht eines 50-jährigen Arbeitnehmers, der in jedem Alter (hier beispielhaft bis zum Alter 60 gezeigt) eine neue Entscheidung treffen kann, ob er ein weiteres Jahr arbeitet. Diese Option der Neuentscheidung bleibt ihm aber nur solange offen, bis er die endgültige Entscheidung trifft, in Rente zu gehen.

Der Optionswertansatz versucht nun weiterhin, diese unterschiedlichen Optionen ökonomisch zu bewerten, indem für alle Entscheidungszeitpunkte die Barwerte der Zahlungsströme ermittelt werden, die sich aus den jeweiligen Arbeit / Ruhestand Entscheidungen ergeben.

\section{Abbildung 5-2: Grundidee des Optionswertes}

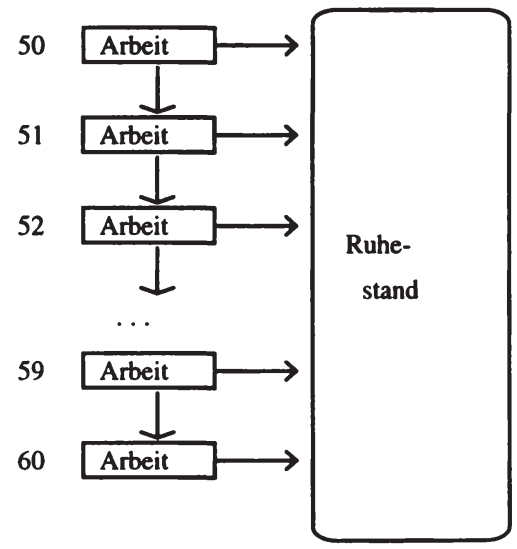




\subsubsection{Grundlegendes Modell von LUMBSDaine, STOCK UND WISE}

Die Wichtigkeit betrieblicher Altersversorgung in den USA ist bereits an verschiedenen Stellen angeklungen. Dies war die Motivation für KOTLIKOFF UND WISE (1985 und 1988), die Betriebsrentenregelungen einer großen amerikanischen Firma genau zu analysieren, indem sie aus diesen Alters-Barwert-Profile errechnen. Solche Profile wurden in Kapitel 3.2.2 beschrieben und für eine deutsche Firma beispielhaft dargestellt.

Dieser Datensatz wird durch LUMBSDAINE, STOCK UND WISE mit Hilfe des Optionswertkonzeptes analysiert. Für die Errechnung des Optionswertes wird dabei zunächst der Barwert $V_{t}$ aller zukünftigen Lohn- und Renteneinkommen eines Arbeitnehmers für jedes denkbare Rentenalter $R$ ermittelt. Dies entspricht Lohneinkünften vom Alter der Entscheidung $\mathrm{t}$ bis zum Jahr vor der Verrentung R-1 und danach Renteneinkommen bis zum Lebensende. Das Arbeitseinkommen $\mathrm{Y}^{\mathrm{L}}$ wird mittels eines AltersEinkommensprofils ermittelt und stellt eine im hohen Alter fallende Funktion dar. ${ }^{42}$ Das Renteneinkommen $Y^{R}$ hängt sowohl vom Renteneintrittstermin $R$ als auch von der Höhe des Arbeitseinkommens ab. Gleichung 5-4 zeigt die Bestimmung dieses Barwertes $V_{t}$ für jedes Entscheidungsalter $t$ und jeden möglichen Rentenzeitpunkt $R .{ }^{43}$

$$
V_{1}(R)=\sum_{s=1}^{R-1} U_{L}\left(Y_{s}^{L}\right) \cdot \delta^{s-1}+\sum_{s=R}^{T} U_{R}\left(Y_{s}^{R}\left(R, Y^{L}\right)\right) \cdot \delta^{s-1}
$$

$\begin{array}{lll}\text { mit: } & \mathrm{V}_{\mathrm{t}}(\mathrm{R}) & \text { Barwert aller Lohn- und Rentenströme in Abhängigkeit von } \mathrm{R} \\ & Y_{s}^{L} & \text { Arbeitseinkommen (Labor income) im Alter } \mathrm{s} \\ & Y_{s}^{R}\left(\mathrm{R}, \mathrm{Y}^{\mathrm{L}}\right) & \text { Renteneinkommen im Alter } \mathrm{s} \\ \mathrm{U}_{\mathrm{L}} & \text { Nutzenfunktion für Arbeitseinkommen } \\ \mathrm{U}_{\mathrm{R}} & \text { Nutzenfunktion für Renteneinkommen (inklusive Freizeitnutzen) } \\ \mathrm{R} & \text { Rentenzugangsalter (Austritt aus dem Erwerbsleben) } \\ \boldsymbol{\delta} & \text { Diskontfaktor mit } \delta=\frac{1}{1+\mathrm{r}} .\end{array}$

Der Optionswert wird nun definiert als die Differenz zwischen dem maximal erzielbaren Barwert $V_{t}\left(R^{*}\right)$ und dem Barwert der Rentenzahlungen bei sofortiger Verrentung:

$$
O V(t)=V_{1}\left(R^{*}(t)\right)-V_{t}(t) \quad \text { wobei: }
$$

\footnotetext{
42 STOCK UND WISE (1990 a), Seite 1178. Die Schätzung ist eine OLS-Regression, die über das Alter, die Firmenzugehörigkeit, die Vorjahreseinkommen in unterschiedlichen Interaktionstermen und Polynomen sowie Jahresdummies definiert ist.
}

${ }^{43}$ STOCK UND WISE (1990 b), Seite 212 ff. 


$$
R^{*}(t)=\underset{r>t}{\operatorname{argmax}} V_{t}(r)
$$

mit: $\quad$ oV

Optionswert (Option Value)

Die Nutzenfunktionen $U_{L}$ und $U_{R}$ werden mit individuenspezifischen Fehlertermen modelliert, auf denen die weitere Entwicklung des Modelles basiert:44

$$
U_{L}\left(Y_{L}\right)=Y_{L}^{\gamma}+\varepsilon^{L}
$$

$$
U_{R}\left(Y_{R}\right)=\left(k \cdot Y_{R}(R)\right)^{\gamma}+\varepsilon^{R}
$$

mit:

$\begin{array}{ll}\gamma & \text { Nutzenparameter } \\ \mathbf{k} & \text { Freizeitnutzen } \\ \varepsilon & \text { Individuenspezifische Fehlerterme. }\end{array}$

Die Wahrscheinlichkeit eines Arbeitsmarktaustrittes im Alter $\mathbf{R}$ ergibt sich als Funktion dieser Fehlerterme. Die zu schätzenden Koeffizienten sind der Nutzenparameter $\gamma$, der Diskontierungsfaktor $r$, ein Varianzparameter sowie der Freizeitnutzen k. Dieser Freizeitnutzen beinhaltet die Annahme, daß ein Arbeitnehmer aus durch Arbeit verdientem Einkommen weniger Nutzen zieht, als aus Transfereinkommen.

Das Optionswertmodell in den Spezifizierungen von STOCK UND WISE (1990 a und b) und LUMBSDAINE, STOCK UND WISE (1992 und 1993) beinhaltet keine Kovariate. Es ist den Autoren sehr wichtig darzustellen, daß das reine Optionswert-Modell (ohne Kovariate) eine bessere Schätzgüte aufweist, als ein mit einem kompletten Satz Altersdummies geschätztes. ${ }^{45}$ Leider ist die Argumentation aus den vorliegenden Veröffentlichungen nicht immer nachvollziehbar, da in vielen Regressionstabellen Gütemaße und Likelihood-Werte fehlen. Auch wird keine Schätzung präsentiert, in der sowohl der Optionswert als auch Altersdummies enthalten sind, so daß durch Insignifikanz der letzteren die Dominanz des Optionswertmodelles untermauert würde. LAZEAR kommentiert die Argumentation, das Modell hätte mit seinen vier Variablen eine bessere Anpassung als 17 Altersvariablen mit den Worten: „Perhaps, but the option value ap-

\footnotetext{
44 Ebenda, Seite 213, Gleichung (3).

${ }^{45}$ Ein Vergleich mit einem Modell, das nur Altersdummies beinhalte, wird in STOCK UND WISE (1990 a, Tabelle II, Seite 1167) zwar genannt, die Schätzergebnisse dieses Modells sind allerdings leider nicht dargestellt, so daß ein echter Vergleich, der einen Vorteil des Optionswertsmodelles zeigen könnte, nicht möglich ist. Es werden lediglich Log-Likelihoodwerte für die Konvergenzparameter angegeben.
} 
proach is not the only way to take economic variables into account" und schlägt in diesem Zusammenhang vor, das Modell mit den gegebenen Daten gegen ein strukturiertes Ruhestandsmodell zu testen. ${ }^{46}$

Tatsächlich erlaubt das hier beschriebene Optionswertmodell keine Kovariate. Dies wird von den Autoren mit der extrem langen Computerlaufzeit begründet, die sich ergeben würde. Die Frage, ob ein Identifikationsproblem vorliegt, kann rein auf Basis der veröffentlichten Literatur nicht beantwortet werden. Die Argumentation der Autoren, die statistische Anpassung sei besser als in einem herkömmlichen Modell mag richtig sein, aber sie ist noch nicht (öffentlich) empirisch belegt. In LUMBSDAINE, STOCK UND WISE (1993) werden zwar eine Probit- und eine dynamische Programmierungsspezifikation als vergleichende Modelle dargestellt, aber diese werden auf die im Optionswertmodell handhabbaren Informationen beschränkt, d.h. es kommen keine weiteren Kovariate hinzu. ${ }^{47}$

Gegeben die Annahme, das hier vorgestellte Modell sei wirklich besser im Sinne der statistischen Schärfe, bedeutet dies, die Handlungen von Arbeitnehmern rein auf ökonomische Anreize zurückzuführen. Diese Art der Modellierung übersieht, das es außer dem „homo ökonomicus“ auch noch andere Aspekte gesellschaftlicher Prozesse gibt. Und auch wenn diese in ökonometrischen Modellen nur durch „Kontrollgrößen“ wie Alter, Geschlecht, Gesundheit, Bildung, Herkunft o.a. berücksichtigt und nicht strukturell interpretiert werden, so bedeutet ihre Implementation doch, die Existenz von über das monetäre hinausgehenden Verhaltensmotivationen zu akzeptieren. Dies ist meines Erachtens für eine adäquate Modellierung gesellschaftlicher Prozesse unerläßlich.

Die Autoren bieten eine sehr komplexe Modellierung eines Sonderfalles. Beobachtet werden Angestellte einer einzigen Firma ${ }^{48}$, deren Verrentungsverhalten durch die Anreizstrukturen des Betriebsrentensystems beschrieben wird. In den diskutierten Arbeiten wird eindrucksvolle Evidenz für die Bedeutung des Optionswert als Indikator für die ökonomischen Anreize eines Rentensystems gegeben. Eine grundlegende Modellierung der Verrentungsentscheidung, die für eine Politikanalyse und Prognose dienen

\footnotetext{
46 LAZEAR (1990), Seite 228.

47 LUMBSDAINE, STOCK UND WISE (1993), v.a. Tabellen 1 und 2 im Anhang.

${ }^{48}$ Mit der Arbeit LUMBSDAINE, STOCK UND WISE (1993) wird eine zweite Firma in die Untersuchung aufgenommen.
} 
soll, muß jedoch über diesen Spezialfall hinausgehen, auch wenn in allgemeineren Daten nicht die gleiche Tiefe der Information vorhanden sein wird.

\subsubsection{Verbindung eines Discrete-Choice Modelles mit dem Optionswertgedan- ken durch BÖRSCH-SUPAN}

In seinen Arbeiten greift BöRSCH-SUPAN die eben angesprochene Idee auf, strukturelle Ruhestandsmodelle mit dem Optionswert als erklärender Variable zur Operationalisierung ökonomischer Anreizstrukturen zu verbinden. Die hier vorgestellten Wahrscheinlichkeitsmodelle mit Berücksichtigung des Optionswertes stellen damit eine Variation zu den oben diskutierten reinen Optionswertmodellen.

In einem empirisch orientierten Übersichtsartikel zur Altersökonomik präsentiert BöRSCH-SUPAN (1991) ein Discrete Choice Modell der Wahrscheinlichkeit, verrentet zu $\operatorname{sein}^{49}$ auf Basis zweier Querschnittsdatensätze aus den USA und der Bundesrepublik, jeweils aus den Jahren 1984. Er analysiert also zeitpunktbezogen für ein Jahr die Aufteilung einer Stichprobe von Personen auf die Zustände "erwerbstätig" und "nicht erwerbstätig"50 und modelliert die Wahrscheinlichkeit für diese Zustände in Abhängigkeit vom Alter, persönlicher Charakteristiken der Arbeitnehmer und des Optionswertes. Die untersuchte Personengruppe sind Arbeitnehmer im Alter zwischen 60 und 70 Jahren.

Das verwendete Logit Modell hat die Struktur:

(5-9) $\operatorname{Prob}($ Ruhestand $)=\operatorname{Ln}\left[\beta_{1} \cdot\right.$ Alter $+\beta_{2} \cdot$ Optionswert $+\beta_{3}$ persönliche Charakteristiken $]$.

Der Optionswert (OV) wird analog der o.a. Darstellung ermittelt mittels:

$(5-10) \mathrm{V}_{1}(\mathrm{R})=\mathrm{E}_{1}\left[\sum_{s=1}^{\mathrm{R}-1} \mathrm{Y}_{s}^{\mathrm{L}} \cdot \delta^{s-1}+\alpha \cdot \sum_{s=\mathrm{R}}^{\mathrm{T}} \mathrm{Y}_{s}^{\mathrm{R}}\left(\mathrm{R}, \mathrm{Y}^{\mathrm{L}}\right) \cdot \delta^{s-1}\right]$

$(5-11) O V(t)=V_{t}\left(R^{*}(t)\right)-V_{t}(t) \quad$ wobei:

$(5-12) R^{*}(t)=\underset{r>1}{\operatorname{argmax}} V_{t}(r)$

mit: $\quad V_{t}(R) \quad$ Barwert aller Lohn- und Rentenströme in Abhängigkeit von $R$,

${ }^{49}$ Die Modelle wurden im Kapitel 5.1 bereits angesprochen.

50 Die Abgrenzung dieser beiden Zustände trifft er über die wöchentlichen Arbeitsstunden: Personen mit weniger als 15 Arbeitsstunden pro Woche gelten als verrentet. Es werden nur Haushaltsvorstände betrachtet (vgl. Börsch-Supan, 1991). 


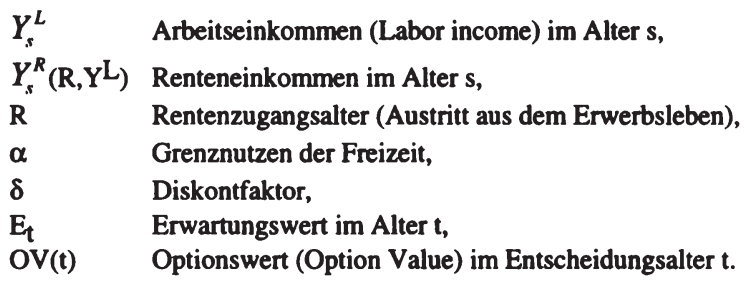

BöRSCH-SUPAN benutzt für die Parameter $\alpha$ und $\delta$ die Werte aus STOCK \& WISE (1990 a) mit $\alpha=1,66$ und $\delta=0,85$ und ermittelt damit die in Tabelle 5-3 angegebenen Elastizitäten.

Tabelle 5-3: Determinanten der Verrentungswahrscheinlichkeit im einfachen LogitModell von BÖRSCH-SUPAN

\begin{tabular}{|l|lc|lc|}
\hline & \multicolumn{2}{|c|}{ USA } & \multicolumn{2}{c|}{ Westdeutschland } \\
Variable & Elastizität & t-Statistik & Elastizität & t-Statistik \\
\hline Konstante & $2,559^{* *}$ & 5,77 & 1,237 & 0,53 \\
Nicht-Weiße & $-0,105^{* *}$ & 5,02 & - &.- \\
Weiblich & $-0,681^{* *}$ & 4,76 & 0,067 & 0,26 \\
Verheiratet & 0,042 & 0,73 & $-0,097$ & 0,87 \\
Kinder & $-0,307^{* *}$ & 3,53 & $-0,001$ & 0,41 \\
Vermögen & $1,394^{*}$ & 0,85 & $-0,805^{* *}$ & 4,39 \\
Grundvermögen & $0,810^{*}$ & 1,72 & 0,200 & 0,74 \\
Optionswert & $-0,463^{* *}$ & 5,32 & $-0,638^{* *}$ & 5,61 \\
Alter 6151 & $-1,313^{* *}$ & 3,01 & 0,605 & 1,23 \\
Alter 62 & $-0,786^{* *}$ & 2,62 & 0,289 & 0,55 \\
Alter 63 & $-0,395$ & 1,37 & $0,1694 * *$ & 2,55 \\
Alter 64 & $-0,849^{* *}$ & 2,64 & 0,575 & 1,04 \\
Alter 65 & $-0,526 *$ & 1,69 & 0,070 & 0,11 \\
Alter 66 & 0,048 & 0,16 & $1,180^{*}$ & 1,73 \\
Alter 67 & 0,160 & 0,50 & $1,714^{* *}$ & 2,97 \\
Alter 68 - 70 & $-0,001$ & 0,01 & $2,080^{* *}$ & 3,13 \\
\hline Likelihood-Wert & $-649,66$ & $(-790,19)$ & $-166,87$ & $(-391,63)$ \\
Prozent richtig & 71,3 & & 89,4 & \\
Beobachtungen & 1.140 & & 565 & \\
$\rho^{2}$ (52) & 17,8 & & 57,4 & \\
\hline
\end{tabular}

Quelle: BOASCH-SUPAN, 1991, Tabelle 9, Seite 116. "*: 95\% Signifikanz, ": $90 \%$ Signifikanz.

${ }^{51}$ Das Alter 60 ist die Referenzkategorie.

52 Das sogenannte "Pseudo $R^{2 "}$ oder "Mc Faddens's $\rho$ " ist definiert als $1-L(\theta) / L(0)$, wobei $\theta$ den Vektor der geschätzten Parameter und $\mathrm{L}$ den Loglikelihood-Wert bezeichnet. 
In dieser Ergebnistabelle wurden die Koeffizienten, bis auf die Konstante und die Alters-Dummies in Elastizitäten umgewandelt. Die wesentlich geringere Schätzgüte für die USA reflektiert das Fehlen wichtiger Einflußgrößen, vor allem der betrieblichen und privaten Zusatzpensionen (BÖRSCH-SUPAN, 1991), deren wichtiger Einfluß in den Vereinigten Staaten bereits in Kapitel 5.2.2 bei der Analyse von STOCK, WISE UND LUMBSDAINE deutlich wurde. Diese Daten liegen allerdings in der Datengrundlage nicht vor, so daß die Schätzqualität auf diese Weise nicht verbessert werden kann. Wesentliche Ergebnisse der Analyse sind:

- Der Optionswert ist in beiden untersuchten Ländern die statistisch wichtigste Einflußgröße zur Erklärung der Verrentungswahrscheinlichkeit;

- Daraus wird zum einen deutlich, daß die Rentenversicherungsgesetzgebung einen erheblichen Anteil an der Tendenz zur frühen Verrentung in beiden Ländern hat;

- Andererseits präsentiert sich der Optionswert als eine adäquate Operationalisierung für diese staatlichen Einflüsse;

- Signifikante Koeffizienten der Altersvariablen implizieren, daß der Optionswert nicht alle Alterseffekte abdecken kann und das die Schätzung entweder um weitere relevante Erklärungsgrößen erweitert werden sollte oder das Modell erweitert werden muß, etwa um die simultane Schätzung der Parameter des Optionswertes oder um die Berücksichtigung der intertemporalen Struktur der Übergänge in den Ruhestand (vgl. Kapitel 5.1 und 5.2).

Die erstgenannte Weiterentwicklung nimmt der Autor in BöRSCH-SUPAN (1992 e) vor, indem er die bisher als konstant angenommenen Parameter $\alpha, \delta$ in einem Logit-Modell simultan mitschätzt und außerdem statt des Einkommens den aus diesem Einkommen resultierenden Nutzen als erklärende Variable aufnimmt. Dieser Nutzen wird als $u(Y)$ $=Y^{\gamma}$ modelliert und auch $\gamma$ wird in der simultanen Schätzung ermittelt. Diese Spezifikation entspricht der von STOCK UND WISE, vergleiche Formeln (5-7) und (5-8).

Die modifizierte Ermittlung des Barwertes $V_{t}$ als Basis des Optionswertes erfolgt dann nach der Spezifikation:

$$
\mathrm{V}_{t}(\mathrm{R})=\mathrm{E}_{\mathrm{t}}\left[\sum_{s=1}^{\mathrm{R}-1} \mathrm{u}\left(\mathrm{Y}_{\mathrm{s}}^{\mathrm{L}}\right) \cdot \mathrm{a}_{\mathrm{s}} \cdot \delta^{s-1}+\alpha \cdot \sum_{s=\mathrm{R}}^{\infty} \mathrm{u}\left(\mathrm{Y}_{\mathrm{s}}^{\mathrm{R}}\left(\mathrm{R}, \mathrm{Y}^{\mathrm{L}}\right)\right) \cdot \mathrm{a}_{\mathrm{s}} \cdot \delta^{s-1}\right]
$$

$$
\begin{array}{lll}
\text { mit: } & \mathrm{V}_{\mathrm{t}}(\mathrm{R}) & \text { Barwert aller Lohn- und Rentenströme in Abhängigkeit von } \mathrm{R} \\
\mathrm{R} & \text { Rentenzugangsalter (Austritt aus dem Erwerbsleben) } \\
Y_{s}^{L} & \text { Arbeitseinkommen (Labor income) im Alter s } \\
& Y_{s}^{R}\left(\mathrm{R}, \mathrm{Y}^{\mathrm{L}}\right) & \text { Renteneinkommen im Alter s }
\end{array}
$$




$\begin{array}{ll}a_{t} & \text { Überlebenswahrscheinlichkeit bis zum Alter t } \\ \alpha & \text { Grenznutzen der Freizeit } \\ \delta & \text { Diskontfaktor } \\ \mathrm{E}_{\mathrm{t}} & \text { Erwartungswert im Alter t } \\ \mathrm{OV} & \text { Optionswert (Option Value) }\end{array}$

Die Nutzenfunktion des Einkommens ist definiert als:

$(5-14) u(Y)=Y^{\gamma}$

wobei die Multiplikation mit dem Freizeitnutzen $\alpha$ in dieser Funktionsform den Nutzen aus Renteneinkommen steigert. Die Regression unterscheidet sich von der oben diskutierten außerdem durch die Aufnahme von Indikatorvariablen für Gesundheit. Die Schätzergebnisse sind in Tabelle 5-4 wiedergegeben.

Tabelle 5-4: Determinanten der Verrentungswahrscheinlichkeit im erweiterten LogitModell von BÖRSCH-SUPAN.

\begin{tabular}{|l|cc|}
\hline Variable & Koeffizient & t-Statistik \\
\hline Konstante & 0,582 & 0,32 \\
$\quad$ Satz Altersvariable & & (insignifikant) \\
Verheiratet & 0,069 & 0,14 \\
Weiblich & $0,846^{*}$ & 1,75 \\
Haushaltsgröße & $-0,247$ & 1,29 \\
Deutsche/r & 0,499 & 0,31 \\
Optionswert & $-0,185^{* *}$ & 3,51 \\
Anzahl Arztbesuche & $0,367 *$ & 1,69 \\
Behinderungsgrad & 0,247 & 1,09 \\
Krankenhaus (ja/nein) & 0,313 & 0,35 \\
Nächte im Krankenhaus & $-2,022^{* *}$ & 2,45 \\
Dauer Krankenhausaufenthalt & 0,066 & 1,15 \\
\hline$\alpha$ & 1,196 & 1,43 \\
$\delta$ & 0,862 & 1,63 \\
$\gamma$ & 1,011 & 1,82 \\
\hline Anzahl Beobachtungen & 479 & \\
Prozent richtig & 84,9 & \\
$\rho^{2}$ & 41,6 & \\
\hline
\end{tabular}

Quelle: BORSCH-SUPAN, 1992 e, Tabelle 9, Seite 550. "*: 95\% Signifikanz, ": 90\% Signifikanz.

Als wichtigste Ergebnisse bleibt festzuhalten:

- Der Optionswert bleibt auch in diesem weiterentwickelten Modell die mit Abstand wichtigste Einflußgröße auf die Wahrscheinlichkeit, verrentet zu sein. 
- Wie bereits in mehreren vorgestellten Modellen tragen auch in dieser Spezifikation Indikatoren eines schlechten Gesundheitszustandes signifikant zur Erklärung des Zustandes „Ruhestand“ bei. Allerdings ergibt sich wieder keine konsistente Aussage, da zwar die Tatsache, ob jemand im Krankenhaus war, nicht signifikant wirkt, wohl aber die Anzahl der dort verbrachten Nächte. Diese Variable wirkt allerdings nicht in die erwartete Richtung. Lediglich die Arztbesuche zeigen mit schwacher Signifikanz das erwartete Vorzeichen.

\section{Parameter des Optionswertes:}

- Der Zusatznutzen für Transfereinkommen (ohne Arbeitsaufwand), $\alpha$, erscheint mit 1,19 eher gering. Dieser Schätzung nach ist ein/e Arbeitnehmer/in zwischen einer DM Arbeits- und 1,20 DM Renteneinkommen indifferent. Mit einem t-Wert von 1,43 ist der Koeffizient allerdings kaum signifikant.

- Im Gegensatz dazu ist der Schätzwert für den Diskontfaktor $\delta$ mit 0,86, was einem internen Zinssatz von $13.8 \%$ entspricht ${ }^{53}$, relativ hoch. Allerdings ist auch dieser Koeffizient nur schwach signifikant, so daß eine Interpretation nicht zu tief gehen sollte.

- Die schwache Signifikanz der beiden Parameter des Optionswert deutet auf ein Identifikationsproblem hin, das bei auch aus der Definition des Barwertes $V_{t}$ deutlich wird. Beide Faktoren wirken multiplikativ auf den Nutzen ein.

- Der Schätzwert für den Parameter der Nutzenfunktion liegt mit $\gamma=1,01$ sehr nahe am Wert 1, so daß der Verlauf der Nutzenfunktion nach diesem Ergebnis auch durch eine lineare Funktion approximiert werden kann.

Beide Analysen von BÖRSCH-SUPAN zeigen den beträchtlichen Einfluß der gesetzlichen Rahmenbedingungen auf die individuelle Wahl des Verrentungsalters und damit auch, daß die Operationalisierung dieser institutionellen Regeln durch den Optionswert ein sinnvoller Weg ist, den es sich lohnt, weiter zu verfolgen. Die Ergebnisse dieser Querschnittsanalysen sind allerdings insofern verzert, als sie auf kumulierten Prozentanteilen verrenteter Individuen beruhen und die Verrentungswahrscheinlichkeiten in höheren Altersstufen -aufgrund der Querschnittsstruktur der Daten- nicht um die schon in jüngeren Jahren getroffenen Arbeitsmarktaustritte bereinigt werden können.

Die wichtigste Weiterentwicklung des beschriebenen Modelles ist in diesem Sinne die Einbindung in eine Zeitdimension unter Messung von Übergangsentscheidungen der

${ }^{53}$ Analog zu den Arbeiten von STOCK und WISE ist der Parameter $\delta$ definiert als $\delta=1 /(1+r)$ mit $r$ als internem Zinssatz (Zeitpräferenzrate). 
Individuen. Nur so ist die Berücksichtigung sich ändernder Gesetze und anderer zeitvarianter Rahmenbedinungen möglich.

Diese Weiterentwicklung wird im empirischen Teil der vorliegenden Arbeit vorgenommen.

\subsection{Zusammenfassung der wesentlichen Ergebnisse und Aufstellung von Arbeitshypothesen}

In den diskutierten Modellansätzen aus der ökonometrischen Literatur wurde die Thematik der Wahl des persönlichen Rentenalters auf unterschiedliche Weise behandelt. Während einige Arbeiten Wert auf die Konstruktion eines anspruchsvollen formalen Modellrahmens legen, behandeln inhaltlich orientierte Ansätze die untersuchte Frage unter verschiedenen Aspekten, wobei nichtökonomische Gesichtspunkte als Kontrollgrößen für die korrekte Messung des Einflusses ökonomischer Indikatoren dienen.

Als Ergebnis dieses Kapitels sind zwei Punkte zu unterscheiden: die Wahl eines geeigneten Modelles zur Abbildung der Ruhestandsentscheidung und die Auswahl der adäquaten Variablen, die innerhalb dieses Modelles die Determinanten der individuellen Abwägung abbilden.

\section{Modellrahmen}

Aus dem Vergleich der Modelle in Kapitel 5.1 und 5.2 wurde deutlich, daß die Wahl des Verrentungsalters einen dynamischen Charakter hat. Der oder die Einzelne plant den Ruhestand nicht zu einem singulären Zeitpunkt, sondern als Auswahl aus möglichen Jahren bzw. Lebensaltern unter Berücksichtigung sich ändernder Rahmenbedingungen wie etwa des Rentenversicherungssystems. Dieser Entscheidungsstruktur muß ein Modell der Ruhestandsentscheidung Rechnung tragen, so daß ein zeitstatisches Modell nicht geeignet erscheint.

Die Diskussion zeitdynamischer Spezifikationen des Abschnittes 5.2 zeigte, daß ein Verweildauermodell rechentechnisch einfacher ist als ein Ansatz der dynamischen Programmierung und durch seine Konstruktion die Natur des modellierten Entscheidungsprozesses richtig abbildet. Weiterhin ist diese Modellklasse bezüglich der Spezifikation relevanter Kovariate flexibler als andere besprochene Modellklassen. Die Klasse der Optionswertmodelle zeigte sich als in der Spezifikation zu inflexibel, da hier keinerlei Berücksichtigung von Kovariaten möglich ist. Die Variable Optionswert hingegen erwies sich als adäquate Messung wichtiger ökonomischer Entscheidungs- 
faktoren (s.u.). Somit bietet sich für die vorliegende Untersuchung ein Verweildauermodell an.

\section{Determinanten der Ruhestandsentscheidung}

Es haben sich vier wesentliche Gruppen von Determinanten der Alterswahl erwiesen:

- persönliche Charakteristiken,

- berufliche Rahmenbedingungen,

- ökonomische Faktoren,

- institutionelle Rahmenbedingungen.

Der letzte Punkt der institutionell vorgegebenen Bedingungen spiegelt sich teilweise in den beruflichen (soweit Betriebsrenten und dort festgelegte Ruhestandsalter involviert sind) und den ökonomischen Faktoren (bezüglich der Rentenberechnung und Terminbestimmung). Die folgende Tabelle 5-5 gibt eine Übersicht über die in der vorgestellten Literatur besprochenen Determinanten. Es ist zu beachten, daß nicht alle Einflußfaktoren genau einem Themenbereich zuzuordnen sind.

Der Vergleich der ermittelten Wirkungsrichtungen der Determinanten von Ruhestandsentscheidungen zeigt eine erfreuliche Eindeutigkeit zwischen den Studien. Sowohl die relative Bedeutung der Faktoren als auch deren Einflußrichtung erweisen sich als stabil. So werden -neben dem Alter- in allen Studien Einkommensindikatoren modelliert, die Zentralvariable ökonomischer Analysen. Die ermittelte Wirkung ist in allen Studien eindeutig: Hohes Arbeitseinkommen senkt die Tendenz zur Verrentung. Dieses Ergebnis kommt auch in Studien zustande, in denen eine gleichzeitige Kontrolle für Ausbildung, Berufsbereich und andere einkommensbestimmende Indikatoren, aber auch das Alter vorgenommen wird. Aus diesem Ergebnis ist zu schließen, daß das Einkommen ${ }^{54}$ als Opportunitätskosten des Ruhestandes interpretiert werden kann. Umgekehrt erhöhen hohe Rentenanwartschaften die Wahrscheinlichkeit einer (frühen) Verrentung. Beide Effekte werden kombiniert in der Variable Optionswert, die Arbeitseinkommen und Rente nicht nur berücksichtigt, sondern beide Zahlungsströme in Beziehung setzt. Die Tatsache, daß BöRSCH-SUPAN (1992 e) durch diese Größe in einem zeitstatischen Modell insignifikante Altersvariablen erzielt, zeigt die Eignung des Optionswertes als Operationalisierung ökonomischer Anreizstrukturen. Diese Variable wird auch in der unten folgenden ökonometrischen Schätzung der ost- und westdeutschen Rentenzugänge verwendet.

\footnotetext{
54 Tatsächlich ist nicht das Einkommen per se, sondern der daraus erzielbare Nutzen die relevante Entscheidungsgröße. Dies wird in der Mehrzahl der Studien modelliert.
} 
Als weitere wichtige Einflußgröße ergeben sich Indikatoren der Gesundheit. Eine objektive Messung des Gesundheitszustandes befragter Individuen erweist sich in ökonometrischen Analysen oft als schwierig. Selbstberichtete Angaben der Befragten müssen mit Vorsicht behandelt werden, wogegen objektive Maße in Mikrodatensätzen in der Regel nicht vorliegen. Die Tatsache, daß die verwendeten Gesundheitsindikatoren trotzdem durchgängig hohe Signifikanz aufweisen, zeigt ihre große Bedeutung. Die in der Tabelle 5-5 (Fußnote 55) und im Kapitel 4 bereits angesprochene Problematik der ungeklärten Kausalität muß jedoch beachtet werden.

Neben diesen Kernvariablen sind vor allem die Regelungen der Rentenversicherung zu beachten, die teilweise bestimmte Ruhestandsalter vorschreiben oder die Rentenhöhe an den Zugangszeitpunkt koppeln. In diesem Sinne muß die Modellierung der ökonomischen Anreize in Verbindung mit den institutionellen Rahmenbedingungen erfolgen, wie dies etwa in der Variable des Optionswertes geschieht. Aber auch weitere Variablen aus allen vier (in der Tabelle) genannten Bereichen weisen signifikante Wirkungen auf das Ruhestandsalter auf. Diese Wirkungen sind in einer Regressionsanalyse gemeinsam zu berücksichtigen.

Tabelle 5-5: In der Literatur untersuchte Determinanten der Verrentung

\begin{tabular}{|c|c|c|}
\hline $\begin{array}{c}\text { Themengebiet / } \\
\text { Variable }\end{array}$ & Anmerkungen & $\begin{array}{l}\text { Modellierung in diskutierten } \\
\text { Studien / (Wirkungsrichtung } \\
\text { auf frühe Verrentung) }\end{array}$ \\
\hline \multicolumn{3}{|c|}{ I. Kontrollvariablen (persönliche Eigenschaften): } \\
\hline Alter & $\begin{array}{l}\text { Das Alter ist der naheliegendste Bestim- } \\
\text { mungsfaktor der Ruhestandsneigung. } \\
\text { Wird in einfachen Modellen direkt (als Satz } \\
\text { einzelner Dummyvariablen oder als Poly- } \\
\text { nom) implementiert, in entsprechend struk- } \\
\text { turierten Ansätzen durch sinngemäB konstru- } \\
\text { ierte Variablen abgedeckt. }\end{array}$ & $\begin{array}{l}\text { [2]: Altersdummies } \\
\text { [3]: Polynom (+) } \\
\text { [4]: Polynom (degressiv) }(+) \\
\text { [6]: Polynom (progressiv) }(-) \\
\text { [6]: Alter·Beschäft.dauer (+) } \\
\text { [8]: Altersdummies } \\
\text { (unterschiedlich) } \\
\text { [9]: Altersdummies } \\
\text { (insignifikant) }\end{array}$ \\
\hline Geburtsjahr & Zur Kontrolle von Kohorteneffekten & (Angabe nicht sinnvoll) \\
\hline Geschlecht & $\begin{array}{l}\text { Indikator für geschlechtsspezifische Präfe- } \\
\text { renzen, gesellschaftliche Ungleichbehand- } \\
\text { lung; unterschiedliche rechtliche Regelun- } \\
\text { gen }\end{array}$ & $\begin{array}{l}\text { [8]: weiblich (USA) } \\
\text { [9]: weiblich (BRD) }\end{array}$ \\
\hline Ausbildung & $\begin{array}{l}\text { In der Regel gemessen als Zahl der Ausbil- } \\
\text { dungsjahre. } \\
\text { Teilweise Indikatorgröße für Arbeitsbedin- } \\
\text { gungen (höhere Ausbildung } \Rightarrow \text { weniger kör- } \\
\text { perliche Arbeit) und Arbeitszufriedenheit. }\end{array}$ & $\begin{array}{l}\text { [4]: Jahre nach 14. Lebensjahr (-) } \\
\text { [5]: Ausbildungsjahre } \\
\text { [6]: Ausbildungsdauer; } \\
\text { Polynom (degressiv) (+) }\end{array}$ \\
\hline
\end{tabular}




\begin{tabular}{|c|c|c|}
\hline $\begin{array}{c}\text { Themengebiet / } \\
\text { Variable }\end{array}$ & Anmerkungen & $\begin{array}{l}\text { Modellierung in diskutierten } \\
\text { Studien / (Wirkungsrichtung } \\
\text { auf frühe Verrentung) }\end{array}$ \\
\hline Gesundheit & $\begin{array}{l}\text { Wichtiger individueller Indikator für die } \\
\text { Wahl eines Ruhestandsalters. } \\
\text { Personen, die aus gesundheitlichen Gründen } \\
\text { ihrem Beruf nicht mehr nachgehen können, } \\
\text { haben die Option des vorgezogenen Ruhe- } \\
\text { standes. } \\
\text { Bereits im vierten Kapitel angesprochen } \\
\text { wurde allerdings auch der Mißbrauchsver- } \\
\text { dacht bezüglich der BU/EU-Renten.55 }\end{array}$ & 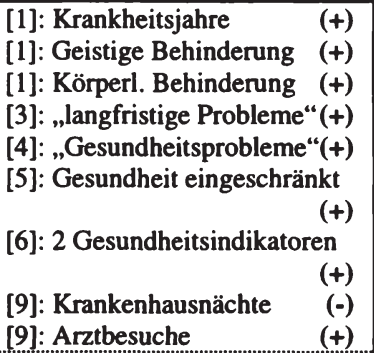 \\
\hline $\begin{array}{l}\text { Familienstand / } \\
\text { Haushaltsgröße }\end{array}$ & $\begin{array}{l}\text { Oft als Indikatorgröße für nicht näher spezi- } \\
\text { fizierte persönliche Einflüsse benutzt. } \\
\text { Gebräuchlich im Zusammenhang mit dem } \\
\text { Erwerbsstatus des Ehepartners (Modellie- } \\
\text { rung gemeinsamer Ruhestandstermines) }\end{array}$ & $\begin{array}{ll}\text { [5]: verheiratet } & (-) \\
\text { [6]: Partner arbeitet noch } & (-) \\
\text { [6]: Anzahl Kinder (USA) } & (-)\end{array}$ \\
\hline $\begin{array}{l}\text { Ethnische Zugehö- } \\
\text { rigkeit }\end{array}$ & $\begin{array}{l}\text { Die Variable "nicht weiß" wird in amerika- } \\
\text { nischen Studien oft als Indikator der Un- } \\
\text { gleichbehandlung unterschiedlicher ethni- } \\
\text { scher Gruppen spezifiziert. }\end{array}$ & $\begin{array}{ll}\text { [4]: nicht weiße Hautfarbe } & (-) \\
\text { [5]: nicht weiße Hautfarbe } & (-) \\
\text { [8]: nicht weiße Hautfarbe } & (-)\end{array}$ \\
\hline \multicolumn{3}{|c|}{ II. Berufsvariablen: } \\
\hline $\begin{array}{l}\text { Branche I } \\
\text { Stellung im Be- } \\
\text { ruf }\end{array}$ & $\begin{array}{l}\text { Indikator für branchenspezifische Bedingun- } \\
\text { gen (Anforderungsprofile, Schwere der Ar- } \\
\text { beit; aber auch spezielle Rentenrechtsrege- } \\
\text { lungen) }\end{array}$ & $\begin{array}{l}\text { [5]: Handwerker } \\
\text { [5]: Arbeiter } \\
\text { [6]: Bürotätigkeit } \\
\text { [6]: Management }\end{array}$ \\
\hline $\begin{array}{l}\text { Firmenspezifische } \\
\text { Regelungen }\end{array}$ & & [1]: Aufstiegschancen \\
\hline $\begin{array}{l}\text { Dauer der Firmen- } \\
\text { zugehörigkeit }\end{array}$ & $\begin{array}{l}\text { Potentieller Indikator für rechtlichen oder } \\
\text { faktischen Kündigungsschutz bzw. Betriebs- } \\
\text { verbundenheit. }\end{array}$ & [4]: Firmenzugehörigkeit \\
\hline \multicolumn{3}{|c|}{ III. Ökonomische Kenngrößen: } \\
\hline Einkommen & $\begin{array}{l}\text { Das Einkommen ist die wichtigste ökonomi- } \\
\text { sche Kenngröße, daher auch in allen Studien } \\
\text { modelliert. Nicht immer erfolgt eine direkte } \\
\text { Schätzung, teilweise gehen Einkommensva- } \\
\text { riablen in die Konstruktion von Indikatoren } \\
\text { ein. } \\
\text { Es muß zwischen dem Arbeits- und dem } \\
\text { Renteneinkommen bzw. dem Verhältnis der }\end{array}$ & $\begin{array}{l}\text { [1]: Einkommensverlust bei } \\
\text { Verrentung } \\
\text { [2]: Rentenanwartschaft } \\
\text { [5]: Einkommenshöhe }(+) \\
\text { [5]: Rentenanwartschaft }(-) \\
\text { [5]: Höhe Gesetzliche Rente mit } \\
\text { 62 Jahren } \\
\text { [5]: Differenzbetrag zu Rente } \\
\text { mit 62 Jahren }\end{array}$ \\
\hline
\end{tabular}

55 Dort wurde die Frage der Kausalität gestellt: Benutzen nur eingeschränkt erwerbsfähige Personen diese Rentenarten oder gehen Arbeitnehmer, die sich aus anderen Gründen einen vorgezogenen $\mathrm{Ru}$ hestand wünschen, verstärkt zu Ärzten und versuchen, eine Erwerbsunfähigkeitsbescheinigung herbeizuführen? 


\begin{tabular}{|c|c|c|}
\hline $\begin{array}{l}\text { Themengebiet / } \\
\text { Variable }\end{array}$ & Anmerkungen & $\begin{array}{l}\text { Modellierung in diskutierten } \\
\text { Studien / (Wirkungsrichtung } \\
\text { auf frühe Verrentung) }\end{array}$ \\
\hline & $\begin{array}{l}\text { beiden (Rentenniveau) unterschieden wer- } \\
\text { den. }\end{array}$ & $\begin{array}{ll}\text { [8]: Optionswert } & (-) \\
\text { [9]: Optionswert } & (-)\end{array}$ \\
\hline Vermögen & $\begin{array}{l}\text { Die Wirkungen von Vermögen auf die Wahl } \\
\text { des Rentenalters sind theoretisch umstritten. } \\
\text { (vgl. Kapitel 2.1) Nach der Lebenszyklushy- } \\
\text { pothese müBte ein hohes Vermögen einen } \\
\text { frühen Renteneintritt ermöglichen. }\end{array}$ & $\begin{array}{l}\text { [5]: Vermögen } \\
\text { [8]: Vermögen (BRD) } \\
\text { [8]: Grundvermögen (USA) }(+)\end{array}$ \\
\hline $\begin{array}{l}\text { Arbeitsmarktsituati } \\
\text { on }\end{array}$ & $\begin{array}{l}\text { Die Tendenz, bei hoher Arbeitslosigkeit, äl- } \\
\text { tere Arbeitnehmer früher zu entlassen, ist in } \\
\text { vielen Staaten zu beobachten. Insofern ist } \\
\text { die Arbeitslosenrate einer Volkswirtschaft } \\
\text { ein potentieller EinfluBfaktor des Rentenal- } \\
\text { ters (vgl. Kapitel 4.1) }\end{array}$ & $\begin{array}{l}\text { [1]: Arbeitslosenrate } \\
\text { [1]: Wiedereinstellungswahr- } \\
\text { scheinlichkeit } \\
\text { [6]: Arbeitslosenrate }\end{array}$ \\
\hline $\begin{array}{l}\text { Betriebliche } \\
\text { Altersversorgung }\end{array}$ & & \begin{tabular}{l} 
[5]: Betriebsrentenzusage \\
\multicolumn{2}{c}{ (insignifikant) } \\
[7]: einzige Variable $\quad((+))$
\end{tabular} \\
\hline \multicolumn{3}{|c|}{ IV. Institutionelle Regelungen: } \\
\hline Pflichtverrentung & $\begin{array}{l}\text { verpflichtende Rentenalter durch Arbeits- } \\
\text { oder Tarifverträge, in Deutschland auch im } \\
\text { öffentlichen Dienst. }\end{array}$ & [5]: Pflichtrentenalter \\
\hline $\begin{array}{l}\text { Mindestalter bei } \\
\text { Rentenbeginn }\end{array}$ & $\begin{array}{l}\text { Früheste Zugangsmöglichkeiten (vgl. Kapi- } \\
\text { tel } 3 \text { und 4). }\end{array}$ & $\begin{array}{l}\text { [10]: vorgegebene Rentenzu- } \\
\text { gangsalter }\end{array}$ \\
\hline
\end{tabular}

Literaturbezüge:
[1] AARTS \& DE JONG (1993)
[6] MEGHIR UND WHITEHOUSE (1993)
[2] KRUEGER UND PISCHKE (1991)
[7] LUMBSDAINE, STOCK UND WISE (1992 und 1993)
[3] GUSTMAN UND STEINMEIER (1991)
[8] BORSCH-SUPAN (1991)
[4] BERKOVEC UND STERN (1991)
[9] BÖRSCH-SUPAN (1992 e)
[5] SUEYOSHI (1989)
[10] Eigene Untersuchung des 4. Kapitels

Ein positives Vorzeichen der 3. Spalte bedeutet im Falle einer Indikatorvariable, daß deren Vorliegen die Wahrscheinlichkeit einer (frühen) Verrentung erhöht. Bei einer kontinuierlichen Variable gilt dies für hohe Werte. Es werden nur Koeffizienten berücksichtigt, die mindestens $90 \%$ signifikant sind.

\section{Arbeitshypothesen für eine empirische Untersuchung}

Durch die unterschiedlichen (landesspezifischen) Voraussetzungen und Modellrahmen der vorgestellten Studien ist es nicht möglich, die dort getesteten Entscheidungsvariablen einer Wahl des Rentenalters direkte zu übernehmen, sondern es gilt, die in der Literatur gewonnenen Erfahrungen in den in dieser Arbeit gewählten empirischen Rahmen umzusetzen. Aus diesem Grunde werden im Folgenden die in einer empirischen Untersuchung der Rentenzugänge zu berücksichtigenden Variablen genannt und die Erwartung an ihre Einflußrichtung in Form von Arbeitshypothesen formuliert, die in der ökonometrischen Untersuchung des siebten Kapitels überprüft werden: 


\section{Tabelle 5-6: Arbeltshypothesen für die Verweildauerschätzung}

Variable

erwartete Wirkungsrichtung: 56

Lebensalter

Mit höherem Lebensalter ist eine steigende Neigung zur Beendigung des Arbeitslebens zu erwarten. Dieser Zusammenhang kann auch durch die Definition altersvariabler ökonomischer Indikatoren modelliert werden.

Geburtskohorte

(unbestimmt)

Eine Berücksichtigung des Geburtsjahrganges ist für eine statistische Kontrolle um Kohorteneffekte nötig, damit eine unverzerrte Ermittlung der Alterseffekte erzielt werden kann.

Geschlecht (weiblich)

Da die Erwerbshistorien von Männern und Frauen stark differieren und weiterhin unterschiedliche gesetzliche Zugangsberechtigungen für die Gesetzliche Rentenversicherung gelten, sollte in der Analyse nach Geschlechtern differenziert werden. Aus den GRV-Regelungen ist ein früherer Rentenzugang von Frauen zu erwarten.

Gesundheit (Gesundheitsprobleme)

Der Gesundheitszustand der Befragungspersonen wirkt direkt auf die Wahl des Rentenalters. Personen, deren Erwerbsfähigkeit eingeschränkt ist, tendieren zu früherem Renteneintritt.

Persönliche Situation

(unbestimmt)

Die familiäre Situation ist als Kontrollvariable in einer Schätzung zu berücksichtigen.

Ausbildung (Dauer)

Die Ausbildungsdauer dient als Approximation der Qualifikation und damit verbunden des beruflichen Status. Von Personen mit höherer Ausbildung wird eine höhere Arbeitsmotivation (durch Verantwortung und Selbstbestimmung), die auf eine Verlängerung der Lebensarbeitszeit hinwirken.

Branche / Stellung im Beruf (gehobenere Stellung)

Branchenindikatoren dienen der Kontrolle um branchenspezifische Effekte. Die berufliche Stellung dient ebenso wie die Ausbildung als Approximation für Arbeitsmotivation und -zufriedenheit. Im Gegensatz zur reinen Bildungsdauer stellt sie eine realisierte Größe dar, unter der Annahme, daß die erreichte Stellung erarbeitet werden mußte und somit eine Bewertung durch den Arbeitgeber darstellt.

56 Interpretation der Vorzeichen analog Tabelle 5-5. 
Firmenspezifika und betriebliche Altersversorgung (Anspruch vorhanden)

Aus der empirischen Tatsache, daß Betriebsrenten früher beginnen als die GRV (Kapitel 3.2.2), ist zu schließen, daß Personen mit einem Anspruch auf eine solchen Rente zu früherem Arbeitsaustritt tendieren. Auch erhöht eine Betriebsrente das Ruhestandseinkommen und verkürzt damit die „Sparphase“ im Lebenszyklus.

Arbeitseinkommen (Höhe; Erwartung der zukünftigen Entwicklung) (unbestimmt) Das Einkommen aus Erwerbstätigkeit stellt Opportunitätskosten der Verrentung dar. Je höher diese Kosten, desto später erfolgt ceteris paribus die Verrentung. Gegenläufiger Effekt: Je höher das Arbeitseinkommen, desto höher der Rentenanspruch (Einkommens- versus Substitutioneffekt) (s.u. Renteneinkommen).

Renteneinkommen (Höhe der Anwartschaft)

Die Rentenhöhe stellt die Grundlage für den Konsum im Alter dar. Unter der Annahme, da $B$ dieser Konsum intertemporal geplant wurde, kann ein Arbeitnehmer umso früher in Rente gehen, je höher das Renteneinkommen ist.

Es ist zu erwarten, daß die Abwägung zwischen Arbeits- und Renteneinkommen auf der Basis eines relativen Vergleiches vorgenommen wird, der in einem Entscheidungsmodell modelliert werden muß. Eine (zeitpunktbezogene) Ersatzquote stellt dabei eine einfache Formulierung dar, wogegen sich in diesem Kapitel die Variable des Optionswertes als dynamische Operationalisierung dieser Abwägung deutlich empfohlen hat. Da dieser spezifiziert ist als der Wert der Option, später in Rente zu gehen, sollten Arbeitnehmer mit einem hohen Optionswert ein späteres Rentenalter wählen. Das erwartete Vorzeichen des Optionswertes ist folglich negativ:

Optionswert

Vermögen

Vermögen (Finanzvermögen und Immobilien) ist aus ökonomischer Sicht eine Einkommensquelle. Daher gilt dieselbe Argumentation wie für Renteneinkommen. Auch private Zusatzversicherungen wirken auf die gleiche Weise.

Arbeitsmarktsituation (hohe Arbeitslosigkeit)

Bei hoher Arbeitslosigkeit werden Entlassungen oft über Sozialpläne vorgenommen, von denen ältere Menschen besonders betroffen sind. Es ist zu erwarten, $\mathrm{da} ß$ Unternehmen in Konjunkturkrisen älteren Angestellten finanziell interessante Angebote für die Unterstützung der Arbeitslosen- / Renteneinkommen machen, damit diese früher ausscheiden. 
Das wird demnächst schon besser gehen, Wenn Ihr lernt, alles zu reduzieren, Und gehörig zu klassifizieren.

\section{Ein Hazardratenmodell der Wahl des Rentenalters}

In diesem Kapitel wird auf Basis der Erfahrungen aus den bisherigen Ansätzen ein Modell der individuellen Verrentungsentscheidung entwickelt, das auf der Grundidee eines Zeitratenmodelles basiert, welches um das Konzept des Optionswertes erweitert wird. Zunächst werden die Grundlagen der Verweildaueranalyse dargestellt und daraus das im folgenden empirischen Teil der Arbeit zu verwendende Regressionsmodell ${ }^{1}$ entwickelt.

\subsection{Zeitraten- oder Verweildaueranalyse}

Die Verweildaueranalyse taucht in der Literatur unter verschiedenen Bezeichnungen auf. So bezeichnen 'Übergangsratenmodell', 'Lebensdaueranalyse', 'Duration (Verweildauer) Model', oder 'Failure Time (Ausfallzeit) Model', 'Event history analysis (Ereignisanalyse)' und ähnliche Termini dieselbe Modellklasse. Die grundlegende Idee ist, daß (Wahl-) Wahrscheinlichkeiten nicht zeitpunktbezogen auftreten, sondern daß sie sich im Zeitablauf ändern, ja vom Zeitablauf selbst oder durch zeitvariate Parameter abhängig sein können. Ein Beispiel für ein zeitkonstantes Wahrscheinlichkeitsmodell sind die beiden Ansätze von BöRSCH-SUPAN (1991, 1992 e, Kapitel 5.3.2), die ein Discrete Choice-Modell benutzen. Discrete Choice Modelle werden in der Ökonometrie oft eingesetzt und beschreiben grundsätzlich das Wahlverhalten von Individuen zwischen 2 oder mehreren Alternativen. ${ }^{2}$ Die Wahl des Verkehrsmittels (modal split), der Automarke oder der Wohnung sind typische Anwendungsbeispiele. Geht es in einer Fragestellung nicht nur um das Was, sondern auch um das Wann einer Entscheidung, muß das benutzte Modell diese Zeitstruktur widerspiegeln. Neben der großen Klasse der Dynamischen-Programmierungs Modelle sind dies vor allem die Zeitratenanalysen.

1 Der Begriff „Regression“ wird hier im eigentlichen Wortsinn des kausalen Zurück-führens (lat. regressio) einer abhängigen Variable auf eine oder mehrere unabhängige benutzt und ist insofern ein allgemeiner Begriff für eine statistische Analyseform. Insbesondere ist er nicht auf lineare Regressionen beschränkt.

2 Siehe Kapitel 5.1, in dem PROBIT und LOGIT Ansätze als typische Beispiele genannt wurden. Vergleiche auch RONNING (1991), Seite 70 ff. 
Die ursprüngliche Anwendung dieser Methode entstammt zum einen der Medizin, wo tatsächliche Überlebensdauern von Patienten nach Operationen oder Behandlungen, aber auch Zeitdauern von Krankenhausaufenthalten gemessen werden. Ein weiteres großes Gebiet ist die Qualitätskontrolle: Haltbarkeit einzelner Komponenten von Maschinen („Failure Time“) oder ganzer Anlagen und Gebäude in Abhängigkeit von der bisherigen Einsatzdauer sind hier wichtige Fragestellungen. In der volkswirtschaftlichen Praxis kommen die meisten Anwendungen aus dem Bereich der Arbeitslosigkeitsforschung, einem der hier behandelten Fragestellung verwandten Themengebiet. Die untersuchten Fragestellungen befassen sich mit verschiedenen Zuständen, zwischen denen die Befragten im Zeitablauf wechseln können. Im vorliegenden Fall sind dies die Zustände der Erwerbstätigkeit oder der des Ruhestandes. Dies ist ein Beispiel für einen absorbierenden Endzustand, da ein Wechsel in den Ruhestand nicht zurücknehmbar ist. Bei der Fragestellung der Arbeitslosigkeit ist dies nicht der Fall. Hier kann eine Person mehrfach zwischen Arbeitstätigkeit und Erwerbslosigkeit hin und her wechseln. Die Zeitdauer, die ein Individuum in einem Zustand verbringt, wird als Episode oder Spell bezeichnet.

\subsubsection{Wahrscheinlichkeitstheoretische Grundlagen}

Im folgenden wird eine einfache und möglichst intuitive Herleitung zentraler Konzepte der Verweildaueranalyse dargestellt. Die konkrete Ausformulierung der Modelle ist in der Literatur an vielen Stellen nachzulesen. Das bekannteste Grundlagenwerk ist das von KALBFLEISCH UND PRENTICE (1980), in Deutschland haben u.a. BLOSSFELD, HAMERLE, MAYER UND TUTZ das Thema bearbeitet (vgl. etwa BlOSSFELD, HAMERLE UND MAYER (1989) oder HAMERLE UND TUTZ (1989) ), sowie SCHNEIDER (1991), der die Präsentation mit einem Modul des Statistik-Programmpaketes GAUSS verbindet.

Im Unterschied zu einem einfachen Wahrscheinlichkeitsmodell ist die Fragestellung von Interesse also:

Wie groß ist die Wahrscheinlichkeit für einen Zustandswechsel, gegeben, daß der Anfangszustand bereits seit einer vorgegebenen Zeit andauert?

Konkretisiert auf die Fragestellung der Ruhestandsentscheidung eines Arbeitnehmers lautet die Frage nach der Wahrscheinlichkeit, daß der Verrentungszeitpunkt $\mathbf{R}$ erst nach einem Zeitpunkt $\tau$ stattfindet, geschrieben werden als:

$$
\operatorname{Prob}(R \geq \tau)=G(\tau)
$$


Zur Symbolik:

R Verrentungszeitpunkt (Lebensalter)

t Zeitachse

$\tau \quad$ ein betrachteter Punkt auf dieser Zeitachse (Ausprägung von $t$ ) im Folgenden bezeichne daher:

f eine Funktion allgemein und

$f(\tau) \quad$ den Funktionswert von $f$ an der Stelle $\tau$

Diese Funktion $G(t)$ wird Überlebensfunktion genannt. Sie läßt sich ausdrücken als $1-F(t)$, wobei $F(t)$ die Verteilungsfunktion angibt, die bekanntlich die umgekehrte Wahrscheinlichkeit

$$
F(\tau)=\operatorname{Prob}(R \leq \tau) \text { angibt. }
$$

Dies sei zur Anschauung graphisch dargestellt. Die Wahrscheinlichkeit, zu einem bestimmten Zeitpunkt in Rente zu gehen, wird durch die Dichtefunktion angegeben. Da allerdings die Wahrscheinlichkeitstheorie zeigt, daß die Wahrscheinlichkeit eines exakt definierten Zeitpunktes immer gleich Null ist, muß ein infinitesimal kleiner Zeitraum betrachtet werden, der hier als $\Delta t$ angenommen sei:

$$
f(\tau)=\operatorname{Prob}(t \leq \tau<t+\Delta t)
$$

Abbildung 6-1 zeigt den Verlauf dieser Funktion für den Fall eines normalverteilten Merkmales. Zur Herleitung der Überlebensfunktion zeigt Abbildung 6-2 zunächst die aus der Dichte abgeleitete Verteilungsfunktion, welche die kumulierte Dichtefunktion (c.d.f) darstellt:

$$
F(\tau)=\int_{-\infty}^{F} f(t) d t
$$

\section{Abbildung 6-1: Wahrscheinlichkeitsdichtefunktion $f$ der Normalverteilung}

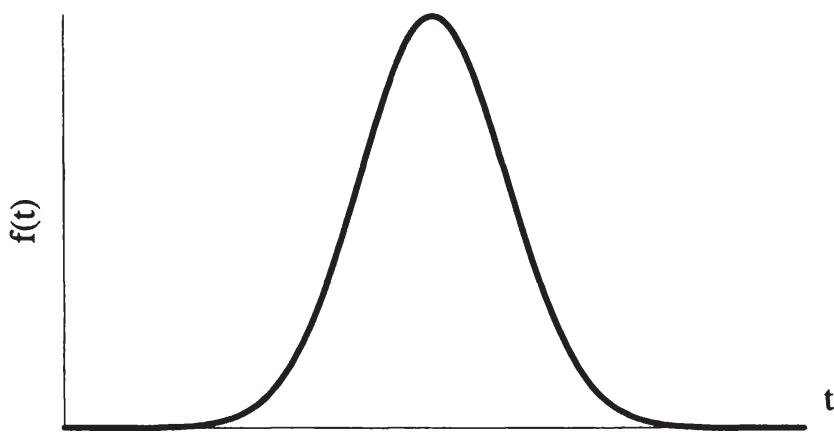




\section{Abbildung 6-2: Verteilungsfunktion F der Normalverteilung}

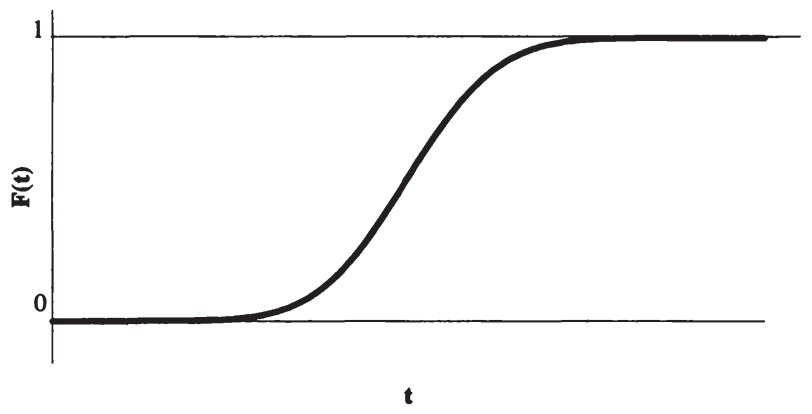

Die Umkehrung dieser Wahrscheinlichkeit, daß die Verrentung $\mathbf{R}$ nach einem Zeitpunkt $\tau$ stattfindet, stellt die Überlebensfunktion dar, die in (6-1) definiert und in Abbildung 6-3 graphisch dargestellt wird. Der Funktionswert G(t) gibt also (am Beispiel) für jedes potentielle Rentenalter $t$ die Wahrscheinlichkeit dafür an, daß bis zu diesem Alter noch nicht in Rente gegangen wurde.

Abbildung 6-3: Überlebensfunktion der Normalverteilung

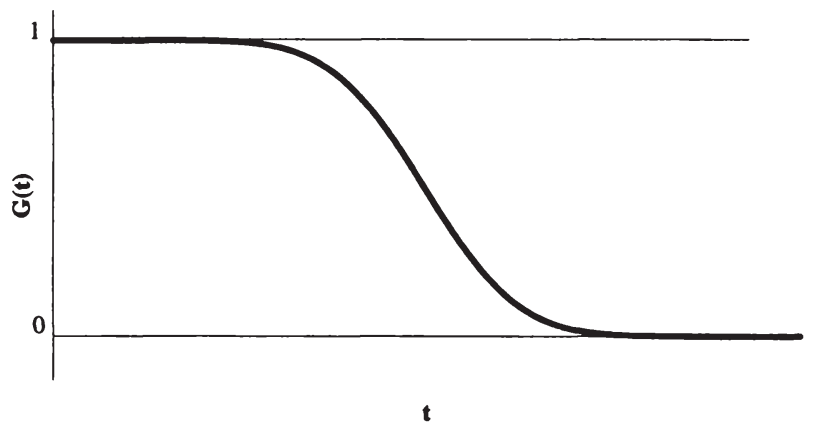

\subsubsection{Die Hazardrate als zentrales Konzept}

Bei der Betrachtung der Verrentung ist aber die Frage nicht nur, ob diese nach einem Zeitpunkt stattfindet, sondern ob sie $z u$ einem Zeitpunkt (z.B. Lebensalter) stattfindet unter der Bedingung, daß die Person bisher noch erwerbstätig ist. Es wurde oben bereits gesagt, daß eine solche Wahrscheinlichkeit für einen Punkt der Zeitachse nicht 
existiert, so da $\beta$ sie als Grenzwertbetrachtung für ein infinitesimal kleines Intervall $\Delta t$ formuliert werden muß:

$$
\lambda(\tau)=\lim _{\Delta t \rightarrow 0} \frac{\operatorname{Prob}(t \leq \tau<t+\Delta t \mid \tau \geq t)}{\Delta t}
$$

Dieser Ausdruck ist das zentrale Konzept der Verweildaueranalyse. Er wird als Hazardrate bezeichnet. Es ist zu beachten, daß dieser Grenzübergang keine Wahrscheinlichkeit ist. Durch die Grenzbetrachtung ist die Hazard zwar noch stets positiv, kann aber auch Werte über 1 annehmen. Es handelt sich um eine latente Variable, die als Intensität des Ausscheidens aufgefaßt werden kann. Je höher diese Hazard ist, desto höher ist die Wahrscheinlichkeit für ein Verlassen des Anfangszustandes. ${ }^{3}$ Die hier beschriebene theoretische Hazard steht damit im Gegensatz der etwa von SUEYOSHI definierten empirischen Hazardrate, ${ }^{4}$ die (für jede Altersgruppe) als Quotient „(Abgänge in Periode $\mathrm{t} /$ Anzahl Personen zu Beginn von $\mathrm{t}$ ) 100 “ definiert war und damit per Definition einen Prozentsatz $\in\{0,1\}$ darstellt. Ein ähnliches Konzept sind die im vierten Kapitel verwendeten Verrentungsraten.

Die theoretische Hazardrate kann aus den o.a. Funktionen direkt ermittelt werden:

$$
\lambda(t)=\frac{f(t)}{G(t)} \quad \text { bzw: } \quad \lambda(t)=\frac{f(t)}{1-F(t)}
$$

mit: $\quad \mathrm{t}$ hier: Verrentungszeitpunkt (Lebensalter)

$\tau \quad$ ein betrachtetes Lebensalter

$\mathrm{f}(\mathrm{t}) \quad$ Wahrscheinlichkeitsdichte

$\mathrm{F}(\mathrm{t}) \quad$ Verteilungsfunktion

$G(t)$ Überlebensfunktion.

\section{Spezifikationen der Übergangsrate}

Der nächste Schritt nach der wahrscheinlichkeitstheoretischen Betrachtung muß die konkrete Ausformulierung eines Regressionsmodelles sein, mit dessen Hilfe empirische Fragestellungen behandelt werden können. In diesem Abschnitt sollen daher die wichtigsten Hazardraten-Modelle vorgestellt werden.

\footnotetext{
3 Vgl. SCHNEIDER (1991), Seite 86.

${ }^{4} \mathrm{Vgl} \mathrm{Kapitel} \mathrm{5.2.2} \mathrm{und} \mathrm{SUEYOSHI} \mathrm{(1989),} \mathrm{Seite} 25$.
} 
Die einfachste Formulierung des Übergangsratenmodelles ist das Exponentialmodell . Es basiert auf der Annahme einer zeitkonstanten Hazardrate über alle Zeitpunkte.

$$
\lambda(\mathrm{t})=\bar{\lambda} \quad \forall \mathrm{t}
$$

so daß sich für die Überlebens- bzw. Dichtefunktion ergeben:

$$
f(t)=\bar{\lambda} \cdot e^{-\bar{\lambda} \cdot t} \quad \text { sowie } \quad G(t)=e^{-\bar{\lambda} \cdot t}
$$

was die Herkunft des Namens Exponentialmodell verdeutlicht.

Für eine Regressionsanalyse muß eine funktionale Abhängigkeit der Hazardrate von erklärenden Variablen definiert werden. Die Matrix der erklärenden Variablen sei als $\mathrm{X}_{\mathrm{t}}$ bezeichnet. Durch deren Parametrisierung hat das Exponentialmodell das folgende Aussehen:

$$
\lambda\left(t \mid X_{t}\right)=e^{X_{t} \cdot \beta}
$$

mit:
t hier: Verrentungszeitpunkt (Lebensalter)
$\mathbf{X}_{\mathbf{t}} \quad$ Matrix der erklärenden Variablen
$\beta \quad$ Parameter-Vektor der zu ermittelnden Regressionskoeffizienten.

Die Schätzparameter $\beta_{\imath}$ werden durch die Regression ermittelt und stellen das relative Gewicht der spezifizierten Erklärungsvariablen dar.

\section{Proportional Hazard Modelle}

Unter Proportional Hazard Modellen werden solche Verweildauerprozesse verstanden, deren Hazard zeitabhängig spezifiziert ist. Die Hazardrate wird in diesem Falls als zweiteilige Funktion formuliert, die eine Baselinehazard $\lambda_{0}(t)$ sowie den multiplikativ verknüpften Teil der erklärenden Variablen, die als Kovariate bezeichnet werden.

Damit ergibt sich (6-10) als Spezifikation der Hazardrate. ${ }^{6}$

5 Gemeint ist der Fall der Spezifikation von Kovariaten. Im folgenden werden nur parametrische Proportional Hazardmodelle betrachtet.

${ }^{6}$ Es wird damit der Formulierung von SCHNEIDER (1991, Seite 136) gefolgt. Dies Formulierung ist eine mögliche Spezifikation der Kovariate, allerdings die weitaus gebräuchlichste. Andere Spezifikation der funktionalen Abhängigkeit von $X$ und $\beta$ sind denkbar, werden aber nicht weiter verfolgt. 


$$
\lambda\left(t \mid x_{t}\right)=\lambda_{0}(t) \cdot e^{x_{1} \beta}
$$

mit:

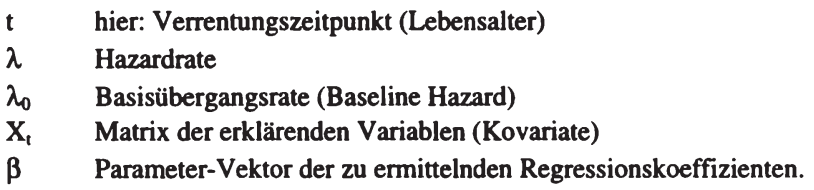

Die Architektur dieser Modellklasse besteht somit in einer Zweiteilung in eine noch näher zu spezifizierende Baselinehazard-Funktion, die nur zeitabhängig spezifiziert ist, und einer Funktion die den Einfluß der Kovariate auf die Gesamtübergangsrate mißt. Die Kovariate erhalten damit den Charakter von Lageparametern für die Basisübergangsrate. Es ist ersichtlich, daß (6-10) den Spezialfall des Exponentialmodelles (6-9) beinhaltet, für den Fall daß $\lambda_{0}$ zeitinvariat ist.

Die Klasse der Proportional Hazard Ansätze macht den größten Teil der gängigen Übergangsratenmodelle aus, die sich durch die Spezifikation der Baselinehazard $\lambda_{0}$ genauer der dieser für Funktion angenommenen Verteilung - unterscheiden.

\subsubsection{Gebräuchliche Verweildauermodelle}

In diesem Abschnitt wird die Spezifikation der Basisübergangsrate $\lambda_{0}$ als Funktion der Verweildauer $\mathbf{t}$ näher betrachtet. Aus den gebräuchlichen Modellen, die in der Regel nach der zugrunde liegenden Verteilungsannahme benannt sind, seien hier zwei, das Weibullmodell und das Gompertzmodell, herausgegriffen, um an ihnen das grundlegende Funktionsprinzip dieser Modelltypen darzulegen.

Die Baseline Hazard im Gompertz Modell folgt einer Gompertzverteilung? und hat somit die Form:

$$
\lambda_{0}(\mathrm{t})=\exp (\gamma \mathrm{t})
$$

Der graphische Verlauf dieser parametrisierten Baseline Hazard hängt von der Größe des Parameters $\gamma$ ab. Er wird in Abbildung 6-4 gezeigt. Es kann also in diesem Modell aus dem Schätzwert für den Parameter $\gamma$ der Funktionsverlauf abgelesen werden.

${ }^{7}$ Dei Gompertz-Verteilung ist ein Sonderfall der rechtssteilen Extremwertverteilung. Vergleiche dazu die Anmerkung in SCHNEIDER (1991), Seite 151. 


\section{Abbildung 6-4: Baseline-Hazard des Gompertz Modelles}

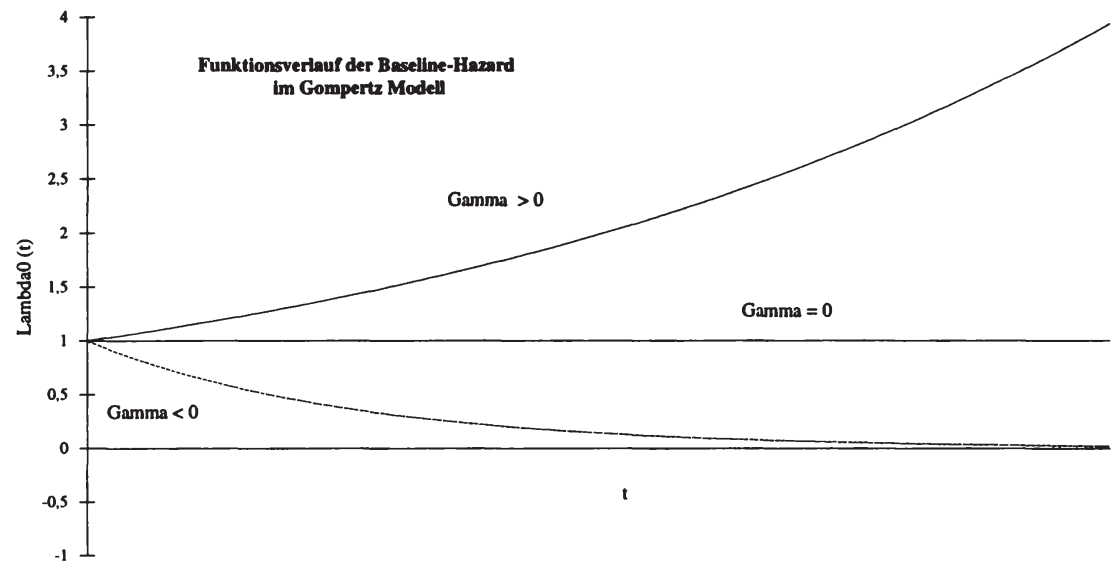

Die gesamte Hazard damit ergibt sich als:

$$
\lambda(t)=\exp (\gamma \cdot t) \cdot \exp \left(X_{t} \cdot \beta\right)
$$

Für den Fall, daß $\gamma$ den Wert Null annimmt, ergibt sich der Sonderfall des Exponentialmodelles. Daraus wird noch einmal die Struktur von Proportional-Hazard Modellen deutlich, in denen zwei Arten von Parametern zu unterscheiden sind:

- die Funktionsparameter, hier $\gamma$, bestimmen den Verlauf der Hazard, Monotonie, Stetigkeit, Nullstellen der Baseline-Hazard $\lambda_{0}$.

- die Lageparameter, $\boldsymbol{B}_{\mathrm{i}}$, die den Einfluß der Kovariaten $\mathrm{X}_{\mathrm{t}, \mathrm{i}}$ messen. In den betrachteten einfachen Modellen sind die Lageparameter unabhängig von der Verweildauer $t$.

Die Überlebensfunktion des Gompertz-Modelles lautet:

$$
G\left(t \mid X_{t}\right)=\exp \left[\frac{\exp \left(X_{t} \beta\right)}{\gamma} \cdot(1-\exp (\gamma \cdot t))\right]
$$

Die Optimierung im Gompertz Modell hängt also von zwei Arten von Parametern ab. Zum einen vom Funktionsparameter $\gamma$ und weiterhin von den Koeffizienten $\beta$ der unabhängigen Variablen $X_{t}$. Dies wird auch in der über diese beiden Parameter(vektoren) zu maximierenden logarithmierten Likelihood-Funktion (6-14) des GompertzModelles sichtbar. ${ }^{8}$

\footnotetext{
${ }^{8}$ In der Modellformulierung von SCHNEIDER (1991) Seiten 150 - 154, der in dieser Darstellung weitgehend gefolgt wurde. Für eine sehr ausführliche Herleitung der Likelihood und die Angabe von deren Ableitungen, vergleiche ROHWER (1993), Seite $19 \mathrm{ff}$.
} 


$$
\ell(\gamma ; \beta)=\sum_{i}\left(\gamma t_{i}+X_{i} \beta\right)-\frac{\exp \left(X_{i} \beta\right)}{\gamma}\left[1-\exp \left(\gamma t_{i}\right)\right]
$$

Durch den Verteilungsparameter g kann das Gompertz-Modell Ermittlung des Verlaufes der Hazardrate benutzt werden. Am Wert des geschätzten Parameters $\hat{\gamma}$ ist dann der Verlauf der Hazard direkt abzulesen.

Dies gilt auch im Weibull Modell, dessen Übergangsrate

$$
\lambda_{0}(t)=\exp (\phi) \cdot t^{\exp (\phi)-1},
$$

ebenfalls einen Parameters $(\phi)$ beinhaltet, dessen Höhe den Funktionsverlauf der Basisübergangsrate bestimmt. Es ist hier neben einer konstanten und einer linear steigenden Rate sogar möglich, je nach Höhe des Parameters, einen konkaven oder konvexen Verlauf zu erzielen, wie Abbildung 6-5 zeigt. Dies resultiert aus der Besonderheit, daß $\lambda_{0}$ sich an der Stelle $\ln (2) \mathrm{zu} \lambda_{0}(\ln (2))=2 \mathrm{t}$ vereinfacht. Neben diesem linear steigenden Verlauf, ist auch hier wieder das Exponentialmodell als Sonderfall für $\phi=0$ enthalten.

\section{Abbildung 6-5: Dle Hazard-Rate des Welbull Modelles .}

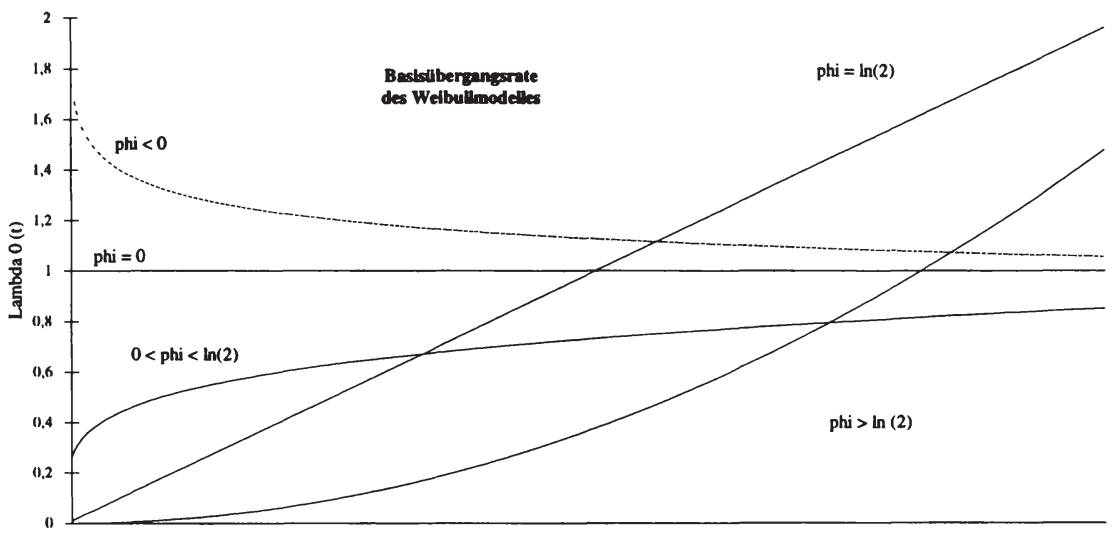


Die Überlebens- und Likelihood-Funktion im Weibull-Modell ergeben sich als:9

$$
\begin{aligned}
& G(t \mid X)=\exp \left[-\exp (X, \beta) t^{\exp (\phi)}\right] \\
& \ell(\gamma ; \beta)=\sum_{i}\left(\gamma t_{i}+X_{i} \beta\right)-\frac{\exp \left(X_{i} \beta\right)}{\gamma}\left[1-\exp \left(\gamma t_{i}\right)\right] .
\end{aligned}
$$

Das Weibull Modell ist in der Klasse der Proportional Hazard Modelle das bekannteste und wird häufig in empirischen Anwendungen verwendet.

Weitere Modelle dieser Klasse sind etwa das Sickle Modell, das seinen Namen dem sichelförmigen Verlauf der Hazard verdankt und zwei Verteilungsparameter aufweist, ebenso wie das log-nomale und das log-logistische, dessen Erweiterung ebenso drei Verteilungsparameter benutzt wie das Gamma Modell und andere. ${ }^{10}$ Den erweiterten Möglichkeiten durch die Verteilungsanpassungen über 3 Parameter steht in diesen Ansätzen der große programmierungstechnische Aufwand gegenüber und ebenfalls die damit verbundene lange Laufzeit, vor allem bei größeren Datenmengen.

In der vorliegenden Arbeit werden die beiden hier diskutierten Modelle, das Gompertz und das Weibull-Modell empirisch getestet und dabei auch die statistische Anpassung der beiden Ansätze verglichen um so zu ermitteln, welche Spezifikation der vorliegenden Fragestellung besser gerecht wird.

\section{Zur statistischen Güte von ML Schätzungen}

Ein $\mathrm{Ma} \beta$ für die Schätzgüte, wie das $\mathbf{R}^{2}$ der linearen Regression, existiert in einer nichtlinearen Maximum Likelihood Regression nicht. Zur Bewertung der statistischen Aussagekraft eines Schätzmodelles haben sich daher verschiedene gebräuchliche Gütemaße herausgebildet, um den statistischen Erklärungsgrad einer solchen Regression zu beurteilen." Eines der bekanntesten Maße ist das aus der Likelihood Ratio (Likelihood Quotienten) abgeleitete $\rho^{2}$ (beziehungsweise „Pseudo $\mathrm{R}^{2 ،}$ ). Diese Bezeichnung lehnt sich an das $\mathbf{R}^{2}$ der linearen Regression an.

\footnotetext{
9 SCHNEIDER (1991), Seite 157.

10 Vergleiche für eine ausführliche und anschauliche Diskussion wiederum ROHWER (1993), aber auch KALBFLEISCH \& PRENTICE (1980).

${ }^{11}$ Für eine Diskussion vergleiche etwa MAIER und WEISS (1990, Seite 91).
} 
(6-18) $\quad \rho^{2}=1-\frac{l^{\max }}{l^{0}}$,

Es wird also berechnet aus einem Quotienten des Log-Likelihoodwertes im ermittelten Maximum an der Stelle der ermittelten Schätzparameter und des Log-Likelihoodwertes, der sich ergeben würde, wenn alle Koeffizienten Null wären. Die Werte der Likelihood sind Wahrscheinlichkeiten, liegen also zwischen 0 und 1 , so daß die logarithmierte Likelihood eine negative Zahl ist, die dem Betrage nach kleiner wird, wenn sich die Likelihood verbessert. Aus diesem Grunde ist der obige Ausdruck ebenfalls auf den Bereich $[0,1]$ normiert, so daß der Wert für $\rho^{2}$-ähnlich wie das $\mathbf{R}^{2}$ - als Prozentsatz interpretiert werden kann. Dies ist allerdings nicht der Prozentsatz der erklärten Varianz wie in der linearen Regression, sondern die prozentuale Verbesserung der Likelihood gegenüber dem Null-Modell.

Ein weiteres häufig in ML-Regressionstabellen angegebenes Maß ist das des Prozentsatzes der richtig vorhergesagten abhängigen Variablen (Percent Correctly Predicted). Hier wird die Regressionsgleichung an der Stelle der optimalen Parameter zu einer Vorhersage der abhängigen Variablen benutzt und diese vorhergesagten Werte mit den tatsächlich beobachteten verglichen.

\subsection{Spezifikation eines parametrischen Proportional Hazard Modelles unter Berücksichtigung des Optionswertes}

Im fünften Kapitel wurden auf Basis der dort vorgestellten Literatur der Rentenzugangsentscheidungen Arbeitshypothesen für eine empirische Untersuchung aufgestellt, die am Ende des Abschnittes 5.4 zusammengefaßt sind. Die Umsetzbarkeit der dort aus theoretischer Sicht geforderten Erklärungsvariablen muß im siebten Kapitel anhand des tatsächlich vorliegenden Datensatzes überprüft werden.

Als adäquate ökonomische Variable wurde dort der Optionswert genannt, der in unterschiedlichen Spezifikationen dargestellt wurde. Im folgenden wird die in der vorliegenden Arbeit benutzte Spezifikation dargestellt:

\section{Definition der Optionswert -Variablen}

Das von LUMBSDAINE, STOCK UND WISE entwickelte Konzept des Optionswertes, d.h. des Wertes der Zunkunftsoptionen, die ein Arbeitnehmer sich dadurch offen läßt, daß er noch nicht in Rente geht, ist nicht an die dort implementierten Modelle gebunden. Diese Modelle waren Optionswertmodelle in dem Sinne, daß der Optionswert modell- 
konstituierend ist. Dies ist, wie in den Ansätzen von BÖRSCH-SUPAN ${ }^{12}$ gesehen, kein notwendiges Vorgehen. Die Variable „Option Value“ ist vielmehr eine Operationalisierung für die ökonomischen Anreize der potentiellen Rentenzugangsalter, die. Sie hat den Vorteil, daß sie die reinen Alterseffekte mit den institutionell durch das Rentenversicherungssytem bedingten Wirkungen kombiniert und zu einer Maßzahl verbindet.

In Analogie zum Modell von BÖRSCH-SUPAN (1992 e) wird der Optionswert hier wie folgt definiert:

Bei der Definition des Barwertes :

$$
V_{1}(R)=E_{1}\left[\sum_{s=1}^{R-1} u\left(Y_{s}^{L}\right) \cdot a_{s} \cdot \delta^{s-1}+\alpha \cdot \sum_{s=R}^{g 9} u\left(Y_{s}^{R}\left(R, Y_{s}^{L}\right)\right) \cdot a_{s} \cdot \delta^{s-1}\right]
$$

$\begin{array}{lll}\text { mit: } & \mathrm{V}_{\mathrm{t}}(\mathrm{R}) & \text { Barwert aller Lohn- und Rentenströme in Abhängigkeit von } \mathrm{R} \\ & \mathrm{R} & \text { Rentenzugangsalter (Austritt aus dem Erwerbsleben) } \\ & Y_{s}^{L} & \text { Arbeitseinkommen (Labor income) im Alter s } \\ & Y_{s}^{R}\left(\mathrm{R}, \mathrm{Y}^{\mathrm{L}}\right) & \text { Renteneinkommen im Alter } \mathrm{s} \\ & \mathrm{a}_{\mathrm{t}} & \text { Überlebenswahrscheinlichkeit bis zum Alter t } \\ \alpha & \text { Grenznutzen der Freizeit } \\ \delta & \text { Diskontfaktor } \\ \mathrm{E}_{\mathrm{t}} & \text { Erwartungswert im Alter t } \\ \mathrm{OV} & \text { Optionswert (Option Value) }\end{array}$

ist die Annahme einer Lebenszeit bis 99 Jahre willkürlich, für die numerische Ermittlung des Barwertes jedoch erforderlich. Hohe Altersjahre gehen ohnehin mit sehr niedrigem Gewicht in die Endsumme ein, da sie mit entsprechend niedrigen Überlebenswahrscheinlichkeiten at multipliziert werden.

Die Nutzenfunktion des Einkommens wird definiert als:

$$
\mathrm{u}(\mathrm{Y})=\mathrm{Y} \sigma
$$

Auf der Basis des optimalen Verrentungsalters

$$
R^{*}(t)=\underset{r>1}{\operatorname{argmax}} V_{t}(r)
$$

wird der Optionswert wie folgt berechnet:

$$
O V(t)=V_{t}\left(R^{*}(t)\right)-V_{t}(t)
$$

12 BÖRSCH-SUPAN (1991 und 1992 e), vergleiche Kapitel 5.3.2). 
Der Optionswert ist groß, wenn ein Arbeitnehmer durch Weiterarbeiten einen hohen Einkommenszuwachs erzielen kann. Aus diesem Grund sollten Personen mit hohem Optionswert nicht zu einer frühen Verrentung tendieren. Der OV liegt in dem Fall, daß der Barwert des optimalen zukünftigen Verrentungszeitpunktes größer ist als der einer sofortigen Verrentung über Null. Ein negativer Wert des OV ist möglich, sollte jedoch nach der Theorie dazu führen, daß die Befragungsperson zum baldmöglichen Zeitpunkt aus dem Erwerbsleben ausscheidet.

Somit ist die erwartete Wirkungsrichtung des Optionswert in einer Regressionsanalyse der Verrentungswahrscheinlichkeit negativ. 
Allein die Welt! Des Menschen Herz und Geist! Möcht jeglicher doch was davon erkennen. $J a$, was man so erkennen heißt!

Wer darf das Kind beim rechten Namen nennen?

\section{Empirische ANALyse der individuellen VerRentungsentscheidung in WEST- UND OSTDEUTSCHLAND}

In diesem Kapitel wird die Rentenalterentscheidung in beiden Teilen der Bundesrepublik Deutschland empirisch untersucht. Dazu werden die in Abschnitt 5.4 aufgestellten Arbeitshypothesen und die Optionswert-Variable auf Basis der vorliegenden Daten in das in Kapitel 6 entwickelte Modell eingebunden.

Zunächst wird die benutzte Datengrundlage, das sozioökonomische Panel SOEP, vorgestellt. Im zweiten Abschnitt werden die zu untersuchenden Variablen gebildet und deskriptiv untersucht. Daran schließt sich die ökonometrische Verweildauer-Analyse für Ost- und Westdeutschland an.

\subsection{Datengrundlage: Das Sozioökonomische Panel (SOEP)}

Das Sozioökonomische Panel ist ein langfristiges Haushaltspanel, das in Deutschland seit 1984 jährlich durchgeführt wird. Es stellt der sozial- und wirtschaftswissenschaftlichen Forschung einen breiten Kranz von Mikrodaten zur Verfügung, indem sowohl persönliche als auch Haushaltsmerkmalen und deren Veränderungen in der Zeit abgefragt werden.' Durch diese „Verfolgung“ von Personen (v.a. deren Arbeitsmarktstatus) über einen Zeitraum von mehreren Jahren, ist das SOEP für die vorliegenden Untersuchung gut geeignet. Es kann nicht nur der Wechsel des Status vom Arbeitnehmer zum Rentner beobachtet werden, sondern die diskutierten Erklärungsgrößen stehen für die Jahre vor und nach dieser Entscheidung zur Verfügung.

Die abgefragten Bereiche des SOEP beinhalten unter anderem:

- Demographie und Bevölkerung

- Arbeitsmarkt und Beschäftigung

- Erwerbs- und Berufsbiographie, Erwerbsstatus, Beschäftigungsmerkmale beim derzeitigen Arbeitgeber, Berufliche Mobilität, Übergang in den Ruhestand, Zukunftspläne und berufliche Erwartungen, Nebenerwerbstätigkeit, Arbeitszeit, Monetäre Gratifikation

- Einkommen, Steuern und Soziale Sicherung

- Einkommensarten und Transfers, Vermögensarten, soziale Sicherung

- Wohnen

- Gesundheit

I Eine Einführung bietet HAHNEFELD (1987). 
- Leistungen privater Haushalte

- Bildung und Qualifikation

- Grundorientierungen, Partizipation und Integration.

Die erste Befragung des SOEP begann 1984 in Westdeutschland mit 5.291 Haushalten, in denen 12.245 Personen lebten. In der siebten Welle 1990 wurden 9.519 Personen in 4640 Haushalten befragt, die Anzahl der Befragten lag also bei 57,9\% der ursprünglichen Anzahl. Da in dieser Anzahl auch die neu befragten Personen enthalten sind (Kinder, die 16 Jahre werden, Haushaltsaufsplittungen), ist die Zahl der über sieben Jahre vollständigen Zeitreihen etwas geringer.

Bereits 1990 wurde die erste Befragung in den neuen Bundesländern durchgeführt.und seit 1991 ist die Zusammenführung der beiden Datensätze Ost und West auf gemeinsame Fragebogen und Codierungen der Variablen im Gange. Die erste Ost-Welle umfaßte 1990 4.453 Personen in 2179 Haushalten, 1992 sind es 4.092 Personen in 2.020 Haushalten entsprechend 82,1\% der Anfangszahl. ${ }^{2}$

Für die vorliegende Untersuchung wurden die ersten 7 Befragungswellen des WestSOEP (1984 - bis 1990, Welle A bis G) und die ersten 3 Wellen der Ost-Stichprobe (1990 bis 1992) verwendet. Aus dem Gesamtbestand wurden diejenigen Personen herausgefiltert, die im jeweilig ersten Beobachtungsjahr 45 Jahre und älter waren. Diese Auswahl stellt sicher, daß auch im letzten beobachteten Übergang West (1989/90) alle 50-jährigen beobachtet werden. In Kapitel 3 war die Notwendigkeit des Einschlusses dieser Altersgruppe deutlich geworden, da einzelne Personengruppen insbesondere im Alter von 50 Jahren vermehrt in Rente gehen (können ${ }^{3}$ ). Es ergeben sich somit für Westdeutschland sechs mögliche Übergangszeitpunkte ${ }^{4}$ und für Ostdeutschland zwei.

\section{Definition der untersuchten Variable}

Zur Aufbereitung der Stichprobe für die in Kapitel 6 beschriebene Verweildaueranalyse mußte zunächst die abhängige Variable "Verrentungsalter" ermittelt werden, da diese nicht explizit im Datensatz enthalten ist. Es wurde daher in jeder Beobachtungs-

2 Diese Angaben beziehen sich auf das gesamte SOEP-Sample aller Altersgruppen und Staatsangehörigkeiten. Quelle: 'SOEPInfo'- elektronisches Datenhandbuch für das Sozioökonomische Panel. Die angegebenen Prozentzahlen sind natürlich wenig vergleichbar, da sie sich einmal auf 7, danach auf 3 Wellen beziehen.

${ }^{3}$ So etwa die Versicherten der knappschaftlichen Rentenversicherung - vgl. Kapitel 3 bzw. 4.

${ }^{4}$ Zeitpunkte des Übergangs vom Status der Erwerbsperson in den Ruhestand. 
le verglichen, ob sich der Erwerbsstatus geändert hatte, d.h. ob Personen, die in der vorigen Welle noch erwerbstätig waren, in den Ruhestand gewechselt waren.

Zur Messung des Erwerbsstatus stehen im Sozioökonomischen Panel prinzipiell vier Möglichkeiten zur Verfügungs:

i. jährlich abgefragter Erwerbsstatus mit den Ausprägungen:

- Vollzeit erwerbstatig

- Teilzeit erwerbstátig (regelmäBig)

- UnregelmäBig erwerbstătig

- In Ausbildung

- Arbeitslos

- Wehr-/Zivildienst

- Nicht erwerbstätig,

ii. monatliche "Kalendarien" des Erwerbsstatus aus dem Kalenderjahr vor der Befragung (ähnliche Kategorien, allerdings expliziter Punkt "Ruhestand"),

iii. monatliche Angabe des Einkommens nach Einzelkategorien (u.a. Renteneinkommen) aus dem Kalenderjahr vor der Befragung,

iv. angegebene Wochenarbeitsstunden.

Die Alternativen ii und iii klingen auf den ersten Blick interessanter, erweisen sich aber als problematisch. Einerseits sind die Angaben in den Kalendarien nicht konsistent. In mehreren Fällen wechseln Personen teilweise mehrmals von Erwerbstätigkeit in Verrentung und zurück. Dies ist aber in Deutschland institutionell unmöglich. Das zweite Problem mit den Optionen ii und iii ist, daß sie im hier benutzten VorabDatensatz der ersten drei Ost-Wellen nicht vorlagen und von daher ein intranationaler Vergleich nicht möglich gewesen wäre.

Die Berechnung des Erwerbsstatus aus den Wochenarbeitsstunden (Punkt iv) wurde von BÖRSCH-SUPAN (1992 e - vgl. Kapitel 5.3.2) gewählt. Sie hat den Nachteil, daß das deutsche Rentenrecht die Sozialversicherungspflicht nicht an Wochenstunden, sondern an Einkommensgrenzen festmacht. Von daher können Personen, die von der Arbeitszeit gesehen in die gleiche Rubrik fallen, entweder (sozialversicherungspflichtig) erwerbstätig oder bereits verrentet sein .

Aus diesem Grunde wurde die Alternative i, der jährliche Erwerbsstatus, als abhängige Variable des Verrentungmodelles gewählt. Als "Ereignis" im Sinne der Verweildaueranalyse (s.u.) zählt der Zustandwechsel von den Status 'voll-' bzw. 'teilerwerbstätig' und 'arbeitslos' in den Status 'nicht erwerbstätig'.

\footnotetext{
${ }^{5}$ Für die erste Befragung in der DDR 1990 gibt es eine zusätzliche Variable "Bezug von Altersruhegeld", die aber weder in der West-Befragung, noch den anderen Ost-Wellen existiert und von daher für eine longitudinale Analyse uninteressant ist.
} 


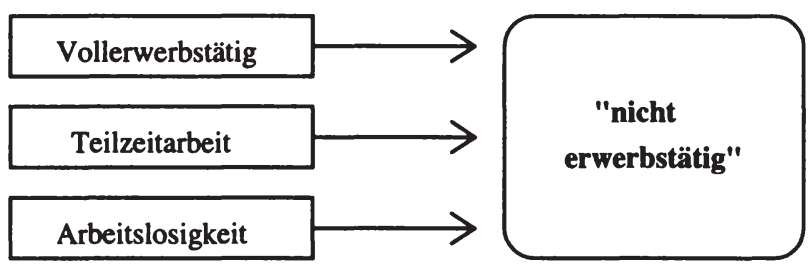

In diesem Sinne ist es angebrachter, von "Austritt aus dem Erwerbsleben" als von "Rentenzugang" zu sprechen, da letzteres nicht tatsächlich gemessen wird. Allerdings liegt bei den hier untersuchten Personen über 50 Jahre die Annahme nahe, daß sie aus der Erwerbstätigkeit ausscheiden, um in den Ruhestand zu gehen.

\section{Ermittlung des Austrittsalters}

Die Analyse der Verrentungsentscheidung auf Basis des SOEP ist allerdings insofern schwierig, daß im Panel der Geburtstermin der Individuen nicht enthalten ist, sondern nur das Geburtsjahr. Der Termin der Befragung ist in den meisten Fällen der April. Eine Ermittlung des Rentenalters über:

$$
\text { Verrentungsalter }=\text { Befragungsjahr }- \text { Geburtsjahr }
$$

ergibt also in 66 - 75 \% der Fälle ${ }^{6}$ einen Fehler der Richtung, daß das Rentenalter ein Jahr zu alt angenommen wird. Diese Meßungenauigkeit ist auf Basis der vorhandenen Daten nicht zu vermeiden. Sie wird in der vorliegenden Arbeit daher durch die Zuweisung:

$$
\text { Verrentungsalter }=\text { Befragungsjahr }- \text { Geburtsjahr }-1
$$

so daß der Meßfehler nun in der Richtung wirkt, daß 25 - $33 \%$ der Fälle ein Jahr zu jung angenommen werden.

Es ist wäre daher sehr wünschenswert, wenn in zukünftige Befragungen des SOEP die Abfrage des Geburtsmonates, mindestens jedoch die Frage "Hatten Sie in diesem Jahr schon Geburtstag?" aufgenommen wird.?

\footnotetext{
6 Je nachdem, wann im April die Befragung vorgenommen wurde.

${ }^{7}$ Die im Grunde angebrachte Erfragung des Geburtstermines im Verbindung mit einer genauen Angabe des Befragungstermines dürfte an Datenschutzgesichtspunkten scheitern.
} 


\section{Übergangsratenstruktur der untersuchten Daten}

In Kapitel 6 wurde erläutert, daß für eine Failure-Time Analyse bestimmte Besonderheiten der Datenstruktur zu beachten sind. Neben der nun vorgenommenen Definition eines Ereignisses sind hier vor allem die Zensurstatus zu beachten.

Unter Zensur wird im technischen Sinne der Verweildaueranalyse verstanden, daß für eine Person kein Ereignis beobachtet wird, weil es entweder vor oder nach dem Beobachtungszeitraum (Beobachtungsfenster) stattfindet. Die Zeitdauer des Verweilens in einem Zustand wird im weiteren als „Spell“ oder „Episode“ bezeichnet. Die verschiedenen möglichen Spells sind in Abbildung 7-1 graphisch dargestellt. Beobachtung A stellt dabei ein ,Ereignis“ dar, eine Person, die während des Beobachtungszeitraumes in den Ruhestand wechselt.

Unter einer Rechtszensur ist ein solcher Beobachtungsfall zu verstehen, der in der letzten beobachten Welle ${ }^{8}$ noch nicht verrentet ist, dessen Verrentung also zeitlich nach dem Beobachtungszeitraum liegt. Dies ist in der Abbildung als Fall B dargestellt. Diese modelltechnische Rechtszensierung muß sorgfältig unterschieden werden von einer datenmäßigen im Sinne des Ausscheidens aus der Stichprobe, also Personen, die eine oder mehrere Befragungswellen nicht realisiert haben. So ist es möglich, da $B$ ein Datensatz mit nur 2 Beobachtungen ein Ereignis beinhaltet, wogegen ein solcher mit 7 Erwerbsperioden rechtszensiert ist.

Die zweite Art der Datenzensierung ist die Linkszensur. Im Grundmodell wird darunter verstanden, daß der Beginn eines beobachteten Spells nicht im Beobachtungszeitraum liegt. In diesem Sinne sind alle betrachteten Personen linkszensiert, denn der Eintritt in die Erwerbstätigkeit liegt jeweils ca. 30 - 40 Jahre zurück und kann insofern in einem 7-Jahres Datenausschnitt nicht erfaßt sein. Die Definition von Linkszensur wird deshalb dahingehend geändert, daß Personen, die bereits in der ersten SOEP-Welle bzw. bei Ihrer ersten Befragung im SOEP verrentet ("nicht erwerbstätig") waren, für eine Schätzung des Überganges zum Zustand 'Rente' nicht relevant sind und daher nicht in die Schätzung eingehen. Diese Personen sind, technisch gesprochen, nicht mehr 'at risk'. Dieser Fall ist als Person C in Abbildung 7-1 veranschaulicht.

${ }^{8}$ Dies ist die letzte realisierte Befragung und muB daher bei Personen, die während der Beobachtungszeit verziehen, versterben oder aus anderen Gründen nicht mehr befragt werden, nicht mit der letzten untersuchten Panelwelle (Welle 7 West bzw. Welle 3 Ost) zusammenfallen. 


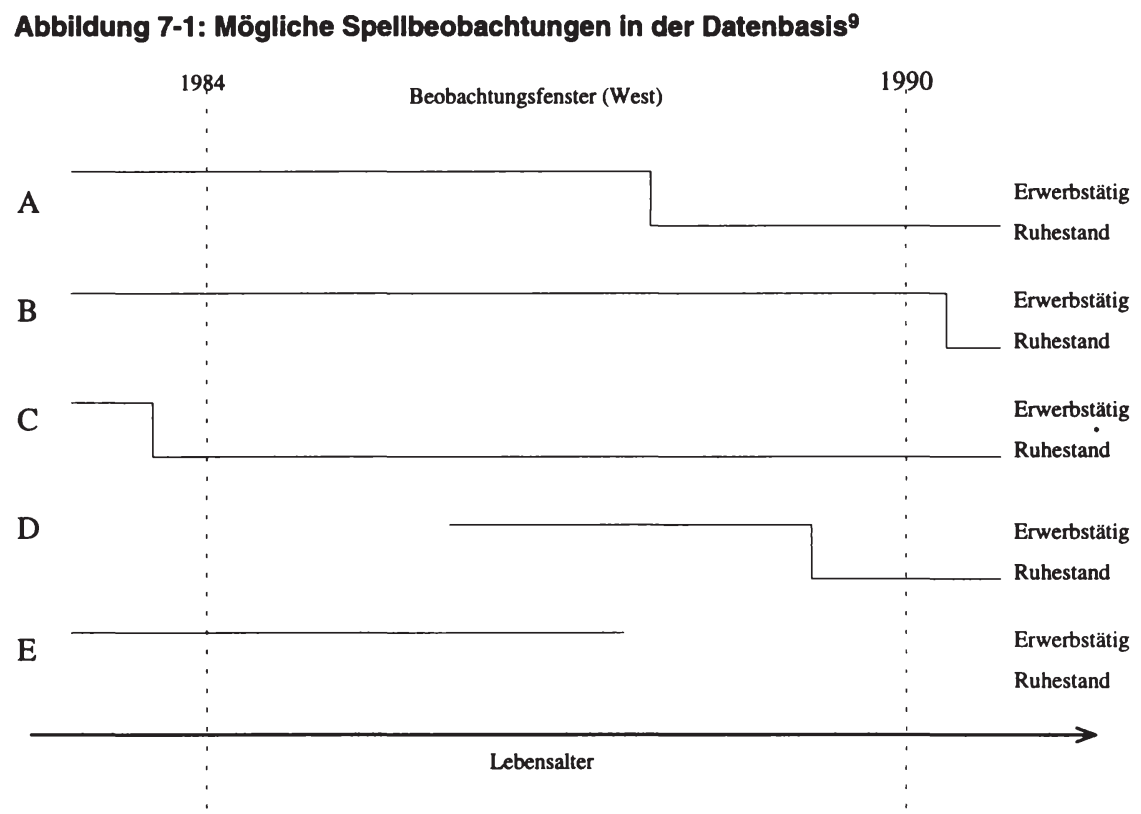

Als mögliche Sonderfälle sind in der Abbildung weitere 2 Fälle unterschieden. Person D wurde zwar nicht in der ersten Welle beobachtet, tritt aber während des „Fensters“ als Erwerbsperson in das Panel ein und setzt sich auch innerhalb der Beobachtungszeit zur Ruhe. Dieser Fall ist somit ein normales „Ereignis“. Hingegen stellt das letzte Beispiel, Fall E, eine Rechtszensur dar, die hier nicht durch ein Herausfallen des Ereignisses aus dem Beobachtungsfenster bedingt ist, sondern dadurch, das die beobachtete Person nicht mehr befragt wird.

Die Zählvariable des Verweildauermodelles - und damit die Entscheidungsvariable des einzelnen Arbeitnehmers - ist hier das Verrentungsalter.

Eine alternative Entscheidungsgröße wäre die (versicherungstechnisch relevante) Lebensarbeitsszeit. $\mathrm{Da} \beta$ diese im vorliegenden Modell nicht verwendet wird, ist darin begründet, daß für eine Simulation der Entwicklung der GRV auf aggregierter Ebene die Kenngröße "Verrentungsalter" die relevante ist, die ebenfalls durch die Altersstrukturverschiebung beeinträchtigt wird.

${ }^{9}$ Die Idee für diese Darstellung stammt von SCHNEIDER (1991), Seite 78. 
Die abhängige Variable des Failure Time Modelles ist also das Alter entweder

- im Jahr vor dem Austritt aus dem Erwerbsleben (bzw. Arbeitslosigkeit) im Fall von Ereignis-Beobachtungen oder

- im letzten Befragungsjahr im Fall rechtszensierter Datensätze.

Gleiches gilt für die unabhängigen Variablen (Kovariate) des Regressionsmodelles, die die entsprechenden Jahreswerte enthalten.

Die so ausgewählte Stichprobe aus dem Sozioökonomischen Panel umfaßte in Westdeutschland 5.326 Personen, von denen gut $49 \%$ noch erwerbstätig waren. Die restlichen Befragten sind im obigen Sinne linkszensiert und gehen daher nicht in die Schätzung ein. In Ostdeutschland erfüllten 1500 Personen das Auswahlkriterium, hier waren 1990 noch mehr als $66 \%$ erwerbstätig, ein Unterschied, der zu einem guten Teil aus der erheblich höheren Erwerbsbeteiligung der Frauen in der ehemaligen DDR zu erklären ist. Tabelle 7-1 zeigt die Aufteilung der verbleibenden Beobachtungspersonen nach Geschlecht sowie die Übergangsraten für den Statuswechsel von "erwerbstätig" zu "nicht erwerbstätig" innerhalb des Beobachtungszeitraumes.

Tabelle 7-1: Untersuchte Personen und Austritte aus der Erwerbstätigkeit im SOEP

\begin{tabular}{|c|c|c|c|c|c|c|}
\hline & \multicolumn{3}{|c|}{$\begin{array}{c}\text { Westdeutschland } \\
\text { (alte Bundesländer) } \\
\text { Welle 1-7: 1984-1990 }\end{array}$} & \multicolumn{3}{|c|}{$\begin{array}{c}\text { Ostdeutschland } \\
\text { (neue Bundesländer) } \\
\text { Welle 1-3: 1990-1992 }\end{array}$} \\
\hline & Männer & Frauen & Gesamt & Männer & Frauen & Gesamt \\
\hline Anfänglich erwerbstätig & 1589 & 780 & 2369 & 483 & 482 & 965 \\
\hline $\begin{array}{l}\text { Übergänge im Beobachtungs- } \\
\text { zeitraum }\end{array}$ & 392 & 216 & 608 & 190 & 210 & 401 \\
\hline $\begin{array}{l}\text { Übergänge in Prozent der } \\
\text { anfänglich Erwerbstätigen }\end{array}$ & $24.7 \%$ & $27.7 \%$ & $25.7 \%$ & $39,34 \%$ & $43.75 \%$ & $41.55 \%$ \\
\hline Übergänge pro Jahr & 65.3 & 46.9 & 101.3 & 95.0 & 105.0 & 200.5 \\
\hline Anteil Übergänge & $4.1 \%$ & $6.0 \%$ & $4.3 \%$ & $19 \%$ & $21 \%$ & $20.0 \%$ \\
\hline
\end{tabular}

Quelle: SOEP (genannte Wellen) - Befragungspersonen, die in der ersten beobachteten Welle 45 Jahre und älter (im Westen, im Osten 48 oder älter) ${ }^{10}$ waren und voll- oder teilzeit erwerbstätig waren und solche, die arbeitslos gemeldet waren.

Gemessen wird der Übergang von einem dieser drei Zustände in den Zustand "nicht erwerbstätig".

Aus Tabelle 7-1 wird deutlich, daß die Austritte aus dem Arbeitsmarkt in West- und

${ }^{10}$ Die Ratio dieser Abgrenzung ist die, daß jede Person, die zu einem potentiellen Übergangszeitpunkt 50 Jahre und älter ist, in die Stichprobe aufgenommen werden soll. Scheidet eine Person in einem jüngeren Alter als 50 aus dem Erwerbsleben aus, zählt dies hier als Austritt, in den Regressionsanalysen des folgenden Abschnittes allerdings nicht als Altersrente. 
Ostdeutschland nur mit sehr großer Vorsicht vergleichbar sind. So sind die Abgangsraten aus dem Arbeitsmarkt in den neuen Ländern fast fünf mal so hoch wie die im Westen beobachteten.

Das Arbeitsmarkt- und Verrentungsverhalten der Bürgerinnen und Bürger der ehemaligen DDR ist stark durch den Systemübergang und die damit verbundenen dramatischen Veränderungen der gesellschaftlichen und persönlichen Situation geprägt. Die reale Arbeitslosigkeit für die einen und die Sorge davor bei den noch nicht betroffenen lassen die Frühverrentung zu einer sicheren Alternative werden, auch wenn der persönliche Wunsch nach Beendigung des Erwerbslebens noch nicht vorhanden war. Ẹine empirische Trennung dieser außerordentlichen Effekte von den persönlichen Kalkülen einer rationalen Verrentungsentscheidung einerseits und den sich permanent ändernden gesetzlichen Rahmenbedingungen andererseits ist sehr schwierig. ${ }^{11}$

\section{Definition des Optionswertes}

Die numerische Ermittlung des in Kapitel 6.2 definierten Optionswertes erfolgt mittels eines Programmes, dessen Struktur als Übersicht in Abbildung 7-2 dargestellt ist. Die Definitionsschritte sind:

\section{Einlesen der persönlichen Merkmale jeder Befragungsperson}

2. Ermittlung des Alters-Einkommensverlaufes

An dieser Stelle wird die Einkommensentwicklung für jede Einzelperson mit Hilfe des unten beschriebenen normalisierten Einkommens wie folgt ermittelt:

- Auf Basis ihrer persönlichen Daten wird jeder Einzelperson ein prognostizierter Einkommensverlauf für alle auf das aktuelle folgende Lebensjahre zugeordnet, indem - alle anderen Eigenschaften konstant gehalten - aus den Koeffizienten der Normaleinkommenschätzung für jedes Lebensalter ein Einkommenswert zugeordnet wird.

- Dieses normalisierte Einkommen wird mit dem für das aktuelle Jahr tatsächlich angegebenen Bruttoeinkommen verglichen und eventuelle Abweichungen korrigiert.

- Aus dem jetzt vorliegenden Brutto-Einkommen wird unter Anwendung des bundesdeutschen Steuertarifes die persönliche Einkommensteuer subtrahiert und somit das persönliche Nettoeinkommen berechnet. ${ }^{12}$

11 Zur Darstellung der Übergangsprozesses und der Vorruhestandsregelungen siehe Kapitel 3.4.

12 Die Routine zur Berechnung der persönlichen Einkommensteuer wurde mir dankenswerterweise von Prof. Börsch-Supan zur Verfügung gestellt. Sie ermittelt den individuellen Steuertarif auf der Peter Schmidt - 978-3-631-75568-6 
- Da dieses Nettoeinkommen nicht bei allen Personen aus dem gleichen Jahr stammt, sondern aus dem Entscheidungsjahr, wird es, mit Hilfe des Preisindexes der allgemeinen Lebenshaltung, auf 1990-DM deflationiert.

\section{Bestimmung der potentiellen Renteneinkommen}

Die Zuweisung eines potentiellen Renteneinkommens erfolgt durch die Multiplikation des persönlichen Einkommens mit einer Ersatzquote. Diese Ersatzquoten sind aus einer Berechnung von CASMIR (1989, Seite 512) entnommen, der sie nach Einkommensklassen und Familienstand ausweist.

\section{Berechnung des Optionswertes}

Die Bestimmung des Optionswertes ist direkt aus den oben angegebenen Formeln zu entnehmen:

- Für jedes mögliche Verrentungsalter wird der -auf das Entscheidungsalter bezogene- Barwert aller Erwerbs- und Renteneinkommen errechnet.

Für diese Berechnung werden die von BÖRSCH-SUPAN (1992 e) geschätzten Werte der Parameter verwendet:
$\alpha$ (Zusatznutzen von Freizeit)
1,196
$\delta$ (Diskont Faktor)
0,862
$\gamma$ (Einkommenselastizität des Nutzens) 1,011

- Aus allen potentiellen Eintrittsaltern R (vom auf das aktuelle Alter folgenden bis zum Alter 99) wird das Alter $R^{*}$ ermittelt, welches den höchsten Barwert $V_{t}\left(R^{*}\right)$ aufweist

- Aus dem Vergleich dieses maximalen $V_{t}\left(R^{*}\right)$ mit dem Barwert bei sofortiger Verrentung $V_{t}(t)$ wird der Optionswert berechnet.

\section{Ermittlung des normalisierten Erwerbseinkommens}

Die Prognose eines normalisierten Erwerbseinkommens ist erforderlich, um die Entscheidungsoptionen des Arbeitnehmers berechnen zu können. Bei der Diskussion der Lebenszyklushypothese (Kapitel 2.1) wurde bereits angesprochen, daß sich das Einkommen eines Menschen im Laufe seines Lebens unterschiedlich entwickelt. Das reale Profil folgt allerdings nicht dem dort beispielhaft angenommenen linearen Verlauf, sondern weist einen konkaven Verlauf auf. Die größten Steigerungen ergeben sich im

Basis des Jahreseinkommens unter Berücksichtigung von Einkommensgrenzen, Familienstand und Freibeträgen. Nicht berücksichtigt werden konnte dagegen das Einkommen des Ehepartners, so daß die individuelle Steuerberechnung von der tatsächlichen Steuerlast abweichen kann. Dies ist eine Ungenauigkeit in den verwendeten Daten, die allerdings alle Personen gleichermaßen betrifft und damit für die in der Schätzung relevanten relativen Unterschiede an Wichtigkeit verliert. 


\section{Abbildung 7-2: Ermittlung des Optionswertes durch FORTRAN-Programm}

Einlesen der persönlichen Merkmale:

- Alter, Geburtskohorte

- Geschlecht, Familienstand

- Bruttojahreseinkommen

- Berufsvariablen (Branche, Status)

- Erwerbsstatus (voll- / teilzeitbeschäftigt)

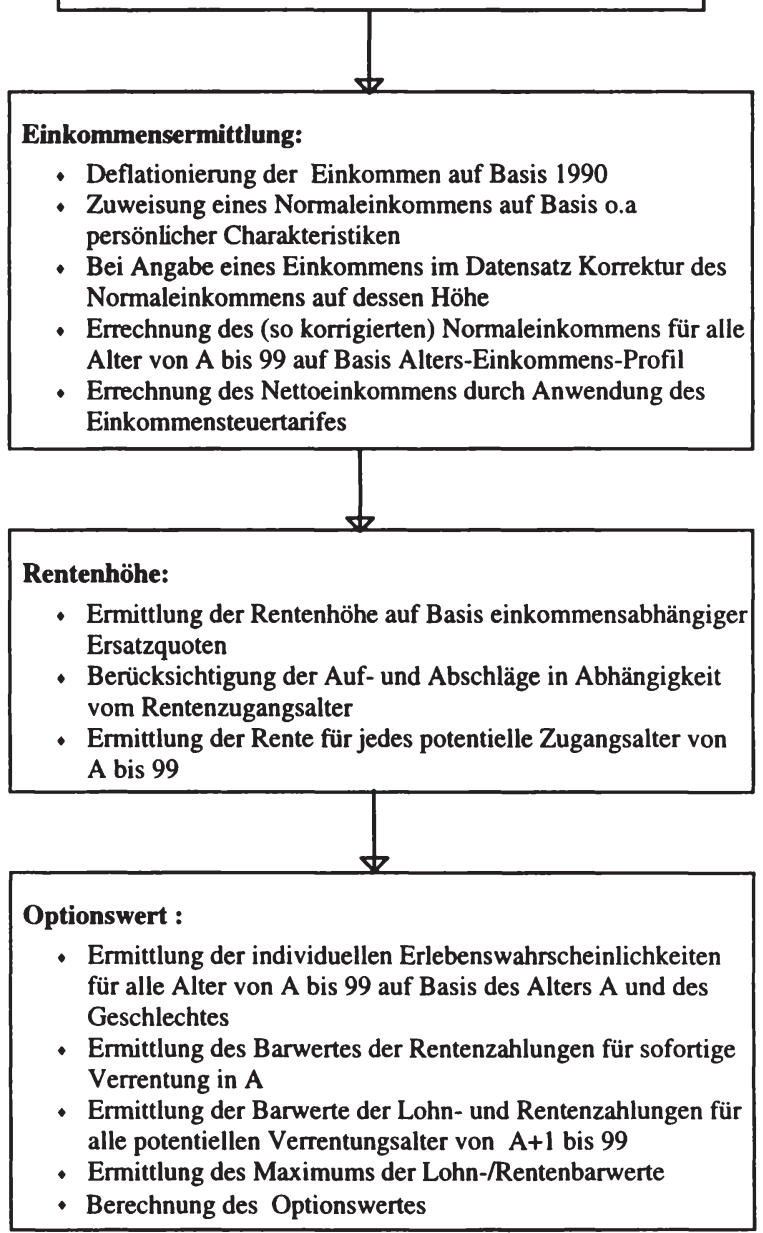

Alter zwischen 20 und 40, in dem sowohl der Berufseintritt, als auch die Etablierung in diesem Beruf und damit verbundene Honorierungen stattfinden. Nach diesem Zeit- 
punkt findet ab dem 40. bis 50. Lebensjahr eher eine Stagnation der Einkommenshöhe statt. ${ }^{13}$ Für eine ökonomische Modellierung dieses Alters-Einkommens-Profiles ist es erforderlich, neben dem Lebensalter auch das Geburtsjahr (Kohorteneffekt) sowie persönliche Faktoren wie Geschlecht, Gesundheit (körperliche Konstitution, Behinderungen), berufliche Stellung und Humankapital (operationalisierbar durch Art und Dauer von Schul- und Berufsausbildung, Abschlüsse) zu berücksichtigen.

Bei dieser Altersabhängigkeit muß sorgfältig zwischen Alters- und Kohorteneffekten getrennt werden. Die Berücksichtigung der Geburtskohorte dient dabei der Kontrolle sowohl gesellschaftlicher als auch ökonomischer Rahmenbedingungen ihrer Mitglieder. Es ist einsichtig, daß eine Kriegsgeneration sich in ihrem Leben mit ganz anderen und grundlegenderen Problemen auseinandersetzen mußte, als etwa die Wirtschaftswundergeneration der 60er Jahre. Eine optimale ökonometrische Messung müßte drei Faktoren berücksichtigen. Einen Alterseffekt, einen Konjunktureffekt und einen Kohorteneffekt, der für sogenannte 'taste shifter' kontrolliert, die nicht ökonomisch bedingt sind, sondern allgemeine gesellschaftliche Veränderungen der Nutzenfunktion widerspiegeln. Die letzteren beiden Faktoren sind in der Regel ökonometrisch nicht identifizierbar. Außerdem macht die Annahme sich permanent wandelnder individueller Nutzen-funktionen (und nicht nur der Parameter derselben) jegliche ökonomische Analyse, die auf Nutzenbetrachtungen beruht, unmöglich. Auf Basis der in der Ökonomie bzw. Ökonometrie regelmäßig getroffenen Annahme zeitkonstanter Nutzenfunktionen kann, wenn diese einen signifikanten Einfluß auf das Verhalten der untersuchten Individuen zeigen, indirekt die Hypothese sich wandelnder Nutzenfunktionen abgelehnt werden.

Die reine Altersabhängigkeit der Einkommensentwicklung ist für das hier zu schätzende Modell insofern relevant, als daß der Optionswert aus einem Vergleich zukünftiger Einkommensverläufe (Erwerbs- und Renteneinkommen) errechnet wird, die naturgemäß nur mit Kenntnis der altersabhängigen Entwicklung des Erwerbseinkommens simuliert werden können. Die Alters-Einkommensfigur wird im vorliegenden Modell mit einer einfachen linearen Regression (7-1) ermittelt.

${ }^{13}$ Für eine ausführliche Diskussion von Alters-Einkommens-Profilen vergleiche etwa FITZENBERGER ET. AL. (1994), die auch eine Trennung von Alters- und Kohorteneffekten vornehmen. 


$$
Y_{t}=\alpha+\beta_{1} A_{1}+\beta_{2} A_{t}{ }^{2}+\beta_{3} A_{t}{ }^{3}+\gamma_{i} X_{t}^{i}+\varepsilon
$$

$\begin{array}{cll}\text { mit: } & \mathrm{Y}_{\mathrm{t}} & \text { Laufendes Einkommen im Jahr t } \\ & \mathrm{A}^{i} & \text { Lebensalter im Jahr t } \\ & X_{t}^{i} & \text { Matrix der weiteren unabhängigen Variablen in t: } \\ & - \text { Kohorte } \\ & - \text { Geschlecht } \\ & \text { - Fanilienstand } \\ & \text { - Gesundheit, gemessen durch Grad der Erwerbsmidnerung } \\ & \text { - Schulische und berufliche Bildung } \\ & \text { - Branche } \\ & \text { - Stellung im Beruf } \\ & \text { - Beschaftigung: teilzeit, vollzeit oder arbeitslos }\end{array}$

Die geschätzten Werte der Koeffizienten $\alpha, \beta$ und $\gamma$ können dem Anhang A-3 entnommen werden. Die Schätzung wurde als gepoolte Regression für 3 Jahre (19881990) durchgeführt, so daß Alters- und Kohorteneffekt getrennt wurden.

\subsection{Deskriptive Betrachtung der Austritte aus dem Arbeitsmarkt in West- und Ostdeutschland}

In diesem Kapitel werden anhand von deskriptiven Analysen und Querschnittsuntersuchungen die wesentlichen Korrelationsstrukturen im untersuchten Datenmaterial dargestellt und auf dieser Basis die in Kapitel 5.4 formulierten Hypothesen für die Zeitraten-Analyse in einer ersten Betrachtung überprüft.

In Kapitel 4 wurde bereits eine umfassende Diskussion der gesamtwirtschaftlichen Entwicklung der bundesdeutschen gesetzlichen Rentenversicherung dargestellt. Inhalt dieses Abschnittes ist es, die untersuchte Stichprobe zu betrachten und das im Panel gefundene Verrentungsverhalten mit dem gesamtwirtschaftlich beobachteten zu vergleichen. Ferner bieten Mikrodaten auch auf deskriptiver Ebene die Möglichkeit einer tieferen Betrachtung, als dies bei aggregierten Zahlen der Fall ist. Im Gegensatz zu Kapitel 4 ist hier eine vergleichende Betrachtung der beiden Teile des wiedervereinigten Deutschland möglich.

Die typischen Altersprofile der bundesdeutschen Rentenzugänge für Männer (in den alten Bundesländern) wurden bereits in unterschiedlichen Kontexten dieser Arbeit dargestellt. Abbildungen 2-3, 2-7 bzw. 4-3 als intertemporaler Vergleich zeigen diese Profile mit den deutlichen Spitzenwerten im Alter von 60, 63 und 65 Jahren. Da sich die Betrachtungen von Kapitel 4 nur auf die männlichen Arbeitnehmer beziehen, zeigt Abbildung 7-3 das Profil für die gesamten Zugänge von Männern und Frauen. Diese 
Gruppe entspricht der Grundgesamtheit, aus der die hier untersuchte Stichprobe des Sozioökonomischen Panels stammt.

\section{Abbildung 7-3: Verteilung der VDR-Rentenzugănge 1988 für Mănner und Frauen aut Elnzelalter}

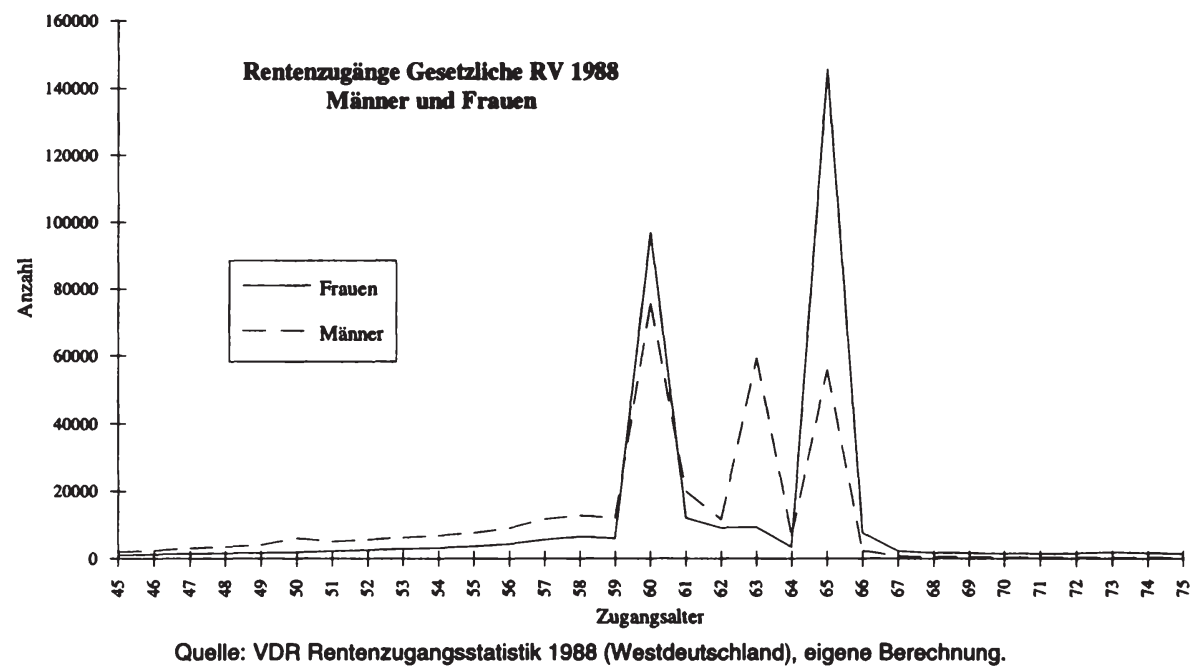

Diese Abbildung 7-3 zeigt für Frauen ein anderes Profil als das bisher für Männer betrachtete. Das Zugangsalter 63 spielt bei den Neurenterinnen nur eine unbedeutende Rolle, die Zugänge im Alter von 65 Jahren sind mehr als doppelt so hoch wie die der Männer. ${ }^{14}$ Es wird deutlich, daß Frauen noch deutlicher in einzelnen Altersjahren in die Rentenversicherung eintreten, als dies bei Männern der Fall. Es sind dies nur $2 \mathrm{Zu}$ gangsalter 60 und 65 und die Anteile der anderen Zugangsalter liegen durchweg unter denen der Männer.

Es stellt sich an dieser Stelle die Frage, ob die Rentenreform 1972, bei der der flexible Ruhestand ab 63 Jahren eingeführt wurde, für Rentnerinnen keine Auswirkungen hatte. Abbildung 7-4 bestätigt dies, zwar stieg der Anteil des Alters 63 im Jahre 1975, also 2 Jahre nach dessen Einführung, leicht an, die Konzentration auf die Zugangsalter 60 und 65 ist aber deutlich dominierend. Die Gründe für diesen Unterschied liegen an

14 Insgesamt gingen 1988 351.000 Frauen und 332.000 Männer in den betracheten Altersjahren 45 bis 75 in Rente. Durch diese gleiche Größe der beiden Gruppen sind auch die relativen Höhen der $\mathrm{Zu}$ gänge pro Einzelalter direkt vergleichbar. 
der unterschiedlichen Erwerbsbeteiligung von Männern und Frauen in (West-) Deutschland. Während die Männer in der Regel aus der aktiven Erwerbstätigkeit heraus in die Rentenphase eintreten, ist die Arbeitsmarktbeteiligung von Frauen erheblich geringer. Trotzdem hat ein erheblicher Anteil der Frauen eine latente Rentenanwartschaft aus früherer Erwerbstätigkeit, die im Regelrentenalter von 65 Jahren in Anspruch genommen werden kann. Die zum Entscheidungszeitpunkt erwerbstätigen Frauen können hingegen auf legalem Wege mit 60 in den Ruhestand gehen, ${ }^{15}$ so daß das Alter des flexiblen Ruhestandes 63 für Frauen wenig Bedeutung hat. Insofern sind das Alter 63 für Männer und das Alter 60 für Frauen vergleichbar in dem Sinne, das beides die Zeitpunkte für den frühestmöglichen Eintritt darstellen. ${ }^{16}$ Das Zugangsalter 60 mit seinen im dritten und vierten Kapitel ausführlich besprochenen Möglichkeiten des vorgezogenen Ruhestandes aus Gesundheitsgründen und wegen Arbeitslosigkeit ist für Frauen damit zeitgleich ihrem vorgezogenen Ruhestandsalter.

\section{Abbildung 7-4: VDR-Rentenzugänge Frauen nach Einzelalter, ausgewählte Kalender- Jahre}

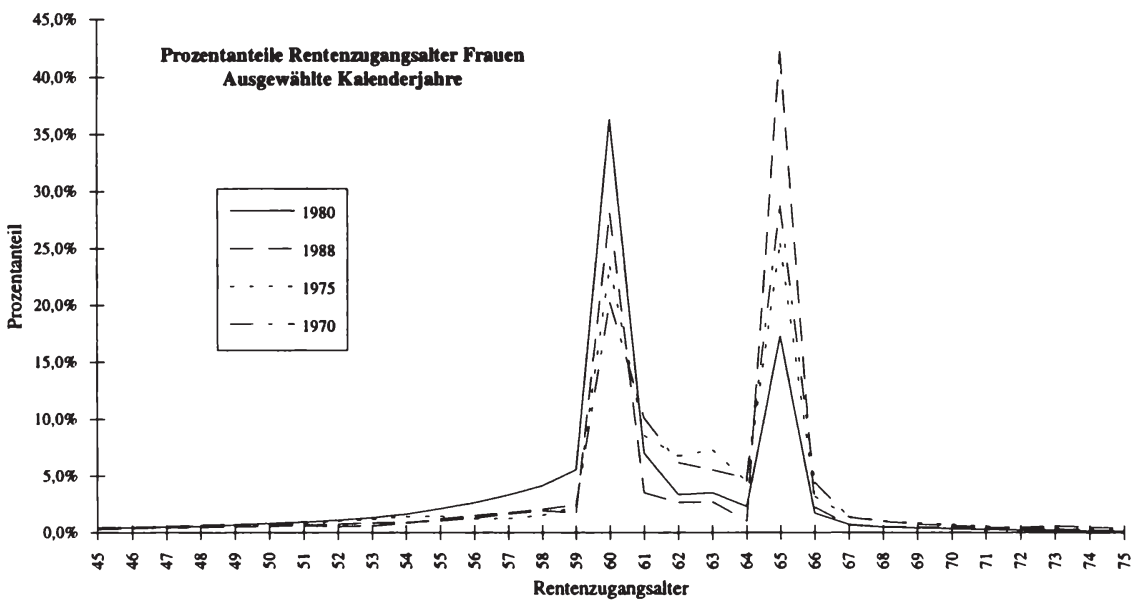

Quelle: VDR Rentenzugangsstatistik angegebene Jahrgånge, eigene Berechnung.

Die vor und zwischen diesen Extrempunkten liegenden höheren Anteile männlicher

15 Unterstellt, daß sie die Anspruchsvoraussetzungen erfüllten, d.h. sie müssen in den letzten 20 Jahren mindestens die Hälfte der Zeit Pflichtbeiträge gezahlt haben, vergleiche Kapitel 3.2.1.2.

16 Für den Flexiblen Ruhestand mit 63 Jahren ist eine Wartezeit von 35 Jahren notwendig, vergleiche ebenfalls Kapitel 3.2.1.2. 
Rentenzugänge reflektieren die Tatsache, da $B$ die in stärkerem Anteil erwerbstätigen Männer damit auch einem höheren Risiko unterliegen, krank oder arbeitslos zu werden.

\section{Vergleich der Rentenalter der GRV mit der Stichprobe des SOEP}

Der Vergleich der Gesamtzahlen des VDR mit den Tabellierungen der hier benutzten Stichprobe aus dem SOEP ist aus zwei Gründen nicht unproblematisch. Erstens wird die Aufteilung mehrerer hundertausend Rentenzugänge des VDR mit nur einigen hundert Befragungspersonen im SOEP vergleichen und zweitens werden vom VDR Rentenzugänge gezählt, wogegen im hier benutzten Datensatz das Ausscheiden aus dem Erwerbsleben als Kriterium benutzt wird. Abbildungen 7-5 und 7-6 zeigen das Analogon zur GRV-Abbildung 7-3 für Ost- und Westdeutschland auf Basis des SOEP mit dem Unterschied, daß hier die Beobachtungen über alle Wellen gepoolt sind, wie sie es auch in der Hazard-Analyse sein werden. Der Nachteil an dieser Darstellung ist, daß sie somit nicht mit mehr mit einer Einzeljahrestabellierung der VDR-Rentenzugänge aus Kapitel 4 vergleichbar sind. ${ }^{17}$

In Abbildung 7-5 ist das in der gesamtwirtschaftlichen Tabellierung typische Altersprofil mit Höchstwerten bei den Austrittsaltern 60, 63 und 65 bereits erkennbar. Die Abweichungen ergeben sich zum Einen aus der bereits angesprochenen Problematik der korrekten Alterszuordnung im SOEP, zum Anderen daraus, daß hier nicht der formale Eintritt in die gesetzliche Rentenversicherung betrachtet wird, sondern die Beendigung der Erwerbstätigkeit. Dies hat vor allem zwei Konsequenzen:

- Die Austrittsalter sind geringer als die Zugangsalter des VDR. Für die vorgezogenen Ruhestände v.a. aus Sozialplänen/59er Regeln ist dies offensichtlich: Der Austritt aus dem Arbeitsmarkt erfolgt mit 57 bzw. 59 Jahren im Fall der Arbeitslosigkeitsregel oder einem andern Alter vor 60, der formale Eintritt in die GRV findet mit 60 Jahren statt. 18

- Der Anteil der Frauen an den Rentenzugängen (in die GRV) ist in der Mikrodatenanalyse dieses Kapitels unterschätzt. Während sie bei den GRV Eintritten etwas mehr als die Hälfte ausmachen, liegt ihr Anteil im westdeutschen SOEP nur bei einem guten Drittel, im Osten hingegen entspricht die Relation der GRV. Auch hierfür ist der Grund naheliegend: Die Renteneintritte der latent versicherten (s.o.) Frauen kann in der Stichprobe nicht als Austritt erfaßt werden, da kein Arbeitsverhältnis vorliegt, das beendet werden kann.

17 Die Betrachtung eines einzelnen Rentenzugangsjahres hätte wegen der zu geringen Fallzahlen allerdings keine Repäsentativität gehabt.

18 Vgl. dazu die Diskussion des 4. Kapitels. 


\section{Abbildung 7-5: Alter bel Beendigung der Erwerbstätigkeit, West-SOEP 1984 - 1990}

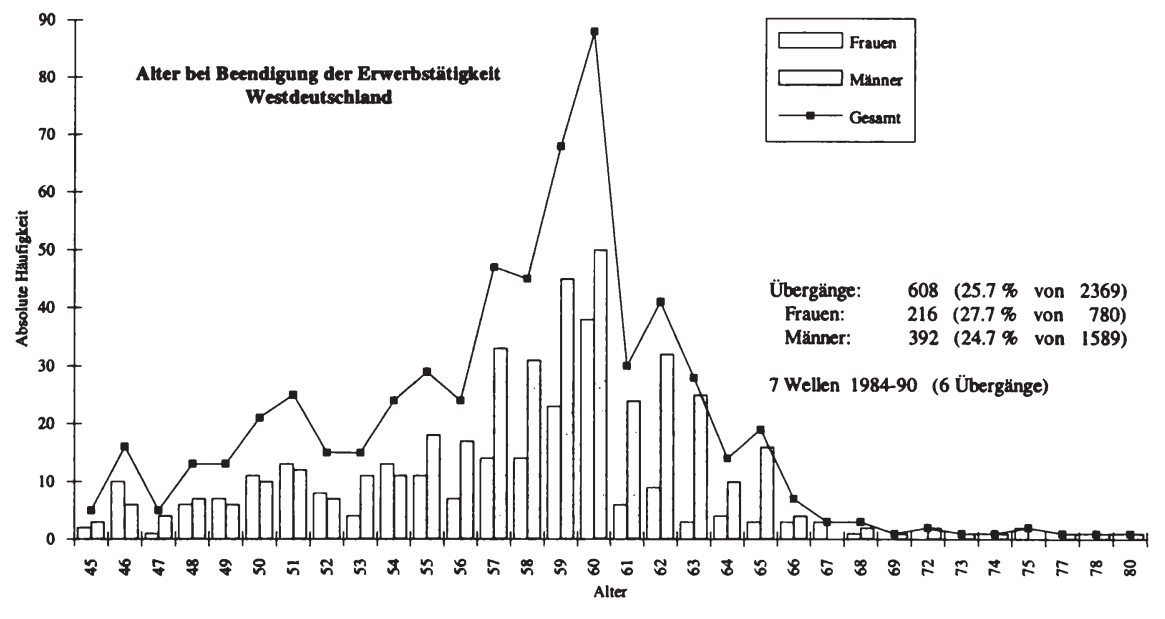

Quelle: West SOEP, Wellen 1 - 7 (1984-1990), eigene Berechnung.

\section{Abbildung 7-6: Alter bei Beendigung der Erwerbstätigkeit, Ost-SOEP, 1990 - 1992}

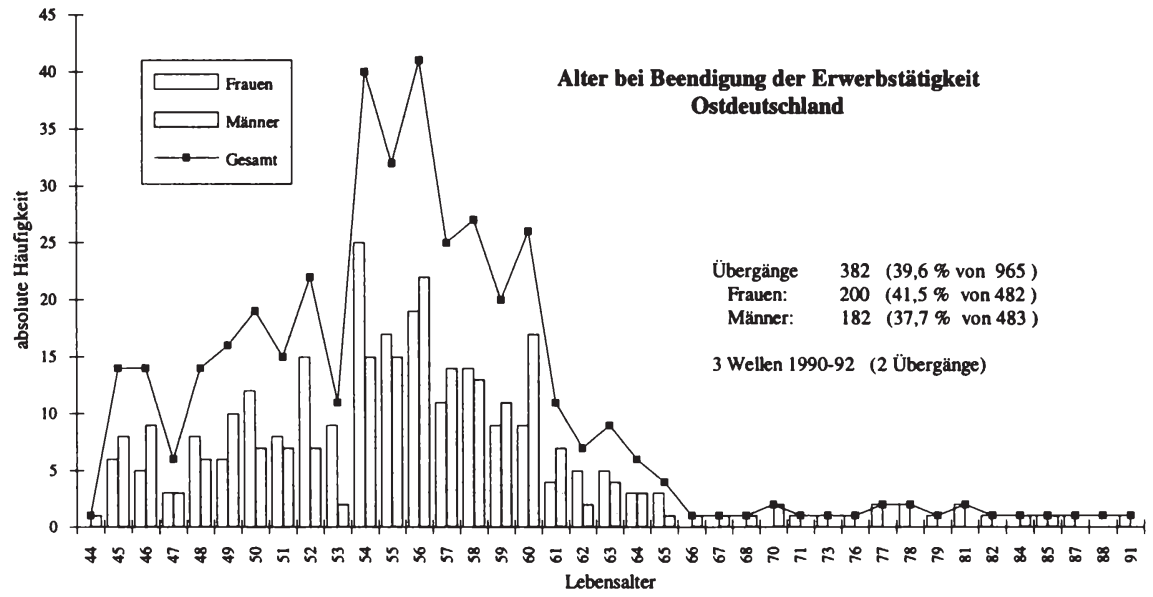

Quelle: Ost SOEP, Wellen 1 - 3 (1990-1992), eigene Berechnung. 
Insgesamt ist die differenzierte Struktur der Zugänge zur deutschen Rentenversicherung in der SOEP-Stichprobe für Westdeutschland erkennbar, aber die deutliche Betonung der gesetzlich fixierten Alter, die sich in den GRV-Zugängen zeigt, wird in den hier betrachteten Abgängen aus dem Erwerbsleben nicht reproduziert. Der Unterschied resultiert vor allem daraus, daß sich die im SOEP gemessenen Arbeitsmarktaustritte erst zu den institutionell vorgegebene Renteneintrittsalter in den VDR-Zahlen realisieren. Daher sind die SOEP-Austrittsalter geringer als die VDR-Zugangsalter. Für die Bewertung der statistischen Analyse ist dieser Niveauunterschied jedoch nicht gravierend, da die Unterschiede der im SOEP befragten Personen untereinander in der Schätzprozedur verwendet werden. Dies gilt auch für die im achten Kapitel durchgeführten Simulationsrechnungen, die die Verschiebung einer vorgegebenen Altersstruktur beschreiben und nicht am Niveau selbst ansetzen.

\section{Unterschiede im Austrittsverhalten in Ost- und Westdeutschland}

Die Austrittsalter für Ostdeutschland 1990-1992 sehen hingegen völlig anders aus. Der Vergleich der Abbildungen 7-5 mit 7-6 zeigt, daß in der ehemaligen DDR im Betrachtungszeitraum völlig andere Muster des Rentenzugangsverhaltens vorherrschen. Zum Verständnis dieser Tatsache ist es erforderlich, sich einerseits die politische und gesellschaftliche Situation in den neuen Bundesländern 1990-92 vor Augen zu führen und andererseits die damit verbunden gesetzlichen (Übergangs-) Richtlinien zu bedenken. Diese Regelungen sind in Kap 3.4 beschrieben. Neben dem Faktum jedoch, daß sehr viele Menschen in relativ jungen Altersjahren ihre Arbeitstätigkeit aufgeben, sollte nicht übersehen werden, daß auch die Alter 60 und 63 lokale Maxima des Graphes sind und die letzten nennenswerten Austrittszahlen mit 65 Jahren zu beobachten sind. Dies illustriert, daß diejenigen älteren Menschen, die zur Beobachtungszeit noch erwerbstätig waren (und somit nicht unter die Vorruhestandsregelungen fielen), an das nun geltenden Recht der Bundesrepublik gebunden waren.

Abbildungen 7-7 und 7-8 zeigen die Prozentanteile der nicht mehr erwerbstätigen ${ }^{19}$ Personen ${ }^{20}$ in den beiden Teilen Deutschlands. Auch hier wird deutlich, daß die Arbeitsmarktaustritte in Ostdeutschland eher durch den Umbruchprozeß und sich wandelnde Rahmenbedingungen gekennzeichnet sind, als durch ein striktes Schema.

19 Die „Erwerbstätigen“ umfassen hier auch die Arbeitslosen. Diese Größe entspricht insofern dem „Erwerbspersonenkonzept“ des Statistischen Bundesamtes.

${ }^{20}$ Es ist zu beachten, daß dies keine kumulierten Werte sind, sondern Prozentanteile für einzelne Altersjahre. Ein Sinken des Anteils mit steigendem Alter ist also nicht inkonsistent, wenn auch auf den ersten Blick überraschend. Es handelt sich dabei um Kohorteneffekte. 


\section{Abbildung 7-7: Prozentanteil nicht erwerbstätiger Befragter - Westdeutschland.}

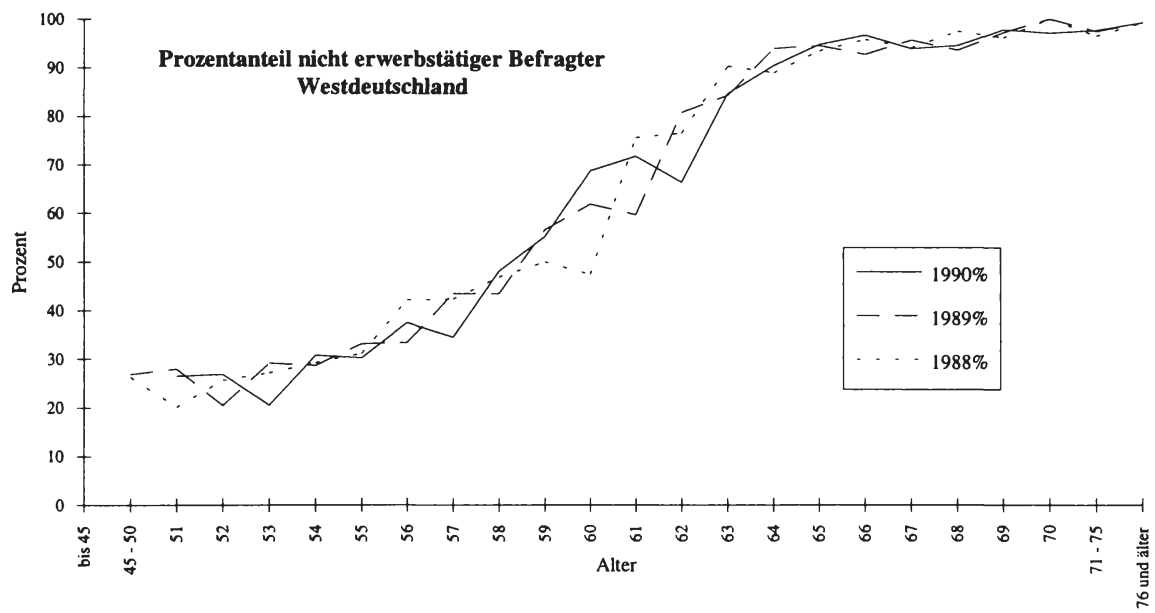

Quelle: West SOEP, Wellen 1 - 7 (1984-1990), eigene Berechnung.

\section{Abbildung 7-8: Prozentanteil nicht erwerbstätiger Befragter - Ostdeutschland.}

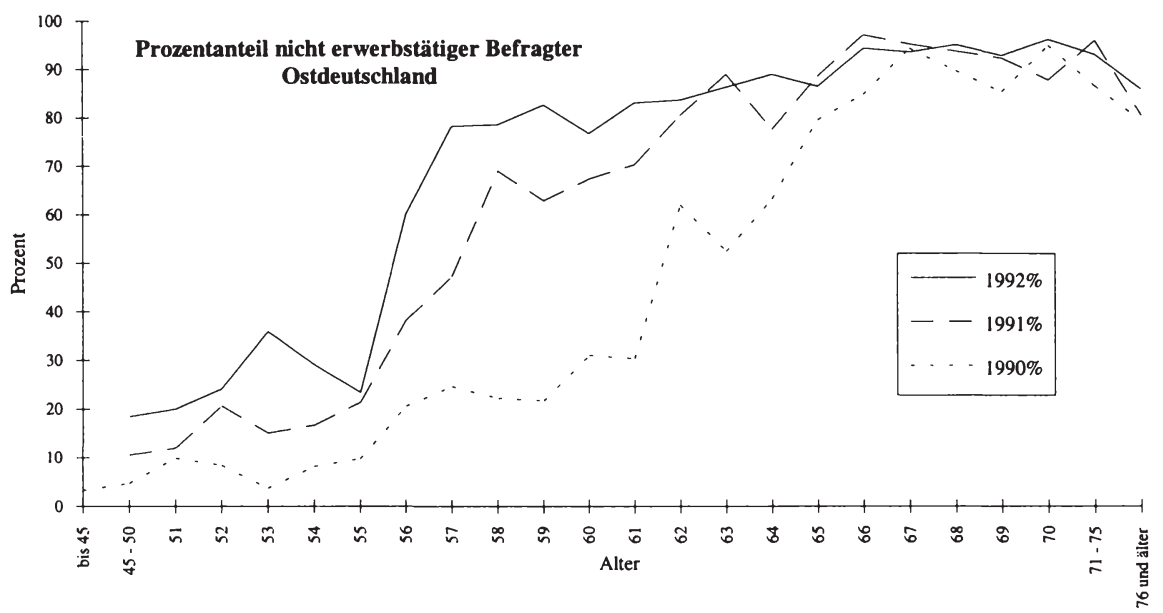

Quelle: Ost SOEP, Wellen 1 - 3 (1990-1992), eigene Berechnung.

Während die Erwerbsbeteiligung in den alten Bundesländern stetig abnimmt und dabei die Kohorteneffekte erkennbar sind, ist die einzige evidente Sprungstelle in der ost- 
deutschen Kurve das Alter 55. Ab diesem Alter gilt die ostdeutsche Vorruhestandsregelung, die in Kapitel 3.4.2 besprochen wurde.

Die überaus komplexe Struktur der ostdeutschen Situation korrekt abzubilden und die einzelnen Determinanten der individuellen Entscheidungen abbilden zu können, die während des Übergangsprozesses getroffen wurden, kann nicht das Ziel dieser Arbeit sein. Der Vergleich der alten Bundesländer mit den neuen kann vielmehr einen Einblick in den Wiedervereinigungsprozeß und seine Probleme geben. Es gilt zu beleuchten, ob auch in der betrachteten Übergangszeit empirische Hinweise auf die Relvanz (rein) ökonomischer Beweggründe für individuelle Entscheidungen meßbar sind. Aus diesem Grunde wird in der folgenden Diskussion partieller deskriptiver Betrachtungen immer auch der Ost-West Vergleich getroffen.

Die arbeitsmarktpolitische Situation war in diesen ersten eineinhalb Jahren nach der Wiedervereinigung vor allem von der Unsicherheit der Arbeitnehmerinnen und Arbeitnehmer geprägt, die sich in einer für sie völlig neuen Situation zurecht finden mußten. Der Status der Arbeitslosigkeit war im sozialistischen Regime fast ausnahmslos wegdefiniert. Eine bis heute nur schwer schätzbare Anzahl von Personen war bereits vor dem Systemwechsel beschäftigungslos, nicht aber arbeitslos im Sinne einer Statistik, da sie formale Arbeitsplätze besetzten und dafür bezahlt wurden, die im Sinne einer Produktivitätsbetrachtung nicht hätten existieren dürfen bzw. in einem marktwirtschaftlichen System nicht existiert hätten.

Das neue Phänomen der Arbeitslosigkeit, die auch die Bürger der neuen Länder richtig antizipierten, führte notwendigerweise zu einer großen Verunsicherung und der Suche nach Möglichkeiten einer sozialen Absicherung. Eine davon war für ältere Menschen das gesetzlichen Angebot der Frühverrentung im Rahmen von Vorruhestandsregelungen und anderem (vgl. Kap 3.4.2). Gleichzeitig wurde die Attraktivität des Ruhestandes dadurch erhöht, daß die schrittweise Anpassung der Rentenhöhe auf Westniveau bald beschlossen wurde und als sichere Option der individuell sehr unsicheren Entwicklung des eigenen Arbeitseinkommens gegenüberstand. Das damit verbundene dramatische Absinken der Erwerbsbeteiligung in Ostdeutschland zeigt sich in Tabelle 7-2.

Tabelle 7-2: Das rapide Absinken der Erwerbsbeteiligung in Ostdeutschland.

\begin{tabular}{|c|ccc|}
\hline & 1990 & 1991 & 1992 \\
\hline Vollzeitbeschäftigt & $56,9 \%$ & $44,5 \%$ & $37,4 \%$ \\
Nicht Erwerbstätig & $33,6 \%$ & $48,3 \%$ & $59,1 \%$ \\
Beobachtungen: & 3,764 & 3,456 & 3,328 \\
\hline
\end{tabular}

Quelle: Wellen 1-3 des SOEP für Ostdeutschland, alle Personen, die 1990 ălter als 44 Jahre waren. 
Tabelle 7-3 quantifiziert den Unterschied der durchschnittlichen Rentenzugangsalter in Ost und West, der schon in den Abbildungen 7-5 und 7-6 erkennbar wurde. Interessanterweise ist ein signifikanter Unterschied nur bei Männern zu erkennen. Ein Blick auf die tatsächlichen Werte des durchschnittlichen Verrentungsalters vom Verband Deutscher Rentenversicherungsträger für Westdeutschland in Tabelle 7-4 zeigt eine geringe Abweichung bei den Männern. Das knapp ein Jahr höhere Durchschnittsalter in der SOEP-Stichprobe resultiert daraus, da $B$ nur Personen ab 50 Jahre betrachtet werden. Auf dieser Basis scheint die erhebliche Abweichung von 3,5 Jahren für Frauen überraschend. Sie ist erneut Auswirkung der gewählten Definition der abhängigen Variable (s.o. bzw. Kapitel 7.1), also des Unterschiedes zwischen der „Austritt aus dem Berufslebens" einerseits und dem „Rentenzugang“ auf der anderen Seite.

Tabelle 7-3: Durchschnittliches Alter bel Beendigung der Erwerbstätigkeit in der SOEP Stichprobe der über 50 -jährigen

\begin{tabular}{|c|ccc|}
\hline & Männer & Frauen & Gesamt \\
\hline Ostdeutschland & 57,7 & 58,2 & 57,9 \\
Westdeutschland & 59,9 & 57,7 & 58,6 \\
t-Wert der Differenz & 2,489 & $-0,732$ & 1,536 \\
\hline
\end{tabular}

Quelle: SOEP, Berechnung für Wellen 1-7 des West-Panels und Wellen 1-3 der Ost-Stichprobe.

Tabelle 7-4: Durchschnittliches Rentenzugangsalter 1980-91 - VDR Statistik für Westdeutschland.

\begin{tabular}{|c|ccc|}
\hline Jahr & Männer & Frauen & Gesamt \\
\hline $\mathbf{1 9 8 0}$ & 58,3 & 60,1 & 59,3 \\
$\mathbf{1 9 8 1}$ & 58,2 & 59,5 & 58,9 \\
$\mathbf{1 9 8 2}$ & 58,4 & 59,5 & 59,0 \\
1983 & 58,6 & 59,6 & 59,1 \\
$\mathbf{1 9 8 4}$ & 58,8 & 60,1 & 59,5 \\
1985 & 58,7 & 60,4 & 59,5 \\
1986 & 58,8 & 61,3 & 60,1 \\
1987 & 59,0 & 61,7 & 60,5 \\
1988 & 59,1 & 61,8 & 60,5 \\
1989 & 59,3 & 61,7 & 60,5 \\
1990 & 59,5 & 61,6 & 60,6 \\
1991 & 59,6 & 61,5 & 60,5 \\
\hline Gewichteter Durchschnitt & $\mathbf{5 9 , 0}$ & $\mathbf{6 1 , 2}$ & $\mathbf{6 0 , 2}$ \\
der Jahre 1984-90 & & \\
\hline
\end{tabular}

Quelle: VDR Rentenzugangsstatistik, verschiedene Jahrgånge; eigene Berechnung 


\section{Varianzanalyse}

Weitere Einblicke in Zusammenhänge zwischen der Verrentungsentscheidung und persönlichen Merkmalen der betrachteten Personen können mit einer Varianzanalyse, d.h. einem Vergleich der durchschnittlichen Verrentungsalter für verschiedene Untergruppen, gewonnen werden. Dies geschieht in den Tabellen 7-5 (Westdeutschland) und 7-6 (Neue Bundesländer). Getestet wird in der Varianzanalyse, ob die bedingten Mittelwerte (hier das durchschnittliche Rentenalter) der betrachteten Untergruppen sich vom Gesamtmittelwert unterscheiden. ${ }^{21}$

Tabelle 7-5: Varianzanalyse des durchschnittlichen Alters bel Beendigung der Erwerbstätigkeit in der SOEP Stichprobe der über 50-jăhrigen - West

\begin{tabular}{|c|c|c|c|c|c|c|c|c|c|c|c|c|}
\hline \multirow[t]{2}{*}{ WEST } & \multicolumn{3}{|c|}{ Gesamt } & \multicolumn{3}{|c|}{ Männer } & \multicolumn{3}{|c|}{ Frauen } & \multicolumn{3}{|c|}{ t-Werte } \\
\hline & $\begin{array}{l}\text { Beob- } \\
\text { ach- } \\
\text { tungen }\end{array}$ & $\begin{array}{c}\text { Mittel- } \\
\text { wert }\end{array}$ & $\begin{array}{l}\text { Standard- } \\
\text { abwei- } \\
\text { chung }\end{array}$ & $\begin{array}{l}\text { Beob- } \\
\text { ach- } \\
\text { tungen }\end{array}$ & $\begin{array}{c}\text { Mittel- } \\
\text { wert }\end{array}$ & $\begin{array}{l}\text { Stan- } \\
\text { dard- } \\
\text { abwei- } \\
\text { chung }\end{array}$ & $\begin{array}{l}\text { Beob- } \\
\text { ach- } \\
\text { tungen }\end{array}$ & $\begin{array}{c}\text { Mittel- } \\
\text { wert }\end{array}$ & $\begin{array}{l}\text { Stan- } \\
\text { dard- } \\
\text { abwei- } \\
\text { chung }\end{array}$ & $\begin{array}{l}\text { Männer } \\
\text { gegen } \\
\text { Frauen: }\end{array}$ & 3,379 & \\
\hline $\begin{array}{c}\text { Gessmt- } \\
\text { Mittel- } \\
\text { wert }\end{array}$ & 556 & 58,62 & 4,52 & 366 & 59,09 & 4,44 & 190 & 57,73 & 4,55 & $\begin{array}{c}\text { Abwe } \\
\text { jeweil }\end{array}$ & Igen & \\
\hline \multicolumn{10}{|c|}{ Jahr der Verrentung } & Gesamt & Mănner & Frauen \\
\hline 1984 & 151 & 58,01 & 5,10 & 94 & 58,23 & 5,69 & 57 & 57,65 & 3,97 & $-1,336$ & $-1,357$ & $-0,124$ \\
\hline 1985 & 78 & 58,90 & 4,74 & 53 & 59,57 & 4,75 & 25 & 57,48 & 4,46 & 0,480 & 0,687 & $-0,259$ \\
\hline 1986 & 83 & 58,53 & 4,81 & 54 & 59,17 & 4,40 & 29 & 57,34 & 5,38 & $-0,167$ & 0,119 & $-0,363$ \\
\hline 1987 & 86 & 59,38 & 3,41 & 61 & 59,84 & 3,01 & 25 & 58,28 & 4,09 & 1,832 & 1,657 & 0,628 \\
\hline 1988 & 77 & 58,45 & 4,07 & 49 & 58,57 & 3,21 & 28 & 58,25 & 5,32 & $-0,338$ & $-1,008$ & 0,495 \\
\hline 1989 & 81 & 58,95 & 4,25 & 55 & 59,65 & 3,85 & 26 & 57,46 & 4,73 & 0,641 & 0,991 & $-0,269$ \\
\hline \multicolumn{13}{|c|}{ Arbeitslos } \\
\hline Nein & 502 & 58,87 & 4,54 & 328 & 59,41 & 4,42 & 174 & 57,87 & 4,59 & 0,891 & 0,936 & 0,295 \\
\hline $\mathrm{Ja}$ & 54 & 56,31 & 3,64 & 38 & 56,37 & 3,60 & 16 & 56,19 & 3,85 & $-4,344$ & $-4,326$ & $-1,512$ \\
\hline \multicolumn{13}{|c|}{ Verheiratet } \\
\hline Nein & 229 & $\mathbf{5 7 , 7 5}$ & 4,55 & 136 & 57,39 & 4,56 & 93 & 58,27 & 4,51 & $-2,461$ & $-3,741$ & 0,947 \\
\hline $\mathbf{J a}$ & 327 & 59,24 & 4,40 & 230 & 60,10 & 4,06 & 97 & 57,21 & 4,55 & 1,982 & 2,840 & $-0,916$ \\
\hline \multicolumn{13}{|c|}{ Berufliche Tätigkeit } \\
\hline ungelernt & 230 & 58,20 & 4,62 & 118 & 58,97 & 4,47 & 112 & 57,39 & 4,67 & $-1,177$ & $-0,263$ & $-0,605$ \\
\hline ausgebildet & 170 & 58,63 & 4,22 & 134 & 58,89 & 4,29 & 36 & 57,67 & 3,86 & 0,014 & $-0,462$ & $-0,082$ \\
\hline gehoben & 65 & 60,43 & 3,16 & 54 & 60,44 & 3,20 & 11 & 60,36 & 3,11 & 4,144 & 2,747 & 2,655 \\
\hline selbständig & 43 & 60,81 & 5,51 & 26 & 61,35 & 5,70 & 17 & 60,00 & 5,28 & 2,540 & 1,976 & 1,719 \\
\hline
\end{tabular}

Quelle: SOEP, Eigene Berechnung aus den Wellen 1.7 des westdeutschen Panels.

t-Werte: Fettdruck: signifikant auf dem 5\%-Niveau; kursiv: signifikant auf dem 10\%-Niveau.

Ablesebeispiel: der t-Wert für die Differenz des Gesamt-Mittelwertes im Jahre $1985(58,01)$ und dem Gesamt-Mittelwert über alle Jahre $(58,62)$ betrăgt $-1,336$ und ist damit insignifikant.

21 Die korrekt formulierte statistische Hypothese lautet: „Die Differenz dieser beiden Mittelwerte ist gleich Null“. Mit einem t-Test wird entschieden, ob diese Null-Hypothese statistisch abgelehnt werden kann. Dies ist bei einem t-Wert, der größer als 1,64 mit 10 prozentiger Fehlerwahrscheinlichkeit und ab einem Wert größer als 1,96 auf einem $5 \%$ Fehlerniveau der Fall. 
In den Tabellen sind zunächst in den ersten 3 Hauptspalten die jeweils notwendigen Angaben, also neben dem durchschnittlichen Rentenalter ('Mittelwert') die 'Anzahl der Beobachtungen' sowie die Standardabweichung der Subpopulation. Die vierte Hauptspalte enthält die $t$-Werte für die Differenzen, wobei die signifikanten Unterschiede gekennzeichnet sind.

Mit Hilfe dieser Tabellierung lassen sich erste Informationen aus der untersuchten Stichprobe ablesen, welche Faktoren die Verrentungsentscheidung besonders beeinflussen. Hier können allerdings nur deskriptive Aussagen gemacht werden, da die Varianzanalyse keine kausale Interpretation erlaubt. Für die alten Bundesländer fallen dabei folgende Zusammenhänge ins Auge:

- Die Wahl des Verrentungsalters ist bei Männern an berufliche Faktoren, aber auch den Familienstand gebunden als bei Frauen.

- Männer, die vor dem Ausscheiden aus dem Erwerbsleben arbeitslos waren, machen diesen Schritt deutlich früher (mehr als 2,5 Jahre). Dieses Verhalten korrespondiert mit dem Rentenversicherungssystem, das es Arbeitslosen erlaubt, bereits ab 60 eine vorgezogene Altersrente zu beziehen (vgl. hierzu Kapitel 3.1, insbesondere die ,57er Regel“")

- Bei Männern -und dadurch bedingt auch in der Gesamtbetrachtung- macht der Familienstand einen beträchtlichen Unterschied im mittleren Rentenalter aus, indem verheiratete Arbeitnehmer mehr als zweieinhalb Jahre später ausscheiden. Dieses Ergebnis deckt sich mit der Untersuchung von SUEYOSHI (1989). Auch MEGHIR UND WhITEHOUSE (1993) kamen zu dem verwandten Ergebnis, daß Personen, deren Partner noch arbeitet, signifikant später in den Ruhestand treten.

Es ist denkbar, daß sich im Familienstand andere, nicht gemessene, Variablen abbilden, wie die Vermögenssituation oder Kinderzahl. Menschen, die Kinder haben, akkumulieren im Verlauf ihres Erwerbslebens weniger Vermögen, da sie es für sich und die Kinder ausgeben, so daß sie am Ende dieser Phase länger ein Erwerbseinkommen benötigen. Ob und wie weit Eltern ein stärkeres Vererbungsbedürfnis haben, ist in der Literatur umstritten (vgl. Kapitel 2.1)

Die Frage, wieweit die Kenngröße Familienstand den Einfluß weiterer Determinanten mißt, kann durch die Regressionsanalyse des folgenden Kapitels geklärt werden, da in der dortigen Regressionsanalyse unter der ceteris paribus Bedingung getestet wird.22

22 Natürlich kann eine statistische Kontrolle nur für Größen erfolgen, die in die Regression eingehen. 
- Eine weitere interessante Einflußgröße stellt die 'Stellung im Beruf' dar. Während die Mehrzahl der Befragten in den Kategorien un- und angelernte Tätigkeiten ('ungelernt') sowie Tätigkeiten, für die eine Berufsausbildung erforderlich ist ('ausgebildet') beschäftigt ist und diese Gruppen daher auch weitgehend den Durchschnitt ausmachen, gehen die beiden Gruppen 'gehobene Tätigkeiten' und 'Selbständige' im Mittel zwei Jahre später in Rente ${ }^{23}$. Es scheint für diese Gruppen weniger der finanzielle Aspekt im Vordergrund zu stehen ${ }^{24}$, als vielmehr das Interesse und Engagement an der Arbeitsstelle. Auch kann davon ausgegangen werden, daß die Gesundheit dieser Gruppen durch den Beruf nicht so stark beansprucht wird, wie in einfachen Tätigkeiten.

Die in Tabelle 7-6 dargestellte Varianzanalyse für Ostdeutschland zeigt wiederum ein von den alten Bundesländern völlig abweichendes Muster. Zunächst fällt das sehr unterschiedliche Verhalten in den beiden betrachteten Jahren auf, ein Einflußfaktor, der im Westen - erwartungsgemäß - keine Rolle spielte.

Tabelle 7-6: Varianzanalyse des durchschnittlichen Alters bel Beendigung der Erwerbstätigkeit der SOEP Stichprobe der über 50-jährigen - Ost

\begin{tabular}{|c|c|c|c|c|c|c|c|c|c|c|}
\hline \multirow[t]{2}{*}{ OST } & \multicolumn{3}{|c|}{ Gesamt } & \multicolumn{3}{|c|}{ Männer } & \multicolumn{3}{|c|}{ Frauen } & \multirow{2}{*}{$\begin{array}{l}\text { t-Werte } \\
\text { Test: } \\
\text { Männer / } \quad-0,6691 \\
\text { Frnuen }\end{array}$} \\
\hline & $\begin{array}{l}\text { Beob- } \\
\text { ach- } \\
\text { tungen }\end{array}$ & $\begin{array}{l}\text { Mittel- } \\
\text { wert }\end{array}$ & $\begin{array}{l}\text { Standard } \\
\text { Abwei- } \\
\text { chung }\end{array}$ & $\begin{array}{l}\text { Beob- } \\
\text { ach- } \\
\text { tungen }\end{array}$ & $\begin{array}{l}\text { Mittel- } \\
\text { wert }\end{array}$ & $\begin{array}{l}\text { Standard } \\
\text { Abwei- } \\
\text { chung }\end{array}$ & $\begin{array}{l}\text { Beob- } \\
\text { ach- } \\
\text { tungen }\end{array}$ & $\begin{array}{l}\text { Mittel- } \\
\text { wert }\end{array}$ & $\begin{array}{c}\text { Standar } \\
\text { d } \\
\text { Abwei- } \\
\text { chung }\end{array}$ & \\
\hline $\begin{array}{c}\text { Gesamt- } \\
\text { Mittel- } \\
\text { wert }\end{array}$ & 317 & 57,99 & 6,41 & 147 & 57,74 & 5,002 & 170 & 58,23 & 7,419 & $\begin{array}{l}\text { Abweichungen vom } \\
\text { jeweiligen Mittelwert }\end{array}$ \\
\hline \multicolumn{10}{|c|}{ Jahr der Verrentung } & Gesamt Männer Frauen \\
\hline 1990 & 200 & 59,07 & 6,77 & 85 & 58,74 & 4,88 & 115 & 59,32 & 7,904 & $\begin{array}{lll}, 805 & 1,491 & 1,192\end{array}$ \\
\hline 1991 & 117 & 56,14 & 5,25 & 62 & 56,37 & 4,88 & 55 & 55,89 & 5,685 & $\begin{array}{lll}-3,056 & -1,840 & -2,431\end{array}$ \\
\hline \multicolumn{11}{|c|}{ Arbeitslos } \\
\hline Nein & 314 & 57,99 & 6,43 & 146 & 57,73 & 5,018 & 168 & 58,21 & 7,451 & \begin{tabular}{|lll}
$-0,006$ & $-0,015$ & 0,003
\end{tabular} \\
\hline $\mathrm{Ja}$ & 3 & 58,33 & 4,04 & 1 & 59 & & 2 & 58 & 5,657 & $\begin{array}{lll}0,144 & 3,051 & -0,052\end{array}$ \\
\hline \multicolumn{11}{|c|}{ Verheiratet } \\
\hline Nein & 57 & 60,47 & 8,19 & 12 & 62,25 & 8,389 & 45 & 60 & 8,177 & $2,168 \quad 1,835$ \\
\hline $\mathrm{Ja}$ & 260 & 57,45 & 5,82 & 135 & 57,34 & 4,415 & 125 & 57,57 & 7,052 & $\begin{array}{lll}-1,066 & -0,715 & -0,758 \\
\end{array}$ \\
\hline
\end{tabular}

Quelle: SOEP, Eigene Berechnung aus den Wellen 1-3 des ostdeutschen Panels.

t-Werte: Fettdruck: signifikant auf dem 5\%-Niveau; kursiv: signifikant auf dem 10\%-Niveau.

Weitere signifikante Unterschiede finden sich lediglich in Einzelfällen. Den einen macht ein (!) Mann aus, der später aus dem Beruf ausschied und auch 12 unverheirate-

${ }^{23}$ Für eine genaue Definition der beruflichen Stellung siehe die Anmerkungen zu Tabelle 7-7.

${ }^{24}$ Es ist davon auszugehen, daß Selbständige und Angestellte in gehobener Position ein überdurchschnittliches Einkommen haben. 
te Männer, die zu späteren Zeitpunkten als der Durchschnitt aufhören zu arbeiten, lassen keine allgemeingültigen Schlüsse zu. Eine Unterscheidung nach Berufscharakteristika kann aufgrund der geringen Anzahl erklärender Variablen nicht durchgeführt werden. Auch bei der in Tabelle 7-6 tabellierten Variable 'Arbeitslosigkeit' zeigt sich eine sehr schiefe Verteilung, indem nur 3 Personen (entsprechend unter einem Prozent der Befragten) den Status „Arbeitslos“ angeben. Dies ist auf Basis der bekannten hohen Arbeitslosigkeit in den neuen Bundesländern überraschend und kann nur daraus resultieren, daß die Bürger der ehemaligen DDR diesen Status zum Befragungszeitpunkt nicht kannten und somit im Falle der eigenen Betroffenheit - bewußt oder unbewußt - nicht angeben. Da hier die Status im Jahr vor der Verrentung angegeben sind, müßte die Zahl erheblich höher liegen, denn die Personen, die in den vorgezogenen Ruhestand ausscheiden wollten, mußten arbeitslos (gemeldet) sein (vgl. Kapitel 3.4.2).

\section{Übersicht über die Erklärungsvariablen des Verweildauermodelles}

In der Tabelle 7-7 sind die Variablen, die in der Verweildaueranalyse des folgenden Kapitels benutzt werden, zusammengefaßt und ihre Mittelwerte abgedruckt. Die Kategorien der Indikatorvariablen mußten teilweise etwas anders definiert werden, da die Variablen des Ost- und Westdatensatzes nicht direkt vergleichbar waren. So ist etwa die Schulbildung im Westen nach Schulart, im Osten nach Schuljahren angegeben. Der Grad der Erwerbsminderung, der im Westen in Prozent angegeben ist und der für die Beantragung einer Erwerbsunfähigkeitsrente erforderlich ist, konnte für die ehemalige DDR nur aus dem Besitz eines in vier Kategorien existierenden (Schwer-) Beschädigtenausweises konstruiert werden. Auch war es für die Menschen im Osten scheinbar schwerer, sich einer der anzukreuzenden (West-) Kategorien des beruflichen Sektors einzuordnen, wie die erheblich niedrigeren Nennungen belegen.

Da die meisten Variablen als Indikatorvariablen konstruiert sind, die nur die Ausprägungen 0 und 1 annehmen können, stellen die entsprechenden Mittelwerte genau den Prozentsatz der Befragungspersonen dar, die den entsprechenden Wert angegeben haben. So sind im Westen $33 \%$ der Befragten weiblich, wogegen in Ostdeutschland dieser Anteil $50 \%$ beträgt. 
Tabelle 7-7: Variablen der Verweildauerschätzung: Mittelwerte für Ost- und Westdeutschland

\begin{tabular}{|c|c|c|c|}
\hline \multirow[t]{2}{*}{ Variable } & \multirow[t]{2}{*}{ Definition } & \multicolumn{2}{|c|}{$\begin{array}{c}\text { Mittelwert } \\
\text { (im Entscheidungsjahr) }\end{array}$} \\
\hline & & West & Ost \\
\hline Weiblich & 1 = weiblich & 0.33 & 0.50 \\
\hline Kohorte & Geburtsjahr $(-1900)$ & 31.97 & 36.11 \\
\hline Familienstand & $1=$ verheiratet, $0=$ sonst & 0.57 & 0.84 \\
\hline Schulbildung & $\begin{array}{l}1=\text { mehr als Hauptschule (West) } \\
1=\text { mehr als } 8 \text { Jahre Schule (Ost) }\end{array}$ & 0.20 & 0.41 \\
\hline Berufsausbildung & $1=$ mehr als Lehre & 0.24 & \\
\hline Behinderung & $\begin{array}{l}\text { Grad der Erwerbsminderung }(0-100 \%) \\
-"-(\text { erzeugt auf Basis von Kategorien) }\end{array}$ & 9.04 & 5.80 \\
\hline $\begin{array}{l}\text { Vollzeit } \\
\text { Teilzeit } \\
\text { Arbeitslos } \\
\text { Referenzkategorie: }\end{array}$ & $\begin{array}{l}1=\text { Vollzeit beschäftigt } \\
1=\text { Teilzeit beschäftigt } \\
1=\text { arbeitslos } \\
\text { unregelmäßig beschäftigt }\end{array}$ & $\begin{array}{l}0.79 \\
0.12 \\
0.04\end{array}$ & $\begin{array}{l}0.86 \\
0.11 \\
0.01\end{array}$ \\
\hline Landwirtschaft & 1 = Landwirtschaftssektor & 0.02 & 0.04 \\
\hline Bergbau & $1=$ Bergbausektor & 0.01 & 0.01 \\
\hline Prod. Gewerbe & $1=$ Produzierendes Gewerbe, Industrie & 0.37 & 0.09 \\
\hline Handel & 1 = Handelssektor & 0.08 & 0.02 \\
\hline Öffentlicher Dienst & $1=$ Angestellter im öffentlichen Dienst & 0.11 & 0.05 \\
\hline Beamte & $1=$ Beamter & 0.07 & 0.00 \\
\hline Dienstleistung & $1=$ Dienstleistung (wenn nicht anders erfaßt) & 0.07 & 0.00 \\
\hline Bank \& Versicherung & 1 = Bank- und Versicherungssektor & 0.02 & 0.00 \\
\hline Ausbildungsberufe & $1=$ Ausbildungsberuf & 0.05 & 0.03 \\
\hline Gesundheit & $1=$ Gesundheitssektor & 0.04 & 0.01 \\
\hline Sozialer Beruf & $1=$ Sozialer Beruf & 0.03 & 0.00 \\
\hline Referenzkategorie: & Baugewerbe & 0.08 & 0.01 \\
\hline Ausgebildete Tätigkeit & $\begin{array}{c}1=\text { Tätigkeit, für die eine Berufsausbildung } \\
\text { erforderlich ist }\end{array}$ & 0.34 & 0.43 \\
\hline Gehobene Tätigkeit & 1 = gehobene Tätigkeit & 0.11 & 0.22 \\
\hline Selbständig & $1=$ Selbständig & 0.11 & 0.06 \\
\hline Referenzkategorie: & un- und angelernte Tätigkeit & 0.45 & 0.00 \\
\hline Hauseigentum & $1=$ Hauseigentum vorhanden & 0.43 & 0.40 \\
\hline Lebensversicherung & 1 = Lebens̀versicherung vorhanden & 0.54 & 0.41 \\
\hline Vermögen & 1 = Besitz von Wertpapieren oder Aktien & 0.18 & 0.07 \\
\hline OV & $\begin{array}{l}\text { Optionswert } \\
\text { [Minimum - Maximum Intervall] }\end{array}$ & $\begin{array}{l}139.65 \\
{[-5,52} \\
1076,82]\end{array}$ & $\begin{array}{l}106.40 \\
{[-9,77-} \\
423,44]\end{array}$ \\
\hline
\end{tabular}

Anmerkungen zur Tabelle: Die Variablen enthalten die Ausprägung im Entscheidungsjahr, d.h. des letzten Arbeitsjahres oder dem letzten Befragungsjahr (vgl. Kapitel 7.1).

Klassifikation der Kategorien zur beruflichen Stellung:

Un- und angelernte Tätigkeiten:

Ungelernter Arbeiter, Angelernter Arbeiter, Angestellte mit einfacher Tätigkeit, Beamte im einfachen Dienst, mithelfende Familienangehörige. 


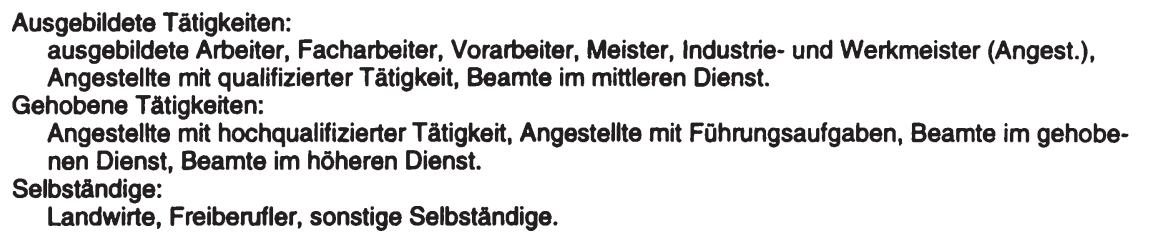

\subsection{Zeitratenanalyse der Wahl des Rentenalters}

Für die Verweildaueranalyse wird das im vorhergehenden Kapitel spezifizierte Verweildauermodell vom Gompertz-Typ verwendet. Zur Beurteilung der Relevanz der in Kapitel 5.4 diskutierten Erklärungsvariablen wurden unterschiedliche Modellspezifikationen vorgenommen. Kernfrage aus ökonomischer Sicht ist, ob der Optionswert bei der Wahl des Rentenalters eine relevante Rolle spielt, oder ob die anderen Variablen (Kovariate) das Verhalten der Befragten bestimmen. Aus diesem Grunde wird im ersten Schritt ein Referenzmodell ohne die Berücksichtigung des Optionswertes geschätzt und dieser anschließend in die Schätzung aufgenommen. Die Regressionsergebnisse sind in Tabelle 7-8 (Seite 186) zusammengefaßt.

\subsubsection{Verweildaueranalyse für Westdeutschland}

\section{Referenzmodell: Spezifikation ohne Optionswertvariable}

Spezifikation W1 beinhaltet die unter 5.3 diskutierten Kovariate zunächst ohne explizite Berücksichtigung ökonomischer Anreize. Der Schätzkoeffizient für den Verteilungsparameter $\Gamma$ ist mit 0,54 größer als Null, was eine steigende Baseline-Hazard impliziert. Dies heißt, daß die bedingte Wahrscheinlichkeit für eine Verrentung mit zunehmendem Alter ansteigt, ein Ergebnis, das der Intuition entspricht. Bei den einzelnen Schätzkoeffizienten sagt ein positiven Vorzeichen, daß ein Vorliegen des entsprechenden Merkmales die Hazardrate erhöht und somit die Wahrscheinlichkeit einer frühen Verrentung steigert. ${ }^{25}$ Es werden im folgenden die in Tabelle 5-6 aufgestellten Arbeitshypothesen überprüft.

Als wichtigste Einflußgröße, gemessen in den t-Werten ${ }^{26}$, ergibt sich das Geburtsjahr (Kohorte). Dies ist allerdings ein statistisches Artefakt, das aus der Zusammensetzung

\footnotetext{
${ }^{25}$ Für nicht $[0,1]$ codierte Variablen wirkt ein hoher Wert erhöhend auf die Wahrscheinlichkeit.

26 Eine direkte Interpretation der Schätzkoeffizienten selbst (über das Vorzeichen hinaus) ist für die vorliegenden nichtlinearen Modelle nicht möglich. Diese werden in den Simulationsstudien des folgenden Kapitels 7.4 quantifiziert. Die relativen Größen der Koeffizienten sind nicht vergleichbar, wenn sie unterschiedlich skaliert sind. Allerdings sind ein Großteil der verwendeten Kovariate Indi-

Peter Schmidt - 978-3-631-75568-6
} 
der Stichprobe resultiert: da nur Personen aufgenommen werden, die noch nicht in Rente sind, müssen diejenigen aus den frühen Kohorten definitionsgemäß sehr alt sein, wenn sie in Rente gehen (Selektionseffekt).

Die statistisch wichtigste inhaltliche Einflußgröße ist die Arbeitslosigkeit (im Jahr vor dem Ausscheiden). Auch dieses Ergebnis war aus theoretischer Sicht zu erwarten, da Arbeitslosen die Möglichkeit einer vorgezogenen Verrentung mit 60 Jahren offensteht. Diese gesetzliche Regelung sowie deren Ausnutzung durch die 57er-Regel wurden in Kapitel 3.1 beschrieben. Der t-Wert von 11,6 zeigt eine deutliche statistische Signifikanz dieser Einflußgröße auf individueller Ebene.

Dieses Ergebnis fügt der gesamtwirtschaftlich orientierten Diskussion des vierten Kapitels (Einführung in Kapitel 4.1 sowie empirische Betrachtung in Abschnitt 4.3.2.3) eine mikroökonomische Sicht hinzu. Allerdings wurde dort die Wirkungsweise der gesamten Arbeitsmarktsituation betrachtet, wogegen hier die persönliche Betroffenheit des Einzelnen durch Arbeitslosigkeit gemessen wird. Auch ist an dieser Stelle, ähnlich wie im bereits angesprochenen Fall der Erwerbsminderung die Frage der Kausalität nicht zu ermitteln:

Ein Sozialplan wird in großen Firmen als Angebot des Unternehmens an die Mitarbeiter eines bestimmten Alters (z.B. 59 bzw. 57 Jahre) aufgestellt. Dies Angebot beinhaltet für den Arbeitnehmer die Möglichkeit eines frühzeitigen Ruhestandes, der zu unterschiedlichen Anteilen durch das Unternehmen, die Bundesanstalt für Arbeit, ab dem 60. Lebensjahr die GRV in Verbindung mit Einkommensverlust durch den Arbeitnehmer selbst finanziert wird. Bei Annahme dieses Angebotes durch den Arbeitnehmer erhält dieser die Kündigung und wird damit arbeitslos. ${ }^{27}$

Diese „freiwillige“ Arbeitslosigkeit ist auch im vorliegenden Mikrodatensatz nicht von der unfreiwilligen Betroffenheit durch eine einseitige Kündigung des Arbeitgebers ${ }^{28}$ trennbar.

katorvariablen, die naturgemäß die gleich skaliert sind, indem sie das Vorhandensein oder nichtVorhandensein eines Merkmales anzeigen. Hierdurch ergibt sich eine Vergleichbarkeit des relativen Gewichtes der Indikatorvariablen untereinander.

27 Die Wahl des einzelnen Beschäftigten ist allerdings auf das Angebot durch die Arbeitgeber beschränkt. BÄCKER UND NAEGELE (1993), Seite 68 ff. heben hervor, daß seitens der Angestellten eine große Nachfrage an Teilzeitarbeit besteht, die aber von den Unternehmen nicht angeboten wird. Somit stellt der vollständige Sozialplan für die Arbeitnehmer nur eine Zweitbestlösung dar.

28 Auch kann der „freiwillige“ Vorruhestand als Reaktion auf erheblichen Druck des Unternehmens gewählt worden sein, das anderenfalls mit Kündigung aus anderen Gründen drohte. 
Die Einflußgröße zur Messung der persönlichen Gesundheit, die Variable Behinderung, zeigt ebenfalls mit hoher Signifikanz das erwartete positive Vorzeichen. Auch hier ist - wie mehrfach angesprochen - nicht zu klären, ob auch hier institutionell bedingte Gründe für den hohen Einfluß dieser Erwerbsminderung verantwortlich zeichnen. Wie bereits angesprochen, ist eine nachgewiesene Erwerbsminderung die Voraussetzung für den Bezug einer vorgezogenen EU/BU-Rente, so daß hier die Kausalität nicht letztlich klar ist: Gehen Menschen früher in Rente, weil ihre Erwerbsfähigkeit eingeschränkt ist oder sind es diejenigen, die früh in den Ruhestand wollen, die eine Erwerbsminderung nachweisen (müssen) und diese dann natürlich auch im Fragebogen angeben?

Die Bildungsvariablen sind beide signifikant, allerdings widersprechen sich die Vorzeichen. Eine Schulbildung, die über die Hauptschule hinausgeht, senkt die Verrentungswahrscheinlichkeit, wie dies erwartet wurde. Dagegen wirkt eine Berufsausbildung "mehr als Lehre“ in Richtung auf eine frühe Verrentung. Der Widerspruch könnte aus einer mangelnden Identifizierbarkeit der beiden Teileffekte allgemeine und berufliche Bildung herrühren, d.h. einer fehlenden statistischen Trennbarkeit der beiden eng korrelierten Merkmale.

Bezüglich der persönlichen Situation kann kein signifikanter Einfluß gemessen werden. Weder wird in dieser Schätzung ein Einfluß des Geschlechtes noch des Familienstandes gemessen.

Dagegen erweisen sich die Einflüsse der beruflichen Rahmenbedingungen als deutlich relevant. Hier sind drei Einflußfaktoren zu unterscheiden:

- Erwerbsstatus:

Alle drei angegebenen Kategorien Vollzeitbeschäftigung, Teilzeitbeschäftigung und Arbeitslosigkeit wirken positiv, d.h. Personen, die eines dieser Merkmale erfüllen scheiden früher aus diesem Status aus als die Referenzkategorie „unregelmäßig Beschäftigte";

- Branchenindikatoren:

Die Referenzgruppe Baugewerbe weist augenscheinlich einen frühen Verrentungszeitpunkt auf, denn fast alle signifikanten Branchen haben ein negatives Vorzeichen, d.h. verlassen den Beruf später (dies sind: Landwirte, Dienstleisungsstellen, Ausbildungsberufe, Gesundheitsberufe und Tätigkeiten im Handel). Die einzige Ausnahme bilden Beamte.

- Stellung im Beruf:

Gegenüber den un- und angelernten Tätigkeiten weisen sowohl Tätigkeiten, die eine Ausbildung erforderten, als auch selbständige Berufe eine spätere Verrentung auf. Das Signifikanzniveau der Selbständigen ist eines der höchsten der Regression und auch der Koeffizient dieser Variable ist mehr als dreimal so groß 
wie der von Ausbildungstätigkeiten und unterstreicht damit den deutlichen Einfluß der Selbständigkeit.

Die Indikatoren für eine intertemporale Sparentscheidung im Sinne der Lebenszyklushypothese, die Fragen nach Hauseigentum und dem Besitz von Wertpapieren oder Aktien zeigen ebensowenig einen signifikanten Einfluß auf die Ruhestandsentscheidung wie das Vorhandensein einer Lebensversicherung, die als Indikator sowohl von Vermögenswerten im Allgemeinen wie auch einer privaten Zusatzrentenversicherung im Besonderen modelliert ist.

Für diese Insignifikanz sind zwei Erklärungen denkbar. Die eine ist, daß die theoretischen Erwartungen der Lebenszyklushypothese in der Tat für die Bundesbürger keine Rolle spielen, zumindest in dieser einfachen Modellspezifikation und von daher - theoretisch wie ökonometrisch - genauer modelliert werden müßten. Die zweite ist, daß die Messung dieser Größen außerordentlich schwer ist und die Vermögensvariablen im SOEP tatsächlich keine sehr genauen Hinweise auf die tatsächliche Situation geben. Die erwähnten Variablen sind reine Indikatorgrößen, es wird im SOEP gefragt, ob ein Haushalt ein Sparbuch, Wertpapiere oder eine Lebensversicherung hat oder nicht. Dies sind natürlich ungenaue Indikatoren, so wurde die Frage nach einem Sparbuch hier nicht als Indikator für Vermögenswerte benutzt, da davon auszugehen ist, das praktisch jeder Haushalt ein Sparbuch besitzt und dies also kein sinnvoller Indikator für Vermögen ist. Ähnliches gilt für die Ja/Nein Fragen nach Wertpapieren oder einer Lebensversicherung ohne Angabe der Höhe des Vermögenswertes. In der fünften Welle des SOEP wurde eine Zusatzbefragung zur Vermögenssituation durchgeführt, deren Hauptergebnis war, daß die Befragten zu diesem Thema keine Auskunft zu geben bereit sind. Aus diesem Grunde bleiben die Vermögensfragen im SOEP auf ein Minimum beschränkt und damit leider auch die Möglichkeiten der vorliegenden Studie.

In diesem Referenzmodell liegt das Gütemaß $\rho^{2}$ bei $47 \%$, dies ist für eine MaximumLikelihood-Schätzung ein relativ hoher Wert. ${ }^{29}$

\section{Messung ökonomischer Faktoren durch den Optionswert}

Die Spezifikation W2 beinhaltet neben den bisher diskutierten Variablen zusätzlich den „Optionswert einer Verlängerung des Erwerbslebens“. Das Vorzeichen dieser

${ }^{29}$ Das $\rho^{2}$ stellt die prozentuale Verbesserung des Erklärungsgehaltes gegenüber dem Null-Modell dar, vergleiche Abschnitt 6.2. 
Einflußgröße ist erwartungsgemäß negativ und weist hohe statistische Signifikanz auf. Damit wird die aus der Literatur erwartete Hypothese bestätigt, daß diese Kenngröße ein sinnvolles Maß für die ökonomischen Anreize des GRV-Systems aus der Sicht des einzelnen Entscheidungsträgers darstellt.

Tabelle 7-8: Verweildaueranalyse für West-Deutschland - Spezifikationen des Gompertz-Modelles

\begin{tabular}{|c|c|c|c|c|c|c|c|c|c|}
\hline \multirow[b]{2}{*}{ erklärende Variable } & \multicolumn{3}{|c|}{$\begin{array}{c}\text { Spezifikation W1 } \\
\text { kein OV }\end{array}$} & \multicolumn{3}{|c|}{$\begin{array}{l}\text { Spezifikation W2 } \\
\text { Optionswert }\end{array}$} & \multicolumn{3}{|c|}{$\begin{array}{l}\text { Spezifikation W3 } \\
\text { nur Optionswert }\end{array}$} \\
\hline & $\beta_{1}$ & t-Wert & & $\beta_{1}$ & t-Wert & & $\beta_{i}$ & t-Wert & \\
\hline$\Gamma$ & 0,5399 & 29,25 & *** & 0,5313 & 28,95 & ** & 0,1765 & 39,77 & *** \\
\hline Konstante & $-44,9684$ & $-29,16$ & ** & $-44,6509$ & $-29,11$ & $* *$ & $-13,9395$ & $-47,93$ & ** \\
\hline Weiblich & 0,1639 & 1,48 & & 0,1162 & 1,05 & & & & \\
\hline Kohorte & 0,3750 & 21,37 & ** & 0,3966 & 22,05 & $* *$ & & & \\
\hline Familienstand & 0,0316 & 0,30 & & 0,0767 & 0,73 & & & & \\
\hline Schulbildung & $-0,4075$ & $-3,14$ & $* *$ & $-0,2818$ & $-2,14$ & $* *$ & & & \\
\hline Berufsausbildung & 0,2382 & 2,32 & ** & 0,2351 & 2,29 & $* *$ & & & \\
\hline Behinderung & 0,0077 & 4,56 & $* *$ & 0,0075 & 4,45 & ** & & & \\
\hline Vollzeit & 0,5668 & 3,04 & $* *$ & 0,3990 & 2,13 & $* *$ & & & \\
\hline Teilzeit & 0,7431 & 4,20 & ** & 0,8933 & 5,02 & ** & & & \\
\hline Arbeitslos & 2,1007 & 11,66 & *** & 2,0061 & 11,05 & ** & & & \\
\hline Landwirtschaft & $-0,7250$ & $-2,19$ & $* *$ & $-0,6858$ & $-2,10$ & $* *$ & & & \\
\hline Bergbau & $-0,1446$ & $-0,34$ & & $-0,0771$ & $-0,18$ & & & & \\
\hline Prod. Gewerbe & $-0,0897$ & $-0,75$ & & $-0,0481$ & $-0,40$ & & & & \\
\hline Handel & $-0,3142$ & $-1,68$ & $*$ & $-0,3607$ & $-1,93$ & $*$ & & & \\
\hline Öffentlicher Dienst & $-0,1500$ & $-0,87$ & & $-0,1348$ & $-0,78$ & & & & \\
\hline Beamte & 0,3852 & 1,85 & $*$ & 0,4831 & 2,32 & ** & & & \\
\hline Dienstleistung & $-0,5985$ & $-2,75$ & ** & $-0,6159$ & $-2,82$ & ** & & & \\
\hline Bank \& Versicherung & 0,0924 & 0,32 & & 0,0909 & 0,31 & & & & \\
\hline Ausbildungsberufe & $-0,6062$ & $-2,52$ & ** & $-0,5860$ & $-2,44$ & ** & & & \\
\hline Gesundheit & $-0,7461$ & $-2,58$ & ** & $-0,6363$ & $-2,20$ & $* *$ & & & \\
\hline Sozialer Beruf & $\begin{array}{l}-0,2529 \\
. \ldots\end{array}$ & $-0,93$ & & $-0,2125$ & $-0,78$ & & & & \\
\hline Ausgebildete Tätigkeit & $-0,3243$ & $-2,95$ & $* *$ & $-0,2540$ & $-2,31$ & $* *$ & & & \\
\hline Gehobene Tätigkeit & $-0,1775$ & $-1,06$ & & 0,0820 & 0,48 & & & & \\
\hline Selbständig & $-1,1151$ & $-5,76$ & $* *$ & $-1,2011$ & $-6,19$ & ** & & & \\
\hline Hauseigentum & $-0,0454$ & $-0,44$ & & $-0,0118$ & $-0,11$ & & & & \\
\hline Lebensversicherung & 0,0693 & 0,73 & & 0,0932 & 0,98 & & & & \\
\hline Vermögen & $-0,0219$ & $-0,18$ & & 0,0213 & 0,17 & & & & \\
\hline OV & & & & $-0,0061$ & $-5,10$ & $* *$ & 0,0035 & 7,64 & $* *$ \\
\hline Pseudo $\mathbf{R}^{2}$ & 0,4743 & & & 0,4782 & & & 0,3302 & & \\
\hline
\end{tabular}

Ein Vergleich der Schätzkoeffizienten und deren Signifikanzen zwischen den ersten beiden Spezifikationen zeigt, daß diese im wesentlichen gleich geblieben sind. Die einzige geringe Veränderung findet bei der Indikatorvariable „Beamte“ statt, deren 
positive statistische Signifikanz deutlicher wird und deren Koeffizient sich deutlich erhöht.

Die auch bei Modellierung des Optionswertes weiter geltende Relevanz der oben beschriebenen nichtökonomischen Einflußfaktoren zeigt, daß die Modellierung (nutzenbewerteter) finanzieller Anreize in Abhängigkeit vom Entscheidungsalter nicht ausreicht, um die Wahl des Ruhestandsalters adäquat zu modellieren. Vielmehr wird die Wahl des Rentenalters in einem persönlichen und gesellschaftlichen Kontext getroffen, der sowohl die in der Vergangenheit getroffenen Entscheidungen der Bildungs- und Berufswahl, als auch die derzeitige berufliche Situation bezüglich Branche, beruflicher Stellung und Arbeitszeit und nicht zuletzt die gesundheitliche Lage umfaßt.

Dieses Bild wird bestätigt durch die Spezifikation W3, in der analog der reinen Optionswertmodellierung von STOCK UND WISE (1990 a) nur die Optionswertvariable als Erklärungsvariable benutzt wird. Das Ergebnis ist, daß diese Variable das - aus theoretischer Sicht - falsche Vorzeichen aufweist, mit deutlicher statistischer Signifikanz. Dies sollte als Hinweis verstanden werden, da $B$ zur Modellierung der deutschen Ruhestandsentscheidung die reinen finanziellen Anreizstrukturen nicht ausreichen. Inhaltlich ist dieses Resultat einleuchtend, denn STOCK UND WISE ermittelten ihre Resultate mit einer sehr homogenen Gruppe, den Arbeitnehmern einer einzigen Firma. Demgegenüber muß die Heterogenität einer Gruppe von Befragungspersonen, die die deutsche Bevölkerung repräsentieren, erheblich höher sein. Die Einflüsse der GRV sind nicht so exakt spezifiziert wie die betriebliche Altersversorgung in der von STOCK UND WISE untersuchten Firma. Das deutlich geringere Gütemaß $\rho^{2}$ zeigt ebenfalls, daß dieses Modell nicht richtig spezifiziert ist.

Ein Vergleich der Optionswert-Variable mit einem Satz reiner Altersdummies ${ }^{30}$ kann aufgrund der Modellstruktur hier nicht vorgenommen werden, da das Alter im Entscheidungszeitpunkt zur Berechnung der abhängigen Variable in die Schätzung eingeht.

\section{Alternative Spezifikation mit einem Weibull-Modell}

Die Anhangtabelle A-7 zeigt die Regressionsergebnisse der bisher diskutierten Spezifikationen mit einem Verweildauermodell des Weibull-Typs. Der Koeffizient der Baseline-Hazard $\Phi$ liegt mit einem Wert von 3,5 deutlich oberhalb des Schwellenwertes

\footnotetext{
${ }^{30}$ Analog zu den beiden vogestellten Modellen von BÖRSCH-SUPAN (1991 und 1992 e). Diese Spezifikation wird auch von STOCK UND WISE (1992 a) als Referenzmodell angegeben.
} 
von $\ln 2$ und zeigt damit ebenso wie das Gompertz-Modell eine im Alter monoton steigende Basisübergangsrate an.

Die Koeffizienten der Kovariate sind aufgrund der unterschiedlichen Hazardraten nicht vergleichbar, wohl aber die Vorzeichen und die statistische Signifikanz. Beim Vergleich der beiden Schätzungen W1 und W2 mit den analogen W4 und W5 fällt als einziger Unterschied ein signifikant positiver Koeffizent von Frauen auf, der aber durch Hinzunahme der Optionswertvariable wieder insignifikant wird. Tatsächlich liegt das durchschnittliche Rentenalter von Frauen mit 57,7 Jahren unter dem der Männer mit 59,9 Jahren, ${ }^{31}$ so daß eine ceteris paribus höhere Hazard durchaus plausibel ist. Da diese durch den Optionswert insignifikant wird, scheint dies ökonomische Gründe zu haben.

Das Gütemaß $\rho^{2}$ liegt mit gut $49 \%$ etwa 2 Prozentpunkte, aber ein Vergleich von Gütemaßen zwischen unterschiedlichen Modellen hat keine starke statistische Aussage.

\subsubsection{Verweildaueranalyse für die neuen Bundesländer}

Tabelle 7-9 zeigt die Ergebnisse der Übergangsratenschätzung für die ersten 3 Panelwellen 1990 bis 1992 in den neuen Bundesländern. Die Spezifikationen entsprechen den für Westdeutschland diskutierten, allerdings sind nicht alle Variablen für Ostdeutschland verfügbar. Dies gilt einerseits für die Angabe beruflicher Bildung, die als Variable nicht vorliegt, weiterhin aber auch für die Branchen Beamte, Dienstleistungsberufe, Bank- und Versicherungstätigkeiten sowie soziale Berufe, die von keiner der befragten Personen genannt wurden. Schließlich ist die Variable Behinderung unterschiedlich definiert. Die einzelnen Variablen und ihre Mittelwerte können der Tabelle 7-7 (Seite 181) entnommen werden.

Die Bestimmtheitsmaße $\rho^{2}$ der Schätzungen 01 und 02 liegen mit über $70 \%$ wesentlich höher als bei den entsprechenden Westregressionen. Dies ist in dieser Deutlichkeit überraschend, bildet jedoch die besondere Situation des deutschen Einigungsprozesses ab. Durch den Zusammenbruch der ostdeutschen Wirtschaft verlor auf einen Schlag ein erheblicher Teil der Arbeitnehmer ihre Arbeitsstelle oder wurde mindestens erheblicher Unsicherheit ausgesetzt. Dies betraf die hier untersuchten älteren Personen in besonderem Maße. Die Folge war ein erhebliches Absinken der Erwerbsbeteiligung in

${ }^{31}$ Siehe Tabelle 7-3, Seite 176. 
Ostdeutschland, die bereits in Tabelle 7-2 zeigte. Die Alternative für ältere Arbeitnehmer lag, wie oben beschrieben, in der Frühverrentung.

Auf diese Weise stellen die ostdeutschen Befragungspersonen, die ausschließlich ältere Arbeitnehmer in dieser beschriebenen Ausnahmesituation sind, eine sehr homogene Gruppe dar, was zur Folge hat, daß eine statistische Messung ein vergleichsweise „scharfes“ (mit geringem Schätzfehler) Bild zeichnen kann. Allerdings ist auch festzuhalten, daß die Schätzergebnisse, die auf einer einmaligen vergangenen Situation beruhen, wenig für Fortschreibungen der Ergebnisse in die Zukunft, also etwa Politiksimualtionen, geeignet sind. Aus diesem Grunde stützen sich die Simualtionsrechnungen des 8. Kapitels ausschließlich auf die West-Ergebnisse, unter der Annahme, daß sich die sozio-ökonomische Situation in Ostdeutschland der westlichen Annähern wird.

\section{Referenzmodell ohne Optionswert (Spezifikation O1)}

Der Koeffizient der Übergangsrate $\Gamma$ ist mit einem Wert von 1,7 dreimal so hoch wie das West-Pendant, was ein erheblich steileres Ansteigen der Baselinehazard anzeigt. In der Tat ist dieser Anstieg in den Abbildungen 7-5 und 7-6 (Seiten 172 und 172) zu unterscheiden. Er ergibt sich daraus, daß die ostdeutschen Befragten bereits erheblich jünger aus dem Erwerbsleben ausscheiden.

Der starke Einfluß der Geburtskohorte ist auch in den neuen Bundesländern zu beobachten. Die Koeffizienten anderer Kovariate weichen jedoch erwartungsgemäß von den Westwerten ab. So ist der im Westen sehr starke Einfluß einer Arbeitslosigkeit in Ostdeutschland nur noch schwach signifikant positiv und auch sein Koeffizient ist, verglichen mit den andern beiden Erwerbsstatus wesentlich geringer als in der Westschätzung. Dies liegt in der geringen Nennung des Status arbeitslos durch die ostdeutschen Befragten (vergleiche dazu die Diskussion zur Varianzanalyse, Seite 179 bzw. Tabelle 7-7) begründet. Auch die beiden anderen Erwerbsstatus Teilzeit- und Vollerwerbstätigkeit sind nicht mehr oder nur schwach signifikant, während diese Gruppe in der Westregression einen wesentlichen Erklärungsgehalt ausmachten. Umgekehrt zeigen sich die Branchenindikatoren in Ostdeutschland wesentlich signifikanter als im Westen, die hohen t-Werte sind in der ganzen Regression dominierend. Bei der Varianzanalyse wurde bereits festgestellt, daß in der Oststichprobe nur wenige Personen eine Branchenangabe gemacht haben. Während sich die Prozentanteile der Branchen (Mittelwerte in Tabelle 7-7) in den alten Bundesländern auf 95\% addieren, haben in der ehemaligen DDR nur 26\% der Befragten eine Angabe gemacht. Somit ist es weni- 
ger die Referenzkategorie Baugewerbe, von der die angegebenen Branchen abweichen, sondern es sind die Personen, die sich überhaupt einer Branche zugeordnet haben, die erheblich höhere Hazardraten aufweisen, als die anderen Befragten. Die Einflußgruppe Stellung im Beruf weist im Gegensatz zum Westen überhaupt keine Signifikanz auf, weder arbeiten die Selbständigen länger als abhängig Beschäftigte noch unterscheiden sich die Rentenalter von Ausbildungsberufen oder gehobenen Tätigkeiten von der Referenzkategorie der un- und angelernten.

Die persönlichen Variablen Geschlecht und Familienstand sind ebensowenig signifikant wie in der Westschätzung, auch ein Einfluß der Schulbildung ist in Ostdeutschland nicht mehr gegeben.

Im Gegensatz dazu sind nun signifikante Einflüsse der Variablengruppe zur Lebenszyklushypothese zu verzeichnen, eine Reaktion, die eher in der Westschätzung und weniger in der Übergangssituation Ostdeutschlands zu erwarten war. Tatsächlich wirken die Einflußgrößen Hauseigentum und Besitz von Wertpapieren verlängernd auf das Erwerbsleben und damit gegen die aus Sicht der intertemporalen Konsumentscheidung zu erwartende Richtung. Lediglich das Vorhandensein einer Lebensversicherung hat das erwartete positive Vorzeichen, daß allerdings nach Hinzunahme des Optionswertes nicht mehr signifikant ist. Diese auf einer sehr langfristig ausgerichteten Theorie basierenden Einflußgrößen sollten eingedenk der Situation in Ostdeutschland an dieser Stelle nicht überinterpretiert werden. Vielmehr ist zu vermuten, daß diese Variablen latente Einflüsse messen, die in der Schätzung nicht modellierbar sind. In diesem Sinne ist eine mögliche Erklärung der signifikant negativen Vorzeichen, daß es sich bei diesen Befragten um Personen handelt, die auch im alten System eine gutgestellte Position hatten, was sich sowohl beruflich auswirkte als auch dahingehend, daß sie die hier angesprochenen Vorsorgen treffen konnten. Wenn diese Personen gleichzeitig aufgrund gesicherter, interessanterer Stellungen und höherer Arbeitsmotivation länger arbeiten, erklärt sich das negative Vorzeichen aus diesem Zusammenhang.

\section{Messung ökonomischer Faktoren durch den Optionswert}

Der Optionswert einer Verlängerung des Erwerbslebens wirkt auch in den neuen Bundesländern signifikant negativ und damit theoriekonform. Wie bereits in der Westschätzung verändert die Hinzunahme des Optionswertes weder die Vorzeichen noch die Koeffizienten der weitaus meisten Koeffizienten, so daß auch hier die Wichtigkeit des Gesamtmodelles unterstrichen wird. Dies gilt auch für die Schätzung $\mathrm{O} 3$, die nur auf dem Optionswert basiert und bei der wie im Westen ein falsches Vorzeichen statistisch signifikant ermittelt wird. 
Der Optionswert verändert die Koeffizienten der Erwerbsstatusvariablen, indem nun eine Vollzeiterwerbstätigkeit eine signifikante Verkürzung des Erwerbslebens bewirkt, wogegen dieser Effekt für Arbeitslose nun völlig verschwindet. Es gilt auch hier das oben zu diesem Themenkomplex gesagte.

Tabelle 7-9: Verweildaueranalyse für Ost-Deutschland - Spezifikationen des Gompertz- Modelles

\begin{tabular}{|c|c|c|c|c|c|c|c|c|}
\hline \multirow{4}{*}{$\begin{array}{l}\text { erklärende Variable } \\
\Gamma \\
\text { Konstante }\end{array}$} & \multicolumn{3}{|c|}{$\begin{array}{l}\text { Spezifikation } 01 \\
\text { kein OV }\end{array}$} & \multicolumn{3}{|c|}{$\begin{array}{c}\text { Spezifikation } 02 \\
\text { Optionswert }\end{array}$} & \multicolumn{2}{|c|}{$\begin{array}{l}\text { Spezifikation } 03 \\
\text { nur Optionswert }\end{array}$} \\
\hline & $\beta_{1}$ & t-Wert & & $\beta_{1}$ & t.Wert & & & \\
\hline & 1,6937 & 20,83 & ** & 1,7228 & 20,79 & ** & 0,1263 & $25,5^{* *}$ \\
\hline & $-153,169$ & $-20,90$ & ** & $-156,15$ & $-20,87$ & ** & $-11,112$ & $-32,9 * *$ \\
\hline Weiblich & 0,1607 & 1,36 & & 0,1585 & 1,34 & & & \\
\hline Kohorte & 1,6600 & 20,55 & ** & 1,7119 & 20,54 & $* *$ & & \\
\hline Familienstand & $-0,0700$ & $-0,46$ & & $-0,0223$ & $-0,15$ & & & \\
\hline Schulbildung & 0,0342 & 0,27 & & 0,1389 & 1,08 & & & \\
\hline Behinderung & 0,4051 & 2,26 & ** & 0,4203 & 2,34 & $* *$ & & \\
\hline Teilzeit & 0,7692 & 2,10 & $* *$ & 0,7135 & 1,93 & $*$ & & \\
\hline Vollzeit & 0,5216 & 1,55 & & 0,6081 & 1,78 & * & & \\
\hline Arbeitslos & 1,0493 & 1,93 & $*$ & 0,7033 & 1,27 & & & \\
\hline Land wirtschaft & 3,2704 & 12,63 & $* *$ & 3,2058 & 12,31 & $* *$ & & \\
\hline Bergbau & 3,4699 & 8,76 & ** & 3,6763 & 9,14 & ** & & \\
\hline Prod. Gewerbe & 3,0351 & 14,75 & ** & 3,0178 & 14,64 & ** & & \\
\hline Handel & 3,0755 & 10,05 & ** & 3,1107 & 10,14 & ** & & \\
\hline Öffentlicher Dienst & 2,7702 & 13,03 & ** & 2,8891 & 13,41 & ** & & \\
\hline Ausbildungsberufe & 2,6624 & 9,76 & ** & 2,8476 & 10,25 & ** & & \\
\hline Ausgebildete Tätigkeit & $-0,1188$ & $-0,95$ & & $-0,0641$ & $-0,51$ & & & \\
\hline Gehobene Tätigkeit & $-15,2847$ & $-0,08$ & & $-15,1109$ & $-0,08$ & & & \\
\hline Selbständig & $-14,7308$ & $-0,04$ & & $-14,7022$ & $-0,04$ & & & \\
\hline Hauseigentum & $-0,2469$ & $-1,95$ & $*$ & $-0,2541$ & $-2,01$ & $* *$ & & \\
\hline Lebensversicherung & 0,2539 & 2,11 & ** & 0,1194 & 0,95 & & & \\
\hline Vermögen & $-1,1116$ & $-3,31$ & ** & $-1,0920$ & $-3,27$ & ** & & \\
\hline ov & & & & $-0,0060$ & $-3,39$ & $* *$ & 0,0074 & $7,96 * *$ \\
\hline Pseudo $\mathbf{R}^{2}$ & 0,7097 & & & 0,7129 & & & 0,2634 & \\
\hline
\end{tabular}

\section{Alternative Spezifikation durch ein Weibull-Modell}

Auch für die ostdeutsche Schätzung wurde mit der gleichen Spezifikation ein WeibullModell geschätzt, dessen Ergebnisse in der Anhangtabelle A-8 abgedruckt sind. Die Ergebnisse zeigen wiederum eine Analogie zum Gompertz/Weibull-Vergleich für Westdeutschland. Das Weibull-Modell weist ebenfalls eine höhere Baseline-Hazard auf als das westdeutsche Weibullmodell. Allerdings identifiziert hier das Weibullmodell teilweise andere Parameter als die Gompertz-Schätzung, ein Zeichen dafür, daB 
die Anpassung des Weibull-Modelles an die steilere Baselinehazard nicht ausschließlich über die Basisübergangsrate geschieht, sondern auch die Lageparameter angepaßt werden.

Auch für die neuen Bundesländer ist es, wie schon in der West-Schätzung, der Fall, daß im Weibull-Modell eine signifikant höhere Übergangsrate für Frauen gemessen wird, die nun allerdings mit einem t-Wert von über 3 hohe Signifikanz aufweist, die auch nach der Hereinnahme des Optionswertes nicht zurückgeht. Dies ist umso überraschender, als daß tatsächlich in Ostdeutschland das durchschnittliche Rentenalter von Frauen mit 58,2 Jahren über dem der Männer $(57,7)$ liegt, ${ }^{32}$ so daß von dieser Sicht eine niedrigere Baseline-Hazard zu erwarten wäre.

Ein weiterer überraschender Effekt im Weibull-Modell ist die nun identifizierte sehr hohe Signifikanz der Indikatoren für Selbständigkeit und gehobene Stellung im Beruf. Dieses plausible negative Vorzeichen, das auch in Westdeutschland Gültigkeit hat, stellt den einzigen gravierenden Unterschied zwischen der Gompertz und der WeibullSpezifikation dar.

\subsection{Zusammenfassung der Schätzergebnisse für Ost- und Westdeutsch- land}

Das wichtigste Ergebnis beim Vergleich der beiden deutschen Landesteile ist, daß die Regressionen trotz eines sehr unterschiedlichen Profils der Rentenzugänge (Abbildungen 7-5 und 7-6) ein überraschend ähnliches Verhalten beschreiben. Der Hauptunterschied zwischen Ost- und Westdeutschland wird durch den erheblich steileren Verlauf der Baselinehazard in der ehemaligen DDR modelliert, aber nach deren statistischer Kontrolle erweist sich der Entscheidungsprozeß beider Gruppen als sehr ähnlich. Dies gilt vor allem für die ökonomischen Anreize des - inzwischen gleichen - Rentenversicherungssystems, die durch die Variable Optionswert modelliert werden.

Es ist aus diesem Grunde langfristig zu erwarten, daß sich das Rentenzugangsverhalten und damit auch das Rentenalter in Deutschland in dem Maße angleicht, wie dies die gesamtdeutsche Arbeitsmarktsituation tut. Die kurz- und mittelfristigen Übergangsprozesse, können dabei für eine Übergangszeit dazu führen, daß Arbeitsmarktprobleme in die Rentenversicherung verlagert werden. Langfristig jedoch gilt, daß für die gesamte

32 Siehe Tabelle 7-3, Seite 176. 
Bundesrepublik das Zugangsverhalten durch eine entsprechende Veränderung der gesetzlichen Rahmenbedingungen verändert werden kann.

Der erste Blick beim Vergleich der Schätzergebnisse für beide Teile des wiedervereinigten Deutschlands zeigt, daß die Schätzung für die ehemalige DDR statistisch besser identifiziert ist, was sich in einem erheblich höheren Gütemaß ausdrückt. Dies erklärt sich aus der besonderen Situation in Ostdeutschland, die die befragten Personen zu einer sehr homogen Gruppe macht.

In beiden Landesteilen erweist sich - neben einer notwendigen statistischen Kontrolle um Geburtsjahrgänge - die Variable Optionswert als adäquate und wichtige Einflußgröße, als Maßzahl für unterschiedliche ökonomische Anreize, die aus dem individuellen Lebensalter und geltenden institutionellen Rahmenbedingungen ermittelt wird. Es wird allerdings ebenso deutlich, daß diese reinen finanziellen Handlungsmotivationen nicht ausreichen, um das Verhalten älterer Arbeitnehmer in Deutschland zu modellieren. Ein ausschließlich auf dem Optionswert basierendes Modell ohne Kovariate zeigt sich ungeeignet, das deutsche Arbeitsmarktaustrittsverhalten abzubilden. Dies steht im Gegensatz zu den Erfahrungen von LUMBSDAINE, STOCK UND WISE (Kapitel 5.3.1), die allerdings Betriebsrentenprofile mit sehr deutlichen Alters-Einkommensstrukturen untersuchen.

Das in dieser Arbeit benutzte Hazardraten-Modell der Ruhestandsentscheidung, das den Optionswert als Operationalisierung der institutionellen ökonomischen Anreize ebenso enthält wie eine Kontrolle um statistische Einflußgrößen, erweist sich als eine adäquate Modellierung der persönlichen Ruhestandsentscheidung für ganz Deutschland.

Die Determinanten der Ruhestandsentscheidung zeigen sich in beiden Landesteilen relativ ähnlich. Als wichtigste technische Kontrollgröße erweist sich jeweils die Geburtskohorte, die einen erheblichen Generationenunterschied offenbart. Zur Begründung dieses unterschiedlichen Verhaltens wurde vor allem der Unterschied zwischen der Kriegsgeneration und den „Wirtschaftswunderjahrgängen“ angeführt.

Ebenfalls in beiden Landesteilen zeigt sich die Variable Behinderung als Gesundheitsindikator, gleichzeitig aber auch formale Voraussetzung für den Bezug einer vorgezogenen BE/EU-Rente, als wichtiger Auslöser einer Frühverrentung. Dagegen ist die im Westen hochsignigikant positiv wirkende Arbeitslosigkeit in der ehemaligen 
DDR nur schwach identifiziert, was aber in der seltenen Angabe dieses Status durch die befragten Personen begründet liegt.

Brancheneinflüsse zeigen sich als notwendige Kontrollvariablen, die allerdings wenig inhaltliche Aussagefähigkeit aufweisen. Die berufliche Stellung ist nur im Westen konstituierend für die Übergangsraten, hier wirkt Selbständigkeit mit hoher Signifikanz verlängernd auf das Erwerbsleben.

Die folgende Tabelle 7-10 zeigt die wichtigsten Determinanten frühen und späteren Rentenalters in beiden Landesteilen in einer Übersicht.

Tabelle 7-10: Signifikante Einflußfaktoren und Wirkungsrichtungen in der Verweildauerschätzung - Vergleich der alten und neuen Bundeslānder

\begin{tabular}{|c|c|c|}
\hline Einflußfaktoren für: & Westdeutschland & Ostdeutschland \\
\hline Frühe Verrentung & $\begin{array}{l}\text { - späteres Geburtsjahr } \\
\text { - Arbeitslosigkeit } \\
\text { - Teilzeitbeschäftigung } \\
\text { - Behinderung } \\
\text { - Vollzeitbeschäftigung } \\
\text { - Berufsausbildung vorhan- } \\
\text { den }\end{array}$ & $\begin{array}{ll}\text { - } & \text { späteres Geburtsjahr } \\
\text { - } & \text { Alle spezifizierten Branchen } \\
\text { hochsignifikant (vgl. Tabelle) } \\
\text { - Behinderung } \\
\text { - Teil-/Vollzeitbeschäftigung } \\
\text { - (Arbeitslosigkeit) }\end{array}$ \\
\hline $\begin{array}{l}\text { Späteren Ruhe- } \\
\text { stand }\end{array}$ & $\begin{array}{l}\text { - Selbständigkeit } \\
\text { - Optionswert } \\
\text { - Ausgebildete Tätigkeit } \\
\text { - Schulbildung } \\
\text { - Diverse Branchen } \\
\text { (vgl. Tabelle) }\end{array}$ & $\begin{array}{l}\text { - Optionswert } \\
\text { - Finanzvermögen } \\
\text { - Grundvermögen }\end{array}$ \\
\hline
\end{tabular}

Quelle: Angabe basiert auf den beschriebenen Regressionsschătzungen, die Variablen sind in der Reihenfolge ihrer statistischen Signifikanz angegeben. Vergleichsbasis sind die Gompertz-Spezifikationen.

Das in diesem Kapitel beschriebene Hazardratenmodell gibt somit Antwort auf die Frage, welche Einflußfaktoren die Ruhestandsentscheidung der Arbeitnehmer in Deutschland bestimmen. Es ist aus den Schätzkoeffizienten direkt jedoch nicht möglich, diese Einflüsse zu quantifizieren und damit aus dem Modell heraus praktische Politikempfehlungen abzuleiten. Hierzu wäre die explizite Berechnung von Elastizitäten nötig, die durch die Kombination des nichtlinearen Zeitdauermodelles mit dem ebenfalls parametrisch spezifizierten, alters- und einkommensabhängig errechneten Optionswert sehr kompliziert. Auch wäre im Hinblick auf die hier interessierende Ein- 
flußgröße des Optionswertes eine reine Elastizitätsbetrachtung nicht die relevante Maßzahl, da der Optionswert seinerseits aus unterschiedlichen Einflußgrößen errechnet wird, so da $B$ nur mittelbare Wirkungen auf die Ruhestandswahrscheinlichkeiten ermittelt werden könnten.

Eine Antwort auf die Frage nach der Auswirkung sich ändernder Rahmenbedinungen kann jedoch aus Simulationsrechnungen gewonnen werden, die auf Basis der hier ermittelten Schätzergebnisse die Ruhestandswahrscheinlichkeiten unter geänderten Erklärungsvariablen vorhersagen. Diese Analyse wird im folgenden achten Kapitel durchgeführt. 
Nachher, vor allen andren Sachen, Müßt Ihr Euch an die Metaphysik machen;

Da seht, daß Ihr tiefsinnig faßt,

Was in des Menschen Hirn nicht paßt.

\section{VOLKSWIRTSCHAFTLICHE UND POLITISCHE IMPLIKATIONEN DER DARGESTELL- TEN ERGEBNISSE}

Die Kernfrage dieser Arbeit, ob der Gesetzgeber durch die Ausgestaltung der GRV die Rentenzugänge entscheidend beeinflussen kann, wurde im vierten und siebten Kapitel empirisch untersucht. Im ersteren lag der Schwerpunkt auf der aggregierten Entwicklung aller Rentenzugänger in der Bundesrepublik, im Kapitel 7 wurde die individuelle Wahl des Rentenalters analysiert. Beide Studien zeigen den erheblichen Einfluß der ökonomischen Anreizwirkungen des Rentenversicherungssytems für die ex post untersuchten Zeiträume auf.

Im vorliegenden Kapitel wird auf Basis der mikroökonometrischen Analyse des 7. Kapitels ex ante die Wirkung von Gesetzesänderungen auf die Rentenzugänge quantifiziert und für den Zeitraum bis 2050 prognostiziert. Es werden Simulationsrechnungen über die Reaktionen der Arbeitnehmer auf Reformen des Rentenversicherungssytems durchgeführt und ihre Konsequenzen für die Finanzierung des Rentenversicherungssytems aufgezeigt. Damit wird hier ein Instrument der Politikberatung entwickelt, das es den Entscheidungsträgern erleichtern soll, die Implikationen der Rentenpolitik abzuschätzen.

\section{Simulationsmethode}

Im sechsten Kapitel wurde die Grundstruktur des Gompertz-Modelles dargestellt, dessen Hazardfunktion sich aus der Baselinehazard $\lambda_{0}$ und Verteilungsparametern zusammensetzt. Letztere quantifizieren den Einfluß der Kovariate. Diese Kovariate werden nun als Optionswert $O V$ und restliche Kovariate $Z_{l}$ getrennt betrachtet:

$$
\lambda\left(t \mid Z_{t}, O V_{t}\right)=\exp (\gamma) \cdot \exp \left(Z_{t} \beta^{\text {Rest }}+O V_{t} \beta^{\text {ov }}\right)
$$

mit: $\mathbf{t} \quad$ Verrentungszeitpunkt (Lebensalter)

$\lambda \quad$ Hazardrate

$\lambda_{0} \quad$ Basisübergangsrate (Baseline Hazard)

$\gamma \quad$ Schätzkoeffizient der Basisübergangsrate

Z Matrix der erklärenden Variablen (Kovariate, ohne Optionswert )

OV Optionswert

$\beta \quad=\left(\beta^{\text {ov }}, \beta^{\text {Rest }}\right)$ : Parameter-Vektor aller Regressionskoeffizienten (Notation Kapitel 6). 
$\begin{array}{ll}X_{t} & \text { Matrix aller erklärenden Variablen } Z_{t} \text { und OV, (Notation Kapitel 6) } \\ \beta^{\text {ov }} & \text { Parameter-Vektor des Regressionskoeffizienten für den Optionswert OV } \\ \beta^{\text {Rest }} & \text { Parameter-Vektor der zu ermittelnden Regressionskoeffizienten für } X_{t}\end{array}$

Die im siebten Kapitel dargestellte Verweildaueranalyse stellte den ersten Schritt der Politiksimulation dar, in dem die Schätzkoeffizienten $\beta$ ermittelt wurden.

Für die Simulationsrechnungen werden die $Z_{\uparrow}$ ceteris paribus an ihrem beobachteten Wert festgehalten, der Optionswert verändert sich durch die unterschiedlich angenommene Rentenformel (GRV-Simulation), so daß sich für jedes Alter $t$ eine simulierte Hazardrate ergibt. Aus dieser werden altersspezifische Verrentungswahrscheinlichkeiten errechnet.

Das Simulationsprogramm ${ }^{1}$ berechnet für jede untersuchte Person des SOEP aus allen altersspezifischen Rentenzugangswahrscheinlichkeiten das erwartete Rentenalter der einzelnen Personen und aus deren Mittelwert das durchschnittliche Rentenalter der Stichprobe. Abbildung 8-1 zeigt den Ablauf der Simulationsrechnung.

Auf Basis dieser durchschnittlichen Rentenalter werden mit Hilfe eines Bevölkerungsprognoseprogrammes ${ }^{2}$ die resultierenden Alterslastquotienten und GRV-Beitragssätze errechnet.

Abbildung 8-1 verdeutlicht, daß für die individuelle Berechnung des Rentenalters die gleichen Kovariate verwendet werden und auch das persönliche Alter und Einkommen in beiden Fällen in die Ermittlung des Optionswertes eingeht, so daß der Unterschied im Rentenzugangsalter ausschließlich durch die Simulation geänderter GRVRegelungen zustande kommt. Die fett gekennzeichneten Linien zeigen den Ablauf dieser Simulation.

Der ermittelte Beitragssatz zur GRV ergibt sich aus der in Kapitel 2 diskutierten Budgetidentiät der gesetzlichen Rentenversicherung (Gleichung 2-1 und 2-2) als (8-2).

1 Zur Simulation wird ein Programm von Prof. BÖRSCH-SUPAN benutzt, das er mir freundlicherweise zur Verfügung stellte und in dem nur geringfügige Änderungen vorgenommen wurden.

2 Das Bevölkerungsprognoseprogramm ist das in BÖRSCH-SUPAN, MEIER UND REIL-HELD (1994) benutzte Modell, das von diesen Autoren gemeinsam entwickelt wurde. 
mit: N Anzahl Beschäftigte

b Beitragssatz zur Gesetzlichen Rentenversicherung

R Anzahl Rentner

RN Rentenniveau,

definiert als: $\frac{\text { Durchschnittsrente }}{\text { Durchschnittslohn }}$.

Abblidung 8-1: Ablaufschema der Simulation des Rentenalters mittels unterschiedlicher GRV-Szenarien

L.Schdit: Yerciellinuersch" troung

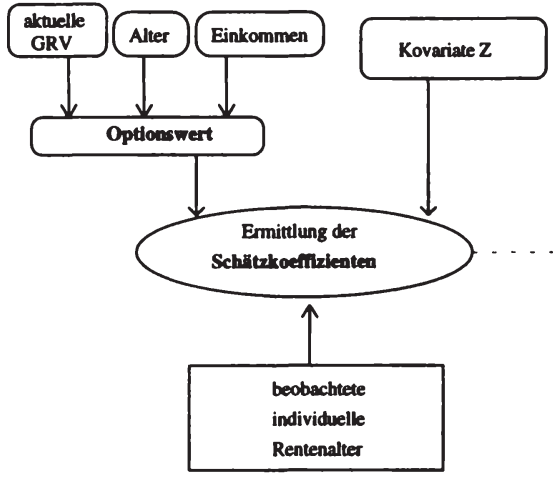

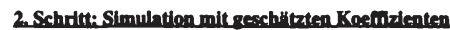

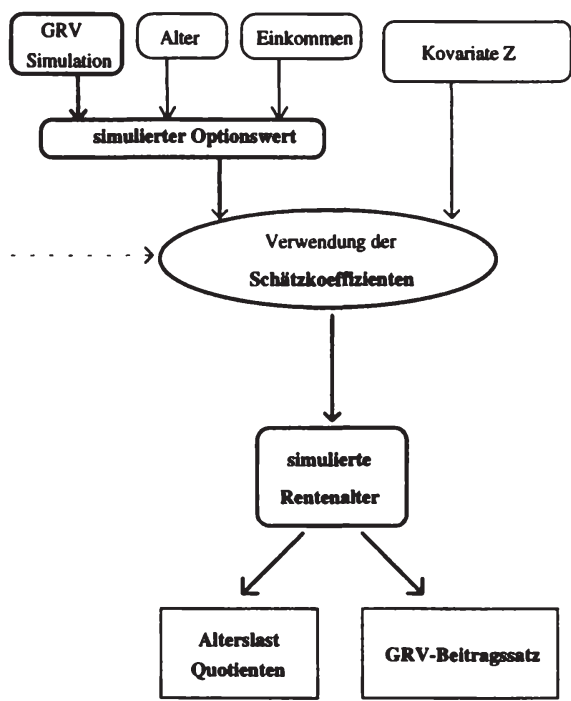

Die Simulation beinhaltet allerdings eine Vereinfachung, da in ihr lediglich ein Simulationslauf durchgeführt wird. Tatsächlich hat der in dieser Simulation ermittelte „GRV-Beitragssatz“ Rückwirkungen auf die erste Stufe (des 2. Schrittes in Abbildung 8-1), die „GRV-Simulation“. Um dieser Tatsache Rechnung zu tragen, müßten wiederholte Programmabläufe durchgeführt werden, um auf iterativem Wege einen gleichgewichtigen Beitragssatz zu ermitteln. Davon wird hier zur Vereinfachung abgesehen. 


\section{Simulationsrechnungen auf Basis der ermittelten Schätzergebnisse}

Zur Simulation potentieller Reformen der gesetzlichen Rentenversicherung werden fünf Szenarien betrachtet:

- heutige GRV (Referenzfall);

- Rentenreform 1992 nach vollständigem Inkrafttreten;

- Versicherungsmathematisch faires System mit einem Diskontfaktor von $5 \% ;^{3}$

- Versicherungsmathematisch faires System mit einem Diskontfaktor von $7 \%$;

- Versicherungsmathematisch faires System mit einem Diskontfaktor von 13,8 \%.

Tabelle 8-1: Kumulierte Wahrscheinlichkeiten eines Ruhestandes in unterschiedlichen GRV-Szenarien für ausgewăhite Alter

\begin{tabular}{|c|c|c|c|c|c|}
\hline \multirow[b]{2}{*}{ Alter } & \multirow[b]{2}{*}{$\begin{array}{c}\text { GRV- } \\
\text { Aufschlag } \\
\text { (vor 1992) }\end{array}$} & \multirow[b]{2}{*}{$\begin{array}{l}\text { Rentenreform } \\
92^{*}\end{array}$} & \multicolumn{3}{|c|}{$\begin{array}{l}\text { Nichtverzerrende Systeme } \\
\text { (versicherungsmathematisch fair) }\end{array}$} \\
\hline & & & $\begin{array}{l}\text { Diskontierungs- } \\
\text { faktor: } 5 \%\end{array}$ & $\begin{array}{l}\text { Diskontierungs- } \\
\text { faktor: } 7 \%\end{array}$ & $\begin{array}{l}\text { Diskontierungs- } \\
\text { faktor: } 13,8 \%\end{array}$ \\
\hline 55 & $39 \%$ & $37 \%$ & $35 \%$ & $34 \%$ & $31 \%$ \\
\hline 56 & $43 \%$ & $42 \%$ & $40 \%$ & $39 \%$ & $35 \%$ \\
\hline 57 & $48 \%$ & $46 \%$ & $45 \%$ & $43 \%$ & $40 \%$ \\
\hline 58 & $53 \%$ & $51 \%$ & $49 \%$ & $48 \%$ & $45 \%$ \\
\hline 59 & $58 \%$ & $55 \%$ & $53 \%$ & $52 \%$ & $49 \%$ \\
\hline 60 & $62 \%$ & $60 \%$ & $58 \%$ & $57 \%$ & $53 \%$ \\
\hline 61 & $66 \%$ & $64 \%$ & $62 \%$ & $61 \%$ & $58 \%$ \\
\hline 62 & $71 \%$ & $68 \%$ & $66 \%$ & $65 \%$ & $62 \%$ \\
\hline 63 & $74 \%$ & $72 \%$ & $70 \%$ & $69 \%$ & $66 \%$ \\
\hline 64 & $78 \%$ & $76 \%$ & $74 \%$ & $73 \%$ & $70 \%$ \\
\hline 65 & $81 \%$ & $79 \%$ & $77 \%$ & $76 \%$ & $73 \%$ \\
\hline 66 & $84 \%$ & $82 \%$ & $81 \%$ & $80 \%$ & $77 \%$ \\
\hline 67 & $87 \%$ & $85 \%$ & $84 \%$ & $83 \%$ & $80 \%$ \\
\hline 70 & $92 \%$ & $91 \%$ & $91 \%$ & $90 \%$ & $88 \%$ \\
\hline 75 & $96 \%$ & $96 \%$ & $96 \%$ & $96 \%$ & $95 \%$ \\
\hline
\end{tabular}

Quelle: Ermittlung aus den Koeffizienten der Gompertz-Regressionsanalyse durch unterschiedliche Berechnung des Optionswertes auf Basis der modellierten GRV-Systeme.

- Modelliert wird die Rentenreform 1992 fūr die Jahre, in denen sie voll in Kraft ist. Durch die Ūbergangsfristen, v.a. betreffend das mögliche Rentenalter, ist dies für Mănner ab dem Jahre 2007 und für Frauen 2013 der Fall.

Tabelle 8-1 zeigt die sich unter den genannten Szenarien ergebenden kumulierten Rentenzugangswahrscheinlichkeiten für ausgewählte Entscheidungsalter. Die angege-

\footnotetext{
${ }^{3}$ Zur Diskussion versicherungsmathematischer FairneB und zur Bedeutung alternativer Diskontfaktoren vergleiche Kapitel 3.5.
} 
benen Werte können interpretiert werden als der Anteil der bereits im Ruhestand befindlichen Personen nach Lebensalter.

Das Alter von 59 Jahren ist herausgehoben, da an diesem der Anteil der bereits vor dem ersten offiziellen Rentenalter 60 ausscheidenden Arbeitnehmer abgelesen werden kann. Dieser beträgt im Status-Quo Szenario bereits $58 \%,{ }^{4}$ und wird durch die gültige Rentenreform von 1992 lediglich um 3 Prozentpunkte gesenkt. Dagegen wird durch das Szenario einer nichtverzerrenden Rentenformel unter Annahme eines Diskontfaktors von 13,8 \%5 ein Sinken um gut 9 Prozentpunkte erreicht, was einem Rückgang um $15,5 \%$ entspricht. Beim Regelrentenalter von 65 Jahren sinkt der Anteil der nicht erwerbstätigen von $81 \%$ im Status-Quo Fall auf 73 \% im unverzerrten System.

\section{Abbildung 8-2: Kumulierte Zugangswahrscheinlichkeiten in die GRV unter verschie- denen GRV-Szenarien}

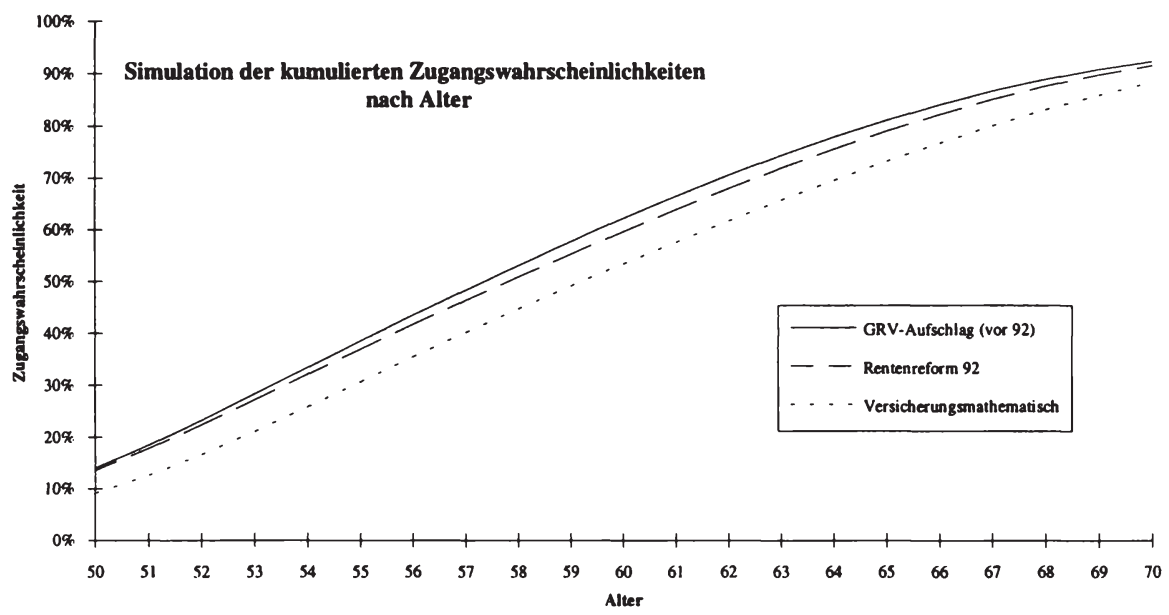

Abbildung 8-2 verdeutlicht dieses Verhältnis und zeigt, daß die Rentenreform 92 die altersspezifischen Zugangswahrscheinlichkeiten nur geringfügig senkt, wogegen ein versicherungsmathematisch faires Rentenberechnungsverfahren den Anteil der Rentner um 8 bis 9 Prozentpunkte nach unten verschiebt.

${ }^{4}$ Dieser Wert entspricht dem aus der SOEP-Stichprobe ermittelten Prozentsatz der Personen des Status „nicht erwerbstätig“ (vgl. Kapitel 7.1), so daß er erheblich über dem Anteil der offiziellen Rentenempfänger liegen dürfte. Da, wie im vierten Kapitel ausgeführt, über die Bezugsgröße der Versicherten oder der Erwerbstätigen jedoch keine genauen Daten verfügbar sind, liegt hier keine Referenzgröße vor.

${ }^{5}$ Dieser Diskontfaktor entspricht dem von BöRSCH-SUPAN (1992 e - Seite 551) ermittelten. 
Umgekehrt wird im unverzerrenden System ein gegebener Anteil von Pensionären erst ca. 2 Jahre später erreicht, so etwa die erwähnten $58 \%$, die im aktuellen System bereits bei einem Alter von 59 Jahren erreicht sind, wogegen dies in der 5 . Spalte erst mit 61 Jahren der Fall ist.

Auf Basis dieser Überlegungen stellt sich die Frage, wie sich die beschriebenen Rentenzugangswahrscheinlichkeiten auf das durchschnittliche Rentenalter auswirken. Diese wird in Tabelle 8-2 beantwortet, deren linke Spalte die Veränderung des Stichprobenmittelwertes unter alternativen Reform-Simulationen zeigt. Auch in dieser Darstellung zeigt sich die Verschiebung des Rentenalters um 2 Jahre, was einer Erhöhung des Rentenalters um 3,4 Prozent entspricht. Diese Zahl wirkt auf den ersten Blick relativ klein. Als Vergleich steigen die realen GRV-Zugangsalter von 57,5 Jahren am „Tiefpunkt“ 1981 auf 59,0 Jahre im Jahre 1991.6 Diese damalige Steigerung um 1,5 Prozentpunkte in einem Zeitraum von 10 Jahren liegt unter der hier prognostizierten.

Tabelle 8-2: Veränderung des durchschnittlichen Verrentungsalters in unterschiedllchen GRV-Szenarien

\begin{tabular}{|c|cc|}
\hline & \multicolumn{2}{|c|}{$\begin{array}{c}\text { Durchschnittliches } \\
\text { Rentenalter }\end{array}$} \\
& $\begin{array}{c}\text { Basis: } \\
\text { Durchschnittsalter des } \\
\text { West-SOEP }\end{array}$ & $\begin{array}{c}\text { Gewichteter VDR- } \\
\text { Durchschnitt }\end{array}$ \\
\hline GRV-Aufschlag (vor 1992) & 58,7 & 60,2 \\
Rentenreform 92 * & 59,0 & 60,6 \\
Nichtverzerrende Systeme & & 61,0 \\
Diskontierungsfaktor: 5\% & 59,5 & 61,3 \\
Diskontierungsfaktor: 7\% & 59,7 & 62,0 \\
Diskontierungsfaktor: 13,8 \% & 60,7 & \\
\hline
\end{tabular}

Quelle: Ermittlung aus den Koeffizienten der Gompertz-Regressionsanalyse durch unterschiedliche Berechnung des Optionswertes auf Basis der modellierten GRV-Systeme. Die angegebenen Basisalter ("GRV-Auischlag") sind die (gewichteten) Durchschnittsalter des Untersuchungszeitraumes 1984-1991.

Die rechte Spalte der Tabelle 8-2 beinhaltet die simulierten Veränderungen auf der Basis des heutigen GRV-Mittelwertes. Der Unterschied zwischen den im SOEP beobachteten Austrittsaltern und den Renteneintrittsaltern der GRV wurde in Kapitel 7.2

${ }^{6}$ Vergleiche Abbildung 2-6 bzw. Tabelle 7-4. 
diskutiert (vgl. Tabelle 7-3 und 7-4). Hauptgrund für die Differenz der beiden Werte ist die unterschiedliche Messung des Ruhestandsalters von Frauen. Während die Durchschnitte der Männer weitgehend übereinstimmen, liegt der Mittelwert der GRVZugänge von Frauen 3,5 Jahre über den im SOEP gemessenen Arbeitsmarktaustritten.

Die Ermittlung der Eintrittsalter in der rechten Spalte erfolgt durch eine Kalibrierung des Simulationsmodelles im Basisfall auf die GRV-Größe von 60,2 \%. Durch die Rentenreform 1992 wird, wenn diese voll in Kraft ist, das durchschnittliche Rentenalter lediglich um 0,4 Jahre erhöht. Eine nichtverzerrende Rentenformel bewirkt ein Ansteigen dieses mittleren Eintrittsalters um 1,8 Jahre.

Im letzten Schritt der Politiksimulation werden mit Hilfe des Bevölkerungsmodelles von BÖRSCH-SUPAN, MEIER UND RELL-HELD (1994) die Auswirkungen der ermittelten Veränderungen im durchschnittlichen Rentenzugangsalter auf die Finanzsituation der GRV quantifiziert. Tabelle 8-3 beinhaltet für drei Szenarien die Alterslastquotienten und die sich ergebenden Beitragssätze zur GRV.7

Während der Altersquotient im Status-Quo Fall in den Jahren ab 2030 teilweise über $100 \%$ steigt und damit anzeigt, daß ein aktiver Arbeitnehmer mehr als einen Pensionär „versorgen“ muß, liegt der entsprechende Wert für das nichtverzerrende System erheblich darunter. Dies bedeutet für das Jahr 2025 eine maximale Entlastung der Beitragszahler um 13,7 Prozent (4,2 Prozentpunkte).

Die Differenzen der Lastquotienten und der Beitragssätze zwischen dem derzeitigen System und einer nichtverzerrenden Rentenformel können aus Tabelle 8-4 abgelesen werden. Es zeigt sich, daß bereits ab dem Jahr 2010 eine Entlastung von über 2 Prozentpunkten entsprechend mehr als 10 Prozent realisiert wird.

7 Die Beitragssätze zur GRV werden auf Basis der im Kapitel 2.2 diskutierten Budgetgleichung und der Annahme eines konstanten Rentenniveaus errechnet. Dort wurden als grundlegende Möglichkeiten einer staatlichen Regulierung des GRV-Systems genannt:

- Beitragssatz,

- Rentenniveau,

- Rentenalter,

so $\mathrm{da}$ sich für festes Rentenniveau und vorgegebenes Rentenalter definitorisch ein Beitragssatz ergibt, der die Einhaltung der Budgetidentität sicherstellt. 
Tabelle 8-3: Implikationen der modellierten GRV-Systeme auf den Alterskoeffizienten und den GRV-Beitragssatz

\begin{tabular}{|c|c|c|c|c|c|c|}
\hline \multirow[b]{2}{*}{ Jahr } & \multicolumn{2}{|c|}{$\begin{array}{l}\text { GRV-Aufschlag } \\
\text { (vor 1992) }\end{array}$} & \multicolumn{2}{|c|}{ Rentenreform 92} & \multicolumn{2}{|c|}{$\begin{array}{c}\text { Nichtverzerrendes } \\
\text { System } \\
\text { Diskontierungsfaktor: } 13,8 \%\end{array}$} \\
\hline & $\begin{array}{l}\text { Alters- } \\
\text { quotient }\end{array}$ & $\begin{array}{c}\text { resultierender } \\
\text { Beitragssatz }\end{array}$ & $\begin{array}{l}\text { Alters- } \\
\text { quotient }\end{array}$ & $\begin{array}{c}\text { resultierender } \\
\text { Beitragssatz }\end{array}$ & $\begin{array}{l}\text { Alters- } \\
\text { quotient }\end{array}$ & $\begin{array}{l}\text { resultierender } \\
\text { Beitragssatz }\end{array}$ \\
\hline 1990 & 0,55 & $18,7 \%$ & 0,55 & $18,7 \%$ & 0,55 & $18,7 \%$ \\
\hline 1995 & 0,57 & $19,5 \%$ & 0,57 & $19,4 \%$ & 0,55 & $18,9 \%$ \\
\hline 2000 & 0,62 & $21,2 \%$ & 0,61 & $20,9 \%$ & 0,58 & $19,9 \%$ \\
\hline 2005 & 0,67 & $22,9 \%$ & 0,66 & $22,5 \%$ & 0,62 & $21,0 \%$ \\
\hline 2010 & 0,71 & $24,3 \%$ & 0,69 & $23,7 \%$ & 0,63 & $21,6 \%$ \\
\hline 2015 & 0,76 & $25,9 \%$ & 0,74 & $25,2 \%$ & 0,67 & $22,9 \%$ \\
\hline 2020 & 0,82 & $27,9 \%$ & 0,79 & $27,0 \%$ & 0,71 & $24,3 \%$ \\
\hline 2025 & 0,90 & $30,7 \%$ & 0,87 & $29,7 \%$ & 0,78 & $26,5 \%$ \\
\hline 2030 & 0,98 & $33,5 \%$ & 0,95 & $32,4 \%$ & 0,86 & $29,2 \%$ \\
\hline 2035 & 1,02 & $34,5 \%$ & 0,99 & $33,7 \%$ & 0,90 & $30,8 \%$ \\
\hline 2040 & 1,01 & $34,2 \%$ & 0,98 & $33,4 \%$ & 0,90 & $30,7 \%$ \\
\hline 2045 & 0,99 & $33,7 \%$ & 0,96 & $32,8 \%$ & 0,88 & $30,0 \%$ \\
\hline 2050 & 0,98 & $33,3 \%$ & 0,95 & $32,4 \%$ & 0,86 & $29,4 \%$ \\
\hline
\end{tabular}

Quelle: Benutzung der in Tabelle 8-2 angegebenen durchschnittlichen Rentenalter in Bevőlkerungsprognosemodell

Tabelle 8-4: Entlastung der GRV durch die Einfuhrung einer unverzerrenden Rentenformel

\begin{tabular}{|c|c|c|c|}
\hline \multirow[t]{2}{*}{ Jahr } & \multicolumn{3}{|c|}{$\begin{array}{l}\text { Differenz zwischen den Werten für } \\
\text { GRV Status-Quo versus unverzerrendes System }\end{array}$} \\
\hline & $\begin{array}{l}\text { Alters- } \\
\text { quotient }\end{array}$ & $\begin{array}{l}\text { resultierender } \\
\text { Beitragssatz }\end{array}$ & resultierender Beitragssatz \\
\hline 1990 & - & - & - \\
\hline 1995 & 2 & 0,6 & $3,1 \%$ \\
\hline 2000 & 4 & 1,3 & $6,1 \%$ \\
\hline 2005 & 5 & 1,9 & $8,3 \%$ \\
\hline 2010 & 8 & 2,7 & $11,1 \%$ \\
\hline 2015 & 9 & 3,0 & $11,6 \%$ \\
\hline 2020 & 11 & 3,6 & $12,9 \%$ \\
\hline 2025 & 12 & 4,2 & $13,7 \%$ \\
\hline 2030 & 12 & 4,3 & $12,8 \%$ \\
\hline 2035 & 12 & 3,7 & $10,7 \%$ \\
\hline 2040 & 11 & 3,5 & $10,2 \%$ \\
\hline 2045 & 11 & 3,7 & $11,0 \%$ \\
\hline 2050 & 12 & 3,9 & $11,7 \%$ \\
\hline
\end{tabular}

Quelle: Differenzen der 1. und 3. Hauptspalte aus Tabelle 8-3. 
Der Vergleich der bereits geltenden Rentenreform 1992 mit dem versicherungsmathematisch fairen System zeigt, daß ersteres zwar eine leichte Entlastung gegenüber dem vorher geltenden Recht darstellt, ${ }^{8}$ die Entlastungswirkungen eines anreizneutralen Systems jedoch nicht annähernd erreicht. Dies ist auch in Abbildung 8-2 erkennbar. Die aktuelle Rentenreform ist somit ein Schritt in die richtige Richtung, der allerdings aus theoretischer wie empirischer Sicht nicht annähernd weit genug geht.

Somit ist aus ökonomischer Sicht ein klares Votum für eine neuerliche Reformierung des Rentensystems durch eine versicherungsmathematisch neutrale Adjustierung der individuellen Rentenhöhe in Abhängigkeit vom Rentenzugangsalter abzugeben.

\section{Tragbarkeit der Sozialversicherung}

Eine weitere Grundfrage der vorliegenden Arbeit war die nach der Tragbarkeit des derzeitigen Rentenversicherungssystems als Umlageverfahren unter Berücksichtigung der demographischen Entwicklung in den kommenden Jahrzehnten.

In diesem Zusammenhang müssen die Ergebnisse der Tabelle 8-3 so betrachtet werden, daß zwar die Steigerung der Rentenversicherungsbeiträge prozentual deutlich gesenkt werden können, wenn die Rentenberechnung geändert wird, die Belastung durch die Sozialversicherung jedoch nichtsdestoweniger in der Zukunft erheblich anwächst. Dies gilt sowohl aus Sicht des einzelnen Arbeitnehmers, der die Abgabenlast als sinkendes Nettoeinkommen spürt, als auch für die Unternehmen, die steigende Lohnnebenkosten verzeichnen werden. Die Steigerung des Beitragssatzes von 18,7 Prozent im Jahre 1990 auf 30,8 Prozent 2035 im Fall eines anreizneutralen Rentenversicherungssystems bedeutet ein Ansteigen dieses Beitragssatzes um knapp 65 Prozent. Für die Beiträge zur Gesetzlichen Krankenversicherung (GKV) muß ebenfalls eine Steigerung angenommen werden, diese dürfte allerdings nicht so hoch ausfallen wie die der GRV. Zwar werden sich die Ausgaben der Krankenversicherung ebenfalls erhöhen, da ältere Menschen höhere Krankheitskosten verursachen als junge Menschen', aber der Ein-

\footnotetext{
${ }^{8}$ Die errechnete Entlastungswirkung ist leicht überschätzt, da die Regelungen der Rentenreform nicht mit deren Inkrafttreten zum 1.1.92, sondern erst im Jahre 2013. Eine Modellierung der sich bis dahin jährlich ändernden Anpassungsregelungen konnte im Rahmen des vorliegenden Modelles jedoch nicht umgesetzt werden.

${ }^{9}$ Vgl. etwa FrERICH (1987), Seite 438 f. Zwischen 1970 und 1985 haben sich die prozentualen Mehrausgaben pro versichertem Rentenempfänger von $9,2 \%$ auf über $58 \%$ erhöht. Die Leistungen der 
nahmeausfall liegt deutlich unter dem der Rentenversicherung, da auch Rentner einen Beitrag zur Krankenversicherung leisten. Auch für den Beitrag zur Pflegeversicherung ist eine Steigerung zu erwarten. Schließlich bleibt auch die steuerliche Belastung nicht unberührt durch die gesellschaftliche Alterung, da vor allem im Falle der GRV ein erheblicher Anteil der laufenden Zahlungen als Bundeszuschuß aus dem öffentlichen Haushalt geleistet werden. 10

BöRSCH-SUPAN, MEIER UND REIL-HELD (1994) beziffern den Anstieg der Krankenversicherungsbeiträge bis 2035 von derzeit auf 12 auf ca. 18 Prozent, der Beitrag zur Pflegeversicherung wird dort für 2035 mit ca. 4 Prozent beziffert." Der Beitrag zur Arbeitslosenversicherung sei auf dem derzeitigen Niveau von 6,8\% fixiert angenommen. Zusammengenommen ergibt sich damit ein Sozialversicherungsbeitrag von 59,6 Prozent, wovon die Hälfte als Arbeitnehmerbeitrag mit knapp $30 \%$ zu Buche schlägt. Die vergleichbare Abgabenlast liegt heute bei 38,612 bzw. 19,3 Prozent (Arbeitnehmeranteil), so daß die prognostizierte Steigerung bis 2035 über $50 \%$ liegt. In Verbindung mit einem durchschnittlichen Steuersatz ${ }^{13}$ von ebenfalls $25 \%$ ergibt sich eine Durchschnittsbelastung für einen Arbeitnehmer von 55 Prozent seines Bruttoeinkommens.

Die Frage, wieweit diese Belastung noch tragbar ist oder nicht, kann rein aus einer empirischen Analyse heraus nicht definitiv beantwortet werden. Vielmehr gilt es an dieser Stelle, die Reaktionen der betroffenen Arbeitnehmer und Unternehmen zu antizipieren, die in Abgabenvermeidungsstrategien in Form von Schwarzarbeit oder anderer Senkung des Arbeitsangebotes liegen können bzw. einer Reaktion der Arbeitsnachfrage durch Substitution von Arbeit durch Kapital. Es ist eine Frage an die Sozialpolitik, wie weit es gelingt, ein gesellschaftliches Verständnis für die demographische

Krankenkassen an Rentner werden nur zu deutlich unter $50 \%$ durch die Beiträge der Rentner gedeckt.

${ }^{10}$ Der Bundeszuschuß liegt zur Zeit bei ca. $20 \%$ der Ausgaben der GRV. 1990 betrug er 45 von 235 Milliarden DM und lag damit bei 19,2\% der GRV-Ausgaben. Gleichzeitig machte er ca. $4,3 \%$ der gesamten Staatsausgaben aus (Quelle: Statistisches Jahrbuch 1992, eigene Berechnung).

11 BORSCH-SUPAN, MEIER UND REIL-HELD (1994), Seite $24 \mathrm{f}$ und Abbildungen 4-10 und 4-12. Die Autoren berechnen sehr unterschiedliche Szenarien in Abhängigkeit von Mobilitäts- und Fertilitätsveränderungen. Es wird hier das einfache Szenario, das auf der Bevölkerungsprognose des Statistischen Bundesamtes beruht, zitiert.

12 Rentenversicherung 19,3, Krankenversicherung im Durchschnitt 12,5, und Arbeitslosenversicherung 6,8 Prozent.

${ }^{13}$ Berechnung aus der Einkommensteuerstatistik 1986, nach Statistischem Jahrbuch 1992, S. 544 ff. 
Problematik zu erzeugen und auf dieser Basis einen Konsens aller Bevölkerungsgruppen zu erwirken.

Der erste Schritt hierzu muß aus ökonomischer Sicht die Umgestaltung des GRVSystems in Richtung einer anreizneutralen Rentenberechnung sein, die in diesem Kapitel beschrieben wurde. Allerdings kann dies nicht die einzige Maßnahme sein, sondern auch die hier konstant gehaltene dritte Einflußmöglichkeit einer staatlichen Regulierung, das Rentenniveau, wird in den Reformprozeß einbezogen werden müssen. Eine intergenerationale Umverteilung ist durch den Alterungsprozeß der Gesellschaft vorgezeichnet und muß durch den Gesetzgeber aktiv gesteuert werden, damit nicht einzelne Altersgruppen größere Lasten tragen als andere.

An dieser Stelle stellt sich die Frage nach der politischen Durchsetzbarkeit der diskutierten Maßnahmen. Der mehrfach diskutierte steigende Alterslastquotient spiegelt auch die Verschiebung politischer Macht auf die ältere Generation wider. Bereits um das Jahr 2020 herum wird die Mehrheit der Wahlberechtigten über 50 Jahre alt und damit entweder Rentner sein oder die Aussicht einer Verrentung in den nächsten 10 Jahren haben. Auf Basis dieser politischen Mehrheitsverhältnisse wird eine ökonomisch sinnvolle und gesamtgesellschaftlich notwendige Umgestaltung der Rentenberechnung immer unwahrscheinlicher. Der ökonomische Schaden aus überproportional steigender Abgabenlast und daraus resultierenden Vermeidungsstrategien ist nicht abschätzbar. 
Ach Gott,

Die Kunst ist lang !

Und kurz ist unser Leben ...

Eh man den halben Weg erreicht, Muß wohl ein armer Teufel sterben.

\section{Abschliebende Betrachtung Und Ausblick}

Die Altersstrukturverschiebung ist in den westlichen Industrienationen durch den Prozeß der doppelten Alterung gekennzeichnet. Einer steigenden Lebenserwartung steht eine geringe Geburtenhäufigkeit gegenüber, so daB sich die ehemalige Alterspyramide inzwischen zu einem Pilz entwickelt hat. Das Verhältnis von Rentenempfängern zu Erwerbspersonen, welche die Renteneinkommen der Ruhestandsgeneration erwirtschaften müssen, ist in der Bundesrepublik Deutschland besonders kraß, in $40 \mathrm{Jahren}$ wird es - als Status-Quo Simulation - mehr Pensionäre als Erwerbstätige geben. Hauptgrund für diese Entwicklung ist neben der demographischen Entwicklung vor allem die starke Tendenz zur Frühverrentung. Auf dieser Basis wurde in der Literatur vermehrt die Frage aufgeworfen, ob das nach dem Umlageverfahren finanzierte deutsche Rentenversicherungssystem überlebensfähig ist.

Die vorliegende Arbeit setzt an dieser Stelle an und stellt die Frage, ob der Gesetzgeber in der Lage ist, innerhalb des bestehenden Systems der GRV durch Änderungen der institutionellen Anreizmechanismen das Zugangsverhalten älterer Arbeitnehmer so deutlich zu verändern, daß die Rentenversicherung spürbar entlastet wird. Diese Frage wird mit einem klaren Ja beantwortet.

Dieses Ergebnis wird erreicht durch eine Erhöhung des Renteneintrittsalters der Arbeitnehmer und damit einer Verschiebung des Verhältnisses von Rentnern zu Erwerbstätigen (Alterslastquotient). Die Steigerung der Rentenzugangsalter kann vom Gesetzgeber durch eine versicherungsmathematisch neutrale Berechnung der persönlichen Rentenhöhe motiviert werden, ohne daB das Recht der persönlichen Wahl des Ruhestandszeitpunktes beschnitten werden muß.

Die Arbeit versteht sich insofern als Plädoyer für eine anreizkompatible Rentenpolitik, die - innerhalb des gegebenen Umlageverfahrens - die Entscheidung jedes einzelnen Arbeitnehmers nicht mehr durch falsche Anreize in Richtung eines möglichst frühen Ruhestandes verzerrt, sondern diesem eine Wahl des individuellen Rentenalters auf Basis seiner persönlichen Präferenzen ermöglicht. 
Es wurde gezeigt, daß das derzeitige System der GRV keine versicherungsmathematische Neutralität aufweist. Diese implizierte, daß aus der Sicht eines Arbeitnehmers für jedes mögliche Rentenalter der Gegenwartswert aller zukünftigen Renteneinkünfte minus den zu leistenden Versicherungsbeiträgen gleich ist. Sie kann durch entsprechende Abschläge von Frührenten erreicht werden. Solche Anpassungsfaktoren existierten in der GRV bis 1991 nicht und wurden durch die Rentenreform 1992 als „Zugangsfaktor“ eingeführt. Die Analyse des dritten Kapitels zeigt jedoch, daß diese Zugangsfaktoren nicht dem Kriterium der versicherungsmathematischen Fairneß genügen, sondern die GRV nach wie vor deutliche finanzielle Anreize für eine frühestmögliche Verrentung setzt. So lag bei einem Ruhestandsalter von 60 Jahren die Rente langjährig versicherter Arbeitnehmer vor 1992 um 48 Prozent „zu hoch“,14 seit der Rentenreform immer noch um 40 Prozent.

Der frühestmögliche Austritt aus dem aktiven Erwerbsleben wird lediglich durch die in der GRV festgeschriebenen Mindestzugangsalter beschränkt. Die makroökonomische Analyse des vierten Kapitels zeigt auf, daß jeder Lockerung dieser Altersbeschränkungen sofort eine deutliche Reaktion nachfolgt, so sinkt das durchschnittliche Rentenalter nach der Rentenreform 1972 von knapp 62 auf 58,4 Jahre. Auch die diskutierten Gesetzesänderungen von 1979/80 (Senkung des vorgezogenen Ruhestandes für Schwerbehinderte, Berufs- und Erwerbsunfähige) und des Haushaltsbegleitgesetzes 1984 zeigen deutliche Auswirkungen auf die altersspezifischen Zugangswahrscheinlichkeiten in die Gesetzliche Rentenversicherung. Die beobachteten Resultate unterstützen die Hypothese, daß Arbeitnehmer einen frühestmöglichen Ruhestand wählen.

Das Resultat der makroökonomischen Analyse ist die qualitative Feststellung, daß eindeutige Verhaltensänderungen auf gesetzliche Maßnahmen folgen.

Aufbauend darauf wird durch eine mikroökonometrische Verweildaueranalyse die einzelwirtschaftliche Wahl des Ruhestandsalters untersucht und die Determinanten dieser Entscheidung quantifiziert. Auch diese Analyse bestätigt den starken Einfluß der gesetzlichen Rentenversicherung. Dieser wird quantifiziert durch den Optionswert, der den nutzenbewerteten finanziellen Anreiz einer Verlängerung des Erwerbslebens abbildet. Die Relevanz der durch den Optionswert operationalisierten Rentenberechnungsmodalitäten ist deutlich signifikant, obwohl in der Schätzung sowohl persönliche Charakteristiken, Ausbildung und Vermögenssituation als auch Indikatoren der Beruflichen Stellung und der Branche berücksichtigt werden. Dieses Resultat erweitert

${ }^{14}$ Im Vergleich zu einem anreizneutralen (versicherungsmathematisch fairen) System. 
damit die Analyse von STOCK UND WISE (1990 a), die den Optionswert entwickelten, in ihrem Modell aber keine Kovariate implementieren können. Weitere wichtige Einflußgrößen sind Arbeitslosigkeit und gesundheitliche Probleme, die verkürzend auf die Lebensarbeitszeit wirken, wogegen selbständige Personen deutlich länger arbeiten.

Der deutliche Einfluß des Optionswertes und damit des Rentenberechnungsverfahrens hat auch in den neuen Bundesländern Gültigkeit, ein Resultat, das auf Basis der beobachteten Übergangsperiode 1990 bis 1992 des gesamten ostdeutschen Gesellschaftssytemes nicht unbedingt zu erwarten war. Zwar weisen die älteren Arbeitnehmer in der früheren DDR ein geringeres Durchschnittsrentenalter auf, nach einer statistischen Kontrolle desselben in Form höherer Basisübergangsraten zeigen die Einflüsse der Kovariate, insbesondere des Optionswertes, in beiden Landesteilen große Übereinstimmung. Somit hat die anschließend durchgeführte Politiksimulation für ganz Deutschland Gültigkeit.

Im letzten Schritt wird aus den so ermittelten Regressionskoeffizienten das zukünftige Verhalten älterer Arbeitnehmer durch Simulationsrechnungen prognostiziert, in denen die Auswirkungen möglicher Rentenreformen ermittelt werden. Diese Reformmaßnahmen umfassen neben der bereits in Kraft befindlichen Rentenreform 92 alternative nicht-verzerrende Systeme auf Basis versicherungsmathematischer FairneB.

Das durchschnittliche Rentenalter kann durch die Einführung einer anreizneutralen Rentenberechung um 2 Jahre erhöht werden, wogegen die Rentenreform 1992, auch wenn sie im Jahre 2013 ihre volle Wirkung erreicht hat, lediglich eine durchschnittliche Verlängerung der Lebensarbeitszeit um 4 Monate bewirkt. Es bestätigt sich somit das Ergebnis der theoretischen Betrachtung versicherungsmathematischer Gesichtspunkte, daß die Rentenreform 1992 zwar ein „Schritt in die richtige Richtung“ ist, aber für einen wirklichen Beitrag zur Lösung des Problems erheblich zu kurz gegriffen wurde.

Die Simulationsrechnungen zeigen, daß der für 2035 prognostizierte Alterslastquotient durch das steigende Rentenalter von 1,02 im Status-Quo Fall auf 0,90 für das anreizneutrale System gesenkt werden kann. Der aus diesem Verhältnis resultierende Beitragssatz zur GRV beträgt 30,8 im Vergleich zu 34,5 Prozent, was eine Senkung um 10,7 Prozent entspricht. Die Auswirkungen der Rentenreform liegen mit einem Altersquotienten von 0,99 und einem resultierenden Beitragssatz von 33,7\% erneut näher am Status-Quo Szenario als an dem in dieser Arbeit vorgeschlagenen anreizneutralen. 
Das Ergebnis der Politikanalyse ist, daß durch eine adäquate Umgestaltung des GRVSystems ein spürbarer Teil der durch die gesellschaftliche Alterung induzierten Probleme abgemildert werden kann. Diese Chance sollte durch die Politik ergriffen werden.

Die Formulierung und Umsetzung einer Rentenreform unter den in dieser Arbeit vorgeschlagenen Gesichtspunkten der Anreizneutralität sollte von den politischen Gremien in naher Zukunft eingeleitet werden, da die demographische Strukturverschiebung auch die intergenerationale Verteilung der politischen Mehrheiten verschiebt. Je weiter der Alterungsprozeß fortschreitet, desto größer wird der Anteil älterer Menschen, zu denen an dieser Stelle auch Arbeitnehmer in der Nähe des Rentenalters gezählt werden müssen, und desto schwieriger wird die politische Durchsetzung einer Umverteilungspolitik, die die Rentnergeneration im Vergleich zum Status-Quo belastet, um die Gruppe der Erwerbstätigen zu entlasten.

Im Ausblick zeigt sich allerdings, daß eine Umgestaltung der Rentenformel allein nicht ausreicht, die aus der Altersstrukturverschiebung resultierende Belastung des Rentenversicherungssystems zu neutralisieren. Die o.a. notwendige Erhöhung des GRVBeitragssatzes der Erwerbstätigen auf $30,8 \%$ im günstigsten Falle bedeutet gegenüber den 19,2\% des Jahres 1994 eine erhebliche Steigerung. Zusammen mit den weiteren Abgaben (Kranken- und Pflegeversicherung, Arbeitslosenversicherung) und der Einkommensteuer ergeben sich durchschnittliche Belastungen der Arbeitnehmer von mehr als 50 Prozent des Bruttoeinkommens. Dies gilt für eine geänderte Rentenformel bei ansonsten gleichen Rahmenbedingungen. Eine aktive politische Gestaltung dieser intergenerationalen Umverteilung ist notwendig. Eine solche Umverteilung von der aktiven Generation hin zur Gruppe der Pensionäre ergibt sich direkt aus der Altersstruktur und fände auch ohne politischen Eingriff statt. Aufgabe der Sozialpolitik ist die umfassende Information der beteiligten Gruppen und die gesellschaftlich und ökonomisch sinnvolle Gestaltung des Diskussions- und Reformprozesses.

Innerhalb dieser Debatte wird als weiteres direktes Mittel der Rentenpolitik über die oben beschriebenen Beitragssätze und das Rentenalter hinaus das - gesellschaftlich erwünschte und finanzierbare - Rentenniveau zu diskutieren sein.

Die politische Analyse der Sozialversicherung darf nicht bei der Ausgestaltung des Rentenversicherungssystems selbst stehenbleiben. Der nächste Schritt muß die einzelstaatliche Sichtweise verlassen und die gemeinsame Entwicklung der Länder Ost- und 
Westeuropas unter der Berücksichtigung der Mobilität von Kapital und Arbeitskräften einbeziehen. Die Arbeiten von FELDERER (1992) und BöRSCH-SUPAN (1994) setzen sich mit der Auswirkung von Migration, letzterer zusätzlich auch internationaler Kapitalmobilität auseinander. In diesem Zusammenhang kann sich die Kooperation von Staaten mit unterschiedlicher Altersstruktur und Kapitalausstattung positiv für alle Beteiligten auswirken. Das naheliegendste Beispiel hierfür ist die europäische Integration, sowohl innerhalb der EU als auch im Zuge der Umgestaltung der osteuropäischen Gesellschaften. 
Peter Schmidt - 978-3-631-75568-6

Downloaded from PubFactory at 01/11/2019 03:28:55AM

via free access 
Grau, teurer Freund, ist alle Theorie und Grün des Lebens goldner Baum.

\section{Anhang}

\section{Inhalt:}

A.1 Regressionsanalysen der Rentenzugänge 1970-1991 ....................................214

A.2 Literatur zur makroökonomischen Entwicklung der GRV................................220

A.3. OLS-Schätzung des normalisierten Einkommens aus dem SOEP für Westdeutschland 1988-1990.

A.4 Regressionsergebnisse der Übergangsratenmodelle für West- und Ostdeutschland - Weibull-Spezifikationen.

\section{Anhangtabellen:}

Tabelle A-1: Übersicht über die verwendeten Variablennamen und -kürzel 214

Tabelle A-2: OLS-Schätzung der Zugangsraten zur GRV, Spezifikationen 1 bis 3 215

Tabelle A-3: OLS-Schätzung der Zugangsraten zur GRV, Spezifikationen 4 und 5 216

Tabelle A-4: OLS-Schätzung der Zugangsraten zur GRV, Spezifikationen 6 bis 8 218

Tabelle A-5: Tabellarische Literaturübersicht.

Tabelle A-6: OLS-Schätzung des Normalisierten Einkommens aus dem SOEP für Westdeutschland 1988-1990

Tabelle A-7: Verweildaueranalyse für West-Deutschland - Spezifikationen des Weibull-Modelles

Tabelle A-8: Verweildaueranalyse für Ost-Deutschland - Spezifikationen des WeibullModelles 


\section{Anhang}

\section{A.1 Regressionsanalysen der Rentenzugänge 1970-1991}

\section{Tabelle A-1: Übersicht über dle verwendeten Variablennamen und -kürzel}

\begin{tabular}{|c|c|}
\hline Symbol & Bedeutung \\
\hline An & 21 Altersvariablen; $n=45, \ldots, 65$ \\
\hline Jn & 22 Jahresvariable; $n=70, \ldots, 91$ \\
\hline Kn & 42 resultierende Kohortenvariablen (Geburtsjahrgänge), $n=5, \ldots, 46$ \\
\hline \multicolumn{2}{|c|}{ Für die Ermittlung der Vektoren gilt: $\mathrm{K}=\mathrm{J}-\mathrm{A}$} \\
\hline AB_Ind & Indikatorvariable Arbeiter \\
\hline BSP & Entwicklung des Bruttosozialproduktes (erste Differenzen) \\
\hline Anpass & jährliche Anpassung (Erhöhung) der GRV-Renten \\
\hline $\mathrm{Alq}_{\mathrm{nn}} \mathrm{mm}$ & Arbeitslosenquote der Altersgruppe von $\mathrm{nn}$ bis mm Jahre \\
\hline G... & $\begin{array}{l}\text { GRV-Indikator: } \\
\text { die G-Variablen sind zusammengesetzte Interaktionstherme: }\end{array}$ \\
\hline \multicolumn{2}{|c|}{ Interaktionsterme } \\
\hline $\mathbf{X}_{\mathbf{n}}$ & $\begin{array}{l}\text { Ausprägung der Variable X }\{\mathrm{A}, \mathrm{J}, \mathrm{K}\} \text { für Index n } \\
\text { Bsp: J73: Jahr } 1973\end{array}$ \\
\hline $\mathrm{X}_{\mathrm{np}}$ & $\begin{array}{l}\text { Ausprägung der Variable X }\{A, J, K\} \text { für Index n und später } \\
\text { Bsp: J73p: Alle Jahre ab 1973; A60p: Alter } 60 \text { und älter }\end{array}$ \\
\hline$u_{n}$ & Ausprägung der Variable $X\{A, J, K\}$ für alle Indizes vor $n$ \\
\hline & Bsp: Ju73: Alle Jahre vor 1973 \\
\hline \multirow[t]{5}{*}{$\mathrm{GJ}_{\mathrm{nn} \_} \mathrm{u}_{\mathrm{mm}}$} & GRV-Dummyvariable, Interaktion durch Multiplikation der Vektoren: \\
\hline & $\mathrm{J}_{\mathrm{nnP}}$ und $\mathrm{Au} \mathrm{u}_{\mathrm{mm}}$ \\
\hline & Bsp: GJ73_u62 $\rightarrow$ Ausprägungen unter Alter 62 ab 1972 \\
\hline & GJ79_63p $\rightarrow$ Ausprägungen ab Alter 63 ab 1979 \\
\hline & 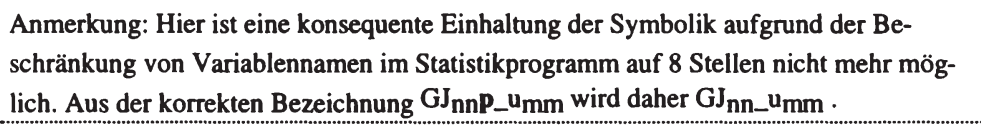 \\
\hline t-Wert & $\begin{array}{l}\text { t-Statistik des Regressionskoeffizienten auf Basis heteroskedastiekonsistenter Standard- } \\
\text { fehler (Korrektur nach White) } \\
* *=\text { signifikant auf dem } 95 \% \text { Niveau } \\
*=\text { signifikant auf dem } 90 \% \text { Niveau }\end{array}$ \\
\hline Beob. & $\begin{array}{l}\text { Anzahl der Beobachtung in der Regression, zusammengesetzt durch die Interaktion von } \\
21 \text { Alters-, und } 22 \text { Jahresdummies für zwei Gruppen (Arbeiter und Angestellte) }\end{array}$ \\
\hline
\end{tabular}


Tabelle A-2: OLS-Schätzung der Zugangsraten zur GRV, Spezifikationen 1 bis 3

\begin{tabular}{|c|c|c|c|c|c|c|c|c|c|c|c|}
\hline \multirow[b]{2}{*}{ Variable } & \multicolumn{2}{|c|}{$\begin{array}{l}\text { Spezif. 1: Nur } \\
\text { Altersvariablen }\end{array}$} & \multirow[b]{2}{*}{ Sign. } & \multicolumn{4}{|c|}{$\begin{array}{c}\text { Spez. 2: } \\
\text { Kohortenvariablen }\end{array}$} & \multicolumn{4}{|c|}{$\begin{array}{c}\text { Spezif. 3: } \\
\text { Nur Jahresvariablen }\end{array}$} \\
\hline & Koeffizient & t-Wert & & Variable & Koeffizient & $t$-Wert & Sign. & Variable & Koeffizient & $t$-Wert S & ign. \\
\hline AB_Ind & 3,579 & 8,02 & ** & AB_Ind & 3,761 & 3,16 & $* *$ & AB_Ind & 3,579 & 2,89 & ** \\
\hline A45 & $-1,578$ & $-4,73$ & ** & K6 & 22,025 & 2,04 & $* *$ & $\mathrm{~J} 70$ & 1,604 & 1,02 & \\
\hline A46 & $-1,552$ & $-4,68$ & $* *$ & K7 & 29,930 & 1,73 & $*$ & J71 & 1,561 & 1,00 & \\
\hline A47 & $-1,517$ & $-4,61$ & $* *$ & K8 & 19,747 & 1,65 & $*$ & J72 & 3,771 & 1,27 & \\
\hline A48 & $-1,476$ & $-4,52$ & $* *$ & K9 & 20,155 & 2,20 & $* *$ & J73 & 4,511 & 1,67 & * \\
\hline A49 & $-1,427$ & $-4,41$ & $* *$ & K10 & 16,567 & 2,40 & ** & J74 & 5,168 & 1,91 & $*$ \\
\hline A50 & $-1,358$ & $-4,26$ & ** & K11 & 16,181 & 2,54 & ** & $\mathrm{J} 75$ & 5,180 & 2,03 & ** \\
\hline A51 & $-1,294$ & $-4,10$ & ** & K12 & 14,310 & 2,62 & ** & $\mathrm{J} 76$ & 5,905 & 2,20 & ** \\
\hline A52 & $-1,203$ & $-3,87$ & ** & K13 & 13,218 & 2,62 & ** & J77 & 5,513 & 2,20 & ** \\
\hline A53 & $-1,092$ & $-3,59$ & $* *$ & K14 & 11,687 & 2,61 & ** & J78 & 5,411 & 2,26 & ** \\
\hline A54 & $-0,945$ & $-3,17$ & ** & K15 & 10,111 & 2,57 & $* *$ & J79 & 5,987 & 2,49 & ** \\
\hline A55 & $-0,712$ & $-2,48$ & ** & K16 & 11,475 & 2,58 & $* *$ & $\mathrm{~J} 80$ & 6,118 & 2,60 & ** \\
\hline A56 & $-0,478$ & $-1,72$ & $*$ & K17 & 10,440 & 2,52 & ** & J81 & 6,522 & 2,40 & ** \\
\hline A57 & $-0,166$ & $-0,62$ & & K18 & 9,936 & 2,64 & $* *$ & J82 & 7,179 & 2,46 & ** \\
\hline A58 & 0,223 & 0,87 & & K19 & 12,317 & 2,63 & $* *$ & J83 & 6,968 & 2,53 & $* *$ \\
\hline A59 & 0,621 & 2,46 & $* *$ & K20 & 9,240 & 2,41 & $* *$ & J84 & 7,644 & 2,30 & ** \\
\hline A60 & 10,259 & 9,08 & $* *$ & $\mathrm{~K} 21$ & 8,742 & 2,30 & $* *$ & J85 & 6,817 & 2,25 & ** \\
\hline A61 & 4,996 & 9,04 & ** & K22 & 9,170 & 2,21 & $* *$ & J86 & 6,807 & 2,14 & ** \\
\hline A62 & 4,808 & 10,73 & ** & K23 & 8,152 & 2,19 & ** & J87 & 7,304 & 2,03 & ** \\
\hline A63 & 34,271 & 14,38 & ** & K24 & 8,595 & 2,09 & ** & J88 & 7,756 & 2,18 & ** \\
\hline A64 & 8,563 & 9,57 & ** & K25 & 8,618 & 2,15 & ** & J89 & 8,653 & 2,13 & ** \\
\hline A65 & 76,613 & 21,19 & $* *$ & K26 & 7,449 & 2,15 & $* *$ & J90 & 8,278 & 2,07 & $* *$ \\
\hline & & & & K27 & 3,058 & 1,65 & $*$ & J91 & 6,874 & 2,03 & ** \\
\hline & & & & K28 & 2,720 & 1,53 & & & & & \\
\hline & & & & K29 & 0,492 & 0,50 & & & & & \\
\hline & & & & K30 & 0,351 & 0,35 & & & & & \\
\hline & & & & K31 & $-0,020$ & $-0,02$ & & & & & \\
\hline & & & & K32 & $-0,956$ & $-1,46$ & & & & & \\
\hline & & & & K33 & $-1,044$ & $-1,59$ & & & & & \\
\hline & & & & K34 & $-1,177$ & $-1,77$ & $*$ & & & & \\
\hline & & & & K35 & $-1,312$ & $-1,95$ & * & & & & \\
\hline & & & & K36 & $-1,375$ & $-2,01$ & ** & & & & \\
\hline & & & & K37 & $-1,434$ & $-2,06$ & $* *$ & & & & \\
\hline & & & & K38 & $-1,490$ & $-2,10$ & $* *$ & & & & \\
\hline & & & & K39 & $-1,524$ & $-2,10$ & $* *$ & & & & \\
\hline & & & & K40 & $-1,571$ & $-2,10$ & $* *$ & & & & \\
\hline & & & & K41 & $-1,569$ & $-2,04$ & $* *$ & & & & \\
\hline & & & & K42 & $-1,605$ & $-1,99$ & $* *$ & & & & \\
\hline & & & & K43 & $-1,618$ & $-1,90$ & $*$ & & & & \\
\hline & & & & K44 & $-1,659$ & $-1,78$ & * & & & & \\
\hline & & & & K45 & $-1,663$ & $-1,57$ & & & & & \\
\hline & & & & K46 & $-1,680$ & $-1,22$ & & & & & \\
\hline Beob. & 924 & & & Beob. & 924 & & & Beob. & 924 & & \\
\hline & $87,2 \%$ & & & & $11,2 \%$ & & & & $1,8 \%$ & & \\
\hline korr. $\mathrm{R}^{2}$ & $86,9 \%$ & & & korr. $\mathbf{R}^{2}$ & $7,1 \%$ & & & korr. $\mathrm{R}^{2}$ & $-0,6 \%$ & & \\
\hline DW & 1,881 & & & DW & 1,825 & & & DW & 1,891 & & \\
\hline
\end{tabular}


Tabelle A-3: OLS-Schätzung der Zugangsraten zur GRV, Spezifikationen 4 und 5

\begin{tabular}{|c|c|c|c|c|c|c|}
\hline \multirow[b]{2}{*}{ Variable } & \multicolumn{3}{|c|}{$\begin{array}{l}\text { Spezif. } 4 \\
\text { Alter, Kohorten, } \\
\text { GRV-Var. }\end{array}$} & \multicolumn{3}{|c|}{$\begin{array}{c}\text { Spezif. } 5 \\
\text { Alter, Kohorten, } \\
\text { GRV, Makro }\end{array}$} \\
\hline & Koeffizient & t-Wert & Sign & Koeffizient & t-Wert & Sign \\
\hline A45 & $-30,212$ & $-1,48$ & & $-27,9$ & $-1,36$ & \\
\hline A46 & $-30,119$ & $-1,48$ & & $-27,9$ & $-1,36$ & \\
\hline A47 & $-29,975$ & $-1,47$ & & $-27,8$ & $-1,36$ & \\
\hline A48 & $-29,833$ & $-1,47$ & & $-27,7$ & $-1,36$ & \\
\hline A49 & $-29,617$ & $-1,46$ & & $-27,6$ & $-1,35$ & \\
\hline A50 & $-29,375$ & $-1,45$ & & $-27,4$ & $-1,34$ & \\
\hline A51 & $-29,243$ & $-1,44$ & & $-27,4$ & $-1,34$ & \\
\hline A 52 & $-29,021$ & $-1,43$ & & $-27,2$ & $-1,34$ & \\
\hline A53 & $-28,790$ & $-1,42$ & & $-27,1$ & $-1,33$ & \\
\hline A54 & $-28,530$ & $-1,41$ & & $-26,9$ & $-1,32$ & \\
\hline A55 & $-28,072$ & $-1,38$ & & $-26,5$ & $-1,31$ & \\
\hline A56 & $-27,624$ & $-1,36$ & & $-26,2$ & $-1,29$ & \\
\hline A57 & $-27,089$ & $-1,33$ & & $-25,7$ & $-1,27$ & \\
\hline A58 & $-26,440$ & $-1,30$ & & $-25,2$ & $-1,24$ & \\
\hline A59 & $-25,759$ & $-1,27$ & & $-24,6$ & $-1,21$ & \\
\hline A60 & $-22,243$ & $-1,10$ & & $-21,2$ & $-1,05$ & \\
\hline A61 & $-22,377$ & $-1,12$ & & $-21,5$ & $-1,07$ & \\
\hline A62 & $-23,408$ & $-1,27$ & & $-23,0$ & $-1,25$ & \\
\hline A63 & $-26,197$ & $-1,42$ & & $-26,2$ & $-1,43$ & \\
\hline A64 & $-20,435$ & $-1,20$ & & $-20,9$ & $-1,23$ & \\
\hline A65 & 42,110 & 4,35 & $* *$ & 41,2 & 4,24 & $* *$ \\
\hline K6 & 11,278 & 0,76 & & 11,0 & 0,75 & \\
\hline K7 & 31,527 & 1,63 & & 30,7 & 1,60 & \\
\hline $\mathrm{K} 8$ & 27,035 & 1,52 & & 25,7 & 1,46 & \\
\hline $\mathrm{K} 9$ & 27,324 & 1,42 & & 25,6 & 1,34 & \\
\hline $\mathrm{K} 10$ & 21,470 & 1,07 & & 19,5 & 0,96 & \\
\hline K11 & 23,564 & 1,15 & & 21,3 & 1,03 & \\
\hline K12 & 24,201 & 1,19 & & 21,8 & 1,06 & \\
\hline K13 & 25,131 & 1,23 & & 22,7 & 1,10 & \\
\hline K14 & 25,467 & 1,25 & & 22,9 & 1,12 & \\
\hline $\mathrm{K} 15$ & 25,320 & 1,25 & & 22,6 & 1,11 & \\
\hline K16 & 27,912 & 1,37 & & 25,1 & 1,23 & \\
\hline K17 & 27,886 & 1,37 & & 25,0 & 1,22 & \\
\hline K18 & 27,680 & 1,36 & & 24,7 & 1,20 & \\
\hline K19 & 29,169 & 1,44 & & 26,0 & 1,27 & \\
\hline K20 & 26,928 & 1,32 & & 23,7 & 1,15 & \\
\hline K21 & 27,104 & 1,33 & & 23,8 & 1,16 & \\
\hline K22 & 28,598 & 1,41 & & 25,2 & 1,22 & \\
\hline K23 & 28,497 & 1,40 & & 25,0 & 1,21 & \\
\hline K24 & 29,427 & 1,44 & & 25,9 & 1,26 & \\
\hline K25 & 30,009 & 1,47 & & 26,4 & 1,27 & \\
\hline K26 & 28,950 & 1,42 & & 25,3 & 1,22 & \\
\hline K27 & 29,209 & 1,43 & & 25,4 & 1,23 & \\
\hline K28 & 29,121 & 1,43 & & 25,3 & 1,22 & \\
\hline K29 & 29,174 & 1,43 & & 25,2 & 1,21 & \\
\hline K30 & 29,289 & 1,44 & & 25,3 & 1,21 & \\
\hline
\end{tabular}




\begin{tabular}{|c|c|c|c|c|c|c|}
\hline \multirow[b]{2}{*}{ Variable } & \multicolumn{3}{|c|}{$\begin{array}{c}\text { Spezif. } 4 \\
\text { Alter, Kohorten, } \\
\text { GRV-Var. }\end{array}$} & \multicolumn{3}{|c|}{$\begin{array}{c}\text { Spezif. } 5 \\
\text { Alter, Kohorten, } \\
\text { GRV, Makro }\end{array}$} \\
\hline & Koeffizient & t-Wert & Sign & Koeffizient & t-Wert & Sign \\
\hline K31 & 29,302 & 1,44 & & 25,2 & 1,21 & \\
\hline K32 & 29,618 & 1,45 & & 25,5 & 1,22 & \\
\hline K33 & 29,802 & 1,46 & & 25,6 & 1,22 & \\
\hline K34 & 29,930 & 1,47 & & 25,6 & 1,22 & \\
\hline K35 & 30,036 & 1,47 & & 25,7 & 1,22 & \\
\hline K36 & 30,158 & 1,48 & & 25,7 & 1,22 & \\
\hline K37 & 30,276 & 1,48 & & 25,7 & 1,22 & \\
\hline K38 & 30,386 & 1,49 & & 25,7 & 1,22 & \\
\hline K39 & 30,527 & 1,49 & & 25,8 & 1,22 & \\
\hline K40 & 30,573 & 1,49 & & 25,9 & 1,22 & \\
\hline K41 & 30,663 & 1,50 & & 25,9 & 1,22 & \\
\hline K42 & 30,723 & 1,50 & & 25,9 & 1,22 & \\
\hline K43 & 30,794 & 1,50 & & 25,9 & 1,21 & \\
\hline K44 & 30,820 & 1,50 & & 25,9 & 1,21 & \\
\hline K45 & 30,879 & 1,51 & & 25,9 & 1,21 & \\
\hline K46 & 30,908 & 1,51 & & 25,9 & 1,21 & \\
\hline GJ73_u62 & $-0,916$ & $-1,93$ & $*$ & $-1,2$ & $-1,90$ & $*$ \\
\hline GJ73_62 & 4,507 & 0,77 & & 4,9 & 0,84 & \\
\hline GJ73_63 & 37,806 & 4,16 & ** & 38,2 & 4,12 & ** \\
\hline GJ73_64 & 6,731 & 0,62 & & 7,5 & 0,67 & \\
\hline GJ73_65 & $-0,861$ & $-0,05$ & & 0,2 & 0,01 & \\
\hline GJ79_u61 & $-0,113$ & $-0,29$ & & $-0,2$ & $-0,28$ & \\
\hline GJ79_61 & 6,982 & 5,29 & $* *$ & 7,1 & 5,54 & ** \\
\hline GJ79_62 & $-1,295$ & $-0,58$ & & $-1,3$ & $-0,57$ & \\
\hline GJ79_63p & $-1,954$ & $-0,71$ & & $-1,8$ & $-0,66$ & \\
\hline GJ80_u60 & $-0,568$ & $-1,55$ & & $-0,4$ & $-0,60$ & \\
\hline GJ80_60 & 10,301 & 12,61 & ** & 10,5 & 13,26 & ** \\
\hline GJ80_61 & $-2,483$ & $-1,61$ & & $-2,3$ & $-1,30$ & \\
\hline GJ80_62p & 1,541 & 0,65 & & 1,8 & 0,71 & \\
\hline GJ84_u60 & $-0,688$ & $-1,75$ & * & $-0,9$ & $-0,90$ & \\
\hline GJ84_60 & 2,404 & 1,96 & ** & 2,4 & 2,01 & $* *$ \\
\hline GJ84_61 & $-5,166$ & $-3,48$ & ** & $-5,4$ & $-2,97$ & $* *$ \\
\hline GJ84_62 & $-6,039$ & $-5,40$ & ** & $-6,2$ & $-4,31$ & $* *$ \\
\hline GJ84_63 & 2,169 & 0,80 & & 2,0 & 0,70 & \\
\hline GJ84_64 & $-9,470$ & $-4,79$ & $* *$ & $-9,6$ & $-4,38$ & $* *$ \\
\hline GJ84_65 & 28,481 & 6,56 & $* *$ & 28,4 & 6,63 & $* *$ \\
\hline GJ73_64 & 9,046 & 1,33 & & 8,9 & 1,29 & \\
\hline AB_Ind & 3,579 & 12,53 & $* *$ & 3,6 & 12,55 & $* *$ \\
\hline$\overline{\mathrm{BSP}}$ & & & & 0,0 & 0,25 & \\
\hline Anpass & & & & 0,1 & 1,21 & \\
\hline Alq_5054 & & & & 0,6 & 0,38 & \\
\hline Alq_5559 & & & & 0,0 & 0,10 & \\
\hline Alq_6064 & & & & 0,3 & 1,12 & \\
\hline Alg_Ges & & & & $-0,4$ & $-0,36$ & \\
\hline Beob. & 924 & & & 924 & & \\
\hline $\mathbf{R}^{2} /$ korr. $\mathbf{R}^{2}$ & $94,8 \%$ & $94,3 \%$ & & $94,79 \%$ & $94,23 \%$ & \\
\hline DW & 1,772 & & & 1,8 & & \\
\hline
\end{tabular}


Tabelle A-4: OLS-Schătzung der Zugangsraten zur GRV, Spezifikatlonen 6 bis 8 - Untersuchung der Arbeltslosigkelt

\begin{tabular}{|c|c|c|c|c|c|c|c|c|c|c|c|}
\hline \multicolumn{4}{|c|}{$\begin{array}{c}\text { Schätzung II } \\
\text { Alter, Kohorten, GRV } \\
\text { Arbeitslosenquote aller Ar- } \\
\text { beitnehmer }\end{array}$} & \multicolumn{4}{|c|}{$\begin{array}{c}\text { Schätzung III } \\
\text { Alter, Kohorten, GRV } \\
\text { Arbeitslosenquote } \\
\text { der } 60-64 \text { jährigen }\end{array}$} & \multicolumn{4}{|c|}{$\begin{array}{c}\text { Schätzung IV } \\
\text { Alter, Kohorten, GRV } \\
\text { Wirkung Arbeitslosenquote } \\
\text { auf Altersgruppen }\end{array}$} \\
\hline Variable & $\begin{array}{l}\text { Koeffi- } \\
\text { zient }\end{array}$ & $\begin{array}{c}\text { t- } \\
\text { Wert }\end{array}$ & Sign| & Variable & $\begin{array}{c}\text { Koeffi- } \\
\text { zient }\end{array}$ & t-Wert & Sign. & Variable & $\begin{array}{c}\text { Koeffi- } \\
\text { zient }\end{array}$ & $\begin{array}{c}\text { t- } \\
\text { Wert }\end{array}$ & Sign. \\
\hline A45 & $-30,0$ & $-1,48$ & & A45 & $-28,7$ & $-1,42$ & & A45 & $-30,5$ & $-1,51$ & \\
\hline A46 & $-30,0$ & $-1,48$ & & A46 & $-28,6$ & $-1,42$ & & A46 & $-30,5$ & $-1,50$ & \\
\hline A47 & $-29,8$ & $-1,47$ & & A47 & $-28,5$ & $-1,41$ & & A47 & $-30,3$ & $-1,50$ & \\
\hline A48 & $-29,7$ & $-1,46$ & & A48 & $-28,4$ & $-1,41$ & & A48 & $-30,2$ & $-1,49$ & \\
\hline A49 & $-29,4$ & $-1,45$ & & A49 & $-28,3$ & $-1,40$ & & A49 & $-30,0$ & $-1,48$ & \\
\hline A50 & $-29,2$ & $-1,44$ & & A50 & $-28,0$ & $-1,39$ & & A50 & $-29,0$ & $-1,43$ & \\
\hline A51 & $-29,1$ & $-1,44$ & & A51 & $-28,0$ & $-1,38$ & & A51 & $-28,9$ & $-1,43$ & \\
\hline A52 & $-28,9$ & $-1,43$ & & A52 & $-27,8$ & $-1,37$ & & A52 & $-28,7$ & $-1,42$ & \\
\hline A53 & $-28,6$ & $-1,41$ & & A53 & $-27,6$ & $-1,37$ & & A53 & $-28,4$ & $-1,41$ & \\
\hline A54 & $-28,4$ & $-1,40$ & & A54 & $-27,4$ & $-1,36$ & & A54 & $-28,1$ & $-1,39$ & \\
\hline A55 & $-27,9$ & $-1,38$ & & A55 & $-27,0$ & $-1,34$ & & A55 & $-26,4$ & $-1,30$ & \\
\hline A56 & $-27,5$ & $-1,36$ & & A56 & $-26,6$ & $-1,32$ & & A56 & $-25,9$ & $-1,28$ & \\
\hline A57 & $-27,0$ & $-1,33$ & & A57 & $-26,1$ & $-1,29$ & & A57 & $-25,3$ & $-1,25$ & \\
\hline A58 & $-26,3$ & $-1,30$ & & A58 & $-25,5$ & $-1,26$ & & A58 & $-24,6$ & $-1,22$ & \\
\hline A59 & $-25,7$ & $-1,27$ & & A59 & $-24,8$ & $-1,23$ & & A59 & $-23,9$ & $-1,18$ & \\
\hline A60 & $-22,2$ & $-1,10$ & & A60 & $-21,5$ & $-1,06$ & & A60 & $-22,0$ & $-1,09$ & \\
\hline A61 & $-22,4$ & $-1,12$ & & A61 & $-21,7$ & $-1,09$ & & A61 & $-22,2$ & $-1,11$ & \\
\hline A62 & $-23,5$ & $-1,28$ & & A62 & $-23,1$ & $-1,26$ & & A62 & $-23,3$ & $-1,27$ & \\
\hline A63 & $-26,5$ & $-1,44$ & & A63 & $-26,3$ & $-1,43$ & & A63 & $-26,4$ & $-1,44$ & \\
\hline A64 & $-21,0$ & $-1,23$ & & A64 & $-20,9$ & $-1,23$ & & A64 & $-21,0$ & $-1,23$ & \\
\hline A65 & 41,4 & 4,26 & $* *$ & A65 & 41,2 & 4,24 & ** & A65 & 41,2 & 4,24 & $* *$ \\
\hline K6 & 11,3 & 0,76 & & K6 & 11,1 & 0,75 & & K6 & 11,2 & 0,76 & \\
\hline K7 & 31,2 & 1,62 & & K7 & 30,8 & 1,60 & & K7 & 31,0 & 1,61 & \\
\hline K8 & 26,4 & 1,49 & & K8 & 25,8 & 1,46 & & K8 & 26,1 & 1,48 & \\
\hline K9 & 26,6 & 1,39 & & K9 & 25,9 & 1,35 & & K9 & 26,1 & 1,36 & \\
\hline K10 & 20,6 & 1,02 & & K10 & 19,7 & 0,98 & & K10 & 20,0 & 0,99 & \\
\hline K11 & 22,6 & 1,10 & & K11 & 21,6 & 1,06 & & K11 & 21,7 & 1,06 & \\
\hline K12 & 23,2 & 1,13 & & K12 & 22,2 & 1,09 & & K12 & 22,1 & 1,08 & \\
\hline $\mathrm{K} 13$ & 24,2 & 1,18 & & $\mathrm{~K} 13$ & 23,1 & 1,13 & & K13 & 23,0 & 1,13 & \\
\hline K14 & 24,5 & 1,20 & & K14 & 23,3 & 1,15 & & K14 & 23,3 & 1,14 & \\
\hline K15 & 24,4 & 1,20 & & K15 & 23,1 & 1,14 & & K15 & 23,3 & 1,15 & \\
\hline K16 & 27,0 & 1,33 & & K16 & 25,7 & 1,27 & & K16 & 26,2 & 1,29 & \\
\hline K17 & 26,9 & 1,32 & & K17 & 25,6 & 1,26 & & K17 & 26,3 & 1,30 & \\
\hline K18 & 26,7 & 1,31 & & K18 & 25,3 & 1,25 & & K18 & 26,2 & 1,29 & \\
\hline K19 & 28,1 & 1,38 & & K19 & 26,7 & 1,32 & & K19 & 27,8 & 1,37 & \\
\hline K20 & 25,9 & 1,27 & & K20 & 24,4 & 1,20 & & K20 & 25,7 & 1,27 & \\
\hline K21 & 26,0 & 1,28 & & K21 & 24,5 & 1,21 & & K21 & 25,9 & 1,27 & \\
\hline K22 & 27,5 & 1,35 & & K22 & 26,0 & 1,28 & & K22 & 27,4 & 1,35 & \\
\hline K23 & 27,4 & 1,35 & & K23 & 25,8 & 1,27 & & K23 & 27,5 & 1,35 & \\
\hline K24 & 28,3 & 1,39 & & K24 & 26,7 & 1,31 & & K24 & 28,7 & 1,41 & \\
\hline K25 & 28,9 & 1,42 & & K25 & 27,3 & 1,34 & & K25 & 29,4 & 1,44 & \\
\hline K26 & 27,9 & 1,37 & & K26 & 26,2 & 1,29 & & K26 & 28,4 & 1,40 & \\
\hline K27 & 28,1 & 1,38 & & K27 & 26,4 & 1,30 & & K27 & 28,7 & 1,41 & \\
\hline K28 & 28,0 & 1,37 & & K28 & 26,3 & 1,29 & & K28 & 28,7 & 1,41 & \\
\hline K29 & 28,1 & 1,38 & & K29 & 26,3 & 1,29 & & K29 & 28,7 & 1,41 & \\
\hline K30 & 28,2 & 1,38 & & K30 & 26,3 & 1,29 & & K30 & 28,8 & 1,41 & \\
\hline
\end{tabular}




\begin{tabular}{|c|c|c|c|c|c|c|c|c|c|c|c|}
\hline $\begin{array}{r}\text { Sc } \\
\text { Alter, } \\
\text { Arbeitslo }\end{array}$ & $\begin{array}{l}\text { chätzung } \\
\text { Kohorten } \\
\text { senquote } \\
\text { eitnehme }\end{array}$ & $\begin{array}{l}\text { II } \\
\text { GRV } \\
\text { aller } \\
\text { r }\end{array}$ & & $\begin{array}{r}\text { S } \\
\text { Alter, } \\
\text { Arb } \\
\text { der }\end{array}$ & $\begin{array}{l}\text { thätzung } \\
\text { Kohorter } \\
\text { eitslosen } \\
0-64 \text { jäh }\end{array}$ & $\begin{array}{l}\text { III } \\
\text { i, GRV } \\
\text { quote } \\
\text { rigen }\end{array}$ & & $\begin{array}{r}\text { S } \\
\text { Alter, } \\
\text { Wirkung } \\
\text { auf }\end{array}$ & $\begin{array}{l}\text { chätzung } \\
\text { Kohorter } \\
\text { Arbeitsl } \\
\text { Altersgry }\end{array}$ & $\begin{array}{l}\text { IV } \\
\text { n, GRV } \\
\text { losenqu } \\
\text { uppen }\end{array}$ & \\
\hline Variable & $\begin{array}{c}\text { Koeffi- } \\
\text { zient }\end{array}$ & $\begin{array}{c}\text { t- } \\
\text { Wert }\end{array}$ & Sign & Variable & $\begin{array}{c}\text { Koeffi- } \\
\text { zient }\end{array}$ & $t$-Wert & Sign. & Variable & $\begin{array}{c}\text { Koeffi- } \\
\text { zient }\end{array}$ & $\begin{array}{c}\text { t- } \\
\text { Wert }\end{array}$ & Sign. \\
\hline K31 & 28,2 & 1,38 & & K31 & 26,3 & 1,29 & & K31 & 28,7 & 1,41 & \\
\hline K32 & 28,5 & 1,40 & & K32 & 26,6 & 1,31 & & K32 & 28,9 & 1,42 & \\
\hline K33 & 28,7 & 1,41 & & K33 & 26,8 & 1,32 & & K33 & 29,1 & 1,43 & \\
\hline K34 & 28,8 & 1,41 & & K34 & 26,8 & 1,32 & & K34 & 29,0 & 1,42 & \\
\hline K35 & 28,9 & 1,42 & & K35 & 26,9 & 1,32 & & K35 & 29,0 & 1,42 & \\
\hline K36 & 29,0 & 1,42 & & K36 & 26,9 & 1,32 & & K36 & 28,9 & 1,42 & \\
\hline K37 & 29,1 & 1,42 & & K37 & 27,0 & 1,33 & & K37 & 28,9 & 1,42 & \\
\hline K38 & 29,2 & 1,43 & & K38 & 27,1 & 1,33 & & K38 & 28,9 & 1,42 & \\
\hline K39 & 29,4 & 1,44 & & K39 & 27,2 & 1,33 & & K39 & 29,0 & 1,42 & \\
\hline K40 & 29,5 & 1,44 & & K40 & 27,3 & 1,34 & & K40 & 29,1 & 1,42 & \\
\hline K41 & 29,6 & 1,44 & & K41 & 27,4 & 1,34 & & K41 & 29,1 & 1,42 & \\
\hline K42 & 29,6 & 1,45 & & K42 & 27,4 & 1,34 & & K42 & 29,1 & 1,42 & \\
\hline K43 & 29,7 & 1,45 & & K43 & 27,4 & 1,34 & & K43 & 29,2 & 1,43 & \\
\hline K44 & 29,8 & 1,45 & & K44 & 27,4 & 1,34 & & K44 & 29,3 & 1,43 & \\
\hline K45 & 29,8 & 1,45 & & K45 & 27,4 & 1,34 & & K45 & 29,3 & 1,43 & \\
\hline K46 & 29,9 & 1,45 & & K46 & 27,4 & 1,34 & & K46 & 29,4 & 1,43 & \\
\hline GJ73_u62 & $-1,1$ & $-1,88$ & * & GJ73_u62 & $-1,2$ & $-1,99$ & ** & GJ73_u62 & $-1,2$ & $-2,04$ & $* *$ \\
\hline GJ73_62 & 4,7 & 0,80 & & GJ73_62 & 4,9 & 0,84 & & GJ73_62 & 4,6 & 0,79 & \\
\hline GJ73_63 & 38,0 & 4,10 & ** & GJ73_63 & 38,2 & 4,14 & $* *$ & GJ73_63 & 37,7 & 4,08 & ** \\
\hline GJ73_64 & 7,1 & 0,64 & & GJ73_64 & 7,5 & 0,67 & & GJ73_64 & 7,0 & 0,63 & \\
\hline GJ73_65 & $-0,3$ & $-0,02$ & & GJ73_65 & 0,1 & 0,01 & & GJ73_65 & $-0,3$ & $-0,02$ & \\
\hline GJ79_u61 & 0,1 & 0,26 & & GJ79_u61 & $-0,1$ & $-0,33$ & & GJ79_u61 & $-0,1$ & $-0,31$ & \\
\hline GJ79_61 & 7,0 & 5,46 & ** & GJ79_61 & 7,1 & 5,54 & ** & GJ79_61 & 6,8 & 5,32 & ** \\
\hline GJ79_62 & $-1,1$ & $-0,49$ & & GJ79_62 & $-1,3$ & $-0,57$ & & GJ79_62 & $-1,2$ & $-0,57$ & \\
\hline GJ79_63p & $-1,7$ & $-0,63$ & & GJ79_63p & $-1,8$ & $-0,67$ & & GJ79_63p & $-1,4$ & $-0,52$ & \\
\hline GJ80_u60 & $-0,8$ & $-1,37$ & & GJ80_u60 & $-0,5$ & $-0,99$ & & GJ80_u60 & $-0,2$ & $-0,39$ & \\
\hline GJ80_60 & 10,4 & 13,11 & ** & GJ80_60 & 10,4 & 13,17 & ** & GJ80_60 & 8,1 & 6,24 & ** \\
\hline GJ80_61 & $-2,7$ & $-1,56$ & & GJ80_61 & $-2,4$ & $-1,44$ & & GJ80_61 & $-4,1$ & $-1,90$ & $*$ \\
\hline GJ80_62p & 1,4 & 0,56 & & GJ80_62p & 1,7 & 0,69 & & GJ80_62p & $-0,2$ & $-0,10$ & \\
\hline GJ84_u60 & $-1,0$ & $-2,44$ & ** & GJ84_u60 & $-0,6$ & $-1,41$ & & GJ84_u60 & $-0,4$ & $-1,02$ & \\
\hline GJ84_60 & 2,4 & 1,99 & ** & GJ84_60 & 2,4 & 2,00 & ** & GJ84_60 & 0,6 & 0,40 & \\
\hline GJ84_61 & $-5,5$ & $-3,65$ & ** & GJ84_61 & $-5,1$ & $-3,29$ & ** & GJ84_61 & $-6,7$ & $-4,06$ & ** \\
\hline GJ84_62 & $-6,3$ & $-5,72$ & ** & GJ84_62 & $-5,9$ & $-5,18$ & ** & GJ84_62 & $-7,6$ & $-5,87$ & ** \\
\hline GJ84_63 & 1,9 & 0,70 & & GJ84_63 & 2,3 & 0,84 & & GJ84_63 & 0,5 & 0,18 & \\
\hline GJ84_64 & $-9,7$ & $-5,01$ & ** & GJ84_64 & $-9,3$ & $-4,78$ & ** & GJ84_64 & $-11,0$ & $-5,45$ & ** \\
\hline GJ84_65 & 28,3 & 6,51 & ** & GJ84_65 & 28,7 & 6,69 & ** & GJ84_65 & 27,0 & 6,26 & ** \\
\hline GJ73_64_ & 8,8 & 1,28 & & GJ73_64_ & 8,9 & 1,28 & & GJ73_64_ & 9,1 & 1,33 & \\
\hline AB_Ind & 3,6 & 12,55 & ** & AB_Ind & 3,6 & 12,55 & ** & AB_Ind & 3,6 & 12,59 & ** \\
\hline BSP & 0,1 & 0,68 & & BSP & 0,0 & 0,39 & & BSP & 0,1 & 0,63 & \\
\hline Anpass & 0,1 & 1,06 & & Anpass & 0,1 & 1,23 & & Anpass & 0,1 & 1,22 & \\
\hline Alq_Ges & 0,2 & 1,51 & & Alq_6064 & 0,2 & 1,95 & * & G_Al4549 & 0,2 & 1,91 & * \\
\hline & & & & & & & & G_Al5054 & 0,0 & $-0,09$ & \\
\hline & & & & & & & & G_Al5559 & $-0,4$ & $-2,33$ & ** \\
\hline & & & & & & & & G_Al60pl & 0,6 & 1,63 & \\
\hline $\begin{array}{l}\text { Beob. } \\
R^{2} / \text { kor. } R^{2}\end{array}$ & $\begin{array}{r}924,0 \\
94,8 \%\end{array}$ & 25 & & $\begin{array}{r}924,0 \\
94,79 \%\end{array}$ & $94,25 \%$ & & & $\begin{array}{r}924,0 \\
94,83 \%\end{array}$ & $94,28 \%$ & & \\
\hline DW & 1,8 & & & 1.8 & & & & 1,8 & & & \\
\hline
\end{tabular}




\section{A.2 Literatur zur makroökonomischen Entwicklung der GRV}

\section{Tabelle A-5: Tabellarische Literaturübersicht}

\begin{tabular}{|c|c|c|c|c|}
\hline Autoren & $\begin{array}{l}\text { Betrachtete } \\
\text { Jahre }\end{array}$ & $\begin{array}{l}\text { Personen- } \\
\text { gruppe }\end{array}$ & Altersgruppen & Methode \\
\hline $\begin{array}{l}\text { MÖR- } \\
\text { SCHEL } \\
\text { und } \\
\text { REHFELD } \\
1981\end{array}$ & 1962-1979 & $\begin{array}{l}\text { Mä/Fr } \\
\text { Ar/An }\end{array}$ & 30-64 in 5er Gruppen & $\begin{array}{l}\text { - Anteil einzelner Rentenarten am Neuzugang } \\
\text { eines Jahres sowie durchschnittliches } \mathrm{Zu} \text { - } \\
\text { gangsalter } \\
\text { - Indikator zugegangene } \\
\text { Invalidenrenten/Anzahl der Versicherten, in } \\
\text { Gruppen über } 5 \text { Altersjahre } \\
\text { - Invalidisierungshäufigkeit, abhängig vom } \\
\text { Alter. }\end{array}$ \\
\hline $\begin{array}{l}\text { MÖR- } \\
\text { SCHEL } \\
\text { und } \\
\text { REHFELD } \\
1982\end{array}$ & $\begin{array}{l}1973- \\
1981, \\
\text { sowie } \\
\text { Kohorten } \\
\text { von } 1907- \\
1916\end{array}$ & $\mathrm{Mä/Fr}$ & Einzelalter 60-64 & $\begin{array}{l}\text { Neuzugang nach Alter/Alle Versicherten der } \\
\text { Altersgruppe } 60-64 \text { nach Mikrozensus } \\
\text { - Neuzugang nach Alter/Wohnbevölkerung. } \\
\text { der Alter } 60-65 \\
\text {. Aus Rentenbestand: in Altersgruppe } 60-65 \\
\text { zugegangene Renten der Jahrgänge } 1907- \\
1916 \text { nach Rentenart und Zugangsalter } \\
\text { - Aus Rentenzugang: in Altersgruppe } 60-65 \\
\text { zugegangene Renten der Jahrgänge } 1913- \\
1916 \text { nach Rentenart und Zugangsalter } \\
\text { Berechnungswerte der neu zugegangenen } \\
\text { Renten. }\end{array}$ \\
\hline $\begin{array}{l}\text { KNOE- } \\
\text { DEL } 1982\end{array}$ & 1981 & $\begin{array}{l}\mathrm{Mä/Fr} \\
\mathrm{Ar} / \mathrm{An} / \mathrm{Kn} / \\
\mathrm{GRV}\end{array}$ & $\begin{array}{l}\text { z.T. in 5er } \\
\text { Altersgruppen }\end{array}$ & $\begin{array}{l}\text { - Keine Kohortenbereinigung } \\
\text { - Absolute Zahl und Häufigkeitsverteilung im } \\
\text { Rentenzugang, Verteilung auf Versiche- } \\
\text { rungszweige, Leistungsart, Geschlecht und } \\
\text { Altersgruppen } \\
\text { - Gegenüberstellung der relativen Anteile } \\
1976 \text { und } 1981 \\
\text { - Nach Rentenart und Versicherungszweig: } \\
\text { Versicherungsjahre, persönl. Bem. } \\
\text { Grundlage, monatl. Nettozahlbeträge } \\
\text { - Anteil Zugänger aus aktiv Pflichtversicher- } \\
\text { ten. }\end{array}$ \\
\hline $\begin{array}{l}\text { KERWAT } \\
1983\end{array}$ & 1982,1981 & An Mä/Fr & $\begin{array}{l}\text { Kohorten } 1912-1922 \\
\text { bei Zugangsalter von } \\
\text { genau } 60,63 \text { oder } 65 \\
\text { Jahren. }\end{array}$ & $\begin{array}{l}\text { - Absolute Zugangszahlen nach Leistungsart } \\
\text { - Indikator: Rentenzugang pro Leistungsart } \\
\text { und Zugangsalters / Wohnbevölkerung } \\
\text { dieses Alters } \\
\text {. Zugänge der Kohorten } 1917 \text { und 1919; } \\
1977 \text { bis } 1982 \text { nach Leistungsart }\end{array}$ \\
\hline $\begin{array}{l}\text { KERWAT } \\
1984\end{array}$ & 1983,1982 & An Mä/Fr & $\begin{array}{l}\text { Kohorten 1912-1923 } \\
\text { bei Zugangsalter } 60,63 \\
\text { oder } 65 .\end{array}$ & $\begin{array}{l}\text { - Absolute Zugangszahlen nach Leistungsart } \\
\text { - Indikator: Rentenzugang pro Leistungsart } \\
\text { und Zugangsalters / Wohnbevölkerung } \\
\text { dieses Alters }\end{array}$ \\
\hline $\begin{array}{l}\text { KNOE- } \\
\text { DEL } 1985\end{array}$ & 1983,1984 & $\begin{array}{l}\mathrm{Mä/Fr} \\
\mathrm{Ar} / \mathrm{An}\end{array}$ & 3 Gruppen, unscharf & $\begin{array}{l}\text { - Zahl eingegangener EU/BU und } 65 \mathrm{er} \\
\text { Anträge } \\
\text {. Bewilligungsquoten für EU/BU }\end{array}$ \\
\hline
\end{tabular}




\begin{tabular}{|c|c|c|c|c|}
\hline Autoren & $\begin{array}{l}\text { Betrachtete } \\
\text { Jahre }\end{array}$ & $\begin{array}{l}\text { Personen- } \\
\text { gruppe }\end{array}$ & Altersgruppen & Methode \\
\hline (Forts.) & & & & $\begin{array}{l}\text { - Relativer Rentenzugang eines Alters } \\
\text { (Zugang/Wohnbevölkerung) } \\
\text { - Frührenten, latent Versicherter und } \\
\text { Rentenumwandlungen }\end{array}$ \\
\hline $\begin{array}{l}\text { REl- } \\
\text { MANN } \\
1985\end{array}$ & $\begin{array}{l}1963-1985 \\
\text { und Vor- } \\
\text { ausschät- } \\
\text { zungen }\end{array}$ & $\mathrm{Mä/Fr}$ & $\begin{array}{l}\text { Kohortenbetrachtung da } \\
\text { ab } 1913 \text {, nach } 1919 \text { bis } \\
1924 \text { z.T. geschätzte } \\
\text { Zugänge. Einzelalter } \\
\text { 50-65 }\end{array}$ & $\begin{array}{l}\text { - Relativer Anteil einzelner Altersgruppen am } \\
\text { Rentenzugang einer Kohorte } \\
\text { - Anteil der } 50-59 \text { jährigen Zugänger in den } \\
\text { Kohorten } 1913 \text { bis } 1924 . \\
\text { - Einzelkohortenbetrachtung zur Auswirkung } \\
\text { von Gesetzesänderungen } \\
\text { - } 7 \text { Kohorten: Verteilung auf Leistungsarten } \\
\text { und Versicherungszweig }\end{array}$ \\
\hline $\begin{array}{l}\text { SCHUN- } \\
\text { TER- } \\
\text { MANN } \\
1986\end{array}$ & 1973-1982 & $\begin{array}{l}\mathrm{Mä/Fr} \\
\mathrm{Ar} / \mathrm{An}\end{array}$ & $\begin{array}{l}\text { unterschiedliche } \\
\text { Einzelalter 20-60, 44 } \\
55,15-60\end{array}$ & $\begin{array}{l}\text { Verfolgt Entwicklung des EU/BU } \\
\text { Berentungsrisikos } 1973 \text { bis } 1981 \text { nach } \\
\text { Geschlecht, Alter, Versicherungszweig und } \\
\text { Versicherungsart }\end{array}$ \\
\hline $\begin{array}{l}\text { MÜLLER } \\
1987\end{array}$ & $\begin{array}{l}\text { Bestände } \\
\text { Altersruhe- } \\
\text { gelder; } 1 . \\
\text { Juli } 1986\end{array}$ & $\begin{array}{l}\mathrm{Mä/Fr} \\
\mathrm{Ar} / \mathrm{An}\end{array}$ & $\begin{array}{l}\text { Nach Kohorte falls } \\
\text { Zugang zwischen Alter } \\
60 \text { und } 65 \text { (1905-1920). }\end{array}$ & $\begin{array}{l}\text { Verteilung der Kohorten 1905-1920, die } \\
1986 \text { noch im Bestand sind (noch leben) auf } \\
\text { Zugangsalter zwischen } 60 \text { und } 65 \text { Jahren } \\
\text {. Verteilung der Kohorten } 1905-1920 \text {, die } \\
1986 \text { noch im Bestand sind (noch leben) und } \\
\text { mindestens } 35 \text { Versicherungsjahre haben }\end{array}$ \\
\hline $\begin{array}{l}\text { JACOBS } \\
\text { KOHLI } \\
\text { REIN } \\
1991 \\
\text { (Kapitel } \\
\text { 6; Seiten } \\
\text { 181-221) }\end{array}$ & $1958-1988$ & $\begin{array}{l}\mathrm{Mä/Fr} \\
\mathrm{Ar} / \mathrm{An}\end{array}$ & $\begin{array}{l}\text { Ser Gruppen 50-64, } \\
\text { Einzelalter 60-65, } \\
\text { Kohorten 1913-1924. }\end{array}$ & $\begin{array}{l}\text { - durchschnittliches Zugangsalter pro Jahr } \\
\text { - Rel. Anteil einzelner Leistungsarten am } \\
\text { Zugang pro Jahr } \\
\text { - Indikator EU/BU Zugang/Bevölkerung in } \\
\text { drei } 5 \text { Jahres Altersgruppen, 1972-1987. } \\
\text { - Verteilung der Kohorten 1913-1924 auf } \\
\text { Zugangsalter (Gruppen und Einzelalter) }\end{array}$ \\
\hline $\begin{array}{l}\text { REHFELD } \\
1991\end{array}$ & $\begin{array}{l}1960 \\
1990 \\
1980-1990\end{array}$ & $\begin{array}{l}\text { Mä/Fr } \\
\text { Ar/An/Kn' } \\
\text { GRV }\end{array}$ & $\begin{array}{l}\text { Ser Altersgruppen für } \\
\text { 45-64, sowie detaillierte } \\
\text { Kohortenbetrachtung }\end{array}$ & $\begin{array}{l}\text { - Globaler Rentenzugang ' } 80-90 \text { in Alters-, } \\
\text { Früh-, Witwen-, und Waisenrenten } \\
\text { - Rentenzugang (absolut) nach Leistungsarten } \\
60-90 \\
\text { - Rentenzugangsstruktur (relativ) nach } \\
\text { Leistungsarten ' } 60,70,80,90 \\
\text { - Frühberentungsquoten nach Altersgruppen } \\
\text { - Indikator: EU/BU Zugang/ Versicherte, in } \\
\text { 5er Altersgruppen für Alter } 45-64,1972- \\
1990 \text {. } \\
\text { - Rentenzugang der Jahrgänge: bis } 1907,20 \text {, } \\
23,25 \text { Verteilung auf Rentenzugangsalter, } \\
\text { Leistungsart }\end{array}$ \\
\hline $\begin{array}{l}\text { REHFELD } \\
1994\end{array}$ & $1945-1990$ & $\begin{array}{l}\mathrm{Mä/Fr} \\
\mathrm{Ar} / \mathrm{An}\end{array}$ & $\begin{array}{l}\text { Kohorten der Geburts- } \\
\text { jahrgänge 1907, 1910, } \\
\text { 1915, 1920, 1925, aber } \\
\text { auch nach Einzelalter } \\
\text { bei Zugang. }\end{array}$ & $\begin{array}{l}\text { - Rentenzugang nach Leistungsart, } \\
\text { kohortenspezifische } \\
\text { Rentenzugangsstrukturen. } \\
\text { - Kumulierte Zugangsstrukturen } \\
\text { - Analyse von Einzeldaten der Versiche- } \\
\text { rungsbiographien }\end{array}$ \\
\hline
\end{tabular}




\section{A.3 OLS-Schätzung des normalisierten Einkommens aus dem SOEP für Westdeutschland 1988-1990}

\section{Tabelle A-6: OLS-Schätzung des Normalisierten Einkommens aus dem SOEP für} Westdeutschland 1988-1990.

Abhängige Variable: $\quad \operatorname{Ln}(\mathrm{Y})$

\begin{tabular}{|c|c|c|c|}
\hline $\begin{array}{c}\text { Erklärende } \\
\text { Variable }\end{array}$ & $\begin{array}{l}\text { Geschätzter } \\
\text { Koeffizient }\end{array}$ & $\begin{array}{l}\text { Standard } \\
\text { Error }\end{array}$ & $\begin{array}{c}\mathrm{t}- \\
\text { Statistik }\end{array}$ \\
\hline Konstante & 3,77549 & 5,33898 & 0,707 \\
\hline Alter & 0,06953 & 0,27313 & $0,255^{*}$ \\
\hline Alter $^{2}$ & $-0,00061$ & 0,00460 & $-0,133^{\circ}$ \\
\hline Alter $^{3}$ & 0,00000 & 0,00003 & $0,150^{*}$ \\
\hline GebJahr (Kohorte) & 0,04848 & 0,00995 & 4,870 \\
\hline weiblich & $-0,33239$ & 0,02158 & $-15,404$ \\
\hline Familienstand & 0,01284 & 0,01788 & 0,718 \\
\hline berufl. Bildung & $-0,01597$ & 0,01916 & $-0,834$ \\
\hline Schule & 0,23594 & 0,02279 & 10,351 \\
\hline Arbeitslos & $-0,21403$ & 0,13049 & $-1,640$ \\
\hline Behindert & 0,00076 & 0,00041 & 1,853 \\
\hline \multicolumn{4}{|l|}{ Berufsfelder: } \\
\hline Land und Forstw. & $-0,57041$ & 0,06622 & $-8,613$ \\
\hline Bergbau, Energie & 0,12294 & 0,06869 & 1,790 \\
\hline Industrie & 0,06605 & 0,02316 & 2,852 \\
\hline Handel & $-0,06608$ & 0,03528 & $-1,873$ \\
\hline Öffentl. Dienst & 0,06414 & 0,03040 & 2,110 \\
\hline Dienstleistung & $-0,00919$ & 0,03621 & $-0,254$ \\
\hline Banken/Versicherung & 0,15849 & 0,05696 & 2,783 \\
\hline Bildung & 0,14046 & 0,04098 & 3,428 \\
\hline Gesundheit & 0,15614 & 0,04209 & 3,710 \\
\hline Soziale Berufe & 0,06807 & 0,05241 & 1,299 \\
\hline \multicolumn{4}{|l|}{ Qualifikation: } \\
\hline angelernt & 0,13700 & 0,01895 & 7,228 \\
\hline gehoben & 0,49856 & 0,02998 & 16,630 \\
\hline selbständig & 0,00372 & 0,02968 & 0,126 \\
\hline Teilerwerbstätig & $-0,77094$ & 0,02906 & $-26,534$ \\
\hline
\end{tabular}

Anzahl der Beobachtungen:

Gütemaß R ${ }^{2}$

Korrigiertes $\mathbf{R}^{2}$

0,51318

Sum of Squared Residuals

654,35500

Standard Error of the Regression

0,44989

Durbin-Watson Statistic

Mean of Dependent Variable

8,02631

*Die drei Altersvariablen sind gemeinsam signifkant auf dem 1\% Niveau. Der partielle F-Test ergibt eine Testgröße von $F_{3,3258}=4,92$ und übersteigt damit die Testgröße $\left(f_{3, \infty}=3,78\right)$, so daß die Nullhypothese ihrer gemeinsamen Insignifkanz verworfen werden kann 


\section{A.4 Regressionsergebnisse der Übergangsratenmodelle für West- und Ostdeutschland - Weibull-Spezifikationen}

Tabelle A-7: Verweildaueranalyse für West-Deutschland - Spezifikatlonen des WeibullModelles

Abhängige Variable: Übergangsrate in Status „Nicht erwerbstätig“

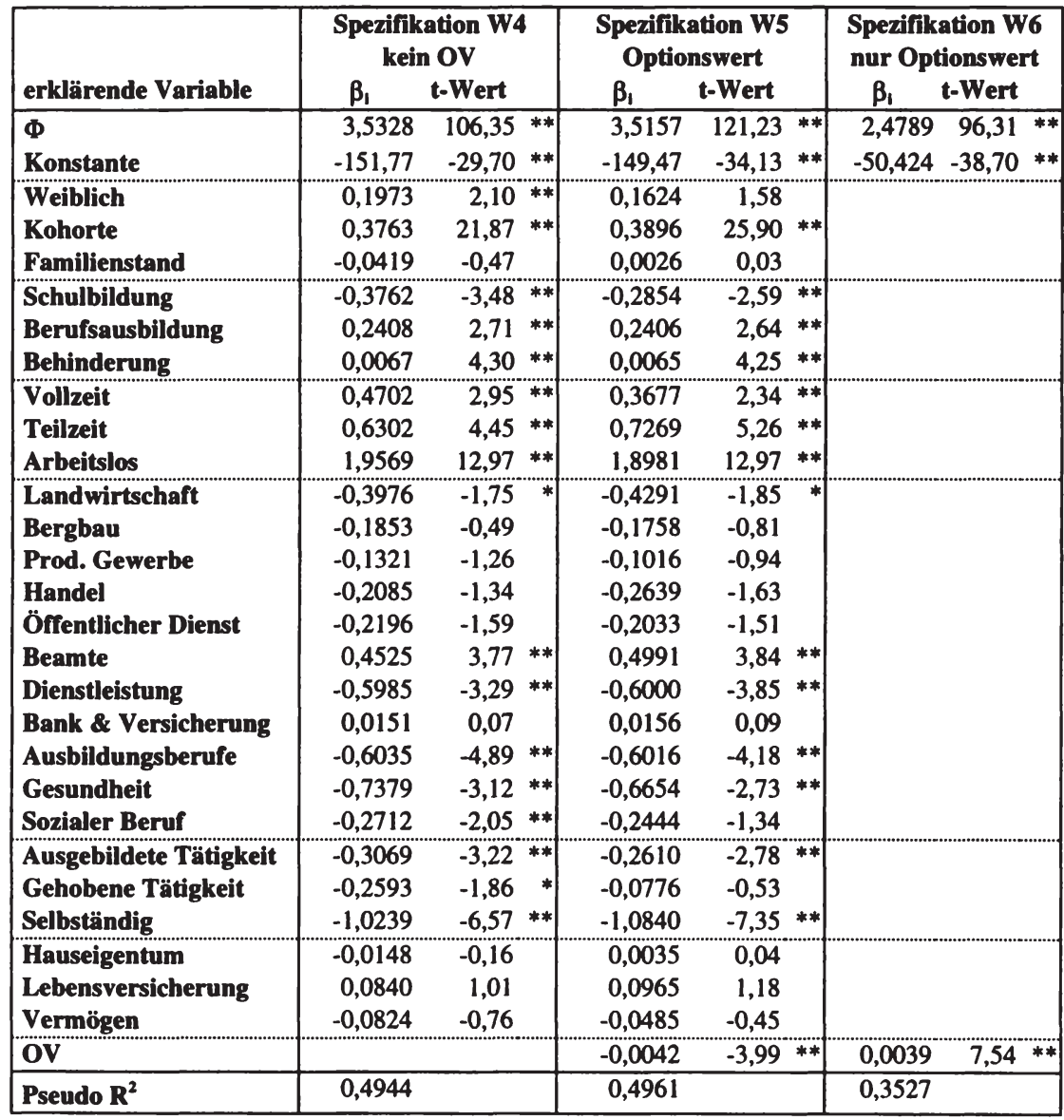


Tabelle A-8: Verwelldaueranalyse für Ost-Deutschland - Spezifikationen des WeibullModelles

Abhängige Variable: Übergangsrate in Status „Nicht erwerbstätig“

\begin{tabular}{|c|c|c|c|c|c|c|c|}
\hline \multirow[b]{2}{*}{ erklärende Variable } & \multicolumn{3}{|c|}{$\begin{array}{c}\text { Spezifikation } 04 \\
\text { kein OV }\end{array}$} & \multicolumn{3}{|c|}{$\begin{array}{c}\text { Spexifikation } 05 \\
\text { Optionswert }\end{array}$} & \multirow{2}{*}{$\begin{array}{c}\text { Spezifikation } 06 \\
\text { nur Optionswert } \\
\beta_{\mathrm{l}} \text { t-Wert }\end{array}$} \\
\hline & $\beta_{\mathbf{I}}$ & t-Wert & & $\beta_{i}$ & t-Wert & & \\
\hline$\Phi$ & 4,5010 & 102,2 & & 4,5060 & 97,8 & & $2,1595 \quad 54,5^{* *}$ \\
\hline Konstante & $-413,31$ & $-22,63$ & $* *$ & $-415,67$ & $-21,67$ & ** & $-36,90-25,01 * *$ \\
\hline Weiblich & 0,3623 & 3,69 & $* *$ & 0,3572 & 3,44 & $* *$ & \\
\hline Kohorte & 1,4282 & 21,59 & ** & 1,4520 & 20,72 & $* *$ & \\
\hline Familienstand & 0,0888 & 0,67 & & 0,1118 & 0,81 & & \\
\hline Schulbildung & $-0,0530$ & $-0,49$ & & 0,0146 & 0,12 & & \\
\hline Behinderung & 0,2802 & 1,83 & $*$ & 0,2746 & 1,76 & $*$ & \\
\hline Teilzeit & 0,6426 & 2,04 & $* *$ & 0,6202 & 2,07 & $* *$ & \\
\hline Vollzeit & 0,4219 & 1,45 & & 0,4572 & 1,63 & & \\
\hline Arbeitslos & 1,0395 & 2,22 & $* *$ & 0,8644 & 1,87 & $*$ & \\
\hline Land wirtschaft & 2,7481 & 11,65 & $* *$ & 2,6979 & 12,43 & $* *$ & \\
\hline Bergbau & 2,4604 & 7,33 & $* *$ & 2,5694 & 7,49 & $* *$ & \\
\hline Prod. Gewerbe & 2,3931 & 14,70 & $* *$ & 2,3658 & 14,07 & $* *$ & \\
\hline Handel & 2,6049 & 10,31 & $* *$ & 2,5844 & 9,76 & ** & \\
\hline Öffentlicher Dienst & 2,3156 & 12,45 & $* *$ & 2,3470 & 11,80 & ** & \\
\hline Ausbildungsberufe & 2,6371 & 11,00 & *** & 2,7038 & 9,98 & $* *$ & \\
\hline Ausgebildete Tätigkeit & $-0,0464$ & $-0,43$ & & $-0,0203$ & $3^{-0,18}$ & & \\
\hline Gehobene Tätigkeit & $-254,069$ & $-22,36$ & $* *$ & $-255,293$ & $-21,35$ & $* *$ & \\
\hline Selbständig & $\begin{array}{r}-113,647 \\
. .113,6-1\end{array}$ & $-22,29$ & $* *$ & $-114,486$ & $5-21,30$ & $* *$ & \\
\hline Hauseigentum & $-0,1731$ & $-1,58$ & & $-0,1860$ & $-1,70$ & $*$ & \\
\hline Lebensversicherung & 0,1075 & 1,01 & & 0,0375 & 0,33 & & \\
\hline Vermögen & $\begin{array}{l}-0,9442 \\
-0,96\end{array}$ & $-3,07$ & $* *$ & $-0,9518$ & $-3,09$ & **... & \\
\hline ov & & & & $-0,0035$ & $-2,26$ & $* *$ & 0,0080 \\
\hline Pseudo $\mathbf{R}^{2}$ & 0,7072 & & & 0,7082 & & & 0,2929 \\
\hline
\end{tabular}


Denn was man schwarz auf weiß besitzt, Kann man getrost nach Hause tragen.

\section{LITERATURVERZEICHNIS}

AARTS, LEO J.M. UND DE JONG, PHIIIP (1993): „Early retirement of older male workers under the dutch social security disability insurance programme", St. Martin's Press, New York.

ANDO, Albert und Modiglian, Franco (1963): „The 'Life-Cycle' Hypothesis of Saving: Aggregate Implikations and Tests“, The American Economic Review 53, Seiten 55-84.

BÄCKER, GERHARD UND NAEGELE, GERHARD (1993): „Alternde Gesellschaften und Erwerbstätigkeit“, Bund Verlag, Köln.

BARRO, ROBERT J. (1974): „Are Government Bonds Net Wealth?“, Journal of Political Economy 82, Seiten 1095-1117.

BARRO, ROBERT J. (1978): „The Impact of Social Security on Private Saving: Evidence from the U.S. Time Series“, Washington, D.C.

BELLMANN, RICHARD (1971): „Introduction to the Mathematical Theory of Control Processes“, Academic Press, New York und London.

Berkovec, JAMES AND STERN, STEVEn (1991): „Job Exit Behavior of Older Men“, Econometrica, 59, Seiten 189-210.

BERTHOLD, NORBERT (1987): „Der Einfluß der Gesetzlichen Rentenversicherung auf die individuelle Ruhestandsentscheidung “, WiSt, Heft 9, Seiten 435-440.

BIRG, HERWIG (1989): „Die demographische Zeitenwende“, Spektrum der Wissenschaft, Januar.

BLOSSFELD, HANS-PETER; HAMERLE, ALFRED UND MAYER, KARL ULRICH (1989): „HazardratenModelle in den Wirtschafts- und Sozialwissenschaften", Allgemeines Statistisches Archiv 73.

BÖRSCH-SUPAN, AXEL (1994): „Migration, Social Security Systems, and Public Finance“, in: Siebert, Horst (Hg): "Migration: A Challange for Europe", Kiel.

BöRSCH-SUPAN, AXEL (1992 a): „Saving and Consumption Patterns of the elderly - The German Case", Journal of Population Economics 5, Seiten 289-330.

BÖRSCH-SUPAN, AXEL (1992 b): „Household Savings in Germany; Part 1: Incentives“, Diskussionspapier, Universität Mannheim.

BÖRSCH-SUPAN, AXEL (1992 c): „Household Savings in Germany; Part 2: Behaviour“, Diskussionspapier, Universität Mannheim.

BÖRSCH-SUPAN, AXEL (1992 d): „Der Wohnkonsum älterer Mitbürger: Wie lange selbständig? Wie oft in Mehrpersonenhaushalten?", in: Hujer, Reinhard; Schneider, Hilmar und Zapf, Wolfgang (Hg.): "Herausforderungen an den Wohlfahrtsstaat im strukturellen Wandel", Campus, Frankfurt, Seiten 143-172.

BöRSCH-SUPAN, AXEL (1992 e): „Population Aging, Social Security Design, and Early Retirement ", Journal of Institutional and Theoretical Economics (Zeitschrift für die gesamte Staatswissenschaft), Vol 148, Seiten 533-557.

BörSCH-SUPAN, AXEL (1991): „Aging Population“, Economic Policy 12, April, Seiten 103-139.

BÖRSCH-SUPAN, AXEL (1990): „Altersökonomik: Unsere Gesellschaft altert - Ökonomische Implikationen der grauen Revolution", Spektrum der Wissenschaft . 
BÖRSCH-SUPAN, AXEL (1989): „Houshold Dissolution and the Coice of Alternative Living Arrangements among Elderly Americans", in: Wise, David (Hg.); "The Economics of Aging", Seiten 119-150, University of Chicago Press, Chicago.

BÖRSCH-SUPAN, AXEL; MEIER, MATTHIAS UND REI-HELD, ANETTE (1994): „Macroeconomic Implications of Population Aging“, in: Bovenberg und van Ewijk (Hg.) "Pensions and Public Finance in an Aging Society" Oxford University Press, Oxford.

BÖRSCH-SUPAN, AXEL UND SCHMIDT, PETER (1995): „Early Retirement in East and West Germany“; in: D. Snower and K. Zimmermann (Hg.): „Employment Policy in the Transition to Free Enterprises - German Integration and its Lessons for Europe", London.

BÖRSCH-SUPAN, AXEL UND SCHMIDT, PETER (1994): „The Impact of the Public Pension System on Retirement Behavior in Unified Germany“, in: DIW Vierteljahreshefte zur Wirtschaftsforschung 1/2, Seite 90-96.

Börsch-SUPAN, AXEL UND STAHL, KONRAD (1991): „Life Cycle Savings and Consumption Constraints: Theory, Empirical Evidence, and Fiscal Implications“, in: Journal of Population Economics 4, Seiten 233-255.

BRAUN, Roland UND KNOEDEl, PETER (1982): „Die Arbeitsmarktlage und die Gewährung von Renten wegen Berufs- und Erwerbsunfähigkeit im Spiegel der Statistik“, Deutsche Rentenversicherung 8, Seiten 448-472.

BREYER, FRIEDRICH (1990): „Ökonomische Theorie der Altersicherung“, Verlag Vahlen, München.

BRUGIAVINI, AGAR; DisNey, RichaRd UND WHITEHOUSE, EDWARD (1993): „Choice of Pension Arrangements under Uncertainity in the UK“, Arbeitspapier zur Präsentation auf dem RES Seminar (Session Pensions and Retirement), Lomdon.

BUNDESANSTALT FÜR ARBEIT (o.J.): „Amtliche Nachrichten der Bundesanstalt für Arbeit“, Periodicum.

BUNDESMINISTER FÜR ARBEIT UND SOZIALORDNUNG (BMA) (1980): „Leitfaden zum 21. RAG“, Bonn.

BUNDESMINISTER FÜR ARBEIT UND SOZIALORDNUNG (BMA) (1989): „Die Rentenbestände in der Rentenversicherung der Arbeiter und Angestellten", Bonn.

BUNDESMINISTER FÜR ARBEIT UND SOZIALORDNUNG (BMA) (1993): „Die Rente 93“, Bonn.

BUNDESMINISTER FÜR ARBEIT UND SOZIALORDNUNG (BMA) (1990 a): „Rentenreform 92 ... denn eins ist sicher: die Rente“, Bonn.

BUNDESMINISTER FÜR ARBEIT UND SOZIALORDNUNG (BMA) (1990 b): „Rentenreformgesetz 1992 Textausgabe“, Bonn.

BUNDESMINISTER FÜR ARBEIT UND SOZIALORDNUNG (BMA) (1990 c): „Die Überführung der Rentenversicherung im Gebiet der ehemaligen DDR“, Faltblatt, Bonn.

BUNDESMINISTER FÜR ARBEIT UND SOZIALORDNUNG (BMA) (Hg.) (1991): „Übersicht über die Soziale Sicherheit", Bonn.

BUNDESMINISTERIUM FÜR FAMILIE UND SENIOREN (1994): „Die Alten der Zukunft - Bevölkerungsstatistische Datenanalyse“, Schriftenreihe, Band 32; Stuttgart.

BURKHAUSER, RICHARD UND QUINN, JOSEPH (1989): „American Patterns of Work and Retirement", in: Schmähl, Winfried: "Redefining the Process of Retirement", Springer-Verlag, Berlin, Heidelberg u.a., Seiten 109-131. 
BUSLEI, HERMANN (1995): „Vergleich langfristiger Bevölkerungsvorausberechnungen für Deutschland", ZEW-Dokumentation 95-01, Zentrum für Europäische Wirtschaftsforschung, Mannheim.

BUSLEI, HeRMANN UND SCHMIDT, PETER (1994): „Staatliche Alterssicherung im demographischen Wandel“, ZEW-Newsletter Nr. 2 - Dezember 1994, Zentrum für Europäische Wirtschaftsforschung, Mannheim, Seiten 15-20.

CASMIR, BERND (1989): „Staatliche Rentenversicherungssysteme im internationalen Vergleich“, Verlag Peter Lang, Frankfurt.

CDU/CSU, FDP \& SPD (1989): „Entwurf eines Gesetzes zur Reform der Gesetzlichen Rentenversicherung (Rentenreformgesetz 1992 - RRG 1992)“, Bundestagsdrucksache 11/4124 v. 7.3.89.

ClarK, RoberT; KREBS, JuANITA AND SPENGleR, JosePH (1978): „Economics of Aging: A Survey“, Journal of Economic Literature XVI, Seiten 919-962.

DEUTSCHES INSTITUT FÜR WIRTSCHAFTSFORSCHUNG (DIW) (1993): „Bevölkerungsentwicklung in Deutschland bis zum Jahr 2010 mit Ausblick auf 2040", DIW Wochenbericht 29, Seiten 393-404.

DEUTSCHE BUNDESBANK (1984): „Betriebliche Altersversorgung in der Bundesrepublik Deutschland“, in: Monatsberichte der Deutschen Bundesbank 8/84, Seiten 30-37.

DiAMOND, PETER (1965): „National Debt in a Neoclassical Growth Model“, The American Economic Review 55, Seiten 1126-1150.

DINKEL, REINER UND LEBROK, UWE (1993): „Können durch Zuwanderungen die Alterung der Bevölkerung und die daraus resultierenden Zusatzlasten gemildert werden?“،, Deutsche Rentenversicherung 6, Seiten 388-400.

DÜRKOP, HARALD (1992): „Alterssicherung in der EG“, Verlag Peter Lang, Frankfurt.

DUDEY, STEFAN (1993): „Die langfristige Entwicklung der Rentenversicherung“, Wirtschaftsdienst 7, Seiten 363-368.

ECKERLE, K., BARTH, H.J.; HOFER, P. UND SCHILING, K. (1987): „Gesamtwirtschaftliche Entwicklungen und Gesetzliche Rentenversicherung vor dem Hintergrund einer schrumpfenden Bevölkerung “, Prognos-Studie, 2 Bände, Basel.

FELDERER, BERNHARD (1992): „Can Immigration Policy Help to Stabilize Social Security Systems?", Diskussionspapier für die ERP-Konferenz "Economics of Ageing", Wien.

FELDERER, BERNHARD (Hg.) (1987): „Kapitaldeckungsverfahren versus Umlageverfahren“, Verlag Duncker \& Humblot, Berlin.

FELDSTEIN, MARTIN (1974): „Social Security, Induced Retirement, and Aggregate Capital Accumulation“, Journal of Political Economy 96, Seiten 983-1004.

FeldDSTEIN, MARTIN (1976): „Social Security and Saving, the Extended Life Cycle Theory“, The American Economic Review 66, Seiten 77-86.

FeldSTEIN, MARTIN (1982): „Social Security and Private Savings: Reply“, Journal of Political Economy 90, Seiten 630-642.

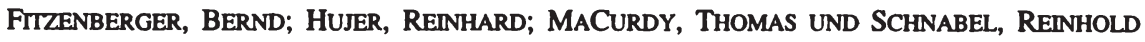
(1994): „Macroeconomic and Microeconomic Wage trends: The Case of West-Germany 1976-1984“, Diskussionspapier, Universität Mannheim.

FRANZ, WOLFGANG (1991): „Arbeitsmarktökonomik“, Springer Verlag, Berlin u.a.

FRERICH, J (1987): „Sozialpolitik“, Oldenbourg-Verlag, München. 
GlOMBIK, MANFRED (1988): „Das System der Sozialversicherung in der Bundesrepublik Deutschland “, Wirtschaftswissenschaftliches Studium, Heft 1.

Goethe, Johann WolfGang von (1951): „Faust - der Tragödie erster Teil“, Droste Verlag, Düsseldorf.

GORDON, MARGARET S. (1988): „Social Security policies in industrial Countries - A comparative Analysis“, Cambridge University Press, Cambridge.

GROHMANN, HEINZ (1980): „Rentenversicherung und Bevölkerungsprognosen“, in: Schriftenreihe des Sonderforschungsbereiches 3 der Universitäten Frankfurt und Mannheim: "Mikroanalytische Grundlagen der Gesellschaftspolitik", Campus Verlag, Frankfurt.

GRUBER, JONATHAN UND MADRIAN, BRIGITTE C. (1993): „Health Insurance Availability and the Retirement Decision“, NBER Working Paper Series, Working Paper No. 4469.

HAHNEFELD, UTE (1987): „Das Sozio-ökonomische Panel - Grundlagen und Konzeption“, Frankfurt, New York.

HAMERLE, ALFRED UND TUTZ GerHARD (1988): „Diskrete Modelle zur Analyse von Verweildauer und Lebenszeiten“, Campus Verlag, Campus Forschung Band 568, Frankfurt, New York.

HEINELT, HUBERT (1991): „Frühverrentung als politischer Prozeß“, Deutscher Universitätsverlag, Wiesbaden.

Heller, Peter S.; Hemming, Richard und Kohnert, Peter W. (1986): „Aging and Social Expenditure in the Major Industrial Countries, 1980-2025“, International Monetary Fund, Washington D.C.

HEMMER, EDMUND (1984): „Die betriebliche Altersversorgung“, Deutscher Institutsverlag (IdW), Köln.

HIRVONEN, PETRI (1993): „Alterssicherung und Alterseinkommensverteilung - Eine empirische Analyse der Einkommenslage der älteren Bevölkerung in der Bundesrepublik Deutschland", Campus, Frankfurt.

HoFFMANN, JoHANNES (1989): „Sparen im Dienst der Altersvorsorge als volkswirtschaftliches Problem“, Institut für Wirtschaftspolitik an der Universität zu Köln.

HOMBURG, STEFAN (1988): „Theorie der Alterssicherung“, Springer Verlag, Berlin.

HOMBURG, STEFAN UND RICHTER, WolfRAM (1990): „Eine effizienzorientierte Reform der GRV“, in: Felderer, Bernhard (Hg.): "Bevölkerung und Wirtschaft", Duncker \& Humblot, Berlin.

HuRD, Michael D. (1990): „The Joint Retirement Decision of Husbands and Wifes“, in: Wise, David (Hg.); "Issues in the Economics of Aging", Cambridge University Press, Cambridge, Seiten 231-258.

JACOBS, KLAUS (1990): „Der Übergang in den Ruhestand“, Wirtschaftsdienst III.

JACOBS, Klaus; Kohli, MARTIN UND REIN, MARTIN (1992): „Germany: The Diversity of Pathways", in: Kohli, Rein, Guillemard and van Gunsteren (Hg.) "Time for Retirement", Cambridge University Press, Cambridge/New York, Seiten 181 - 221.

JÄHNKE, BIRGIT (1993): „Verrentungsanreize der betrieblichen Altersversorgung“, Diplomarbeit, Universität Mannheim, unveröffentlicht.

JÄHNKE, BIRGIT (1994): „Gesamtprofile der Verrentungsanreize aus betrieblicher und gesetzlicher Altersversorgung“, unveröffentlichter Arbeitsbericht, Universität Mannheim, mimeo. 
JAHNKE, WILFRIED (1990): „Wirkungen der Bevölkerungsentwicklung bis zum Jahre 2000“, in: Felderer, Bernhard (Hg.): "Bevölkerung und Wirtschaft", Duncker \& Humblot, Berlin.

KALBFleisCH, J.D. UND PRENTICE, R.L. (1980): „The statistical Analysis of Failure Time Data“, New York, Chichester, Brisbane, Toronto.

KAMIEN, MORTON AND SCHWARTZ, NANCY (1981): „Dynamic Optimization: The Calculus of variations and Optimal Control in Economics and Management", North Holland.

KERWAT, MARTIN (1983): „Der Rentenzugang der Angestelltenversicherung im Jahr 1982“, Deutsche Angestelltenversicherung 5, Seiten 187-190.

KERWAT, MARTIN 1984 (1984): „Der Rentenzugang 1983“, Deutsche Angestelltenversicherung 5, Seiten 250-254.

KIM, SANGHO (1992): „Sozialversicherungskapital und das Sparen der privaten Haushalte in der Bundesrepublik Deutschland“, Verlag Dr. Kovac, Hamburg.

KLEINHENZ, GERHARD (Hg.) (1991): „Sozialpolitik im vereinten Deutschland I“, Duncker und Humblot, Berlin.

KNOEDEL, PETER (1985): „Das Berentungsgeschehen nach dem Haushaltsbegleitgesetz1984“, Aus der Statistik der deutschen gesetzlichen Rentenversicherung Seite: 181-194.

KoHLI, MARTIN UND REIN, MARTIN (1991): „The Changing Balance of Work and Retirement“, in: Kohli, Rein, Guillemard und van Gunsteren (Hg.) "Time for Retirement", Cambridge University Press, Cambridge/New York, Seiten 1-35.

KoHli, M. ;REIN, M.; Gulllemard A.-M. und van Gunsteren, H. (Hg.) (1991): „Time for Retirement: Comparative Studies of Early Exit from the Labor Force", Cambridge University Press, Cambridge/New York.

KOMMISSION DER EG (1989): „Vergleichende Darstellung der Systeme der sozialen Sicherheit in den Mitgliedstaaten der EG“, 15. Auflage Brüssel-Luxemburg.

KotuIKofF, LAURENCE J. AND Wise, DAVID (1988): „Employee Retirement and a Firms Pension Plan“, Wise, David (Hg.), "The Economics of Aging", University of Chicago Press, Chicago, Seiten 279-333.

KOTLIKOFF, LAURENCE J.; WISE, DAVID A. (1985): „Labor compensation and the structure of private pension plans: Evidence for contractual versus Spot labor markets", In Wise, David (Hg.): "Issues in the Economics of Aging", NBER, Chicago.

KRUEGER A.B. AND PISCHKE, J-S. (1991): „The Effect of Social Security on labor Supply: a Cohort Analysis of the Notch Generation", National Bureau of Economic Research, Working Paper No. 3699.

LAMPERT, HEINZ (1985): „Lehrbuch der Sozialpolitik“, Springer Verlag, Berlin.

LAZEAR, EDWARD (1988): „The Pension Inducement to Retire: An Option Value Analysis -Comment", in: Wise, David (Hg.); "Issues in the Economics of Aging", Cambridge University Press, Cambridge, Seiten 224 - 229.

LEHR, UrSUla (1987): „Psychologie des Alterns“, Quelle und Meyer, 6. Auflage.

LEIBFRTI, WILlY; KRUMPER, ARTHUR; NIERHAUS, WOLFGANG UND PARSCHE, RÜDIGER (1986): „Sicherung der Altersvorsorge durch Aufgabenverteilung zwischen Individualversicherung und Rentenversicherung “, Ifo-Studien zur Finanzpolitik 37, München.

LÖTSCH, MANFRED (1992): „Systemtransformation und soziale Strukturbrüche in der (ehemaligen) DDR“, in: Schmähl, Winfried: "Sozialpolitik im Prozess der deutschen Vereinigung", Campus Verlag, Frankfurt. 
LuMSDAINe, Robin L., STOCK, JAMES H. UND WiSE, DAVId A. (1990): „Efficient Windows and Labor Force Reduction", Journal of Political Economics 43.

LUMSDANE, ROBIN L.; STOCK, JAMES H. UND WISE, DAVID A. (1993): „Pension plan provisions and retirement: men \& women, medicare, and models“, NBER Working Paper Series, Working Paper.

LUMSDAINE, Robin L; STOCK, JAMES H. UND WISE, DAVID A. (1992): „Three Models of Retirment: computational Complexity versus Predictive Validity", in: Wise, David (Hg.); "Topics in the Economics of Aging", Cambridge University Press, Cambridge, Seiten 19-60.

MADDALA, G.S. (1983): „Limited Dependent and Qualitative Variables in Econometrics“, Cambridge University Press, Cambridge.

MAIER, GUNTHER UND WEISS, PETER (1990): „Modelle diskreter Entscheidungen -- Theorie und Anwendung in den Sozial- und Wirtschaftswissenschaften“, Springer Verlag, Wien, New York etc.

MAYDELL, BERND vON (1986): „Lexikon des Rechts“, Neuwied, Darmstadt.

MCFADDEN, DANIEL (1989): „A Method of Simulated Moments for Estimation of Discrete Response Models without numerical integration“, Econometrica 57, Seiten 995 - 1026.

MEGHIR, COSTAS UND WHITEHOUSE, EDWARD (1993): „The Job Exit Behaviour of Older Men in the UK: Evidence from Event History Data“, Institute for Fiscal Studies and University College, London.

Mrtchell, Olivia S. AND Fields, GaRy S. (1984): „The Economics of Retirement Behavior“, Journal of Labor Economics 2.

MÖRSCHEL, R. UND REHFELD, U. (1981): „Untersuchungen der Rentenzugänge im Zeitablauf, Teil I“, Deutsche Rentenversicherung 4, Seiten 234-253.

MÖRSCHEL, R. UND REHFELD, U. (1982): „Untersuchungen der Rentenzugänge im Zeitablauf, Teil II“, Deutsche Rentenversicherung, 8, Seiten 448-472.

MÜLlER, HORST-WOLF UND ROPPEL, ULRICH (1990): „Eine Abschätzung des Kapitalbedarfes bei einer vollständigen Kapitaldeckung der Gesetzlichen Rentenversicherung“", in: Felderer, Bernhard (Hg.): "Bevölkerung und Wirtschaft", Duncker \& Humblot, Berlin.

MülLER, H.W. (1987): „Zur Verkürzung der Rentenlaufzeiten- Möglichkeiten und finanzielle Konsequenzen“, Deutsche Rentenversicherung, Seite: 30-55.

MURRAY, ROBERT F (1968): „Economic aspects of pensions: A summary report“, NBER Working Paper Series, Working Paper.

OECD (1977): „Old Age Pension Schemes“, Paris.

OECD (1992): „Private Pensions and Public Policy “, OECD, Paris.

OECD (Hg.) (1988 a): „Reforming Public Pensions“, OECD Social Policy Studies No. 5.

OECD (1988 b): „Ageing Population: The Social Policy Implications“, OECD, Paris.

ORBACH, HAROLD UND TibBITS, ClaRK (Hg.) (1963): „Aging and the Economy“, Ann Arbor, University of Michigan Press.

PISCHNER, RAINER UND WAGNER, GERT (1992): „Zwei Aspekte der Flexibilität beim Übergang vom Erwerbsleben in den Ruhestand“, in: Hujer, Reinhard; Schneider, Hilmar und Zapf, Wolfgang (Hg.) "Herausforderungen an den Wohlfahrtsstaat im strukturellen Wandel, Campus Verlag, Frankfurt, Seiten 143-172. 
PRESSE- UND INFORMATIONSAMT DER BUNDESREgIERUNG (1993): „Almanach der Bundesregierung 1993/94“, Bonn.

RAFFELHÜSCHEN, BERND UND KITTERER, WOLFGANG (1990): „Übergangsprobleme eines Systemwechsels in der sozialen Alterssicherung. Eine dynamische Systemanalyse“, in: Felderer, Bernhard (Hg.): "Bevölkerung und Wirtschaft", Duncker \& Humblot, Berlin.

REHFELD, UWE (1994): „VerrentungsprozeB und versicherungsbiographische Fakten für Geburtsjahrgangskohorten - Analyse der Rentenzugangsdaten 1973 bis 1990 für Versichertenrenten“, Deutsche Rentenversicherung, 7, S. 471-526.

REHFELD, UWE (1991): „Das Rentenzugangsgeschehen im Zeitablauf“, Deutsche Rentenversicherung, 10-11, Seite: 682-712.

REIMANN, AXEL (1985): „Trend zur Frühberrentung noch ungebrochen“, Deutsche Angestelltenversicherung, 10, Seite: 406-413.

RIPHAHN, REGINA UND SCHMIDT, PETER (1995): „Die langfristige Entwicklung der Gesetzlichen Rentenversicherung“, Discussion Paper No. 95-10, Zentrum für Europäische Wirtschaftsforschung, Mannheim.

RIPHAN, REgINA (1995): „Disability Retirement Among German Men in the 1980s“, Dissertation, University of North Carolina.

ROHWER, GÖTZ (1994): „Parametric Transistion Rate Models“, in: "TDA: A statistical Program for Transition Data Analysis", Working Paper 5-3, Universität Bremen.

RONNING, GERD (1991): „Mikroökonometrie“, Springer Verlag, Berlin, Heidelberg.

RULAND, FRANZ (1989): „Langfristig erzielbare Leistungen aus der Gesetzlichen Rentenversicherung in einzelwirtschaftlicher Betrachtung", in: Zeitschrift für Versicherungswissenschaft; Seite: 591-611.

RULAND, FRANZ UND MAYDELL, BERND vON (1989): „Sozialrechtshandbuch“, Neuwied, Darmstadt.

RUST, JOHN (1990): „A Dynamic Programming Model of Retirement Behavior“, in: Wise, David (Hg.) "Issues in the Economics of Aging", University of Chicago Press, Chicago.

SACHVERSTÄNDIGENKOMMISSION ALTERSSICHERUNGSSYSTEME (1983): „Vergleich der Alterssicherungssysteme und Empfehlungen der Kommission", Gutachten der Sachverständigenkommission, Band 1, Bonn.

SAMUELSON, P.A. (1958): „An Exact Consumption - Loan Model of Interest With or Without the Social Contrivance of Money“, Journal of Political Economy 66, Seiten 467-482.

SCHMÄHL, WINFRIED (1992 AIN): „Sozialpolitik im Prozeß der deutschen Vereinigung“, Campus Verlag, Frankfurt.

SCHMÄHL, WINFRIED (1992 b): „Public Pension Schemes in Transition: Germany's Way to cope with the Challenge of an Ageing Population and the German Unification", Paper to be presented at the ERP Conference on Economics of the Aging, Barcelona.

SCHMÄHL, WINFRIED (1991 a): „The Future of Basic and Supplementary Pension Schemes in the European Community-1992 and beyond“", Nomos Verlagsgesellschaft, Baden-Baden.

SCHMÄHL, WINFRIED (1991 b): „On the Future Development of Retirement in Europe. Especially of Supplementary Pensio Schemes - An introductory Overview“, in: Schmähl, Winfried, "The Future of Basic and Supplementary Pension Schemes in the European Community-1992 and beyond", Nomos Verlagsgesellschaft, Baden-Baden. 
SCHMÄHL, WINFRIED (1991 c): „Alterssicherung in der DDR und ihre Umgestaltung im Zuge des deutschen Einigungsprozesses - einige verteilungspolitische Aspekte“, in: Kleinhenz, Gerhard: "Sozialpolitik im vereinten Deutschland I"; Duncker und Humblot, Berlin.

SCHMÄHL, WINFRIED (Hg.) (1989): „Redefining the Process of Retirement - An International Perspective", Springer Verlag, Berlin, Heidelberg u.a.

SCHMÄHL, WINFRIED (1988): „Beiträge zur Reform der Rentenversicherung“, Tübingen.

SCHNEIDER, HIMMAR (1991): „Verweildaueranalyse mit GAUSS“, Campus.

SCHNIEWIND, HANS JÜRGEN (1989): „Gesetzliche Rentenversicherung und Konsum - Eine Untersuchung zur Situation in der Bundesrepublik Deutschland“, Rudolf Haufe Verlag, Freiburg.

SCHOLZ, PETER (1993): „Ausgestaltung und Reform der gesetzlichen Alterssicherung“, Dissertation, Dortmund.

SchulZ, James H. (1988): „The Economics of Aging“, Auburn House Publishing Company, Dover, 4. Auflage.

SCHUNTERMANN, MiCHAEL F. (1986): „Das Berrentungsrisiko wegen Erwerbsminderung:Begriff, Struktur und Entwicklung in der Zeit von 1973-1982", Deutsche Rentenversicherung 3/4, Seiten 237-256.

SCHWTTZER, KLAUS (1990): „Zur sozialen Lage von AltersrentnerInnen in der DDR vor der Währungs-, Wirtschafts- und Sozialunion“, WSI-Mitteilungen 8.

SNOWER, DENNIS J. UND ZIMMERMANN, KLAUS (Hg.) (1994): „Employment Policy in the Transition to Free Enterprise - German Intergrations and its Lessons for Europe“, London.

SOMMER, BETTINA (1994): „Entwicklung der Bevölkerung bis 2040“ Wirtschaft und Statistik 7 , Seiten 497-503.

SOMMER, BETTINA (1992): „Entwicklung der Bevölkerung bis 2030“ Wirtschaft und Statistik 4, Seiten 217-222.

STAHL, KONRAD (1989): „Housing Patterns and Mobility of the Aged: The United States and West Germany“, in: Wise, David (Hg.); "The Economics of Aging", Seiten 93-118, University of Chicago Press, Chicago.

STATISTISCHES BundeSAMT (1991): „Im Blickpunkt: Ältere Menschen“, Metzler-Poeschel, Stuttgart.

STATISTISCHES BUNDESAMT (diverse Jahrgänge): „Statistisches Jahrbuch“, Metzler-Poeschel, Stuttgart.

STATISTISCHES BUNDESAMT (diverse Jahrgänge): „Statistisches Jahrbuch für die Bundesrepublik Deutschland“, Metzler-Poeschel, Stuttgart.

STATISTISCHES BundeSAMT (Hg.) (1990): „Familien Heute - Strukturen, Verläufe und Einstellungen“, Metzler-Poeschel Verlag Stuttgart.

STIFTUNG WARENTEST (1992): „Richtig Vorsorgen“, Reihe: "Ratgeber Geld”, Stiftung Warentest, Berlin.

StOCK, JAmes H. AND WiSE, DAVID A. (1990 a): „Pensions, the Option Value of Work, and Retirement", Ecomometrica 58.

Stock, James H. AND WiSe, DAvid A. (1988): „The Pension Inducement to Retire: An Option Value Analysis“, in: Wise, David (Hg.): "Issues in the Economics of Aging", Seiten 205230. 
SUEYOSHI, GLENN T. (1989): „Social Security and the Determinants of Full and Partial Retirement: A Competing Risk Analysis“, National Bureau of Economic Research, Working Paper No. 3113.

TEGTMEIER, WERNER (1989): „Die Gesetzliche Rentenversicherung in der Gesamtwirtschaft“, in: Zeitschrift für Versicherungswissenschaft; Seiten: 647 - 657.

THELEN, KARL-PETER (1990): „Ökonomische Analyse der betrieblichen Alterssicherung“, Verlag Josef Eul.

THELEN, KARL-PETER (1990): „Pensionsrückstellungen: Zur Diskussion um eine steuerliche Vergünstigung des Arbeitgebers“, in: Der Betrieb 43, Seiten $437 \mathrm{ff}$.

VENTI, STEVEN UND WISE, DAVID (1990): „But they Dont want to Reduce Housing Equity“, in: Wise, David (Hg.): "Issues in the Economics of Aging", University of Chicago Press, Chicago, Seiten 13-43.

VERBAND DEUTSCHER RENTENVERSICHERUNGSTRÄGER (VDR) (1992): „Fragen und Antworten zur Rentenversicherung in den neuen Bundesländern“, Broschüre, VDR, Frankfurt.

VERBAND DEUTSCHER RENTENVERSICHERUNGSTRÄGER (VDR) (1992): „Rentenversicherung in Zeitreihen“, VDR, Frankfurt.

VERBAND DEUTSCHER RENTENVERSICHERUNGSTRÄGER (VDR) (diverse Jahrgänge): „Statistik Rentenzugang", VDR, Frankfurt.

WiSE, DAVID (Hg.) (1985): „Pension, Labor, and Individual Choice“, University of Chicago Press, Chicago.

WISE, DAVID (Hg.) (1989): „The Economics of Aging“, University of Chicago Press, Chicago.

WISE, DAVID (Hg.) (1990): „Issues in the Economics of Aging“, NBER, University of Chicago Press, Chicago.

ZIMMERMANN, HANS GeORG (1988): „Privates Sparen versus Sozialversicherung“, Springer, Berlin.

ZWEMMÜLLER, JOSEF; WINTER-EBMER, RUDOLF UND FALKINGER, JOSEF (1993): „Joint retirement of Spouses and Social Security Reform", Arbeitspapier, Universität Linz. 
Oh glïcklich, wer noch hoffen kann, Aus diesem Meer des Irrtums aufzutauchen, Was man nicht weiß, das eben brauchte man Und was man weiß, das kann man nicht gebrauchen.

\section{SCHLAGWORTINDEX}

3

3-Säulen Modell der Rentenversicherung 33

$\boldsymbol{A}$

absorbierender Endzustand 128

aktueller Rentenwert 61

Allgemeine Bemessungsgrundlage (BG) 61

Altersgrenzen 58

Alterslastquotient 8

Altersruhegeld

flexibles 38

flexibles für Schwerbehinderte, Berufs- und Erwerbsunfähige 39

hinausgeschobenes 38

vorgezogenes 38

vorzeitiges für Frauen 38

vorzeitiges wegen Arbeitslosigkeit 38

Anpassungsfaktoren für

versicherungsmathematische Fairne $B \mathbf{7 2}$

Anrechnungsfähige Versicherungsjahre

(Vj) 60

Anrechnungszeiten 59

Arbeitslosenversicherung 53

Arbeitslosigkeit 38

Auffüllbetrag 67

Ausfallzeiten 38; 59

Austrittsalter im SOEP 160

$\boldsymbol{B}$

Berücksichtigungszeiten 59

Berufsunfähigkeit 39

betriebliche Altersversorgung $45 ; 143$

Betriebsrenten 45

Betriebsrentengesetzes 45

BfA 36

Bundeszuschuß 59
BU-Rente 39

D

DDR-Rentenversicherung 62

Dependency-Ratio 1;8

Dichtefunktion 146

Discrete Choice Modelle 110; 144

Double Aging 5

Duration-Modell 144

Durchschnittsentgelt 60

Dynamische Programmierung 115

Dynamisierung 42; 61

$\boldsymbol{E}$

Einkommen 140

Einwanderungen 19

Endzustand, absorbierender 128

Entgeltpunkte 60

Ersatzquote 23

Ersatzzeiten 38

Ersparnisbildung 13

Erwerbspersonen 90

Erwerbsquoten 91

Erwerbsunfähigkeit 39

EU-Rente 39

Exponentialmodell 149

$\boldsymbol{F}$

Feldstein-Barro 12

flexibler Ruhestand $15 ; 28 ; 38$

Freiwillige 62

freiwillige Versicherung 37

Frühverrentung 39

$\boldsymbol{G}$

Geburtenziffern 7

Generationenvertrag 30

Gesundheit als Einflußfaktor 142

Gompertz Modell 150 
H

Halbbelegung 39

Hazardrate 147 empirische $121 ; 148$ theoretische 148

Hazardratenmodelle 120

Hinterbliebenenrenten 38

$\boldsymbol{K}$

Kapitaldeckungsverfahren 30

Kindererziehungszeit 59

Kohorteneffekt 16

Krankenversicherung 52

Krankenversicherung der Rentner 52

L

Lastquotienten 6

latent Versicherte 38

Lebensversicherung 51

Lebenszyklusmodell 10

Leistungsarten 38; 84

Likelihood Ratio 153

Linkszensur 161

Logit-Modell 110

LVA 36

M

Maximum-Likelihood Gütemaß 153

$\boldsymbol{N}$

Nettoanpassung 59

o

Optionswert 128; 154

Definition 155

Optionswertmodelle $127 ; 132$

$\boldsymbol{P}$

Persönlicher Vom-Hundert-Satz (Vhs) 60

Pflichtversicherte 37

Probit-Modell 110

Proportional Hazard Modelle 150

$\boldsymbol{R}$

Rechtszensur 161

Regelrentenalter 15

Regelrentenalters 38

Regression 144

Renten nach Mindesteinkommen 54; 57

Rentenalter

Vergleich BRD - USA 15
Rentenangleichungsgesetz 67

Rentenarten 38

Rentenartfaktor 60

Rentenformel 59

aktueller Rentenwert 61

Allgemeine Bemessungsgrundlage (BG) 42; 61

Anrechnungsfähige Versicherungsjahre (Vj) 42

Anrechnungsfähige Versicherungsjahre (Vj) 60

Durchschnittsentgelt 60

Entgeltpunkte 60

Persönlicher Vom-Hundert-Satz (Vhs) 42; 60

Rentenartfaktor (St) 60

Steigerungssatz (St) 42; 60

Zugangsfaktor 61

Rentenniveau 22

Rentenversicherungsträger 36

Rentenzugang von Frauen 169

Rentenzugangsalter 38

Risikoausgleich 31

Ruhestandsmodelle 109

$S$

Schätzgüte für Maximum-Likelihood-

Schätzungen 153

Schwankungsreserve 22; 30

Schwerbehinderung 39

Selbständige 37

Sicherungsziel 34

Simulationsrechnungen 199

Sozialhilfe 54

Sozialversicherungspflicht 36

Sozialversicherungspflichtig Beschäftigte 90

Sozialzuschläge 68

Sozioökonomische Panel (SOEP) 158

Standardrentner 24

Steuerliche Behandlung von Leistungen und Beiträgen 54

Subsidiaritätsprinzip 34

$\boldsymbol{T}$

Teilverrentung 59

ปे

Übergangsratenmodell 144

Überlappende Generationen Modell 12

Überlebensfunktion 146; 147 
$\boldsymbol{U}$

Umlageverfahren 22; 29

Umverteilung 31

Unverfallbarkeit 45

$\boldsymbol{V}$

VBL 36

VDR $36 ; 77$

Vermögen 141

Verrentungsalter

Definition im Datensatz 158

Verrentungsfenster $28 ; 38 ; 57$

Verrentungsraten $77 ; 81 ; 92 ; 94$

Versichertenrenten 38

versicherungsmathematisch fair 70

Verteilungsfunktion 146
Verweildauermodell 144

Vorruhestandsmodell DDR 69

Vorruhestandsregelung 40; 53

W

Wahrscheinlichkeitsmodelle zeitdynamische 114 zeitkonstante 110

Wartezeiten 38

Wege in den frühen Ruhestand 28; 39

Weibull Modell 152

Z

Zugangsfaktor 61

Zurechnungszeit 39

Zwangsversicherung 30 


\section{STAATLICHE ALLOKATIONSPOLITK IM MARKTWIRTSCHAFTLICHEN SYSTEM}

Band 1 Horst Siebert (Hrsg.): Umweltallokation im Raum. 1982.

Band 2 Horst Siebert (Hrsg.): Global Environmental Resources. The Ozone Problem. 1982.

Band 3 Hans-Joachim Schulz: Steuerwirkungen in einem dynamischen Unternehmensmodell. Ein Beitrag zur Dynamisierung der Steuerüberwălzungsanalyse. 1981.

Band 4 Eberhard Wille (Hrsg.): Beitrăge zur gesamtwirtschaftlichen Allokation. Allokationsprobleme im intermediären Bereich zwischen öffentlichem und privatem Wirtschaftssektor. 1983.

Band 5 Heinz König (Hrsg.): Ausbildung und Arbeitsmarkt. 1983.

Band 6 Horst Siebert (Hrsg.): Reaktionen auf Energiepreissteigerungen. 1982.

Band 7 Eberhard Wille (Hrsg.): Konzeptionelle Probleme offentlicher Planung. 1983.

Band 8 Ingeborg Kiesewetter-Wrana: Exporterlösinstabilităt. Kritische Analyse eines entwicklungspolitischen Problems. 1982.

Band 9 Ferdinand Dudenhöfer: Mehrheitswahl-Entscheidungen über Umweltnutzungen. Eine Untersuchung von Gleichgewichtszustănden in einem mikroökonomischen Markt- und Abstimmungsmodell. 1983.

Band 10 Horst Siebert (Hrsg.): Intertemporale Allokation. 1984.

Band 11 Helmut Meder: Die intertemporale Allokation erschöpfbarer Naturressourcen bei fehlenden Zukunftsmărkten und institutionalisierten Marktsubstituten. 1984.

Band 12 Ulrich Ring: Öffentliche Planungsziele und staatliche Budgets. Zur Erfüllung öffentlicher Aufgaben durch nicht-staatliche Entscheidungseinheiten. 1985.

Band 13 Ehrentraud Graw: Informationseffizienz von Terminkontraktmărkten für Wăhrungen. Eine empirische Untersuchung. 1984.

Band 14 Rüdiger Pethig (Ed.): Public Goods and Public Allocation Policy. 1985.

Band 15 Eberhard Wille (Hrsg.): Öffentliche Planung auf Landesebene. Eine Analyse von Planungskonzepten in Deutschland, Österreich und der Schweiz. 1986.

Band 16 Helga Gebauer: Regionale Umweltnutzungen in der Zeit. Eine intertemporale Zwei-Regionen-Analyse. 1985.

Band 17 Christine Pfitzer: Integrierte Entwicklungsplanung als Allokationsinstrument auf Landesebene. Eine Analyse der offentlichen Planung der Länder Hessen, Bayern und Niedersachsen. 1985.

Band 18 Heinz König (Hrsg.): Kontrolltheoretische Ansätze in makroökonometrischen Modellen. 1985.

Band 19 Theo Kempf: Theorie und Empirie betrieblicher Ausbildungsplatzangebote. 1985.

Band 20 Eberhard Wille (Hrsg.): Konkrete Probleme öffentlicher Planung. Grundlegende Aspekte der Zielbildung, Effizienz und Kontrolle. 1986.

Band 21 Eberhard Wille (Hrsg.): Informations- und Planungsprobleme in öffentlichen Aufgabenbereichen. Aspekte der Zielbildung und Outputmessung unter besonderer Berücksichtigung des Gesundheitswesens. 1986.

Band 22 Bernd Gutting: Der Einfluß der Besteuerung auf die Entwicklung der Wohnungs- und Baulandmärkte. Eine intertemporale Analyse der bundesdeutschen Steuergesetze. 1986.

Band 23 Heiner Kuhl: Umweltressourcen als Gegenstand internationaler Verhandlungen. Eine theoretische Transaktionskostenanalyse. 1987. 
Band 24 Hubert Hornbach: Besteuerung, Inflation und Kapitalallokation. Intersektorale und intemationale Aspekte. 1987.

Band 25 Peter Müller: Intertemporale Wirkungen der Staatsverschuldung. 1987.

Band 26 Stefan Kronenberger: Die Investitionen im Rahmen der Staatsausgaben. 1988.

Band 27. Armin-Detlef Rieß: Optimale Auslandsverschuldung bei potentiellen Schuldendienstproblemen. 1988.

Band 28 Volker Ulrich: Preis- und Mengeneffekte im Gesundheitswesen. Eine Ausgabenanalyse von GKV-Behandlungsarten. 1988.

Band 29 Hans-Michael Geiger: Informational Efficiency in Speculative Markets. A Theoretical Investigation. Edited by Ehrentraud Graw. 1989.

Band 30 Karl Sputek: Zielgerichtete Ressourcenallokation. Ein Modellentwurf zur Effektivitätsanalyse praktischer Budgetplanung am Beispiel von Berlin (West). 1989.

\section{ALLOKATION IM MARKTWIRTSCHAFTLICHEN SYSTEM}

Band 31 Wolfgang Krader: Neuere Entwicklungen linearer latenter Kovarianzstrukturmodelle mit quantitativen und qualitativen Indikatorvariablen. Theorie und Anwendung auf ein mikroempirisches Modell des Preis-, Produktions- und Lageranpassungsverhaltens von deutschen und französischen Unternehmen des verarbeitenden Gewerbes. 1991.

Band 32 Manfred Erbsland: Die öffentlichen Personalausgaben. Eine empirische Analyse für die Bundesrepublik Deutschland. 1991.

Band 33 Walter Ried: Information und Nutzen der medizinischen Diagnostik. 1992.

Band 34 Anselm U. Römer: Was ist den Bürgern die Verminderung eines Risikos wert? Eine Anwendung des kontingenten Bewertungsansatzes auf das Giftmüllrisiko. 1993.

Band 35 Eberhard Wille, Angelika Mehnert, Jan Philipp Rohweder: Zum gesellschaftlichen Nutzen pharmazeutischer Innovationen. 1994.

Band 36 Peter Schmidt: Die Wahl des Rentenalters. Theoretische und empirische Analyse des Rentenzugangsverhaltens in West- und Ostdeutschland. 1995. 